\title{
Structure and Evolution of Star Clusters in the Vicinity of the Magellanic Clouds
}

\author{
by \\ Timothy Stuart Banks
}

A thesis

submitted to the Victoria University of Wellington

in fulfilment of the

requirements for the degree of

Doctor of Philosophy

in Physics.

(C) Victoria University of Wellington

December 1994 
To Hà and our Parents -

for their patience and encouragement. 


\section{Abstract}

This thesis describes the collection, reduction, and analysis of Charge Coupled Detector (CCD) images of star clusters. The objects studied are primarily in the Large Magellanic Cloud (LMC), a nearby galaxy. The study of these groupings can provide information such as the initial dynamic state of Globular Clusters, the heavy-element enrichment rate of the LMC, the distribution of masses that stars form with, and the validity of given stellar evolution models.

The majority of the observations were collected at Mount John University Observatory (NZ). Procedures for the collection and transfer of the data are described, along with an overview of the analysis facility and CCDs. Statistical moment-based ellipse fitting was applied to the observations, confirming that trends are evident in the position angles and ellipticities of the clusters, as had been reported in the literature. Artificial images of clusters with known parameters were generated and subjected to the same analysis techniques, revealing apparent trends caused by stochastic processes. Caution should therefore be exercised in the interpretation of observational trends in the structure of young LMC clusters. Isochrones were used to date the 19 clusters. The resulting ages are in good agreement with the literature, as are results from profile modeling. There is no evidence for tidal truncation of the young clusters.

Observations were made of two LMC and two Galactic star clusters in a test of imaging clusters with the Vilnius photometric system and a CCD. The colour-magnitude diagrams, distances and interstellar reddenings of the clusters were derived and found to be in agreement with the literature. This is the first time that the standard Vilnius filter set has been used with a CCD. Use of the system for direct imaging of star clusters appears promising.

Johnson BV CCD observations were made of the young LMC cluster NGC 2214 and a nearby field using the Anglo-Australian Telescope. It has been suggested in the literature that this elliptical cluster is actually two clusters in the process of merging. No evidence was found from profile fitting or the colour-magnitude diagrams to support this contention. Completeness factors were estimated for the CCD frames. These values were used in conjunction with luminosity functions to estimate the Initial Mass Function (IMF) for NGC 2214. A powerlaw $\mathrm{M}^{-(1+x)}$ was assumed for the IMF (where M is stellar mass relative to that of the Sun $\mathrm{M}_{\odot}$ ), with a good fit being found for $x=1.01 \pm 0.09$. There is some indication that the low mass end $\left(\lesssim 3 \mathrm{M}_{\odot}\right.$ ) has a smaller gradient than the high mass end of the derived IMF. The value of $x$ is in reasonable agreement with literature values for other Magellanic IMFs, and not substantially different from the poorly determined Galactic IMFs, suggesting the possibility of a 'universal' IMF over the Magellanic Clouds and our Galaxy in the mass range $\sim 1$ to $\sim 10 \mathrm{M}_{\odot}$. 


\section{Acknowledgments}

$\prod$ he author is grateful for the service observing of NGC 2214 by the Anglo-Australian Telescope and Dr. Gary Da Costa, to Dr. D. Schaerer for kindly making available machinereadable isochrone tables, Dr. Phillipe Fischer for his elliptical-aperture photometry program, Dr. Don Wells for his help with FITS, and to the Foundation for Research, Science and Technology of New Zealand for partial funding of this project in conjunction with the VUW Internal Research Grant Committee. I gratefully acknowledge partial support during this study by the inaugural R.H.T. Bates Postgraduate Scholarship of the Royal Society of New Zealand, together with the financial support kindly provided by the Physics Department (VUW) via a Teaching Assistantship. Substantial access to computers was made available by Dr. Ray Brownrigg and the Institute of Statistics \& Operations Research, Information Technology Services, and Acorn New Zealand Ltd. - which lent an R260 computer for use. It is a pleasure to thank Prof. V. Straižys and the Lithuanian Institute of Theoretical Physics and Astronomy for supplying the Vilnius filter set used by this study.

In particular, thanks are due to my Supervisors, Drs. Denis Sullivan and Richard Dodd, who have both been sources of friendly guidance, prepared to freely give time even when their schedules were tight.

Many others have provided help during this project. These include the MJUO staff, Dr. William Tobin, Dr. John Scalo, Dr. Mike Fitzgerald of the IRAF Support Team, ITS Operations and UNIX Support staff, the staff of Carter Observatory, Mr. Grant Carter, and Mr. Murray Forbes - my fellow astrophysics PhD student. Finally, I thank my family. Without their help and encouragement I could not have followed this course of study.

Rất nhiều lời cám on đển Hà, nêu không có sự giúp đỡ

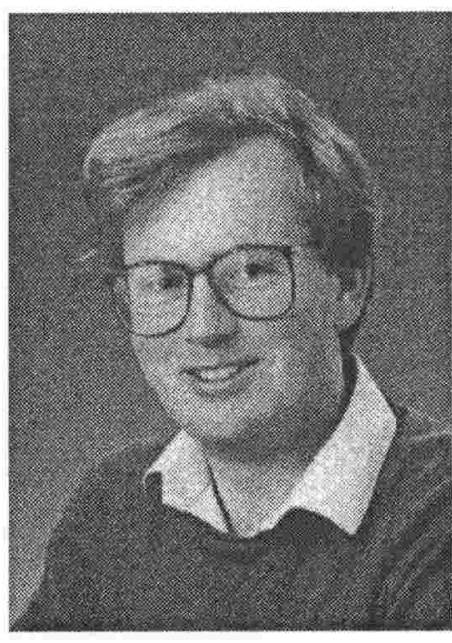

Figure 0-1: The Author của Hà cái luận đề này sẽ không thành công như ý muốn! 


\section{Contents}

$\begin{array}{ll}\text { Abstract } & \text { iii }\end{array}$

$\begin{array}{ll}\text { Acknowledgments } & \text { v }\end{array}$

1 Introduction $\quad 1$

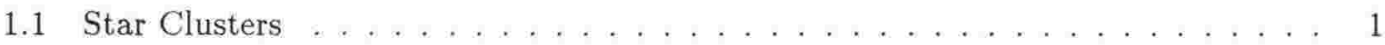

1.2 Stellar Evolution . . . . . . . . . . . . . . . . . . . . . . 5

1.3 The Magellanic Clouds . . . . . . . . . . . . . . . . . . . . . . 11

1.4 The Large Magellanic Cloud . . . . . . . . . . . . . . . . . . . . . . . . . 12

1.5 The LMC Clusters . . . . . . . . . . . . . . . . . . . . . . . . . . 18

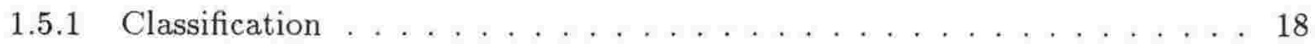

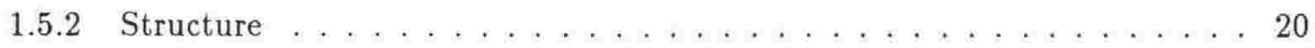

1.5 .3 Mass Functions . . . . . . . . . . . . . . . . . . 21

1.5 .4 Ages and Metallicity ........................ 21

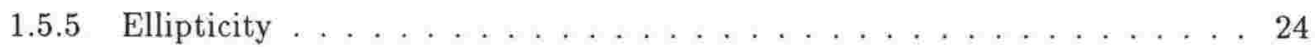

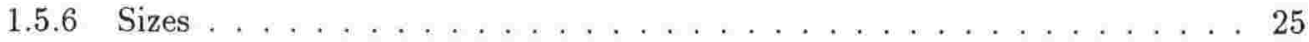

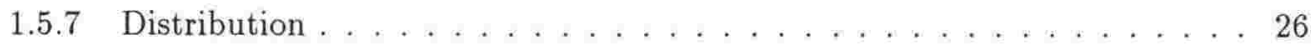

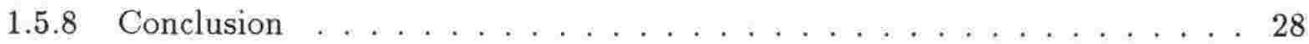

2 Data Collection $\quad 29$

2.1 Charge-Coupled Devices . . . . . . . . . . . . . . . . . . . 29

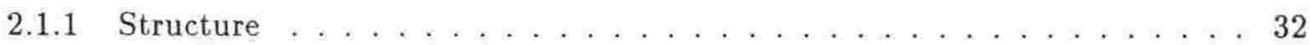

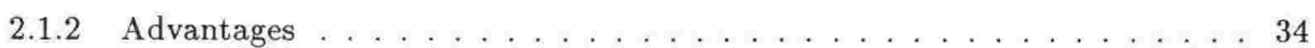

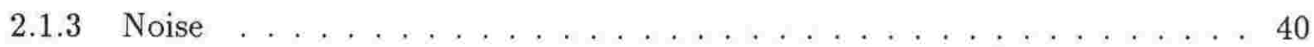

2.1.4 Cosmic Ray Strikes . . . . . . . . . . . . . . . . . . . . 42

2.2 Mount John University Observatory . . . . . . . . . . . . . . . . . . . . . . . . 42

2.3 The MJUO CCD System . . . . . . . . . . . . . . . . . . . . . . . . . 44

2.4 Photometric Systems . . . . . . . . . . . . . . . . . . . . . 45

2.5 Standard Stars . . . . . . . . . . . . . . . . . . . . 48

2.6 Preparation for an Observing Run . . . . . . . . . . . . . . . 52 
2.7 Observing Procedures $\ldots \ldots \ldots \ldots \ldots \ldots \ldots \ldots$

3 The Data Pathway 63

3.1 Tape Reading . . . . . . . . . . . . . . . . . . . . . 63

3.1 .1 Subroutines and Functions . . . . . . . . . . . 65

3.1.1.1 Subroutine Decode_U ................. 65

3.1 .1 .2 Function JD . . . . . . . . . . . . 67

3.1.1.3 Function GMST . . . . . . . . . . . . . . 67

3.1 .1 .4 Function HJD_JD . . . . . . . . . . . . . 68

3.1 .1 .5 Function $\mathrm{R} 4 \ldots \ldots \ldots \ldots \ldots \ldots \ldots \ldots$

3.1.1.6 Function UT_CCD . . . . . . . . . . . . . . 72

3.1 .2 Program PTM_HARD . . . . . . . . . . . . . . . . 72

3.1 .3 Program CCD_TO_V . . . . . . . . . . . . . . . 78

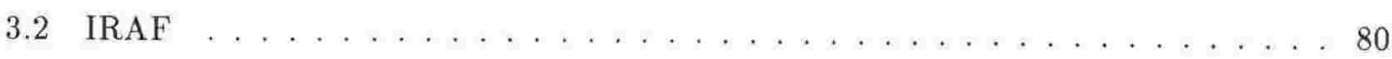

3.2 .1 Major Components . . . . . . . . . . . . . . . . . . 81

3.2 .2 Implementation . . . . . . . . . . . . . . . . . 84

4 Photometry $\quad 85$

4.1 Aperture Photometry . . . . . . . . . . . . . . . . . 85

4.1 .1 Centres . . . . . . . . . . . . . . . . . . . 86

4.1 .2 Background . . . . . . . . . . . . . . . . 87

4.1 .3 Summing the Light . . . . . . . . . . . . . . . . . 88

4.2 Aperture Corrections . . . . . . . . . . . . . . . . . . . . . 89

4.3 Profile Fitting . . . . . . . . . . . . . . . . . . . 91

4.3 .1 Method . . . . . . . . . . . . . . . . . . . . . . 92

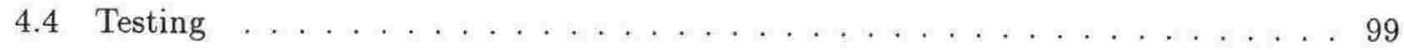

5 The Survey 103

5.1 The Observations . . . . . . . . . . . . . . . . . . . . . 103

5.1 .1 Reductions . . . . . . . . . . . . . . . . . . . . . . . 108

5.2 Colour-magnitude Diagrams . . . . . . . . . . . . . . . . . . . 108

5.3 Profile Fitting . . . . . . . . . . . . . . . . . . . . . . . . . . . 123

5.4 Ellipse Fitting . . . . . . . . . . . . . . . . . . . . . . . . . . . . . 128

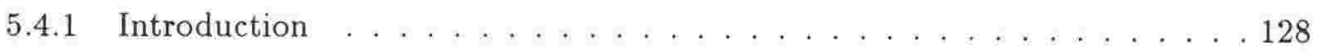

5.4 .2 Method . . . . . . . . . . . . . . . . . . . . . . . . . . . . .

5.4 .3 The Fitting Software . . . . . . . . . . . . . . . . . . 132

5.4 .4 Observations . . . . . . . . . . . . . . . . . . . . . . . 134

5.4 .5 Simulations . . . . . . . . . . . . . . . . . . . . . . . . . . 
5.4 .6 Conclusions . . . . . . . . . . . . . . . . . . . . . . . 141

5.5 Concluding Remarks . . . . . . . . . . . . . . . . . . . . 141

6 Vilnius Photometry 143

6.1 The Vilnius Filter Set . . . . . . . . . . . . . . . . . . . . . . . . . 143

6.1.1 Bandpasses for Early-Type Stars . . . . . . . . . . . . . . 145

6.1 .2 Bandpasses for Late-Type Stars . . . . . . . . . . . . . . . . . 146

6.2 The Southern System . . . . . . . . . . . . . . . . . . . . . 148

6.3 Tests . . . . . . . . . . . . . . . . . . . . . . . . . . . . 149

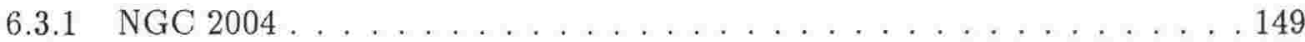

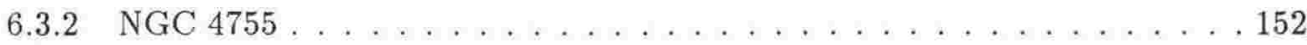

6.3 .3 Discussion . . . . . . . . . . . . . . . . . . 155

6.4 The February 1994 Run . . . . . . . . . . . . . . . . . . . 156

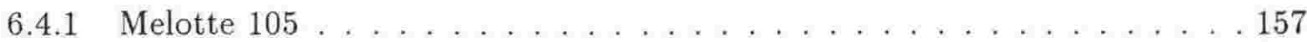

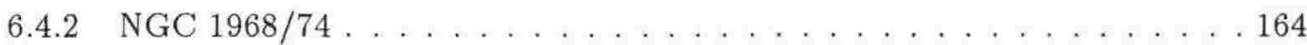

6.5 Conclusions . . . . . . . . . . . . . . . . . . . . . . 164

7 AAT Observations of NGC $2214 \quad 171$

7.1 Introduction . . . . . . . . . . . . . . . . . . . . . . . . 171

7.2 Observations . . . . . . . . . . . . . . . . . . . . . . . . 174

7.3 Results. . . . . . . . . . . . . . . . . . . . . . . . . 177

7.3 .1 Colour-magnitude Diagrams . . . . . . . . . . . . . . . . 177

7.3 .2 Contours . . . . . . . . . . . . . . . . . . . . . . 180

7.3 .3 Ellipse Fitting . . . . . . . . . . . . . . . . . . . . . . . . . . . . 182

7.3 .4 Isochrone Fits . . . . . . . . . . . . . . . . . . . 183

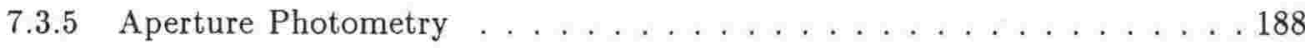

7.3 .6 Completeness . . . . . . . . . . . . . . . . . . . 190

7.3.6.1 Tests . . . . . . . . . . . . . . . . . . . 192

7.3.6.2 Application . . . . . . . . . . . . . . . . . 198

7.3 .7 Field Star Subtraction . . . . . . . . . . . . . . . . . . . . . . 200

7.3 .8 Luminosity Functions ． . . . . . . . . . . . . . . . . . . . 201

7.3 .9 Mass Functions . . . . . . . . . . . . . . . . . . . . . . . . 202

7.4 Conclusions . . . . . . . . . . . . . . . . . . . . . . . . 207

A Flexible Image Transport System 209

A.1 Preface . . . . . . . . . . . . . . . . . . . . . . . . . . . 209

A.2 Introduction . . . . . . . . . . . . . . . . . . . . . . . . 209

A.3 The Primary Header . . . . . . . . . . . . . . . . . . . . . . . . 211 
A.4 The Data Unit . . . . . . . . . . . . . . . . . . . . . . . . 212

A.5 Extensions . . . . . . . . . . . . . . . . . . . . . 213

A.5.1 ASCII Table Extension . . . . . . . . . . . . . . . . . 214

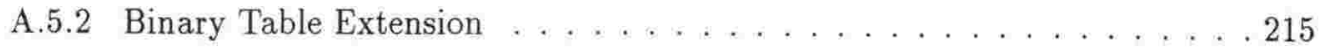

$\begin{array}{ll}\text { B IRAF Documentation } & 217\end{array}$

$\begin{array}{ll}\text { Glossary of Acronyms } & 223\end{array}$

$\begin{array}{ll}\text { References } & 225\end{array}$ 


\section{List of Figures}

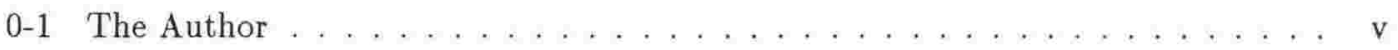

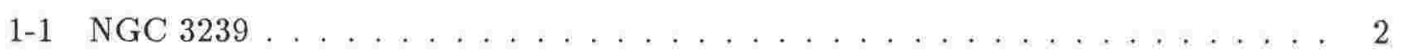

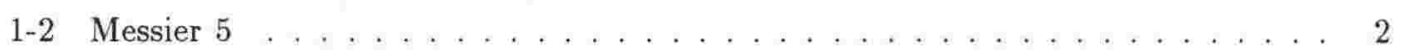

1-3 Colour-magnitude Diagram for M $3 \ldots \ldots \ldots \ldots \ldots \ldots$

$1-4$ The Helix Nebula . . . . . . . . . . . . . . . . . . . . . . . 8

1-5 The Small Magellanic Cloud . . . . . . . . . . . . . . . . . . 11

1-6 The Large Magellanic Cloud . . . . . . . . . . . . . . . . . . . . . 11

1-7 The Magellanic Stream . . . . . . . . . . . . . . . . . . . . . 16

1-8 The LMC Cluster Hodge $11 \ldots \ldots \ldots$

1-9 Integrated $(\mathrm{B}-\mathrm{V})$ Colours of LMC Clusters . . . . . . . . . . . . 19

1-10 The Age-Abundance Relation for LMC Clusters . . . . . . . . . . . . . . . 22

1-11 LMC Cluster Rotation Curves . . . . . . . . . . . . . . . . . . . 27

2-1 CCD Exposure and Readout . . . . . . . . . . . . . . . 30

2-2 CCD Readout and General Structure . . . . . . . . . . . . . . . . . 31

$2-3$ Charge Coupling . . . . . . . . . . . . . . . . . 33

2-4 Virtual Phase CCD Architecture ... . . . . . . . . . . 36

2-5 Example MJUO Flat-field Frame . . . . . . . . . . . . . . . . . 38

$2-6$ Typical Cosmic Ray Strike . . . . . . . . . . . . . . . . . . . . . . . . 41

2-7 The Peak of Mount John . . . . . . . . . . . . . . . . . . 42

2-8 MJUO 1 metre Telescope Dome and Observers' Quarters . . . . . . . . . . . 43

2-9 MJUO 1 metre with CCD System Attached . . . . . . . . . . . . . . . 43

2-10 Strömgren Filter Placement . . . . . . . . . . . . . . . . . . 47

2-11 Johnson BVI Filter Responses . . . . . . . . . . . . . . . . . . . . . 50

2-12 Example Airmass Diagram . . . . . . . . . . . . . . . . . . 52

2-13 Sample Finding Chart . . . . . . . . . . . . . . . . . 54

2-14 Offset Guider Overlay ... . . . . . . . . . . . . . . 5 55

2-15 The Equatorial Coordinate System . . . . . . . . . . . . . . . 56

2-16 Filling the CCD Dewar . . . . . . . . . . . . . . . . 57 
3-1 The Data Pathway and IRAF . . . . . . . . . . . . . . 64

$3-2$ Anomaly. . . . . . . . . . . . . . . . . . . . . . . 70

3-3 Single Precision 32 Bit IEEE Floating Point Standard . . . . . . . . . . . 71

3-4 Example Output from PTM_HARD . . . . . . . . . . . . . . 77

3-5 An Example IRAF Display . . . . . . . . . . . . . . . . . . . . 80

4-1 An Example Growth Curve . . . . . . . . . . . . . . . . . . . . . 9 90

4-2 One Dimensional Profiles of Astronomical Objects and Erroneous Events _. . . 94

$4-3$ Colour-Magnitude Diagrams for NGC $1978 \ldots \ldots$. . . . . . . . . . . 100

5-1 Primary Extinction Coefficients at MJUO . . . . . . . . . . . . . . . 105

$5-2$ Simulated $1^{\prime \prime}$ Seeing . . . . . . . . . . . . . . . . . . 107

$5-3$ Simulated $2^{\prime \prime}$ Seeing . . . . . . . . . . . . . . . . . 107

$5-4$ Simulated $3^{\prime \prime}$ Seeing . . . . . . . . . . . . . . . . . . . 107

$5-5 \quad$ Simulated $4^{\prime \prime}$ Seeing . . . . . . . . . . . . . . . . . . 107

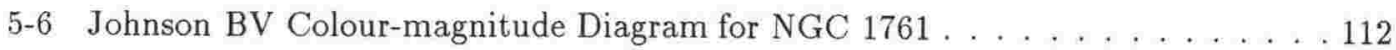

5-7 Johnson BV Colour-magnitude Diagram for NGC $1774 \ldots \ldots \ldots . . \ldots . . .112$

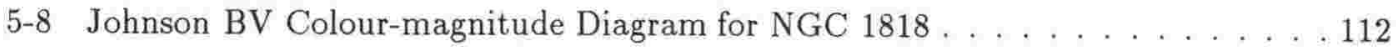

5-9 Johnson BV Colour-magnitude Diagram for NGC $1834 \ldots \ldots \ldots 112$

5-10 Johnson BV Colour-magnitude Diagram for NGC $1835 \ldots \ldots$. . . . . . . . . 113

5-11 Johnson BV Colour-magnitude Diagram for NGC $1839 \ldots \ldots . \ldots 113$

5-12 Johnson BV Colour-magnitude Diagram for NGC $1847 \ldots \ldots$. . . . . . . . 113

5-13 Johnson BV Colour-magnitude Diagram for NGC $1850 \ldots \ldots$. . . . . . . . . 113

5-14 Johnson BV Colour-magnitude Diagram for NGC 1854 . . . . . . . . . . . 114

5-15 Johnson BV Colour-magnitude Diagram for NGC $1863 \ldots \ldots . . . . . . . .114$

5-16 Johnson BV Colour-magnitude Diagram for NGC 1968 . . . . . . . . . . . 114

5-17 Johnson BV Colour-magnitude Diagram for NGC 2004 . . . . . . . . . . . 114

5-18 Johnson BV Colour-magnitude Diagram for NGC 2018 . . . . . . . . . . . 115

5-19 Johnson BV Colour-magnitude Diagram for NGC 2031 . . . . . . . . . . 115

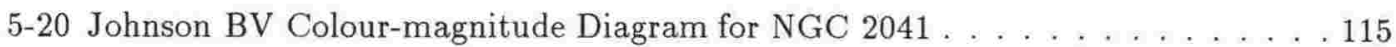

5-21 Johnson BV Colour-magnitude Diagram for NGC 2058 . . . . . . . . . . 115

5-22 Johnson BV Colour-magnitude Diagram for NGC $2157 \ldots \ldots . \ldots \ldots$

5-23 Johnson BV Colour-magnitude Diagram for NGC 2164 . . . . . . . . . . . 116

5-24 Johnson BV Colour-magnitude Diagram for NGC 2214 . . . . . . . . . . 116

5-25 Johnson BV Colour-magnitude Diagram for SL 268 . . . . . . . . . . . 116

5-26 Elson (1991) Johnson BV Colour-magnitude Diagram for NGC 1818 . . . . . 117

5-27 Elson (1991) Johnson BV Colour-magnitude Diagram for NGC $1850 \ldots 117$ 
5-28 Elson (1991) Johnson BV Colour-magnitude Diagram for NGC 2004 . . . . . . 117

5-29 Elson (1991) Johnson BV Colour-magnitude Diagram for NGC 2157 . . . . . 117

5-30 Elson (1991) Johnson BV Colour-magnitude Diagram for NGC 2164 . . . . . . 117

5-31 Elson (1991) Johnson BV Colour-magnitude Diagram for NGC 2214 . . . . . 117

5-32 Long Exposure CMD for NGC 2214 . . . . . . . . . . . . . . . . . . 121

5-33 CMD for NGC 1968/74 Based on Co-added Images . . . . . . . . . . . . 123

$5-34$ King Model Fit to NGC 2164 V Data . . . . . . . . . . . . . . 126

5-35 Cluster Core Radius Against Log Age . . . . . . . . . . . . . . . 127

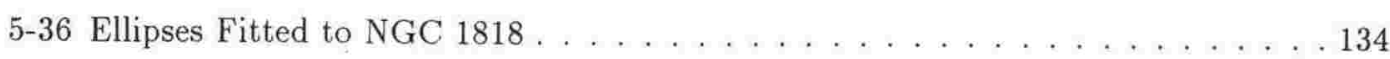

$5-37$ Flat Distribution . . . . . . . . . . . . . . . . . . . 138

$5-38$ Star Counts in a Sliced Flat Distribution . . . . . . . . . . . . . . 138

6-1 Vilnius Bandpasses Superimposed on Example A3 and G5 Spectra . . . . . . . 144

6-2 Spectra of Example B4 Main Sequence and B5 Supergiant Stars about the Balmer Jump . . . . . . . . . . . . . . . . . . . . . . . . . 146

6-3 Definition of the Q Parameter . . . . . . . . . . . . . . . . 147

6-4 Spectra of Example K0 Main Sequence and Giant Stars . . . . . . . . . . 148

$6-5$ Vilnius Filter Response for the TH7882 Chip . . . . . . . . . . . . . . . 149

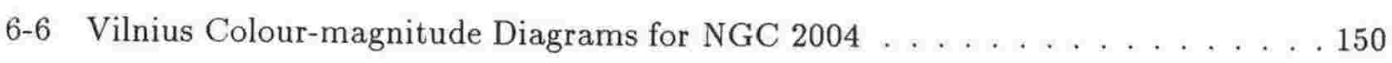

6-7 Vilnius Colour-magnitude Diagrams for NGC $4755 \ldots \ldots$. . . . . . . . 153

6-8 P-V, V-Y Colour-Colour Diagram for the $\kappa$ Crucis Cluster . . . . . . . . . . . 154

6-9 Reddening and Distance Modulus Estimates for NGC $4755 \ldots \ldots$. . . . . . . 156

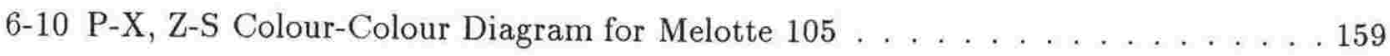

6 -11 Vilnius Colour-magnitude Diagrams for Melotte $105 \ldots \ldots$. . . . . . . 160

$6-12$ Vilnius VS CMD for Melotte $105 \ldots \ldots$. . . . . . . . . . . . 161

6-13 Reddening Free $Q(\lambda)$ Functions for Luminosity Class V Stars . . . . . . . . 162

6-14 Positions and Identifications of Stars in Melotte $105 \ldots \ldots$. . . . . . . 163

6-15 P-X, Z-S Colour-Colour Diagram for NGC 1968/74 . . . . . . . . . . . . . 164

6-16 Vilnius Colour-magnitude Diagrams for NGC 1968/74 . . . . . . . . . . . 165

6-17 Vilnius VS Colour-magnitude Diagrams for NGC 1968/74 . . . . . . . . . 166

7-1 The 3.9 metre Anglo-Australian Telescope . . . . . . . . . . . . . . . . . . 172

$7-2$ The Anglo-Australian Telescope Building . . . . . . . . . . . . . . 173

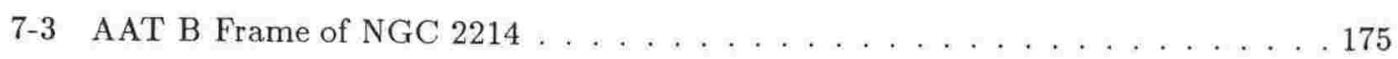

$7-4$ AAT B Frame of NGC 2214 Field . . . . . . . . . . . . . . . 175

$7-5$ Colour-magnitude Diagram for NGC $2214 \ldots \ldots \ldots \ldots$. . . . . . . . . . . . . .

$7-6$ Colour-magnitude Diagram for the Field . . . . . . . . . . . 176 
7-7 Photometric Errors for the 300 second V Exposure of NGC $2214 \ldots \ldots \ldots \ldots$

$7-8$ Contours for the Inner Regions of NGC $2214 \ldots \ldots \ldots \ldots$

7-9 Contour Maps of the 30 second NGC 2214 V Frame . . . . . . . . . . . . . . 179

7-10 NGC 221430 second V Frame Star-counts in 19.5 arcsecond Bins . . . . . . . . . 180

7-11 Grid Diagram of the Blended Region . . . . . . . . . . . . . . . . 181

7-12 Ellipse Fits to the 60 second B Frame . . . . . . . . . . . . . . . 182

$7-13 \mathrm{Z}=0.02$ Isochrones Superimposed onto the BV CMD of NGC $2214 \ldots \ldots . . .184$

7 -14 Comparison between Different Isochrones . . . . . . . . . . . . . 185

7-15 NGC 2214 Luminosity Profile . . . . . . . . . . . . . . . . . . . . 187

7-16 NGC 1978 Johnson B Band Aperture Photometry Comparison . . . . . . . . 187

7-17 King Model fit to NGC 1978 . . . . . . . . . . . . . . . . . . . . 188

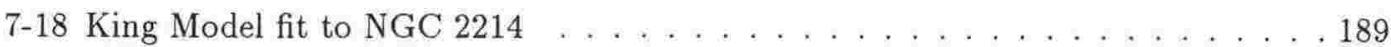

7-19 Monte-Carlo Luminosity Function . . . . . . . . . . . . . . . . . . 192

7-20 The Test Frames Magnitude Relationship . . . . . . . . . . . . . . . . 193

7-21 Mateo (1988b) Ratio for the Test Frames . . . . . . . . . . . . . . 195

7-22 Observational V Band Luminosity Functions . . . . . . . . . . . . . 198

7-23 Long Exposure Frames Mass Function for NGC 2214 . . . . . . . . . . . . . . . 204

$7-24$ Mass Function for NGC $2214 \ldots \ldots$. . . . . . . . . . . . . . . 204

A-1 MJUO FITS Header . . . . . . . . . . . . . . . . . . . . 210 


\section{List of Tables}

3.1 MJUO Frame Header Records . . . . . . . . . . . . . . . . . . . . . . 73

3.2 Photometrics Header Records . . . . . . . . . . . . . . . . . . . . . . . . 74

4.1 Comparison of Transformation Equations . . . . . . . . . . . . . . 101

5.1 Seeing Estimates at MJUO . . . . . . . . . . . . . . . . . . . . . . 104

5.2 Dates of Observing Runs at MJUO . . . . . . . . . . . . . . . . . . . 104

5.3 Transformation Equation Coefficients for the MJUO Survey . . . . . . . . . 105

5.4 Standard Star Colour and Airmass Ranges . . . . . . . . . . . . . . . 106

5.5 Isochrone Age Estimates for Survey Clusters . . . . . . . . . . . . . . . 110

$5.6 \mathrm{E}(\mathrm{B}-\mathrm{V})$ Estimates for the Survey Clusters . . . . . . . . . . . . . 111

5.7 Optimum Test King Model Parameters . . . . . . . . . . . . . . . . . 124

5.8 Profile Fit Parameters . . . . . . . . . . . . . . . . . . . . . 125

5.9 Literature Values for Ellipticities and Position Angles of LMC Clusters . . . . . . 128

5.10 Test Ellipticity and Orientation Values . . . . . . . . . . . . . . . 132

5.11 Variation of Elliptical Parameters for Selected LMC Clusters . . . . . . . . . 135

5.12 Comparison of Ellipticity Estimates . . . . . . . . . . . . . . 136

6.1 Mean Wavelengths and Half-Widths of the Vilnius Filters . . . . . . . . . . 145

6.2 Vilnius Transformation Coefficients . . . . . . . . . . . . . . . . . 151

6.3 Transformation Parameters for NGC $4755 \ldots \ldots \ldots$. . . . . . . . . . . 152

6.4 Literature Estimates for Reddening and Distance Modulus of NGC $4755 \ldots$. . . . 155

6.5 February 1994 Transformation Coefficients . . . . . . . . . . . . . . . 158

6.6 Comparison of Melotte 105 Photometry . . . . . . . . . . . . . 158

6.6 Melotte 105 Photometry . . . . . . . . . . . . . . . . . . . . . . . 168

6.7 Vilnius Photometry of NGC $1968 / 74 \ldots \ldots \ldots \ldots$

7.1 Adopted Error limits for NGC Cluster and Field Photometry . . . . . . . . . . 178

7.2 NGC 2214 Isochrone Fit Ages . . . . . . . . . . . . . . . . . . . . . . 183

7.3 Expected Star Counts . . . . . . . . . . . . . . . . . . . . . 186

7.4 Test Frame Artificial Stars Recovery Rates . . . . . . . . . . . . . . . . . 194 
7.5 B Test Frame Completeness . . . . . . . . . . . . . . . . . . . . . . . . 194

7.6 V Test Frame Completeness . . . . . . . . . . . . . . . . . . . . . . 195

7.7 Dual Frame Completeness Test Results . . . . . . . . . . . . . . . . . . . . 196

7.8 Long Exposure Field Completeness Factors . . . . . . . . . . . . . . . . . . . . 199

7.9 Long Exposure Cluster Completeness Factors . . . . . . . . . . . . . . . . 200

7.10 Short Exposure Field Frames Completeness Factors . . . . . . . . . . . . . . 201

B.1 IRAF Reference Manuals . . . . . . . . . . . . . . . . . 220

B.2 Names and Network Archives for External IRAF Packages . . . . . . . . . . 221 


\section{Chapter 1}

\section{Introduction}

$\mathrm{T}$

his chapter is intended to act as an introduction to the objects studied in this project and to some of the terminology associated with these objects. The chapter begins by defining what a cluster is, and introducing terms that will be used later. Throughout this chapter definitions for terms that might be unfamiliar are either provided as a quick aside, or given in a footnote if the flow of the text would otherwise be disrupted. The use of footnotes removes the disruption to reading involved when a glossary at the end of a book must be consulted to understand a term, although a glossary of acronyms is provided (beginning on page 223).

After the introduction to star clusters in general, colour-magnitude diagrams and their features are discussed with reference to basic stellar evolution. Next, the Magellanic Clouds, and in particular the Large Magellanic Cloud, are discussed, leading on to the actual objects of interest themselves - star clusters in the Large Magellanic Cloud.

\section{$1.1 \quad$ Star Clusters}

star cluster is a grouping of stars whose members are sufficiently close enough to each
other for the stars to be gravitationally bound together as a unit. The number of stars congregations of many thousands of stars. There are two basic types of cluster -

- The galactic or open clusters, which contain a modest number of Population I stars that range from around 20 to no more than a thousand in number. Population I stars are young and metal-rich, as opposed to the older Population II which are metal-deficient stars. In astronomical parlance metals are all elements heavier than hydrogen and helium. There is a continuum between these two classes of star.

In our galaxy, these diffuse clusters are found in or near the plane of the galactic disk, and new examples are continually being formed. They are relatively loosely bound systems, 


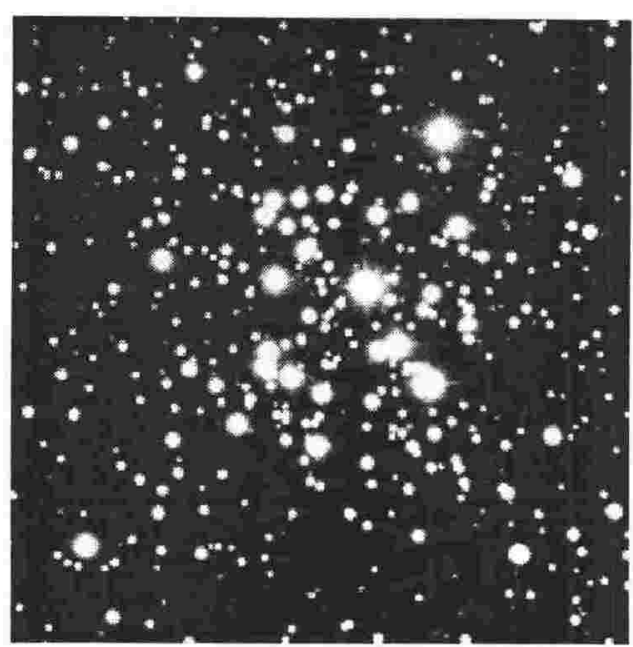

Figure 1-1: NGC 3239. An example of a young open cluster in the Milky Way. Photograph taken by D. Malin with the AAT (C)AAO. Reproduced with permission).

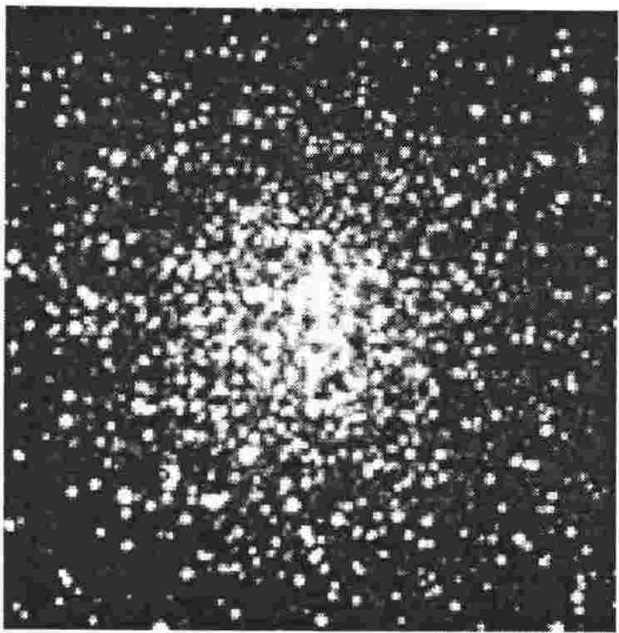

Figure 1-2: Messier 5. An example of a globular cluster in the Milky Way. Photograph taken by David Malin with the AAT (C)AAO. Reproduced with permission).

that tend to be gradually dispersed by a combination of (King 1980) -

- Stellar encounters between component stars. Equipartion of energy will lead to the velocities of the lower mass stars involved in such interactions being increased, until at some point they reach escape velocity and are ejected from the cluster. Such a loss reduces the gravitational binding of the cluster, lowering the necessary escape velocity. The more massive stars sink towards the cluster centre.

$\mathrm{N}$-body simulations have shown that the formation of hard (strongly gravitationally bound) binaries ${ }^{1}$ from the most massive stars is typical. Once formed, such binaries dominate the evolution of a star cluster (Heggie 1975). Alteration of the orbital velocities of the component stars in these binaries acts as a sink for the binding energy of the cluster. Through encounters with other stars, the binaries become more tightly bound, leading to the velocities of the other stars involved in each encounter being increased.

- Mass loss from evolving stars. Some of this material is ejected from the system, decreasing the binding energy of the cluster.

- The differential rotation of the Galaxy across the extent of the cluster, which will tend to stretch the cluster out over several rotations of the cluster about the Galactic centre.

- Tidal shocking by interstellar cloud complexes that the cluster passes near to. Details

\footnotetext{
${ }^{1} \mathrm{~A}$ pair of stars that revolve about a common centre of mass under the influence of their mutual gravitational attraction is called a Binary Star.
} 
of this interaction may be found in Hartwick (1973).

Open clusters are typically disrupted within a few Galactic rotations, although some survive much longer, e.g. M67, which has been dated as 3.2 billion years old (Astronomical Almanac 1990). One Galactic rotation takes 200 million years. See Figure 1-1 for an example of an open cluster.

- Globular clusters are spherically symmetric, populous, tightly packed halo (Population II) objects believed to have formed some 12-13 billion years ago, before the main body of the Galactic disk had evolved. These objects move in huge elliptical orbits about the Galactic centre. The furthest known globular clusters are further away from the Galaxy than the Magellanic Clouds are. The diameters of globular clusters range in size up to a few hundred light years across, opposed to the typical open cluster which is less than 50 light years across. Radii of open and globular star clusters were calculated from the tables in the Astronomical Almanac (1990), which are in turn based on Lynga (1981) and Harris \& Racine (1979). A mean diameter of $6 \pm 5^{1}$ parsecs $^{2}$ was calculated for the nuclei of open clusters. The maximum diameter was 50 parsecs. Globular clusters are typically larger with a mean diameter of $28 \pm 21$ parsecs, ranging up to the largest tabulated value of 141 parsecs.

There are two basic types of globular cluster (King 1989)-

- The halo globular clusters, which as a group has a nearly spherical distribution about the Galactic centre. The motions of the individual clusters are random, and the system of clusters as a whole has little or no rotation. The most distant of these objects, the so-called Palomar clusters, are extremely sparse. Their longevity appears to be due to their remote distance which lowers both the disruptive Galactic tidal forces and the dynamical friction caused by gravitational interaction with field stars in the Galactic halo - as there are few field stars at their distances from the Galaxy.

- The Disk globular clusters, which are in a more flat spatial distribution with a mean rotation half that expected from Galactic rotation. While their metal abundance is low, it is greater than the halo globulars.

Globular clusters contain upwards of thousands of stars, whose concentration increases towards the centre of the cluster (see Figure 1-2). Central densities may be as much as a

\footnotetext{
${ }^{1}$ While standard deviation errors are given, both the open and globular cluster distributions are skewed towards small diameters and are not Gaussian.

${ }^{2} 1$ parsec $=3.2616$ light years. Light travels through a vacuum at the finite velocity of $\sim 3 \times 10^{8} \frac{\mathrm{m}}{\mathrm{s}}$. Therefore one light year is both a measure of time and also distance, being how far light travels in a year. A parsec is short for a parallax second, and is the distance at which the mean radius of the Earth's orbit subtends an angle of one arcsecond.
} 
thousand stars per cubic parsec (Illingworth 1979). Because of their larger populations, globular clusters have longer lifetimes than open clusters.

Evolutionary mass loss plays a large role in the structural evolution of young globular clusters, causing the grouping to expand - possibly to the point of disruption (Larson 1992). By the time stars more massive than the Sun have evolved off the main sequence ${ }^{1}$ (or, in other words, when hydrogen fusion reactions no longer occur in their cores), evolutionary mass loss becomes relatively unimportant compared to two-body gravitational interactions. As with the open clusters, these interactions will result in mass segregation as massive stars sink towards the core of the cluster, and the less massive stars are ejected to the outer regions of the cluster and beyond. Ejections out of the cluster cause the cluster to contract, leading to it becoming increasingly centrally concentrated, possibly in a runway process. Modeling some twenty years ago (e.g. Lynden-Bell \& Wood 1968, Spitzer \& Hart 1971) suggested that the central density would approach infinity after a finite period, with the complete disruption of the cluster ensuing. For a typical cluster such 'core collapse' is expected to occur at an age of 2 billion years, with complete destruction within a billion years more. However, globular clusters are still observed today. They contain stars that models of stellar evolution indicate are much older than the times calculated for disruption of the clusters. Therefore, some process or processes must have prevented the destruction of the clusters. Observations indicate that around one-quarter of globular clusters have already undergone core collapse, exhibiting central cusps in their surface density profiles. These cusps are not as steep as those predicted for collapsed cores, which could be due to the cores being dominated by dark matter in the form of stellar remnants. The remnants would be more massive and more centrally concentrated than the visible stars.

Core collapse is believed to be halted, and to some extent even reversed, by the formation of binary star systems in the core of the cluster. As in open clusters, the binaries act as an energy source that stirs stellar velocities up through close gravitational interactions with other stars. These interactions lead to the velocities of the stars that interacted with the binaries being increased, countering the effects of core collapse. N-body models indicate that eventually a violent encounter will occur that ejects such a binary from the star cluster. After enough such ejections, core collapse resumes until further binaries are either generated or, in the case of primordial binaries, transferred into the core region. Core collapse will again be countered, and the cycle of events described here begins again. Large oscillations in the core density are predicted in this post-collapse phase (see e.g. Bettwieser \& Sugimoto 1984).

\footnotetext{
${ }^{1}$ Defined below.
} 
However, while the complete collapse of the central regions is delayed by these oscillations, the outer layers of the cluster continue to evaporate via two-body encounters and tidal shocking as the cluster passes through the Galactic disk (which strips the low mass stars that are preferentially found in the outer regions). These processes inevitably lead to disintegration of the cluster. The evaporation time for a cluster is proportional to the square root of its mass, and so the larger a cluster the longer its lifetime. Some 25 billion years is required for the complete evaporation of a typical globular cluster observed now, or longer than the age of the universe (Larson 1992). Clusters with masses much smaller than the mass of a typical globular cluster (as of now) have been disrupted.

Associations were not included in this list as their space density is generally too low for the grouping to be gravitationally bound. However it should be noted that their disruption times are comparable to the lifetimes of the most massive component stars.

Clusters are often regarded as being a homogeneous unit. All the component stars of a cluster are effectively the same age, are usually the same chemical composition due to their common origin, and are at the same distance from the Sun. While clusters do have a spatial extent, it is typically small compared with the distance from the Sun to the cluster. Populous clusters are especially useful for studies of stellar evolution where, due to the large number of stars in the cluster, examples of short-lived stages of stellar evolution can be observed. The colour-magnitude diagram (CMD) of a cluster is a snapshot showing the distribution stars of different masses have at a given time, for a given chemical composition.

\section{$1.2 \quad$ Stellar Evolution}

The CMD is one form of the Hertzsprung-Russell (H-R) diagram, which demonstrates the correlation between spectral type and the luminosity of stars. Stars are classified according to their spectra, and fall into a given spectral type, reflecting the temperature of the star. The majority of stars can be divided into seven spectral types - O, B, A, F, G, K, and M, given in order of decreasing temperature.

A CMD plots the observable colour ${ }^{1}$ index, which is a temperature indicator, against magnitude. Magnitude is plotted on the vertical axis so that brightness increases upwards, while colour is plotted so that lower temperatures (redder colours) fall on the right. For histori-

\footnotetext{
${ }^{1}$ In photometry colour is defined as the difference between two bandpasses. If the $\mathrm{V}$ magnitude is defined to be $m_{V}=-2.5 \log I_{v}$, where $I_{v}$ is the intensity as measured through the $V$ filter, and the $B$ magnitude is defined similarly, then the B $-\mathrm{V}$ colour is:

$$
\mathrm{C}_{\mathrm{B}-\mathrm{V}}=\mathrm{m}_{\mathrm{B}}-\mathrm{m}_{\mathrm{V}}=-2.5 \log \frac{\mathrm{I}_{\mathrm{B}}}{\mathrm{I}_{\mathrm{V}}} .
$$
}


cal reasons, the relative brightnesses of stars and other celestial objects are measured using a logarithmic scale, rather than the linear one of intensities. An increase of one magnitude corresponds to a decrease in intensity of 2.512 times. The apparent magnitude of an object is the brightness it appears as seen from Earth, while its absolute magnitude is how bright it would appear if the object were 10 parsecs away.

Instead of a random distribution across a CMD, stars fall into well-defined groups or bands (see Figure 1-3). These grouping are due to stellar evolution. The majority of stars lie on the main sequence, which corresponds to those stars fusing hydrogen to helium in their cores. The main sequence is the longest evolutionary phase that a star experiences. The zero age of a star is defined to be when its core temperatures become hot enough for nuclear fusion reactions to commence. Hence, the sequence formed on an H-R diagram by zero age stars of different masses is known as the Zero Age Main Sequence (ZAMS). As the hydrogen in the core of a star is converted, the chemical composition and structure of the star change, causing it to shift on the H-R diagram off the ZAMS and slightly to the right (redward).

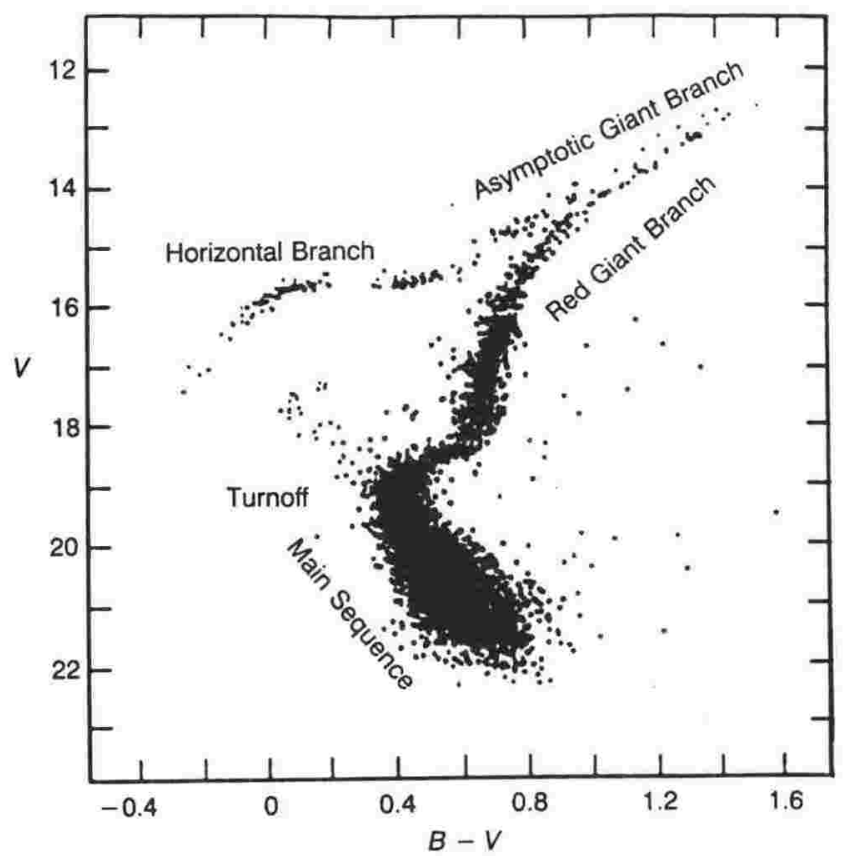

Figure 1-3: Colour-magnitude Diagram for M3. Evolutionary features are indicated on the CMD. This figure is taken from Da Costa (1992)
The position of a star in the main sequence depends primarily on the mass of the star. The more massive a star, the more luminous it is, the faster its nuclear reactions run, and the higher up the main sequence the star appears. Therefore the time a star stays on the main sequence is also a function of the star's mass. After $\sim 12 \%$ of the hydrogen in a (solar mass) star has been consumed, the star will evolve off the main sequence to eventually become a red giant. The main sequence of a star cluster terminates at the main sequence turnoff point where main sequence stars begin to evolve towards the red. The absolute brightness of stars at this point is an important quantity, since comparison with results from stellar evolution models allows the age of a cluster to be estimated. The models are used to compute isochrones. An isochrone is a sequence on the H-R diagram indicating the 
positions of stars with the same age but a continuum of masses. These isochrones are computed as a function of the effective temperature $\mathrm{T}_{\mathrm{eff}}$ and $\log \frac{\mathrm{L}}{\mathrm{L} \odot},{ }^{1}$, which can be transferred to the observational values of absolute $\mathrm{V}$ and $\mathrm{B}-\mathrm{V}$. The distance modulus is the difference between the apparent $(\mathrm{m})$ and absolute magnitudes $(\mathrm{M})$ of a star, and is therefore a measure of distance, i.e.,

$$
\mathrm{m}-\mathrm{M}=5 \log \frac{\mathrm{d}}{10}=5 \log \mathrm{d}-5
$$

where $\mathrm{d}$ is the distance in parsecs. The distance modulus for a cluster can be estimated from a CMD, being the shift in $\mathrm{V}$ required to match the best-fit isochrone with the data (This is a simplification, which is refined on page 159). The turnoff point of a cluster lowers with time towards fainter magnitudes in the H-R diagram, as the higher mass stars reach the end of their main sequence lifetimes and evolve off the Sequence. As time goes by, increasingly lower mass stars reach this state. A small number of stars brighter and more blue than the turnoff can be seen in Figure 1-3. These are blue stragglers. Their origin is not fully understood, although plausible explanations involve mass transfer between components of close binary star systems.

Red giants are stars in which the fusion of hydrogen takes place in shells about the core, which results in an inflation of the star's outer layers. These stars have low effective temperatures of 2000-3000 kelvin, which means that they appear red to the eye. The diameters of red giant stars are around 10 to 100 times that of the Sun - explaining the name of this class of star. The colour of a red giant is sensitive to the metal abundance of the star, becoming more red with increasing metal abundance. This can make red giants a useful diagnostic of the abundance and homogeneity of metals within a cluster. The red giant evolutionary phase is short compared with the main sequence, and so the number of red giants in a cluster is much less than that of main sequence stars. The number of red giants in a cluster decreases with the increasing absolute magnitude of the giants. This is because the evolutionary scales become shorter for more massive red giant stars. During this phase the helium core is being heated by the fusion reactions occurring in shells about it.

For low mass $\left(\sim \mathrm{M}_{\odot}\right)$ stars, evolution up the Red Giant Branch (RGB) terminates when the central helium core of the giant becomes hot enough for the helium to ignite via the triplealpha process $\left(3^{4} \mathrm{He} \rightarrow{ }^{12} \mathrm{C}+\gamma\right)$. This event is called the 'helium flash', and corresponds to the tip of the RGB. After the helium flash, the star drops in absolute magnitude and shifts blueward on the CMD. This ignition is rapid, due to the degenerate nature of the core which

\footnotetext{
${ }^{1}$ Effective Temperature is the surface temperature of a star expressed as the temperature of a black body having the same radius as the star and radiating the same total amount of energy per unit area per unit time, i.e.,

$$
\mathrm{T}_{\mathrm{eff}}=\frac{\mathrm{L}}{4 \pi \mathrm{r}^{2} \sigma}
$$

where $\sigma$ is the Stefan-Boltzmann Constant, $\mathrm{r}$ is the stellar radius, and $\mathrm{L}$ is the luminosity of the star. Luminosity is the total energy radiated per unit time. The symbol $\odot$ stands for Sun, hence the solar luminosity is given by $\mathrm{L}_{\odot}$.
} 
can not expand and cool to counteract this new heat source. Hence the reaction takes place rapidly throughout the core, leading to the core becoming non-degenerate and the site of stable helium fusion reactions that generate carbon. The hydrogen shell burning continues, although its generation rate is less than that of the core. After the central helium fuel supply in the core is exhausted, a solar mass star is not massive enough that further collapse will increase temperatures in its core to where further fusion reactions can commence. Fusion of carbon will therefore not be possible. For a short period two shells burn hydrogen and helium respectively and the star becomes a red giant again. The outer layers of the star will be ejected during this phase. This is because the triple-alpha fusion process is temperature sensitive. The outer layers of the star pulsate. Gravitational collapse increases temperatures in the helium shell, causing the $3 \alpha$ process to increase in activity. The resulting increase in flux 'blows' the outer layers of the star out. Temperatures drop, and the reaction rate slows, leading to the cycle recommencing. Some of the pulses will be more violent, leading to the outer envelope of the star reaching escape velocity.

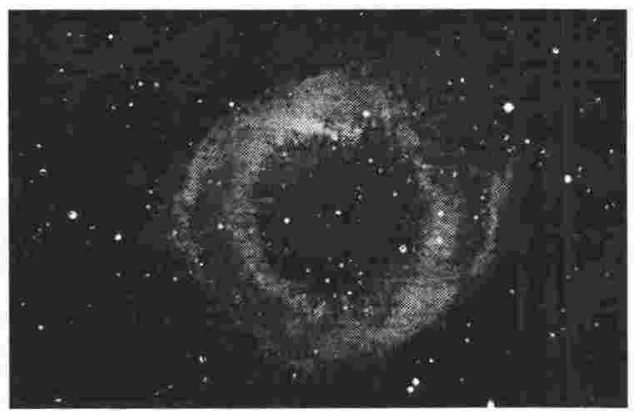

Figure 1-4: The Helix Nebula is an example of a planetary nebula. The central star was once the core of a red giant. The nebula looks like a series of rings, which are actually spheres offset by the motion of the red giant. The observable optical density is at a maximum at the sides of the sphere. Photograph taken by David Malin with the AAT (CAAO. Reproduced with permission).
These pulsations lead to the formation of a convective zone in the outer layers of the star. This convection can 'dredge up' processed material from deeper regions inside the star. This material can then be subjected to further reactions resulting in the formation of heavier elements. Dredge-ups occur each time a star enters the giant branch. A strong stellar wind, driven by the thermal pulsations, can rip off the outer layers of the star, forming a planetary nebula (see Figure 1-4) and blast this processed material back into space. Such debris will make up a large proportion of the material from which later generations of stars form.

After finally exhausting its available fuel supplies, a low mass star will collapse to form a white dwarf. These are compact bodies which form a cooling sequence in the lower left of the Hertzsprung-Russell diagram. The stellar core is degenerate, and so no amount of contraction will increase its temperature to the point at which fusion can recommence - indeed, degeneracy pressure will resist any attempts at compression. However the self-gravitation of white dwarf remnants of more massive stars that have a remnant mass greater than $\sim 1.4 \mathrm{M}_{\odot}$ can overcome this pressure, causing the star to collapse further. Above this limit and below $\sim 3 \mathrm{M}_{\odot}$ the collapse forces protons and electrons to merge together 
as neutrons. The resulting object is supported against further collapse by neutron degeneracy pressure, and is called a 'neutron star'. Beyond $\sim 3 \mathrm{M}_{\odot}$ even this degeneracy pressure is not enough to stop the collapse, and a black hole is formed. As an aside, it should be noted that the existence of black holes has not been definitively proven, although the evidence points towards their existence. The most convincing evidence for these objects is binary star systems where radial velocity measurements point to the masses of the second and unseen companions being in excess of the maximum (theoretical) mass for a neutron star. See Cowley (1992) for a review of the evidence for black holes in stellar binary systems.

The hotter cores of massive stars result in rapid reaction rates, which in turn result in the quick exhaustion of the available core hydrogen. The Sun will remain on the main sequence for some 10 billion years, while a $15 \mathrm{M}_{\odot}$ star stays on the main sequence for only $\sim 12$ million years (e.g., see Figure 1 of Lindoff 1967). However the high internal temperatures of massive stars allow heavier elements to be used in fusion reactions. After reaching the turnoff, the core of a massive star contracts, drawing new hydrogen down into the star's inner regions. hydrogen fusion reactions then commence in a shell, as for the low mass stars, and the star becomes a red giant. However, unlike low mass stars the core of a massive star is not degenerate. Stars with masses greater than $2 \frac{1}{4} \mathrm{M}_{\odot}$ do not develop degenerate helium cores because the stellar core does not become dense enough before helium ignition takes place. Therefore, when the core is heated enough to commence helium fusion no helium flash occurs, but a relatively gentle ignition instead. The star shifts blueward on the CMD, remaining nearly constant in luminosity - rather than drops dramatically as with low mass stars - moving in a so-called giant loop.

For a short time the star 'burns' helium in its core. When adequate supplies run out, the core contracts and shell burning again commences around the core, but this time of helium. The star moves back onto the red giant branch. Depending on the mass of the star, this process can continue through further giant loops as heavier elements are used as fuel. Successive reaction stages can occur in a series of concentric shells about a star's core, rather resembling an onion, with the final element depending (to a first approximation) on the mass of the star. The older shells do not cease activity, but simply move radially outwards as the star contracts. Nuclear fusion of iron is an endothermic reaction. Given that a star is a balance between the inwardly directed gravitational force and the outward radiation pressure generated by the fusion reactions, silicon is the last element that can be used by a star as fuel. Such a star is a pre-supernova (Type $\mathrm{II}^{1}$ ). Its iron core is incapable of generating energy. The star then collapses catastrophically, liberating vast amounts of gravitational potential energy. The outer layers of the star are blasted into space. The supernova progenitor should be a red giant,

\footnotetext{
${ }^{1}$ There are two basic types of supernova. Type I explosions are believed to be a white dwarf in a close binary star system that has accreted enough mass from a companion star for it to exceed the Chandrasekhar limit of $1.4 \mathrm{M}_{\odot}$. The dwarf then collapses, violently igniting the accreted material.
} 
although SN1987A was a blue supergiant thought to be a Wolf-Rayet star. These are massive stars which have lost their outer layers as dense high velocity winds, thereby exposing the hot stellar cores. Other theories which attempt to explain why a blue star went supernova centre around the presumably low metallicity of the progenitor and the effects this may have on late stages of stellar evolution. For a review of SN1987A see Arnett et al. (1989).

Metallicity affects the evolution of a star in that low metallicity stars fusing helium in their cores lie in a different region of a CMD from extreme Population I stars at the same evolutionary phase. Regardless of the metallicity of a star, the general trends of stellar evolution remain the same. Once equilibrium is restored after the helium flash, the brightness and colour of a low metal abundance, low mass star are such that it now lies on the horizontal branch (HB) on the CMD. The horizontal branch is so-called because it is a roughly horizontal feature on the diagram (see Figure 1-3). The branch typically lies some 3.5 magnitudes above the main sequence turnoff, and around 2 magnitudes below the tip of the RGB. The horizontal branch for M3 (Figure 1-3) is well populated, with both blue and red stars. The apparent gap at B - V $\sim 0.3$ is not an actual feature of the branch. The HB stars in this region of the H-R diagram are known to be variables (RR Lyrae stars), whose outer layers radially pulsate in the lower spherical harmonics with periods typically between 0.2 and 0.8 days. All RR Lyrae stars have the same mean absolute magnitude of $\sim 0.5$, making them useful distance indicators out to about 200 kiloparsecs.

The morphology of the HB varies between old clusters, with some clusters having only blue HB stars while others have branches that are more red. Stellar evolution models have shown that the HB morphology is dependent on metal abundance. A metal-rich cluster generally has a horizontal branch more red than that of a metal-poor cluster. However the HB is different between clusters with the same metallicity, indicating the need for at least a second parameter to explain the variations. Many parameters have been suggested to explain what has come to be known as the "second parameter problem". These have included the age of the cluster, which is the most promising, and variations of carbon, nitrogen, and oxygen abundances relative to each other. For a good fit to cluster CMDs, models of stellar evolution require that HB stars are around $20 \%$ less massive than the main sequence turnoff mass. It therefore appears that mass must be lost during the red giant phase, or during the structural realignment of the star following the helium flash.

Once HB stars exhaust their core helium, they again evolve towards higher luminosities and redder colours. The extent of evolutionary movement across the CMD is determined by the envelope mass above the hydrogen-burning shell. HB stars with small envelopes evolve almost vertically before becoming white dwarfs, while stars with larger envelopes evolve towards and above the RGB outlining the asymptotic giant branch (AGB). The evolution of a star along 
the $\mathrm{AGB}$ ends when the mass of the outer envelope of the star reaches a lower limit. During the AGB stage, this envelope is being stripped either by gradual mass loss or the dramatic loss associated with the formation of a shell of a planetary nebula. When the mass limit is met, the internal pressure of the shell beneath the envelope is too low to support fusion reactions.

\subsection{The Magellanic Clouds}

Both the Large Magellanic Cloud (L$\mathrm{MC}$ ) and Small Magellanic Cloud (SMC) are large naked-eye objects, resembling detached portions of the Milky Way. This is just the appearance one might expect of the Clouds, which are the closest ${ }^{1}$ large galaxies to our own (see Figures 1-5 and 1-6 for photographs of the SMC and LMC). Being close to the South Celestial Pole with declinations of $\sim-70$ degrees, the Clouds are best placed for ob-

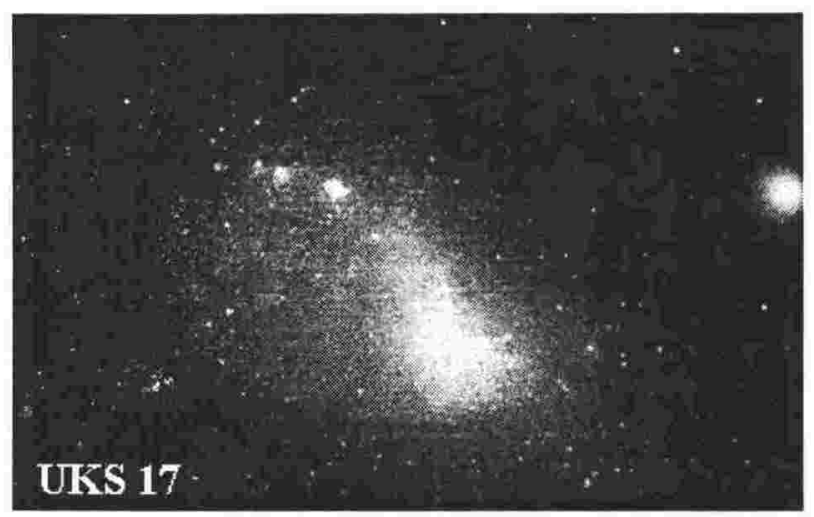

Figure 1-5: The Small Magellanic Cloud. Photograph taken by the 1.2 metre UK Schmidt Telescope (C)ROE/AAO. Reproduced with permission).

serving in the Southern Hemisphere, although they can be seen from the lower northern hemisphere latitudes.

The Clouds are named after the Portuguese explorer Ferdinand Magellan, who mentioned them in his 1521 log-book during the first circumnavigation of the world (Bok 1969a, b). While the Clouds may be named in honour of the leader of the first European expedition that saw these galaxies, they were well known to the established southern hemisphere cultures. For instance, one Maori tradition held that the Clouds warded

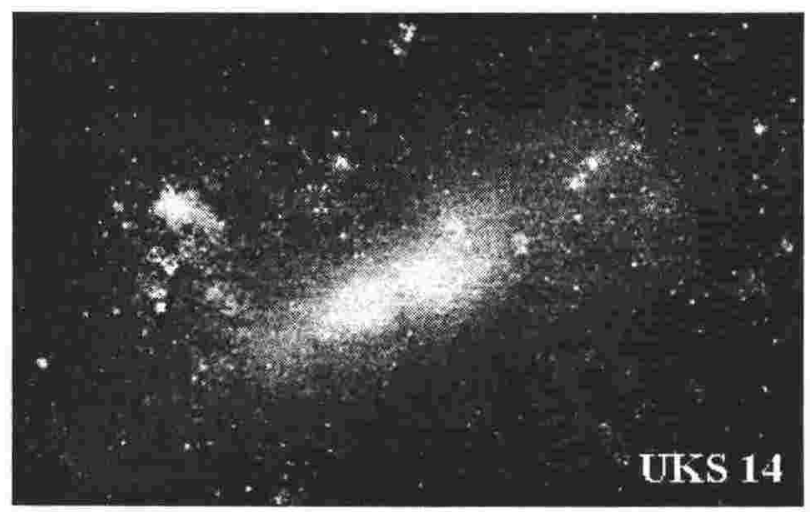

Figure 1-6: The Large Magellanic Cloud Photograph taken by the 1.2 metre UK Schmidt Telescope (CROE/AAO. Reproduced with permission).

off strong winds, and also acted as aids for weather forecasting. A supposed sign of fair weather was if the LMC was the closer of the two galaxies to the direction of the prevailing wind (Best 1987).

\footnotetext{
${ }^{1}$ The newly discovered dwarf spheroidal in Sagittarius now has the honour of being the closest external galaxy (Ibata et al. 1994, Mateo et al. 1994).
} 
The Magellanic Clouds are believed to be satellites of our own galaxy. Their proximity provides us with a unique laboratory for the study of the evolution and dynamics of stars, clusters, and galaxies. It is especially convenient that they are placed well above the disk of the Milky Way with a Galactic latitude $\mathrm{b} \sim-34^{\circ}$, lowering the amount of interstellar dust that we observe them through to almost negligible values $(\mathrm{E}(\mathrm{B}-\mathrm{V}) \sim 0.10)$.

\subsection{The Large Magellanic Cloud}

The Large Magellanic Cloud appears on first examination to be an irregular galaxy, but possesses a bar and some indication of spiral structure (de Vaucouleurs 1963, Smith et al. 1988) leading to its classification by de Vaucouleurs \& Freeman (1972) as the $\mathrm{SBm}^{1}$ prototype a class between late spiral and irregular galaxies. The main characteristics of this group are (Freeman 1984) :-

- A small bar, which is optically the most conspicuous feature.

- A disk, which is the main light source. The disk exhibits the expected exponentially decaying (with radius) distribution in brightness.

- A strong asymmetry of the spiral structure, with the bar and disk offset by a typical distance of 0.5 to $1.0 \mathrm{kpc}$.

- The disk is not as thin as those of normal spirals. Wielin \& Fuchs (1984) suggested that the cause could be some mechanism heating the disk of the LMC. A source that has been suggested for this mechanism might be the asymmetry of the bar itself, via a resonant excitation of the motion perpendicular to the Galactic plane (Binney 1981). However the disk is still quite thin, as indicated by the small average stellar velocity dispersion of $15.1 \pm 1.9 \mathrm{~km} / \mathrm{s}$ (which increases with object age and proximity to the centre of the LMC (Feast 1989)) derived after compensating for the galactocentric motion of the Earth and the rotation of the LMC (see e.g. Feast et al. 1960).

Rotation of the LMC was initially suggested by the radial velocities of 17 emission nebulae ${ }^{2}$ by Wilson (1918). Velocities become more positive to the south. The high mean velocity of $276 \mathrm{~km} / \mathrm{s}$ indicated clearly for the first time that the LMC was external to the Milky Way; the velocity being well above the rotation speed of the Milky Way in its outer regions (see Fich \& Tremaine (1991) for a galactic rotation curve). Hertzsprung $(1920,1923)$ argued that the velocity gradient was not due to rotation, as Wilson (1918) had suggested, but rather

\footnotetext{
${ }^{1} \mathrm{SBm}$ : which in Hubble notation stands for the 'Spiral, Barred - Magellanic' subset of galaxies.

${ }^{2}$ Emission nebulae are regions of hot interstellar gas and dust that shine by their own light. The constituent atoms of such nebulae are ionized. There are three classes: H II regions where the hydrogen is ionized by ultraviolet radiation from nearby $O$ and $B$ stars; planetary nebulae where the exciting source is the central dying star; and supernova remnants.
} 
experimental error with all parts of the LMC having the same motion. This was a view widely accepted for three decades (Wilson 1944), although a re-analysis of the Lick data (of Wilson 1918) indicated rotational motion of the LMC even if a large radial velocity was assumed (Kaburaki 1931). The latter view was finally supported by the $21 \mathrm{~cm}$ (Kerr et al. 1954) and stellar (Feast et al. 1955, Feast 1964) rotation curves, although they indicated different centres of rotation.

The $21 \mathrm{~cm}$ centre is at the (Epoch 1950) equatorial coordinates of $5^{\mathrm{h}} 20^{\mathrm{m}},-69.0$ (Hindman 1967 ) in an optically empty region $\sim 1^{\circ}$ north of the bar, while the optical one (based on isophotes of the outer regions) lies at $5^{\mathrm{h}} 25^{\mathrm{m}},-69.5$ (Feast 1964) - again, outside the bar. Other centres lie in a range between these two points, e.g. the stellar and $\mathrm{H}$ I nebulae radial velocities centre is close to that for radio observations (Feast et al. 1961), while the planetary nebula (Webster 1965) and the cluster centres lie closer to the optical centre (see Figure 11 of de Vaucouleurs \& Freeman 1972). Unfortunately formal errors for the centre positions are not given by these early studies. However, there is little disagreement about the position angle of the maximum differential velocity, with $171^{\circ}$ (Feast et al. 1961), $165^{\circ}$ (de Vaucouleurs 1957), and $170^{\circ}$ (Elsässer 1959). See Westerlund (1990) for a more complete list of derived inclinations and position angles. The greatest extension of the cluster distribution is found in this region (Westerlund 1964). If rotational symmetry is assumed then an inclination, rotational period, and mass $\left(\sim 10^{10} \mathrm{M}_{\odot}\right)$ can be calculated for the LMC, with uncertainties in the inclination and overall distance complicating the mass estimates. Due to the certainly distorted nature of the LMC, a large range of $22-45^{\circ}$ has been reached for the inclination (see Feast 1989 for a comprehensive summary of these studies). The de Vaucouleurs \& de Vaucouleurs (1963) value of $27^{\circ}$ is widely adopted, although this analysis truncated the southern side of the LMC. The rotational and circular orbital (about the Galaxy) periods of the LMC are approximately the same length at $10^{9}$ years, leading to the suggestion of tidal locking (Bok 1969a).

Initially it was believed that the Magellanic Clouds were at the same distance of $\sim 32$ kpc (Thackeray 1963). This distance was based on the long period Cepheid work of Shapley $(1918,1924)$. Cepheids are supergiant stars that are pulsating variables, rather like the RR Lyraes. The log of their pulsation periods increases linearly with magnitude, making them a useful gauge of distance. If the pulsation rate and apparent magnitude of a given Cepheid are known then the absolute magnitude of the star can be calculated. Confusion in early work using Cepheids to determine distances was resolved by the discovery that there are two types of Cepheids: 
- Classical Cepheids, which belong to Population I, and

- W Virginis stars, which are older and less massive Population II stars. The W Virginis stars are about 1.7 magnitudes fainter than classical Cepheids.

By the 1940s observational improvements had been made that allowed magnitudes 17-18 to be reached. RR Lyrae stars should have been observed at these magnitudes, given the estimated distances of the Clouds. They were not, and therefore the Clouds had to be further away than they were believed to be. Thackeray \& Wesselink (1953) finally observed RR Lyrae stars at magnitude $\sim 19$ in the SMC star cluster NGC 121. This and later work led to new distance estimates of 55 and $63 \mathrm{kpc}$ for the LMC and SMC respectively (Thackeray \& Wesselink 1955, see also Sandage \& Tammann 1974). These distance are in reasonable agreement with distances derived using:

- The peak intensities of novae ${ }^{1}$ (Shapley 1953),

- The apparent diameters of H II regions (Gum \& de Vaucouleurs 1953), and

- The integrated magnitudes of star clusters (Shapley \& Nail 1953a, b).

Problems have been encountered with this 'classic' LMC distance modulus in isochrone fits to cluster CMDs (e.g. Walker 1974, Schommer et al. 1984, Andersen et al. 1985, Mateo \& Hodge 1986). The major problem with the CMD studies is that the masses of red giant clump stars are in excess of the corresponding main sequence turnoff mass for the 'classic' distances to the Clouds. Stellar evolution models indicate that mass loss must have been experienced by these clump stars (Seggewiess \& Richtler 1989). A modulus shorter by $\sim 0.5$ magnitudes has therefore been suggested, which provides a better fit of the observations to theory. Bertelli et al. (1986) introduced convective overshooting at the $\mathrm{H}$ and $\mathrm{He}$ cores in an attempt to resolve the problem. This lowered the maximum helium flash mass, extending both advanced evolutionary state lifetimes and the core $\mathrm{H}$ burning phase considerably by $\sim 3$ times (Chiosi \& Pigatto 1986). Such models would place the clusters at a slightly greater distance away.

Convection is not rigourously understood, with the 'mixing length' approximation being widely used. This method checks if a temperature gradient is greater than the adiabatic one. If it is, then energy transport is attributed to convection, with 'blobs' rising or falling through a given height at speeds dependent on their relative densities compared to their surroundings. This height, or the mixing length, is typically one or two pressure scale heights. One problem with this approach is that at the edge of the convective zone, the force on a blob goes to zero, yet

\footnotetext{
${ }^{1}$ Novae are stars that suddenly and temporarily brighten by 10 magnitudes or more. They are believed to be close binary stars, where one component is a red giant star streaming matter onto an extremely evolved star, such as a white dwarf. When a 'critical' mass of hydrogen is accreted onto the white dwarf, nuclear reactions commence violently and last briefly.
} 
its velocity is likely not to be zero at this point. Such overshooting will expand the theoretical convective zone, mixing in more fuel for a star.

Mateo \& Hodge (1987) found that overshooting did not resolve the distance problems with LMC star clusters. Graham (1984) noted that the resolution of the problem could lie in the metal-poor LMC RR Lyrae stars being a magnitude fainter than their Galactic counterparts, together with the possibility of a discrepancy in the zero point of the Cepheid period-luminosity relation. The distance modulus remains controversial.

The Magellanic Clouds are part of an interacting system consisting of the two Clouds, the Milky Way, and nine spheroidal dwarf galaxies. Interactions between the Clouds and the Galaxy are believed to have caused tidal distortions of the Clouds and stripping of gas from them. Mathewson et al. (1987) have suggested that the SMC has been ripped into two by tidal forces, and that this galaxy in the process of irreversible disintegration. A warp in the disk of the Milky Way was suggested by Kerr (1965) to be caused by the Clouds. However later work (see Binney 1992, Gilmore 1989) showed that such warps were common in spiral galaxies. Arp (1961) noted that SBm galaxies are often found in free space as pairs (e.g. NGC 4618/4625 and NGC 4027/A) and have been proposed as fission remnants from events in the early universe. A small sample survey of 31 spiral galaxies from the Shapley-Ames (1932) catalog showed that for up to spiral type Sb, 30\% of companion galaxies are of the Magellanic class. The remaining $70 \%$ of companions are 'normal' irregular galaxies. All the companions to Sc class galaxies are standard irregular galaxies. This survey suggests that the type of satellite galaxies could be influenced by the primal conditions of the 'parent' galaxy.

There are three main propositions for the origin of the Clouds -

1. A chance meeting with the Milky Way, resulting in a hyperbolic orbit about the Galaxy (Mathewson \& Ford 1984).

2. Formation as part of our hyper-galaxy (Einasto et al. 1976). The Clouds have therefore always been satellites of our Galaxy.

3. Formation as part of the Local Group with capture by the Milky Way reasonably early (Westerlund 1990). This scenario would explain why the orbital plane of the Clouds coincides with that corresponding to the Local Group.

The main problem with the second hypothesis is that the Clouds are still rich in gas 15 Gyrs after formation, while the more distant satellite galaxies and globular clusters are nearly completely stripped of gas (Westerlund 1989). The third hypothesis is currently enjoying popularity, particularly due to the work of Murai \& Fujimoto (1980) who regressed the positions of the three major bodies (LMC, SMC, and the Galaxy) up to $10 \mathrm{Gyr}$ into the past. There was considerable success in reproducing observational features such as the Magellanic Stream. Dif- 
ficulties in determining the velocities of the Magellanic Clouds lie with problems compensating accurately for the motion of the Earth. This means that the first possibility for the origin of the Clouds can not yet be discarded.

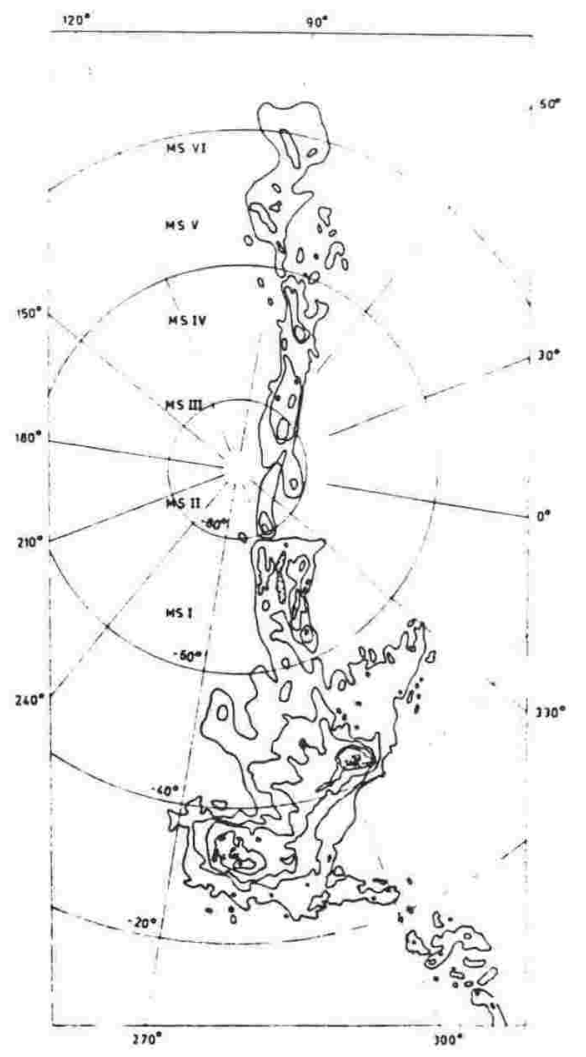

Figure 1-7: The Magellanic Stream is given as contours of H I gas in galactic co-ordinates. The outer contour is the $10^{-19}$ atoms per $\mathrm{cm}^{2}$ isophote, with the others arbitrarily chosen to emphasize features. The LMC is at $(1, b) \sim$ $\left(280^{\circ},-34^{\circ}\right)$, while the SMC is $(1, \mathrm{~b}) \sim$ $\left(300^{\circ},-43^{\circ}\right)$. (Diagram from Mathewson \& Ford 1984)

well the observational stream velocities of Mathewson et al. (1979). The steep H I gradient on the eastern side of the LMC is explained as being due to halo ram pressure, i.e., the Clouds orbit anti-clockwise about the Galaxy.

Support for a shorter period of 'capture' can be seen in that the LMC is archetypical of its class, and has not experienced as severe damage as could be expected after several close 
interactions with the Milky Way. There is no evidence for a bridge of material connecting the Clouds to the Milky Way, despite the suspicions of Herschel (1847), de Vaucouleurs (1954), and Elsässer \& Haug (1960). The Murai \& Fujimoto $(1980,1986)$ model predicts that the last encounter between the Clouds coincided with perigalacticon. According to the model, this was 200 million years ago with a centre to centre separation of $3 \mathrm{kpc}$ between the two Clouds. This event would have caused severe tidal shocking of these smaller bodies. Gunn (1980) suggested that this could be responsible for bursts of star formation. However, the star formation bursts and enrichment rates of the two Clouds do not coincide (Da Costa 1991a), casting doubt on such an external cause for these events being common to the two galaxies.

Problems with the tidal models include (Moore \& Davis 1994):

1. The expectation of stars in the Magellanic Stream. None have been detected, although stars should have been stripped as easily as gas from the Clouds (Mathewson 1985). The lack of stars in the Stream means that the distance to it can not be calculated.

2. The lack of a leading stream as expected in tidal models.

3. High resolution numerical simulations indicate that material tidally stripped from the LMC would retain the internal LMC velocities, and so form a thick plane about the Milky Way rather than the tight wake that is observed.

Discrete ram pressure models suggest that the Stream is material swept from the Magellanic Cloud environs by collisions with HVCs. Mathewson et al. (1987) explain HVCs as being the capture products of a collision between our Galaxy and another galaxy some $10 \mathrm{Gyr}$ ago. While this explains the clumpy nature of the Stream, the model requires six collisions of the Clouds with the HVCs (one collision for each clump) to occur in rapid succession over the last 500 million years. Continuous ram pressure models, such as Liu (1992), require a high halo density ( $>10^{-3}$ atoms per cubic $\mathrm{cm}$ ). This density is not confirmed by the low energy X-ray emission that would be expected from such a mass (Moore \& Davis 1994), nor can these models easily explain the subclumps in the stream.

The transverse motion of the LMC has been measured by Jones et al. (1991) and Tucholke \& Hiesgen (1991), who compared current star positions with those on old photographic plates. While measurement errors are large, the fact that the Stream in on the trailing edge of the Clouds is confirmed. The orbit of the LMC appears to be circular, which does not fit well will the model of Murai \& Fujimoto $(1980,1986)$.

Unfortunately the pan-Magellanic flow field, which has proven to be so useful in establishing the dynamics of the LMC, disturbs the motion of objects in the outer regions of the Cloud, such as H II regions. These objects could have been used to establish data points at large radii in rotation curve studies. Hence the assumption of Murai \& Fujimoto (1980) of a dark massive 
corona, necessary in their model for the formation of the Stream, is not directly provable. However the rotation curve solution of Carignan (1983) for the SBm galaxy NGC 3109 required such a corona of approximately seven times the mass of the luminous disk (within $10 \mathrm{kpc}$ ). This does lend support to the existence of such masses about the Clouds.

\subsection{The LMC Clusters}

\subsubsection{Classification}

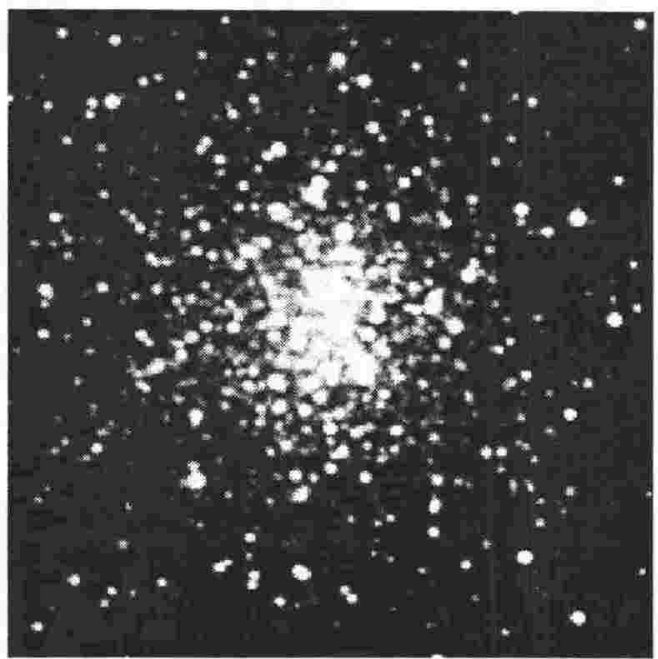

Figure 1-8: The LMC Cluster Hodge 11. Photograph taken by David Malin with the AAT (C)AAO. Reproduced with permission).
The dichotomy that exists for clusters in the Milky Way does not occur in the Magellanic Clouds. There is no such bivariance (Gascoigne 1966), with objects that resemble globular clusters (see Figure 1-8) spanning a large interval of ages. The clusters also show a large heavy element abundance spread at any given age (Batinelli \& Capuzzo-Dolcetta 1989). The latter was explained by Freeman (1981) as being possibly due to the clusters forming in the outer and low density regions of the LMC. This would result in a somewhat unpredictable Initial Mass Function ${ }^{1}$ (IMF).

However, the star clusters in the Large Magellanic Cloud can be divided into two groups on the basis of their integrated colours (Gascoigne 1966). The distribution of colours is not uniform, being bimodal (see Figure 1-9a). The blue clusters outnumber the red clusters by 5 to 1 . Several explanations have been suggested for the cause of the gap, including that it is:

- Really an age gap, due to separate bursts of cluster formation,

- An effect of cluster disruption rates being different for clusters of different masses, or

- Due to red giant or asymptotic branch phase transitions (Chiosi 1989). The sudden appearance of stars in either of these two evolutionary stages would drastically redden the colour of a cluster (Gascoigne 1980), although the lack of a bimodal distribution for the SMC and M33 (Christian \& Schommer 1982) suggests that this reason is not the cause of the gap. The gap is apparent in M31, but red clusters outnumber blue clusters instead (Fusi Pecci 1988)

\footnotetext{
${ }^{1}$ Discussed below
} 


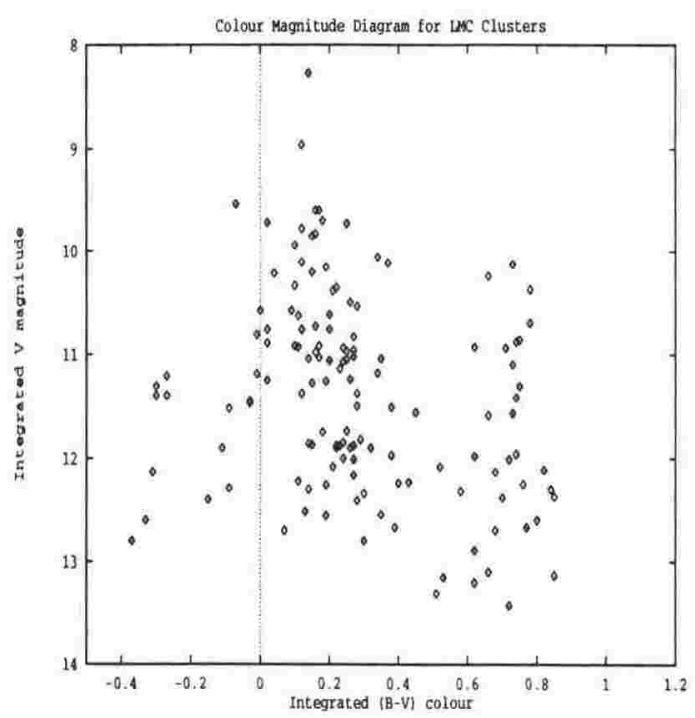

(a)

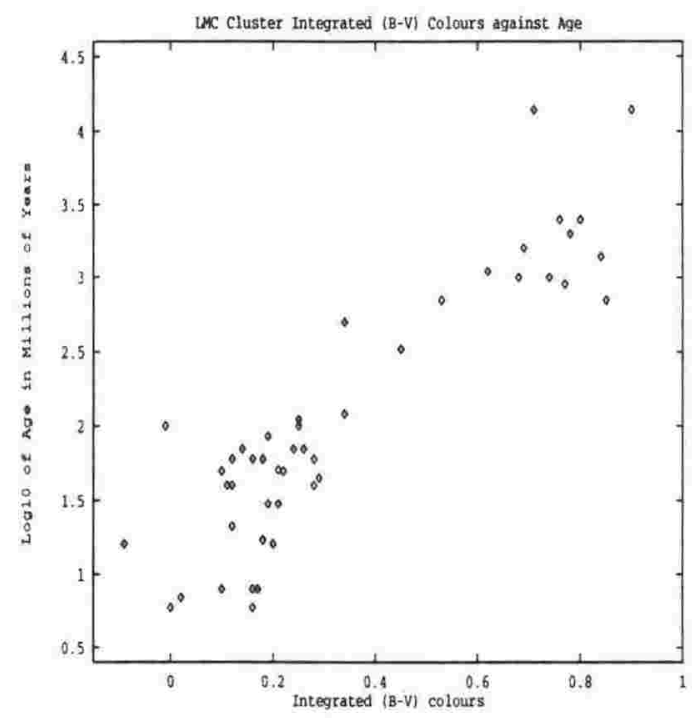

(b)

Figure 1-9: Integrated (B - V) Colours of LMC Clusters from Van den Bergh (1981) are plotted against the integrated $\mathrm{V}$ magnitudes for the clusters in sub-figure (a), and against age in sub-figure (b). The ages from Seggewiss \& Richtler (1989).

The gap is most likely to be due to two bursts of star formation, given the age-colour relation shown in Figure 1-9(b).

The two classes of star clusters in the Large Magellanic Cloud are called the:

- Red Clusters - which are old objects, with their brightest stars being red giants. Radial star counts by Geyer et al. (1979) found the clusters to be remarkably similar, with the blue (HB, and originally more massive) stars centrally condensed. This indicates that the clusters are dynamically well relaxed. There are seven clusters which appear to be genuine globular clusters, being of the right mass, size, age, and mean absolute magnitude $M_{v}$ of -7.1 (Van den Bergh 1991). These are the clusters Hodge 11 and NGC numbers $1466,1786,1835,1841,2210$, and 2257. They do not appear to be Galactic globular clusters merely superimposed on the LMC. If a double symmetry of the system of globular clusters, about the Galactic disk and the Galactic longitude of $0^{\circ}$, and a mass for the LMC of $10^{10} \mathrm{M}_{\odot}$ are assumed then the gravitational range of the LMC can be calculated. The number of globular clusters falling into this region can be compared with the number in the other three 'mirror' regions. Two genuine Galactic globular clusters are superposed onto the LMC. These are Sersic 1 and ESO 3, which are both at the gravitational limit of the LMC, if they are assumed to be at the same radial distance as the LMC. The three mirror regions contain 0,3 , and 4 globular clusters, as opposed to 9 if all the globular clusters in the direction of the LMC belong to the Galaxy and are 
merely in our line of sight. Given a mean of $2.3 \pm 2.1,9$ is beyond the $3 \sigma$ confidence level, and very unlikely. It is therefore more probable that the seven clusters are indeed in the LMC, a view supported by radial velocity studies such as Meylan (1988) and Freeman et al. (1983), which show that clusters such as NGC 1835 have the systemic radial velocity of the LMC.

- Blue Clusters - which are young objects, with high mass stars still on the main sequence and generally only a few evolved stars (Geyer \& Hänel 1984). From the viewpoint of stellar evolution the clusters resemble open clusters, but morphologically they are globular clusters (Geyer et al. 1979) although their ages are shorter than their relaxation times and their masses may be ten times smaller than those of globular clusters. This has led to the suggestion these clusters are relaxed at formation, perhaps providing a clue about the initial dynamics of globular clusters. Such evidence would have been obliterated by two-body processes in the Milky Way globular clusters over their long lifetimes. Some authors have found no sign of mass segregation (e.g. Geyer et al. 1979) with an apparently uniform distribution of the stellar types, as might be expected in young clusters which have not had enough time to relax dynamically. However Geyer \& Hänel (1984) did find some evidence for mass segregation in the young cluster NGC 1818 with the more massive stars already more centrally concentrated (see also Chrysovergis et al. 1989). Hodge 11 is an example of the blue cluster group (see Figure 1-8 on page 18), but it is an exception. The blue integrated colour of the cluster is due to the brightest member stars not being young but rather on the horizontal branch. Hodge 11 is actually an old cluster.

\subsubsection{Structure}

The radial light curves and star counts for both classes of LMC star cluster are fairly well represented by globular cluster King $(1962,1966 \mathrm{a}, \mathrm{b})^{1}$ models (Chun 1978, Freeman 1974). Around $15 \%$ of the younger clusters exhibit abnormalities in their outer regions such as bumps or shoulders (Elson 1991). Cluster masses can be estimated from the tidal radii if circular cluster orbits about the centre of the LMC are assumed, as the tidal field of the LMC is known from rotation curves. This has led to estimates of cluster masses of between $10^{4}$ to $10^{5} \mathrm{M}_{\odot}$, together with corresponding mass-luminosity ratios of [0.02-0.56], which is about $10 \%$ that of our Galaxy (Seggeweiss \& Richtler 1989). Such masses are low in comparison with Milky Way globular clusters, although the concentrations (tidal/core radii) are similar for the LMC and Galactic globular clusters. The concentrations are between 1 and 1.5. Whether the LMC populous clusters are 'real' globular clusters is hotly debated - as they are in general younger

\footnotetext{
${ }^{1}$ King models are discussed in more detail in Chapter 5 , where terms such as tidal and core radii are explained.
} 
and less massive than Galactic globular clusters.

Meylan (1988) argued that the King $(1962,1966 \mathrm{a}, \mathrm{b})$ models are an unphysical oversimplification with their single mass and isotropic approach which also ignores the effects of any rotation of the cluster. A further complication for the application of King models to young LMC clusters is that the clusters do not appear to be tidally limited, with a halo of unbound stars (Elson et al., 1987). Presumably similar halos about the young globular clusters in the early Galaxy were the origin of the bulk of the halo stars ${ }^{1}$. Together with the assumption of a circular orbit for a cluster about the LMC centre, Meylan (1988) considered that studies such as Elson \& Freeman (1985) only provided a lower limit to the mass of a given cluster. Meylan (1988) therefore applied a multi-mass anisotropic distribution model, already used on some Galactic globular clusters, in conjunction with fitting of the internal velocity dispersion of the star cluster. Meylan (1989) derived a mass of $10^{6} \mathrm{M}_{\odot}$ for NGC 1835 , with a mass-luminosity (M/L) ratio of 3.4. These values are comparable to a rich Milky Way globular. Similarly Meylan et al. (1991) calculated a M/L ratio of 1.2 to 4.2 for NGC 1978 , along with a mass estimate of $0.4-1.4 \times 10^{6} \mathrm{M}_{\odot}$ for the cluster. These results suggest that perhaps the difference between the masses of 'globular' clusters in the LMC and our Galaxy lies in the techniques being used on the LMC clusters.

\subsubsection{Mass Functions}

The distribution of masses with which stars form is known as the Initial Mass Function (IMF). It is often described using a power law of form $\mathrm{M}^{x-1}$, where $\mathrm{M}$ is mass in solar units (see Chapter 7 for further details on the IMF). The IMFs derived for the LMC clusters by Elson et al. $(1987,1989)$ and Freeman (1977) are of shallow gradient, with $x$ values in the range -0.2 to 0.8. Other investigators used CCDs (Charge-Coupled Devices) and techniques to account for counting incompleteness, rather than the photographic plate and drift photometry techniques of Elson et al. $(1987,1989)$. The CCD-based studies have derived higher values of $x$ from a maximum of around 2.5 (Mateo 1988) to a minimum near 1.1 (Cayrel et al. 1988, Sagar \& Richtler 1991), depending on the techniques used to estimate the completeness of the data. This topic is further discussed in greater detail in the introduction to Chapter 7 .

\subsubsection{Ages and Metallicity}

Cluster ages can be estimated by several techniques including:

- The extension of the asymptotic giant branch (Mould \& Aaronson 1983),

- Luminosity of the main sequence turnoff point (Hodge 1983),

\footnotetext{
${ }^{1}$ Although escape from young globular clusters can not explain the early-type stars found in the halo.
} 


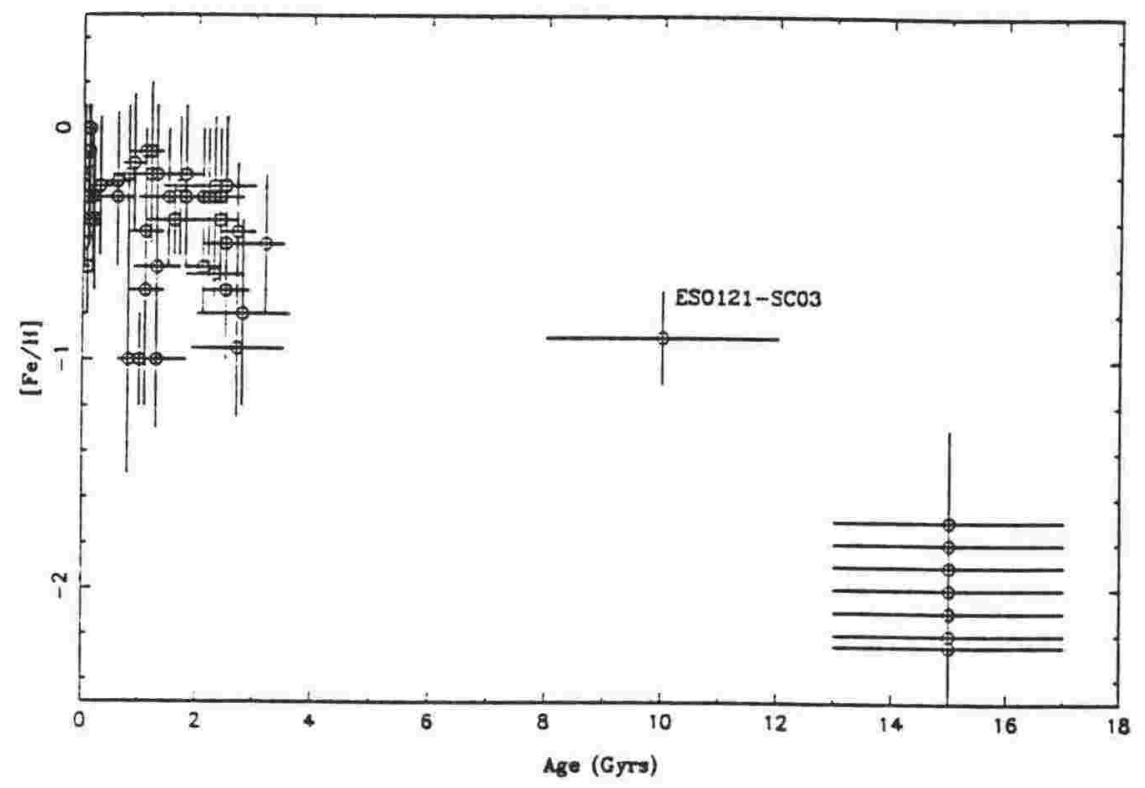

Figure 1-10: The Age-Abundance Relation for LMC Clusters whose ages have been determined using photometry and fitting of the main sequence turnoff. The old clusters have been arbitrarily assigned an age of $15 \pm 2 \mathrm{Gyr}$. Metallicity is plotted on the y axis. (Figure taken from Da Costa 1991a)

- The distribution of red giant stars in a colour-colour diagram (although this method is only suitable for young clusters, Flower 1984), and

- The fitting of isochrones based on stellar evolution theories to colour magnitude diagrams (e.g. Van den Bergh 1981, Sweigart \& Gross 1976, Maeder \& Mermilliod 1981, Bertelli et al. 1986, Seidel et al., 1987).

The age distribution of the Magellanic clusters is bimodal. The first peak in the number of clusters formed is some $13 \mathrm{Gyr}$ ago, while the other started about $3 \mathrm{Gyr}$ ago and continues to the current day (Geyer \& Cassatella 1984, Stryker 1984, Mateo 1988a, Olszewski 1988). Only one cluster in between these two groups, with an age of about $8 \mathrm{Gyrs}$, has yet been dated (see Figure 1-10).

The age-metallicity relation derived from clusters indicates that the LMC enrichment rate has generally been slower than that of the Milky Way, leading to the present day metallicity of the LMC being approximately three-quarters the Solar value (Searle 1984). Smith et al. (1988) presented evidence that the LMC chemical enrichment rate over the last 6-8 Gyrs has been faster than either in the SMC or the Solar Neighbourhood in the Milky Way, with the inner regions of the LMC still forming stars (Hardy et al. 1984, Mateo 1988a). A radial trend towards the LMC centre can be seen also in the few comprehensive field studies yet performed 
(Stryker 1983, Butcher 1977, Hardy et al. 1984), with a $9 \mathrm{kpc}$ (distance from the LMC centre for cluster rotation) bulk field age of 6-7 Gyr, a 4-5 kpc radius being 3-5 Gyr old, and the bar being 0-3 Gyr old. Da Costa et al. (1985) noted that the cluster ages given by Gascoigne et al. (1976) and their study also implied a radial abundance gradient, although this involved only two clusters! The spectroscopy of individual stars by Cohen (1982) indicated a monotonic growth in abundance, but more recent work on field asymptotic giant branch stars (Frogel 1984, Frogel \& Blanco 1983) indicated two recent bursts of star formation $10^{8}$ and 3-5 x $10^{9}$ years ago. The weight of the argument, based mainly on cluster data, is away from monotonic enrichment.

Searle et al. (1980) used intermediate band photometry to order LMC clusters by their hydrogen lines, resulting in a smooth but not monotonic series in metal line strengths. This was unlike Danziger (1973), who ordered LMC clusters by metal strength alone. Searle et al. (1980) defined 7 age groups, which Frenk \& Fall (1982), Hodge (1983), and Mould \& Aaronson (1983) attempted to date using the age techniques discussed above. Unfortunately there is poor agreement between these studies. The main sequence turnoff ages are much smaller than those derived using the asymptotic giant branch. This difference is presumably due to theoretical difficulties estimating the mass loss rate experienced in the advanced evolutionary state of AGB stars (Hodge 1983). However, the work of Searle et al. (1980) had a large impact on the field, as it acted as a check on colour-magnitude diagram based ages. For instance, NGC 418 and NGC 419 were dated the same age via their CMDs (Hodge 1983). While these clusters possessed similar metal line strengths, their hydrogen lines were very different indicating that they really are different ages (Searle 1984). This was confirmed to be the case by later CMDs of greater photometric accuracy and limiting magnitude.

The metallicities of young LMC clusters are lower than their Milky Way counterparts, being $0>[\mathrm{M} / \mathrm{H}]>-1.2$ (see Figure 1-10) where $\mathrm{M}$ stands for metals and $\mathrm{H}$ stands for hydrogen. ${ }^{1}$ This leads to the suggestion that perhaps the low environmental enrichment encourages rich 'globular' formation (Nelles \& Richtler 1984), which would explain why such clusters are not being currently formed in the Milky Way. Richtler \& Seggeweiss (1988) argued that metalpoor proto-clusters are more stable against mass loss, partially because they are smaller and more gravitationally bound (if thermal equilibrium is assumed). Abott (1982) noted that the strength of stellar winds decreases with metallicity. Therefore, it would be more difficult for metal-poor clusters to be disrupted by stellar winds. However, there is a problem in that while young blue LMC clusters are rarely embedded in any remnant interstellar matter, neighbouring

\footnotetext{
${ }^{1}$ To specify abundances it is usual to give the logarithmic abundance of iron, or all the metals, to the hydrogen in the object. This ratio is then related to that of the Sun. For iron, the relation is:

$$
[\mathrm{Fe} / \mathrm{H}]=\log \frac{\mathrm{N}_{\mathrm{Fe}}}{\mathrm{N}_{\mathrm{H}}}-\log \left(\frac{\mathrm{N}_{\mathrm{Fe}}}{\mathrm{N}_{\mathrm{H}}}\right)_{\odot} .
$$

$[\mathrm{Fe} / \mathrm{H}]$ has been observed to vary from nearly +1 for some stars in the Galactic Bulge down to -2.3 and beyond for the most metal-poor globular clusters and halo stars.
} 
loose stellar associations of similar age are often coupled to dense H II regions. Hence either star formation in LMC star clusters is a very efficient process, or the combined ultraviolet radiation of the many early spectral type members 'blows' the gas away. Some young clusters are located completely outside the neutral hydrogen surrounding the LMC (Olszewski 1984), raising the problem of their formation. Similar observations apply for a substantial lack of nearby $\mathrm{CO}$ (Cohen et al. 1981), $\mathrm{H}_{\alpha}$ emission, and even dense molecular Clouds (McGee \& Milton 1966). Additionally there are many cases of the fields surrounding LMC clusters being distinctly younger or older than the cluster (e.g. Stryker 1983, Mould et al. 1984). Kontizas et al. (1987) claimed old clusters tended to be in an old field, the intermediate in young, and the young in old. In other words, Kontizas et al. (1987) argued that the star cluster is a completely separate unit from its immediate surroundings. This could simply be due to movement of the cluster from the site of its formation, although this would not be possible for young clusters. Richtler \& Nelles (1983), Spite et al. (1986), and Wolf \& Reiermann (1989) have noted that stars in the young populous clusters have lower abundances than the field, which is well-mixed across the LMC. Spite (1989) suggested that this indicates that perhaps the clusters formed from masses of gas and dust which escaped mixing for a substantial time, forming stars from the under-processed material after a delay. This has led to the role of clusters as tracers of the chemical evolution of the LMC being queried.

\subsubsection{Ellipticity}

The Magellanic clusters appear to have a higher ellipticity than the Milky Way globular clusters (Geisler \& Hodge 1980, Geyer et al. 1983, Frenk \& Fall 1982), with the SMC clusters being the most elliptical (Kontizas et al. 1991). Hodge (1984) suggested that the ellipticity increased with age. Fall \& Frenk (1984) found that the greatest change in ellipticity for a cluster occurred at an age of $\sim 10^{8}$ years, and suggested aspherical collapse and violent relaxation at formation to be the cause of the ellipticities. Such mechanisms would produce the observed ellipticity without inducing mass segregation. Frenk \& Fall (1982) commented that variation in ellipticity between clusters was due to the individual internal evolution of each cluster. Internal variation of ellipticity is common (Geyer \& Richtler 1984, Geyer et al. 1983). Zepka \& Dottori (1987) found that along with such an internal change in ellipticity, generally decreasing radially outwards, the major axis of the best-fit ellipses also varied with radius in about half their sample. Norris (1983) suggested that the ellipticity is related to the second parameter problem, noting that those Galactic globulars with blue horizontal branches have higher ellipticities, while those with red horizontal branches are almost spherical. This trend, if real, does not apply to the Magellanic clusters as Gascoigne (1966) found NGC 121 to have a high ellipticity and a red horizontal branch. Rather the ellipticity appears to be a function of the cluster luminosity 
(Van den Bergh, 1984). Once this dependence is removed there is no age dependence (Van den Bergh \& Morbey 1984).

Kontizas et al. (1989) argued that the higher ellipticity of the Magellanic star clusters is due to the lower masses of the LMC and SMC, which would reduce the effect of gravo-thermal shocks on the clusters. They found no age dependence, explaining the internal variations in ellipticity as being due to the increasing predominance of the external tidal field with radius from the cluster centre. This field would distort the cluster from spherical symmetry. Rotation acquired during the formation of the star cluster in the disk of the LMC has also been suggested as the cause of the ellipticities (Geisler \& Hodge 1980).

\subsubsection{Sizes}

The young Magellanic star clusters tend to be a variety of sizes, while the old ones are all small presumably because they are more tightly bound and so will survive as a unit longer. Bhatia \& MacGillivray (1989) used the COSMOS automatic photographic-plate measuring machine in an automated search of some $1.2 \mathrm{~m}$ UKST plates. They estimated that their survey was $\sim 99 \%$ complete for clusters down to radii of about $5 \mathrm{pc}$. The median cluster diameter was $8 \mathrm{pc}$, with a mean of $11 \mathrm{pc}$. These values are systematically larger than those for Galactic open clusters, whose size distribution is of a similar form. This suggested that the same formation mechanism is at work, with the size difference being caused by the different gravity of the respective parent galaxy.

A unimodal and asymmetric size distribution, with increasingly less numbers of smaller sized clusters, was unexpected although the trend can be seen in the re-binned survey data of Hodge (1980) and Shapley (1930a). Rather it had been expected that as the radius decreased the number of clusters would increase. The extensive tail towards the larger radii that was observed is presumably due to the low mass of the LMC, which also seems to encourage many binary clusters (Hatzidimitriou \& Bhatia 1988). Some of these binary clusters could be in the process of merging (Bhatia \& MacGillivray 1988). Such merging could be the cause of the unusual features, such as condensations, seen in profiles of some young clusters. The COSMOS survey found no strong dependence of ellipticity on cluster size, although there was some indication that major (diameters $>20 \mathrm{pc}$ ) clusters may possess smaller ellipticities. The mean ellipticity of the LMC clusters was confirmed as being larger than that of Milky Way globular clusters. There is an anti-correlation between the LMC bar and the major axes of the clusters, with the effect being weaker for the larger clusters. The axes of neighbouring clusters tend to be parallel, suggesting that the cause of the alignment is not tidal in nature, but possibly related to the fragmentation of the proto-clouds that the clusters formed from. 


\subsubsection{Distribution}

Morgan et al. (1989) reported that a new LMC cluster catalogue was in progress, and confirmed the double ellipse distribution of LMC star clusters as reported in previous surveys. The inner ellipse is the more densely populated of the two, and is centred close to the optical centre of the LMC. It has a major axis $\sim 8^{\circ}$ long, and an ellipticity of 0.9 . The outer ellipse is centred $\sim 1^{\circ}$ east of the optical centre. It is about $\sim 13^{\circ}$ long, and of 0.7 ellipticity. Both distributions share the same western boundary. The reason for these distributions is not known. There is an indication of structure in the cluster distribution with an 'arm' of clusters extending from the south-west corner, accompanied by a faint hint of another arm extending from the north-east side.

The old clusters are smoothly distributed as individuals across the face of the LMC. The young clusters congregate more in groupings, which themselves tend to concentrate towards the bar of the LMC (Van den Bergh 1991). While the apparent lack of a concentration of the older clusters in the bar might be a selection effect, with photometry of the older clusters being much more difficult in crowded regions, such an interpretation is unlikely given that no novae have been found in this region either.

The work of Freeman et al. (1983) on the radial velocities of LMC clusters overturned previous work which had expected the red and blue cluster populations to be halo and disk groups (e.g. Arp 1961, Cowley \& Hartwick 1982). Freeman et al. (1983) demonstrated that the young clusters rotate in a disk along with $\mathrm{H}$ I and II regions. The older clusters also move in a disk of similar rotational amplitude, but with a position angle of $41 \pm 5$ degrees as opposed to $1 \pm 5$ degrees (i.e., the nodes differ). This was taken to outline the old LMC disk. However, Freeman et al. (1983) noted the sparcity of the old cluster data, and commented that the result was far from clear cut (see Figure 1-11). The older cluster system also had a systematic velocity $\sim 18 \mathrm{~km} / \mathrm{s}$ lower than the young disk. Schommer (1991) presented velocities for 75 LMC clusters, and found this difference in velocities was actually a systematic error. The intermediate age clusters were found to rotate in a disk with an intermediate position between the young and old cluster disks. The disk for the oldest clusters was still appreciably rotated relative to that of the youngest clusters by $\sim 40^{\circ}$, although again the solution for the old clusters was poorly constrained.

The age of the youngest 'disk' agrees well with the time of the last sizeable tidal interaction in the model of Murai \& Fujimoto $(1980,1986)$, suggesting that a tidal mechanism could be responsible for the skewing of the LMC. 


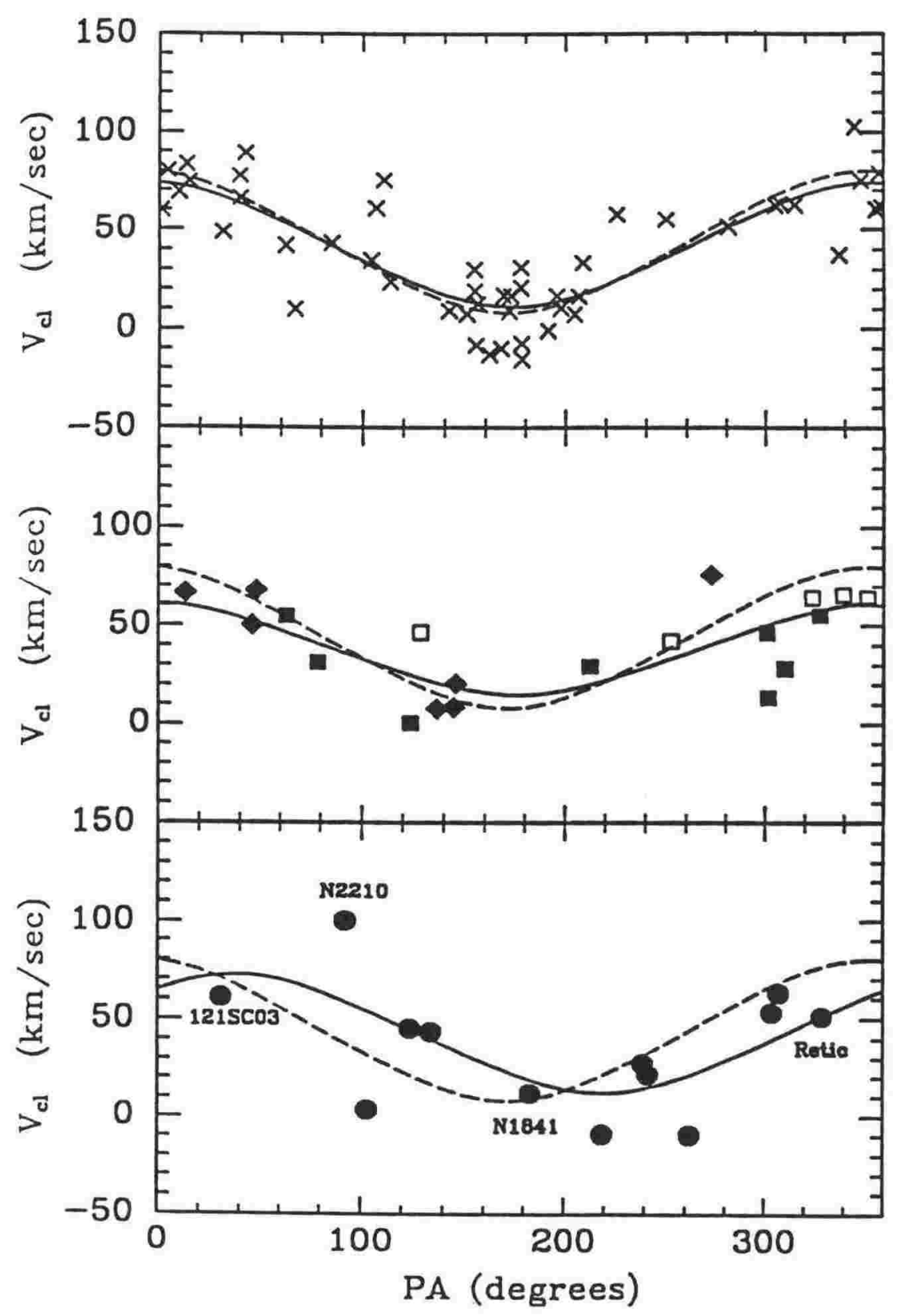

Figure 1-11: LMC Cluster Rotation Curves. The velocity of LMC clusters is plotted against position angle (which is measured in degrees in an easterly direction from north). The top diagram plots data points from the survey by Schommer (1991), the middle one plots intermediate age clusters, while the bottom one plots data for the oldest true globular clusters plus 121SCO3 (which is the isolated cluster in Figure 1-10). The lines are the models of Schommer (1991) fitted to the various data (Figure from Schommer 1991). 


\subsubsection{Conclusion}

The populous Magellanic clusters are important in that they can provide clues to the initial dynamic state of globular clusters, the enrichment rates of the Magellanic Clouds, the possibility of a universal IMF, and the stellar evolution of metal-poor stars. The populous nature of these clusters means that stars can be observed occupying short-lived (and therefore unusual) positions in the H-R diagram. In addition, we can refine the more general integrated observational methods via comparison with observations of individual stars using photometry or spectroscopy. The Clouds are close enough that individual stars can be studied in the clusters, but not so close that integrated studies of the clusters can not easily be undertaken. Refinement of the integrated methods by such comparisons with studies based on individual stars will allow greater confidence in results when the integrated techniques are applied to more distant galaxies. 


\section{Chapter 2}

\section{Data Collection}

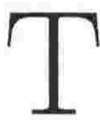

he collection of good quality data requires an understanding of both the equipment used to collect the observations and the problems, such as the dimming of starlight by the Earth's atmosphere, that have to be overcome. Solutions to these problems involve the collection of 'special' observations. The observing procedures themselves are to a large extent defined by the reduction methods.

The bulk of the data was collected at the Mount John University Observatory (MJUO) using the charge-coupled device (CCD) system installed there. Therefore this chapter begins with a general discussion about CCDs. MJUO and its CCD system are discussed next. Following this, an excursion is made covering various photometric systems. The choice of an appropriate filter system depends on the aim of the intended observations, given that different spectral regions can be singled out by different passbands. For example, it would be senseless to use the Strömgren system to observe late-type stars given that this filter set focuses on the spectral features of early-type stars. Finally, standardisation requirements are covered, leading to a discussion of the practical techniques required in field work.

\subsection{Charge-Coupled Devices}

harge-coupled devices were invented by Willard Boyle and George Smith of Bell Labs $\mathcal{~ i n ~ 1 9 6 9 , ~ a n d ~ w e r e ~ p a t e n t e d ~ o n ~} 31$ December 1974. CCDs were initially intended as a serial-in, serial-out metal-oxide semiconductor (MOS) memory circuit for computers, similar to magnetic bubble memory (Janesick \& Blouke 1987). Data were to be stored as charge in a twodimensional array of potential wells formed in a silicon substrate. A shift register (essentially a one-dimensional CCD) was placed immediately adjacent to this array, and led to either an output amplifier where the charge was 'translated' into data, or to an input amplifier at the other end of the register where charge was injected back into the CCD at a level proportional to the value of the input datum. Readout of a particular charge packet therefore involved its movement along a column to the shift register, and then along the register to the amplifier 


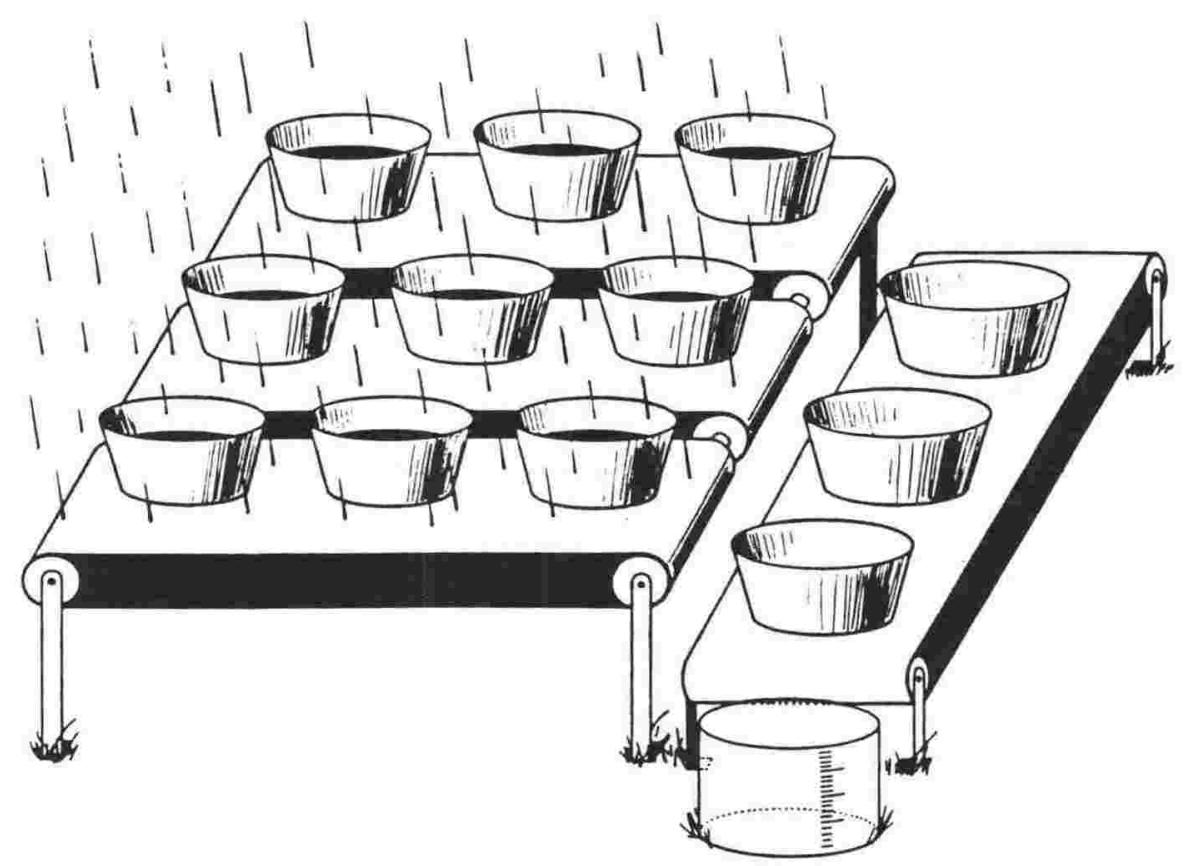

Figure 2-1: CCD Exposure and Readout can be likened to measuring the amount of rain falling onto a field with an array of buckets. After the rain has ended, the buckets in each row are moved horizontally across the field on conveyer belts. When each bucket reaches the end of its conveyer belt, its contents are emptied into another bucket. This second conveyer belt is then moved so that each of its buckets is shifted in turn down to a station at the end of the belt, where the amount of rainfall is measured (Diagram from Janesick \& Blouke 1987).

This resulted in relatively slow latency (or readout) times of $\sim 400$ microseconds. In addition, the charge packets had to be shifted at some minimum rate, as otherwise the empty wells would fill to saturation level with thermally generated electrons. Periodic refreshment was therefore required. Recirculating columns were developed to combat this thermal noise problem. However data degradation still resulted as the charge transfer process was not $100 \%$ efficient, leaving a residue of charge in each location. These problems led to the use of the CCD as a memory circuit being completely displaced by the later development of the faster Dynamic RAM.

Although not a success in their initially envisaged role, the light sensitive nature of silicon has led to CCDs revolutionizing direct imaging. Silicon is a semiconductor, with an energy gap of $\sim 1.1 \mathrm{eV}$ between the conduction and valence bands. Incident photons with wavelengths shorter than 1.1 microns can supply this amount of energy, creating an electron-hole pair. Under constant illumination an equilibrium is reached between the incoming flux rates and recombination in the lattice. An applied field will cause conduction in both bands, with valence vacancies being 'holes' or positive charge carriers. N-type semiconductors have more negative charge carriers available as they contain atoms with more electrons than are required to complete bonds with neighbouring atoms, i.e., there are extra energy levels near the conduction band. 


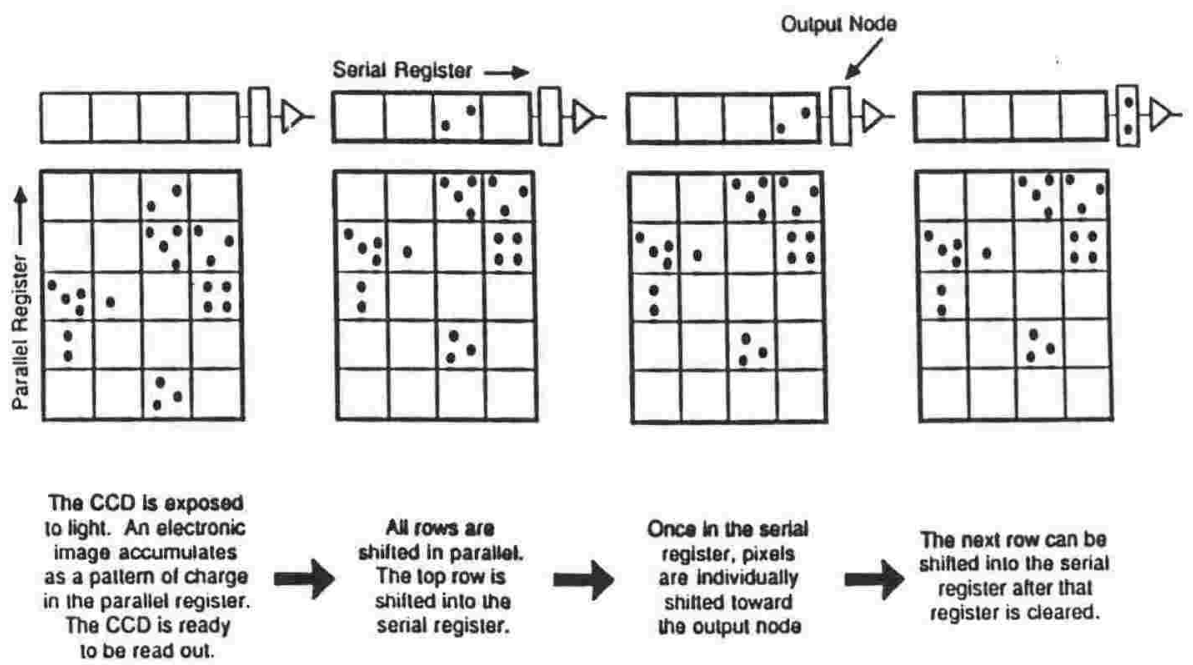

Figure 2-2: CCD Readout and General Structure. The amount of charge in a pixel is indicated by the number of dots in it.

P-types are the opposite case, having atoms with fewer valence electrons than required for bonding. Electrons are therefore readily accepted, leaving mobile holes. The charge carriers in most CCDs are electrons.

CCDs are primarily a parallel register consisting of $\sim 10 \mu \mathrm{m} x \sim 10 \mu \mathrm{m}$ pixels (picture elements), or charge collection sites, laid out in a grid rather like a chess board. When an object is imaged by a CCD, each pixel collects the light from a small square (the usual pixel shape) proportional to the light emitted by the corresponding element of the object, i.e, an Image $^{1}$ focused onto a CCD produces a charge pattern in proportion to the total integrated flux incident on each pixel. To rephrase, during an exposure the incoming photons cause a charge buildup in each pixel, recording a direct representation of the brightness distribution of the object. At the end of an exposure, the charge is read out line by line. The first row of pixels transfer their charge into an adjacent shift register (see Figure 2-1 for an analogy of the readout process, and Figure 2-2), which in turn moves the data along to an amplifier where the charge is measured. Readout is rather similar to a bucket brigade. As the first row is being read out, the second row of pixels transfer their data to the first, the third row to the second, and so on. Eventually all the pixels are read out and the data stored in the memory of the computer. The bucket analogy continues in the case of too much charge being collected by a pixel. Like a bucket collecting too much rain-water, it will overflow onto its neighbouring pixels. Generally, it only overflows down its column as these channels are separated by narrow barriers called channel stops, which prevent the sideways movement of charge.

\footnotetext{
${ }^{1}$ There is potential confusion between a CCD Image (or frame) and a star image. Throughout this thesis, Image will refer to the former.
} 


\subsubsection{Structure}

A CCD is made by first forming the channel stops, typically 5 microns wide, in a block of crystalline silicon. The channel stops prevent the charge carriers from moving sideways, along the length of a given electrode. Channel stops are usually regions of heavily doped ${ }^{1} n$-type material. The negative charge of the stops repels electrons, and prevents movement of charge between columns. This simplifies the final structure of the CCD. The substrate itself is usually a p-type material. An insulating layer of $\mathrm{SiO}_{2}$ is deposited over the substrate, preventing contact with the electrodes that will be grown above this layer. A thick $(\sim 500 \mathrm{~nm})$ layer of poly-silicon is grown by gradual vapour deposition onto the insulating layer, and heavily doped to improve its conductivity (Kristian \& Blouke 1982). The first set of electrodes or gates is made from this layer by etching away the unwanted material. A process of etching and regrowing a poly-silicon layer is used to build up the gate structure (which will be explained later), with an insulating oxide layer between them. A final oxide layer is deposited over them, holes etched through to each layer, and aluminium vapour deposited over the entire surface. This is then suitably etched to form leads to the electrodes, and to form connections with peripheral bonding pads which will link with external circuitry.

An electric field is generated inside the silicon when positive voltage is applied to an electrode. This field repels holes and creates a region depleted of charge - a potential well. When an electron-hole pair is formed by the absorption of a photon, the hole will be pushed out of this region and the electron attracted to the electrode. The electrons are stored and transferred at the surface of the substrate, explaining why such CCDs are known as surface-channel devices.

The boundary between the $\mathrm{SiO}_{2}$ and the substrate can have many lattice defects with slow release times. This will lead to an Image being smeared during readout. Surface defects can also inject spurious charges. Buried-channel devices, such as the MJUO CCD, avoid this problem by using a potential profile with a minimum (where the charge is most likely to reside) away from the surface. The difference between buried and surface-channel CCDs lies in the former having a highly doped n-type layer of silicon grown above the p-type substrate. A pn junction is formed. Some electrons from the $\mathrm{n}$ region diffuse across the junction into the $\mathrm{p}$ region, making it more negative. The opposite occurs for holes in the p region. A depletion zone is formed on each side of the junction, where the charge carrier density in either material is lower than the density well away from the junction. Further diffusion is prevented by the previous charge transfer itself. The charge of the electrons that have already diffused across to the p material will tend to repel any later electron diffusion. If a positive voltage is applied to the p side of the diffusion zone, this potential barrier will be lowered, and more electrons will be able to diffuse across the boundary. This is called forward biasing. Reverse bias is the application of negative

\footnotetext{
${ }^{1}$ Doping is the addition of impurity atoms into a material to achieve a given polarity and conduction.
} 


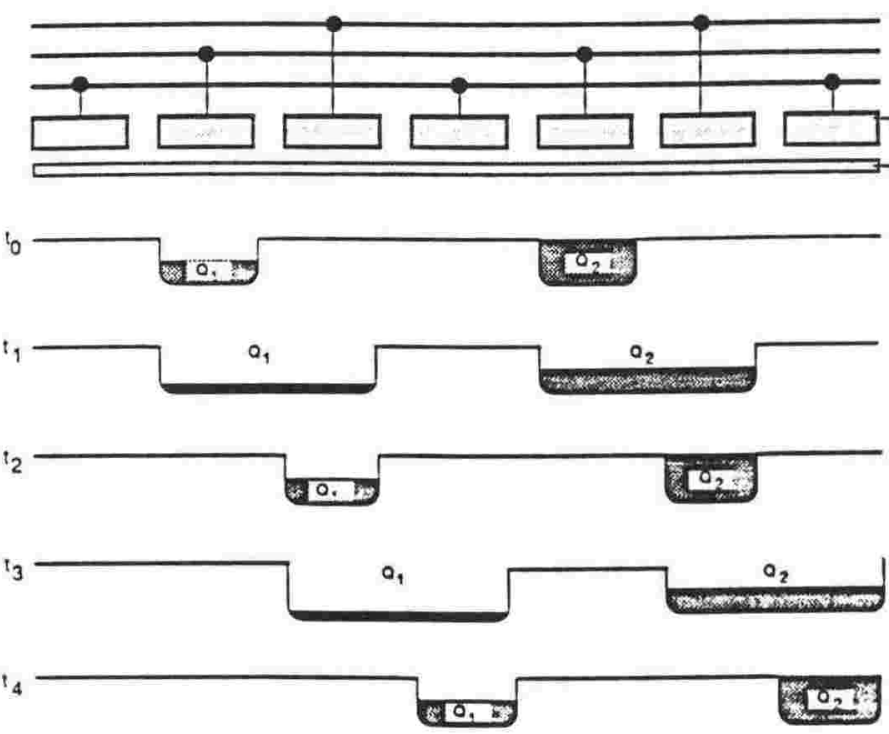

Direction of Transier

Figure 2-3: Charge Coupling. This schematic relates a three-phase gate structure to the movement of charge between pixels. At time $t_{0}$ two pixels have charges $Q_{1}$ and $Q_{2}$ respectively. At phase one $\left(t_{1}\right)$ of the readout cycle, gates immediately adjacent to the collection zones are biased, allowing the charge to spread out over a larger region. Phase two $\left(t_{2}\right)$ involves the smooth removal of the bias in the original collection zones, transferring the charge over by one gate. Then biases are applied immediately adjacent to the charge again, with the subsequent removal of the phase two bias. Using a three gate sequence allows smooth charge movement with each charge packet safely separated from the others (Diagram from Photometrics 1989).

voltage instead, which increases both the potential barrier and the width of the depletion zone. Buried-channel CCDs are simply pn devices with a voltage applied across them. The potential well is usually deepest on the $\mathrm{n}$ side of the junction, but still within the depletion zone. When an electron-hole pair is formed, the potential difference pulls them apart before they can recombine. The price for the enhanced charge mobility of these devices is a reduction in the charge storage capability of the pixels due to the reduced depth of the collection zone.

The gates of a CCD overlap, so the charge can be transferred by forming a potential well immediately adjacent to the original one. Charge in the first well is then spread between the two. By increasing the potential of the first well all the charge is transferred into the second well (see Figure 2-3). This coupling between charge, applied potentials, and charge transfer gives CCDs their name. The charge transfer efficiency (CTE) must be high to ensure that the majority of charge is shifted, particularly as transfer from pixels far from the output amplifier may involve hundreds of transfers. A scientific grade CCD has a CTE of approximately 0.99999 . $\mathrm{CTE}$ is of considerable concern at low charge levels, where significant degradation of the data 
occurs with the loss of a little charge.

A multiple gate structure is required to ensure that the separation of individual charge is maintained during charge transfer. If the well under a given electrode is nearly full, its cumulative charge interferes with that of the gate, degrading the CTE. Hence the MJUO CCD is operated with a recommended maximum charge value of around three-quarters the level for saturation of a pixel.

There are three basic structures for CCDs:

1. Full frame, where the entire imaging surface is used. During readout, a shutter is closed to prevent smearing of the Image as more 'data' is collected. Readout may take up to several minutes for very large CCDs, as each pixel is digitized to 14 or even 16 bit precision. There is no dead space between pixels, since charge generated between pixels diffuses to the nearest potential well.

2. Frame transfer devices, where there is a masked storage array the same size as the image array. At the end of an exposure, the data in the imaging array is rapidly $(\sim 1 \mathrm{~ms})$ shifted into its 'twin', where readout proceeds at leisure while more data is being collected in the former array. Smearing is low, due to the speed of the first transfer.

3. Interline Devices have opaque storage registers between pixel columns. At readout the entire Image is shifted by a pixel sideways to the pixels beneath these interline masks, with readout continuing in the normal manner along the columns. Unfortunately the masks degrade the Image quality, acting rather like a Venetian blind.

The Mount John CCD was initially designed as a frame transfer device, which is evident in the two separate chip regions seen in flat-field frames (see Figure 2-5 on page 38). The mask was removed, effectively doubling the field of view for Image collection. This is an important consideration given the small physical size and field of the devices. The generally low light levels involved in astronomical applications invalidate the advantages of the frame transfer design over the full frame one.

\subsubsection{Advantages}

Some of the advantages CCDs have over other available methods of direct imaging in astronomy include:

- A high quantum efficiency (Q.E.), which is the ratio of the number of photons entering a recording medium to the number actually recorded. The Q.E. of photographic films is $\sim 1-3 \%$, while that of CCDs can be up to $\sim 80 \%$ depending on the wavelength. Hence more 'light' is collected, and a comparable CCD exposure to one using a photographic 
plate is of shorter duration. It is the combination of the high Q.E. with low noise that makes CCDs so powerful. A Reticon array typically has a higher Q.E. than a CCD, but a poorer performance because of its much greater noise. A $60 \mathrm{~cm}$ telescope equipped with a CCD can, in a one hour exposure, reach the faintest objects detectable in a similar length photographic exposure on a $5 \mathrm{~m}$ telescope. A further advantage over photographs is the lack of reciprocity failure, where response slows during an exposure, although pixel saturation (and its approach) are problems.

- A broad spectral response from $\sim 400$ (ultraviolet) to $1,100 \mathrm{~nm}$ (near-infrared). This can be compared with the $\sim 300$ to $500 \mathrm{~nm}$ range of the commonly used IIIa-J photographic plate. CCDs respond well to even the longer wavelengths at the red end of the spectrum where a photo-cathode has problems. However, when illuminated from the front, CCDs are rather insensitive to the ultraviolet and $\mathrm{X}$-rays as the pixel electrodes are opaque to these photons. 'Solutions' include:

- Over-coating the front of the device with a phosphor which absorbs the incident photons, and re-emits photons at longer wavelengths where the CCD is more efficient. This results in only about $\mathrm{a} \sim 20 \%$ improvement, with the daughter photons being radiated in all directions.

- thinning the CCD and back illuminating the device so that Images fall onto the rear side of the chip. Photons therefore enter the active charge collection region without passing through the electrodes. The CCD must then be further treated to avoid the possibility of the photo-electrons being trapped by a potential well which is formed by the rear surface becoming charged. The shorter the incident wavelength, the shorter the depth of penetration a photon can make into the CCD. Silicon is transparent at $\sim 1,100 \mathrm{~nm}$, and essentially opaque at less than $400 \mathrm{~nm}$. Hence UV and blue photo-electrons form close to the surface of a thinned and back-illuminated CCD. They are often unable to break through such a potential well. The positive charge buildup is due to the growth of a Silicon dioxide layer on the surface when it is exposed to air. One solution to this problem is the unusually named technique of backside charging, where the chip is flooded with intense UV, generating excess photo-electrons which fill up the potential well. The CCD is then cooled to normal operating temperatures $\left(\sim-100^{\circ} \mathrm{C}\right)$, immobilising the charge and preventing the decay of the negative surface potential that has been formed. This potential will repel any photo-electrons, driving them towards the depletion zone. Other solutions countering the positive surface charging include the chemical alteration of the surface by exposure to strongly oxidising agents (such as nitric oxide), the application of a 

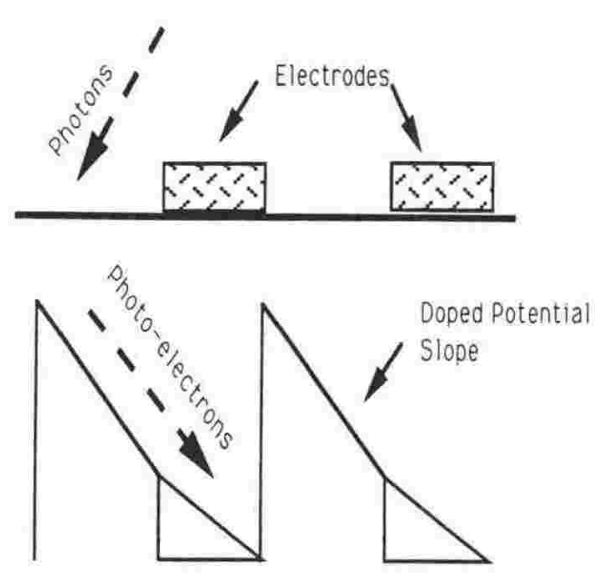

Phase One

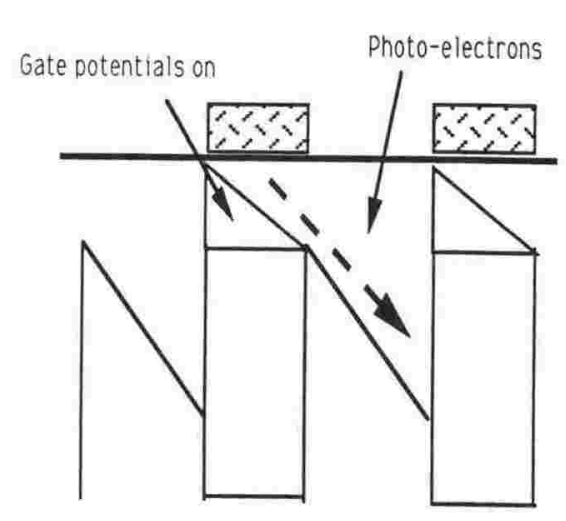

Phase Two

Figure 2-4: Virtual Phase CCD Architecture. The first diagram shows the potential wells of such a chip during an observation. Photons hit the CCD creating photo-electrons which then run down a potential slope (which may be stepped). During the readout phase the electrode's potential is cycled up above that of the slope, allowing the trapped electrons to run down the next slope (as shown in the diagram on the right). By repeatedly cycling the potentials in this manner the charge can be transferred across the CCD for readout. In astronomical applications the photons will usually arrive at a near perpendicular angle to the surface of the CCD.

thin $(0.4 \mathrm{~nm})$ transparent electrode across the surface which can be biased to counter the potential, and acid etching to remove many of the surface traps followed by the growth of a more homogeneous oxide layer.

- The use of a virtual phase architecture where only one poly-silicon electrode is used for each pixel, covering half of it. Different levels of surface doping create potential steps which direct the flow of charge as the electrode voltage is varied above and below that of these 'virtual' electrodes (refer to Figure 2-4).

The MJUO Thomson TH7882 CCD is a thick device that has been over-coated with Metachrome II to enhance the ultraviolet response, while the Hubble Space Telescope chips are coated with the organic phosphor Coronene and thinned to improve performance.

- A large dynamic range, which is the ratio between the brightest and the faintest objects that can be imaged simultaneously. Photographic plates have a maximum brightness range of around 100:1. The larger the size of a CCD pixel, the greater the number of electrons it can contain before saturation. The TH7882 384 x 576 pixels are $23 \mu \mathrm{m}$ across, and are capable of holding approximately $5 \times 10^{5}$ electrons (McCall et al. 1989). Output register pixels are larger than the imaging pixels so that they can contain more charge. The risk of saturation is reduced, with the advantage that pixels can be binned together should the user require it. Incidentally, both this register and the amplifier are light 
sensitive, and must be shielded.

The smallest detectable charge packet in a CCD may be $\sim 10$ electrons, and so the dynamic range is up to $10^{5}: 1$. Such a large dynamic range is particularly useful for objects such as distant galaxies, with their faint outer regions and brilliant nucleus all being recorded in one Image.

- Good linearity. If an object is twice as bright as another, then their images should be too. This is not the case for photographs - even if the reciprocity failure was eliminated (see Ch. 14, Sterken \& Manfroid 1992) as developing is a chemical and non-linear process. A CCD can be linear to within $0.1 \%$ over its dynamic range. The MJUO chip has been found to be linear within $0.5 \%$ over the range of 10 to 15,900 analogue to digital units ${ }^{1}$ (ADU) (Pollard 1989a), but the individual pixels have different responses. The analogue to digital converter (ADC) converts the voltage signal from a CCD pixel into a binary number (the ADU). The value assigned to a pixel by the ADC will not usually be the same as the number of electrons that were in the pixel (readout is a destructive process), although it must always be proportional. The number of electrons per ADU is tunable (see Tobin (1992a) for a discussion of gain and noise for the MJUO CCD). The gain of the MJUO CCD was set to 0 during the project, corresponding to $\sim 16$ electrons per ADU.

Illumination of a CCD by a uniform light source (a 'flat-field') maps the variations in pixel sensitivity (see Figure 2-5). A frame divided by such a flat-field will ideally compensate for these variations in pixel sensitivity, resulting in an Image of uniform sensitivity across its constituent pixels. In practice $\sim 1 \%$ uniformity is achieved. As the response of a CCD varies with wavelength, a flat-field needs to be collected for each filter used. Ideally the flat-field should be of the same spectral nature as the object being observed. Flat-field calibrations assume that this 'object' is the night sky, given that it will illuminate most of a CCD frame collected during an observing session. At best flat-fielding is a compromise, since although with narrow passband filters it is possible to match the colour of the night sky well, if the object being observed dominates the CCD frame then the flat-field will be inappropriate. A worst case scenario would be to have a very red object in the same field as a very blue one! The problem of mimicking the sky worsens with the broad band filters. Generally observers use either a twilight sky, or a specially illuminated board inside the telescope dome itself. Some average a night's worth of observations, reaching a 'perfect' spectral composition, although of somewhat low signal. Flat-fielding is possibly the largest source of uncertainty with CCD Images.

\footnotetext{
${ }^{1}$ Gain was set to zero in these tests.
} 


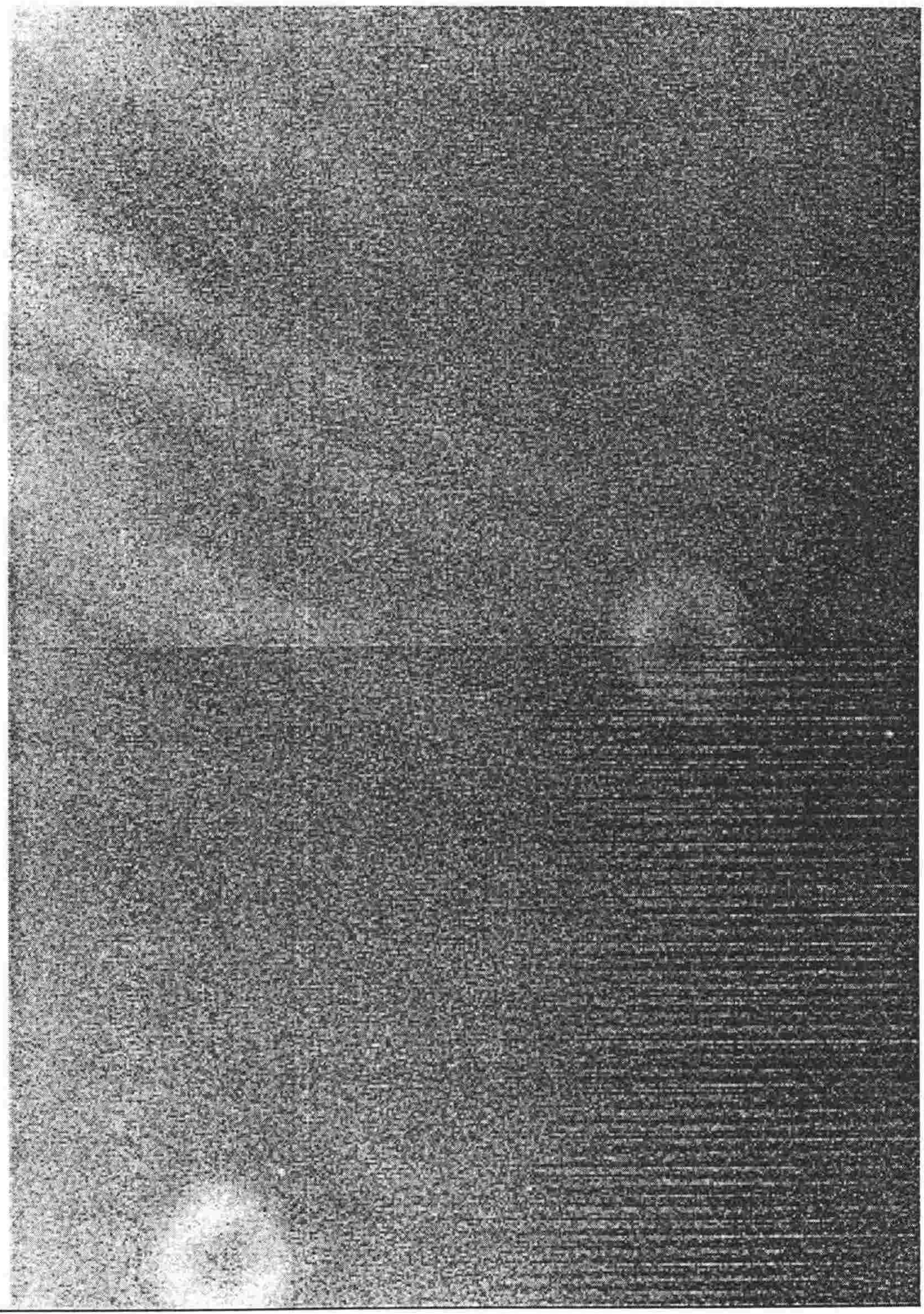

Figure 2-5: Example MJUO Flat-field Frame taken using the Johnson B filter. The doughnut-like features are caused by dust specks in the optical path of the telescope. The larger the ring, the higher the speck is in the optical train. The striped region in the lower right is likely to be a fabrication fault, with rows of pixels alternating in width. The assumption of uniform pixel size leads to a plot of the Flat-field having rows alternating bright (the physically larger pixels) and dim. The horizontal line half way up the frame is the boundary between the original imaging and storage sections of the CCD. Signal variation across the chip, barring the two columns discussed in the text, is typically $\sim 10 \%$. 
The technique of drift scanning attempts to circumvent flat-fielding problems. To avoid image smear, the CCD readout is slowly clocked relative to the rate of movement of the object through the field of view, so that light from the object is collected along an entire column. Intra-pixel, variations, fringes ${ }^{1}$ and so on are then averaged out. There is no need for a special flat-field frame, as the actual sky level is recorded during the observation, and an accuracy of $\sim 10^{-4}$ is possible. Some CCD surveys involve halting the tracking of a telescope and fixing the readout rate of a CCD to the movement of the observed field. Stars then enter into the field of view, and as they track across the CCD the electrodes are cycled so that the charge accumulated from the star follows the movement of the star image across the CCD. Two-dimensional strips of the sky are therefore observed. This technique is not suitable for deep frames of star clusters.

Examination of individual rows and columns across the MJUO chip indicated no largescale cyclic variation between neighbouring pixels, although gain did increase along rows, while columns gently peaked in gain near the middle. The first row seems to be faulty, with very low readings $\sim 1 \%$ those of the following row, while the gain of the final row was also slightly lower than its predecessor (by $\sim 6 \%$ ). In addition, a small change of gain is discernible halfway along the columns, which is not too surprising as the TH7882 was originally a frame transfer device. The MJUO CCD seems to be free of cosmetic defects, such as the many 'hot' and 'cold' pixels detected in a similar TH7882 at the University of Toronto Southern Observatory (McCall et al. 1989).

- Impersonal image centering: Subsequent CCD frame processing can include the use of synthetic apertures, which can be centred on the star more precisely than the diaphragm of a photometer could be. Variations in telescope tracking rates mean that apertures in photoelectric photometry should be somewhat conservative, and therefore larger than can be used in synthetic aperture photometry. Sky measurements can be made from the same Image, making them strictly simultaneous.

Resolution is ideally limited by the pixel size. With $\sim 3^{\prime \prime}$ seeing commonplace at MJUO, the MJUO CCD pixel size somewhat over-samples stellar images (a pixel corresponds to $0.6^{\prime \prime}$ at the $\mathrm{f} / 7.9$ Cassegrain focus of the $\mathrm{M}^{\mathrm{c}}$ Lellan telescope) except in the occasional excellent conditions. This increases the total noise ${ }^{2}$ as more pixels are covered by larger stellar images, proportionally increasing both the photon and readout noises. The increase in noise needs to be balanced with the requirement for good spatial sampling. If the

\footnotetext{
${ }^{1}$ An interference pattern due to the thickness of the active silicon layer being comparable to the wavelength of the incoming light. It is generally insignificant in broadband work, and can be ignored if the background is fitted and subtracted locally as with the PSF-based packages such as DAOphot (Stetson 1987). Fringing is particularly severe in the Infrared.

${ }^{2}$ Noise is discussed in the Section 2.1.3.
} 
pixels are too large with respect to the width of a stellar image, then spatial information is degraded.

A major disadvantage of CCDs is that they are physically small devices only a few centimetres across, as opposed to photographic plates which can be $\sim 50 \mathrm{~cm}$ wide. This limits their field of view.

\subsubsection{Noise}

There are three main sources of noise with CCDs:

1. Dark current is due to carriers being formed in the silicon by thermal effects. It is socalled because it can be generated in the absence of any light. The buildup of dark current sets an upper limit to the available exposure length before pixel saturation occurs. Dark current can be reduced by lowering the operating temperature of the CCD, although it cannot be lowered as far as is actually possible since CTE drops for temperatures below $-190^{\circ} \mathrm{C}$. At room temperature around 50 bonds are broken and recombined every second in a $1 \mu \mathrm{m}^{3}$ volume of silicon (Sterken \& Manfroid 1992). At $-60^{\circ} \mathrm{C}$ there is approximately 1 'dark' electron per pixel per second for the MJUO CCD. The dark signal of the MJUO CCD is negligible when the chip is cooled to its usual operating temperature of $-110^{\circ} \mathrm{C}$. The dark current in 20 minute-long exposures is equal to the preflash noise. There is no practical difference between the MJUO bias and dark frames ${ }^{1}$ except for long exposures. Bias frames are zero-length exposures which measure the bias of the output electronics, the preflash offset, and any variations in bias levels between pixels.

A further effect influencing dark current at MJUO is the temperature of the Camera Electronics (CE) 200 control unit. Gilmore \& Tobin (1991) found that the temperature of the dome, and hence this unit, was related to the count offset (bias level) by the relation:

$$
\text { bias } \simeq 302+(0.67 \times \mathrm{T})
$$

where $\mathrm{T}$ is degrees Celsius. If the dome temperature changes substantially during an observing session, a new dark current frame should be collected. The MJUO software monitors the bias levels for such changes and notifies the user when a new frame should be collected.

2. Preamplifier noise, which is generated by the on-chip output amplifier and sets the lower detection limit for a CCD. It is also called readout noise.

\footnotetext{
${ }^{1}$ Amateur astronomers typically estimate the dark signal in an exposure by collecting a dark frame (where the CCD is covered) of the same exposure length as the observation.
} 


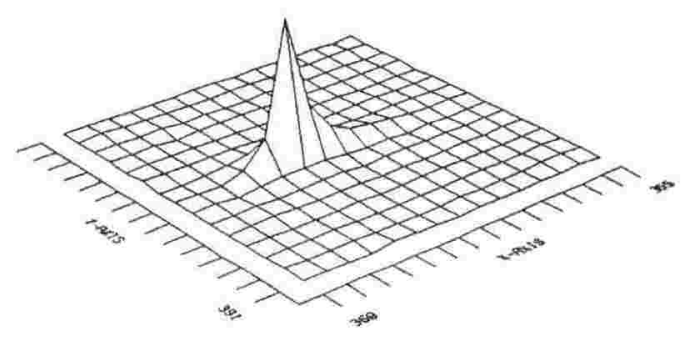

Figure 2-6: Typical Cosmic Ray Strike experienced at MJUO during observations of LMC clusters.

3. Photon Noise - which is due to the total number of photons emitted over any time interval by a 'steady' source actually varying according to a Poisson Distribution.

Total noise $\mathrm{N}_{\mathrm{T}}$ is given in terms of the preamplifier noise $\mathrm{N}_{\mathrm{P}}$ and the number of electrons $\mathrm{E}$ collected in a given pixel as:

$$
\mathrm{N}_{\mathrm{T}}=\sqrt{\mathrm{N}_{\mathrm{P}}^{2}+\mathrm{E}}
$$

given that the two sources of noise are independent. At high light levels the system noise is overshadowed by photon statistics, while at the other extreme preamplifier noise dominates. Illuminating the CCD with a brief burst of light (preflashing) before actually exposing the CCD to image an object can help in the latter case where the anticipated data level will be low. Small pockets of charge may be trapped in potential well irregularities, and so by adding a constant amount of charge which will fill in these holes, the CTE of the device can be improved. A charge pocket between the parallel (the CCD) and serial registers of a TH7882 CDA CCD affects the transfer of signal levels lower than 50 electrons. To improve the CTE a 'flash ring' of 8 green LEDs is sandwiched between the mechanical one inch aperture, 5 petal Uniblitz shutter and the MJUO cryostat window. The ring is typically illuminated for $70 \mathrm{~ms}$ before the shutter is opened and the exposure begins. Some 300 electrons are deposited in each pixel. While the CTE is improved, this technique does increase the noise level of a frame, as could be expected from Equation 2.1.

After a science frame $\left(I_{S}\right)$ has been obtained, the dark current and any bias $\left(I_{D}\right)$, such as due to preflashing, must be subtracted from it and the total divided by the flat-field $\left(\mathrm{I}_{\mathrm{F}}\right)$ to ensure that each pixel is an independent linear photometer, i.e.,

$$
\text { Processed frame }=\frac{I_{S}-I_{D}}{I_{F}-I_{D}} .
$$




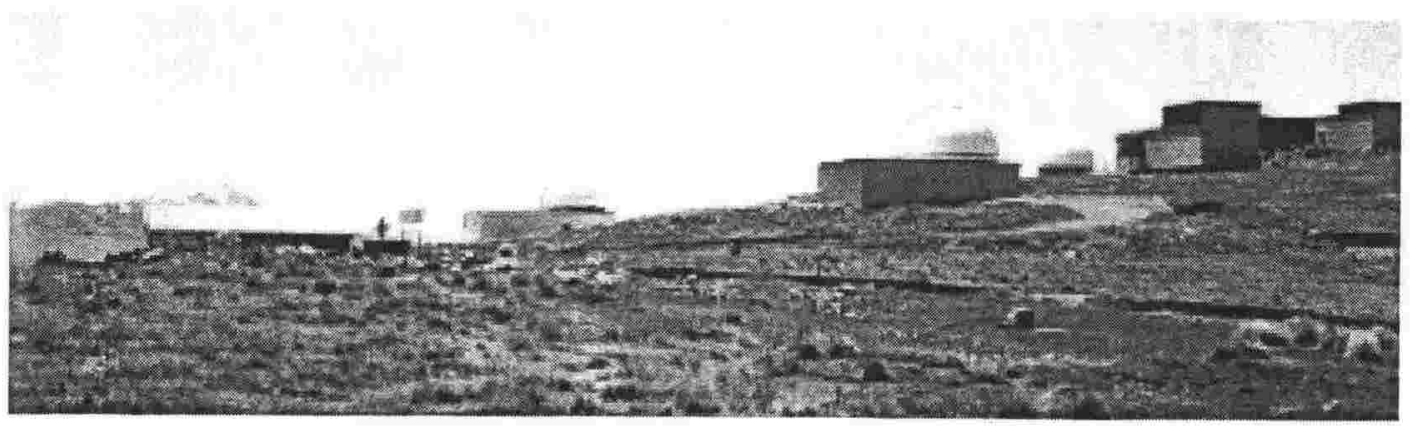

Figure 2-7: The Peak of Mount John. The majority of the MJUO facilities lie $\sim 100 \mathrm{~m}$ north of the Observers' Quarters, scattered around the peak of the hill. The astrograph and cameras are housed in the roll-off-roof buildings on the right, the Optical Craftsman telescope is in the middle dome, and the Boller \& Chivens telescope in the left dome. The building on the far left was originally the Observers' Quarters, but now is used as a workshop and offices. The right dome is currently empty.

\subsubsection{Cosmic Ray Strikes}

High-energy radiation, such as protons, on impact with nuclei in the upper atmosphere form pions, which subsequently decay to form muon or cosmic ray showers. When a muon strikes a CCD it delivers a large signal concentrated into a small region (Leach \& Gursky 1979). Typically this is within a pixel, but the number of pixels affected depends on the angle the impacting ray makes with the detector (see Figure 2-6). Radio-active decay of the surroundings of the CCD have a similar effect on the detector as cosmic rays. Some glasses used in UV and blue filters, such as Schott UBK7, emit X-rays and even the metal in the cryogenic dewar is contaminated by radio-active isotopes, leading to spurious events (Mackay 1986, p.123 Mclean 1989). Since the effect of a cosmic ray strike is limited to a few pixels, it can easily be detected and 'removed' from a frame (see Section 5.1.1, which begins on page 108, for details of this algorithm).

\subsection{Mount John University Observatory}

Mount John University Observatory is situated on Mount John, which is a hill ${ }^{1}$ rising some 300 metres above Lake Tekapo and the tussock-filled plateau of the Mackenzie Basin. The basin itself is 700 metres above sea level. This region is in southern Canterbury in the South Island of New Zealand, $230 \mathrm{~km}$ south-east from Christchurch. The location was selected as one of the best observing sites in New Zealand following a site survey conducted between 1961 and 1963 (Bateson 1962, 1964), leading to the founding of the Observatory as a joint facility by the Universities of Pennsylvania, Florida, and Canterbury. The US participation in the observatory has been dormant for many years.

\footnotetext{
${ }^{1}$ Mount John is a relic from the last glaciation, being a Roche Moutoneé (see p.207 of Press \& Siever (1982) for details on the formation of this type of feature).
} 
By the standard of the New Zealand maritime climate, the site enjoys a high number of clear nights with good transparency. The nearby village of Tekapo has a population of 300 people, and does not produce significant light pollution. The Southern Alps to the west of Tekapo, and the Two Thumb Range to the east of the Basin, act as barriers stopping much cloudy weather. This leads to Tekapo having one of the highest mean annual sunshine hours in New Zealand, together with a low mean annual rainfall of $575 \mathrm{~mm}$.

The Observatory is operated by the Department of Physics and Astronomy of the University of Canterbury (UoC) in Christchurch. The Observatory was established in 1963, and officially opened in July 1965 (see Scott (1976) for a summary history of MJUO). From 1969 to 1983 a US BakerNunn Satellite Tracking Station was also at Mount John. After its closure, the station building was leased by the University

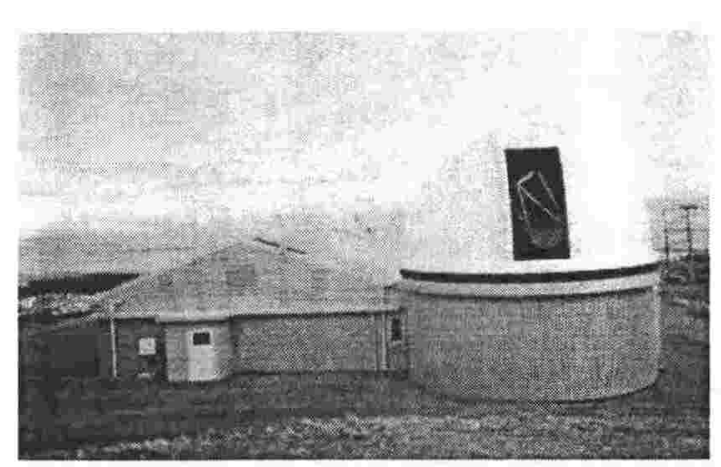

Figure 2-8: MJUO 1 metre Telescope Dome and Observers' Quarters.

of Canterbury and extensively refitted to accommodate the $\mathrm{M}^{\mathrm{c}}$ Lellan Dall-Kirkam ${ }^{1}$ one metre telescope. This telescope was built by the University of Canterbury over a five year period from 1981.

The observatory also has two $61 \mathrm{~cm}$ reflecting telescopes which were installed in 1970 and 1975 respectively (see Figure 27), Harvard and Bamberg cameras, and a Cooke astrograph. A wide variety of programmes are active at MJUO (see Hearnshaw (1992) for a detailed discussion about the majority of these programmes), many of which make use of the three resident service observers and the fact that MJUO is the southern-most professional observatory in the world. Its position gives it better ac-

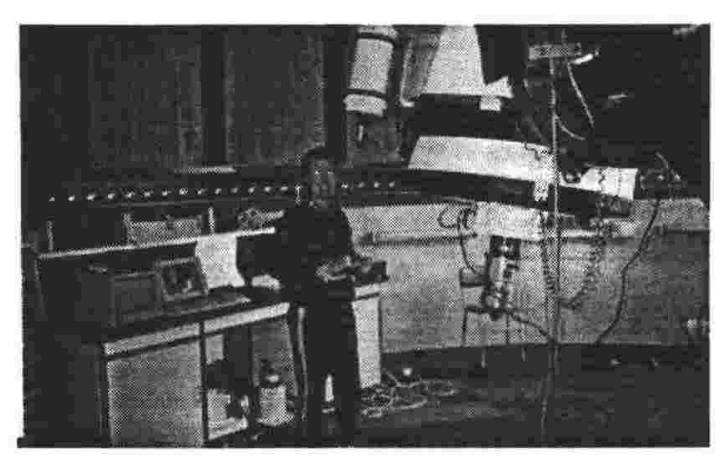

Figure 2-9: MJUO 1 metre with CCD System Attached. The CCD Dewar is mounted beneath the telescope. The CE-200 control box is the white object immediately above the dewar. A control console is on the left of the figure. cess (i.e., observations at lower airmasses) to many southern objects for longer observing seasons than other observatories, although there is a cost for summer programmes (such as a project centred on the LMC) in that the summer nights are even shorter than for lower latitudes (see also page 53).

The bulk of the data for this project was collected at MJUO using the McLellan one metre

\footnotetext{
${ }^{1}$ See Nankivell \& Rumsey (1986) for details on the specifications of the 1 metre telescope.
} 
telescope equipped with a charge-coupled detector system (see Figures 2-8 and 2-9), which is described in the following section.

\subsection{The MJUO CCD System}

In December 1988 the University of Canterbury took delivery of a Photometrics 3000 cryogenic CCD system. Initially the system was attached to the $61 \mathrm{~cm}$ Boller \& Chivens telescope, but is now available for use with only the one metre telescope. The system consists of (Tobin 1990):

- A cryogenically cooled CH210 camera head initially designed for medical imaging, equipped with a four phase, front illuminated, buried channel Thomson CSF TH7882 CDA CCD. The chip is mounted behind a quartz window within the evacuated camera head. Photometrics recommends that the optimum operating temperature lies between $-90^{\circ}$ and $-130^{\circ} \mathrm{C}$ due to the facts that dark current must be minimised, but that long wavelength quantum efficiency and the CTE deteriorate at temperatures colder than these values. The optimum operating temperature also depends on the wavelengths being observed maximum sensitivity in the red $(\sim 655 \mathrm{~nm})$ is at $\sim-90^{\circ} \mathrm{C}$ and in the green $(\sim 555 \mathrm{~nm})$ at $-140^{\circ} \mathrm{C}$ (Pollard 1989b). Practical experience has shown that operating significantly ( $\sim 10$ degrees) above or below $-110^{\circ} \mathrm{C}$ leads to faster boil-offs of the cooling Nitrogen or the appearance of several bad Q.E. columns. Pollard (1989b) notes that temperatures below $-110^{\circ} \mathrm{C}$ are also hard to maintain if the telescope position is being altered often, as the liquid nitrogen changes position in the dewar.

- A CE200 camera electronics unit with a $50 \mathrm{kHz} 14$ bit analogue to digital converter.

- A PM3000 control computer running a FORTH (Moore 1974) operating system for data acquisition. An M4-9903 1600 BPI 9-track half-inch tape drive is attached to this computer, along with a 9-pin dot-matrix printer which provides a hard-copy record of observing sessions. FORTH is an interpreted language developed at the National Radio Astronomy Observatory specifically for control of instrumentation. The main advantage of this language, which led to its adoption at MJUO, is the ease with which 'keywords' can be defined. This allows each observer to define functions tailored to their observing programme. FORTH also gives users easy access to control and loop structures, allowing automation of repetitive tasks. However, the fragile nature of the FORTH Operating System meant that users could not be allowed access to ForTH files, and so had to type in keywords at the beginning of each observing session - or after the system crashed. Regular users could be allocated files for the storage of their command words, but these files had to be maintained by Dr. William Tobin, who was responsible for the system. 
Photometrics also supply a UNIX system as an alternative to FORTH, with the option of the user being able to select either system when they boot the computer.

A control console consisting of a terminal capable of Tektronix graphics and a monochrome video monitor (for Image display) is available in the control room, with another console on the 'floating floor' of the telescope dome. ${ }^{1}$

\subsection{Photometric Systems}

Ideally stars would be measured at many monochromatic wavelengths simultaneously, with examination of spectral absorption or emission lines providing dynamical, thermal and chemical information about the star, its surroundings, and the interstellar material between the star and the observer. The low photon count-rate per unit wavelength means that even with large telescopes and long integration times, only relatively bright stars can be observed spectroscopically. ${ }^{2}$ Given the difficulty in collecting enough light for this high resolution work, and the fact that there is much redundant information contained in a stellar spectrum, a practical alternative is to observe a star through a number of wide passband filters. These are centred on important regions of a star's spectrum, and despite the lower resolution, can in principle recover the gross information ${ }^{3}$ provided by spectroscopy with the advantage of a fainter limiting magnitude.

Strömgren (1963) categorised photometric systems into three types:

- Narrow Band, where the mean system bandpass is less than or equal to $9 \mathrm{~nm}$ across. An example is the $\mathrm{H}_{\beta}$ system, introduced by Crawford (1958), which comprises two interference filters centred on the $\mathrm{H}_{\beta}$ line at $486.1 \mathrm{~nm}$. The $\mathrm{H}_{\beta}$ line is a strong absorption feature in early-type stars. The strength of $\mathrm{H}_{\beta}$ line indicates the luminosity class of $\mathrm{O}$ to A stars, and is a temperature indicator for the spectral types A to G.

The 'narrow' filter has a halfwidth of $\sim 3 \mathrm{~nm}$, while the 'wide' filter has a corresponding width of $15 \mathrm{~nm}$. The narrow filter measures the $\mathrm{H}_{\beta}$ strength. A low intensity through the narrow filter could be due to either a strong $\mathrm{H}_{\beta}$ absorption, or simply that the star is faint. To discern between these two cases a second (wide) filter is needed to measure the adjacent continuum. The ratio of the two filters gives the strength of the $\mathrm{H}_{\beta}$ line relative to the continuum. The system is not dependent on atmospheric or interstellar extinction since the filters have the same effective wavelength.

\footnotetext{
${ }^{1}$ As a telescope is moved it can alter its height above the floor of the dome, particularly if it has an equatorial mount. If the dome floor is unable to be moved, access to the telescope will be difficult when the telescope is placed in certain orientations. A solution is to be able to shift a floor vertically, for instance by using hydraulic jacks. Such a floating floor was built for the dome of the $M^{c}$ Lellan telescope.

${ }^{2}$ For instance, the Morgan \& Keenan (1973) spectroscopic catalogue only extends to $\mathrm{V} \sim 15$.

${ }^{3}$ I.e., not the finer details such as radial velocities.
} 
- Intermediate Band systems, with a mean passband width greater than $9 \mathrm{~nm}$ but less than or equal to $30 \mathrm{~nm}$. Two examples of such a system would be the Vilnius (Straižys 1992a,b) and Strömgren $(1963,1966)$ systems. The Vilnius filter set is described in detail in Chapter 6 and so shall not be discussed further here. The latter system consists of 4 filters (uvby) intended for use with early-type stars. It was designed to be nearly filterdefined, or insensitive to the detector being used, and to require no second-order terms in the extinction and transformation equations. ${ }^{1}$ The Strömgren u filter lies between the atmospheric extinction cutoff and the Balmer Jump, $v$ lies after the Balmer Jump being centred on the $\mathrm{H}_{\delta}$ lines, $\mathrm{b}$ is positioned in a region free of strong lines bar $\mathrm{H}_{\beta}$, and $\mathrm{y}$ is as close as possible to the Johnson V (see below) to tie in with that system (Jaschek \& Jaschek 1987). Often the $\mathrm{H}_{\beta}$ filters are included to complement the system. The following indices are commonly used:

$\diamond(b-y)$, which indicates temperature,

$\diamond \mathrm{c}_{1}=(\mathrm{u}-\mathrm{v})-(\mathrm{v}-\mathrm{b})$ is a measure of the Balmer discontinuity, and

$\diamond \mathrm{m}_{1}=(\mathrm{v}-\mathrm{b})-(\mathrm{b}-\mathrm{y})$ measures line blanketing, or the net effect of some absorption lines on the spectra of a star, and so the metallicity of the star. Line blanketing is caused by absorption lines of metals, and becomes stronger for spectral types later than A0, A colour index is effectively measuring the slope of the continuum (see Figure 2-10), and so a comparison of the $(v-b)$ colour, which is affected by blanketing, with $(b-y)$, which is not affected by the blanketing, indicates the level of blanketing. If no blanketing is present, the two colours would be nearly the same as the continuum gradient is roughly constant over the appropriate wavelength range.

$c_{1}$ is nearly free of line blanketing effects as the $v$ filter suffers about half the blanketing encountered by the $u$ filter.

- Broad Band systems, where the mean passband width is greater than $30 \mathrm{~nm}$ across. An example is the Johnson UBV system (Johnson \& Morgan 1953, Johnson 1963), which is defined by a combination of the Earth's atmosphere together with the responses of the filters and the detector. The U (ultraviolet), B (blue), and V (visual) passbands have effective wavelengths around 350,430 , and $550 \mathrm{~nm}$ with FWHM of 50,85 , and $90 \mathrm{~nm}$ respectively. Historical reasons are partly responsible for this choice of passbands. V corresponds roughly with visual magnitude, and B to the mean of the nineteenth century photographic magnitudes. The U filter was chosen to collect as much ultraviolet light as possible, and positioned so that the $(\mathrm{U}-\mathrm{B})$ colour gives an indication of the strength of

\footnotetext{
${ }^{1}$ These terms are explained in the following section.
} 


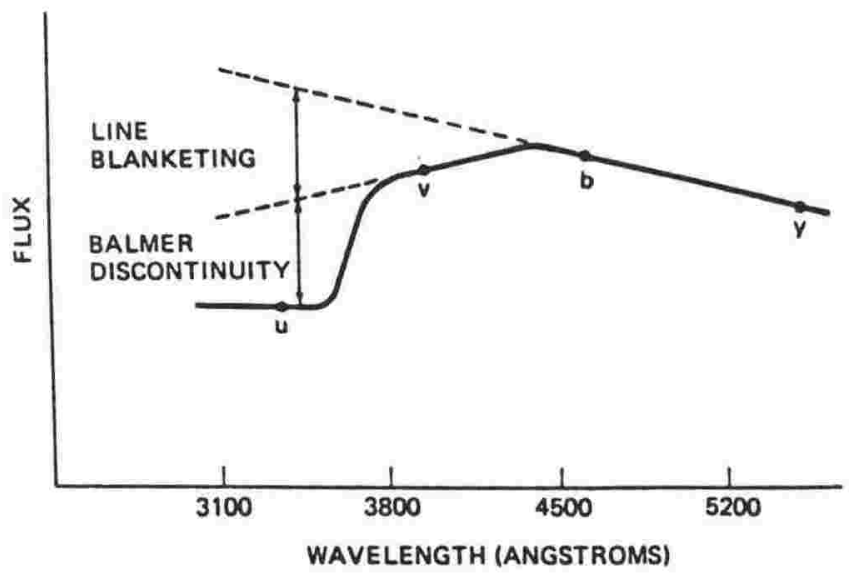

Figure 2-10: Strömgren Filter Placement is indicated on a simplified spectrum of an earlytype star. The stellar absorption lines have been omitted for clarity (Diagram from Hendon \& Kaitchuk 1989)

the Balmer discontinuity for a given star. The system is closely tied to the Morgan-Keenan (1973) spectral classification system.

The main problems with this system lie in that the short wavelength cut-off of the U filter is defined by the atmospheric extinction cutoff (which is variable and also depends on the altitude of an observatory), and that the higher wavelength cut-off for the $\mathrm{V}$ filter is defined by the red cut-off of the photo-cathode used by Johnson to establish the system. The use of the V filter with red sensitive detectors will result in 'red leak', where too much red light is detected by the combined system. In such cases an additional cutoff filter is added to remove the red leak, although such filters can alter the shape of the passband unless care is taken. The UBV system is not filter defined.

Together with the RIJKLMH passband extensions ${ }^{1}$ into the red (Johnson 1965), the Johnson system is the most popular one in use today. This popularity is essentially due to the broadness of its passbands. This allows the rapid collection of photons with even small telescopes, with a subsequent lowering of percentage noise in an observation as the signal level increases. The limiting magnitude of the Johnson system for a given instrument, detector, and exposure length is fainter than for narrower photometric systems. Despite the spectral coarseness of the system, estimates of stellar temperature and interstellar extinction can be made using it.

\footnotetext{
${ }^{1} \mathrm{R}$ and $\mathrm{I}$ are the most frequently used of these filters, with the remainder requiring specialised detectors (p. 55, Hendon \& Kaitchuk 1989).
} 


\subsection{Standard Stars}

Only in a few applications, such as time-series photometry (e.g. Sullivan 1993), are observations kept in the instrumental system as defined by the telescope, filters, and detector. Normally, the observations are transformed to a standard system to allow comparisons between measurements collected by different instrumental systems. Standard systems are defined by sets of standard stars measured in the original instrumental system of the 'standard'.

The derivation of accurate transformations from the instrumental system to the 'standard' one (although see Young (1993), who contends this to be a difficult task) requires:

- photometric weather,

- an observable sequence of standard stars that have accurate standard system magnitudes,

- a filter set reasonably close to the standard system when the response of the detector is considered, and

- the observation of many standard stars with a good range in colour and magnitude. If extinction stars (see below) are not being used, then the standards (as a group) should be observed over a wide range of airmasses.

Ideally, around 20 complete sets of observations of standards should be made over a night. The colour range of the standards should encompass that of the programme objects, so that extrapolations are not being made. Some observers combine standards observations from several nights of a run together to derive the transformation equations. While this may be possible at the best photometric sites in the world, the variable New Zealand weather conditions mean it is not typically true for MJUO.

The term 'atmospheric extinction' is used to describe the combination of atmospheric scattering and absorption suffered by starlight. Extinction is due to several physical effects, such as simple absorption by molecules and dust or water particles in suspension, Rayleigh scattering by molecules, and aerosol scattering off small water droplets and dust. The level of aerosol scattering is unpredictable. For instance, it was substantially enhanced by the eruption of Mt. Pinatubo in 1991. Extinction depends not only on wavelength but also the amount of atmosphere the starlight has to pass through to reach the observer. Consider a model of a flat earth with a static plane-parallel layer atmosphere overlying it. Light from a star immediately overhead will pass through only one thickness of the atmosphere, and be dimmed appropriately. As the angle a star makes with an observer's zenith increases, the starlight passes through an increasing amount of atmosphere. Therefore the extinction experienced by the starlight will increase as well. Hence a secant function will be applicable. This simple approximation is often used for zenith angles up to 60 degrees. Even at this angle the airmass calculated by the secant 
law is different by only 0.005 airmasses from values calculated by better models, such as Hardie (1962) or Young (1974). Both these authors fitted functions to the extinction data based on the model atmosphere of Bemporad (1904).

Extinction is commonly measured by following the change in apparent magnitude of a star (an 'extinction' star) as it passes through a large range in airmass. Forbes (1992) describes and contrasts several extinction star methods, and recommends the use of two stars - one rising and the other setting - for the most accurate results. Observations of single stars with CCD systems are often wasteful of telescope time due to considerations such as CCD readout times, which may be minutes for the largest arrays. Such observations are also wasteful of computing resources such as disk and tape space, with many redundant sky measurements in frames of single standard stars. These problems often lead to CCD observers not measuring as many standard stars as photoelectric observers would (e.g. Elson 1991). There is an urgent need for the establishment of CCD-sized fields across the sky, containing several reasonably faint standards. Many of the E-region and equatorial standards (Menzies et al. 1989, 1991) are too bright for CCDs attached to $1 \mathrm{~m}$ and larger telescopes. Bessel (1993) notes that the suitably faint stars listed in the Menzies et al. $(1989,1991)$ catalogues have an inadequate range in colour — as do the standard fields in Graham (1982). ${ }^{1}$ Landolt (1992) has provided a list of UBVRI equatorial standards suitable for CCDs. Being equatorial stars, they are not as convenient for southerly observatories (such as MJUO) as the E-region fields which effectively pass through the zenith. Field standards need to be close enough to each other to be imaged simultaneously by a CCD, but not so close that accurate aperture photometry ${ }^{2}$ is not easy and therefore that their standard magnitudes would not be accurately measured. This last point means that there is little to be gained from observing 'standards' in crowded fields such as in globular clusters (e.g. Alcaino \& Liller 1984, 1985, 1986) if accurate photometry is the object. Da Costa (1991b) points out that it is rare for the magnitudes of such stars to be known to $1 \%$, or even for their measurements to be accurately on the standard system.

Standard star magnitudes are typically determined using large aperture photoelectric photometry. Often CCD observations reveal faint companions near standard stars. It is important that the CCD photometry of such stars be made with a large aperture that will include the light of any companion stars, just as it was with the original photoelectric photometry which determined the standard values for the star, and not by profile fitting or small aperture photometry. It would be preferable to use CCD profile fitting photometry instead of photoelectric photometry to set up standard fields intended for use with CCDs. In such work it would be important to ensure that the filter passbands used for the CCD photometry are as close as possible

\footnotetext{
${ }^{1}$ Bessel (1993) also notes that there are systematic differences between the values of Graham (1982) and Menzies et al. (1989) for the reddest Graham (1982) stars.

${ }^{2}$ Aperture and Profile Fitting photometry are discussed in the Chapter 4.
} 


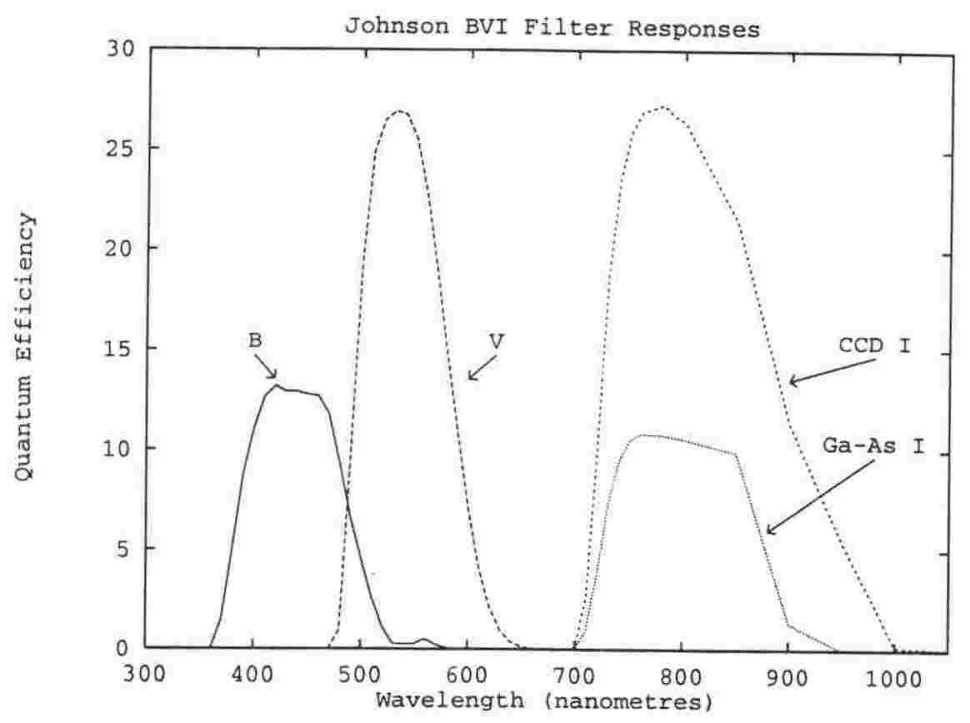

Figure 2-11: Johnson BVI Filter Responses. The Schott glass prescriptions of the MJUO BVI filters were convolved with the quantum efficiency of the Thomson CCD. The I filter was also convolved with the Q.E. of an RCA-31034 Gallium-Arsenide photomultiplier as provided by the specifications of the manufacturer. The rapid cutoff of the tube itself was used to define the I band, but CCDs have a superior red response. The combined transmittance of the I band is a poor fit to both of the Johnson and Cousin I bandpasses (West et al. 1992).

to the standard photoelectric system, taking into account the different wavelength responses of different CCDs. Bessel (1990) describes the design of filter systems via synthetic photometry, in which filter responses (given the transmittances and spectral responses of their components) are convolved with data from spectro-photometric catalogues such as the Vilnius (Straižys \& Sviderskiené 1972) and Gunn-Stryker (Gunn \& Stryker 1983) catalogues. The importance of such filter design was emphasized during this project when the spectral properties of the MJUO Johnson I filter were synthesized, using the Schott catalogue, and convolved with the quantum efficiency of the MJUO CCD. A non-standard I filter was revealed, with a substantial extension towards the red (see Figure 2-11). West et al. (1992) convolved the MJUO B and V filters against the Q.E. of the TH7882 CCD, finding a good agreement with the standard passbands as given by Lamla (1982).

The 'inertia' of CCD systems often leads to CCD observers shying away from the technique of following extinction stars, across to the technique of observing standard stars (or fields) at a variety of different airmasses. Extinction and instrumental coefficients are then derived using a least-squares solution to all the standard star observations obtained during a night (see Harris et al. 1981). This technique is very efficient in terms of telescope time, which is an important consideration for summer nights, which can be as short as 4 hours around the summer solstice at MJUO. It is this technique that was used for CCD observations during this project, using the IRAF 2.10 photcal package and custom transformation computer programs to determine 
the transformation equations.

The transformation equations may take many forms, but a typical one is:

$$
\mathrm{m}=\mathrm{M}+a_{1}+a_{2}(\text { colour })+a_{3} X+a_{4} X(\text { colour })+\ldots
$$

where

- $\mathrm{m}$ is the instrumental magnitude.

- $\mathrm{M}$ is the standard magnitude.

- colour is the standardized colour.

- $X$ is the airmass of the object when it was observed.

- $a_{1}$ is the zero point coefficient. The zero point is the extra-atmospheric magnitude corresponding to $1 \mathrm{ADU}$ per second for a star of zero colour.

- $a_{2}$ is the colour coefficient, which indicates how well the instrumental system matches the standard one. Typically $a_{2}$ should be below 0.1 (Da Costa 1991b), with a perfect match being indicated by $a_{2}=0$.

- $a_{3}$ is the first-order extinction coefficient, which depends on the atmospheric transmission, and so primarily on the thickness of the atmosphere that the object was observed through.

- $a_{4}$ is the second-order extinction coefficient. Extinction can vary across a filter bandpass which, when combined with the change in the effective wavelength of a filter with spectral type, leads to a variation in extinction with colour. It is largely a function of the width and shape of a passband, and can therefore be assumed to be constant through a night. The properties of filters can change with age. Therefore this coefficient needs to be redetermined every half year or so.

Further terms, such as n-th power colour extinctions, are sometimes applied in precise photometry (Stetson 1990), but are not common. A colour-squared dependence will only occur if the instrumental system is very different from the standard one. Only the minimum number of coefficients required for a satisfactory fit should be used. Colour terms should remain constant throughout a run, as should the zero points given that the overall sensitivity of the instrumentation should not change over the short term. However, extinction is likely to change from night to night as atmospheric conditions change, reinforcing the need to obtain many standard star observations over a good airmass range every night. This assumption of a stable instrument and varying extinction is the opposite of that often used in photoelectric photometry, where mean extinction coefficients are assumed and the zero point allowed to vary (Da Costa 1991b). 
NGC 2214 Airmass Diagram

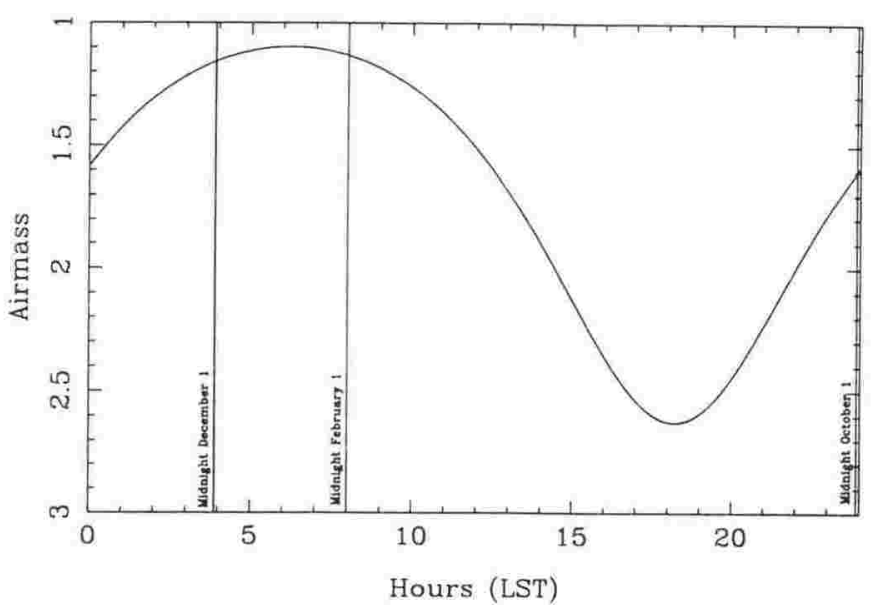

Figure 2-12: Example Airmass diagram. Airmass for the LMC Cluster NGC 2214 is plotted against local sidereal time (LST) at MJUO. Midnight (Standard Time) is indicated for the first days of October, December, and February. These months cover most of the LMC observing season. Another program allowed the airmass of an object to be plotted against Universal Time. This helped in planning an observing sequence for a night.

\subsection{Preparation for an Observing Run}

Telescope time must be applied for, and gained, before any data can be collected. The application may involve substantial paperwork, such as with the Anglo-Australian Telescope (AAT), through to the less intense application procedure at MJUO where most intending observers and their projects are already well known. No matter what level of official paperwork is involved, each observing run must be carefully planned as telescope time is a valuable commodity and should not be wasted. The majority of professionally equipped telescopes are over-subscribed, with over-subscription generally increasing with aperture size. Even after an application has been submitted, accepted, and telescope time allocated, it may be necessary to go through the planning process again due to the allocated time not being as long, or at the same time, as that applied for. Topics that require consideration include:

- The Phase of the Moon: it is no use trying to observe faint objects near a Full Moon, as they will be washed out in the lunar glare.

- The intended detector-filter combination, which defines the instrumental response, must be a good approximation to the appropriate standard system bandpasses. Non-standard bandpasses (e.g. too wide or narrow, or with different central wavelengths) may appear to produce satisfactory transformations until observations are made of objects with spectral distributions sufficiently different from the standards, which are typically solar abundance dwarfs (Da Costa 1991b). Systematic differences from the standard system magnitudes 
will then result. If new, modified, or long unmeasured filters are being used it may be worth remeasuring their transmittances with a spectro-photometer. If a new detector is being used it may be worthwhile convolving its response curve with the filter transmittances to investigate whether the instrumental system is similar to the standard one. The relative efficiencies of the instrumental passbands are provided by such work, which may be helpful in selecting suitable exposure times for each passband.

- The position of the project objects above the horizon must be considered. Firstly the object must be above the horizon! While the LMC is circumpolar from New Zealand latitudes, it still can sink below two airmasses. Observations are best made at the low airmasses of small zenith angles, where atmospheric absorption is small (see Figure 212 for an example airmass diagram). Seeing is also a function of airmass, and has an important effect on the direct imaging of crowded fields such as LMC clusters. The data of Pritchard (1993) indicates that seeing decreases by $\sim 0.6$ arcseconds per airmass. If only relatively high airmasses are available for a programme studying LMC star clusters, then more open objects such as $\mathrm{OB}$ associations may be more suitable targets than tight populous clusters.

- Finding charts will need to be prepared. The $\mathrm{M}^{\mathrm{c}}$ Lellan Telescope has a pointing accuracy of a few arcminutes. Therefore recognition of apparent patterns of stars, with reference to the finding chart, aids the observer in orienting the telescope onto the correct object - and confirming that the target is being observed. The Hodge \& Wright (1966) photographic atlas of the LMC is suitable for targets such as the LMC star clusters, with a good scale and sufficient detail to make identification of objects and their surroundings easy. The Hubble Space Telescope Guide Star Catalog (GSC) (Lasker et al. 1990) can be used to generate finding charts tailored to the needs of a specific observing programme (see Figure 2-13). This is an advance over published star atlases which are often to the wrong scale or an unsuitable limiting magnitude.

- Estimate the exposure times necessary to reach the desired limiting magnitude. It may be necessary to use an offset guider to ensure that long exposure observations are accurately guided. While it is possible to drive the offset guider about during an observing session in order to find a suitable guide star, this will waste telescope time. Instead overlays can be prepared (see Figure 2-14) which are scaled appropriately for given charts, and used to select suitably bright stars available to the offset guider when the telescope is centred on the target object.

- Standard (and possibly extinction) stars will need to be selected. Standards should have precise values (ideally to better than $0.5 \%$ ) and cover the colour range of the programme 


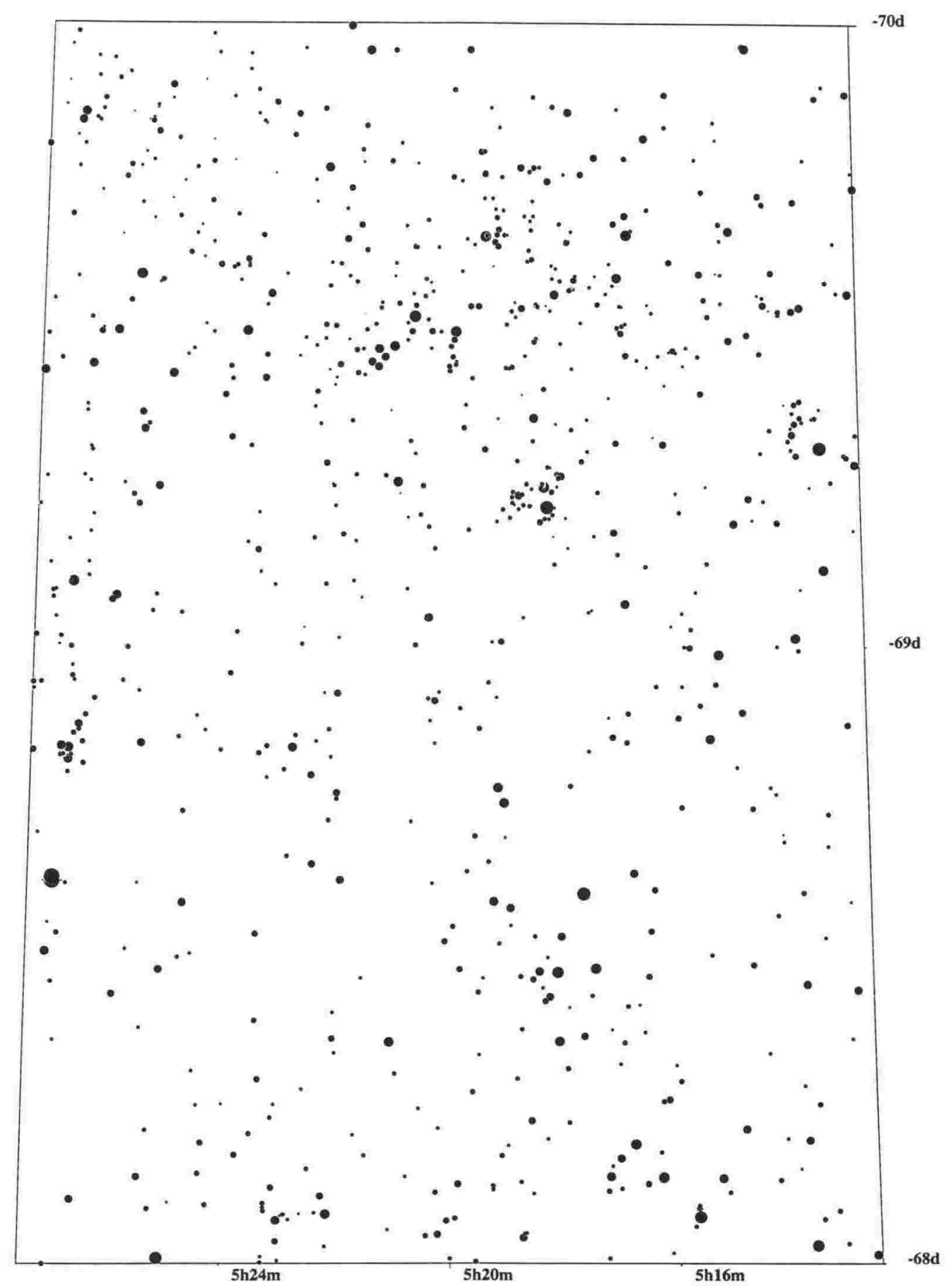

$21 \mathrm{~cm}$ LMC centre; $5 \mathrm{~h} 20 \mathrm{~m} .-69 \mathrm{~d}$ lim: 16.0

Figure 2-13: Sample Finding Chart depicting the star field at the centre of the LMC based on the $21 \mathrm{~cm}$ rotation curves. The brighter a star is visually, the larger the radius of the dot depicting it in the finding chart. The limiting magnitude of this chart is $\mathrm{V}=16$. 
University of Canterbury

Department of Physics \& Astronomy

Mount John University Observatory

CCD Photometer Head

offset Guider Co-ordinate Overlay

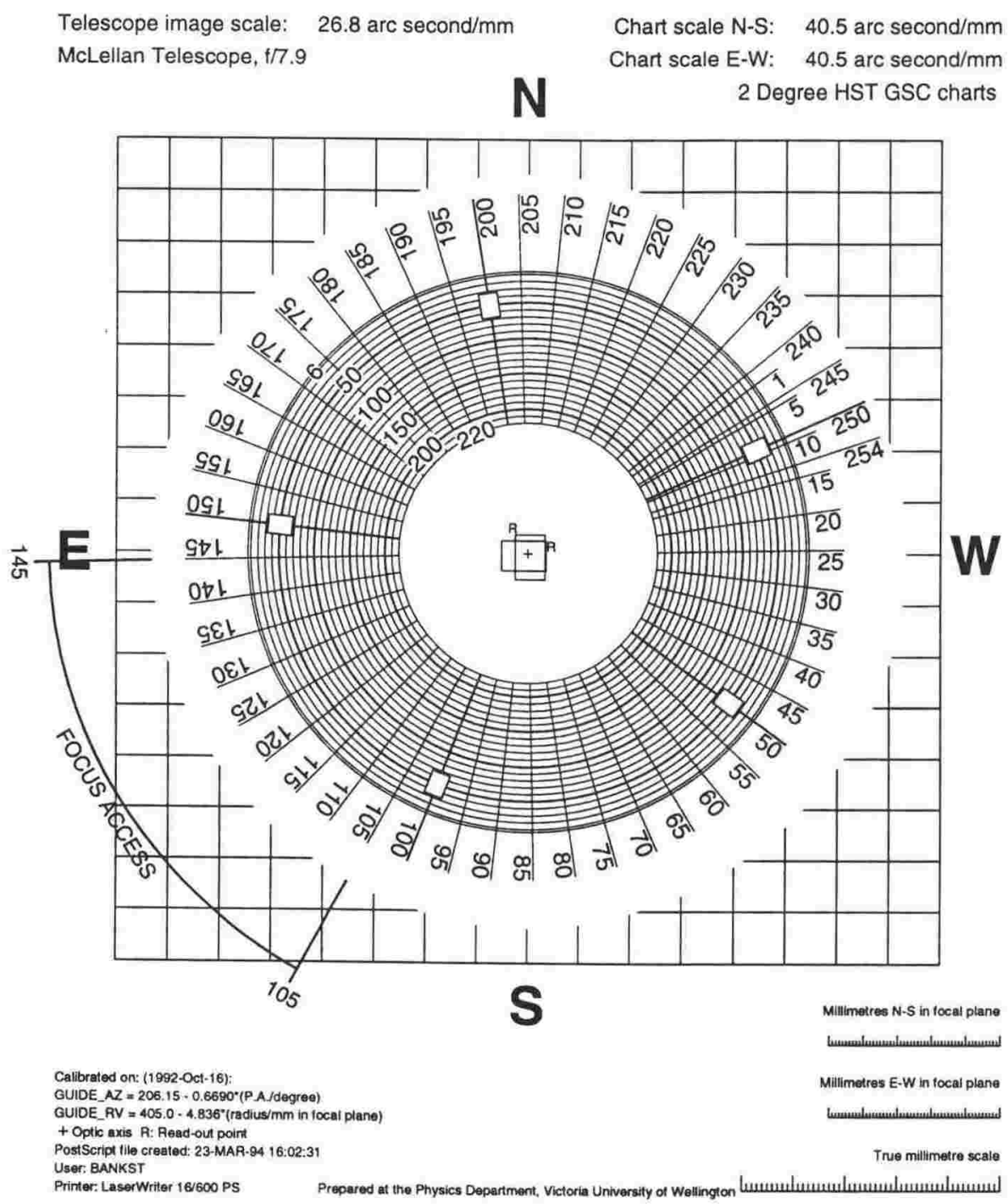

Figure 2-14: Offset Guider Overlay. Dr. William Tobin (University of Canterbury) kindly made available a FORTRAN program which generates a PostScript overlay for the MJUO offset guider customised for a given chart. This example overlay is for two degree charts, generated by the starchart software using the Hubble Space Telescope Guide Star Catalogue data. It has been reduced in size for inclusion into the thesis. When overlaid onto a chart, the overlay can be used to determine the two coordinates (azimuth and radius) required to move the offset guider CCD around to image a selected guide star. 


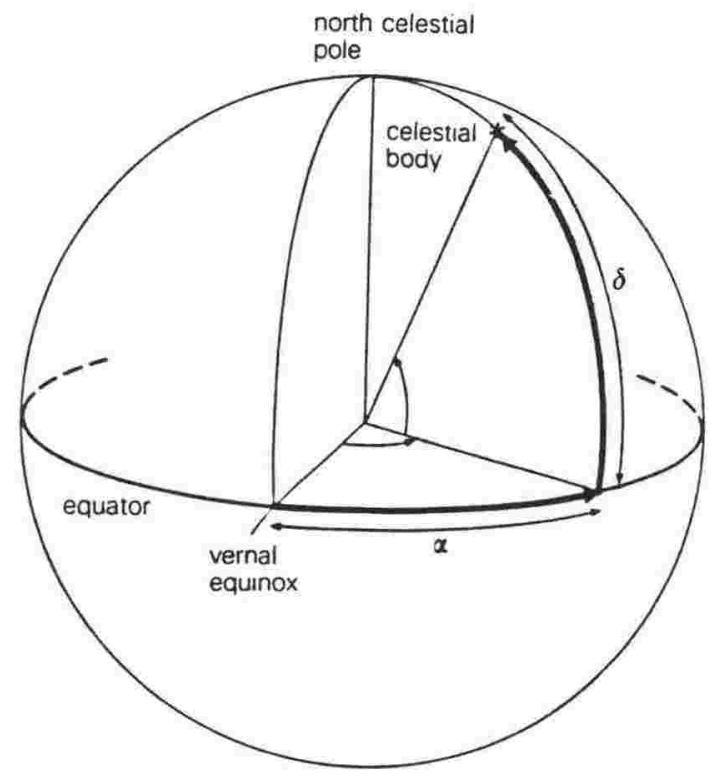

Figure 2-15: The Equatorial Coordinate System uses two coordinates called Right Ascension (R.A.) and Declination (Dec) to indicate the position of an astronomical object. The fundamental reference circle is the celestial equator, which is an arc across the celestial sphere immediately above the equator of the Earth. The celestial sphere is an imaginary sphere of infinite radius on which celestial objects are supposedly fixed. Declination $(\delta)$ is angular distance north (counted as positive) or south of the celestial equator. Right ascension $(\alpha)$ is angular distance along the celestial equator, measured eastwards from the Vernal Equinox. The two Equinoxes are the intersections of the ecliptic with the celestial equator. The ecliptic is the great circle formed by the intersection of the Earth's orbit with the celestial sphere, and is effectively the annual apparent path of the Sun across the sky (if the gravitational effect of the Moon on the Earth is ignored). The vernal equinox is around March 21. Declination is measured in degrees, arcminutes, and arcseconds, while right ascension is measured in hours, minutes, and seconds (i.e., 24 hours $=360^{\circ}$ ). (Figure from Illingworth, 1979).

stars. These will need to observed every clear night of the run. If the method of Harris et al. (1981) is being used, then the standards should be observed over an airmass range greater than the target objects, and ideally at least one airmass. Blue and red standards should be observed at both extremities of the airmass range, allowing the colour extinction terms to be definitively derived. The stars should not be too bright, which would result in short exposures which in turn would lead to shutter timing problems. For short exposures the time that the shutter diaphragm takes to open and close becomes an appreciable fraction of the exposure length. The central regions of the MJUO CCD are exposed for longer periods, being exposed first and covered last. The MJUO Uniblitz shutter takes between $\sim 5$ and $\sim 20 \mathrm{~ms}$ to open and close (Tobin 1993). Therefore observations should be longer than 10 seconds in length. 


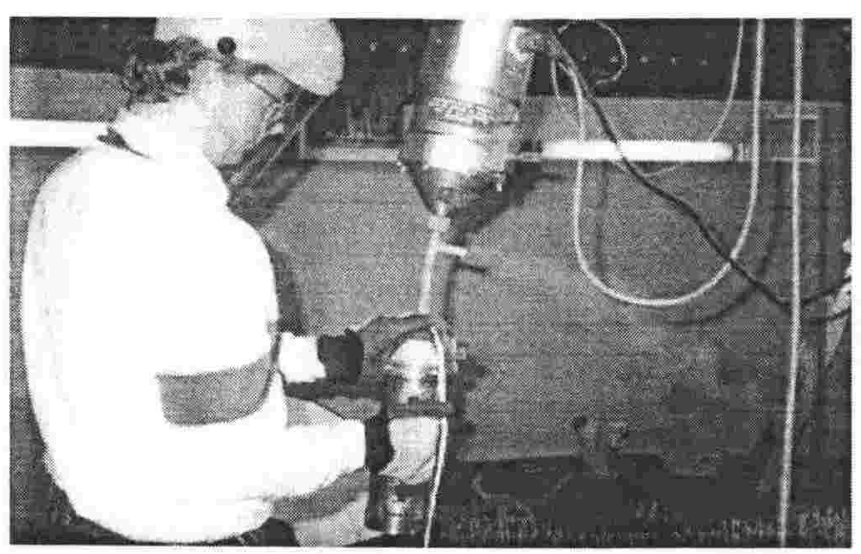

Figure 2-16: Filling the CCD Dewar with liquid nitrogen under pressure. As with all dealings with cryogenic material, safety precautions must be followed. Note the plastic face-plate in case of nitrogen spurting out past the rubber bung of the flask, or perhaps due to the rubber hosing up to the dewar breaking. Sturdy gloves are worn, and the excess nitrogen flow turned away. Photograph courtesy of Murray Forbes.

Observations should be scheduled so that sufficient standard and program observations are made. This may involve following selected standards as they rise or set during the night, with target observations being made in between these observations. Scheduling will involve consideration of airmass and exposure times, as well as leaving sufficient time for slewing the telescope, acquiring the target and identifying it via the finding charts, and the inevitable mistakes and breakdowns.

It is also helpful to consider what observing programme should be followed should weather at the site clear part-way through a previously unusable night.

- Coordinates of objects may need to be precessed ${ }^{1}$ across to a more suitable epoch. Failure to precess old coordinates can lead to the telescope being aimed far from where the desired object really is. The equatorial coordinate scheme, used for the positions of astronomical objects, is described in Figure 2-15.

\subsection{Observing Procedures}

This section is intended to act as a check-list of procedures that should be followed during an observing session using the $\mathrm{M}^{\mathrm{C}}$ Lellan telescope. FORTH command sequences are not discussed as the basic commands are thoroughly documented in the manuals supplied with the system. Copies of these manuals are kept with the computer.

\footnotetext{
${ }^{1}$ Precession is a slow periodic change in the direction of the rotational axis of a spinning object due to the application of an external force. In the case of the Earth, its axis precesses completely every 25,800 years with the principal external forces being the gravitational attraction of the Sun and Moon on the Earth's equatorial bulge (which attempt to align the bulge in their directions).
} 
On arrival at MJUO:

- Mount the CCD dewar and control electronics onto the telescope. Rebalance the telescope to ensure smooth tracking. Connect cabling to the CE200, terminals, monitors, and computer. Confirm that the system operates correctly.

- Cool the CCD to the normal operating temperature of $-110^{\circ} \mathrm{C}$. The CCD dewar was originally intended for medical imaging applications, facing downwards. The dewar is mounted onto the telescope facing upwards, leading to problems filling it with liquid nitrogen (LN). An electric resistor is placed into a flask of LN and used to boil off some of the liquid. Pressure is built up in the flask, forcing the LN up into the dewar (see Figure 2-16). Cooling will take several litres of LN, with the fillings being staggered over several hours. These refills should be timed to take place when the temperature of the CCD has reached a minimum, and is on the verge of warming. This will avoid excess boil off of the LN. Once cooled, the dewar has a hold time of $\sim 14$ hours.

- Insert the filter wheel into the CCD Photometer Head, after ensuring that the filters are clean and securely seated at right angles to the plane of the filter wheel since:

- Peak transmittance through interference filters varies according to the incident angle of light.

- Dust particles on a filter or the quartz window of the cryostat do not occult much light. A 'doughnut' (see Figure $2-5$ on page 38 ) is usually only a $\sim 3 \%$ drop in intensity. However, flexure of the telescope or photometer head can cause the dust rings in flat-field frames not to line up with the rings in observations, and so they will not be divided out completely by flat-fielding. The photometer head was designed to minimise dust contamination (see Tobin et al. 1993).

Tobin et al. (1993) report that bubbles and striations in the filters appear to be the principal cause of the $\sim 1 \%$ variations between flat-fields of the same passband. If the filter position (relative to the CCD chip) changes by greater than $\sim 100 \mu$ m between two flat-field observations there will be development of structure commencing at the $0.2 \%$ level. Therefore the resetting precision of the filter wheel is within $90 \mu \mathrm{m}$.

- Switch on the offset camera, which is slowed Peltier-cooled Philips 56470 camera. 1 second exposures will reach $\mathrm{V} \sim 11$. The guider has $\sim 1.2$ diameter field of view, ensuring that a suitable guide star will nearly always be available for a given target. 
On a good night (for observing):

- Open the dome a few hours before observations will commence. This will allow time for the temperature inside the dome to equalise with that outside. Close the doors into the dome, to prevent warm air flowing in.

- Perhaps top up the cryostat with LN if the last refill was long ago or the next refill would occur during observations.

- Ensure that the computer and consoles are functioning correctly. In particular reset the clock register, which can jam. Switch the printer on so that a permanent record is made of the sequence of observations. Load a magnetic tape, and ready it for use.

- When the sky intensity is nearly at a suitable level for flat-fielding, remove the dust covers from the telescope (and finder telescopes), enable the dome tracking, and switch on the telescope drives. If the telescope is going to be remotely controlled from the data room, drive the floating floor below the base of the window to the data room. The telescope can not be driven into the floor when it is at this position or lower.

- Slew the telescope to the east, where the intensity gradient of the sky is less. The tracking of the telescope can be switched off. Collect three dark frames and take the median. When the sky is dark enough, collect three flat-field frames of the twilight sky for each filter, subtract the median dark frame from each, and take the median. Exposure lengths for flat-fields should be such that their mean intensity is 10,000 ADU. Ensure that the flatfield exposures are long enough to avoid shutter timing problems. Examine the frames for any unusual features (such as severe cosmic ray strikes), and discard any bad frames. Save these frames to tape.

Note: If the telescope has not been focused yet, do not collect twilight flat-fields. The effect on Images of dust granules in the optic path alters with focus, and so flat-fielding should be left until the dawn.

- When it is dark enough for bright stars to be seen in the evening sky, re-enable the telescope drives, and slew the telescope across to one. Check the focus of the telescope, line up the finder telescopes, and calculate the offset of the star from the coordinates displayed on the telescope console. This offset should applied to all coordinates during the observing session.

- Commence observations of standard stars and the target objects when the sky is dark enough. Dark frames should be subtracted from observations as soon as they are collected, since the subtraction will be aborted if the dark frame is no longer appropriate. This will 
allow a new dark frame to be collected - which can not be done if this step is left until later. Flat-fielding an observation is time consuming, and should be left to a later time. If the offset guider is being used, and the telescope is aimed at the target, align the guider CCD with the guide star and adjust the integration rate so that a well-defined image is displayed with good time resolution. The focus of the offset guider may need to be adjusted.

- Collect flat-fields in the morning twilight, preferably of the western sky.

- Once the sky is too bright for observing, disable the dome tracking, close the dome, and turn it to its parking position of facing south-east, which is usually down-wind. Top up the cryostat, and park the telescope on the western side of the dome, at an hour angle ${ }^{1}$ of zero. The telescope should be tilted slightly to the north, so that any rainwater leaks will run off the covers. Switch off the telescope drives, and put the covers on all the telescopes. Ensure that the telescope is not beneath the motor for the dome shutter, so that oil can not drop onto the telescope mirror. Rewind and close the magnetic tape. Switch off the tape drive and printer, but do not switch the computer off as it controls the temperature of the CCD.

If the night becomes unusable before dawn and flat-fields have not yet been collected for the night, dome flat-fields should be taken. Flat-field observations must be collected close to the science observations to avoid problems with the movement of any dust in the optic path. To obtain dome flat-fields the telescope is focused to infinity, and aimed at an illuminated screen attached to the inside of the dome. To correctly position the dome, markers on it must be aligned with twin markings on the dome wall. The telescope should be slewed to a declination $-79.5^{\circ}$ under the pole and an hour angle of zero (Clark 1994). The screen is supposed to be so far out of focus that the telescope focal plane is uniformly illuminated. The paint used on the screen has a flat reflectivity across the observational wavelengths. Many white paints contain 'optical brighteners', which are fluorescent dyes that absorb the wavelengths 300-400 nm and re-emit the radiation at longer wavelengths. This makes whites that appear more white to the human eye. A good reflector is Eastman 6080 paint, which is a nearly perfect diffuse reflector throughout the visible and into the infrared. Absolute reflectance is greater than $98 \%$ over the range $300-1300 \mathrm{~nm}$, being over $99 \%$ for the interval $350-1100 \mathrm{~nm}$. It is used to paint the interiors of integrating spheres, which are designed to provide an optical source which is uniform and proportional to the amount of light input but independent of the viewing angle. Such spheres are used in the calibration of small detectors.

\footnotetext{
${ }^{1}$ Hour Angle is the angle measured westward along the celestial equator from an observer's meridian to the hour circle (or R.A.) of an object.
} 
Two projectors are available for flat-fielding, and are used to illuminate the screen. The first is a $1200 \mathrm{~W}$ theatrical spotlight with a quartz-halide bulb. Cooling of such lamps assumes that they are installed facing downwards. The reverse is true at MJUO, and so care must be taken not to overheat the lamp. The second lamp is available for red and infrared flat-fields, as the first is too bright in this part of the spectrum.

Dome and twilight flats agree at the $\sim 1 \%$ level. In a test that flat-field frames are indeed flat to this level, Tobin (1992b) observed two E-region standards with a separation less that the field of view of the CCD. The magnitude difference between the two stars varied in a reproducible manner by up to $\sim 4 \%$ depending on the positions of the stellar images on the CCD. Hence both types of flat-field Image are warped. Tobin (1992b) suggested that this was due to light scattered off the chimney baffle of the primary mirror, and has since installed annuli in the baffles to reduce it.

At the end of an observing run the filter wheel should be removed for return to Wellington, the PM-3000 shut down, and the dewar allowed to return to room temperature. The photometer head, temperature control unit, and associated hardware should be switched off and removed from the telescope. The removal and storage of the equipment is usually performed by the resident MJUO staff. 


\section{Chapter 3}

\section{The Data Pathway}

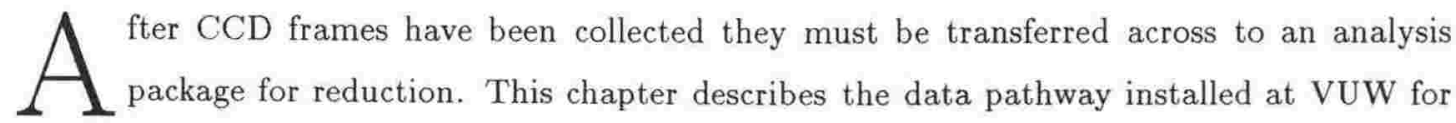
this purpose. Figure 3-1 is a schematic of the pathway.

CCD frames are written at MJUO onto 9-track magnetic tapes in a proprietary format that no analysis package supports. Therefore, not only must the observations be transferred from tape onto disk, they must also be converted into a format acceptable to the chosen package. Often this format is the Flexible Image Transport System (FITS), which is described in Appendix A (page 209).

Data reduction is simplified when useful information about an observation is included into the header records of its Image file. These records contain information such as the airmass of the observation, the exposure length, and when the exposure was made. The tape reader program converts this information into FITS format, as well as calculating new information from 


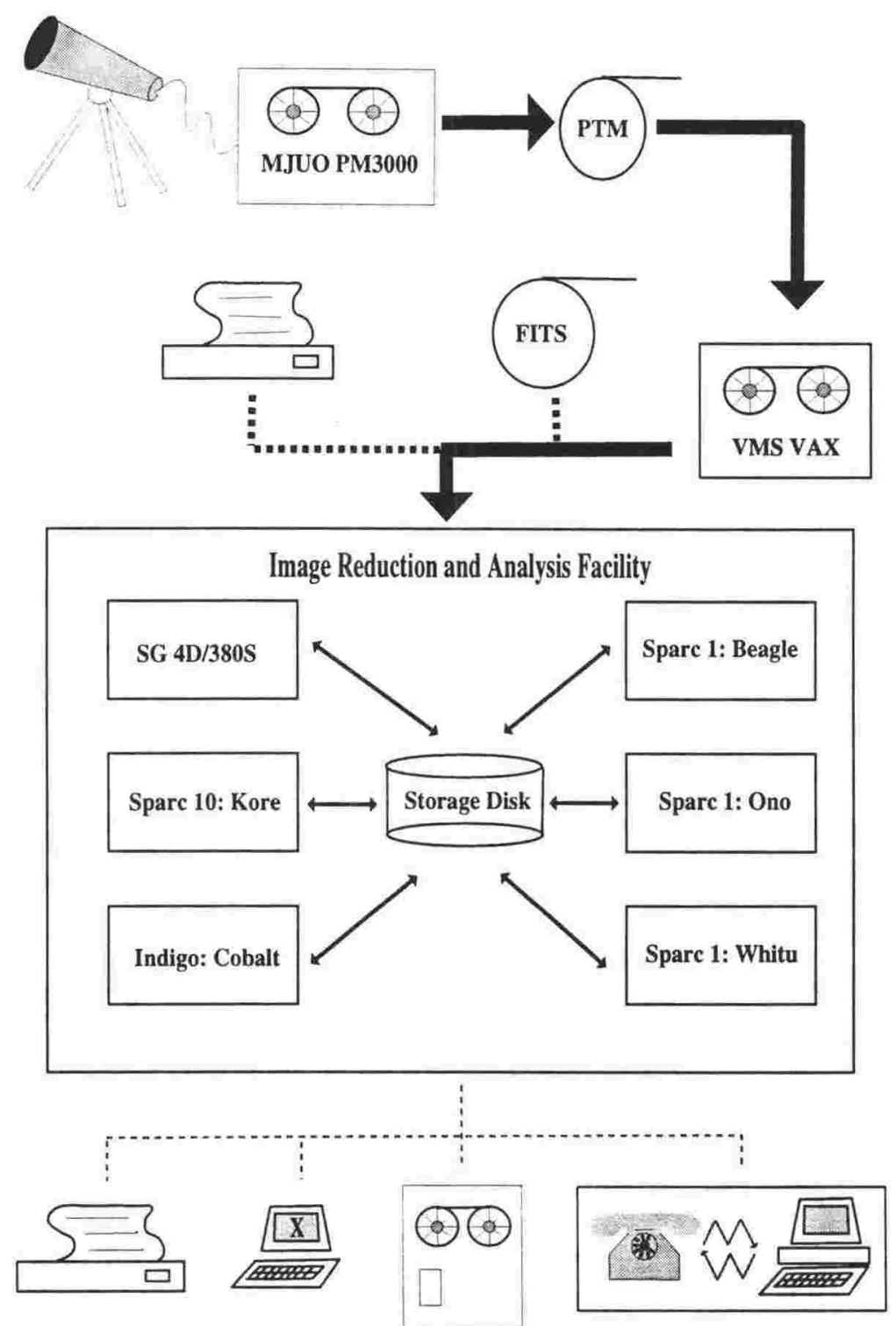

Figure 3-1: The Data Pathway and IRAF. Data is collected at the telescope using the PM-3000 computer and transferred to 9-track magnetic tape. The tape files are written in the proprietary PTM (PhoToMetrics) format. The tape is transported back to Wellington, read in by the VUW VAX running under the VMS Operating System, and the individual files converted into FITS format. The Image files are written to backup tapes in this format. Hard-copy of the CCD frames can also be printed at this stage. The data are then read into the Image Reduction and Analysis Facility, which includes a central $300 \mathrm{Mb}$ hard disk for the storage of pixel data that can be accessed by the six computers making up the local IRAF installation. Considerable effort has gone into giving IRAF access to a variety of tape drives (9-track, DAT, Exabyte, and Qic - all of which can be used to directly import FITS files), different printers, other sites off-campus using the UUCP and SLIP protocols across telephone lines, and various terminals (X-terms, Visual 500s, etc.). This effort has led to the VUW installation being able to provide a complete virtual Operating System. 
PM-3000 CCD Image acquisition computer onto a VMS VAX, where the second program may be used to generate a PostScript file of the Image. PostScript is a widely supported graphics programming language, which is thoroughly documented by Adobe (1985a, b, c).

Dr. Tobin kindly provided copies of these programs, which have been slightly modified for use at VUW and are described below. The algorithms could be further optimised. The discussion begins with the subroutines and functions which are common to both of the programs, before turning to the programs themselves.

\subsubsection{Subroutines and Functions}

\subsubsection{Subroutine Decode_U}

The PM-3000 computer stores the start and end times of an observation in the UNIX time format. This format is the number of seconds elapsed since $0^{\mathrm{h}} 0^{\mathrm{m}} 0^{\mathrm{s}}$ January 11970 (Universal Time). The start and end times are stored in bytes 105-108 and 113-116 of the Header (see Table 3.1 on page 73 ). The subroutine 'decodes' the 32 bit unIX representations into a more usable form, although it can only convert one value each time it is called. Therefore it must be called twice to decode the two records. The observation date, time, Epoch, Julian Date, and Local Sidereal Time are returned by the subroutine. The terms given in this paragraph will be explained below.

The subroutine performs the following steps:

1. The UNIX time is assembled from the 4 bytes passed to it:

$$
\text { Byte }_{4}+2^{8}\left(\text { Byte }_{3}+2^{8}\left(\text { Byte }_{2}+2^{8} \text { Byte }_{1}\right)\right)
$$

where Byte ${ }_{1}$ is the lowest order byte (i.e., Header Records 108 and 116).

2. The resulting time value is divided by 86,400 , or the number of seconds in a day. The time is now given as the fractional number of days since the beginning of 1970 .

3. The year of observation is calculated by a loop which:

- Initially checks to see if the number of days is less than in the year 1970. If so, the year of observation is 1970 - a somewhat unlikely event!

- Otherwise the number of days in the year being tested are removed from the total, and the subsequent year checked. The loop ends when the number of days remaining is less than in a given year.

Leap years are accounted for. 
4. Next the month of observation is determined. Each month of the year is compared in turn with the number of days remaining. If the day number is smaller than the length of the month, then the month of observation has been determined, and the subroutine moves on to the next step. Otherwise, the length of the month is subtracted from the day number and the next month checked. Variation in the length of February is accounted for.

5. The date number (i.e., $1^{\text {st }}, 9^{\text {th }}$ ) is the integer of the time value. The integer is subtracted from the time value.

6. All that remains of the time value is a fraction representing how far through a given day the observation was, which is converted into hours, minutes, and seconds. The Universal Time (U.T.) of observation has now been calculated. U.T. is the mean solar time at the meridian of zero longitude (the Greenwich Line). Mean solar time is measured with reference to the uniform motion of the mean sun. The mean solar day is the interval between two successive passages of the mean sun through the meridian. ${ }^{1}$ The apparent motion of the real Sun across the sky is variable. This non-uniformity is mainly due to the elliptical orbit of the Earth, which causes the orbital speed of the planet to vary and hence the apparent speed of the Sun in the sky to change as well. The mean sun 'follows' a circular orbit along the celestial equator (not the ecliptic), moving eastwards at a constant speed so that one complete revolution takes one tropical year. ${ }^{2}$

7. The Epoch of the observation is calculated by the addition of a fraction to the integer year. The fraction is the number of days through the year that the observation was made on, divided by the length of that year in days. A value similar to 1991.52 is produced.

8. Next the Julian Date (JD) of observation is calculated using the algorithm given by Duffett-Smith (1984):

$$
\mathrm{JD}=17,209,945+\operatorname{int}(365.25 \mathrm{x} \text { year })+\operatorname{int}(30.6001(\text { month }+1))+\text { day } .
$$

The Julian Date is the number of days elapsed since noon U.T. January $1^{\text {st }} 4713$ B.C., including the fractional day. The advantage of this system is that days are numbered consecutively, simplifying investigations involving periodicity. It is independent of the length of a month or a year. The system was devised by Joseph Scalinger in 1582, and named in honour of his father. Modified Julian Date (MJD) is the Julian Date with $2,400,000.5$ subtracted from it. It is widely used as the higher magnitude digits are effectively redundant given the time frame of the data and any usage of it.

\footnotetext{
${ }^{1}$ The observer's meridian is the great circle passing from the point on the horizon due south through the zenith, which is the point directly overhead at anytime, to due north on the observer's horizon.

${ }^{2}$ The tropical year is the time between two successive passages of the Sun through the vernal equinox.
} 
9. Finally, the Local Sidereal time (LST) at MJUO is calculated. The first step involves the function GMST (described in Section 3.1.1.3), which calculates the Greenwich Mean Sidereal Time (GMST is the mean sidereal time at longitude zero). 11.36433 hours are then added to the GMST to reach LST for MJUO. The unusual number being added on to GMST is the longitude of Mount John expressed in hours, rather than the normal format of $+170^{\circ} 27^{\mathrm{m}} \mathrm{9}$ East. Sidereal Time is measured by noting the rotation of the Earth relative to the stars. The orbital motion of the Earth about the Sun causes the sidereal day to be $\sim 3^{\mathrm{m}} 56^{\mathrm{s}}$ shorter than the mean solar day.

\subsubsection{Function JD}

This function returns the Julian Date given an integer input of year, month, day, minutes, and real seconds. $J D$ first checks that the input is correct, i.e., no negative numbers or hours $=$ 25. The difference between the year of observation and 1988 is calculated. The number of days corresponding to this difference is either added to, or subtracted from, the number $2,447,160.5$ depending on whether the year of observation is before or after 1988. This number is the Julian Date for Jan $00^{\mathrm{h}} 0^{\mathrm{m}} 0^{\mathrm{s}} 1988$ (U.T.). Leap years are accounted for. The number of days elapsed through the year of observation is calculated, using the month and day values passed to the function. Again, variation in the length of February is noted. The resulting value is added onto the total, as is the fraction:

$$
\frac{1}{24}\left(\text { hour }+\frac{1}{60}\left(\text { minute }+\frac{1}{60} \text { second }\right)\right) .
$$

\subsubsection{Function GMST}

This function returns Greenwich Mean Sidereal Time in decimal hours given input in the form of integer year, month, day, hours, minutes, and real seconds. Firstly the time 'elapsed' since Jan 1.52000 (U.T.) as measured in Julian centuries, $t_{u}$, is calculated by:

$$
\mathrm{t}_{\mathrm{u}}=\frac{\mathrm{JD}-2,451,545.0}{36,525}
$$

where JD is the appropriate Julian Date found using the subroutine $J D$. The Julian Century is not related to the Julian Date by name. Instead the name commemorates Julius Caesar, who established this calendar in 46 B.C. with the technical advice of Sosigenes of Alexandria. In the Julian Calendar, three years of 365 days length are followed by a leap year 366 days long. Leap years were not correctly inserted into the Julian Calendar until 8 A.D, when Augustus revised the system. Unfortunately the mean Julian year is $11^{\mathrm{m}} 14^{\mathrm{s}}$ longer than the tropical year, leading to the 'appearance' of an extra day every 128 years. The system was used in general throughout Europe until 1582, when the Gregorian calendar was introduced. The new 
system was instituted by Pope Gregory XIII, with the immediate 'elimination' of 10 days in October 1582 to bring the calendar back into accord with the seasons. If the last year of a century has four hundred as a factor, then that year is a leap year. Otherwise a century year is not a leap year. Great Britain and its colonies did not adopt this system until 1752 (p.43 Coveney \& Highfield 1990, p.145 Abbot 1925), as they were not Roman Catholic countries and were therefore reluctant to adopt an improvement advocated by a Pope. Russia did not change systems until 1918.

$t_{u}$ is then substituted into:

$$
\mathrm{GMST}_{0^{\mathrm{h}} \mathrm{UT}}=24,110^{\mathrm{s}} .5481+8,640,184^{\mathrm{s}} .81266 \mathrm{t}_{\mathrm{u}}+0.093104 \mathrm{t}_{\mathrm{u}}^{2}-6^{\mathrm{s}} .2 \times 10^{-6} \mathrm{t}_{\mathrm{u}}^{3}
$$

producing the GMST at 0 hours (U.T.). Equations 3.4 and 3.5 are taken from the Astronomical Almanac (1990). A loop is then entered to test whether the value of GMST is positive. If the value does not meet this condition, then the number of seconds in a day is added to the total, and the test performed again. Once the condition is met, a second loop is entered which performs the opposite, subtracting 86,400 seconds from the value. These two loops ensure that the final value of GMST is between 0 and 86,400 seconds, and allows the function to handle dates before and after Jan 1.5 2000. Finally, GMST is converted from seconds into hours.

\subsubsection{Function HJD_JD}

This function calculates the difference between the Heliocentric Julian Date and the Julian Date on which a star was observed. This difference is called the heliocentric correction. It is necessary to account for the variation in the distance of the Earth from the Sun. The time for light to travel 1 A.U. ${ }^{1}$ is about $8^{\mathrm{m}} 19^{\mathrm{s}}$. Observations collected six months apart will be up to $16^{\mathrm{m}} 38^{\mathrm{s}}$ out of phase, depending on the position of the star in the sky. This difference in light travel time should be removed, particularly if time dependent objects (such as eclipsing binaries) are being studied.

The function is passed the equatorial coordinates of the object, and the Julian Date of observation. The heliocentric correction (H.C.) to the time of observation is given by (Landolt \& Blondeau 1972):-

$$
\text { H.C. }=-0^{d} .0057755 \mathrm{R}(\cos \theta \cos \alpha \cos \gamma+\sin \alpha(\sin \xi \sin \delta+\cos \xi \cos \delta \sin \alpha))
$$

where:

- $\mathrm{R}$ is the radius vector for the Earth at the time the observation was collected,

\footnotetext{
${ }^{1} 1$ Astronomical Unit, which is equal to the mean distance between the Sun and the Earth.
} 
- $\theta$ is the true longitude of the Sun at the time of observation,

- $\alpha$ is the precessed right ascension of the object,

- $\delta$ is its precessed declination, and

- $\xi$ is the instantaneous obliquity of the ecliptic.

Celestial longitude and latitude are coordinates in the Ecliptic System. The former is the angular distance of an astronomical object from the vernal equinox, measured eastwards along the ecliptic to the intersection with the object's circle of longitude. Celestial latitude is defined as the angular distance of an object north (positive) or south of the ecliptic. Both coordinates are measured in degrees. Observations in the ecliptic system are typically corrected to account for the fact that they were collected at a point on the surface of the Earth. Such corrected coordinates are said to be the geocentric values. Once again, there is a difference between apparent and mean values, with correction to the mean being assumed.

The geocentric mean longitude of the Sun is:

$$
\mathrm{L}=279 \circ 69668+36,000 \div 76892 \mathrm{~T}+0 \% 0003025 \mathrm{~T}^{2}
$$

where $\mathrm{T}$ is the time of observation from January 0.51900 , as measured in Julian Centuries, i.e.:

$$
\mathrm{T}=\frac{\mathrm{JD}-2,415,020.0}{36,525}
$$

where JD is the Julian Date, as before.

The Instantaneous Obliquity of the Ecliptic is given (empirically) as:

$$
\xi=23: 452294-090130125 \mathrm{~T}-0.000000164 \mathrm{~T}^{2}+0.000000503 \mathrm{~T}^{3},
$$

while the distance of the Earth from the Sun in Astronomical Units is:

$$
\mathrm{R}=1.00000022(1-\mathrm{e} \cos \nu)
$$

where $e$ is the eccentricity of the Earth's orbit:

$$
\mathrm{e}=0.01675104-0.0000418 \mathrm{~T}-0.000000126 \mathrm{~T}^{2}
$$

and $\nu$ is the True Anomaly (see Figure 3-2). The value of T substituted into Equations 3.9 and 3.11 is calculated using Equation 3.8. $\nu$ is equal to $\mathrm{M}+\mathrm{C}$, where $\mathrm{C}$ is the Sun's Equation of 


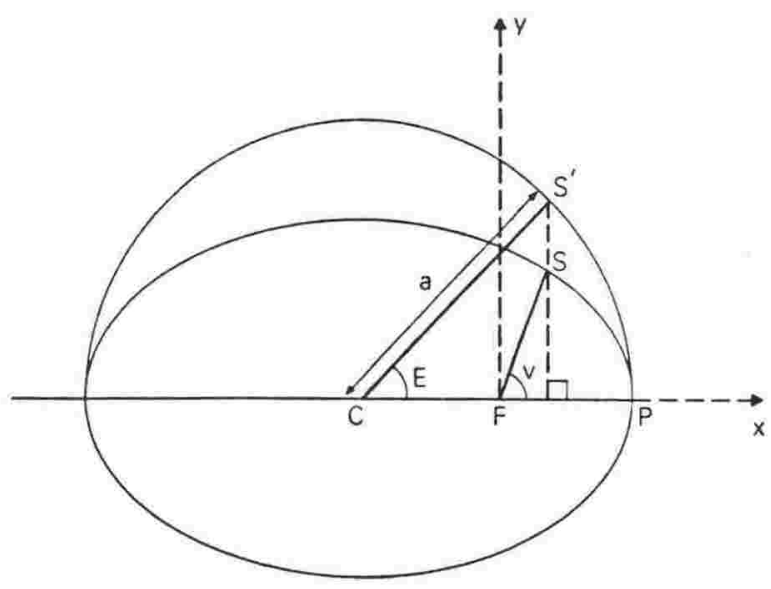

Figure 3-2: Anomaly. There are three different anomaly angles for a body moving in an elliptical orbit. The true anomaly is the angle $v$ between the body at $\mathrm{S}$, the focus $\mathrm{F}$, and the point of nearest approach to the focus (periapsis), $\mathrm{P}$. The eccentric anomaly is angle $\mathrm{E}$ between the lines $\overline{\mathrm{CS}^{\prime}}$ and $\overline{\mathrm{CP}}$, where $\mathrm{C}$ is the midpoint of the ellipse's major axis. $\mathrm{S}^{\prime}$ is on a circle with radius equal to that of periapsis, and has the same (vertical) projection onto the major axis as point $\mathrm{S}$ does. The mean anomaly is the difference between object $\mathrm{S}$ and an hypothetical object moving along the same orbit at the mean velocity of S. (Figure from Illingworth 1979).

Centre:

$$
\begin{array}{r}
C=\left(1.919460-0.004789 \mathrm{~T}-0.000014 \mathrm{~T}^{2}\right) \sin \mathrm{M}+ \\
\quad(0.020094-0.000100 \mathrm{~T}) \sin 2 \mathrm{M}+0.000293 \sin 3 \mathrm{M},
\end{array}
$$

and $M$ is the Mean Anomaly of the Sun (see Figure 3-2):

$$
\mathrm{M}=358 \div 47583+35999 \div 04975 \mathrm{~T}^{2}-0 \div 000150 \mathrm{~T}^{2}-0 \div 00000033 \mathrm{~T}^{3} .
$$

Equations 3.7 to 3.13 are taken from Meeus (1982). Given these formulae, HJD_JD calculates the HJD using the following steps:

1. The date of the observation measured in Julian Centuries $(\mathrm{T})$ is calculated using Equation 3.8 .

2. The solar geometric mean longitude (L) is calculated using Equation 3.7. A series of conditional statements and loops ensure that the final value for the longitude is smaller than 360 degrees.

3. The mean anomaly of the Sun (M) is then given by Equation 3.13. A set of loops and conditional statements ensure that the final value of $\mathrm{M}$ is less than 360 degrees.

4. Equation 3.11 is evaluated, providing the eccentricity (e) of the Earth's orbit for the date of observation. 


\begin{tabular}{|l|l|l|}
\hline S & Exponent & Significand \\
\hline 0 & 1 & 9
\end{tabular}

Figure 3-3: Single Precision 32 Bit IEEE Floating Point Standard for the storage of numbers. 'S' stands for the sign bit, while the numbers indicate the offset of a given bit from the first bit. The first eight bits make up the high byte.

5. Next to be evaluated is Equation 3.12, which requires the values of $\mathrm{T}$ and $\mathrm{M}$. C is output.

6. Now that $\mathrm{C}$ is known, the true longitude of the Sun $(\theta)$ is given by adding $\mathrm{C}$ onto $\mathrm{L}$. The true anomaly $(\nu)$ of the Sun is calculated by adding $\mathrm{C}$ with $\mathrm{M}$.

7. The radius of the Earth's orbit $(R)$ is calculated using Equation 3.10 and the values for e and $\nu$.

8. Equation 3.9 is evaluated, providing the instantaneous obliquity of the ecliptic $(\xi)$. The variables $\mathrm{R}, \theta$, and $\xi$ are now available for substitution into the heliocentric correction equation (Equation 3.6), which the function does. This correction is applied to the JD, giving the heliocentric Julian Date.

\subsubsection{Function R4}

The Io-ForTH control language of the PM-3000 computer uses the single precision 32 bit IEEE floating point standard, which is a protocol designed to facilitate the portability of programs (see Figure 3-3 and pp. 270 Stallings 1990). The standard initially focused on microcomputers with their more limited capabilities. The implied base is 2, and the lowest order bit (of the high byte) is the sign bit. The sign bit is set to zero if the number is positive. The next 8 bits are the exponent of 2 , with 128 (the bias) added to ensure it is always positive. This is known in Computer Science as a biased representation. The remaining 23 bits represent a number between 1 and 0.5 , with the 24 th bit being a hidden normalisation bit. This first bit would always be 1 , so it can be assumed to be so and used as the lowest order bit in the exponent.

$R_{4}$ is passed four bytes, which is converted into a real number by the following steps:

- The sign bit is examined.

- The exponent is formed by taking the remaining 7 bits of the high byte, multiplying them by 2 , adding in the high bit of the next byte, and subtracting the bias.

- The number is constructed from the remaining bits, scaled appropriately using the exponent. 
As an example, consider that the exponent is 1 . Since the significand lies between 0.5 and 1 , numbers from 1 to 2 can be represented. If the exponent is set to two, numbers between 2 and 4 can be represented, and so on.

\subsubsection{Function UT_CCD}

This routine originally utilised a look-up file of clock corrections for the internal clock of the acquisition system. This feature has been suspended at VUW, as observers reset against a master clock or the time-keeping radio station WWV, and the variable 'ut_ccd' that contained the correction has been removed.

\subsubsection{Program PTM_HARD}

This program takes PTM format Image files and produces PostScript programs as output. These program files can be sent to a suitable printer to produce a printed copy of the CCD Image. The printout of the Image includes information about the target, such as its name and coordinates.

PTM files have a record length of 768 bytes, with the first record being the header. Only the first 160 bytes of the first record are used. The format for the customised MJUO PTM header is given in byte order as Table 3.1. The format of the original Photometrics header, as supplied with the system, is given as Table 3.2. The 'pads' were designed to accommodate locally defined functions, explaining why the instrument parameters are not consecutive and there are blanks left for expansion. Subsequent records contain the data for individual CCD rows as 16 bit integers. Hence there is one 768-byte record per row of the CCD read out. If a sub-array of the CCD has been stored (i.e., less than 384 pixels in a row), then trailing bytes in the records are ignored.

The program first asks the user for the name of the file to be converted into a PostScript version. This file, and files containing names and coordinates of the observed objects, are opened before the user is prompted for automatic or manual selection of print parameters. Next the exposure time, beginning and end of observation, column origin, row origin, number of columns and rows, column and row binning factors, gain factor, and image type key-words are retrieved from the header records. The subroutine Decode $U$ is used to convert the time measurements into usable values.

The software used to acquire the frames is then determined. If the basic Photometrics system was used, then the start time for the observation is stored in the header bytes 112-115. The contents of these bytes will have been assigned to incorrect variables by the program, which assumes that the UoC customized system was being used. Therefore the program shuffles data about so that it is correctly connected with the right variables. The exposure length is then 


\begin{tabular}{|c|c|}
\hline Bytes & Purpose \\
\hline 001-079 & ASCII Target name and comments field. \\
\hline 080-098 & ASCII flags indicating which (if any) of the reduction steps such as \\
\hline$\cdots$ & flat-fielding and dark subtraction have been performed on the Image. \\
\hline 099 & An ASCII null \\
\hline 100 & Unused \\
\hline $101-104$ & A 4 byte exposure time, which is set by the acquisition commands \\
\hline $\begin{array}{c}\cdots \\
105-108\end{array}$ & $\begin{array}{l}\text { dark and obs, but not readout. } \\
\text { The commencement time of the observation in } 32 \text { bit uNIX format. }\end{array}$ \\
\hline $109-112$ & $\begin{array}{l}\text { The commencement time of the observation in } 32 \text { bit UNIX format. } \\
\text { Four ASCII characters for the initials of the Observers }\end{array}$ \\
\hline $113-116$ & For Images acquired with the readout command this gives the end \\
\hline$\cdots$ & of the observation time in UNIX format. For obs and dark commands \\
\hline$\cdots$ & this field contains the exposure commencement time. \\
\hline $\begin{array}{c}117-118 \\
\ldots\end{array}$ & $\begin{array}{l}\text { Two bytes giving the column origin - useful when the Image is a subarray } \\
\text { of the original. }\end{array}$ \\
\hline $119-120$ & Two bytes giving the row origin. \\
\hline $121-122$ & Number of rows in the Image. \\
\hline $123-124$ & Number of columns in the Image. \\
\hline $125-126$ & Column binning factor. \\
\hline $127-128$ & Row binning factor. \\
\hline $129-130$ & Amplifier gain. \\
\hline $\begin{array}{l}131 \\
\ldots\end{array}$ & A one byte numeric code for the instrument used: \\
\hline$\ldots$ & $11=$ Pentax bayonet fitting for large filters that were manually changed. \\
\hline$\cdots$ & $12=$ CCD top hat and filter wheel. \\
\hline$\cdots$ & $13=$ Photometer \\
\hline$\ldots$ & $14=$ Échelle spectrograph \\
\hline ... & $15=$ The spectrograph on the Boller \& Chivens telescope. \\
\hline 132 & One byte numeric code for the telescope used. \\
\hline$\ldots$ & $0=$ unknown \\
\hline$\cdots$ & $1=$ Boller \& Chivens $61 \mathrm{~cm}$ \\
\hline$\cdots$ & $2=\mathrm{M}^{\mathrm{c}}$ Lellan $1 \mathrm{~m} \mathrm{f} / 7.9$ \\
\hline$\cdots$ & $3=\mathrm{M}^{\mathrm{c}}$ Lellan $1 \mathrm{~m} \mathrm{f} / 13$ \\
\hline 133-134 & Not used, left for expansion. \\
\hline $135-138$ & Floating point number giving average offset plus dark current. \\
\hline $139-142$ & The filter name in ASCII. \\
\hline $143-146$ & Parameters specific to the instrument used. \\
\hline $147-150$ & Parameters specific to the instrument used. \\
\hline $151-152$ & Two bytes specifying the image type $(0=$ bias, $1=$ dark, $2=$ Image $)$. \\
\hline $153-156$ & Parameters specific to the instrument used. \\
\hline $157-160$ & Parameters specific to the instrument used. \\
\hline
\end{tabular}

Table 3.1: MJUO Frame Header Records 


\begin{tabular}{||c|l||}
\hline Bytes & Purpose \\
\hline $001-100$ & Pad \\
$101-104$ & 32 bit Exposure Time in deciseconds. \\
$104-111$ & Exposure length in deciseconds. \\
$112-115$ & Header creation time in seconds. \\
$116-117$ & Column origin. \\
$117-118$ & Row origin. \\
$120-121$ & Column length. \\
$122-123$ & Row length. \\
$124-125$ & Column binning factor. \\
$126-127$ & Row binning factor. \\
$128-129$ & Gain factor, as a 16 bit integer. \\
$130-149$ & Pad \\
$150-151$ & Image type (as above). \\
$152-160$ & Pad \\
\hline
\end{tabular}

Table 3.2: Photometric Header Records

used to calculate the end of exposure time, and the appropriate variable set. Otherwise, if the customised acquisition software was used, the program sets the character variables 'telescope' and 'instrument' using the appropriate header bytes.

Regardless of which Image format was used, the average offset is decoded by $R 4$. Next, the name of the observed object is examined. Information about the objects observed is stored in three files on the computer. The first file contains calibration object names (e.g. FLAT), the second file contains the object names together with an identifying number (the "key"), and the third file contains the "key" on a line with coordinates for the object at a specified Epoch. Different names may be used for the same object during different observing runs. All these variations are entered into the second file and have the same key. Hence they address the same coordinate information in the third file.

The object name, as read from the tape, is compared against the first file. If a match is found then the frame is a calibration, and so no coordinates apply to it. If a match is not found then the second file is checked in turn for a match. If a match is successfully made in the second file, then the individual 'key' for the object is determined and used to look up the appropriate data from the third file. If no match is found in either the first or the second file the user is prompted to input the values for the object. The object name, epoch, and coordinates are displayed for the user to check, and replace if necessary.

Once the coordinates of the (non-calibration) object are determined for a given Epoch, they are precessed using the non-rigourous equations:

$$
\Delta \alpha=\mathrm{m}+\mathrm{n} \sin \alpha \tan \delta
$$


to the Epoch of the observation (which will have been determined by the subroutine Decode_U). The adopted $m$ and $n$ are 3.0742 and 20 ".0383, which are the values for Epoch 2000.0 (Meeus 1982).

If the exposure is under five minutes in length, and not a calibration, then the airmass $(\mathrm{X})$ is calculated using the equation given by Young (1974):

$$
X=\sec (z)\left(1-0.0012\left(\sec ^{2}(z)-1\right)\right)
$$

where $\sec (z)$ is given by:

$$
(\sin (\phi) \sin (\delta)+\cos (\phi) \cos (\delta) \cos (\text { H.A. }))^{-1}
$$

H.A. is the hour angle, $\phi$ is the latitude of the observing site, and $\mathrm{z}$ is the zenith angle. If the exposure is longer than five minutes, the approximation of Stetson (1989) for the mean airmass of a long exposure is used:

$$
X=\frac{X_{0}+4 X_{\frac{1}{2}}+X_{1}}{6}
$$

$\mathrm{X}_{\mathrm{O}}, \mathrm{X}_{\frac{1}{2}}$, and $\mathrm{X}_{1}$ are the airmasses at the beginning, mid-way, and end of the exposure (calculated using Equation 3.14).

Regardless of the exposure length, the Heliocentric Julian Date of observation for the midpoint of the exposure is calculated using Hjd_jd.

Once the header has been read in, the actual CCD Image data is read into a 384 by 576 array called 'image'. A smaller-sized CCD Image would occupy the lower portion of 'image', with its dimensions defined by the appropriate keywords from the header. The user is then prompted for how many rows are to be output. An input of ' 0 ' indicates that all of the Image is to be output. This choice is selected under the automatic print option. The maximum, minimum, mean, and standard deviation of the pixel intensities are calculated. These values are used to calculate the range in intensity represented by each of the 32 available grey-scale levels (tiles). The program offers a choice of three grey-scale techniques:

- Linear, where 32 shadings are equally spaced between user-specified minimum and maximum intensities, e.g. if the minimum intensity was 100 counts in a pixel, and the maximum intensity was 419 , then each shade (or tile) would represent a range of 10 counts. The first tile would cover $100-109$, the second $110-119$, and so on. The user can specify the minimum and maximum intensities for this technique and the next method.

- Power-law mapping. The maximum intensity represented by a tile is given by:

$$
\text { minimum }+(\text { maximum }- \text { minimum })\left(\frac{8 n-1}{256}\right)^{\frac{1}{\beta}}
$$


where $\beta$ is input by the user, maximum is the maximum intensity to be represented by any tile, minimum is the minimum intensity, and $n$ is from 1 to 32 in steps of one. The numerator is of such a form to prevent the value 256 being formed. Power-law mapping causes the intensity range covered by each subsequent tile to uniformly either increase or decrease. Detail is accentuated near the maximum or minimum intensity values, depending on the sign of $\beta$.

- Pixel Area scaling. The intensity range of each tile is specified by the number of pixels it is permitted to represent, i.e., in the special case of uniform areas each tile will represent the same number of pixels:

$$
x=\frac{576 \times 384}{32} \text { pixels. }
$$

When the pixels are sorted into an order of increasing intensity, the first tile would represent the first $x$ pixels. Its intensity range would be from the intensity of the first pixel up to that of pixel 6,912 . The second pixel would represent the pixels 6,913 to 13,824 , and so on.

In the general case, the number of pixels per tile is given by:

$$
\frac{1-x^{\mathrm{n}}}{1-x^{32}}
$$

for $\mathrm{n}=1$ to 32 . The choice of $x$ can bias the tiling towards either extreme. Values of $x$ greater than 1 will put more white into a positive Image, and vice versa for a negative Image.

An integer array represents all the possible intensity values, ordered in a numerically increasing sequence. The values in the array are initialised to zero. A value in the array is incremented for every pixel of a given intensity. To allocate the intensity ranges for each tile, the program begins at the lowest value in the array. The number of pixels (and hence the intensity range) that should be allocated to the tile is calculated, and the program stepped along the array until the cumulative pixel count is equal to or greater than the required number of pixels. The cumulative count is reset, the range of the next tile calculated, and the process continued for the remaining tiles in turn. This method avoids the problem of more than one tile being assigned to a single intensity.

The user is prompted for which type of grey-scale is to be used, and whether the Image is positive or negative (if the automatic option was not selected at the start of the program). If the automatic print option was selected and if the object is a calibration frame then the output Image will be positive, otherwise the printed Image will be negative. The automatic option uses the technique of pixel-area scaling with $x=1$ if the Image is to be positive, and $x=0.95$ 


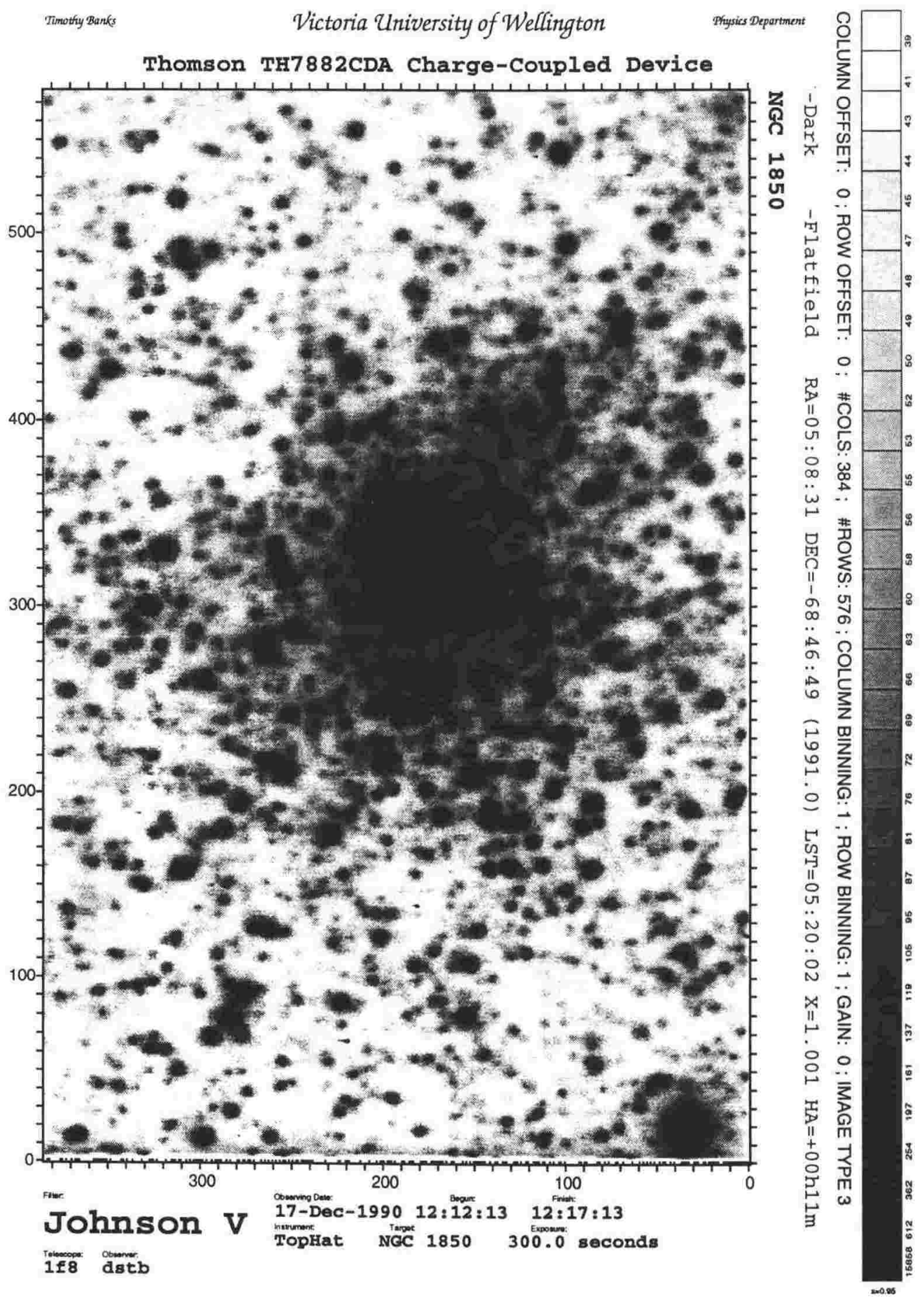

Figure 3-4: Example Output from PTM_HARD showing a Johnson V Image of the cluster NGC 1850. Note the unusual 'limb' extending from the upper right of the main body of the cluster. 
otherwise. Depending on which grey-scale scheme was chosen, the appropriate 32 shades are selected and the necessary parameters prompted for.

The output of the PostScript program to a file is commenced. When this file is submitted to a PostScript printer, it will be run as an interpreted program and produce the Image as output. Statements generating a series of 32 boxes indicating the selected grey-scale are then written to the file. The underlying idea in this section of PTM_HARD is to check variables read in from the header of the input file, and write to the output file this information as appropriate statements that make up a suitable PostScript program. Hence, the instrument type can be checked, and the appropriate lines (with variables) written out. After the basic details such as object name, coordinates, airmass, etc. are written, the necessary code to handle the CCD Image is put in the file, followed by the data itself which is treated as a PostScript "image". The actual values written for each pixel depend on which grey-scale type was selected. Pixels are processed one at a time by the appropriate grey-scale function in an order proceeding along rows and then columns. The entire Image is then written to the file, row by row. Closing PostScript commands (such as showpage) are written to the output file, all files used by the program closed, and the program terminated. Figure 3-4 is an example of the output of PTM_HARD.

\subsubsection{Program CCD_TO_V}

This program reads the MJUO PM-3000 data files from a 9-track 1600 BPI magnetic tape. It can output a tape listing, FITS format Image files, Photometrics format Image files, or both file types onto a VMS VAX. The code (like PTM_HARD) has been formatted, internally commented, and rewritten in various areas.

As soon as the program is started it obtains the logical unit number of the tape. The number of files on the tape is determined from the tape header. The program uses the letter $\mathrm{V}$ (for VUW) as the first character for all the output files. The user is prompted for three characters which will be written to the second, third, and fourth characters of the output file names, e.g. if 'Dec' was entered the log-file would be called vdec.log. Log-files provide a record of which tape files were retrieved by the program as well as observational details about the Images. The output FITS files would be called Vdec_nnn.MT, where nnn is how many files into the tape the input file was. Blanks are filled with zeros, e.g. Vdec_009.MT would be the name for a FITS file converted from the ninth CCD Image file on a tape.

Next, the user is prompted for what type of output file they want. The log-file is then opened and general information about the transfer (such as when it was) written to the file. Further details, such as if FITS files are to be written, are also recorded in the log. The user is then asked which individual files are to be read from the tape. Files are designated by their relative position along the tape for this choice. An input of 0 indicates that all the tape is 
to be read. Only 5 lines, of eighty characters length each, are available for input, with no embedded spaces being allowed. Commas and dashes (e.g. 12-25 or 1,5,6,7-10) are allowed, and the numbers must be in ascending order. The input is then checked to ensure that these conditions are obeyed, and the file numbers to be read are stored in an array.

A loop is commenced, which will repeat as many times as there are selected files to read. If all the tape was selected, the maximum number of files that can be read is $999 .^{1}$ The tape is wound on to the start of the first selected file. The data read loop is broken if the subroutine $M T A C C E S S$, which reads the tape records, returns with the 'end of tape' flag indicator set. If the read fails, it is repeatedly tried again. A successful read leads to the opening of the appropriate files (FITS or Photometric format) with suitably named file extensions such as MT for the FITS files, and PTM for the Photometric format files. The header is then read and processed in an identical manner to PTM_HARD.

The tape header is directly copied into the first 768 bytes of the Photometrics file, assuming that this output option was selected. The header of the FITS output file is somewhat more complicated, being constructed of 80 character length lines containing keywords and their values (see Appendix A for details on the FITS format). A 80 by 36 array is filled with the appropriate keyword pairs. If the object is not a calibration, then its epoch, airmass, and coordinates are written together with the other keyword pairs. After the common keywords, those specific to a given instrument are inserted. Subroutine dofitshdr ensures that once 36 lines are filled, they are written to the FITS output file and the array blanked out in preparation for the next block. Once all the keywords have been paired with the appropriate values, the array storing the FITS header is then written to disk, if it hasn't just been. This is to handle tape headers with lengths that are not multiples of 36 .

The pixel data is read in from the tape (unless only a tape inventory is required, in which case this step is skipped). The output buffers for each format type are cleared, and the 768 byte records read up from tape one by one. In each iteration the complete record is written to the Photometrics format file, while 2880 byte records are assembled and written to the FITS output file. Once the end of file indicator is returned by MTACCESS, the read loop terminates, and the final record of each output format is filled with the last data values, and padded with zeros to maintain the fixed formats. The Image output files are closed. A check is made to see if there are any more tape files to be read up. If so, the program branches back to the start of the loop for reading tape files.

Once all the selected tape files have been read, all the files (including the tape) are closed,

\footnotetext{
${ }^{1}$ This limit will not be reached given current tape lengths and the fact that only a density of 1600 BPI is possible with the MJUO tape unit. The $\sim 1200$ foot long 9-track tapes sometimes used by observers should ideally contain $\sim 23 \mathrm{Mb}$. Practical experience has shown that these tapes only store $15.5 \mathrm{Mb}$, which corresponds to 35 full frame files (West 1991). Substantial inter-record gaps appear to be the cause of this discrepancy.
} 


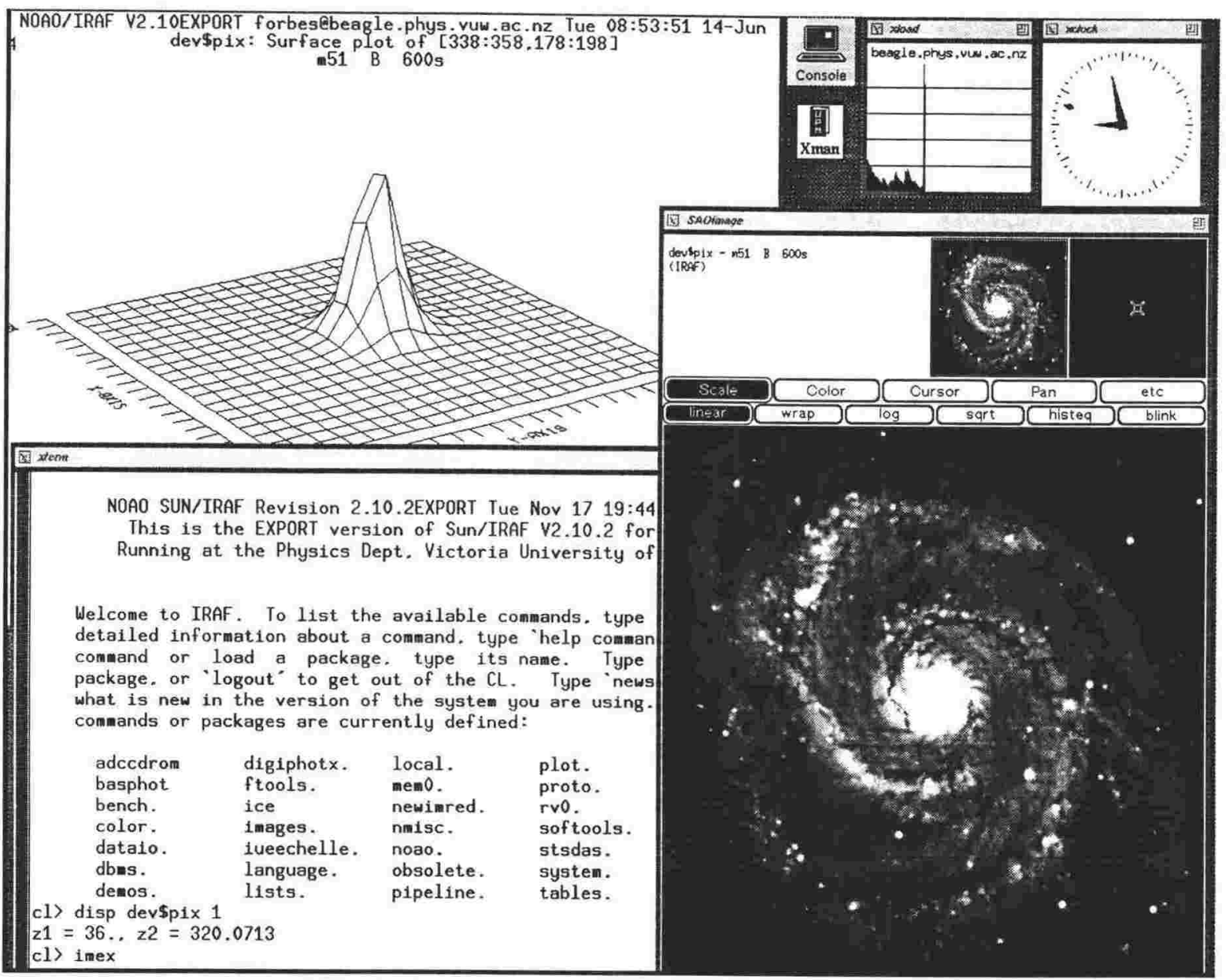

Figure 3-5: An Example IRAF Display showing the Command, Image Display, and Graphics windows open. The IRAF test Image of the spiral galaxy M51 is shown in the 'SAOImage' Display, while a three dimensional representation of a star is displayed in the Graphics Window. Intensity is plotted against the $\mathrm{x}$ and $\mathrm{y}$ axes of a sub-array in the CCD frame.

and the program ends. The FITS files are now ready to be read up by IRAF, using its data input/output package dataio, for subsequent analysis.

\subsection{IRAF}

The Image Reduction and Analysis Facility (IRAF) is a general purpose software system for the reduction and analysis of astronomical data. It provides a wide range of programs for general Image processing and graphics applications, as well as a large selection of specialised programs for the reduction and analysis of astronomical data. IRAF is intended to provide a complete scientific programming environment that is as portable and device independent as possible, allowing the use of the system on a wide range of host computers and operating systems with a large variety of supported display devices.

The IRAF project commenced at Kitt Peak National Observatory in 1981. Since then, a 
programming team has continuously developed IRAF into a very thorough environment, as well as providing excellent user support. This has led to the widespread popularity of IRAF. The system is extremely well documented, with manuals ranging in level from those aimed at novice users through to advice on how to port IRAF across to an unsupported computing platform (see Appendix B). These factors, combined with the facts that IRAF contained CCD reduction packages essential to the project (such as DAOphot, Stetson 1987), and that the software and documentation were easily available across the international computer networks, led to the adoption of the system at VUW.

\subsubsection{Major Components}

IRAF was designed to provide a convenient, efficient and portable analysis system primarily for Images (see Figure 3-5 for an example of an IRAF Display). In particular the system is designed to handle astronomical Images, but it has general facilities which can be applied to many other types of data - such as plotting data and statistical analysis. IRAF was designed as an open system which can easily be extended to add new analysis capabilities or be customised for specific applications by a user.

There are three major components of the IRAF system. The first is the Command Language (CL) which is the user interface used to run the system utilities and application programs. Both this software and the CL depend on the facilities provided by the Virtual Operating System (VOS) to function. The VOS depends on the kernel, or the run-time component of the Host System Interface (HSI), for all communications with the operating system of the host computer. All software above the HSI is completely portable, and may be transferred to any IRAF host. IRAF looks and behaves the same on many different systems, with only the sections that interface directly with the operating system of the host computer being different. Currently supported operating systems include various flavours of UNIX (Sun, Ultrix, HPUX, and AUX), AOS V/S, and VMS 5.

The $\mathrm{CL}$ is designed to serve both as a command language and as an interpreted programming language via scripts. The basic IRAF command syntax is the same as the concise format of the UNIX C-shell, with similar input/output (I/O) redirection and pipe facilities, extended to provide support for the graphic streams. Other major features of the CL are:

- Package Structure for software organisation. A package is a collection of logically related tasks, which is loaded by entering its name into the CL. Loaded packages are organised as a linear list, with the order being that each package was loaded in. Task name references are resolved by a circular search of this list, beginning with the most recently loaded package. If there is an ambiguity with more than one task sharing the name (or the abbreviation used), the user is notified of the competing task names. Either an unam- 
biguous abbreviation or the use of the appropriate package name as a prefix will remove the ambiguity.

- Hidden user-accessible menus for both utilities and applications.

- Extensive online help, including interactive tutorials.

- Parameter prompting and defaults.

- Minimum match abbreviations for utility and application task names.

- Direct access to host system commands.

- Background job submission, queuing, and control.

- UNIX-style virtual filename and directory structure.

- Graphics and Image display cursor facilities, providing a built-in graphics or Image control capability that can be operated whenever a cursor is read by an applications program, without the need to exit that application.

- A history mechanism allowing previous commands to be re-used or edited.

- Maintenance of a 4-slot process cache of connected but idle subprocesses, reducing the process spawn overhead. The first slot always contains system utilities, while the remaining three are dynamically assigned according to the actions of the user.

All user interaction with IRAF is through the CL. The CL executes concurrently with any application processes, responding to parameter requests from them and managing I/O. In essence, the CL and the application are one program, except that binding occurs at the process connect time rather than during a link. This allows interactive and sophisticated user interfaces, without the need to link large amounts of code into an executable. New tasks and packages can be easily added to the CL at any time, using the same mechanisms used for the standard IRAF tasks and packages. No change has to be made to the standard system.

While IRAF provides a wide range of software, it is unrealistic to expect it to provide everything a project might need. A useful system should make it easy for users to add, or modify, software. IRAF provides three programming environments ${ }^{1}$, tailored to particular software and the experience of the programmer. The first is the CL, where the programming language for scripts is the command language, and the environment is the packages and tasks callable by the CL. The second is IMFORT (IMage FORTran), where IRAF provides libraries giving the FORTRAN compiler of the host system access to the CL, cursors, and Images. The third is

\footnotetext{
${ }^{1}$ The term programming environment refers to the languages, $\mathrm{I} / \mathrm{O}$ libraries, software tools, etc. making an environment for programming
} 
defined by the VOS, and was used to implement most of the IRAF system and application software using the SPP (Subset PreProcessor) language.

In addition to the provision of the programming environments, the VOS performs the standard facilities expected of conventional operating systems, such as file I/O, dynamic memory allocation, terminal control, and exception handling. Most of the capabilities of the VOS are present in already existing operating systems or libraries. The IRAF programming team decided to invest the considerable effort in writing the VOS, rather than use these existing solutions, due to concerns about the reliability, completeness, and portability of these solutions (Tody 1986). It is hard to produce a self-consistent, efficient, reliable, well structured and understood system by patching together independently developed systems. The VOS has all these features, and is tailor-made for IRAF's tasks. At some sites users are logged directly into the CL, and never see the local operating system, using IRAF's facilities completely. The team considered portability to be an important feature, rather than forcing potential users to adopt a given computer, operating system version, and compiler version. Porting IRAF across to other hosts is much easier with the VOS, as the HSI is small, well-isolated, and well-defined. Through the VOS, IRAF is also device independent. Various text files contain device capabilities, allowing virtually any device to be interfaced to without the need to modify code or relink executables. IRAF references these files to determine the capabilities of a given device, and then acts within the specified limits for it.

The HSI is made up of several sections:

- The Kernel, which is a subroutine library containing all the host-dependent primitive functions. It is the most machine-dependent part of the HSI, and its conversion is the major portion of a port attempt.

- Bootstrap Utilities required to compile and maintain IRAF. These are fairly portable, being written in C. These include tools to strip binaries from packages and a tar archive handler.

- The Hlib library, which is a directory containing various host and site-dependent look-up tables.

- The AS assembler library module.

- The Gdev directories which contain the host-dependent I/O interfaces for binary graphics devices otherwise supported by the VOS machine-independent kernels.

Only those few attempting ports need to be aware of the HSI and its structure, especially given that the IRAF Support team provides binaries for a large range of platforms, making installation straight-forward. 


\subsubsection{Implementation}

The VUW installation of IRAF has evolved over the three years the system has been installed. Initially IRAF was installed on only one Sparc Station 1. Banks (1993) describes the system as it was in mid-1992, when IRAF version 2.9 was running under Sunview SunOS Release 4.1. By late 1993 the system had ceased to evolve.

A gigabyte disk on the VUW Silicon Graphics 4D/380S Parallel Processor contains both the IRIX and Sun IRAF v 2.10.2 binaries, together with many of the add-on packages such as STSDAS $^{1}$ and $\mathrm{X}$-windows support. This software was retrieved across the Internet by use of the Anonymous File Transfer Protocol (for usage instructions see Banks \& Jansen (1992), for example), built, and maintained by the author as the IRAF site manager. The Sun version of IRAF is NFS ${ }^{2}$-mounted across to three Sparc Station 1 computers and a Sparc Station 10, with this disk appearing as if it is local to the machines it is exported to. The IRIX binaries are used by the Parallel Processor itself, as well as being exported to a Silicon Graphics Indigo. IRAF contains support for network access to any physical resource resident on a remote node running IRAF, although the node must be explicitly identified. This facility is provided by the VOS, so the nodes do not have to be architecturally similar. Networking between all six IRAF nodes was implemented. IRAF stores CCD Images as two files - a modified FITS header and a byte stream containing the pixel data. These files need not be in the same directory, and it is usual to store the large pixel files elsewhere on a disk reserved for the purpose. One Sparc 1 NFS-exports to the other machines a 300 megabyte disk for the storage of the pixel data, allowing the same CCD Image to be transparently manipulated from any node. Each installation of IRAF was tuned for the host machines and peripherals, such as printers, tape drives, and graphics devices. X-terms are the preferred interactive devices by users, although the host consoles, Visual 500 graphic terminals, VT 100s (usually modem emulations), and Visual 220 terminals are available.

\footnotetext{
${ }^{1}$ Space Telescope Science Data Analysis System, courtesy of the Space Telescope Science Institute, Baltimore.

${ }^{2}$ Network Filing System
} 


\section{Chapter 4}

\section{Photometry}

his chapter discusses the reduction techniques used by this project to analyse CCD
frames, and in particular the crowded field photometry package DAOphot (Stetson 1987).

\subsection{Aperture Photometry}

mall holes, or apertures, are used in photoelectric photometry to isolate a star from its
surroundings. Not all the light of the star can pass through this aperture ${ }^{1}$, but the same
fraction of light is lost for all the observed stars given the same seeing. Typically a range of
apertures is available. The choice of which aperture to use on a given night primarily depends
on the seeing, although the program may require a variety of different apertures to be used on
an object, e.g. for intensity profiles. Consideration will also have to be given to whether the
target stars are in a crowded region, such as a star cluster, or not. A smaller aperture will be
required for photometry in a crowded cluster, as well as a night with good seeing. However,
the chosen aperture must not be too small since there will be problems:

- Initially lining the telescope and photometer up on the star.

- Keeping the telescope aligned on the star, given that the tracking of the telescope will not be perfect.

- Stars at different airmasses will have different profiles due to the different levels of seeing. This could become important with extinction or standard stars observed at high airmasses.

While accurate tracking is required for long CCD exposures, the other two concerns are not a problem given the extended field of view of a CCD. A smaller aperture can therefore be used for the photometry of stars in CCD frames than would be applicable in photoelectric photometry.

\footnotetext{
${ }^{1}$ King (1971) noted that for his photographic work the intensity profile of a star extended arcminutes in radius before the background values were reached.
} 
Aperture photometry is simply the integration of the light received by a detector, or array of detectors, from a solid angle of sky which includes the object of interest. In photoelectric photometry, the amount of light received from the background in a given solid angle is estimated by observing a nearby region of sky 'free' of stars. The difference between the two observations is taken to be the contribution from the observed object. While it is possible to follow the same procedure in synthetic aperture photometry of CCD frames, as is often done for the reduction of standard star frames, it would be very time consuming when many star images were visible on a frame. Hence there is a need for a computer program which can automatically perform photometric measurements on a frame of a crowded field. Such a program is discussed in Section 4.3 .

The derivation of the magnitude of a star in a frame involves:

- Determination of where the centre of the star image is,

- Estimation of the sky background level, and

- Summation of the light within a given aperture, and removal of the background.

These steps are discussed in turn.

\subsubsection{Centres}

Many methods for estimating the centre positions of stellar images have been developed for astrometry ${ }^{1}$. Two simple techniques are widely used in CCD photometry. These are the 'marginal sum' and 'image centroid' methods.

The first technique simply performs the following steps on a stellar image:

1. An initial guess is made of where the centre of the stellar image is. Often this estimate is made by the user, although the IRAF task Daofind will calculate estimates automatically. Daofind is described in Section 4.3.1.

2. A sub-array of arbitrary size is taken around this estimate of the centre. This sub-array must extend far enough that the background is reached. A reasonable axis length would be $\sim 5$ times the Full Width Half Maximum (FWHM) of the stellar image.

3. The marginal sums along each axis:

$$
\Upsilon\left(x_{i}\right)=\sum_{j=1}^{m} I_{i j} \text {, and } \Upsilon\left(y_{j}\right)=\sum_{i=1}^{n} I_{i j}
$$

\footnotetext{
${ }^{1}$ The study of accurate celestial positions.
} 
are determined. $I_{i j}$ is the intensity of pixel $(x=i, y=j)$ in a sub-array of $x=n, y=m$ extent.

4. The image centre is estimated by fitting a one-dimensional function, such as a Gaussian, to each of the marginal distributions.

This method will give an acceptable estimate provided that the stars in the frame are well separated from each other. If the effect of neighbour stars is present in the sub-array then the derived centre will be skewed, and a more complicated method of estimating the central position of an image should be used. One example of a more robust technique is the "centroid method', which uses the following algorithm:

1. Estimate the centre $(x, y)$ of the image, perhaps using marginal sums.

2. Using this centre, calculate the $\mathrm{x}$ and $\mathrm{y}$ axis marginal sums over a square sub-array of dimension 2a:

$$
\Upsilon\left(x_{i}\right)=\sum_{j=-a}^{a} I_{i j} \text {, and } \Upsilon\left(y_{j}\right)=\sum_{i=-a}^{a} I_{i j} .
$$

The value of ' $a$ ' should be similar to the FWHM of the image.

3. Calculate the mean marginal intensities:

$$
\overline{\mathrm{I}}_{\mathrm{x}}=\frac{1}{2 \mathrm{a}+1} \sum_{\mathrm{i}=-\mathrm{a}}^{\mathrm{a}} \Upsilon\left(\mathrm{x}_{\mathrm{i}}\right) \text {, and } \overline{\mathrm{I}}_{\mathrm{y}}=\frac{1}{2 \mathrm{a}+1} \sum_{j=-\mathrm{a}}^{\mathrm{a}} \Upsilon\left(\mathrm{y}_{\mathrm{i}}\right)
$$

4. Fit a one-dimensional function to each marginal distribution, using only the points with an intensity above the mean. These points will have good signal levels. A new estimate for the centre of the stellar image is made in this step.

5. If the new estimate for the centre does not fall within an arbitrary distance (usually one pixel) from the immediately previous estimate, then loop back to the second step until this condition is satisfied or the maximum number of permissible iterations is reached. In the latter case the fit should be rejected.

If the field is even more crowded then one of the various point-spread function (PSF) profile fitting techniques should be used. These methods are discussed in Section 4.3 beginning on page 91 .

\subsubsection{Background}

A photometric measurement of a star is the sum of the apparent magnitude of the star and a background signal. This background is itself a sum, being the product of many factors including: 
- Air glow - which is light released by chemical reactions in the atmosphere.

- Zodiacal Light - sunlight scattered off minute particles concentrated into a belt along the ecliptic.

- Light Pollution. This can be both natural (e.g. the Moon) or artificial (e.g. Street Lights).

- Aurorae.

- Scattered light inside the CCD camera.

- Faint stars and galaxies

An annulus about the image centre is often used to determine the background signal. This is not possible with objects such as comets, where specialist packages such as Basphot ${ }^{1}$ should be used. These packages allow a background estimate to made in several uncrowded regions of the frame. However, care needs to be taken to account for any gradients in the background signal across the frame. The use of an annulus about a stellar image cancels out any linear trends in the background, explaining the popularity of this method. This annulus should have an inner radius of at least 5 times the FWHM of the image, reducing the contribution of the star itself to the background estimate. The width of the annulus should be large enough that the annulus contains a sizeable number of pixels, of the order of at least a few hundred. This will ensure that the statistical error in the background estimate is small. The background is typically estimated by the mode ${ }^{2}$ of the annulus, since this represents the most probable value of a pixel chosen randomly out of this region (Stetson 1987). Outliers skew the mean and median more than the mode. Often pixels with intensities greater than 2.5 or 3 standard deviations from the mean are excluded from the pixel list, and then the mode calculated from this trimmed distribution (Stetson 1987). This can even be an iterative process, looping until the background estimated in one iteration is different by less than a user-defined limit from the background value estimated in the previous iteration.

\subsubsection{Summing the Light}

Once the image centre and background are determined, the intensities of the individual pixels falling within an arbitrary radius of the centre are summed together. The background intensity is scaled by the number of pixels in the aperture $\left(\mathrm{N}_{\mathrm{pix}}\right)$, and subtracted from the aperture intensity to give the signal from the star alone, i.e.:

$$
\text { Magnitude }=\text { zero point }-2.5 \log \left(\sum \mathrm{I}_{\mathrm{ij}}-\left(\mathrm{N}_{\mathrm{pix}} \mathrm{I}_{\text {sky }}\right)\right)
$$

\footnotetext{
${ }^{1}$ Courtesy of Dr. Marc Buie, Lowell Observatory.

${ }^{2}$ mode $=(3 \times$ median $)-(2 \times$ mean $)$.
} 
for all $(i, j)$ inside the aperture. $I_{\text {sky }}$ is the (mode) background sky value per pixel, and $I_{i j}$ is the intensity of a pixel falling within the aperture.

Pixels have a finite size. This fact becomes important with apertures that are relatively small compared to the pixel size, e.g. a radius of a dozen pixels. In such cases the inclusion, or exclusion, of individual pixels can have a substantial effect on the summed intensity. The aperture is an irregular polygon which is not a good approximation to a circular aperture. The method of partial pixels is widely adopted to counter this problem. A boundary pixel is divided up into sub-pixels, often in a 10 by 10 grid where each sub-pixel is assumed to contain $1 \%$ of the total intensity detected by the pixel. Each of the sub-pixels is checked in turn to see if it falls within the aperture. If so, its 'intensity' is included in the aperture summation. This technique is used by the aperture photometry section of DAOphot (Stetson 1987). The program checks the radius $\left(\mathrm{r}_{\mathrm{ij}}\right)$ a given pixel is from the image centre. If ${ }^{1}$

- $\mathrm{r}_{\mathrm{ij}}<\mathrm{R}-0.5$, where $\mathrm{R}$ is the aperture radius, then the pixel is completely within the aperture. Its full intensity can be added to the aperture intensity.

- $\mathrm{r}_{\mathrm{ij}}>\mathrm{R}+0.5$, then the pixel is completely outside the aperture, and contributes no light to it.

- $\mathrm{R}+0.5>\mathrm{r}_{\mathrm{ij}}>\mathrm{R}-0.5$, then the pixel is on the boundary, and the method of partial pixels is used to estimate its contribution to the aperture.

\subsection{Aperture Corrections}

The appropriate radius of an aperture partly depends on the FWHM of the stellar images in a frame. Consider an aperture in which there is a total measured intensity I. If the gain of the CCD system is D electrons per ADU, then the total number of photo-electrons inside the aperture is I x D. Given that photons follow a Poisson distribution together with a readout noise $r$ (in electrons), the magnitude error is:

$$
\delta \mathrm{m}_{1}=\frac{1.09 \sqrt{\mathrm{ID}+\mathrm{r}^{2} \mathrm{n}}}{\mathrm{ID}}
$$

where $\mathrm{n}$ is the number of pixels in the aperture. As the radius of an aperture increases, the number of pixels inside it must increase as well, which in turn increases the contribution of the readout noise. In most cases the total noise is not dominated by the readout component. In these cases, the intensity increases with aperture radius, and so the magnitude error decreases. At the outer limits of an image, the fractional increase in intensity will not offset the increase

\footnotetext{
${ }^{1}$ Rotation has been ignored to simplify this explanation.
} 


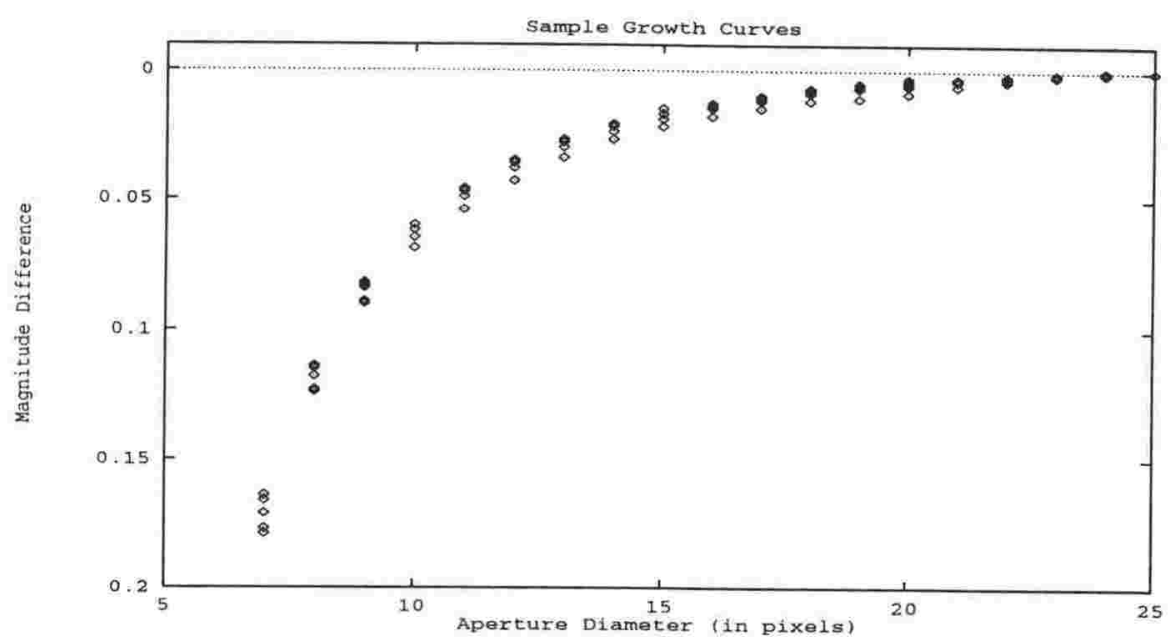

Figure 4-1: An Example Growth Curve. The differential magnitudes for some standard stars are plotted against the diameters of the various apertures. The diameters are measured in pixels. In this example the magnitudes are all relative to the magnitude of the largest aperture, although it is more usual to use the mean value of the outermost apertures. See Stetson (1990) for a discussion of the automatic derivation of growth curves from an entire night or observing run using the program DAOGROW.

in readout noise from the extra pixels. Therefore, it would seem sensible to find this boundary radius, and use that as the aperture radius. However, there is another component to the noise, being due to the sky background:

$$
\delta \mathrm{m}_{2}=\frac{1.09}{\mathrm{I}} \sqrt{\frac{\mathrm{nI}_{\mathrm{sky}}}{\mathrm{D}}}
$$

which increases as the aperture radius becomes larger. This component is clearly minimized for small apertures. Thus if both $\delta \mathrm{m}_{1}$ and $\delta \mathrm{m}_{2}$ are considered, it can be seen that there are two limiting cases:

- Stars that are bright in a given frame, having ID $\gg n\left(\mathrm{r}^{2}+\mathrm{DI}_{\text {sky }}\right)$, would be best measured using a large aperture.

- Faint stars where ID $\ll n\left(r^{2}+D_{\text {sky }}\right)$. Small apertures are preferable in this case.

Hence, the appropriate aperture in a given frame will depend on the apparent brightness of a star. Standard stars tend to have bright images as the observer would want to collect as much signal as possible for these calibration objects. The target stars may not be so bright, or in the case of crowded fields, be measured with a small aperture in an attempt to exclude neighbours. The magnitude determined for a star will depend on its aperture. Given that the apertures used for photometry of different sets of stars can be different diameters, there is a need for 'corrections' to bring all the measured magnitudes onto the same scale. 
The basis for such 'aperture corrections' lies in the fact that the two-dimensional intensity distribution (the PSF) produced by a point source on the plane of a detector, such as photographic plate or a CCD, will be the same for all stars regardless of their observed intensity. This assumes that there are no optical distortions in the equipment used to acquire the Image. Therefore, the images of bright and faint stars only differ in intensity and not in form. The fraction of light excluded by a given aperture will be the same regardless of the intensity of the image being measured. While faint stars should be measured with a small aperture, bright stars can be measured using both a large aperture for photometric accuracy and the small aperture. The difference in the magnitudes estimated using the two apertures will be the same for the fainter stars, and so the small aperture magnitudes can be 'corrected' across to the same scale as the bright stars (see Figure 4-1).

\subsection{Profile Fitting}

Many interesting fields are too crowded for standard aperture photometry. Such fields include the LMC star clusters. An appropriate technique for such frames is PSF photometry. Some PSF fitting programs, such as STARMAN (Penny \& Dickens 1986) and DoPHOT (Schecter et al. 1993, Mateo \& Schecter 1989), fit an analytic PSF. Typically a Gaussian, Modified Lorentzian, or a Moffat function is used (for expressions of these functions see Da Costa 1991b). The advantage of this method is that an analytic PSF can be integrated numerically over each pixel in a stellar image, avoiding problems with the size of the pixels relative to the dimensions of the PSF itself. The major disadvantage of this technique is that the chosen model profile may not be a good representation of the observational data, particularly if the real PSF is not symmetric. The integrations themselves are also time consuming to perform.

These problems have led to other software being written which construct empirical PSFs from the data. VISTA (Lauer 1983) and WOLF (Lupton \& Gunn 1986) are examples of this type. These programs make no assumption about the shape of the actual PSF. Interpolation of the PSF is much faster than the evaluation of the two-dimensional numerical integrals used in the analytic functions. However, the construction of an empirical PSF from several image profiles requires the accurate superposition of each profile to avoid broadening the global (model) PSF. Each stellar image is defined on the pixel grid. Interpolation of the PSF to another set of coordinates will introduce difficulties, particularly when the PSF radius is small and the images are nearly critically sampled. The resulting interpolation may not adequately represent the actual profile.

Neither of these methods is ideal. DAOphot (Stetson 1987) combines the two techniques in an attempt to gain the benefits of both. The PSF is modeled using two components: 
- A bivariate Gaussian, which is the basis of the profile. The Gaussian typically fits the central regions of the observed PSF well. It serves as a first-order approximation to the PSF.

- An array is formed which contains the residuals of the actual PSF to the Gaussian. A look-up table is calculated from these residuals. The table is centred on the centroid of the stellar image, with the look-up corrections being tabulated at half pixel intervals via bivariate cubic interpolation.

The profiles of several stars are combined together to define the model PSF, which will be used to reduce the frame. The signal to noise ratio of the model will be improved through a judicious choice of several stellar images. As each star is added, the current PSF is shifted and scaled to a best fit with the stellar image. The scaled Gaussian is then subtracted from the image, producing an array of residual intensities. These residuals are then interpolated to half pixel intervals, scaled, and incorporated into the look-up table.

Hence an analytic function represents the large-scale variations, making for more accurate interpolations than the empirical methods since the residuals tend to be small. This study used DAOphot for the photometry of crowded fields.

\subsubsection{Method}

While CCD aperture photometry is fairly simple, being very similar to the procedures of photoelectric photometry at a telescope, the reduction of crowded fields is more complicated. The basic steps are:

1. Estimate the initial positions of the stars in the frame. This task involves locating small, positive enhancements in a frame, and distinguishing those due to stars from other features such as random noise peaks, galaxies, nebulosity, cosmetic defects of the CCD, and cosmic ray strikes.

The DAOphot task Daofind performs this search, once given the frame and several parameters:

(a) The typical FWHM for the frame. This is estimated as the mean FWHM of the available bright and isolated stars in the frame.

(b) The readout noise of the CCD (in ADU)

(c) The number of photo-electrons per ADU.

(d) The signal (in ADU) above which the CCD no longer behaves linearly. Data above this value will be ignored. 
(e) The approximate level of signal due to the diffuse sky background.

Daofind then steps through the frame, pixel by pixel, calculating how bright a star would be if it was centred at each pixel, i.e., a bivariate Gaussian is fitted to the intensities in a sub-array about each pixel in turn. If a star is centred on the pixel being examined, then the fit of the analytic function to the data will be good. The brightness of the star will be proportional to the central height of the fitted Gaussian. If a star is not centred on the pixel, with the pixel being either empty sky or in the wings of a stellar image, then the central height will be zero or even negative.

In more detail, the process is the following. The unit height bivariate Gaussian is given by:

$$
\mathrm{G}(\Delta \mathrm{i}, \Delta \mathrm{j}, \sigma)=\mathrm{e}^{-\frac{\Delta \mathrm{i}^{2}+\Delta \mathrm{j}^{2}}{2 \sigma^{2}}}
$$

and the intensity of a pixel $(i, j)$ is given as $D_{i j}$. The central brightness of a Gaussian which best fits the pixel intensities about a point $\left(\mathrm{i}_{\mathrm{o}}, \mathrm{j}_{\mathrm{o}}\right)$ is $\mathrm{H}_{\mathrm{i}_{o}, \mathrm{j}_{\mathrm{o}}}$, and the local background intensity is b. $\mathrm{D}_{\mathrm{ij}}$ can be used as the 'solution' of a least-squares fit to the equation:

$$
\mathrm{H}_{\mathrm{i}_{o}, \mathrm{j}_{\circ}} \mathrm{G}\left(\mathrm{i}-\mathrm{i}_{\circ}, \mathrm{j}-\mathrm{j}_{\circ}, \sigma\right)+\mathrm{b}
$$

for pixels $(i, j)$ in a sub-array about, and including, $\left(i_{o}, j_{0}\right)$. $H_{i_{o}, j_{\circ}}$ is given by a linear least-squares fit to:

$$
\frac{\sum\left(G_{i j} D_{i j}\right)-\frac{\left(\sum G_{i j}\right)\left(\sum D_{i j}\right)}{N}}{\sum G_{i j}^{2}-\frac{\left(\sum G_{i j}\right)^{2}}{N}}
$$

where $\mathrm{N}$ is the number of pixels in the sub-array. The position $i_{o}, j_{\circ}$ is varied across all the pixels in the frame, with the resulting values of $H$ stored in an array. This array is slightly smaller than the original pixel array as a margin equal to the fitting radius for $\mathrm{H}$ is left about the perimeter of the frame. The reason for this is given below. Figure 4-2 demonstrates the procedure for the simplified case of a one-dimensional intensity distribution.

The advantages of searching for local maxima in $\mathrm{H}$, rather than in $\mathrm{D}$, are:

- The search is optimised for stellar images. The FWHM defines the value of $\sigma$ used in the fitting function, as is the extent of the sub-array that is being fitted. Objects with radii substantially different from $\sigma$ will have smaller values of $H$ than stars.

- The sky background is not a factor in $\mathrm{H}$, as $\mathrm{H}$ is an estimate of the central brightness of a 'star' above the local background. Since the Gaussian is symmetric, any slope in the sky background cancels out in $\mathrm{H}$.

- Peaks tend to be narrower, which helps to de-blend some double stars. 

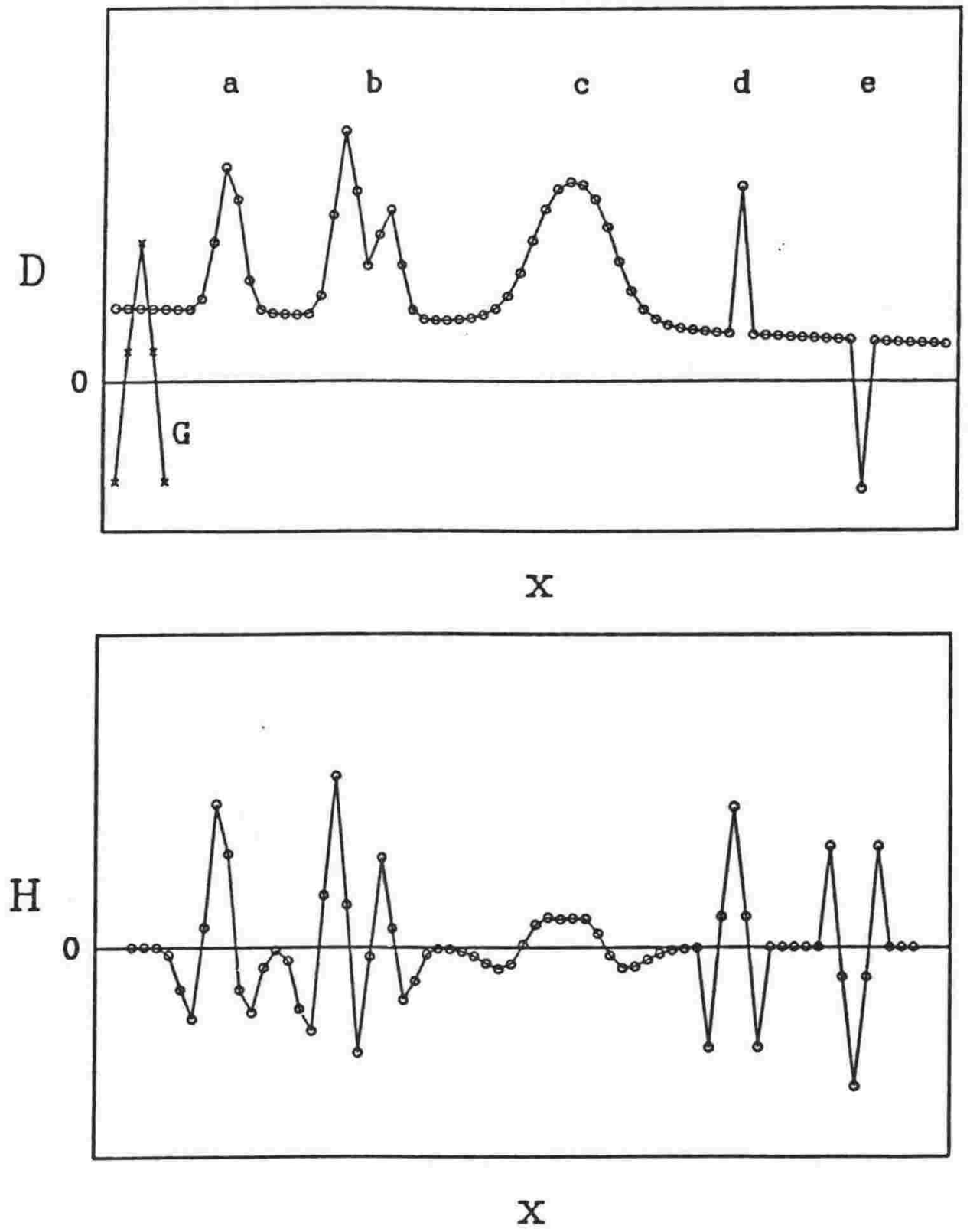

Figure 4-2: One Dimensional Profiles of Astronomical Objects and Erroneous Events. Intensity profile (a) is that of a star, (b) is a blended pair of images, (c) is a barely resolved galaxy, (d) a cosmic ray strike, and (e) is a bad pixel. G is the lowered and truncated Gaussian with which the intensity profiles are 'convolved', producing the profile $\mathrm{H}$. Bad pixels are omitted from the fits for $\mathrm{H}$, making the process not a true convolution, but really a series of least-squares fits. The Gaussian has been lowered so that it has a zero integral. 
- The significance level of a 'detection' can be easily estimated. If $\mathrm{s}_{1}$ is the random error in intensity for a given pixel located in a region free of stars, then the random scatter in $\mathrm{H}$ will be:

$$
\mathrm{s}_{\mathrm{H}}{ }^{2}=\frac{\mathrm{s}_{1}{ }^{2}}{\sum\left(\mathrm{G}_{\mathrm{ij}}^{2}\right)-\frac{\left(\sum \mathrm{G}_{\mathrm{ij}}\right)^{2}}{\mathrm{~N}}}
$$

if no star is present (Stetson 1987). The value of $s_{1}$ can either be estimated directly from the frame by using regions free of stars or as:

$$
\mathrm{s}_{1}{ }^{2}=(\text { Readout noise in ADU })^{2}+\frac{\text { Sky Background in ADU }}{\text { Photons per ADU }} .
$$

A minimum confidence level that a given detection is actually a star can then be based on $\mathrm{H}$. Typically this level, $\mathrm{H}_{\min }$, is set at the five sigma level.

The array $\mathrm{H}$ is examined pixel by pixel in a search for local maxima with intensities greater than $\mathrm{H}_{\min }$. A list of candidates is produced which contains mainly stars, but also some less obvious flaws and non-stellar objects. The majority of the flaws are more narrow in profile than genuine stars. A measure of sharpness is therefore used to prune the defects from the list. The sharpness $(\chi)$ value of a detection compares the intensity of the best-fitting Gaussian $\left(\mathrm{H}_{\mathrm{i}_{\mathrm{o}}, \mathrm{j}_{\mathrm{o}}}\right)$ to the ratio between the intensity of the central pixel of the proposed stellar image and the mean of the remaining pixels in the sub-array $(i, j)$, i.e.:

$$
\chi=\frac{\frac{\mathrm{D}_{\mathrm{i}_{0}, \mathrm{j}_{0}}}{\left\langle\mathrm{D}_{\mathrm{i}, \mathrm{j}}\right\rangle}}{\mathrm{H}_{\mathrm{i}_{\mathrm{o}}, \mathrm{j}_{\circ}}} \text { for }(\mathrm{i}, \mathrm{j}) \neq\left(\mathrm{i}_{\mathrm{o}}, \mathrm{j}_{\circ}\right) .
$$

A cosmic ray strike has a very narrow profile with the majority of its intensity contained in one or two pixels. This leads to a $\chi$ value of greater than one. The reverse occurs for spurious detections centred on low-valued pixels, with $\chi$ being close to zero. For moderately peaked objects, such as stars, $\chi$ should scatter about a value in between these two extremes. Generally the constraint $0.2 \leq \chi \leq 1.0$ is adequate for the selection of stars.

However, the $\chi$ test will not remove overflow columns which have bled from over-exposed pixels. A large number of spurious detections will therefore remain. Daofind employs a second test called 'roundness', which compares the profile of a detection in the $\mathrm{x}$ direction to the profile in the y direction. One-dimensional Gaussians are fitted to each direction. Roundness is defined as:

$$
2\left(\frac{h_{y}-h_{x}}{h_{y}+h_{x}}\right)
$$

where $h_{x}$ and $h_{y}$ are the central heights of the Gaussians fitted along the $\mathrm{x}$ and $\mathrm{y}$ directions. A charge overflow column will be narrow in one direction, and long in the other. Hence 
its 'roundness' will be far from zero, which is the value expected for round profiles such as star images. Roundness can only be calculated when there is a sufficient number of pixels on all sides of an image. Hence, it can not be calculated for images adjacent to the perimeter of the frame, explaining why the array $\mathrm{H}$ is smaller than the original intensity array. Typically the constraint $\mid$ roundness $\mid \leq 1.0$ is used.

Finally, Daofind records the positions of possible stars in a file.

2. Perform Aperture Photometry of the candidate stars, together with a sky annulus about each star. The magnitudes are used as initial estimates for the PSF profile fitting, while the annulus is used to estimate the sky background. This photometry is performed by DAOphot task Phot.

3. Establish the Global PSF using isolated bright stars on the frame and the task PSF. The PSF is colour and seeing dependent, so a PSF should be constructed for each frame. Often there are not enough bright stars without neighbours for the construction of a good model PSF. An iterative method can be adopted to cope with this:

(a) An initial estimate of the global PSF is made using the bright stars, regardless of whether they have any faint neighbouring stars or not. There should be no defects, such as bad columns, near any of the PSF stars.

(b) The PSF estimate is then used to subtract the neighbour stars out of the frame.

(c) A new estimate of the PSF is made from the bright, and now isolated, stars.

(d) If these stars can be cleanly subtracted from the frame, leaving no significant residuals, then an adequate PSF has been constructed. Otherwise, the PSF is regarded as only preliminary, and the process loops back to the second step (using the original frame).

4. Convolve the Frame with the Model PSF using the $\chi^{2}$ test as a measure of the goodness of fit. Starting estimates of the centroids and instrumental magnitudes of the stars are provided from the previous steps. A PSF is then suitably scaled at the position of each star. These profiles are then subtracted from the frame, out to one image radius from their centroids. The image radius is the radial distance from the centroid of a star where the profile disappears into the background noise. The residuals from this subtraction are examined and used to refine the fit via the least-squares solution of:

$$
s k y+\sum_{\mathrm{k}=1}^{\mathrm{N}^{\prime}}\left(\Delta \mathrm{x}_{0, \mathrm{k}}\left(\frac{\partial \mathrm{P}_{\mathrm{k}}}{\partial \mathrm{x}_{0, \mathrm{k}}}\right)_{\mathrm{i}, \mathrm{j}}+\Delta \mathrm{y}_{0, \mathrm{k}}\left(\frac{\partial \mathrm{P}_{\mathrm{k}}}{\partial \mathrm{y}_{0, \mathrm{k}}}\right)_{\mathrm{i}, \mathrm{j}}+\Delta \mathrm{h}_{\mathrm{k}}\left(\frac{\partial \mathrm{P}_{\mathrm{k}}}{\partial \mathrm{h}_{\mathrm{k}}}\right)_{\mathrm{i}, \mathrm{j}}\right)
$$

where 
- $\mathrm{P}_{\mathrm{k}}$ is the model PSF which has been fitted to the proposed star ( $\mathrm{k}$-th in the list of stars),

- $\mathrm{x}_{0, \mathrm{k}}$ and $\mathrm{y}_{0, \mathrm{k}}$ are the estimated $\mathrm{x}$ and $\mathrm{y}$ centroids of the star,

- $h_{k}$ is the estimated intensity of the image,

- $(i, j)$ is the set of pixels within one fitting radius of the centroid, and

- $\mathrm{N}^{\prime}$ is the number of possible stars in the frame.

A fitting radius is the region about a centroid that is considered in the least-squares fit determining the brightness and position of a star. Equation 4.11 is solved for each pixel (within one fitting radius from any star in a group) in turn. The derived parameters are used to fit the data array again, until the corrections become negligible. At the end of this iterative procedure another routine, called Substar; is used to subtract the best-fit profiles from the frame. This second frame contains only the residuals, and is examined in Step 5.

There are several subtleties that have been glossed over. The crowded field task, Allstar, breaks the candidate star list up into smaller groups. This is to make the problem more tractable computationally. Allstar involves the repeated solution of a system of simultaneous equations involving $3 \mathrm{~N}^{\prime}$ unknowns. By breaking the problem down into $\beta$ subgroups, the number of unknowns drops to $\frac{\mathrm{N}^{\prime 3}}{\beta^{2}}$. Allstar breaks the overall list into subgroups using a critical distance. Two stars separated by less than this distance are reduced together. This separation is taken to be the sum of the fitting and image radii, as the image radius indicates how far the effect of the first star can be detected. The image radius of a star must not fall within the fitting-radius of the second star if the two stars belong to two different groups. The actual algorithm for creation of the groups is:

- A star is transferred to the group list. The remaining stars in the candidate list are checked to see if their centroid falls within the critical radius of the first star.

- If no such stars are found, then the group list is complete.

- Otherwise each 'neighbour' star is added to the group list.

- Those stars not assigned are then checked to see if they fall within the critical radius of the second group star. Those that do, are shifted from the candidate list across to the end of the group list.

- The remainder of the stars in the group list are checked in a similar manner. Additional stars may be added to the group list, and checked in turn. At the end of this process, all stars 'associated' with the original star have been found and assigned to the group. 
- The first star in the unassigned list is now used as the basis for the next group. The process continues until there are no stars remaining in the unassigned list.

Another subtlety is the derivation of the sky background. Some profile fitting software, such as Romafot (Buonanno et al. 1979, 1983), simultaneously fit a model for the sky background and the PSF to the stellar images. Typically a polynomial function of $\mathrm{x}$ and $\mathrm{y}$ is used. Allstar uses a second method in which the sky annulus, measured by the aperture photometry, defines the sky background. This estimate for the sky includes the contribution of detectable stars - which will be modeled with profiles themselves. Stetson (1987) performed empirical tests of the two methods and found that explicitly solving the background contribution resulted in greater scatter in the final magnitudes, with the additional drawback of more computations being required. Stetson attributes the decrease in precision to be due to sky fitting requiring a larger fitting-radius so that the background is adequately sampled. This increases the likelihood that fits will be disturbed by unresolved stars in the outer regions of the profile, slightly abnormal pixels, and errors in the shape of the model PSF at its extremities.

The convergence of some of the groups may have to be assisted. A case might be an oscillating solution where a pixel one fitting-radius away from a star causes problems. When this pixel is included in the group containing the star, the subsequent profile fit causes the centroid of the star to shift slightly, removing the pixel from the fitting radius of the star. However, the next fit leads to the centroid being shifted so that the pixel is included again, and so on indefinitely. Allstar weights pixels according to their radius from an image centroid, with the weight falling to zero at the fitting radius. The pixels are dynamically re-weighted during the iterations. The pixel weights are altered according to the size of their residuals after several iterations of profile fitting. Pixels that fit a profile well are given higher weightings. This is an attempt to account for mildly erroneous data.

Allstar limits the size of any magnitude or position corrections. The sign of each parameter correction is stored, and if the latest correction has the opposite sign to its predecessor, then the maximum step size for the parameter concerned is halved. Hence oscillating solutions are strongly damped, but solutions steadily heading in one direction can proceed as far as is necessary, though the size of each step is constrained.

It is not possible to detect every star in the frame. For instance, the resolution of the CCD system will certainly not be enough to split all double stars. It is also important that Allstar does not include peaks in the random noise as potential stars since another profile will be fitted to this 'star', affecting any neighbouring profiles. Allstar therefore has a mechanism for testing stars, with subsequent rejection of the failures from the star 
list. The standard error in intensity for a star is derived from the inverse normal ('error') matrix (see Bevington 1969). The tests commence after the fourth iteration. The most uncertain detection in a group and any star with a centroid within one FWHM of a brighter star are tested and removed if:

- At the fourth loop and afterwards, the standard error in intensity of the star is greater than its intensity.

- At the eighth iteration and afterwards its intensity is less than 1.5 times the standard error.

- At the twelfth loop and beyond, or if the group converges before the twelfth iteration, the intensity is less than twice the standard error.

Only one star per group can be removed from the candidate list per iteration. Any star within $40 \%$ of the FWHM of a brighter star is automatically rejected.

5. Inspect the Residuals. Some stars that were lost by being blended in with another star should be visible now. The positions of these stars should be added to the list of stars made in the first step, and the previous steps (bar the first) repeated.

6. Construct a Listing of the Photometry. The magnitudes, positions, and associated uncertainties will have been output to a file. This list should be merged with lists for the same object, but for frames collected using different passbands. While the Anglo Australian Telescope (AAT, see Chapter 7) Images are steady, those acquired at Mount John can exhibit substantial linear translations ${ }^{1}$ which must be accounted for.

More detailed descriptions may be found in Massey \& Davis (1992), which is a user manual for the IRAF photometry packages, and in Stetson (1987), which is a detailed description of DAOphot.

\subsection{Testing}

Johnson BV frames of the LMC star cluster NGC 1978 were used to check that the version of DAOphot installed at VUW was performing correctly, and that there were no significant biases being introduced by the analyst. These observations were obtained by Dr. Philippe Fischer (who kindly made them available) on January 201991 with the Las Campanas $1 \mathrm{~m}$ telescope equipped with the TEK2 $1024^{2}$ CCD acquisition system. Seeing was good, being $\sim 2^{\prime \prime}$. A colour magnitude diagram was derived using the techniques discussed above, and is shown in

\footnotetext{
${ }^{1}$ Investigation revealed that no rotation was present.
} 

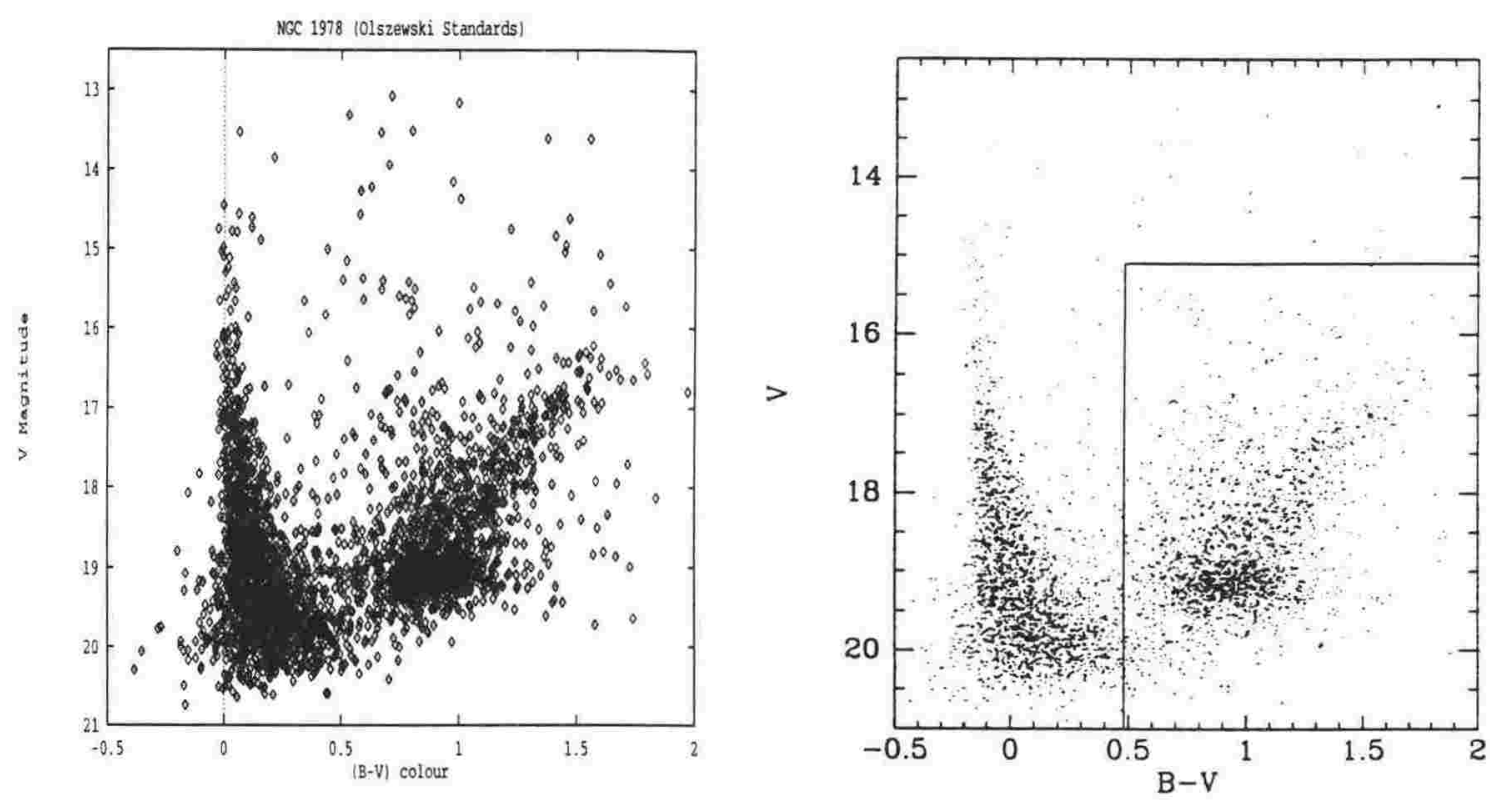

Figure 4-3: Colour-Magnitude Diagrams for NGC 1978. The same CCD frames were independently reduced using two different installations of IRAF, and by two different investigators. Very similar CMDs are arrived at, providing reassurance that the analysis techniques are being applied in a similar manner. The left-hand CMD is based on the VUW reductions, while the other is from Fischer et al. (1992b). The V exposure was 100 seconds long, while the B frame was collected over 200 seconds.

Figure 4-3. It compares well with the CMD of Fischer et al. (1992b), which was based on the same observations.

There is a systematic difference in ( $\mathrm{B}-\mathrm{V}$ ) between the two CMDs (see Figure 4-3). This is due to the current study using those photoelectric standards of Olszewski (1984) which were located inside the frames. This led to a slightly more red CMD (as can be seen in the CMD of Olszewski) than Fischer et al. (1992b), who observed 9 E-region standards of Menzies et al. (1989) with a colour spread of -0.08 to 1.77 , over an airmass range of 1.1 to 2.0 . The standard deviations of the transformation equations derived by Fischer et al. (1992b) are 0.017 and 0.023 magnitudes for $\mathrm{B}$ and $\mathrm{V}$ respectively. However, the essential point is that the crowded field reduction techniques employed by the current study resulted in a CMD similar to that of another group using the same data. Demonstrations of this include bright stars falling in the same position on the CMD relative to the other stars, and that the same general features of the CMDs are seen in both diagrams. This is subjectively reassuring.

It was planned to use the IRAF interactive non-linear least-squares task Fitparam to derive the standardisation equations during the project. Fitparam fits a given (user-defined) transformation equation to a set of data points, and iterates until the change in $\chi^{2}$ is less than a tolerance level set by the user, less than the numerical precision of the computer, or when the maximum number of allowed iterations is reached. After an initial fit is computed, a user may 


\begin{tabular}{||c|c|l|l|l|c||}
\hline Program & Filter & Zero Point & Extinction & \multicolumn{1}{|c|}{ Colour } & RMS \\
\hline Fitparam & B & $19.94 \pm 0.03$ & $0.255 \pm 0.016$ & $0.07 \pm 0.02$ & 0.033 \\
Hendon \& Kaitchuk & B & 20.03 & 0.262 & 0.04 & - \\
\hline Fitparam & V & $19.28 \pm 0.03$ & $0.138 \pm 0.015$ & $0.007 \pm 0.015$ & 0.032 \\
Hendon \& Kaitchuk & V & 19.30 & 0.145 & 0.003 & - \\
\hline
\end{tabular}

Table 4.1: Comparison of Transformation Equations derived by the IRAF Fitparam task and the Hendon \& Kaitchuk (1989) program suite. The zero point, first order extinction, and colour coefficients are given for each solution. The root mean square (RMS) errors in the IRAF solutions are given. 23 pairs of observations were made. The Hendon \& Kaitchuk (1989) software does not provide error estimates.

examine it and include or reject data from subsequent fits. Fitparam is based on the standard Levenberg-Marquadt algorithm, details of which may be found in Press et al. (1992).

In order to test Fitparam, and the usage of it, photoelectric observations of Graham (1982) E2 region stars were made on the 9th of February 1991 using the MJUO $61 \mathrm{~cm}$ Boller \& Chivens telescope. Six standards (stars C, n, s, q, p, and f) were repeatedly observed throughout the night over an airmass range of 1.22 to 3.07 . The $(B-V)$ colour range of these stars is from 0.18 to 1.61 , while the $\mathrm{V}$ magnitudes lie between 7.64 and 9.50. The transformation coefficients derived by Fitparam and the program suite of Hendon \& Kaitchuk (1989) are given in Table 4.1. There is good agreement between the two methods. 


\section{Chapter 5}

\section{The Survey}

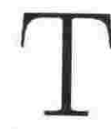

his chapter discusses the survey of LMC star clusters based on observations collected at MJUO. Isochrones have been fitted to the derived colour magnitude diagrams, resulting in estimates for the ages and reddenings of the clusters. Models were fitted to profiles of the clusters, and the radial variations of ellipticity inside the clusters were also studied.

\subsection{The Observations}

The bulk of the Johnson BV survey of LMC clusters took place in the summer of 1991.
The Images did not suffer from pixel saturation problems since the exposure lengths typically were 5 minutes long. The survey aim was to determine which type of LMC cluster (in age, size, population and field contamination) was suitable given the available equipment.

The age classifications of Searle et al. (1980), Freeman et al. (1983), Seggewiss \& Richtler (1989), and Meurer et al. (1990) were used to produce a listing of young LMC clusters. Descriptions of these clusters were taken from the Shapley \& Lindsay (1963) and Revised New General (Sulentic \& Tifft 1973) catalogues. Reference lists were compiled using the SIMBAD-III (Wenger et al. 1990) bibliographic database in Strasbourg, and the appropriate papers retrieved from the Carter Observatory Library. Finally, the clusters were located on the Hodge \& Wright (1966) photographic atlas of the LMC. All this information was used to select suitable targets, using the following criteria:

- Young, and therefore bright. Representative clusters of the first three Searle et al. (1980) classes were selected. This was to assess which classes could be successfully observed given the available acquisition equipment. Class $I^{1}$ objects were given priority, with Class II second, since one of the initial goals of the project was the study of mass functions for different clusters. Such work requires a large continuous observed main sequence in each cluster. The younger a given LMC star cluster is, the greater the observable portion of

\footnotetext{
${ }^{1}$ The youngest of the seven classifications of Searle et al. (1980).
} 


\begin{tabular}{||l|l||l|l||l|l||c|c||c|c||}
\hline $14 / 12 / 90$ & $4.0^{\prime \prime}$ & $17 / 12 / 90$ & $3.3^{\prime \prime}$ & $04 / 11 / 91$ & $2.2^{\prime \prime}$ & $05 / 11 / 91$ & $2.0^{\prime \prime}$ & $09 / 11 / 91$ & $3.1^{\prime \prime}$ \\
\hline $13 / 12 / 91$ & $4.0^{\prime \prime}$ & $14 / 12 / 91$ & $4.0^{\prime \prime}$ & $16 / 12 / 91$ & $2.4^{\prime \prime}$ & $30 / 10 / 92$ & $3.4^{\prime \prime}$ & $31 / 10 / 92$ & $3.3^{\prime \prime}$ \\
\hline & & $27 / 12 / 92$ & $3.5^{\prime \prime}$ & $15 / 03 / 93$ & $2.7^{\prime \prime}$ & $05 / 04 / 93$ & $2.6^{\prime \prime}$ & $15 / 02 / 94$ & $3.0^{\prime \prime}$ \\
\hline
\end{tabular}

Table 5.1: Seeing Estimates at MJUO. These are values of the best seeing experienced on nights that were at least partially clear, out of a total of 88 nights allocated at MJUO (see Table 5.2 for a calendar of all the observing runs at MJUO for this project). Two more of these 88 nights were clear and used for single channel photometry, but seeing estimates were not made on them. The seeing given above is based on the Full Width Half Maxima of several bright stars per frame.

\begin{tabular}{||c|l|l|c||}
\hline Run \# & \multicolumn{1}{|c|}{ Start Date } & \multicolumn{1}{|c|}{ End Date } & \# Days \\
\hline 01 & 14 December 1990 & 23 December 1990 & 10 \\
02 & 09 February 1991 & 17 February 1991 & 09 \\
03 & 11 October 1991 & 17 October 1991 & 07 \\
04 & 02 November 1991 & 10 November 1991 & 09 \\
05 & 11 December 1991 & 16 December 1991 & 06 \\
06 & 26 October 1992 & 01 November 1992 & 07 \\
07 & 21 December 1992 & 27 December 1992 & 07 \\
08 & 13 January 1993 & 17 January 1993 & 05 \\
09 & 23 February 1993 & 28 February 1993 & 06 \\
10 & 13 March 1993 & 18 March 1993 & 06 \\
11 & 12 Novernber 1993 & 17 November 1993 & 06 \\
12 & 09 February 1994 & 18 February 1994 & 10 \\
\hline \multicolumn{3}{|r|}{ Total: } & 88 \\
\hline
\end{tabular}

Table 5.2: Dates of Observing Runs at MJUO including those attempting to obtain Vilnius Photometry.

the main sequence. NGC 1835 is an exception to this rule, being a Class VII cluster and therefore old. It is the only 'true' globular cluster in the LMC not to have a published CMD. It was therefore included as a target of opportunity.

- Large Diameter and Populous, which would lead to well-populated cluster sequences on a CMD. Towards the end of the survey, the large young clusters had already been imaged, and so smaller young clusters, such as NGC 1839, were observed. Unfortunately, on reduction some of the earlier nights were found to be of poor quality, and the data were rejected. Rejected clusters included objects such as NGC 1711 and NGC 1944.

- Preferably unobserved with a CCD. This criterion became increasingly difficult with the subsequent publishing of studies such as Elson (1991) and Sagar \& Richtler (1991) after the commencement of the survey. Some of the clusters had photographic CMDs in the literature (such as in Alcaino \& Liller 1987), but it was hoped that the CCD-based CMDs produced by the survey would be of higher photometric quality, more populous, and to fainter limiting magnitudes.

The project appeared to be particularly viable given: 


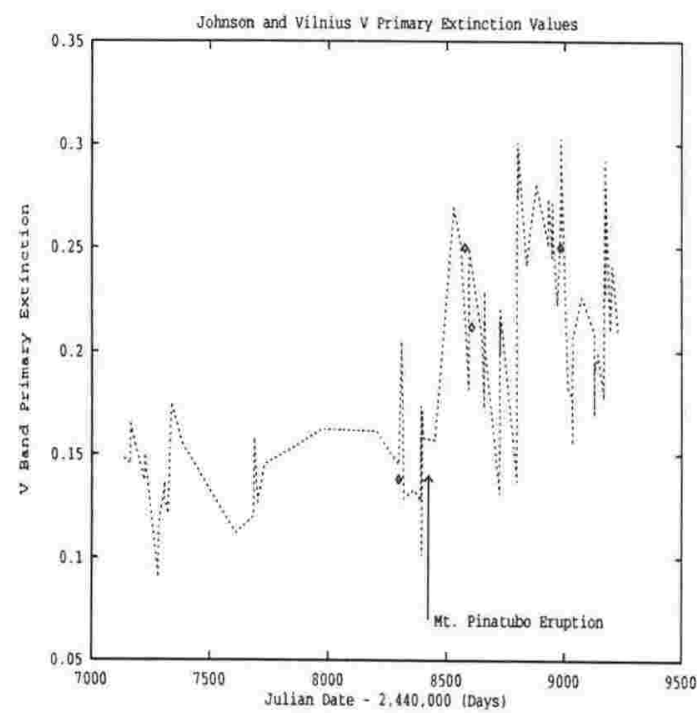

(a)

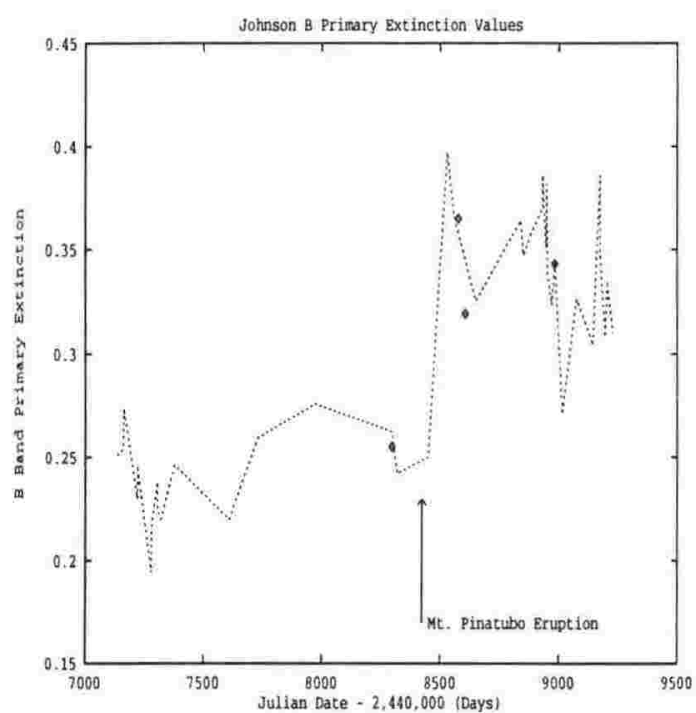

(b)

Figure 5-1: Primary Extinction Coefficients at MJUO for the Johnson and Vilnius V filters are shown joined as a dotted line in (a), while the Johnson B coefficients are plotted in (b). The Johnson values were obtained as part of the MJUO service observing program (Gilmore \& Kilmartin 1994). The Vilnius observations are from Forbes (1994). Johnson coefficients derived by this study are plotted as individual points, and are in good agreement with the other data. The primary eruption of the Phillipine volcano Mt. Pinatubo, on June 14/15 1991, is indicated. A further discussion of the variations in extinction at MJUO may be found in Forbes et al. (1994). The February 1991 data points are calculated from the photo-electric photometry discussed in Section 4.4 (which begins on page 99).

\begin{tabular}{||c|c|c|c|c||}
\hline Run & v1 & v2 & v3 & RMS \\
\hline Dec 1990 & $18.99 \pm 0.05$ & $0.04 \pm 0.05$ & 0.13 & 0.045 \\
Nov 1991 & $19.06 \pm 0.02$ & $0.03 \pm 0.03$ & $0.250 \pm 0.008$ & 0.037 \\
Dec 1991 & $19.33 \pm 0.06$ & $-0.04 \pm 0.07$ & $0.212 \pm 0.005$ & 0.023 \\
Dec 1992 & $19.36 \pm 0.01$ & $0.08 \pm 0.01$ & $0.250 \pm 0.006$ & 0.011 \\
\hline Run & b1 & b2 & b3 & RMS \\
\hline Dec 1990 & $18.27 \pm 0.05$ & $0.13 \pm 0.05$ & 0.25 & 0.045 \\
Nov 1991 & $17.90 \pm 0.02$ & $0.06 \pm 0.03$ & $0.365 \pm 0.007$ & 0.035 \\
Dec 1991 & $18.34 \pm 0.06$ & $0.08 \pm 0.07$ & $0.319 \pm 0.007$ & 0.026 \\
Dec 1992 & $18.40 \pm 0.01$ & $0.10 \pm 0.01$ & $0.343 \pm 0.008$ & 0.013 \\
\hline
\end{tabular}

Table 5.3: Transformation Equation Coefficients for the MJUO Survey. The equations are of the form used in Equation 2.2 on page 51 (i.e., $\mathrm{v}_{3}$ and $\mathrm{b}_{3}$ are the extinction coefficients). The extinction coefficients for December 1990 were provided from concurrent service photometry at MJUO (Kilmartin 1992). Unfortunately power cuts reduced the number of hours usable on this Observing Run. An insufficient airmass range was therefore covered by the standards, and the extinction coefficients could not be derived. Information on the observed standards is given in Table 5.4 . 


\begin{tabular}{||c|c|c|c|c|c|l}
\hline Run & \# Stds & \# V & \# B & Airmass Range & B-V Colour & Standards \\
\hline Dec 1990 & 09 & 09 & 09 & $1.09-1.10$ & $-0.05-1.58$ & Nelson \& Hodge (1983), Hodge \& Lee (1984) \\
Nov 1991 & 07 & 17 & 21 & $1.00-1.81$ & $0.17-0.89$ & Graham (1982) \\
Dec 1991 & 05 & 20 & 25 & $1.01-1.82$ & $0.17-0.89$ & Graham (1982) \\
Dec 1992 & 10 & 18 & 28 & $1.07-2.30$ & $0.17-1.61$ & Graham (1982), Menzies et al. (1989) \\
\hline
\end{tabular}

Table 5.4: Standard Star Colour and Airmass Ranges. The column headed '\# Stds' lists the number of individual standards observed, while the columns '\# V' and '\# B' list the number of individual V and B observations of the standard stars. 'Standards' indicates the source of the standard stars used.

- The recent acquisition of a CCD camera and ancillary support equipment by the University of Canterbury, and its subsequent installation on the $\mathrm{M}^{\mathrm{c}}$ Lellan telescope.

- That excellent CMDs were being acquired of LMC clusters using similar sized telescopes to the MJUO $1 \mathrm{~m}$, such as the Las Campanas $1 \mathrm{~m}$ (see Figure 4-3 on page 100 for a CMD produced from data acquired with this instrument).

- Considerable observing time could be, and was, allocated by the University of Canterbury to the project.

- The acquisition of a Sparc computer by the Physics Department, on which IRAF could be installed for the reduction of the Images.

During the project, the atmospheric extinction was dramatically increased by the Mt. Pinatubo eruption in 1991 (see Figure 5-1). $3-5 \mathrm{~km}^{3}$ of rock was ejected in this event, with the Plinian column reaching a height of $30 \mathrm{~km}$ (Grouthues \& Gochermann 1992), well into the stratosphere, which 'starts' at a height of $\sim 11 \mathrm{~km}$. While the larger, and therefore heavier, material fell out quickly, the $\sim 20$ megatonnes of sulphuric acid particles formed from the volcanic $\mathrm{SO}_{2}$ was expected to linger for years (Stowe et al. 1992). This has proven to be the case, with the aerosols leading to enhanced levels of extinction through to at least mid-July 1994 (see Forbes et al. 1994). The cyclic variation in atmospheric extinction observed at MJUO after the eruption (see Figure 5-1) could be the normal annual oscillation superposed on the enhanced extinction levels. Data prior to June 1991 are too sparse to show this effect clearly. See Wadsworth \& Cole (1994) for data obtained at Black Birch Observatory (Blenheim, NZ), which shows the seasonal variation in atmospheric extinction before and after the eruption.

Weather during the 1991/92 and 1992/93 observing seasons was worse than usual. Forbes (1994) reports that only $\sim 8 \%$ of the 65 nights scheduled for the Vilnius Standards Program at MJUO during this period were photometric - a figure comparable with the conditions experienced by this study (see Tables 5.1 and 5.2). When the skies did clear, the atmosphere was unstable and consequently the seeing poor. The clearance was often short-lived, not even lasting throughout a short summer night. On two previously good nights this deterioration led 


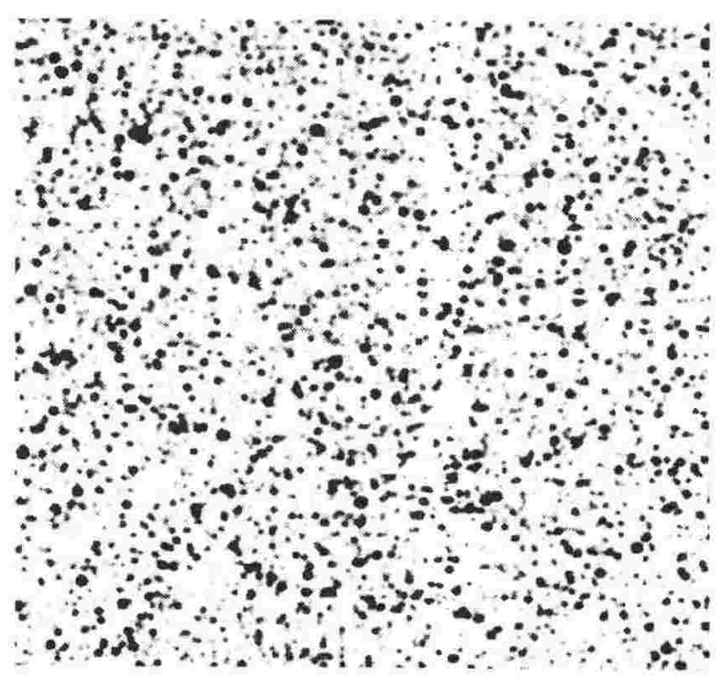

Figure 5-2: Simulated 1" Seeing

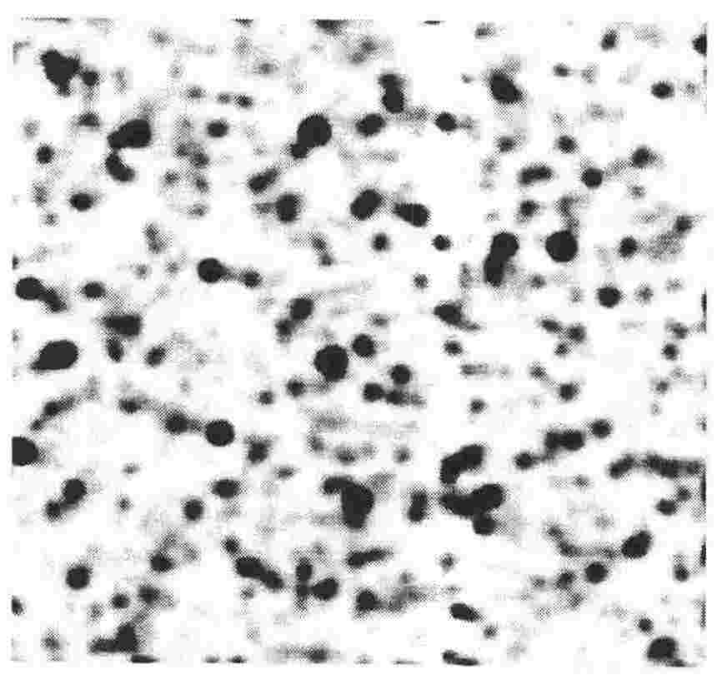

Figure 5-4: Simulated 3" Seeing

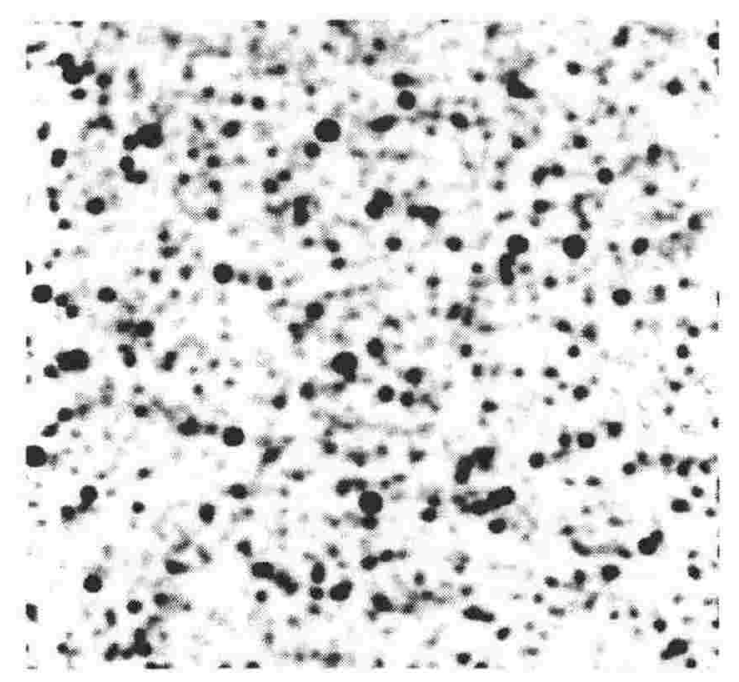

Figure 5-3: Simulated $2^{\prime \prime}$ Seeing

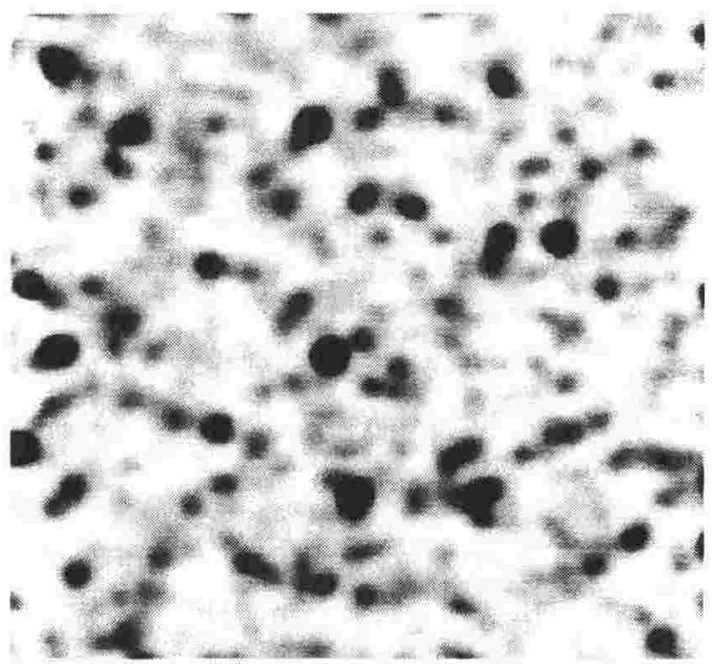

Figure 5-5: Simulated $4^{\prime \prime}$ Seeing

to poor calibrations, with a limited colour range in the observed standard stars (see Tables 5.3 and 5.4).

Seeing has a dramatic effect on the imaging of astronomical objects, with anything worse than $3.5^{\prime \prime}$ being effectively useless for these crowded fields ${ }^{1}$. Figures 5-2 to 5-5 depict the same artificial field of stars, but in increasingly worse seeing from $1^{\prime \prime}$ to $4^{\prime \prime}$. Figures 5-4 and 5-5 are representative of the $3^{\prime \prime}$ to $4^{\prime \prime}$ seeing typically experienced at MJUO during this project, whereas 1" seeing is frequent at excellent sites such as in Chile or South Africa. As seeing worsens, the limiting magnitude of an Image decreases, and the stellar images increasingly merge with nearby images. In bad seeing, care has to be taken to ensure that the model PSF is

\footnotetext{
${ }^{1}$ NGC 1761 was observed in worse conditions. It is a young open cluster, and therefore not so badly affected by the seeing. Further open clusters were therefore scheduled for observation, but no clear nights were available during the run scheduled for this.
} 
based on unambiguously single stars. Even if the model PSF is accurate, photometry of merger products will be misleading, producing a confused CMD. Good seeing results in narrow and well-defined sequences in a CMD, as in Figure 4-3 (on page 100 in Chapter 4).

\subsubsection{Reductions}

The Images were transferred from the MJUO Photometric system, along the data pathway described in Chapter 3, and into IRAF. Bias frames were subtracted from the Images at the time of acquisition, with flatfielding being performed by IRAF. The IRAF task cosmic was used to detect and remove cosmic ray strikes from frames, using the following algorithm:

- A 'window' of an arbitrary size, typically 5 by 5 pixels, is passed through the Image.

- The flux in the brightest pixel is compared with the mean of the rest of the pixels in the window. The mean excludes the second brightest pixel which may be another ray strike.

- If the flux in the brightest pixel is more intense than the mean by greater than a given threshold value, then the pixel is flagged as a potential strike.

- Once the window has passed through all the Image, the potential strikes are displayed in a graph plotting the background subtracted flux of the 'strike' against the ratio of the mean background to the flux of the candidate strike. A threshold level in the flux ratio can be set, dividing the sharp cosmic ray strikes from the smooth intensity distributions of faint stars. Typically there is a distinct division between these two populations.

- Given the user-defined flux ratio threshold, a new (shorter) list of likely cosmic ray strikes is formed. Pixels with intensities above the threshold are replaced by the mean of the four neighbouring pixels. If the second brightest pixel is one of these neighbours, it is excluded from the mean.

Finally, the frames were reduced using the software discussed in Chapter 4. The LMC star clusters and fields are strongly populated, requiring use of the crowded field photometry package $D A O p h o t$. Standards were reduced using synthetic aperture photometry.

\subsection{Colour-magnitude Diagrams}

Figures 5-6 to 5-25 are colour-magnitude diagrams based on observations collected during the survey. An offset guider was not available for the $\mathrm{M}^{\mathrm{C}}$ Lellan telescope during the 1990 and 1991 observing seasons, being still under construction. It was therefore decided to obtain shallow CMDs of many clusters, from which interesting objects could be selected for further investigation once the offset guider was available. The short (five minute) exposure times avoided problems 
with the telescope tracking, which is known to be unreliable for periods greater than $\sim 10$ minutes (Sullivan 1990), as well as the possibility of pixel saturation.

To act as a comparison with the CMDs produced by this study, some CMDs from Elson (1991) are given as Figures 5-26 to 5-31 (on page 117). Elson (1991) observed 18 young LMC clusters using the coated GEC \#2 CCD on the Siding Spring Observatory $1 \mathrm{~m}$ telescope with similar exposure times, though typically with slightly worse seeing conditions. The general agreement between clusters observed in both studies is good. Usually more stars were detected by the current study. Agreement with other studies (such as Alcaino \& Liller 1987) was also good.

Isochrones based on the $Z=0.008$ stellar evolution models of Schaerer et al. (1993) were fitted to the CMDs. $Z=0.008$ is the current metallicity of the LMC, and so presumably close to that of the young clusters observed. A distance modulus of $18^{\mathrm{m}} \cdot 4$ was assumed (Feast 1991). Results of these fits are given in Table 5.5. The reddenings $\mathrm{E}(\mathrm{B}-\mathrm{V})$ of the clusters, based on the shifts needed to fit the isochrones to the data, are given in Table 5.6. These results are compared with those from other studies, and generally are in good agreement.

A brief discussion of each CMD is given below:

- $N G C 1761$ is an open cluster that has no colour-magnitude diagram in the literature (see Figure 5-6). The Revised New General Catalog (RNGC, Sulentic \& Tifft 1973) description for the cluster describes it as a large object, with a compressed middle region. It appears to be a young cluster, with a strong main sequence. The cluster does not have a welldefined supergiant branch (SGB). There is one candidate for a star on that branch, being to the red of the turnoff point, but it could be a field star. The cluster would be a good candidate for a mass function study.

- NGC 1774 (SL 141) is another young cluster with no published CMD (see Figure 5-7). It is classified as a Class II cluster by Searle et al. (1980). It has a well-defined main sequence, together with a sparsely populated supergiant branch and red loop.

- NGC 1818 is a Class I cluster (Searle et al. 1980). The RNGC describes NGC 1818 as a very bright, round cluster some 50 to 60 arcseconds across, with a very compressed centre. The CMD is given as Figure 5-8 and is very similar to that of Elson (1991), which is given as Figure 5-26. The main sequence is well defined to $\mathrm{V} \sim 16$, with the upper main sequence and SGB being sparsely populated. A clump of red supergiants is apparent.

Cayrel et al. (1988) presented a BV CMD extending to $V=20$, based on CCD Images obtained with the ESO $2.2 \mathrm{~m}$ telescope in 1.6" seeing. The main sequence is revealed to be strongly populated, narrow, and well-defined. However, the SGB is again sparse, with some clumping at the red end of the SGB loop. Similarly, the field is weak. 


\begin{tabular}{||l|c|c|c|c|c|c||}
\hline Cluster & \multicolumn{5}{|c||}{ Age (Log yrs) } & Date \\
\cline { 2 - 6 } Name & Current & A\&L & Elson & Meurer & Other & \\
\hline NGC 1761 & $7.4 \pm 0.2$ & & & & & $04-11-91$ \\
NGC 1774 & $7.4 \pm 0.1$ & & & 7.8 & & $04-11-91$ \\
NGC 1818 & $7.2 \pm 0.1$ & & 7.2 & 7.4 & 7.2 & $17-12-90$ \\
NGC 1834 & $7.7 \pm 0.1$ & 7.7 & & 7.9 & 7.6 & $16-12-91$ \\
NGC 1839 & $7.6 \pm 0.1$ & 7.5 & & & & $16-12-91$ \\
NGC 1847 & $7.4 \pm 0.2$ & 7.4 & & 7.4 & 7.4 & $17-12-90$ \\
NGC 1850 & $7.7 \pm 0.1$ & 7.3 & 7.5 & 7.8 & 7.8 & $17-12-90$ \\
NGC 1854 & $7.4 \pm 0.1$ & 7.4 & 7.4 & 7.7 & 7.5 & $17-12-90$ \\
NGC 1863 & $7.4 \pm 0.1$ & 7.8 & & 7.9 & 7.8 & $16-12-91$ \\
NGC 1968 & $7.0 \pm 0.1$ & & & 7.0 & & $05-11-91$ \\
NGC 2004 & $7.1 \pm 0.1$ & & 7.1 & 7.1 & 6.9 & $05-11-91$ \\
NGC 2018 & $7.5 \pm 0.1$ & & & 7.0 & & $05-11-91$ \\
NGC 2031 & $7.7 \pm 0.1$ & & & & & $04-11-91$ \\
NGC 2041 & $7.6 \pm 0.2$ & & & & & $04-11-91$ \\
NGC 2058 & $7.6 \pm 0.2$ & & & & & $05-11-91$ \\
NGC 2157 & $7.7 \pm 0.1$ & & 7.6 & 7.7 & 7.5 & $05-11-91$ \\
NGC 2164 & $7.8 \pm 0.2$ & & 7.6 & 7.9 & 8.3 & $05-11-91$ \\
NGC 2214 & $7.7 \pm 0.1$ & & 7.5 & 7.8 & 7.6 & $04-11-91$ \\
SL 268 & $7.2 \pm 0.1$ & & & & & $16-12-91$ \\
\hline
\end{tabular}

Table 5.5: Isochrone Age Estimates for Survey Clusters. The $Z=0.008$ metallicity isochrones of Schaerer et al. (1993) were fitted to the CMDs, being appropriate to the current metallicity of the LMC and presumably for the young clusters as well. The column labeled 'Current' lists the ages determined for the clusters by this study. The isochrone age estimates of Alcaino \& Liller (1987) are given in the column titled 'A\&L', while those of Elson (1991) are given in the column headed 'Elson'. The age estimates of Meurer et al. (1990) are also listed. The date of observation is listed under the column 'Date'. The reddenings derived from these fits are given in Table 5.6.

The ages listed in the column titled 'Other' were estimated using two basic techniques. The first was isochrone fitting — by Nelson \& Hodge (1983) for NGC 1847, by Vallenari et al. (1994) for NGC 1850, and by Vallenari et al. (1991) for NGC 2164. The last paper used stellar evolution models incorporating convective overshoot. The two main results of models incorporating overshooting are that the lifetime of the core hydrogen burning phase is considerably enhanced, and that the maximum initial mass for the core helium flash is lowered (Chiosi \& Pigatto 1986). The second method used the age calibration of Hodge (1983) of the V magnitude for the brightest main sequence stars. These ages are listed in the catalogue of Seggewiss \& Richtler (1989).

Considering the quality of the observations, there is good agreement between the results of this study and the published values. Half the range in age and reddening (for equally good fits of the models to the data) was taken as the uncertainty in each parameter. However, the age and reddening estimates should only be considered as preliminary, pending at least three-colour photometry in better seeing conditions. 


\begin{tabular}{||l|c|c|c|c|c||}
\hline \multirow{2}{*}{ Cluster } & \multicolumn{2}{|c|}{ E(B-V) } & \multirow{2}{*}{ B Seeing } & V seeing \\
\cline { 2 - 4 } & Current & A\&L & Elson & & \\
\hline NGC 1761 & $0.12 \pm 0.05$ & & & $4.0^{\prime \prime}$ & $4.4^{\prime \prime}$ \\
NGC 1774 & $0.08 \pm 0.01$ & & & $2.4^{\prime \prime}$ & $2.4^{\prime \prime}$ \\
NGC 1818 & $0.16 \pm 0.05$ & & 0.10 & $3.8^{\prime \prime}$ & $3.3^{\prime \prime}$ \\
NGC 1834 & $0.09 \pm 0.03$ & 0.10 & & $2.9^{\prime \prime}$ & $2.6^{\prime \prime}$ \\
NGC 1839 & $0.28 \pm 0.04$ & 0.27 & & $2.8^{\prime \prime}$ & $2.6^{\prime \prime}$ \\
NGC 1847 & $0.18 \pm 0.04$ & 0.25 & & $3.3^{\prime \prime}$ & $3.5^{\prime \prime}$ \\
NGC 1850 & $0.14 \pm 0.03$ & 0.18 & 0.15 & $3.0^{\prime \prime}$ & $3.0^{\prime \prime}$ \\
NGC 1854 & $0.19 \pm 0.04$ & 0.20 & 0.12 & $3.7^{\prime \prime}$ & $3.3^{\prime \prime}$ \\
NGC 1863 & $0.20 \pm 0.02$ & 0.20 & & $2.7^{\prime \prime}$ & $2.3^{\prime \prime}$ \\
NGC 1968 & $0.08 \pm 0.01$ & & & $2.0^{\prime \prime}$ & $2.0^{\prime \prime}$ \\
NGC 2004 & $0.04 \pm 0.01$ & & 0.06 & $2.9^{\prime \prime}$ & $2.8^{\prime \prime}$ \\
NGC 2018 & $0.22 \pm 0.03$ & & & $2.3^{\prime \prime}$ & $2.5^{\prime \prime}$ \\
NGC 2031 & $0.19 \pm 0.03$ & & & $3.1^{\prime \prime}$ & $2.7^{\prime \prime}$ \\
NGC 2041 & $0.12 \pm 0.03$ & & & $2.8^{\prime \prime}$ & $2.7^{\prime \prime}$ \\
NGC 2058 & $0.33 \pm 0.03$ & & & $2.4^{\prime \prime}$ & $2.4^{\prime \prime}$ \\
NGC 2157 & $0.15 \pm 0.07$ & & 0.10 & $2.2^{\prime \prime}$ & $2.2^{\prime \prime}$ \\
NGC 2164 & $0.05 \pm 0.03$ & & 0.10 & $2.3^{\prime \prime}$ & $2.2^{\prime \prime}$ \\
NGC 2214 & $0.08 \pm 0.04$ & & 0.10 & $3.4^{\prime \prime}$ & $2.7^{\prime \prime}$ \\
SL 268 & $0.03 \pm 0.02$ & & & $3.0^{\prime \prime}$ & $2.7^{\prime \prime}$ \\
\hline
\end{tabular}

Table 5.6: E(B - V) Estimates for the Survey Clusters derived from isochrone fits. The reddenings of Alcaino \& Liller (A\&L, 1987) and Elson (1991) are given together with those estimated by the current study. The seeing during the observations is also listed.

- $N G C 1834$ is a small cluster in a very populated field, as evidenced by the well-populated field giant branch stretching diagonally from near the base of the observed main sequence of the cluster. The features are better defined than the CMD based on the photographic work of Alcaino \& Liller (1987), with more stars present in Figure 5-9 and the main sequence extending to slightly brighter magnitudes.

- NGC 1835 is the last of the known true LMC globular clusters not to have a published CMD. This is due to the fact that NGC 1835 is located in the bar of the LMC, making for a particularly crowded field - as can be seen in Figure 5-10. The tip of the giant branch and AGB of the cluster are lost in the field stars of this CMD.

- $N G C 1839$ is a small cluster, which is rather irregular in shape. Some nebulosity is present, suggesting that the cluster is very young. Again the CMD is better defined and more populated than that given in Alcaino \& Liller (1987), although the general features in the photographic CMD are confirmed (see Figure 5-11). Both CMDs extend to the same depth in V.

- NGC 1847: Alcaino \& Liller (1987) noted what appeared to be a second supergiant branch, with a turnoff at $\mathrm{V} \sim 16.5$, in their CMD for this cluster. Figure 5-12 does not provide evidence to either confirm or disprove this hypothesis. A feature which could 
NGC 1761

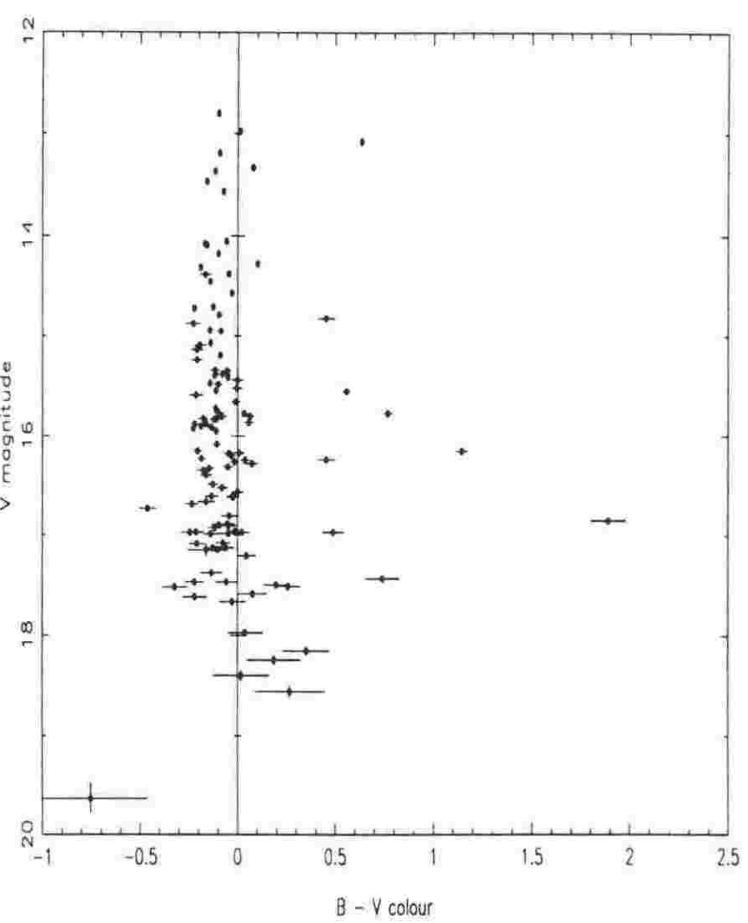

Figure 5-6: BV Colour-magnitude Diagram for NGC 1761.

NGC 1818

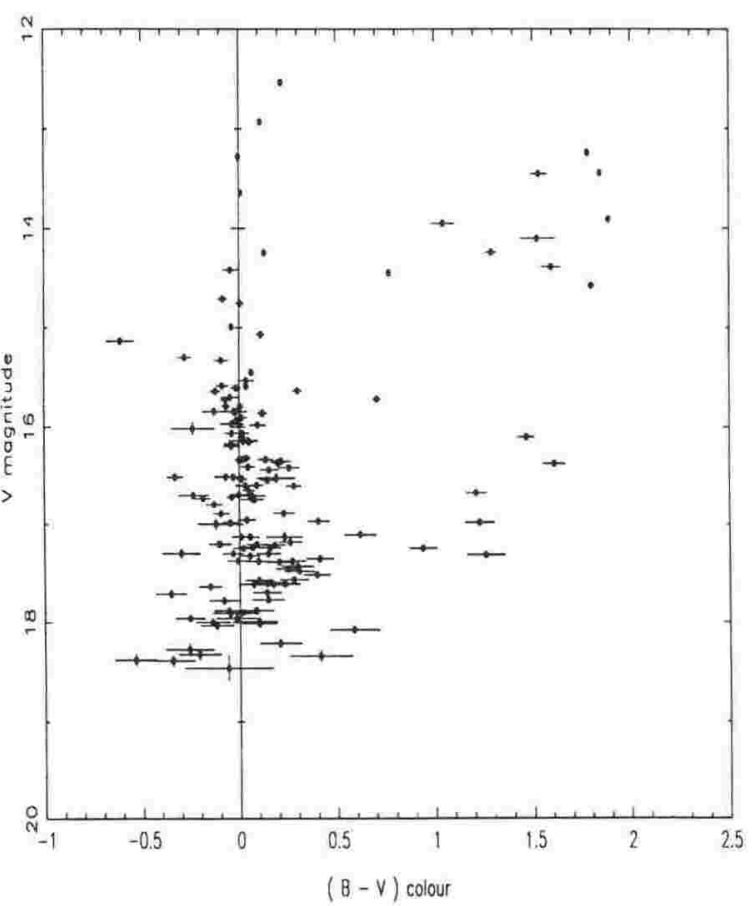

Figure 5-8: BV Colour-magnitude Diagram for NGC 1818.
NGC 1774

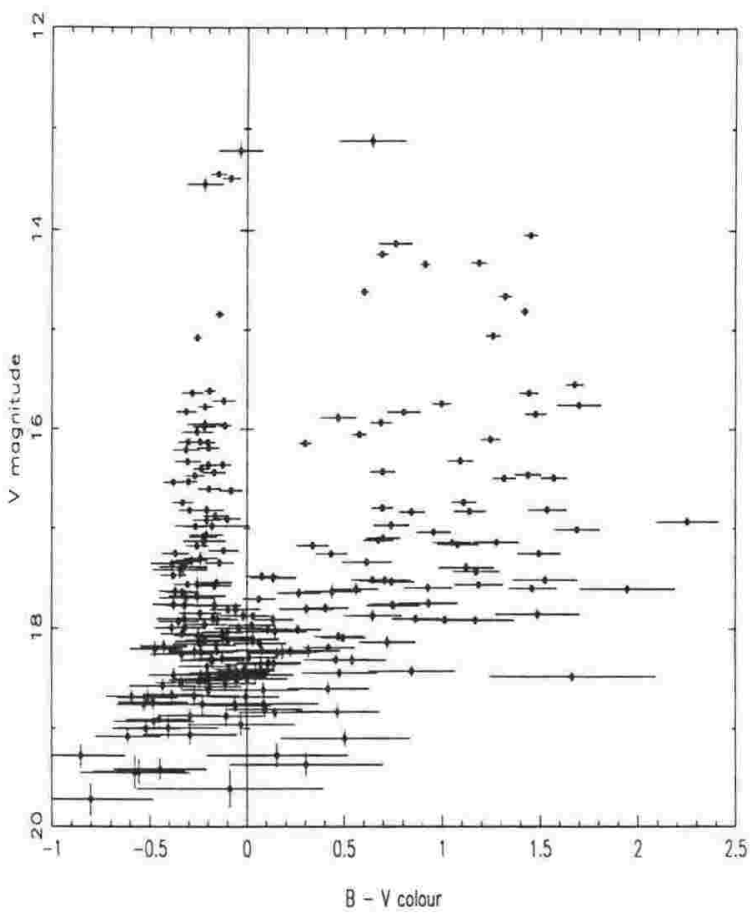

Figure 5-7: BV Colour-magnitude Diagram for NGC 1774.

NGC 1834

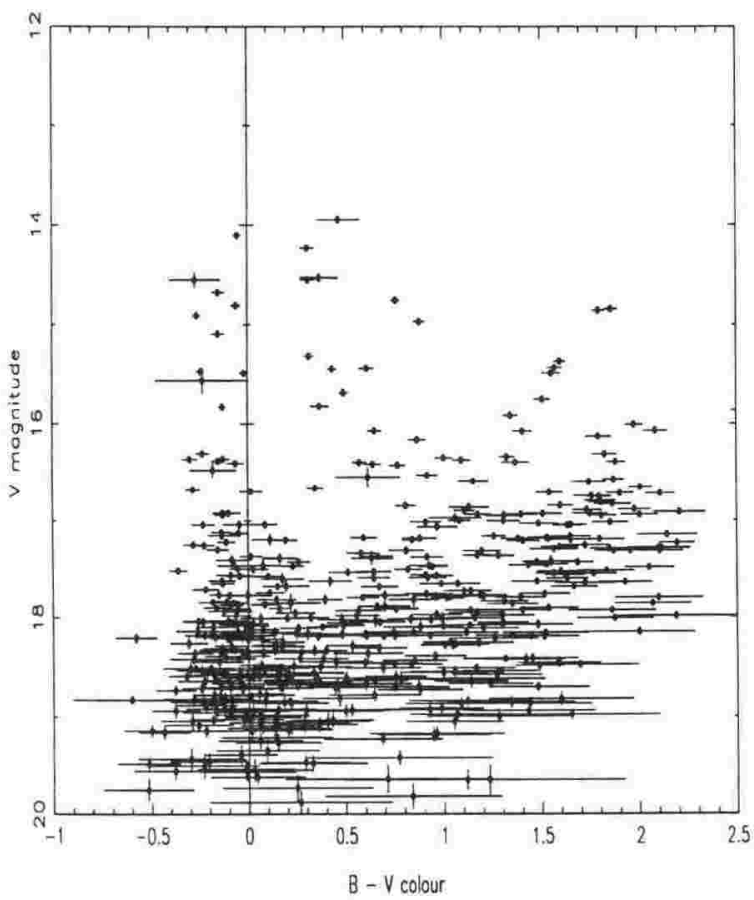

Figure 5-9: BV Colour-magnitude Diagram for NGC 1834. 
NGC 1835

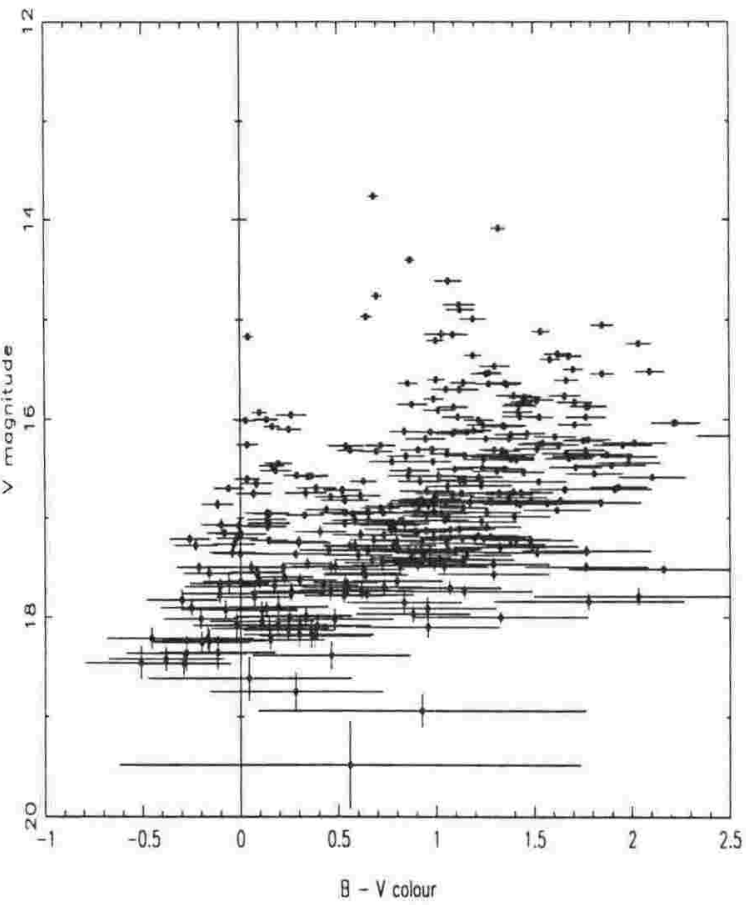

Figure 5-10: BV Colour-magnitude Diagram for NGC 1835.

NGC 1847

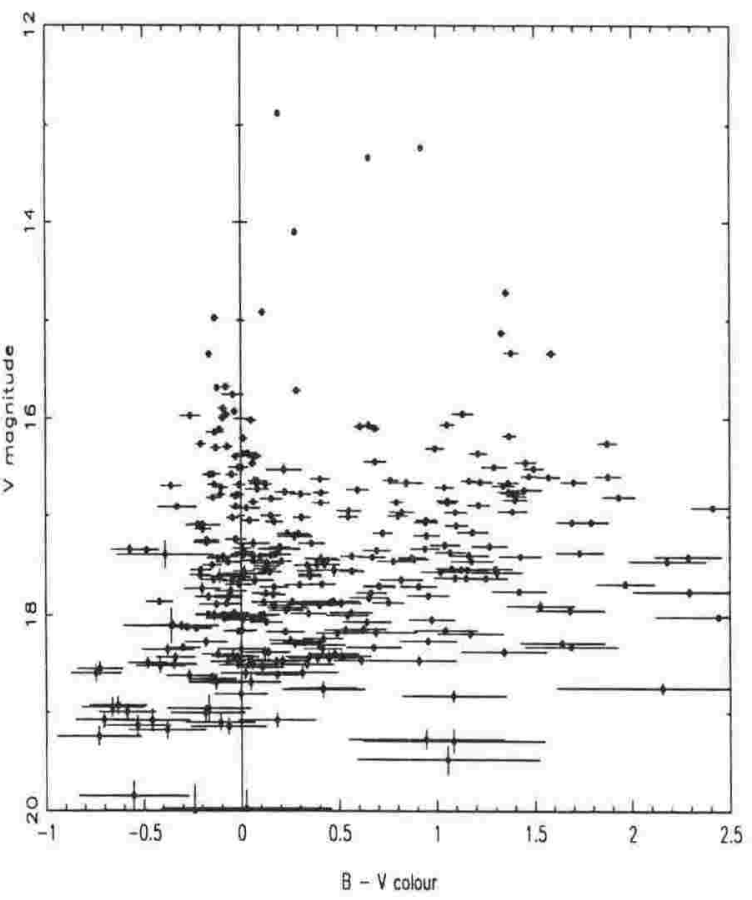

Figure 5-12: BV Colour-magnitude Diagram for NGC 1847.
NGC 1839

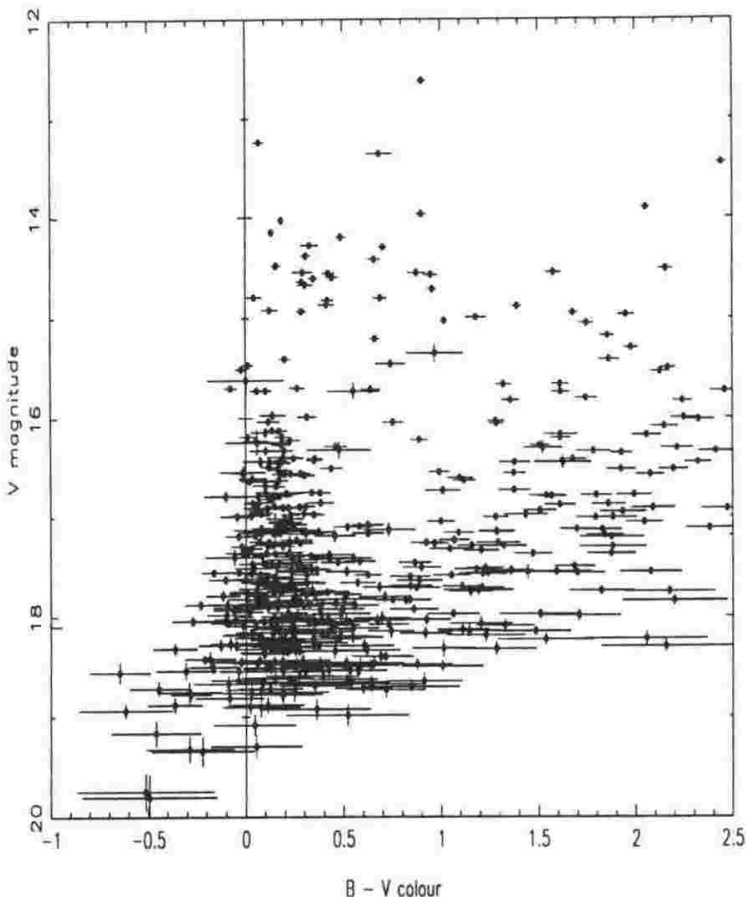

Figure 5-11: BV Colour-magnitude Diagram for NGC 1839.

NGC 1850

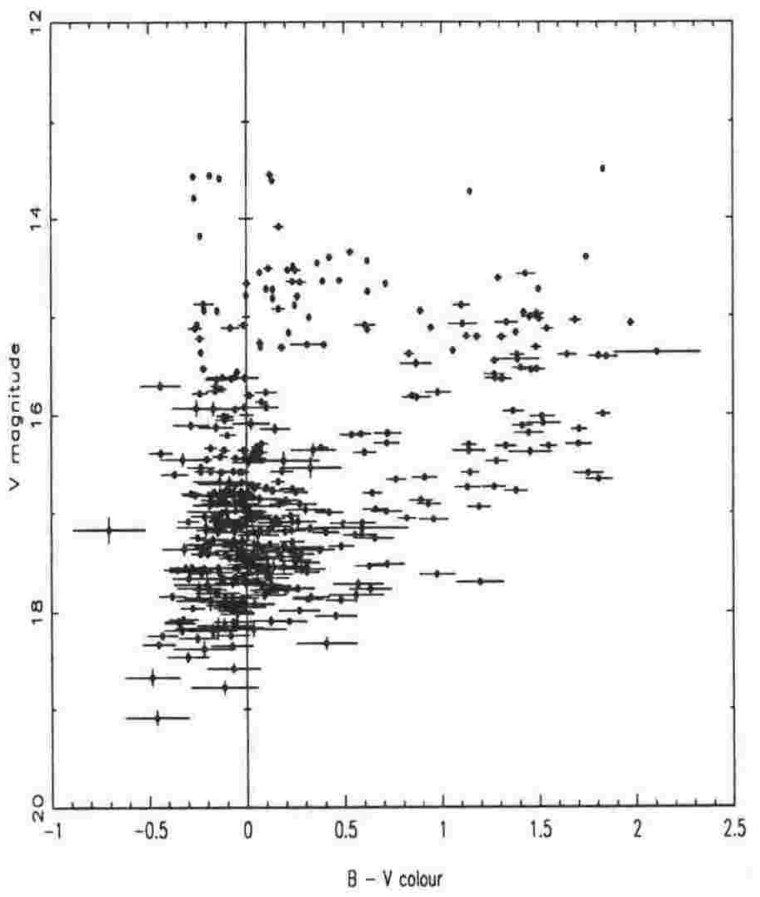

Figure 5-13: BV Colour-magnitude Diagram for NGC 1850. 
NGC 1854

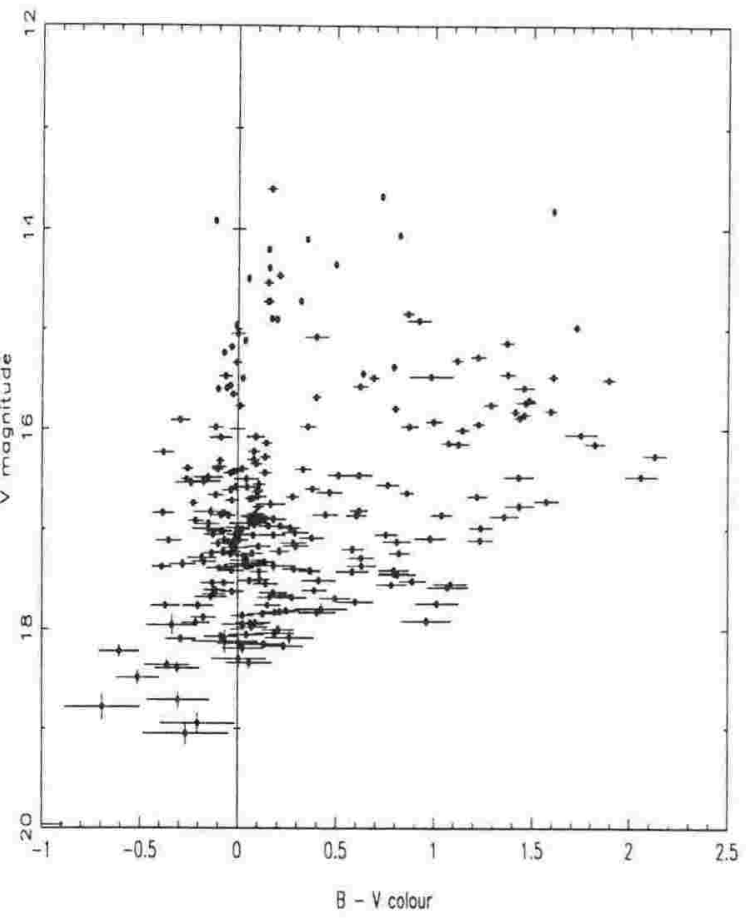

Figure 5-14: BV Colour-magnitude Diagram for NGC 1854.

NGC 1968/NGC 1974 (Nov 91)

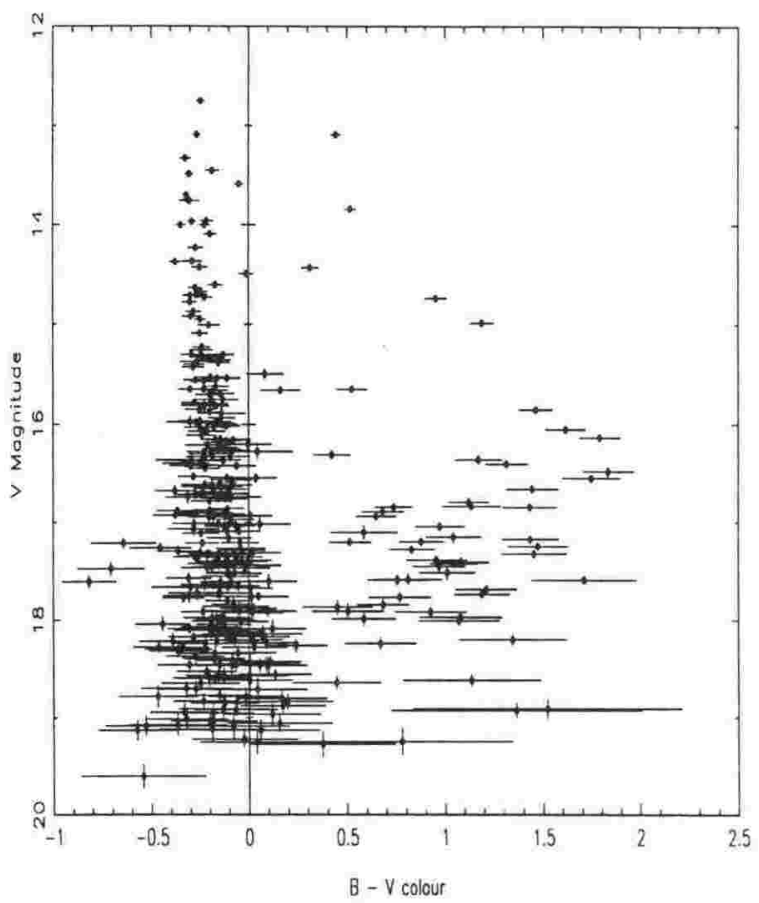

Figure 5-16: BV Colour-magnitude Diagram for NGC 1968 and NGC 1974.
NGC 1863

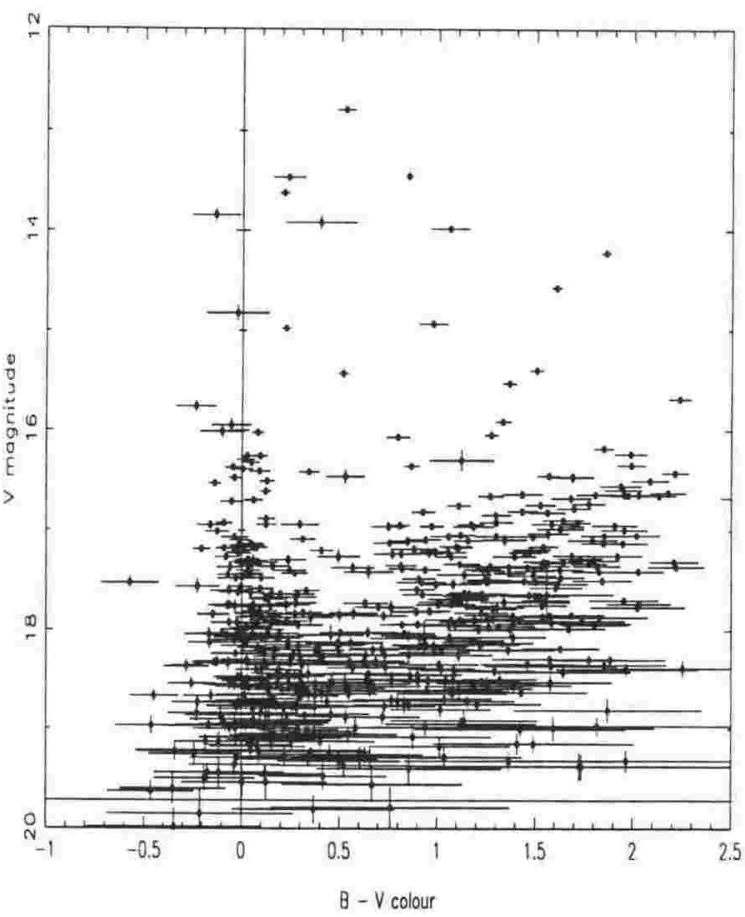

Figure 5-15: BV Colour-magnitude Diagram for NGC 1863.

NGC 2004

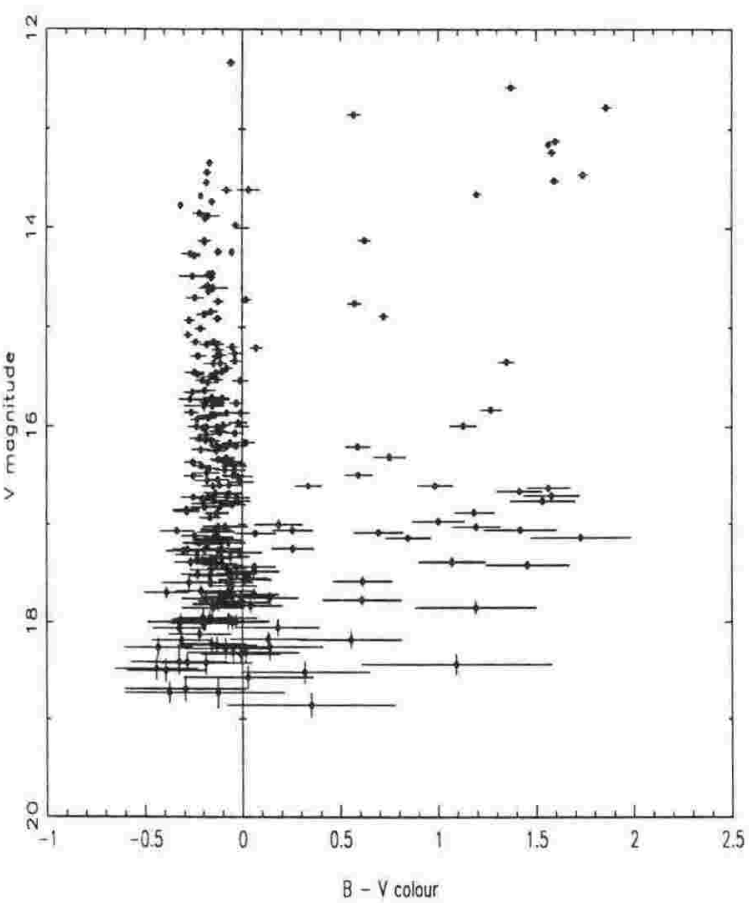

Figure 5-17: BV Colour-magnitude Diagram for NGC 2004. 
NGC 2018

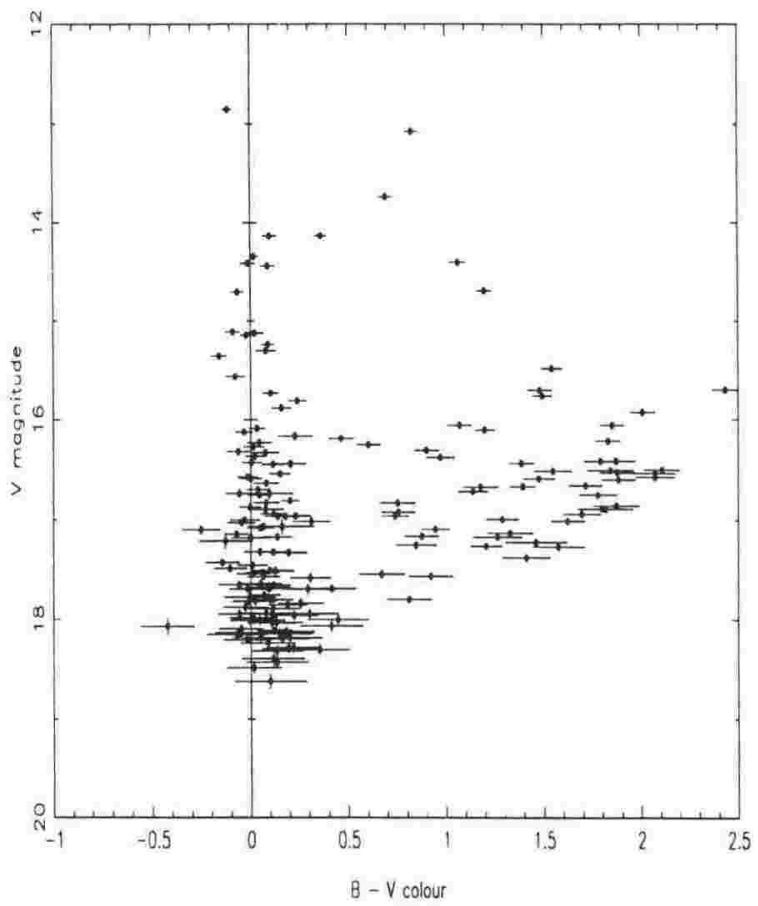

Figure 5-18: BV Colour-magnitude Diagram for NGC 2018.

NGC 2041

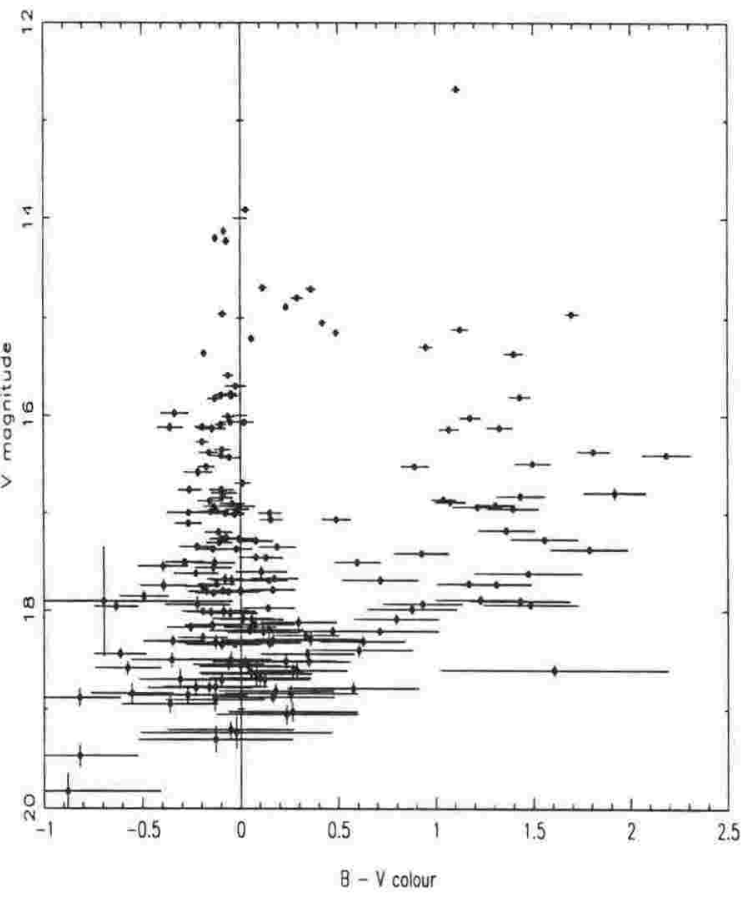

Figure 5-20: BV Colour-magnitude Diagram for NGC 2041.
NGC 2031

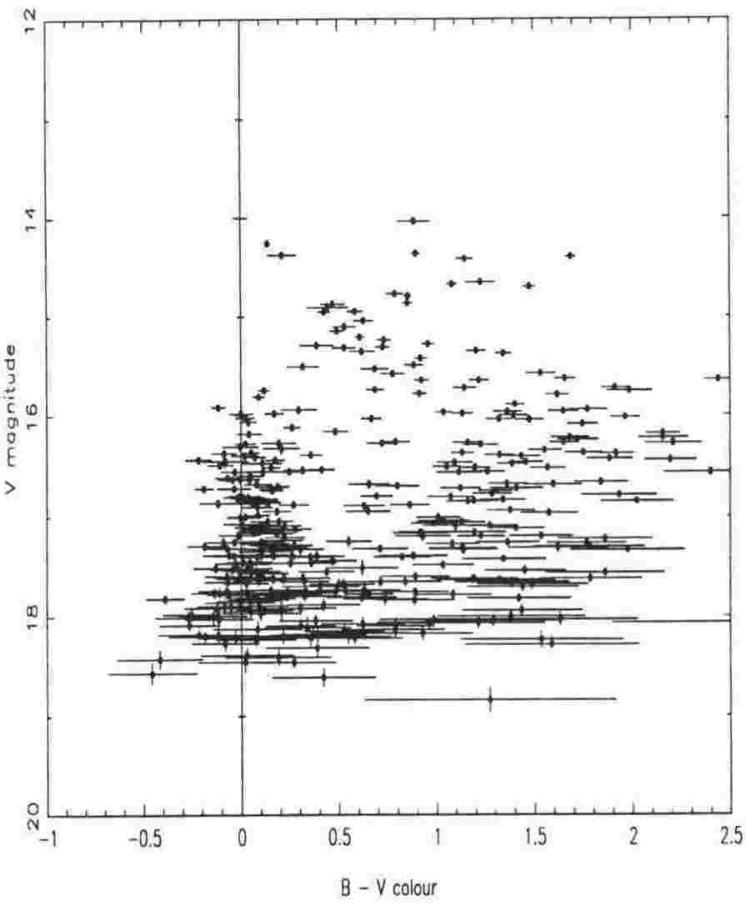

Figure 5-19: BV Colour-magnitude Diagram for NGC 2031.

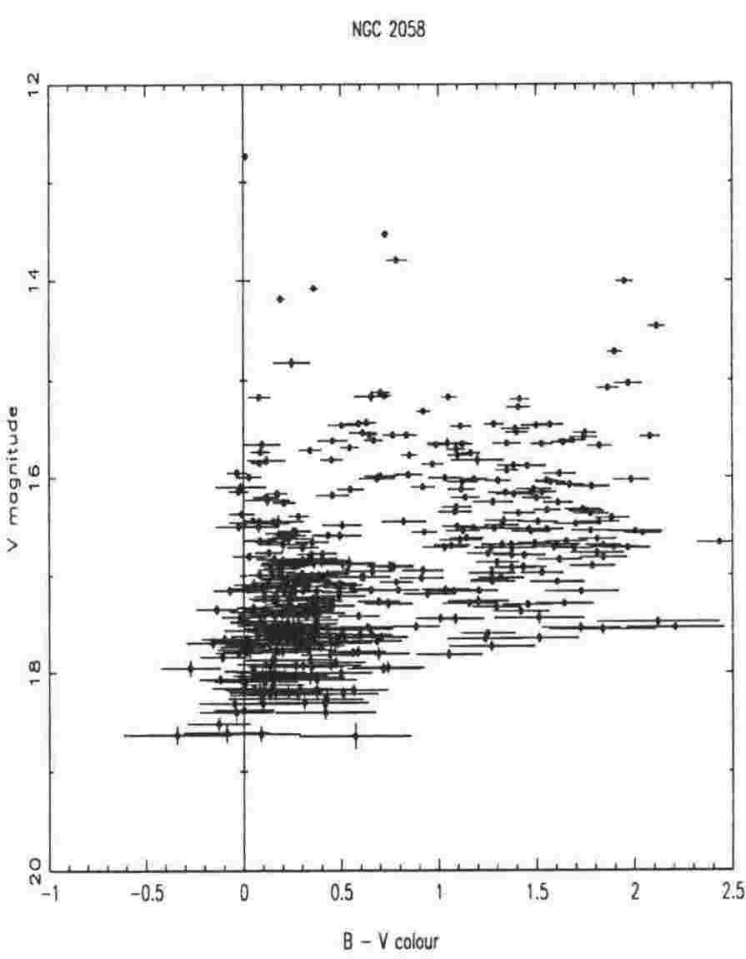

Figure 5-21: BV Colour-magnitude Diagram for NGC 2058. 
NGC 2157

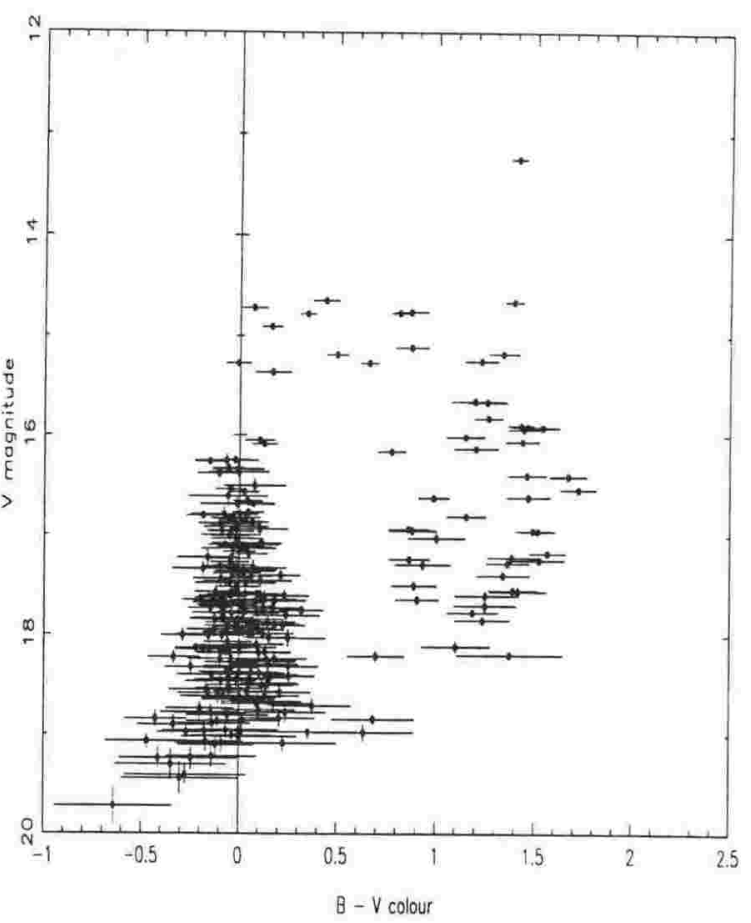

Figure 5-22: BV Colour-magnitude Diagram for NGC 2157.

NGC 2214

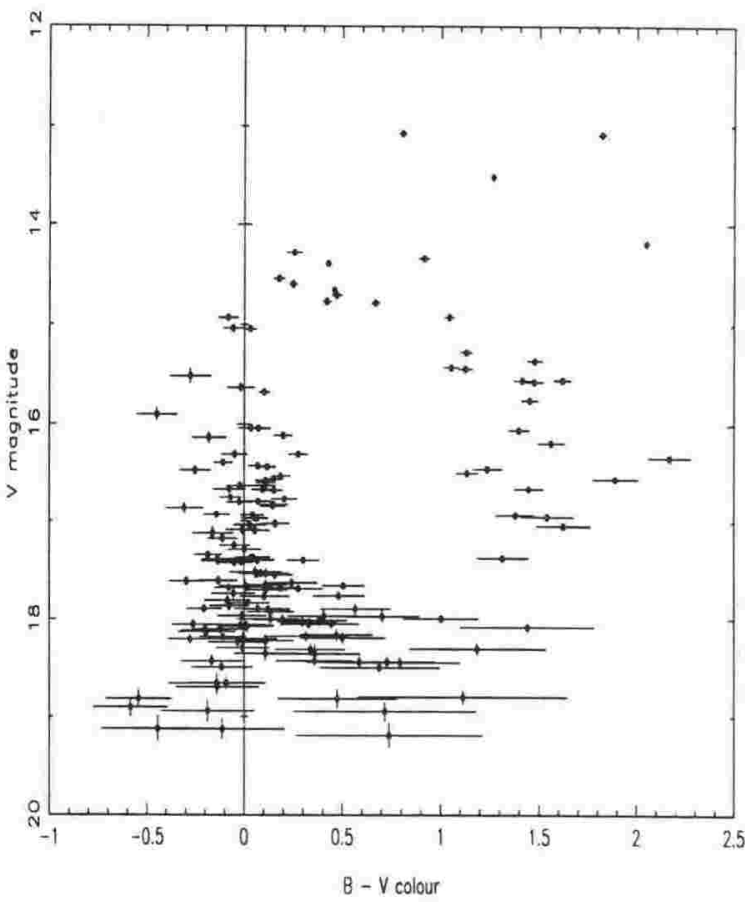

Figure 5-24: BV Colour-magnitude Diagram for NGC 2214.
NGC 2164

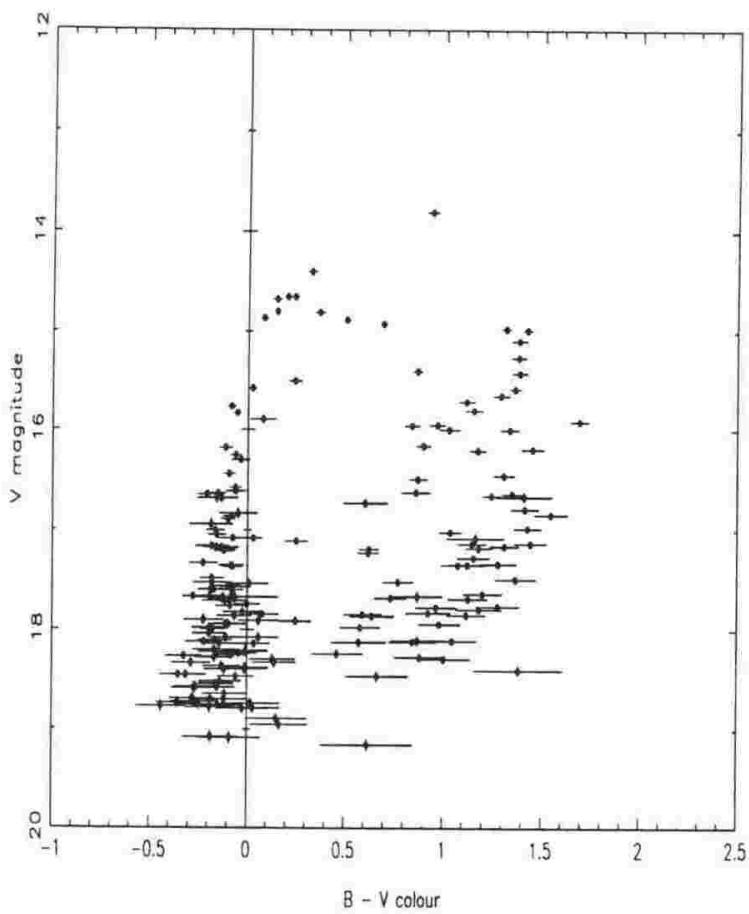

Figure 5-23: BV Colour-magnitude Diagram for NGC 2164.

SL 268

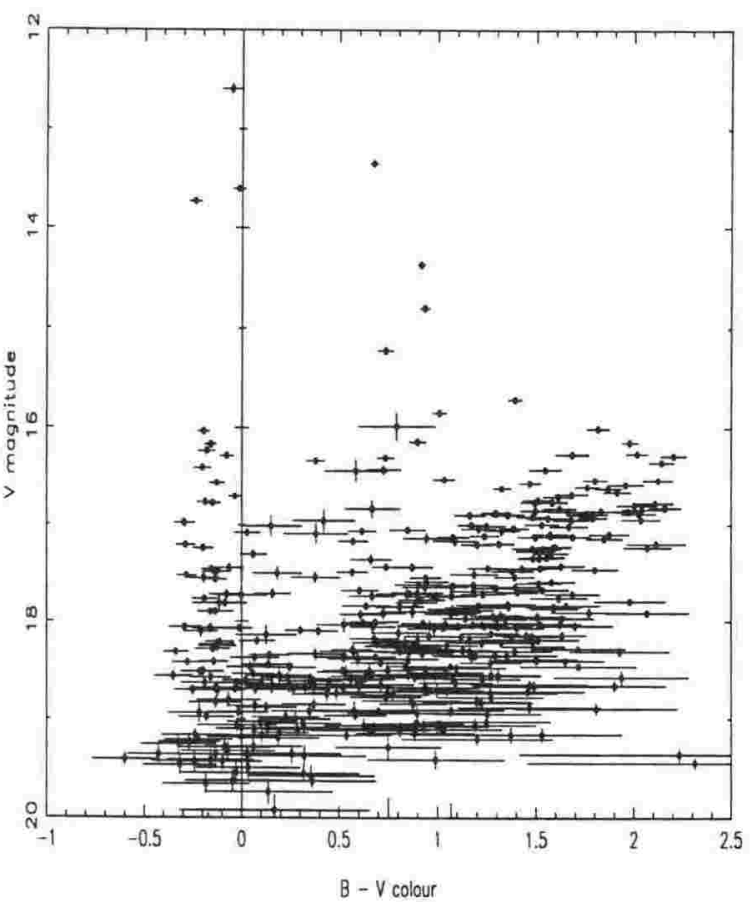

Figure 5-25: BV Colour-magnitude Diagram for SL 268. 


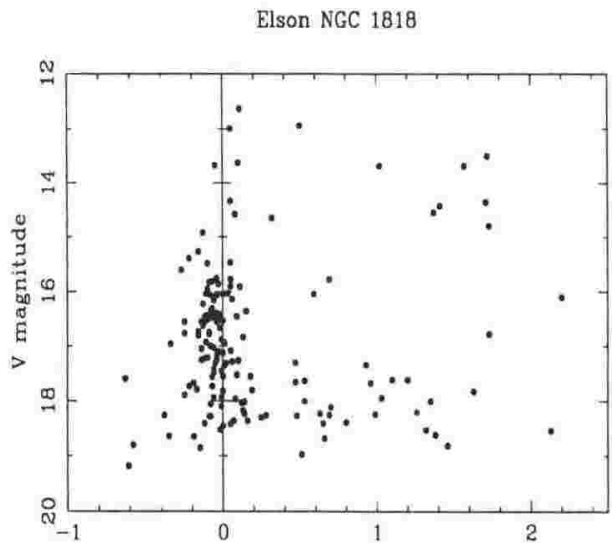

B - V colour

Figure 5-26: Elson (1991) Johnson BV Colour-magnitude Diagram for NGC 1818.

Elson NGC 2004

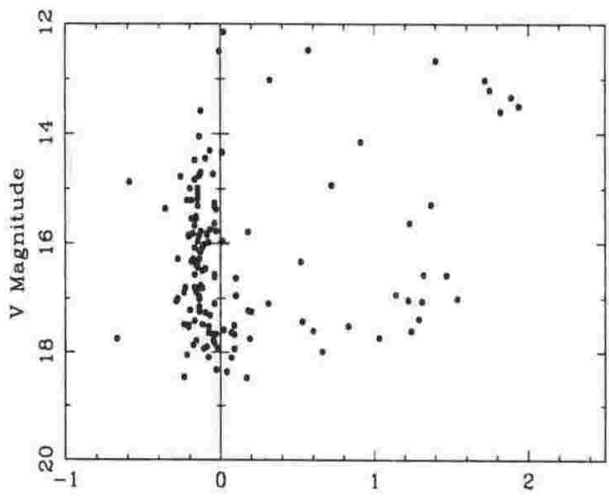

B - V colour

Figure 5-28: Elson (1991) Johnson BV Colour-magnitude Diagram for NGC 2004.

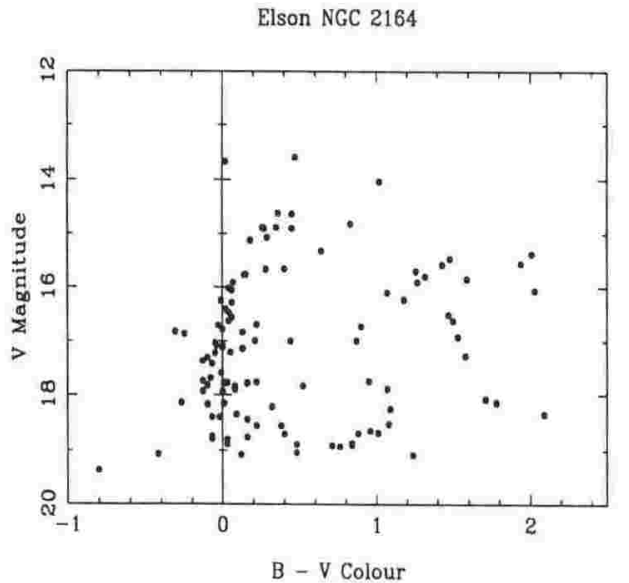

Figure 5-30: Elson (1991) Johnson BV Colour-magnitude Diagram for NGC 2164.

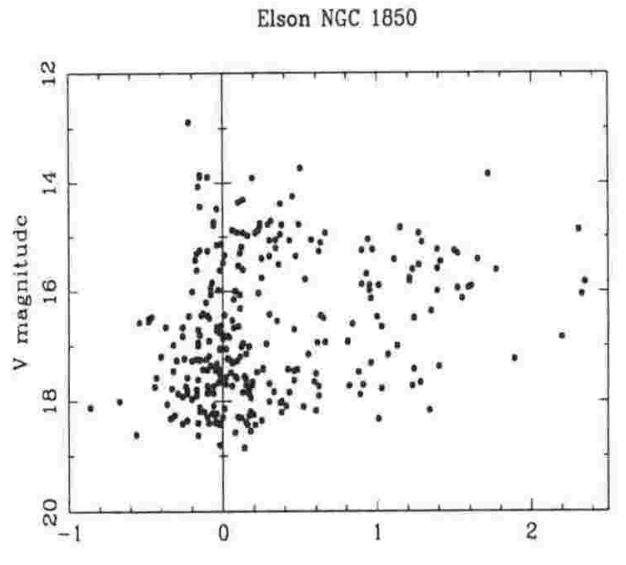

Figure 5-27: Elson (1991) Johnson BV Colour-magnitude Diagram for NGC 1850.

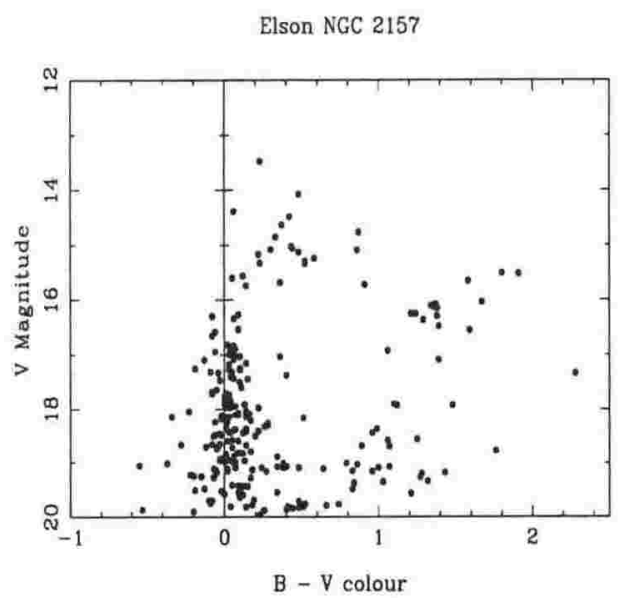

Figure 5-29: Elson (1991) Johnson BV Colour-magnitude Diagram for NGC 2157.

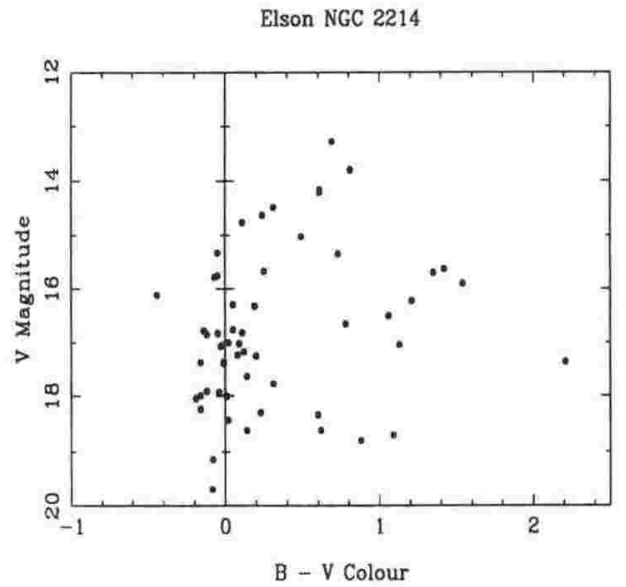

Figure 5-31: Elson (1991) Johnson BV Colour-magnitude Diagram for NGC 2214. 
correspond to this older branch can be seen, but the data are simply not of high enough quality to be sure. The CMD is similar to that of Alcaino \& Liller (1987) with the main sequence climbing to $\mathrm{V} \sim 16$, and a sparsely populated SGB. Nelson \& Hodge (1983) presented a photographic BV CMD for the cluster. The strong turnoff evident in their CMD is not confirmed. The Nelson \& Hodge (1983) CMD has a very weak SGB and a reasonably strong field component, similar to Figure 5-12. By comparison with the synthetic cluster CMDs of Harris \& Deupree (1976), Nelson \& Hodge (1983) estimated the cluster age to be $25 \pm 10$ million years $(\log 7.4)$, in good agreement with the age estimated through isochrone fits by this study (see Table 5.5 on page 110).

- NGC 1850: Figure 5-13 reveals a cluster with a strongly populated SGB and many blue stragglers. The same features may be seen in the CMDs of Alcaino \& Liller (1987) and Elson (1991). The CMD of Elson (1991) for NGC 1850 is given as Figure 5-27. The blue stragglers are scattered across the cluster, and are not associated with a lump protruding from the main body of the cluster (see Figure 3-4 on page 77 of Chapter 3), which Bhatia \& MacGillivray (1987) suggested was an interaction tail due to the presence of a binary companion. There is some evidence for a second and older SGB extending from a main sequence turnoff at $\mathrm{V} \sim 16$. The stars in this suggested SGB are located inside the lump (on the frame), suggesting that it is actually another star cluster. Given the apparent lack of tidal distortions of the main body, this second cluster could be in the line of sight (but still within the LMC). However, this work contradicts Fischer et al. (1993), who found that the blue stragglers were associated with the lump, which would then be a cluster younger than the main body. Further observations are needed.

- NGC 1854 is described in the RNGC as a small, round, and rather bright globular cluster which gradually brightens towards its centre. Robertson (1974) and Elson (1991) presented CMDs for this cluster, although Elson denoted the cluster as NGC 1855 and claimed that it had not been observed before. This is incorrect, with Shapley \& Lindsay (1963) noting that NGC 1854 and NGC 1855 are in fact the same object. The CMD given in Elson (1991) is similar to that in Figure 5-14. The clump of bright stars slightly to the red of the main sequence turnoff appears to be the blue loop of the SGB. Olszewski et al. (1991) note that the extent of the loop is strongly dependent on both age and metallicity for low metallicity clusters.

- The CMD of NGC 1863 derived by the current study is not very similar to the CMD of Alcaino \& Liller (1987), which had a turnoff around $V=16$. The main sequence extends much higher in Figure 5-15, with a sparsely populated SGB extending to $\mathrm{V} \sim 13$. This branch has a very extended blue loop, bending back to nearly the same colour as the main 
sequence. Alcaino \& Liller (1987) commented that it is a sparse cluster, and that it was hard to determine where the tip of the main sequence is.

- NGC 1968 and 1974 are two open clusters, described in the RNGC as a loose grouping of stars in nebulosity. There is no clear division between them, and there is no age difference apparent between the two objects since their colour magnitude diagrams are identical. They were therefore treated as one object in this study. Figure 5-16 shows this open cluster to be a very young object, with a steep and well-defined main sequence. A few stars appear to have passed the turnoff point.

- NGC 2004: The main sequence of this cluster is strongly populated and well-defined (see Figure 5-17), together with a sparsely populated SGB that has a red clump. Again, the CMD is very similar to that of Elson (1991), although there are less stars in Elson (1991) (see Figure 5-28). The field is not strong in either CMD. Balona \& Jerzykiewicz (1993) presented a BV CMD for NGC 2004 based on CCD observations collected with the SAAO $1 \mathrm{~m}$ telescope. The median seeing was $2^{\prime \prime}$. This CMD has better defined sequences, and extends to $\mathrm{V}=20$, but has the same morphology as Figure $5-17$.

- $N G C 2018$ has no published CMD. It is described in the RNGC as a reasonably large and bright globular cluster. A scattering of bright stars over a significantly fainter cluster body gives NGC 2018 an irregular appearance. Figure 5-18 shows the cluster to be young, with a bright SGB and reasonably strong main sequence.

- NGC 2031: Mould et al. (1994) presented a CMD based on Johnson B and Kron-Cousins R observations obtained with the CTIO $4 \mathrm{~m}$ telescope. A BV CMD was given by Olszewski et al. (1993), who found that a single isochrone could not explain the large luminosity spread of the Cepheid stars. The main sequence is broad in all three studies, terminating at slightly fainter than $\mathrm{V} \sim 16$, with a confused supergiant branch to the upper right of the turnoff. The CMDs are in good agreement, including confirmation of the strong field component. Mould et al. (1994) fitted the $\mathrm{Z}=0.008$ isochrones of Bertelli et al. (1990) to their data, and concluded that $150 \mathrm{Myr}(\log 8.14)$ is an upper estimate for the age of the cluster. Mould et al. (1994) adopted a reddening of $\mathrm{E}(\mathrm{B}-\mathrm{V})=0.18 \pm 0.05$. This value was calculated using the H I map of Mathewson \& Ford (1984), and is well within error of that derived by the current study from isochrone fits.

- NGC 2041: There is no published CMD for this cluster, and it is simply described in the RNGC as a bright, small, round globular cluster. Figure 5-20 shows the cluster to have a well-populated main sequence, with a well-defined turnoff point.

- NGC 2058 was observed by Flower (1982), who published a CMD based on photographic 
plates. An age of $1.2 \times 10^{8}$ years ( $\log 8.1$ ) was estimated using the isochrones of Flower (1976), which is older than the estimate made by this study (see Table 5.5). However, Flower (1982) estimated that the reddening $\mathrm{E}(\mathrm{B}-\mathrm{V})$ was 0.22 , well below the value of 0.33 calculated from Figure 5-21. The two CMDs are in reasonable agreement, showing the main sequence up to $\mathrm{V} \sim 16$, a well populated SGB with red clump, and a strong field. The broad main sequence apparent in Figure 5-21, and the subsequent difficulty in fitting isochrones to the CMD, could be the cause of the differences between the two studies.

- $N G C 2157$ has a very densely populated main sequence, but a relatively weak SGB. The SGB is not as well defined as in the CMD of Elson (1991), although the main sequence does terminate at the same position in the CMD. See Figure 5-29 for the CMD of Elson (1991).

- NGC 2164 has been observed by Elson (1991), Sagar et al. (1991a, b), and Hodge \& Flower (1973). All these studies produced CMDs, although the last study (in the list) was based on photography rather than CCD photometry. The morphology of the CMD (given as Figure 5-23) agrees well with that of Elson (1991), although it is shifted slightly blueward. The Elson (1991) CMD for NGC 2164 is given as Figure 5-30. The main sequence turnoff is at the same position in Figure 5-23 as that in the CMD of Sagar et al. (1991b). Both CMDs have the same morphology, complete with upturn at the end of the lower supergiant branch, with the one exception in that an older and poorly populated SGB is not visible in the former CMD. An old SGB is present in several of the CMDs given by Sagar et al. (1991b), but no comment was made of these features except for NGC 2214. The CCD-based CMD of Vallenari et al. (1991) for NGC 2164, based on observations with the ESO $2.2 \mathrm{~m}$ in $1^{\prime \prime}$ seeing, did not show the older branch either (see below for a discussion about the cause of such branches), but agreed well with Figure 5-23.

- NGC 2214: The CMD given as Figure 5-24 is similar in morphology to that given in Elson (1991), although it is better populated and sequences are better defined. The CMD of Elson is given as Figure 5-31. These differences are likely to be due to the observations of Elson (1991) being made in worse seeing than the MJUO observations, at 5.5". The CMD for NGC 2214 given by Sagar et al. (1991b) is even better defined and deeper than Figure 5-24, however there is an anomalous older SGB.

Bhatia \& MacGillivray (1988) argued that the rather elliptical cluster NGC 2214 is actually a binary star-cluster in an advanced stage of merging. The claim of Sagar et al. (1991a) to have detected two well-defined supergiant branches supported this notion. The older turnoff was slightly brighter than $\mathrm{V}=17$. A bump at this magnitude seemed to be 


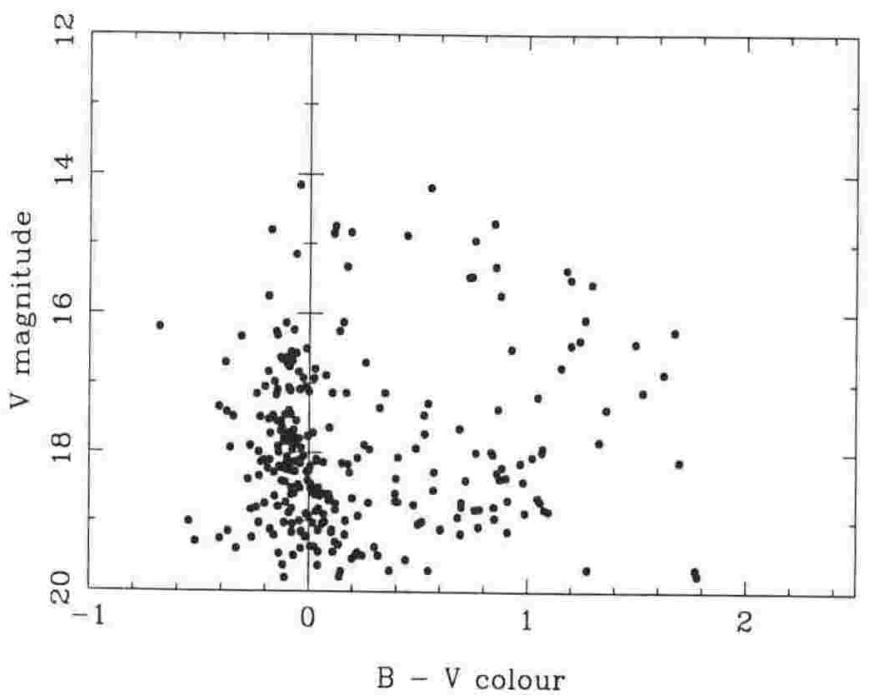

Figure 5-32: Long Exposure CMD for NGC 2214 obtained using the offset guider. Exposure lengths were 40 and 24.5 minutes for the Johnson B and $V$ filters respectively. The observing conditions were extremely poor.

apparent in the MJUO data.

The observations of Sagar et al. (1991a,b) were collected in $2^{\prime \prime}$ seeing with ESO $1.54 \mathrm{~m}$ Danish telescope. Poorer seeing combined with the fact that stars populating the older proposed SGB were centrally concentrated in the cluster, could have explained why this branch is not apparent in the CMD of Elson (1991) or of the current study. In October 1992 long exposures were taken at MJUO of the cluster, revealing what could be the second turnoff. Observing conditions were far from ideal, due to high winds combined with a dense cloud front covering most of the sky about the LMC. Seeing was $3.7^{\prime \prime}$. No standards were observed, and the transformation to the CMD given as Figure 5-32 was simply by matching 26 bright stars with Sagar et al. (1991b). The accuracy of these transformations was poor with uncertainties of 0.06 and 0.08 for the $\mathrm{V}$ and B magnitudes respectively. Once again, a second branch could almost be discerned. The idea that the branch was made of field stars was countered by the fact that a nearby field CMD had no stars in this region. When the positions of stars in these two 'branches' were examined it was found, in apparent contradiction of Sagar et al. (1991a), that the younger giants were centrally concentrated, while the fainter older giants were out at the edges. Features in the CMD of Sagar et al. (1991b) not seen in the MJUO CMD, such as a bright red clump of stars, were found to be due to the stars not being in the MJUO field of view, being lost due to the seeing through being in the dense centre of the cluster, or being blended with a close neighbour to the point of being so elliptical that DAOphot rejected 
them. The second branch was still in contention, with no conclusive evidence one way or the other.

The older supergiant branch did not appear in the CMD of Lee (1992), based on CCD observations obtained in $1.1^{\prime \prime}$ to $1.6^{\prime \prime}$ seeing with the Las Campanas du Pont $2.5 \mathrm{~m}$ telescope. To settle the problem, BV Images of NGC 2214 and E region standards were collected for the current study by the AAT Service Observing Program on March 1 1993, with seeing around $\sim 2.2^{\prime \prime}$. Not even a hint of an older branch is visible in the resulting CMD, confirming the results of Lee (1992). The AAT data are discussed further in Chapter 7 .

The problem appears to lie with the reduction techniques of Sagar et al. (1991a). One indicator of poor reductions are many bright stars blueward of the main sequence. There are plenty of such stars in their CMD, but few in the CMD of Lee (1992). If the existence of a second branch had not been suspected, the clump seen in the MJUO CMDs would have been rejected as being due to low number statistics.

- $S L 268$ has no published CMD. It appears to be a very young and sparsely populated cluster.

The survey results were then used to select the clusters with NGC numbers 2214, 1850, 1968/74, 2004, 2018, and 2041 as a short list of targets for the following observing season. The clusters are given in order of priority for subsequent observing. NGC 2214 was scheduled for further investigation in light of the suggestion that the cluster is actually two clusters in the process of merging together. NGC 1850 was selected due to its unusual structure and the presence of many blue stragglers. The remainder of the clusters were chosen as they possessed well-defined and reasonably populated main sequences, suitable for preparation of luminosity (and subsequent mass) functions over a large magnitude range. A mass function has already been derived for NGC 2004 by Sagar et al. (1991a). The inclusion of this cluster was therefore to act as a check via comparison of the derived mass function with that of Sagar et al. (1991a). Clusters that may have appeared to be suitable from lists such as Searle et al. (1980), or Freeman et al. (1983), often proved not to be so by being too densely packed for DAOphot to resolve stars well given the typical seeing at MJUO, or being too small, or in too dense a field. Preference was given in this list to clusters that had no published CMDs or showed interesting properties.

The offset guider was available for the 1992/93 observing season, making possible attempts to collect long exposure observations. Attempts were also made to collect short exposures with the same positioning relative to a cluster, which could then be added together to synthesize longer exposure Images. Unfortunately the weather was very poor over this summer, being 
NGC 1974 / NGC 1968 Dec '92

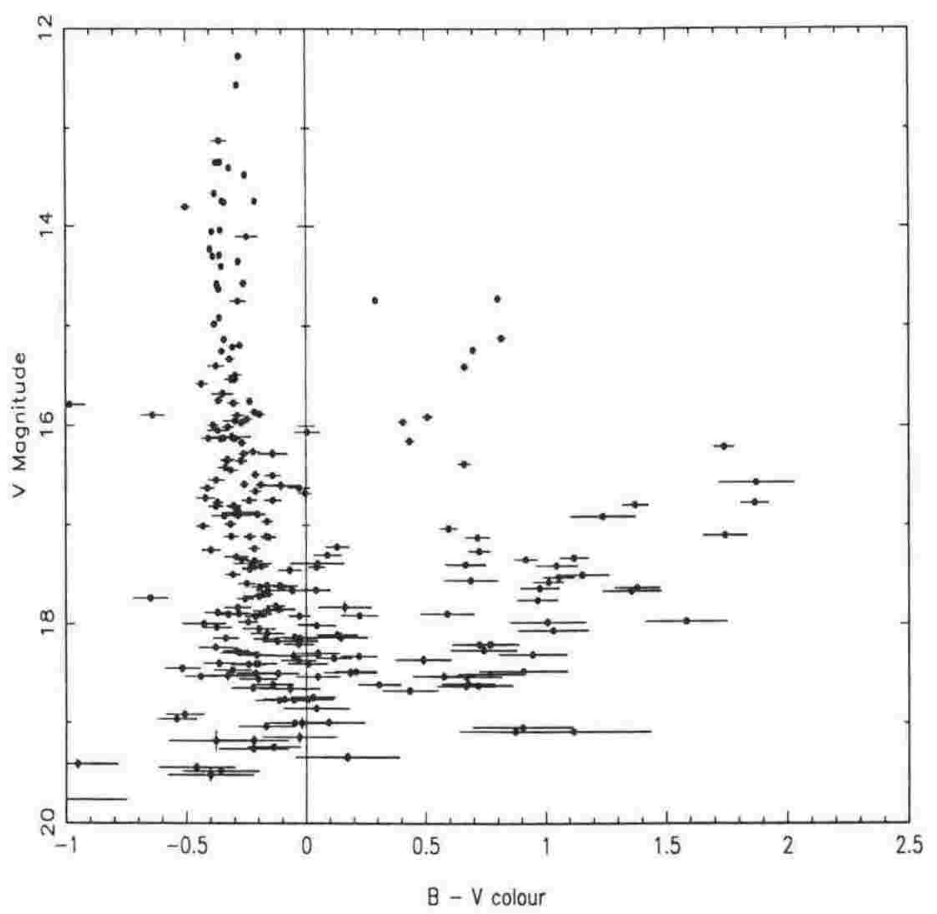

Figure 5-33: CMD for NGC 1968/74 based on co-added images which were individually of five minutes exposure, and were manually guided. The total exposures were 30 and 45 minutes in $\mathrm{B}$ and $\mathrm{V}$ respectively.

detrimentally affected by the Pinatubo eruption. Attempts to acquire guided short exposure Images of NGC 1968/74 for addition into longer exposure Images, led to a CMD which was not a substantial improvement over the CMD based on survey Images (see Figure 5-33), although the photometric uncertainties of the brighter stars were reduced. These observations were collected in poor $3.5^{\prime \prime}$ seeing on December $27^{\text {th }} 1992$, as opposed to the $2^{\prime \prime}$ seeing of the November 1991 data. The differences between Figures 5-33 and 5-16 lie in the CCD being attached in December 1992 at right angles to its orientation the year before. Therefore, some different stars were imaged, and others excluded.

Only one other night during this season was partially clear for Johnson CCD imaging, and was of worse quality. The results of an attempt to acquire guided long exposure Images of NGC 2214 on that night have been discussed above.

\subsection{Profile Fitting}

King (1962) showed that three parameters can describe images of globular clusters:

- A core radius $\left(\mathrm{r}_{\mathrm{c}}\right)$, specifying the size of a region of constant density near the cluster centre; 


\begin{tabular}{||l|c|c|c|c|c|c|c||}
\hline \multirow{2}{*}{ Cluster } & \multicolumn{4}{|c|}{ Linear Least Squares Technique } & \multicolumn{2}{c||}{ Kinman \& Rosino } & \multicolumn{2}{c||}{ Petersen \& King } \\
\cline { 2 - 8 } & $\mathrm{k}$ & $\mathrm{r}_{\mathrm{c}}$ & $\mathrm{r}_{\mathrm{t}}$ & $\mathrm{r}_{\mathrm{c}}$ & $\mathrm{r}_{\mathrm{t}}$ & $\mathrm{r}_{\mathrm{c}}$ & $\mathrm{r}_{\mathrm{t}}$ \\
\hline Abell 1 & $0.42 \pm 0.01$ & $0.54 \pm 0.01$ & $2.85 \pm 0.03$ & 0.5 & 2.5 & 0.6 & 5.0 \\
Abell 5 & $2.56 \pm 0.06$ & $3.34 \pm 0.06$ & $8.18 \pm 0.07$ & 2.0 & 10 & 2.9 & $10^{+}$ \\
Abell 7 & $2.29 \pm 0.06$ & $1.99 \pm 0.11$ & $7.27 \pm 0.26$ & - & 6.0 & - & - \\
Abell 11 & $1.69 \pm 0.02$ & $1.08 \pm 0.01$ & $5.79 \pm 0.05$ & 1.0 & 5.3 & 1.3 & 7.4 \\
Abell 12 & $1.14 \pm 0.02$ & $0.62 \pm 0.01$ & $3.71 \pm 0.08$ & 0.4 & 3.6 & 0.5 & $5^{+}$ \\
Abell 13 & $2.77 \pm 0.09$ & $0.59 \pm 0.02$ & $1.07 \pm 0.01$ & 0.3 & 1.05 & 0.5 & $1.3^{+}$ \\
Anon & $2.71 \pm 0.05$ & $1.43 \pm 0.03$ & $3.74 \pm 0.03$ & 1.4 & 4.2 & - & - \\
\hline
\end{tabular}

Table 5.7; Optimum King Model Parameters. Kinman \& Rosino (1962) fitted King models to photographic star counts. These data were used as part of the testing of the linear least-squares program. Results of the tests are given, along with the parameters derived by Kinman \& Rosino (1962), and Petersen \& King (1975). The errors given by the program were calculated assuming a data accuracy of $1 \%$, which is certainly over-optimistic, although Kinman \& Rosino (1962) gave the stellar densities to four decimal places implying a greater accuracy still. Neither of the two comparison papers gave error limits for the derived parameters. The cluster names are as in Kinman \& Rosino (1962). All radii are in arcminutes. All solutions were determinate.

- A tidal radius $\left(\mathrm{r}_{\mathrm{t}}\right)$, being the tidal limit defined by the gravity of the galaxy that the cluster is orbiting about;

- and a scaling factor $\mathrm{k}$.

The empirical formulation of King (1962) is:

$$
\mathrm{f}=\mathrm{k}\left(\frac{1}{\sqrt{1+\left(\frac{\mathrm{r}}{\mathrm{r}_{\mathrm{c}}}\right)^{2}}}-\frac{1}{\sqrt{1+\left(\frac{\mathrm{r}_{\mathrm{c}}}{\mathrm{r}_{\mathrm{c}}}\right)^{2}}}\right)^{2}
$$

where $\mathrm{f}$ is the intensity per pixel (or surface density), and $\mathrm{r}$ the radius.

King (1966a) commented that his later dynamical models agree closely with the empirical curves. This formula was used as the fitting function for a non-linear least-squares computer program. The program iterated automatically, selecting each iteration to alter one parameter to improve the $\chi^{2}$ goodness of fit after examining variations in all the available parameters. Iterations ceased once the decrease in $\chi^{2}$ fell within a user-set limit, or when the number of permissible iterations was exceeded. The Hessian matrix was then evaluated. This matrix is made up of the second-order partial derivatives of the fitting function. Breakdown of the matrix (indeterminacy) occurs when zero or negative numbers enter any of the diagonal values of the matrix, indicating that the solution is not a closed minimum in $\chi^{2}$-space. The Hessian, and in particular its inverse or the error matrix (Bevington 1969), can be used to derive a standard deviation error for the optimised parameters once given an estimate of the accuracy of the data. See Table 5.7 for tests of the program with real data. 


\begin{tabular}{||c|c|r|r|r|r|c|c|c||}
\hline Cluster & Filter & \multicolumn{1}{|c|}{$\mathrm{r}_{\mathrm{c}}$} & Elson $\mathrm{r}_{\mathrm{c}}$ & \multicolumn{1}{c|}{$\mathrm{r}_{\mathrm{t}}$} & \multicolumn{1}{c|}{$a$} & EFF $a$ & $\gamma$ & EFF $\gamma$ \\
\hline NGC 1818 & $\mathrm{V}$ & $6.9 \pm 2.4$ & 7.9 & $390 \pm 220$ & $9.2 \pm 4.1$ & 9 & $2.5 \pm 0.4$ & $2.45 \pm 0.25$ \\
NGC 1818 & $\mathrm{B}$ & $7.9 \pm 1.8$ & 7.9 & $350 \pm 30$ & $8.6 \pm 5.3$ & & $2.9 \pm 1.2$ & \\
\hline NGC 1850 & $\mathrm{V}$ & $10.7 \pm 1.5$ & 8.8 & $260 \pm 180$ & $9.5 \pm 3.1$ & & $1.9 \pm 0.3$ & \\
NGC 1850 & $\mathrm{B}$ & $11.1 \pm 2.1$ & 8.8 & $300 \pm 90$ & $11.9 \pm 3.7$ & & $2.2 \pm 0.3$ & \\
\hline NGC 1856 & $\mathrm{V}$ & $8.7 \pm 0.7$ & & $1130 \pm 320$ & $8.1 \pm 1.4$ & & $2.1 \pm 0.1$ & \\
NGC 1856 & $\mathrm{B}$ & $7.1 \pm 1.0$ & & $1250 \pm 100$ & $7.2 \pm 1.4$ & & $1.9 \pm 0.1$ & \\
\hline NGC 2004 & $\mathrm{V}$ & $5.1 \pm 2.7$ & 4.3 & $700 \pm 570$ & $8.6 \pm 2.6$ & 5 & $1.8 \pm 0.3$ & $2.20 \pm 0.20$ \\
NGC 2004 & $\mathrm{B}$ & $5.0 \pm 3.2$ & 4.7 & $1310 \pm 500$ & $8.1 \pm 4.4$ & & $2.1 \pm 0.4$ & \\
\hline NGC 2031 & $\mathrm{V}$ & $10.2 \pm 1.4$ & & $1150 \pm 190$ & $6.3 \pm 2.3$ & & $1.6 \pm 0.2$ & \\
NGC 2031 & $\mathrm{B}$ & $9.0 \pm 1.8$ & & $900 \pm 160$ & $8.7 \pm 2.6$ & & $2.0 \pm 0.3$ & \\
\hline NGC 2041 & $\mathrm{V}$ & $5.5 \pm 1.2$ & & $1370 \pm 620$ & $6.7 \pm 1.8$ & & $2.2 \pm 0.2$ & \\
NGC 2041 & $\mathrm{B}$ & $5.6 \pm 1.5$ & & $630 \pm 560$ & $7.1 \pm 2.1$ & & $2.3 \pm 0.3$ & \\
\hline NGC 2157 & $\mathrm{V}$ & $7.1 \pm 1.0$ & 8.6 & $1840 \pm 80$ & $9.6 \pm 3.1$ & 7 & $2.3 \pm 0.4$ & $2.90 \pm 0.27$ \\
NGC 2157 & $\mathrm{B}$ & $6.8 \pm 1.5$ & 7.9 & $980 \pm 50$ & $8.0 \pm 2.2$ & & $2.2 \pm 0.2$ & \\
\hline NGC 2164 & $\mathrm{V}$ & $5.4 \pm 1.5$ & 5.7 & $630 \pm 190$ & $6.7 \pm 2.8$ & 9 & $2.3 \pm 0.3$ & $2.80 \pm 0.30$ \\
NGC 2164 & $\mathrm{B}$ & $5.3 \pm 2.7$ & 5.9 & $340 \pm 130$ & $5.7 \pm 4.2$ & & $2.3 \pm 0.9$ & \\
\hline NGC 2214 & $\mathrm{V}$ & $9.8 \pm 2.9$ & 8.5 & $770 \pm 250$ & $9.4 \pm 6.3$ & 11 & $2.0 \pm 0.6$ & $2.40 \pm 0.24$ \\
NGC 2214 & $\mathrm{B}$ & $9.5 \pm 2.5$ & 8.6 & $750 \pm 110$ & $10.4 \pm 3.9$ & & $2.2 \pm 0.4$ & \\
\hline
\end{tabular}

Table 5.8: Profile Fit Parameters. The values of $\mathrm{r}_{c}, a$, and $\gamma$ derived by the current study are compared with the values given by Elson et al. (1987) and Elson (1991). Elson et al. (1987) is abbreviated as EFF. All radii are given in arcseconds. Chun (1978) fitted King models to NGC 1818 and NGC 2157. The resulting core and tidal radii were $\left(5.6^{\prime \prime}, 178^{\prime \prime}\right)$ and $\left(7.2^{\prime \prime}, 188^{\prime \prime}\right)$ for each cluster respectively. The core radii are in good agreement with the present study, while the tidal radii are not. The core radii of the current study agree well with the results of Elson (1991).

Subramaniam et al. (1993) listed $\gamma$ values derived by Elson (1991) for the clusters NGC 2004, 2164 , and 2214 . These values were $2.0,2.8$, and 2.3 respectively, while Subramaniam et al. (1993) derived $2.4 \pm 0.1,2.5 \pm 0.3$, and $2.4 \pm 0.1$ for the same clusters. The $\gamma$ values are in reasonable, but not good, agreement between the three studies. Possibly the differences are because the exposure lengths of Elson (1991) and the current study were too short to well define the outer regions of the clusters. $\gamma$ therefore could not be confidently determined. This is presumably responsible for the difference between the $\gamma$ values derived for NGC 2214 and those based on the AAT data (see Chapter 7 ), although the uncertainties overlap.

Synthetic aperture photometry was performed on the largest of the LMC clusters observed in the survey. Many of the clusters were too small for good aperture photometry, and so were omitted. Circular apertures were used, rather than slightly elliptical apertures. Tests revealed no significant differences between circular and elliptical aperture profiles, supporting the contention of Elson (1991). Each aperture ring was divided into eight segments. The standard deviation of the intensity of these segments was taken to be the photometric uncertainty for the annulus.

Elson (1991) noted that most of the noise in the cluster profiles is due to the presence of young, and therefore bright, stars. Elson (1991) therefore subtracted the bright stars using the appropriate PSF for a CCD Image, and claimed that there was no difficulty in achieving the detection and removal of such stars even in the centre of a cluster. This is not supported by the tests described in Section 5.4 .5 where bright stars were 'removed' from clusters. The subtractions were not clean. Therefore, this technique was not used by the current study. 
The results of King model fits are presented in Table 5.8. See Figure 5-34 for an example profile and model fit. The tidal radii were not well constrained, with the clusters not appearing to be tidally truncated. Elson et al. (1987) and Elson (1991) noted the same fact in their profiles of young LMC clusters, and instead fitted an untruncated power-law model (whose form was chosen purely for mathematical convenience):

$$
\mu(\mathrm{r})=\mu_{0}\left(1+\frac{\mathrm{r}^{2}}{a^{2}}\right)^{\frac{-\gamma}{2}} .
$$

Therefore, this model was used as the fitting function in the program, producing results listed in Table 5.8. The $\chi^{2}$ for these fits was on average $\sim 15 \%$ better than for the corresponding best-fit King models. Elson et al. (1987) suggested that the clusters were surrounded by a halo of unbound stars. Such a halo would be expected if a cluster expanded across its tidal limit because of mass loss. The unbound halo of stars would last several orbits of the cluster about the parent galaxy before they were stripped from the vicinity of the cluster. The clusters studied by Elson et al. (1987), and the current study, have not yet completed a single orbit about the LMC.

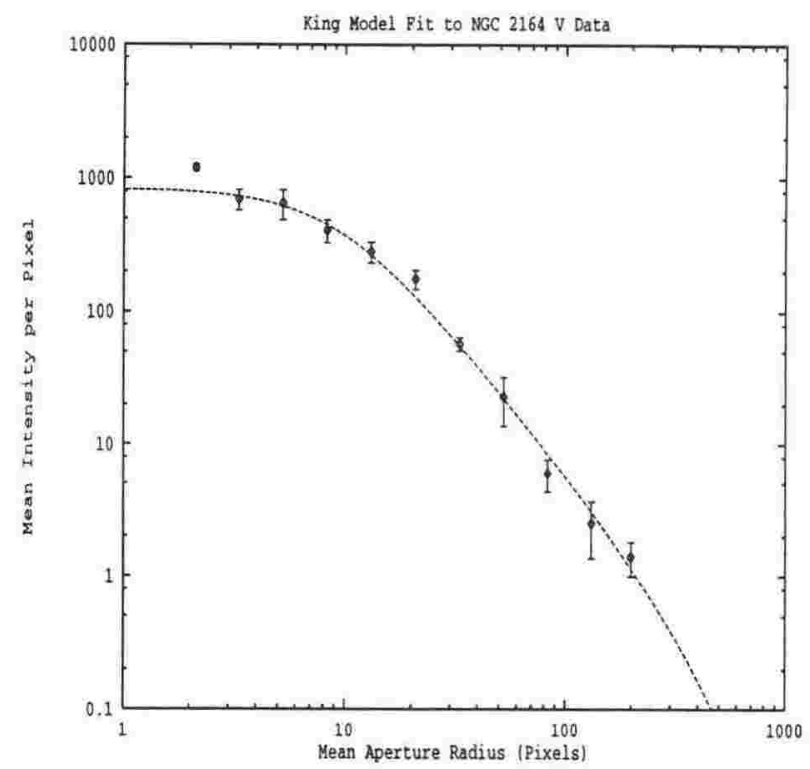

Figure 5-34: King Model Fit to NGC 2164 V Data. The best-fitting model profile is given as the smooth line. Note the unusually high intensity in the centre of the cluster which was due to a single bright star.
Elson (1991) did not present values for $\gamma$ for the profile fits to her CCD data, instead commenting that the values fell into the range $2.2 \leqq \gamma$ $\leqq 3.2$. The profiles were less extensive than those of Elson et al. (EFF) (1987), which were based on aperture photometry and star counts from photographs. Elson (1991) therefore considered that the outer regions of the profiles would not be as accurate as those of EFF, and concentrated on the core radii. The same comment can be made on the reliability of the values of $\gamma$ derived here, which are in general less than those of EFF. They are also less well constrained, and extend below the lower limit of the previous study. The values of $a$ derived by the current study are not in good agreement with those of EFF, but nor do the values given by EFF agree well with those of Elson (1991). 
EFF commented the smallest values of $a$ (i.e., 7 " to $9^{\prime \prime}$ ) were "very poorly determined", while they considered that their intermediate values $\left(9-11^{\prime \prime}\right)$ could be uncertain by up to $50 \%$. The lack of agreement between EFF and the current work for the radii $a$ is therefore not of great concern.

The Images have too bright a limiting magnitude for the technique of Subramaniam et al. (1993) to be adopted. This method investigated the clusters for signs of mass segregation. Subramaniam et al. (1993) applied this technique to the CCD Images of Sagar et al. (1991a, b) in order to derive the spatial distribution of different stellar masses in the clusters. Out of 5 clusters, only one exhibited evidence for mass segregation. ${ }^{1}$ Similarly, Elson et al. (1987) found that only one of their clusters showed any evidence for mass segregation. Subramaniam et al. (1993) confirmed that the density profiles of young LMC clusters decrease in the outer radii of the clusters as power-laws with indices $\gamma+1 \sim 3.5$. The current study finds $\gamma$ to be $2.16 \pm 0.29$, which is consistent with the results of Elson et al. (1987), Elson (1991), and Subramaniam et al. (1993).

Elson et al. (1987) found that the core radii of LMC clusters appeared to increase from $\sim 0$ to $\sim 5$ parsecs between the cluster ages of $\sim 10^{6}$ and $10^{9}$ years, before decreasing again. The core expansion was proposed to be driven by mass loss from evolving stars. According to the cluster evolution models of Chernoff \& Weinberg (1990), the observed variation in the core radii favoured an initial mass function for the clusters with $x \sim 1$, although there was considerable scatter in radii between clusters of approximately the same age. To investigate this age effect, the core radii listed in Table 5.8 were plotted against the ages given in Ta-

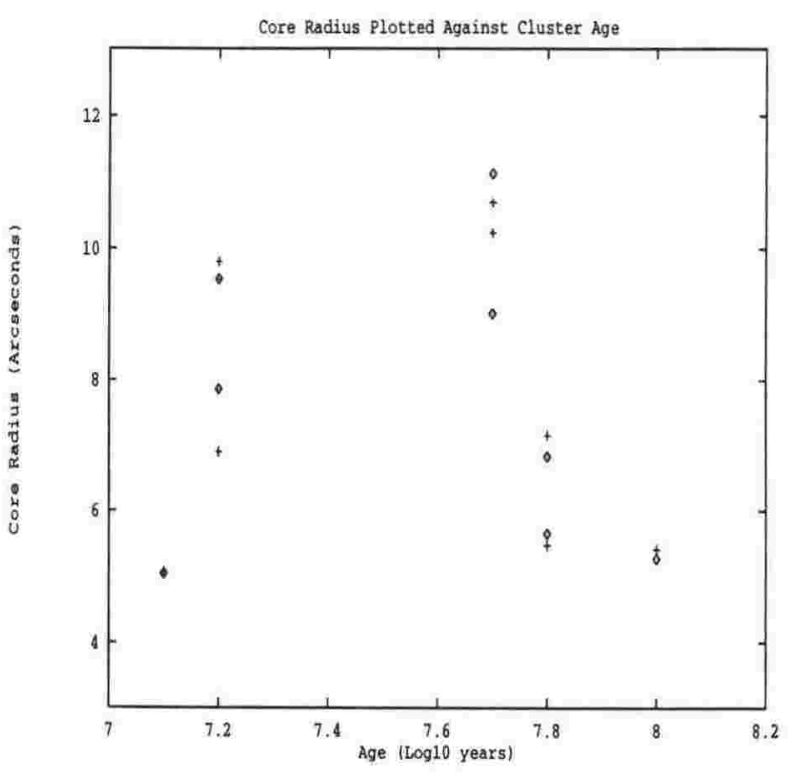

Figure 5-35: Cluster Core Radius Against Log Age. The core radii were estimated from the profile fitting, while the ages are based on the isochrone fits described above. The + symbols indicate values derived from $\mathrm{V}$ frames, while the $\mathrm{B}$ values are indicated by the $\diamond$ symbols.

ble 5.5. Only a short age range was available, being between 10 and 100 million years (see Figure 5-35). Linear least-squares fitting indicated a gradient of $0.01 \pm 0.04$ arcseconds per log year. The current observations therefore can not confirm the relation of Elson et al. (1987) and Elson (1991). This is interesting in the

\footnotetext{
${ }^{1}$ It would be interesting to prepare CCD frames of artificial star clusters both with and without mass segregation in order to test how accurately any trends are recovered by such techniques.
} 


\begin{tabular}{|c|c|c|c|c|c|c|c|}
\hline \multirow{2}{*}{$\begin{array}{c}\text { Cluster } \\
\text { Name }\end{array}$} & \multicolumn{2}{|c|}{ Frenk \& Fall } & \multicolumn{2}{|c|}{ Geisler \& Hodge } & \multicolumn{2}{|c|}{ Kontizas et al. } & \multirow{2}{*}{$\begin{array}{l}\text { Geyer et al } \\
\text { Ellipticity }\end{array}$} \\
\hline & Ellip & P.A. & Ellip & P.A. & Ellipticity & Radius & \\
\hline NGC 1651 & 0.03 & - & 0.31 & 126 & - & - & 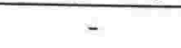 \\
\hline NGC 1751 & 0.12 & 55 & 0.26 & 133 & 0.12 & $0.9^{\prime}$ & - \\
\hline NGC 1751 & - & - & - & - & 0.16 & $0.5^{\prime}$ & - \\
\hline NGC 1754 & 0.06 & 38 & 0.11 & 108 & - & - & - \\
\hline NGC 1783 & 0.25 & 64 & 0.19 & 72 & - & - & - \\
\hline NGC 1786 & 0.02 & - & 0.09 & 166 & 0.12 & - & 0.00 \\
\hline NGC 1806 & 0.09 & 9 & 0.19 & 159 & 0.07 & $1.8^{\prime}$ & 0.05 \\
\hline NGC 1806 & - & - & - & - & 0.12 & $0.9^{\prime}$ & - \\
\hline NGC 1835 & 0.21 & 88 & 0.17 & 77 & 0.14 & $0.13^{\prime}$ & 0.21 \\
\hline NGC 1835 & - & - & - & - & 0.21 & $0.16^{\prime}$ & - \\
\hline NGC 1835 & - & - & - & - & 0.19 & $0.30^{\prime}$ & - \\
\hline NGC 1846 & 0.08 & 151 & 0.23 & 129 & 0.07 & $1.9^{\prime}$ & 0.13 \\
\hline NGC 1846 & - & - & - & - & 0.16 & $0.11^{\prime}$ & - \\
\hline NGC 1917 & 0.07 & 168 & 0.29 & 4 & 0.15 & - & 0.00 \\
\hline NGC 1978 & 0.33 & 150 & 0.30 & 159 & - & - & - \\
\hline NGC 2019 & 0.07 & 139 & 0.20 & 124 & 0.17 & $1.0^{\prime}$ & - \\
\hline NGC 2019 & - & - & - & - & 0.23 & $0.5^{\prime}$ & - \\
\hline NGC 2108 & 0.11 & 80 & 0.18 & 115 & - & - & - \\
\hline NGC 2121 & 0.18 & 21 & 0.32 & 64 & - & - & - \\
\hline NGC 2154 & 0.17 & 36 & 0.13 & 42 & - & - & - \\
\hline NGC 2155 & 0.08 & 102 & 0.27 & 76 & - & - & - \\
\hline NGC 2173 & 0.06 & 70 & 0.27 & 141 & - & - & - \\
\hline NGC 2210 & 0.07 & 78 & 0.12 & 82 & - & - & - \\
\hline NGC 2213 & 0.01 & - & 0.26 & 91 & - & - & - \\
\hline
\end{tabular}

Table 5.9: Literature Values for Ellipticities and Position Angles (P.A.s) of LMC clusters common to Frenk \& Fall (1982) and Geisler \& Hodge (1980). Where available, the ellipticity values of Kontizas et al. (1989) and Geyer et al. (1983) are also shown. Note the frequent discrepancies, even for clusters which are given as very elliptical in one catalogue (e.g. NGC 2121,2155 , \& 2173). Kontizas et al. claim that such differences are due to internal variations in the clusters, and so the radii that an ellipticity is measured at must also be specified. Where Kontizas et al. have given more than one value for a cluster, the extra values are given on subsequent lines. Generally they measured ellipticity at the half mass radius (see King 1966a), which is a constant throughout the dynamical evolution of a cluster.

light that the observations of Elson (1991) are of similar, if not worse, quality to those of the current study.

\section{$5.4 \quad$ Ellipse Fitting}

\subsubsection{Introduction}

Globular clusters belonging to the Galaxy are effectively spherical in shape, with a mean ellipticity (defined as for elliptical galaxies) of 0.12 (Shapley 1930b). One of the most elliptical Galactic globular clusters is $\omega$ Centauri with ellipticity estimates of 0.14 (Dickens \& Woolley 1967) and 0.19 (Van den Bergh 1983, Frenk \& Fall 1983). 
It has been known for many years that some of the brightest old clusters in the Magellanic Clouds are strongly flattened (Van den Bergh 1983). Geisler \& Hodge (1980) used microdensitometry of photographic plates for 25 LMC star clusters, and found a mean ellipticity of 0.22 , which they commented was far above the Galactic mean. They also noted some internal variations in the position angle of the fitted ellipses as well as in the ellipticity itself. Radial variations were also reported by Geyer et al. (1983) and Zepka \& Dottori (1987), and commented on by Kontizas et al. (1989) as a possible explanation for the discrepancies in the elliptical parameters derived by different investigators for the same clusters (see Table 5.9).

Van den Bergh (1983) noted that the more luminous LMC clusters of any age are more flattened that fainter clusters. Frenk \& Fall (1982) estimated by eye 52 LMC cluster ellipticities, although their mean value was $0.12 \pm 0.07$, which should be compared with their mean estimate for 93 Galactic globular clusters of $0.08 \pm 0.05$. Using the age classes of Searle et al. (1980) Frenk \& Fall noted an apparent $97 \%$ correlation of ellipticity with age, with the younger clusters being flatter on average than the older clusters, which were similar to Galactic globulars in shape. They suggested that this was the result of internal evolution of the clusters. Van den Bergh \& Morbey (1984) demonstrated that this correlation was not statistically significant after the dependence of ellipticity on luminosity was removed, given that the brightest blue clusters are more luminous that the brightest red clusters, so making these two correlations not mutually exclusive. A two-tailed Kolmogorov-Smirnov test showed that the hypothesis that the LMC clusters and Galactic globulars are from the same parent population with the same ellipticity distribution can be rejected at the $99.2 \%$ confidence level.

The aim of this section is to investigate the internal variations of elliptical parameters in a sample of LMC clusters. Initially, a standard package was used to fit ellipses to the clusters, but dissatisfaction with its results led to the use of a moments-based technique (as outlined by Stobie (1980a, b) and described below).

\subsubsection{Method}

A major advantage in using moments (see Larson, 1982, for a general background) for image analysis lies in their ease of calculation, e.g. the summation for the second moment can be calculated without knowledge of the mean, which is only needed in the final step:

$$
\sum_{i=1}^{n}\left(x_{i}-\bar{x}\right)^{2} f_{i}=\sum_{i=1}^{n} x_{i}^{2} f_{i}-\bar{x}^{2} \sum_{i=1}^{n} f_{i}
$$

where $f_{i}$ is the intensity of the $i$ th background subtracted $x$ pixel. The $x$ axis zeroth and normalised first-order moments: 


$$
\sum_{i=1}^{n} f_{i} \text { and } \frac{\sum_{i=1}^{n} x_{i} f_{i}}{\sum_{i=1}^{n} f_{i}}
$$

can be easily interpreted as the total intensity of the object and the normalised intensityweighted $x$ centroid. The second-order moments are harder to interpret, but give information on the structure of the pixel cluster, as will be shown for the general elliptical case. Consider an ellipse described by:

$$
\frac{x_{1}^{2}}{a^{2}}+\frac{y_{1}^{2}}{b^{2}}=1
$$

in the arbitrarily rotated Cartesian frame $\left(x_{1}, y_{1}\right)$. For simplicity in the following, it is also assumed that $f_{(i, j)}$ is uniform for all $x$ and $y$, making the zeroth moment simply the area of the elliptical distribution. For a continuous two-dimensional distribution, the second-order moments are:

$$
\begin{gathered}
U_{x x}=\iint \frac{\left(x_{1}-\overline{x_{1}}\right)^{2} d x_{1} d y_{1}}{A}, \\
U_{y y}=\iint \frac{\left(y_{1}-\overline{y_{1}}\right)^{2} d x_{1} d y_{1}}{A}, \text { and } \\
U_{x y}=\iint \frac{\left(y_{1}-\overline{y_{1}}\right)\left(x_{1}-\overline{x_{1}}\right) d x_{1} d y_{1}}{A}
\end{gathered}
$$

where the area $A$ of the ellipse is $\pi a b$. The ellipse is assumed to be centred on the origin, i.e., $\overline{x_{1}}=\overline{y_{1}}=0$. Noting the four-fold symmetry of an ellipse, the second-order moment in $x$ can be written as:

$$
\iint x_{1}^{2} d x_{1} d y_{1}=4 \int_{0}^{a} x_{1}^{2} y_{+} d x_{1}=4 b \int_{0}^{a} x_{1}^{2} \sqrt{1-\frac{x_{1}^{2}}{a^{2}}} d x_{1}
$$

where $y_{+}$refers to the function in the first quadrant. Substituting $x_{1}=a \sin \theta$, the second-order moment is given by:

$$
\frac{a^{3} b}{8} \int_{0}^{\frac{\pi}{2}}(1-\cos \theta) d \theta=\frac{\pi a^{3} b}{4}
$$

Similarly, it can be shown that for an ellipse the other second-order moments are:

$$
\iint x_{1} y_{1} d x_{1} d y_{1}=0
$$

and

$$
\iint y_{1}^{2} d x_{1} d y_{1}=\frac{\pi a b^{3}}{4}
$$

Rotation of the $\left(x_{1}, y_{1}\right)$ co-ordinate axes back to the standard $(x, y)$ co-ordinates is given by: 


$$
x=x_{1} \cos \theta-y_{1} \sin \theta
$$

and

$$
y=x_{1} \sin \theta+y_{1} \cos \theta
$$

where $\theta$ is the rotation about the common centre of origin between the two reference frames (see p.701, Anton 1980). As the area element $d A=d x_{1} d y_{1}=d x d y$ then

$$
\begin{gathered}
\iint x^{2} d x d y=\iint\left(x_{1} \cos \theta-y_{1} \sin \theta\right)^{2} d x_{1} d y_{1}, \\
\iint x y d x d y=\iint\left(x_{1} \cos \theta-y_{1} \sin \theta\right)\left(x_{1} \sin \theta+y_{1} \cos \theta\right) d x_{1} d y_{1}, \text { and } \\
\iint y^{2} d x d y=\iint\left(x_{1} \sin \theta+y_{1} \cos \theta\right)^{2} d x_{1} d y_{1} .
\end{gathered}
$$

If the normalised continuous moments are defined as:

$$
\begin{aligned}
U_{x x} & =\frac{\iint x^{2} d x d y}{A} \\
U_{y y} & =\frac{\iint y^{2} d x d y}{A}
\end{aligned}
$$

and

$$
U_{x y}=\frac{\iint x y d x d y}{A}
$$

then the equations can be re-expressed, using the transformation given as Equations 5.10 and 5.11, as:

$$
\begin{gathered}
U_{x x}=U_{x_{1} x_{1}} \cos ^{2} \theta-2 U_{x_{1} y_{1}} \cos \theta \sin \theta+U_{y_{1} y_{1}} \sin ^{2} \theta \\
U_{y y}=U_{x_{1} x_{1}} \sin ^{2} \theta+2 U_{x_{1} y_{1}} \cos \theta \sin \theta+U_{y_{1} y_{1}} \cos ^{2} \theta \\
U_{x y}=\left(U_{x_{1} x_{1}}-U_{y_{1} y_{1}}\right) \cos \theta \sin \theta+\left(\cos ^{2} \theta-\sin ^{2} \theta\right) U_{x_{1} y_{1}} .
\end{gathered}
$$

Substituting Equations 5.7, 5.8, and 5.9 into Equations 5.18, 5.19, and 5.20 gives:

$$
\begin{aligned}
4 U_{x x} & =a^{2} \cos ^{2} \theta+b^{2} \sin ^{2} \theta \\
4 U_{y y} & =a^{2} \sin ^{2} \theta+b^{2} \cos ^{2} \theta \\
4 U_{x y} & =\left(a^{2}-b^{2}\right) \cos \theta \sin \theta,
\end{aligned}
$$




\begin{tabular}{||c|c|r|r|r||}
\hline \multicolumn{3}{||c|}{ Ellipticity $e$} & \multicolumn{2}{c||}{ Angle $\theta$} \\
\hline In & Out & \multicolumn{1}{|c|}{ Ratio } & \multicolumn{1}{|c|}{ In } & Out \\
\hline 0.033 & 0.034 & 103.0 & 90.0 & 89.5 \\
0.200 & 0.199 & 99.7 & 0.0 & 0.0 \\
0.250 & 0.250 & 100.0 & 0.0 & 0.0 \\
0.500 & 0.495 & 98.9 & 115.0 & 114.5 \\
0.571 & 0.569 & 99.3 & 110.0 & 109.9 \\
0.631 & 0.626 & 99.2 & 45.0 & 45.0 \\
0.792 & 0.784 & 98.9 & 145.0 & 145.0 \\
0.800 & 0.794 & 99.3 & 175.0 & 175.1 \\
\hline
\end{tabular}

Table 5.10: Test Ellipticity and Orientation Angle values. Input and output ellipticities $(e)$ and orientation angles $(\theta)$ are shown for a few selected tests, showing that the input values are well recovered by the moment analysis technique. Ratio gives the output ellipticity as a percentage of the input value. The angles are given in degrees.

or three equations for three unknowns. Solving results in:

$$
\begin{gathered}
\theta=\frac{1}{2} \arctan \left(\frac{2 U_{x y}}{U_{x x}-U_{y y}}\right) \\
a=\sqrt{2\left(\left(U_{x x}+U_{y y}\right)+\sqrt{\left(\left(U_{x x}-U_{y y}\right)^{2}+4 U_{x y}^{2}\right)}\right)} \\
b=\sqrt{2\left(\left(U_{x x}+U_{y y}\right)-\sqrt{\left(\left(U_{x x}-U_{y y}\right)^{2}+4 U_{x y}^{2}\right)}\right)} .
\end{gathered}
$$

Thus the size, orientation, and ellipticity (as $e=1-b / a$ ) of the ellipse can be obtained. Similar equations can be derived for weighted moments. Weighted first-order moments were used to determine the centre of a pixel distribution (Dodd \& MacGillivray 1986), while the ellipse fitting applied no weighting. The latter results in the determined shape and orientation of the distribution being more representative of the overall distribution, rather than being skewed by the brighter central regions. In asymmetric distributions, the former point means that the derived centre is the centre of mass.

\subsubsection{The Fitting Software}

A FORTRAN program was written based on Equations 5.24, 5.25, and 5.26, using the IRAF IMFORT libraries for the Image manipulation routines. Threshold values were read from a file. The background of an Image was estimated using the IRAF imex tool in clear (star and cosmic strike free) regions of the Image.

The Image pixel array was scanned starting at pixel $(1,1)$. The array dimensions were automatically determined by the software. The first direction of search was along the $x$ axis. When the end of this row was reached, the $x$ position was reset to 1 and the $y$ position incre- 
mented. This continued until the opposite corner of the Image was met. When the intensity of the search's current pixel was above the threshold set for the ellipse fitting, an interior-defined seed-fill algorithm (see p.86, Rogers 1988) was commenced, and the $x$ and $y$ positions of the pixel were pushed onto a stack. While this stack was not empty, the following occurred. The pixel positions were popped from the stack, and the corresponding $(x, y)$ position flagged in a boolean array of identical dimensions to the Image. Eight-way connectivity (see p.84, Rogers 1988) was assumed, so all pixels around the current one were examined in turn. If the new pixel had an intensity above the threshold and had not been marked as detected, its position was pushed on to the stack. The end result was that each contiguous set of pixels with intensities above the chosen threshold value was taken as an individual distribution. This sub-array was then passed to the moments analysis subroutine, to evaluate $a, b$, and $\theta$. The size, centre, orientation, and ellipticity of the distribution were then written to disk. Once all pixel distributions had been detected and measured, the next value in a file containing the intensity threshold values was read. The detection array was cleared, and the search commenced again from position $(1,1)$. In practice, the threshold step direction was towards the background, corresponding to an increase in the dimensions of the pixel distribution. This allowed examination of the variability of the ellipse parameters describing the star cluster with radius from the centre of the cluster. Further software prepared isophotal maps of the ellipses (see Figure 5-36), as well plots of the detected pixels for a given threshold.

This program was carefully tested. Initially, uniformly weighted ellipses were generated and placed into an array with an arbitrary background level. These were then submitted as noiseless Images to the program. 757 trials with different input $a, b$, and $\theta$ values showed that $a$ and $b$ were generally slightly overestimated by $\sim 0.08 \pm 0.06$ pixels (see Table 5.10 for some representative tests). The variations with angle are due to the discrete nature of the fitted ellipse as pixels. As the ellipticity of the cluster became less in tests, it was found that although the scatter was constant, the difference for $a$ decreased while that for $b$ increased, as might be expected. The ellipse generating function of the testing program used the parametric equations for an ellipse to define the boundary, and then block-filled the interior. Real numbers for the boundary's $x$ and $y$ positions were converted into integers, indicating a boundary pixel. In comparison with the superior approach of recognizing that pixel $(x, y)$ extends \pm 0.5 in both dimensions, the $x$ and $y$ centroids are displaced by -0.5

The typical FWHM of the programme images was 5 pixels and the ellipse fitted to the star clusters ranged out to 100 pixels. The trials showed that $\theta$ was recovered by the program to within $0.10 \pm 0.27$ degrees and the ellipticity to $0.006 \pm 0.003$. Figure $5-36$ is an example of the ellipse fitting by the program. 


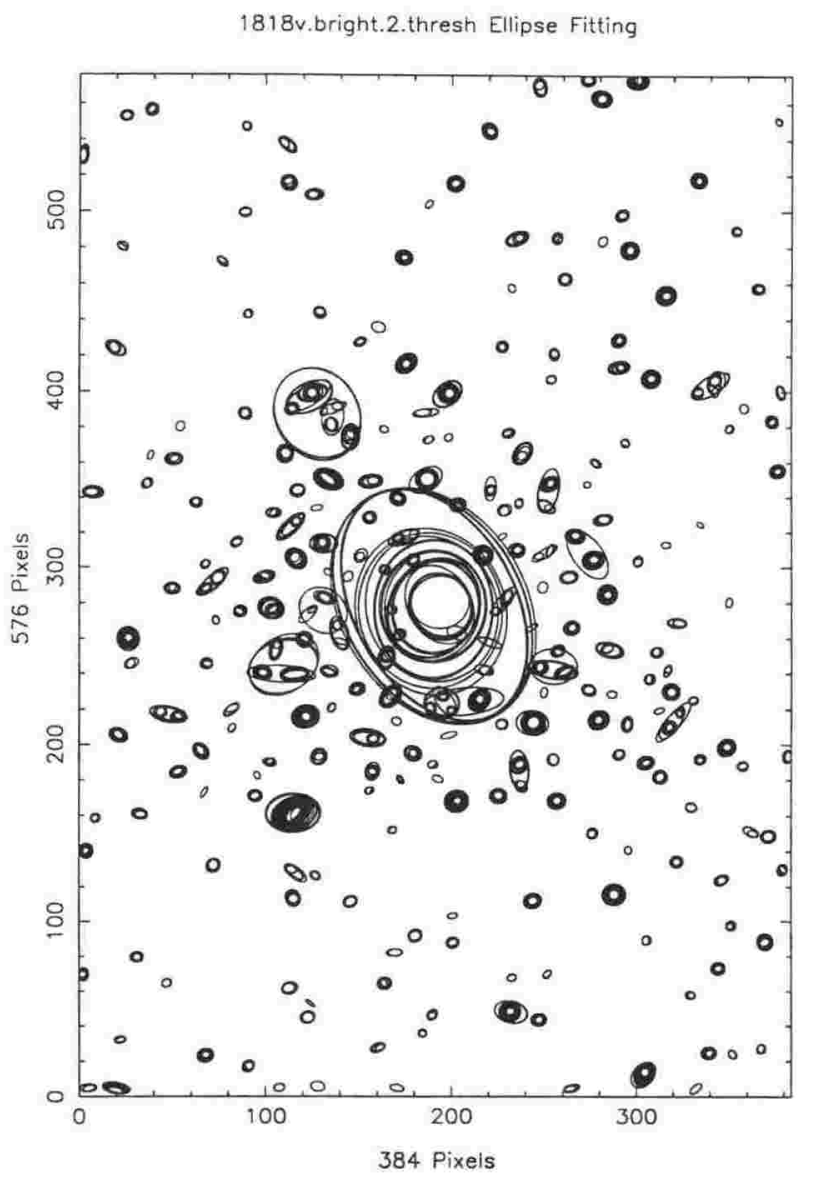

Figure 5-36: Ellipses Fitted to NGC 1818 using the moments analysis technique.

\subsubsection{Observations}

The STSDAS ellipse function, based on Jedrzejewski (1987), was initially used to fit elliptical contours to V CCD Images of the clusters. Attempts were made to use DAOphot (Stetson 1987) to subtract the resolvable stars which would skew the ellipses, and to boxcar smooth these $\mathrm{V}$ band residuals over an area of comparable dimensions to the FWHM. Even when these steps were taken, ellipse often failed to find solutions over a wide range of radii. However, successful solutions were found for some clusters, such as NGC 1818. When the brighter stars (above a peak intensity of 300 counts) were subtracted from NGC 1818 an angle $\theta$ of around 32 degrees was derived. The ellipticity increased from 0.07 (at a radius of $14.4^{\prime \prime}$ ) to a maximum of 0.28 at $19.8^{\prime \prime}$, before dropping to $\sim 0.15$ at a $24^{\prime \prime}$ radius. Frenk \& Fall (1982) give an ellipticity of 0.24 and a position angle of 115 degrees (or a $\theta$ of 25 degrees), in reasonable agreement with the current results. They do not give a radius for this ellipse, but the ellipses were fitted by eye in the range between the burnt-out centres and the peripheries defined by the background. It should be noted that when ellipse was run on the "raw" image of NGC 1818 a uniform ellipticity of $\sim 0.2$ was found over the radius $6^{\prime \prime}$ to $24^{\prime \prime}$. 


\begin{tabular}{||c|c|c|r||}
\hline Cluster & Theta & Ellip & \multicolumn{1}{|c||}{ Radius } \\
\hline NGC 1818: & 0 & + & $15-137^{\prime \prime}$ \\
NGC 1835: & 0 & + & $17-90^{\prime \prime}$ \\
NGC 1836: & - & - & $3-130^{\prime \prime}$ \\
NGC 1839: & 0 & + & $17-145^{\prime \prime}$ \\
NGC 1847: & - & - & $5-72^{\prime \prime}$ \\
NGC 1850: & 0 & + & $3-117^{\prime \prime}$ \\
NGC 1856: & + & 0 & $30-107^{\prime \prime}$ \\
NGC 2004: & 0 & + & $7-133^{\prime \prime}$ \\
NGC 2031: & 0 & - & $20-120^{\prime \prime}$ \\
NGC 2133: & - & 0 & $15-125^{\prime \prime}$ \\
NGC 2164: & - & 0 & $15-105^{\prime \prime}$ \\
NGC 2214: & 0 & 0 & $15-150^{\prime \prime}$ \\
\hline
\end{tabular}

Table 5.11: Variation of Elliptical Parameters for selected LMC Cluster V Images. A + indicates that the parameter increased with radius, a - the opposite, and 0 stands for constant with radius. The typical ranges of the trends were of several tens of degrees in angle and 0.4 in ellipticity. Only general trends are discussed, as in Zepka \& Dottori (1987). Noting that the table captions are reversed in Zepka \& Dottori (1987), the current results agree with them for the common clusters NGC 1835, 2004, and 2214, but not for NGC 2031 (which they found to vary in both parameters). Trends evident in NGC 1835 were smooth. The column 'Radius' gives the range of radii that ellipses were fitted over.

Similar problems to the NGC 1818 fits were found with NGC 1850, whose raw image resulted in a noisy but effectively constant ellipticity, but whose star subtracted image had a linearly increasing ellipticity with radius, and NGC 1856 which exhibited the opposite behaviour in both cases. Zepka \& Dottori (1987) fitted ellipses to isophotal contours, and had also noted radial variations in ellipticity and/or axis orientation in all but 4 of their 17 LMC clusters, with a preference for ellipticity to decrease with radius. Fischer et al. (1993) used the ellipse program to fit CCD observations of NGC 1850. Although not numerically giving the results, they commented that the ellipses did not fit the distribution well, that the elliptical parameters varied rapidly with radius, and that there was poor agreement between the $\mathrm{B}$ and $\mathrm{V}$ band fits despite no radial colour gradient being evident in the cluster. The latter contradicts Geyer et al. (1983).

Concerned at this lack of reliability and unsure if any derived trends were real, the moments technique outlined above was adopted, which appeared to be more robust. As a trial, the first Image to be fitted was of M81, obtained by Michael Richmond (Princeton) who kindly made it available. Ellipse fitting to the smooth distribution of this Sb galaxy showed constant values of around 0.3 and $150^{\circ}$ for the ellipticity and the position angle. If the disk of the galaxy is assumed to be circular, then M81 is tilted to the line of sight by some 46 degrees. The ellipticity is somewhat less than the 0.5 given in Allen (1973), as the current measure is for the inner nuclear region of the galaxy, not including the spiral arms. The position and inclination angles are in good agreement with the $150^{\circ}$ and $\sim 35^{\circ}$ values of Boggis (Jones 1968). 


\begin{tabular}{|c|c|c|c|c|}
\hline \multirow[b]{2}{*}{ Cluster } & \multirow{2}{*}{$\begin{array}{c}\text { Radius } \\
\text { (Arcsec) }\end{array}$} & \multicolumn{3}{|c|}{ Ellipticity } \\
\hline & & Current & $\mathrm{K}$ & ZD \\
\hline NGC 1835 & 27 & 0.09 & & $0.16 \pm 0.09$ \\
\hline$\cdots$ & 34 & 0.11 & & $0.21 \pm 0.12$ \\
\hline$\cdots$ & 46 & 0.12 & & $0.26 \pm 0.12$ \\
\hline$\cdots$ & $48-90$ & $0.14-0.24$ & 0.14 & \\
\hline NGC 1847 & $18-30$ & $0.48-0.62$ & 0.29 & \\
\hline$\cdots$ & $30-54$ & $0.15-0.62$ & 0.20 & \\
\hline NGC 1850 & 30 & 0.20 & 0.19 & \\
\hline$\cdots$ & $48-192$ & $0.18-0.35$ & 0.10 & \\
\hline NGC 1856 & $24-84$ & $0.01-0.10$ & 0.16 & \\
\hline$\cdots$ & 96 & 0.05 & 0.05 & \\
\hline NGC 2004 & $42-60$ & $0.02-0.21$ & 0.20 & \\
\hline$\cdots$ & $66-96$ & $0.18-0.28$ & 0.16 & \\
\hline NGC 2031 & $12-54$ & $0.21-0.44$ & 0.20 & \\
\hline$\cdots$ & 17 & 0.44 & & $0.46 \pm 0.09$ \\
\hline$\cdots$ & 22 & 0.30 & & $0.28 \pm 0.13$ \\
\hline$\cdots$ & 30 & 0.27 & & $0.21 \pm 0.13$ \\
\hline$\cdots$ & 51 & 0.29 & & $0.21 \pm 0.08$ \\
\hline$\cdots$ & $60-114$ & $0.11-0.26$ & 0.11 & \\
\hline NGC 2214 & (a) & 0.45 & & $0.40 \pm 0.14$ \\
\hline$\cdots$ & 19 (b) & 0.45 & & $0.38 \pm 0.12$ \\
\hline$\cdots$ & $26 \quad$ (b) & 0.37 & & $0.39 \pm 0.12$ \\
\hline$\cdots$ & $27 \quad$ (a) & 0.36 & & $0.34 \pm 0.13$ \\
\hline$\cdots$ & $36 \quad$ (b) & 0.34 & & $0.46 \pm 0.09$ \\
\hline$\cdots$ & (a) & 0.32 & & $0.30 \pm 0.07$ \\
\hline
\end{tabular}

Table 5.12: Comparison of Ellipticity Estimates for clusters in common with the current study, Kontizas et al. (1989), and Zepka \& Dottori (1987). Frenk \& Fall (1982) and Geisler \& Hodge (1980) did not state what radii ellipticities were measured at. The column 'Radius' lists the radii, in arcseconds, that Kontizas et al. (1989) or Zepka \& Dottori (1987) estimated ellipticities over. 'Current' gives the ellipticities derived by the current study for the radii, while 'K' and 'ZD' list the ellipticity given by Kontizas et al. (1989) or Zepka \& Dottori (1989). The latter paper presented two different profiles for NGC 2214, which have been indicated as (a) and (b) in 'Range'. Kontizas et al. (1989) estimated an uncertainty of 0.03 for their ellipticity values.

Having demonstrated the stability of the fitting program on a smooth distribution, it was then applied to the LMC cluster observations, with their more irregular morphology partly due to the presence of bright young stars. The general results of the fitting program on Images with the resolvable bright stars subtracted are given in Table 5.11, while Table 5.12 presents a comparison for the derived ellipticities with those of Zepka \& Dottori (1987) and Kontizas et al. (1989). While overall trends were apparent, large radial variations in $\theta$ and $e$ were present even when bright stars were removed and smoothing employed (as before). The results given in Table 5.12 reveal a somewhat weak agreement between the three studies, which used different techniques on different data sets collected under different seeing conditions. In the case of the current study, the poor seeing experienced would increase the difficulty of detecting and removing bright stars from the clusters. Both the previous studies used digital microdensitometric 
scans of photographic plates. Zepka \& Dottori (1987) performed least-squares fits to isophotal contours, while Kontizas et al. (1989) used a computer-aided interactive procedure where ellipses were fitted by eye to scanned images. No mention is made of seeing by either group of authors. The ellipticities of Kontizas et al. (1989) appear to be biased towards the values at the maximum radius. There are major differences between Kontizas et al. (1989) and Zepka \& Dottori (1987) for some clusters, as well as discrepancies with the current study.

\subsubsection{Simulations}

Given the uncertainty in the reliability of the analysis techniques, artificial elliptical star cluster Images with known parameters were generated. This was to test if the input parameters could be successfully derived by the two methods. The magnitude distribution was calculated using the IRAF starlist function which allowed power-law, uniform, Salpeter model (a best-fit function to the data of McCuskey (1966)), and Bahcall \& Soneira $(1980,1984)$ functions to be used. It was decided to use an adjusted spherical King $(1962,1966 \mathrm{a}, \mathrm{b})$ model for the placement of stars inside the cluster, on the basis that spherical King models are algebraically simple and have been widely applied to the LMC clusters (e.g. Chun 1978, Elson 1991, Fischer et al. 1992a, b, 1993). $x$ and $y$ positions were generated using a pseudo-random number generator (which tests revealed to have no bias). These values were then converted across to polar coordinates centred on the (user-set) artificial cluster centre. The tidal, or limiting, radius of the cluster was assumed to vary elliptically. Given the polar co-ordinates of the starting position, a tidal radius was generated for the point. If the point did not fall within this limit, another random position was generated. Once a point fell within its appropriate tidal limit, another random number between 0 and 1 was generated. This was compared against a radial distribution scaled by the tidal radius at the polar angle $\theta$, allowing the ellipticity to be included. If the random number fell below the probability of the distribution at the point's radius, a star image was assumed to exist. See Figure 5-37 for a test distribution. Finally, the elliptical distribution could be rotated about its centre. This allowed two 5000 stellar image $e=0.5 \mathrm{King}$ distributions to be placed at right angles to each other. Reduction of the resulting Image by the automatic ellipse fitting software was expected to produce results of zero ellipticity. A slightly greater ellipticity of 0.03 was achieved. A higher ellipticity in the centre of this test distribution was due to the greater effect of the positions of large individual bright stars in the small fitting region, where they are also more probable due to the increased stellar density towards the centre of the cluster. Above a radius of some 3 full width half maxima reliable results seem to be obtained.

As a further test, 'flat' (uniform probability within the ellipse boundary) distributions were sliced up in $\theta$ (e.g. halves, eighths, etc.), with each slice being cut into equal-area segments, so as to check that the radial distribution was being scaled correctly with $\theta$ (see Figure 5 - 38 ). The 


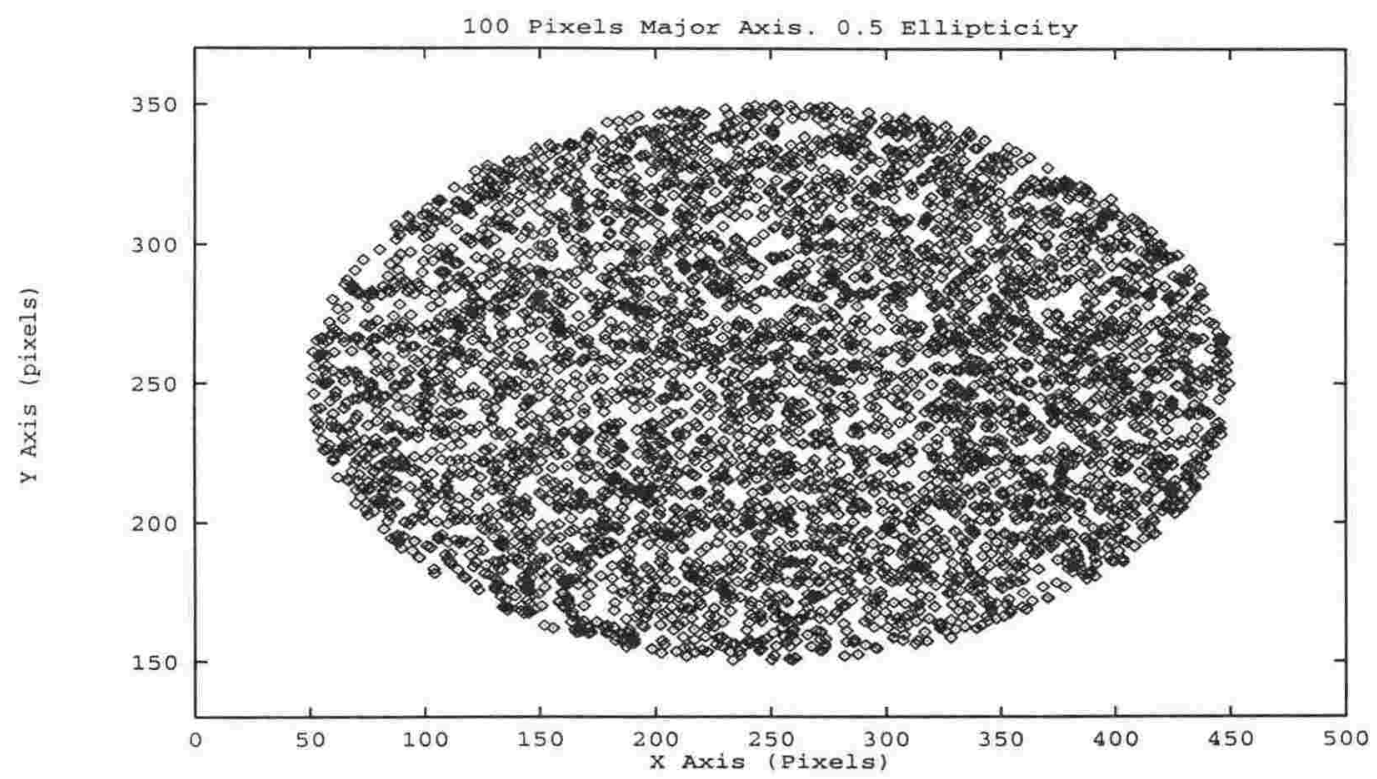

Figure 5-37: Flat Distribution. Star positions for a 'flatly distributed' ellipse of $0.5 e$ are shown. By a 'flat' distribution it is meant that if a randomly generated $(x, y)$ position ranging freely over the CCD Image fell within the boundary of the ellipse, a star was considered to occupy that point with $100 \%$ probability. Outside the boundary a $0 \%$ probability of 'existence' prevails.

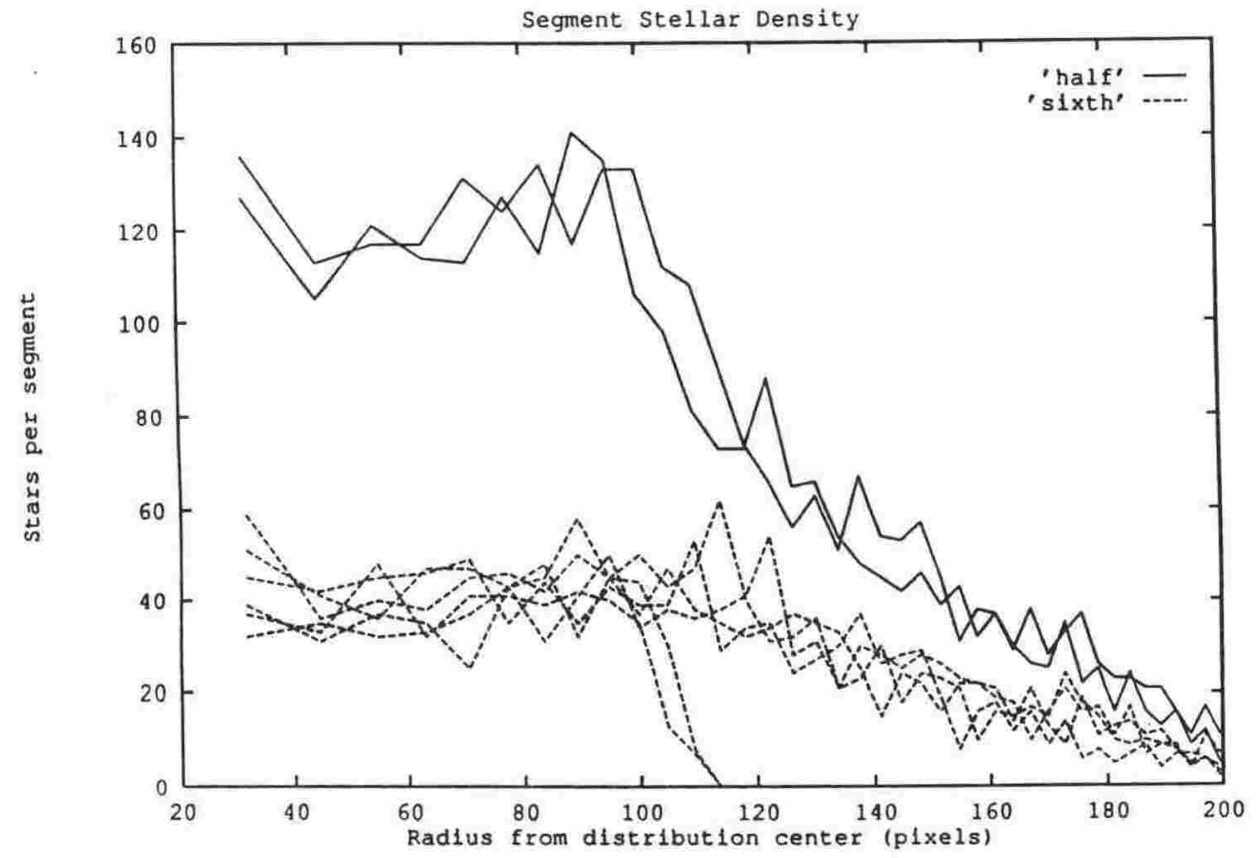

Figure 5-38: Star counts in a sliced flat distribution. 5000 stars were randomly distributed in a flat probability, elliptical distribution with $a=200$ and $e=0.5$. 
solid lines in this figure plot the stellar density when the cluster had been split into two halves containing 40 equal-area segments each. The segments were considered to be sectors of a circle. Given that the inner segment radius was 31.5 pixels, a density of 124.4 stars was expected for those segments inside a 100 pixel radius (the $b$ axis). Examination of the 18 segments within a 95 pixel radius gave a mean of $122.5 \pm 10.1$ stars per segment, and a median of 123 . The mean density over the 80 segments was $62.5 \pm 41.9$, as expected. When the distribution was sliced into sixths (the dashed lines), the density fell by the expected third. Also note the drop-off of the two sectors centred on the $y$ axis is more rapid due to the smaller radial size of the sectors, and that they have fallen off completely just above the $b$ axis length as could be expected for circular regions there.

Given the success of the flat distribution in these tests, and that the King function used did approach the empirical inner and outer radii functions of King (1966a), it appears that the Monte-Carlo placement of the stars was performed correctly. Since the co-ordinates and magnitudes of the stars were available, the IRAF mkob task was used to create artificial frames. A Gaussian profile was used for the point spread function of the 20,000 stars per frame, together with a read noise value of 7.35 electrons and a gain of 4.27 electrons per decimal unit (being the values for the TH7882 chip used to collect the real observations). $2.4^{\prime \prime}$ seeing was assumed in the tests, corresponding to the best seeing conditions experienced (by the project) at MJUO. An aim was to have a smooth distribution of faint stars, with a few bright ones scattered around in it.

The first test frame was of a 0.5 ellipticity cluster, with scaled King radii based on the values of Chun (1978) for the old LMC cluster NGC 1835. The values were 500 and 15 pixels for the tidal and core radii respectively. These values for the radii were chosen for realism, and used in all the simulations presented below. Trials varying the magnitude zero point had no effect on the derived trends and results. The lowest threshold value used was barely above the background value, being at three standard deviations of the noise above the background. The derived position angle agreed well with the input value, falling within 5 degrees of it. Ellipticity increased linearly from an inner value of 0.3 , reaching the input value at the outer radii, and then dramatically dropping at the last "isophotes". Such a low ellipticity halo containing an elliptical cluster was noted for NGC 2214 by Bhatia \& MacGillivray (1988), although the cause in the simulation was simply that the cluster distribution extended off the right boundary of the Image (due to slightly asymmetric centring of the cluster in it). This also resulted in the

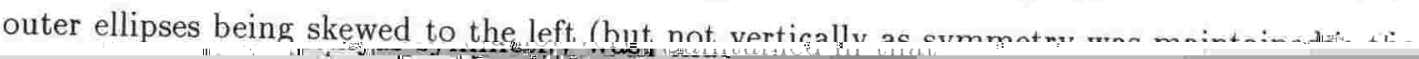


magnitudes excluded. 441 stars were 'lost' by this process. Fitting showed that throughout the cluster the ellipticity of this smooth distribution was within 0.03 of the input ellipticity, until the boundary problem mentioned above was met. The position angle was stable, although systematically overestimated by one degree, while the $x$ and $y$ cluster centres were stable about the input values. Similar trends were found for simulations with all 20,000 stars set to a faint magnitude, although they were slightly closer to the input values (as might be expected since the combined luminosity distribution gradients are more uniform across the Image). Such results are in line with what could be expected for smooth distributions, such as in old LMC clusters where the bright stars have long since evolved. Further trials using the original magnitude distribution (i.e., with the bright stars), but with different star placements and ellipticities, showed the following :

- The major axis angle could vary by $\sim 80^{\circ}$ with radius, becoming more variable as $e$ approached zero. However, large variations of $\theta$ over small radial distances were found in a very $(e=0.5)$ elliptical distribution, due to the random placement of bright unresolved stars.

- No particular trend in ellipticity with radius appeared to be preferred. Examples were found where ellipticity increased, remained constant, or decreased with radius.

- Generally the input ellipticity was reached in the outer regions. However, without prior knowledge of this value, it would be difficult to ascertain that the 'real' value of the cluster had been determined. Often, in the simulations it was only met for one or two points.

However, such tests removing bright stars are not very realistic as they are correctly 'removed' from the cluster Image. In practise, it is common (see e.g. Fischer et al. 1992a, b, 1993, Elson 1991) to use a PSF to subtract out the bright stars from a frame. This is because it has been widely recognized that such stars will bias ellipse (and profile model) fits by their placement and intensity. The IRAF tasks daofind and substar were therefore used. Bright stars in uncrowded areas were used in an iterative process to construct the empirical corrections to a Gaussian PSF. This process concluded when tests showed that stars in uncrowded regions were being cleanly subtracted from the frame. All the stars in the frame that could be identified by daofind were, and the bright ones (above a user-set intensity) limit subtracted off using the modeled PSF. The moment analysis software was then run on the Image. Ellipticity was found to increase with radius, reaching the input value at $\sim 4$ times the FWHM of the PSF, and then dropping away before the outer isophotes were met. The range of the trend was 0.15 in $e$, which is comparable with the trends found in the Survey observations and Zepka \& Dottori (1987). The position angle was always within 3 degrees of the input value, excluding severe disturbance in the cluster centre. Similar results can be seen in the results of Zepka \& Dottori (1987), 
contributing to their gradients. These trends are presumably due to the unresolved bright stars in the cluster centre. It is interesting to note that moment fitting of the original frame showed the ellipticity was recovered more rapidly with radius than the star subtracted frame, and that this ellipticity was more constant until the boundary problem was met. It is likely that poor background estimates in crowded regions leads to either positive (under-subtraction) or negative (over-subtraction) residuals being left behind after a bright star has been subtracted, and in turn to spurious radial gradients.

Ellipse was also run on some of the test frames, to see how well it agreed with the moments technique in light of the concerns mentioned above. Even in clusters of 0.4 and 0.5 ellipticity trends were evident in $e$, accompanied by $\theta$ ranging up to 70 degrees. Low ellipticities were found for cluster centres. The input ellipticity was not recovered over substantial radial intervals, and was not obvious in plots of $e$ against radius (i.e., substantial plateaux corresponding to the value of the input ellipticity were not seen). Problems were often encountered with ellipse failing to iterate towards a solution. It does not appear that ellipse is suitable for young clusters, with their clumpy distributions, even when measures are taken to exclude the brightest pixels from given ellipse fits.

\subsubsection{Conclusions}

Radial variations in the ellipticity and position angle of LMC star clusters have previously been taken to indicate that they are triaxial structures in equilibrium (Zepka \& Dottori 1987). In light of the simulations, it is reasonable that caution should be exercised in the interpretation of ellipse fitting of young populous clusters. Stochastic effects in the placement of bright stars, which can not all be both detected and cleanly subtracted from a CCD Image using standard (and previously used) techniques, will result in spurious trends. Such problems will not be evident in large bodies observed at low resolution (such as M81) or evolved old clusters (such as NGC 1835), both with smooth radial distributions. The fact that trends were observed in NGC 1835 suggests that its radial variations may be real.

\subsection{Concluding Remarks}

Experience has shown the MJUO is not a good site for crowded field photometry, ${ }^{1}$ with generally poor seeing due to the New Zealand maritime climate. Future direct imaging projects at this

\footnotetext{
${ }^{1}$ It should be noted that there is evidence that the seeing on the MJUO $0.61 \mathrm{~m}$ telescopes is better than that experienced by the $\mathrm{M}^{c}$ Lellan telescope, e.g. the mean seeing of the CCD photometry of West et al. (1992) using the $0.61 \mathrm{~m}$ Boller \& Chivens telescope in 1990 was $2.2 \pm 0.5$ arcsec. A linear regression to these data gave a relation for the seeing of $1.46 \pm 0.12+0.49 \pm 0.07$ (airmass), where seeing is in arcseconds and airmass is unity at the zenith. Therefore, the surroundings of the $\mathrm{M}^{\mathrm{C}}$ Lellan telescope could be at least partially responsible for the poor seeing encountered by this project.
} 
site should centre around more open fields, preferably with photo-electric standards "on the chip", that is, in the same field of view for the CCD as the target objects. While the quality of the survey data is not high, the survey still fulfilled its objectives of providing CMDs for the generation of a list of suitable targets for later detailed investigation, experience in observing and data reduction techniques, and the impetus to establish a reliable data pathway. On the basis of observations collected at MJUO and the experience gained from them, service observing was successfully requested on the Anglo-Australian Telescope for the clusters NGC 1850, 1968/74, and 2214. The NGC 2214 observations are discussed in Chapter 7 (and Banks et al. 1994c). The observing programme for the other two targets is still active at a high priority. Observations should be collected during the 1995 summer, with the primary objective of mass functions being derived for the clusters. The MJUO observations were also used to select targets for observation with the Vilnius filter set, as described in the next chapter. 


\section{Chapter 6}

\section{Vilnius Photometry}

$\mathrm{T}$

his chapter discusses attempts to image star clusters using the Vilnius photometric system, in conjunction with the CCD Image Acquisition System at MJUO. This is only the second time that CCD observations have been collected using the Vilnius filters. Vilnius photometry with a CCD has been performed before by Boyle et al. $(1990,1992)$, whose observations were of open fields in the directions of Lyra and the globular cluster M71. However the filter set used by Boyle et al. was not the standard Vilnius photometric system. Boyle et al. was part of a program studying the distribution of spectral types and extinction at different Galactic longitudes and latitudes. The data were collected in September 1986, using the KPNO $0.9 \mathrm{~m}$ telescope, and were reduced with DAOphot. Nor is the current study the first time the Vilnius system has been applied directly to a star cluster. Dzérvítis \& Paupers (1993) derived a V versus X-Z colour-magnitude diagram for the open cluster NGC 752, and estimated the spectral types of the component stars, together with the reddening and distance modulus of the cluster, from seven filter photometry. In essence, this chapter details a feasibility study of the use of the Vilnius system for cluster studies using the MJUO CCD frame acquisition system.

\subsection{The Vilnius Filter Set}

$\mathrm{T}$ he seven filter intermediate passband Vilnius system was designed to allow the purely photometric determination of spectral types and absolute magnitudes of normal stars, whilst also correcting for interstellar reddening (Straižys \& Sviderskienė 1972, Straižys 1973, 1992a, b). The system can separate out peculiar stars (from normal stars) such as sub-dwarfs, white dwarfs, blue horizontal branch stars, Be stars ${ }^{1}$, Herbig Ae/Be $\operatorname{stars}^{2}$, T Tauri type ${ }^{3}$, metal-poor stars, carbon and barium stars, and some types of unresolved binaries. From the

\footnotetext{
${ }^{1} \mathrm{~B}$ type stars with strong hydrogen emission lines, surrounded by an expanding shell of material which is drawn into a disk via rotation.

${ }^{2}$ Small bright concentrations of gas and dust, believed to represent the final stages of the evolution of protostars.

${ }^{3}$ Irregular variable stars of F, G, or K spectral types believed to be the last evolutionary phase of a proto-star before ZAMS.
} 


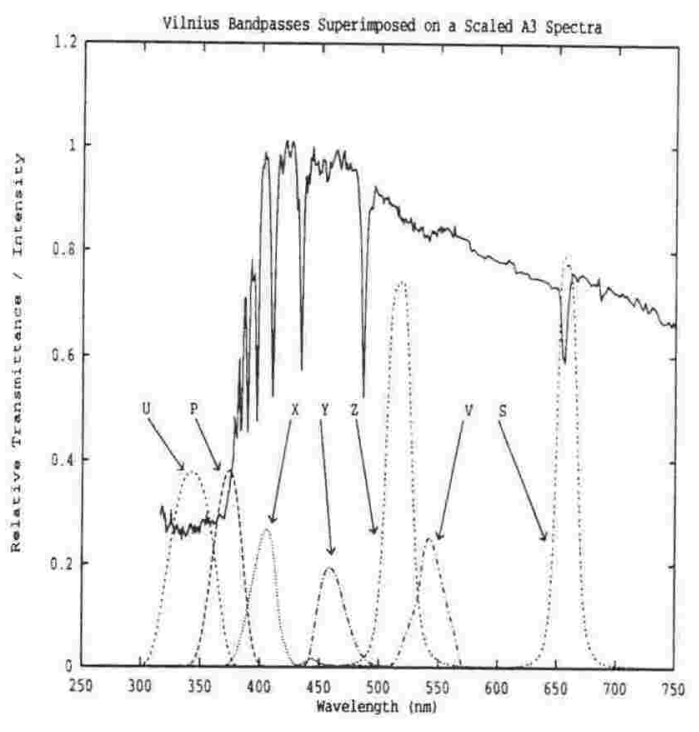

(a)

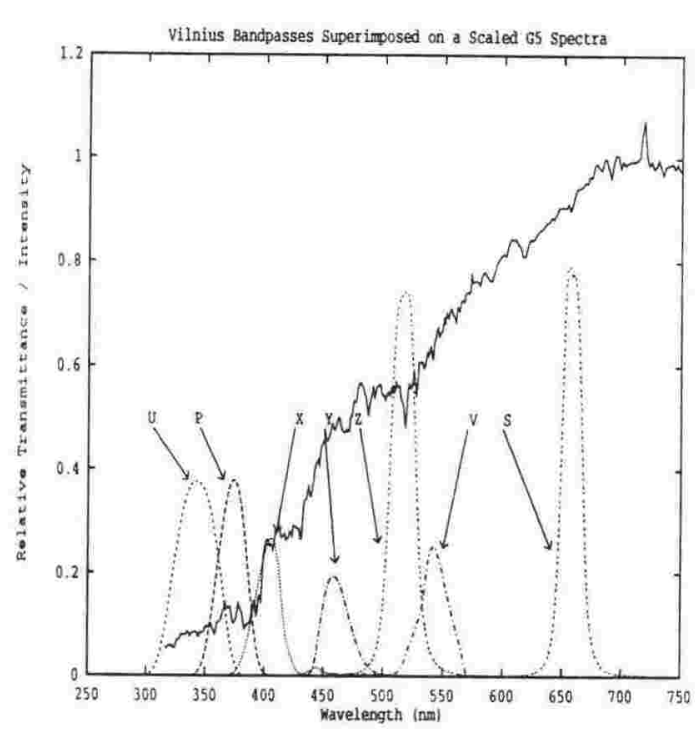

(b)

Figure 6-1: Vilnius Bandpasses Superimposed on Example A3 (a) and G5 Spectra (b). The spectra were obtained from the spectrophotometric atlas of Gunn \& Stryker (1983). The Vilnius passbands are the standard ones given in Straižys (1992a)

original conception of the filter system, it was decided to use a medium, rather than a narrow, band system to ensure that photometry of relatively faint stars was possible. The fact that the bandpasses were of intermediate width meant that careful thought had to be given to the positioning and widths of the filters relative to the spectral features of all luminosity classes, if the system was to meet the objectives given above. The bandpasses of the Vilnius filter set are plotted against early and late-type spectra in Figure 6-1. Reference should be made to this diagram when considering the following list, which introduces the filters of the Vilnius system and their positioning relative to important spectral features:

- The U filter measures the ultraviolet intensity of a star at a range of wavelengths shorter than that corresponding to the Balmer jump.

- The P filter is placed on the jump itself, allowing luminosity determinations for early-type stars.

- The $\mathrm{X}$ filter measures a wavelength range longer than the Balmer jump, between the $\mathrm{H}_{\delta}$ and $\mathrm{H}_{\epsilon}$ lines. This filter measures the continuum intensity after the Balmer jump for early-type stars, and the metallic-line blanketing for late-type stars.

- $\mathrm{Y}$ is centred around $466 \mathrm{~nm}$, excluding the interstellar band at $433 \mathrm{~nm}$ and the $\mathrm{H}_{\gamma}$ line.

- $\mathrm{Z}$ is near the bottom of an absorption feature in late-type stars. The absorption intensity of the $\mathrm{Mg}$ II triplet lines and the $\mathrm{Mg} \mathrm{H}$ band is measured. 


\begin{tabular}{||c|c|c|c|c|c|c|c||}
\hline & U & P & X & Y & Z & V & S \\
\hline Mean Wavelength & 345 & 374 & 405 & 466 & 516 & 544 & 655 \\
Half Width at Half Maximum & 40 & 26 & 22 & 26 & 21 & 26 & 20 \\
\hline
\end{tabular}

Table 6.1: Mean Wavelengths and Half-Widths of the Vilnius Filters as given in Straižys \& Sviderskiene (1972) and Straižys (1992a). All units are nanometres.

- V measures the continuum at nearly the mean wavelength of the Johnson V filter. In conjunction with the $\mathrm{Y}$ filter, a direct conversion may be made to the Johnson BV system (Forbes 1994).

- $\mathrm{S}$ is centred on the $\mathrm{H}_{\alpha}$ line at $656 \mathrm{~nm}$. It is used to separate the $\mathrm{B}$ emission (Be) stars from normal B stars. The filter measures the absorption or emission intensity of the $\mathrm{H}_{\alpha}$ line in early-type stars, or the pseudo-continuum (due to metallic lines) in late-type stars. Unlike the other filters, which are Soviet-made coloured glasses cemented with Canadian Balsam, the $\mathrm{S}$ filter is an interference filter.

The Vilnius colour indices U-P, P-X, X-Y, Y-Z, Z-V, and V-S are set to zero for unreddened O-type stars. Colours for all normal stars are therefore positive. The mean wavelengths and widths of the standard Vilnius photometric filter set ${ }^{1}$ are presented in Table 6.1.

\subsubsection{Bandpasses for Early-Type Stars}

The classification of early-type stars centres on the Balmer jump. To measure the jump photometrically, one filter $(\mathrm{U})$ is placed blueward of the jump, while another $(\mathrm{X})$ is placed after the jump. Time-dependent variation of ozone extinction extends over the range 300 to $330 \mathrm{~nm}$. There is therefore not much room for the placement of the $\mathrm{U}$ filter, which is located between the Balmer jump and the strongest ozone absorption lines. Placement of the $\mathrm{X}$ filter is even more difficult, with the only line-free region immediately redward of the jump being between the $\mathrm{H}_{\delta}$ and $\mathrm{H}_{\epsilon}$ lines. Unfortunately, these two lines are close enough to each other that a medium-width filter extends across them. While the position of the filter could be shifted redward, this would reduce the important X-Y colour term.

$P$ was placed on the Balmer jump itself. It is very sensitive to the both the height and position of the jump, making it an excellent indicator for luminosity class determinations of early-type stars (see Figure 6-2). The position of the P filter on the Balmer jump was determined by the analysis of synthetic U-P, P-Y colour-colour diagrams, giving the best separation of luminosity classes in this diagram.

\footnotetext{
${ }^{1}$ Which is distinct from the photographic Vilnius filter system (Straižys 1992a).
} 


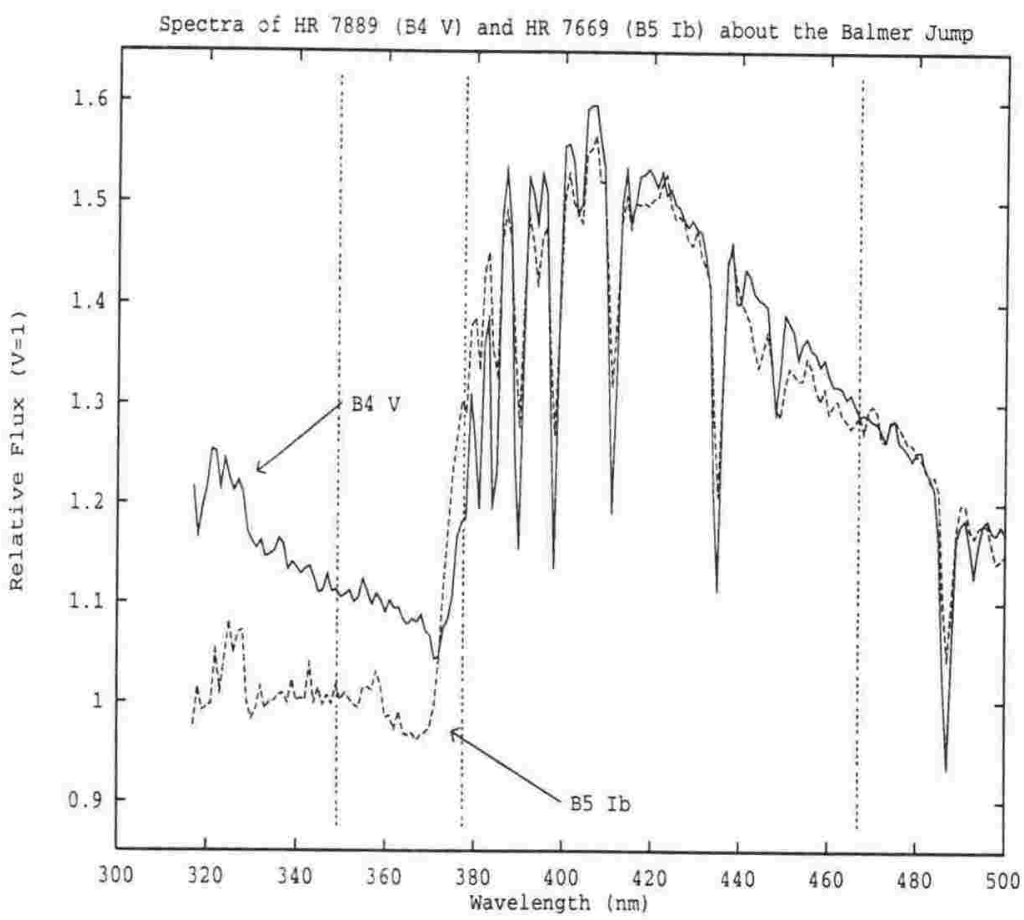

Figure 6-2: Spectra of Example B4 Main Sequence and B5 Supergiant Stars about the Balmer Jump demonstrating that the U, P and Y bandpasses can act as excellent indicators of luminosity class for early-type stars. The mean wavelengths of the U, P, and Y filters are indicated as dotted lines.

Over the wavelength range $0.3-1.5 \mu \mathrm{m}$ interstellar extinction can be approximated ${ }^{1}$ as being two straight lines intersecting at $435 \mathrm{~nm}$. The shorter wavelength (blue) segment has the steeper of the two gradients. The $\mathrm{Y}$ filter is placed at this intersection, with the $\mathrm{X}$ and $\mathrm{Z}$ filters on opposite sides of the point in order to measure the two gradients. Unreddened stars will fall in a line on a X-Y, Y-Z colour-colour diagram that does not turn back on itself, being a one-to-one function. Such a diagram can therefore be used to determine the spectral types of stars. The further the $\mathrm{X}$ bandpass is from the intersection, the greater the $\mathrm{X}-\mathrm{Y}$ colour index, and so the greater the variation in $\mathrm{X}-\mathrm{Y}$ with spectral type. However, placing the $\mathrm{X}$ filter even partially onto the Balmer jump will cause the X-Y, Y-Z sequence to bend back onto itself (except for very late-type stars). Therefore, the $\mathrm{X}$ filter was placed as far blueward as possible, without overlapping onto the jump.

\subsubsection{Bandpasses for Late-Type Stars}

The classification of late-type stars centres around the $Q_{x z s}, Q_{x y z}$ diagram. The ' $Q$ parameter' $\mathrm{Q}_{\mathrm{m}_{1} \mathrm{~m}_{2} \mathrm{~m}_{3}}$ is defined to be:

\footnotetext{
${ }^{1}$ The individual emission and absorption features are being neglected in this discussion.
} 


$$
\left(m_{1}-m_{2}\right)-\frac{E_{m_{1}-m_{2}}}{E_{m_{2}-m_{3}}}\left(m_{2}-m_{3}\right)
$$

where $\mathrm{m}$ is a magnitude through a given bandpass and

$$
E_{m_{1}-m_{2}}=A_{1}-A_{2} .
$$

$A_{1}$ and $A_{2}$ are the interstellar absorption terms for the two different bandpasses, indicating how much interstellar dust and gas the starlight has passed through. $\mathrm{E}$ is called the colour excess. Filter systems with three or more filters have an important parameter - the colour excess ratio. This ratio:

$$
\frac{E_{m_{1}-m_{2}}}{E_{m_{2}-m_{3}}}
$$

is the reddening gradient on a colour-colour diagram (e.g. Figure 6-3).

Knowledge of the colour excess ratio allows the removal of the effect of interstellar reddening, and the subsequent derivation of the intrinsic colour indices of the observed stars. The $Q$ parameter (Equation 6.1) is the value on the $m_{1}-m_{2}$ axis when it is intercepted by the reddening line or its extension. In Figure 6-3 the positions of three stars are indicated. (1) is unreddened, while (2) and (3) have different amounts of interstellar reddening. All three stars are of the same spectral type and have different colour excesses, but they have the same value of $Q$. This explains the usefulness of $\mathrm{Q}$ parameters, since they are independent of interstellar reddening.

$\mathrm{Q}_{\mathrm{xzs}}$ is sensitive to the luminosity of late-

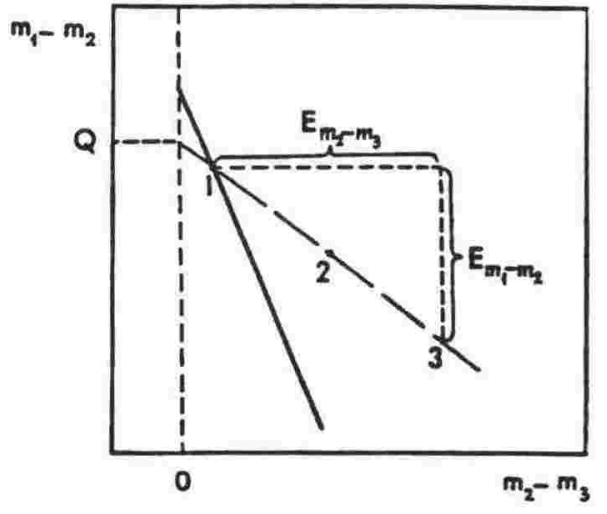

Figure 6-3: Definition of the Q Parameter. The bold line represents an unreddened sequence of stars in the colour-colour diagram. Points 1 , 2 , and 3 lie along a reddening line. The colour excess ratio is the gradient of this line, while the $\mathrm{Q}$ parameter represents the $y$ axis intercept of the line. Figure from Straižys (1992a).

type stars, while $Q_{x y z}$ is sensitive to spec-

tral type. The positioning of the Vilnius $\mathrm{V}$ and $\mathrm{S}$ filters was based on the analysis of such Q-Q diagrams with various positions of the two filters (Straižys 1992a). These positions were refined by observational work. The $\mathrm{Z}$ filter is positioned on a wide absorption band present in $\mathrm{G}, \mathrm{K}$, and $\mathrm{M}$ spectra. The $\mathrm{X}-\mathrm{Z}$ colour is a good indicator of luminosity class (see Figure 6-4), with the colour being more positive for a giant than a dwarf star. 


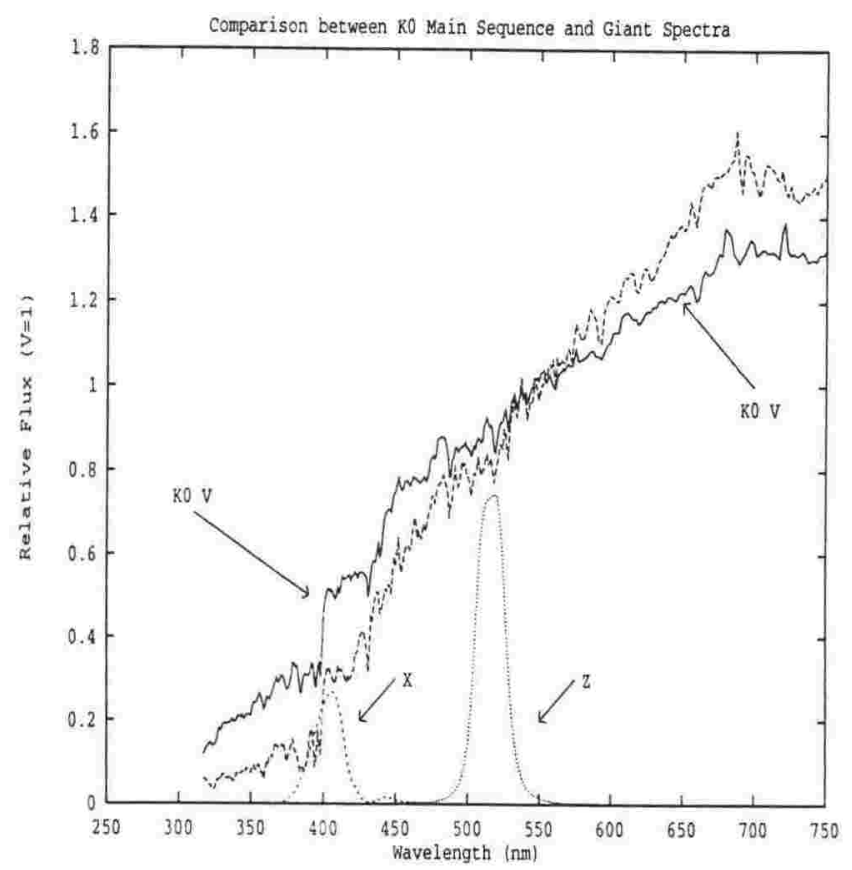

Figure 6-4: Spectra of Example K0 Main Sequence and Giant Stars.

The mean wavelength of the $\mathrm{V}$ filter nearly coincides with that of the Johnson $\mathrm{V}$ bandpass. No colour term is required for the transformation of magnitudes between the two filters, until the early M-type stars. Qxzs and QXzv have similar properties, allowing them to be interchanged. The Z-V colour measures the depth of the Mg I triplet lines. The careful placement of the passbands allows the classification of nearly all spectral types with the six filter UPXYZV system (see p. 414, Straižys 1992a). Unfortunately, early M-type stars can not be distinguished from $\mathrm{K}$ stars with this system - although the original Vilnius system included a $\mathrm{T}$ filter to allow this by measuring the strength of the Ti0 band.

\subsection{The Southern System}

In light of the capabilities of the Vilnius system, the idea of extending it to the Southern Hemisphere was first considered in 1985 at the Royal Observatory Edinburgh, given that none of the already established standard regions (e.g. Zdanavičus et al. 1969, Černies et al. 1989, and Černies \& Jasevičus 1992) extended south of the celestial equator. This programme commenced in 1988 using the $61 \mathrm{~cm}$ telescopes at MJUO, with the initial goal of establishing standards near the South Celestial Pole and also bright $(\mathrm{V}<7)$ stars generally distributed south of -20 degrees. To date some 90 cluster, 109 primary standard, and 225 secondary standard stars have been observed at least once (Dodd et al. 1993). 
Combination of TH7882 CCD and Vilnius filters

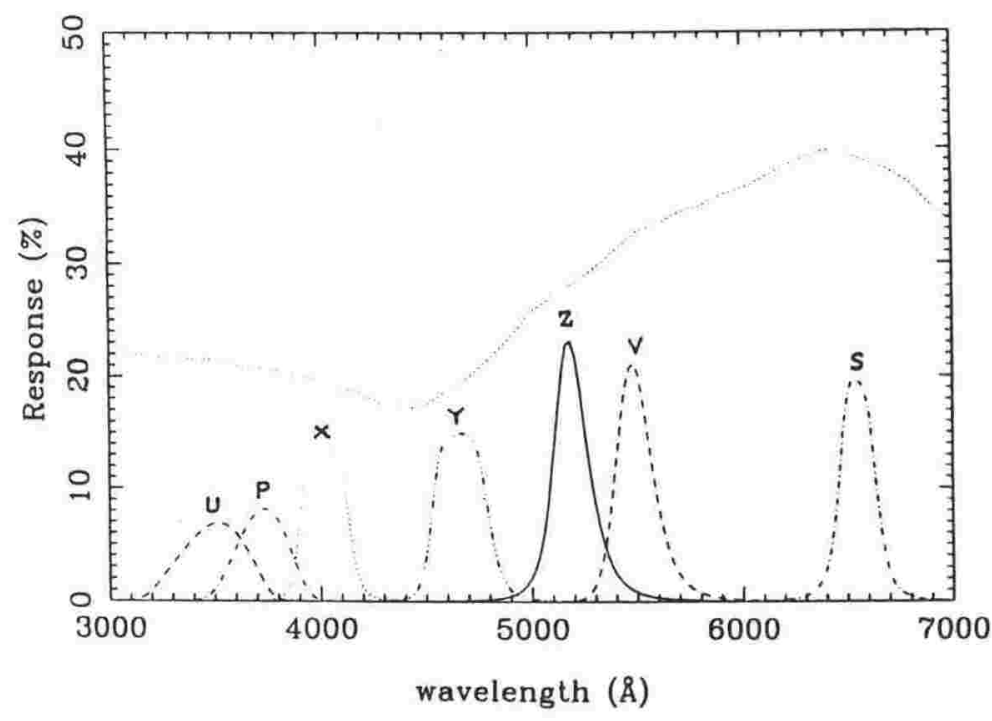

Figure 6-5: Vilnius Filter Response for the TH7882 Chip. Measured transmittances of the Vilnius filters and the manufacturer's quantum efficiency specifications for the TH7882 CCD used in this study (given as a dotted line), were combined to show the filter responses with this chip. For a flat spectrum, percentage transmittances for the (U, P, X, Y, Z, S) filters are respectively $(61,53,74,95,106,87)$ relative to $\mathrm{V}$.

\subsection{Tests}

Given the Southern Vilnius standard star network, the abilities of the Vilnius system, and the availability of both an established Image reduction system and a medium-size telescope equipped with a CCD, UPYV test Images were acquired of the open (Galactic) cluster NGC 4755 and the young populous LMC cluster NGC 2004. Convolution of the quantum efficiency of the Thomson CCD with the transmittances of the Vilnius filters revealed that the $\mathrm{U}$ and $\mathrm{P}$ exposures would need substantially longer exposures than the other filters, if the same sample of stars was to be observed through all the filters (see Figure 6-5). These observations were collected on March 151993.

\subsubsection{NGC 2004}

NGC 2004 is a young ( $\sim 8 \times 10^{7}$ years according to Hodge 1983) star cluster in the Large Magellanic Cloud, making it an attractive object for the study of stellar evolution models. Several studies have derived Johnson BV colour-magnitude diagrams for it, including Bencivenni et al. (1991), and Balona \& Jerzykiewicz (1993). The UV, PV, and YV CMDs based on 19.1 minute exposures ${ }^{1}$ for the $\mathrm{P}, \mathrm{V}$ and $\mathrm{Y}$ exposures, and 4.8 minutes for the $\mathrm{U}$ frame ${ }^{2}$ are given

\footnotetext{
${ }^{1}$ The unusual exposure lengths are due to the Photometrics Acquisition System running $4.3 \%$ fast for some observation commands (see Tobin 1991a).

${ }^{2}$ The reason for the $U$ exposure being short compared to the others, despite the need for it to be longer given the combined response of the filter and CCD Q.E., is given below.
} 


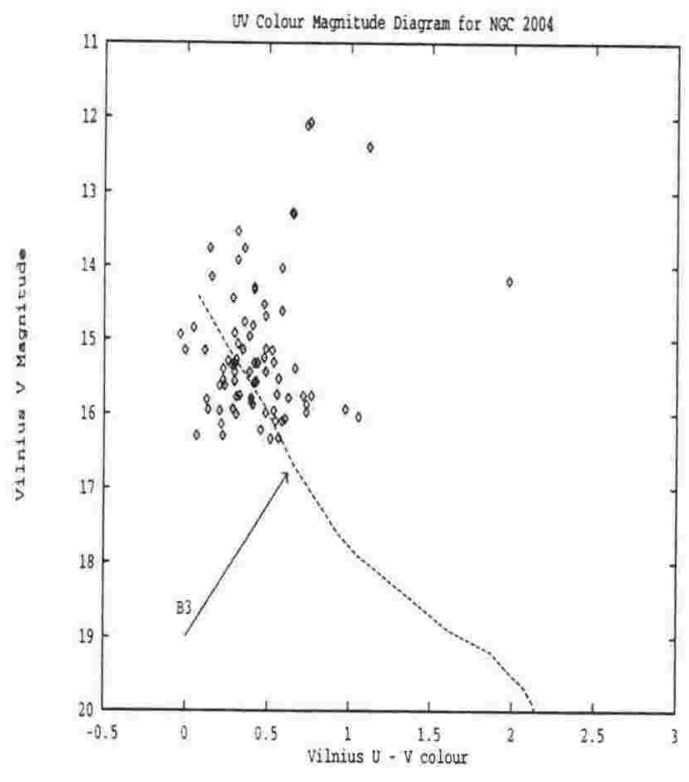

(a)

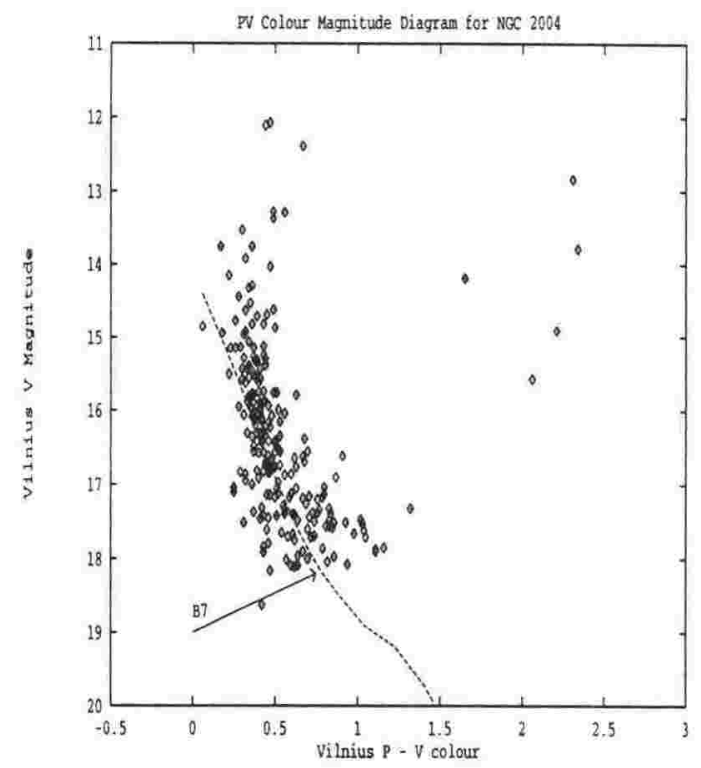

(b)

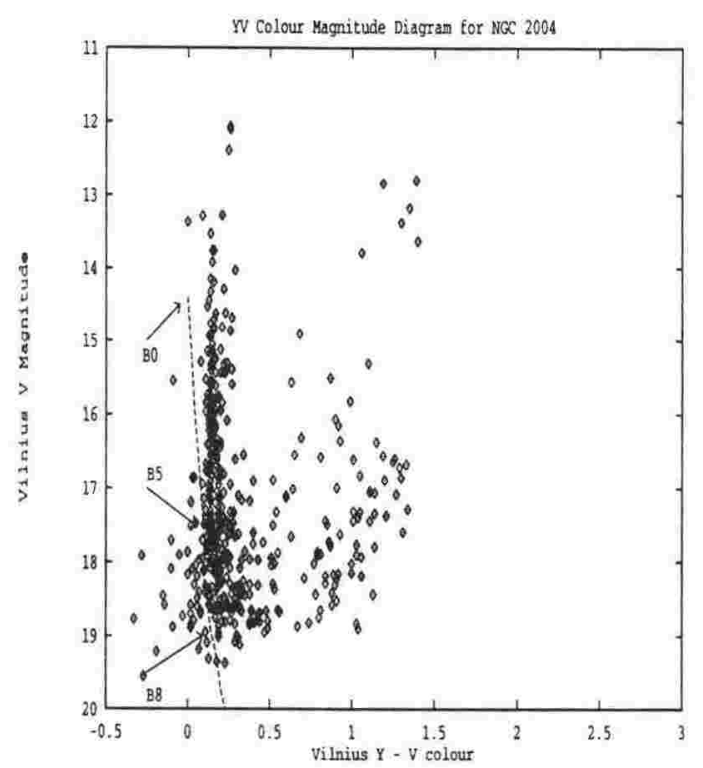

(c)

Figure 6-6: Vilnius Colour-magnitude Diagrams for NGC 2004. Lines representing the main sequence, as given in Straižys (1992a), are plotted on each of the colour magnitude diagrams. These sequences have not been corrected for reddening, which is small for this cluster. Reddening was estimated from a P-V, Y-V colour-colour diagram as $\mathrm{E}(\mathrm{B}-\mathrm{V})=0^{\mathrm{m}} 08 \pm 0^{\mathrm{m}} 03$. This is somewhat larger than other estimates for this cluster (e.g. see Table 5.6 on page 111), however Lee (1991) gives the same reddening and uncertainty based on Johnson UBV CCD photometry. The positions of various spectral classes are shown. Photometric uncertainty increases with magnitude, leading to some faint stars with unrealistic negative colours. A distance modulus of $18^{\mathrm{m}} \cdot 4$ was adopted. 


\begin{tabular}{||c|c|c|c|c||}
\hline Colour & $\mathrm{q}_{1}$ & $\mathrm{q}_{2}$ & $\mathrm{q}_{3}$ & $\mathrm{RMS}$ \\
\hline $\mathrm{U}$ & $17.388 \pm 0.056$ & $-0.865 \pm 0.131$ & $0.455 \pm 0.024$ & 0.015 \\
$\mathrm{P}$ & $17.609 \pm 0.028$ & $+0.121 \pm 0.033$ & $0.530 \pm 0.024$ & 0.025 \\
$\mathrm{Y}$ & $18.091 \pm 0.018$ & $-0.028 \pm 0.018$ & $0.282 \pm 0.010$ & 0.017 \\
$\mathrm{~V}$ & $17.874 \pm 0.018$ & $-0.079 \pm 0.025$ & $0.198 \pm 0.014$ & 0.023 \\
\hline
\end{tabular}

Table 6.2: Vilnius Transformation Coefficients for the March 1993 observing run, derived using Fitparam. The coefficients are those given in Equation 6.2.

as Figure 6-6.

Banks et al. (1994c) presented preliminary transformation equations and a YV colourmagnitude diagram for NGC 2004. Their results are superseded here. Ten standard stars were observed. 15 sets of UPYV observations of standard stars were used to derive the standardisation equations. The (Y-V) colour spread of the standards was from 0.09 to 1.08 , with an airmass range 1.0 to 3.3 . Curvature of the Earth and refraction was taken into account in the calculation of the airmasses. Nine stars were primary standards ${ }^{1}$, being members of the northern hemisphere Vilnius standards, and so were all below 1.5 airmasses. The last was BS 4293 , listed in Forbes et al. (1993a) as a southern hemisphere Vilnius standard, which was observed several times at low airmasses. Seeing was good during the observations, being $\sim 2.8^{\prime \prime}$. Unfortunately, the development of cloud in the early morning led to other collected observations of standards being rejected. This development of cloud took place during the observations of NGC 4755 .

The IRAF Fitparam task was used to fit equations of the form:

$$
\mathrm{Q}_{\mathrm{o}}=\mathrm{Q}+\mathrm{q}_{1}+\left(\mathrm{q}_{2} \times(\mathrm{Y}-\mathrm{V})\right)+\left(\mathrm{q}_{3} \times \text { Airmass }\right)
$$

where the subscript ' $O$ ' indicates the observational magnitudes, $q$ are the coefficients, and $Q$ the 'standard' magnitude. The coefficients of fits and their root mean squares (RMS) may be found in Table 6.2. Extinction and instrumental coefficients were determined by the IRAF Photcal package using a least-squares solution to all the standard star data (see Harris et al. 1981). The $\mathrm{V}$ and $\mathrm{Y}$ equations are in good agreement with the preliminary transformations of Banks et al. (1994c). A further uncertainty in the zero point is caused by the aperture corrections. The uncertainties in the aperture corrections are $0.040,0.025,0.020$, and 0.018 magnitudes in $\mathrm{U}$, P, V and Y. Da Costa (1991b) notes that this correction is the largest source of systematic uncertainty in the entire CCD photometry process.

The major features of the YV CMD are the near-vertical and well-defined main sequence (MS), a few evolved stars in a red giant clump around $\mathrm{V} \sim 13$ and $(\mathrm{Y}-\mathrm{V}) \sim 1.4$, and the

\footnotetext{
${ }^{1}$ Stars HD 90994, 91612, 95345, 97585, 104181, 107070, 108985, 111765, and 77445 from North (1980).
} 


\begin{tabular}{||c|c|c|c|c|c||}
\hline Filter & Zero Point & Colour Term & RMS & Uncertainty & Standards \\
\hline U & $16.143 \pm 0.116$ & $-0.500 \pm 0.293$ & 0.039 & 0.03 & 11 \\
P & $16.679 \pm 0.098$ & $+0.873 \pm 0.253$ & 0.037 & 0.03 & 17 \\
Y & $17.178 \pm 0.077$ & $-0.042 \pm 0.192$ & 0.025 & 0.04 & 17 \\
V & $17.076 \pm 0.088$ & $+0.294 \pm 0.237$ & 0.052 & 0.04 & 13 \\
\hline
\end{tabular}

Table 6.3: Transformation Parameters for NGC 4755. The zero point and color term parameters are $\mathrm{q}_{1}$ and $\mathrm{q}_{2}$ in Equation 6.2. The Root Mean Square of each fit is given in the column 'RMS' as magnitudes. 'Uncertainty' lists the standard deviation based on the formal errors of the standard stars magnitudes themselves, as given by Forbes (1994). The column 'standards' indicates how many secondary Vilnius standards were used to derive the transformation parameters.

field evident at the fainter magnitudes to the red of the MS. These features, and their relative number densities, are identical to those seen in the literature for this cluster (see e.g. Elson (1991) for a BV CMD acquired with a $1 \mathrm{~m}$ telescope equipped like the MJUO $1 \mathrm{~m}$ ). The stars to the blue of the MS are artifacts of the crowded field reduction and the poor (by international standards) observing conditions. The widening of the MS towards the fainter magnitudes is a good reflection of the photometric errors (see Mateo \& Hodge 1987). Direct comparison between the CMD presented and Johnson BV CMDs in the literature is aided by the fact that Vilnius $\mathrm{V}$ coincides with the Johnson $\mathrm{V}$ magnitude for all unreddened or slightly reddened stars of spectral types O to K and all luminosity classes (p. 489, Straižys 1992a).

\subsubsection{NGC 4755}

NGC 4755 (IAU 1250-600, OCL 892) is a very young ( $8-11.5 \times 10^{6}$ years: Dachs \& Kaiser 1984) open star cluster surrounding the M supergiant $\kappa$ Crucis, leading to ' $\kappa$ Crucis' being one of the popular names for the cluster. The other popular name of the 'Jewel Box' stems from the statement of Sir John Herschel that the cluster resembled a superb piece of jewellery.

Many southern Vilnius standard stars have been established in NGC 4755. It was therefore originally intended to use the secondary standards in this cluster, in conjunction with the low-altitude primary standards, to derive the transformation equations. When observed, the cluster standards were at low airmasses. Unfortunately, the above mentioned formation of cloud prevented this plan, forcing the use of a direct transformation based on the "on chip" local standards. Seeing during the observations of NGC 4755 was $\sim 2.7^{\prime \prime}$, as estimated by the mean FWHM of PSF stars in the Images. The root mean square errors for the transformations are large (see Table 6.3), which is at least partially due to the brighter, and more frequently measured, standards being saturated or approaching pixel saturation, and so the fainter and less frequently measured stars being used. 


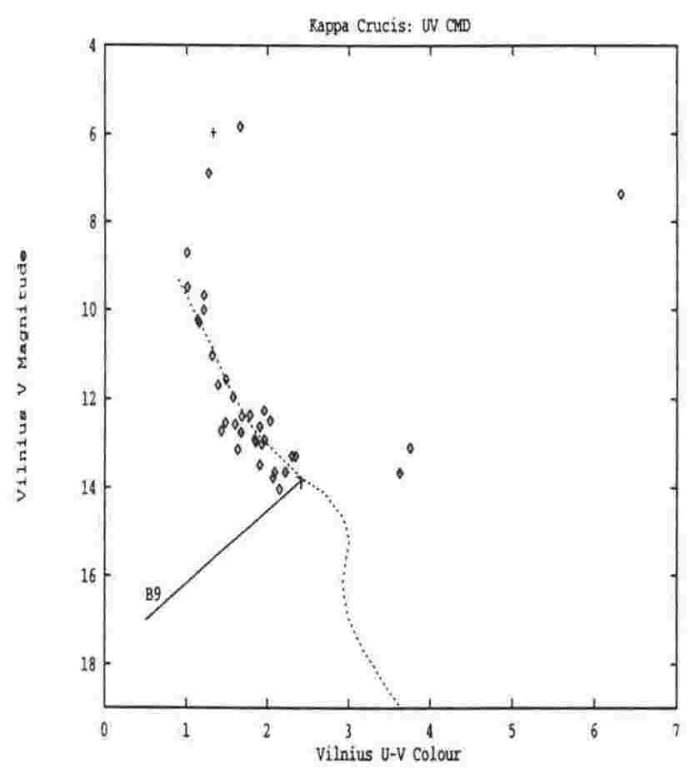

(a)

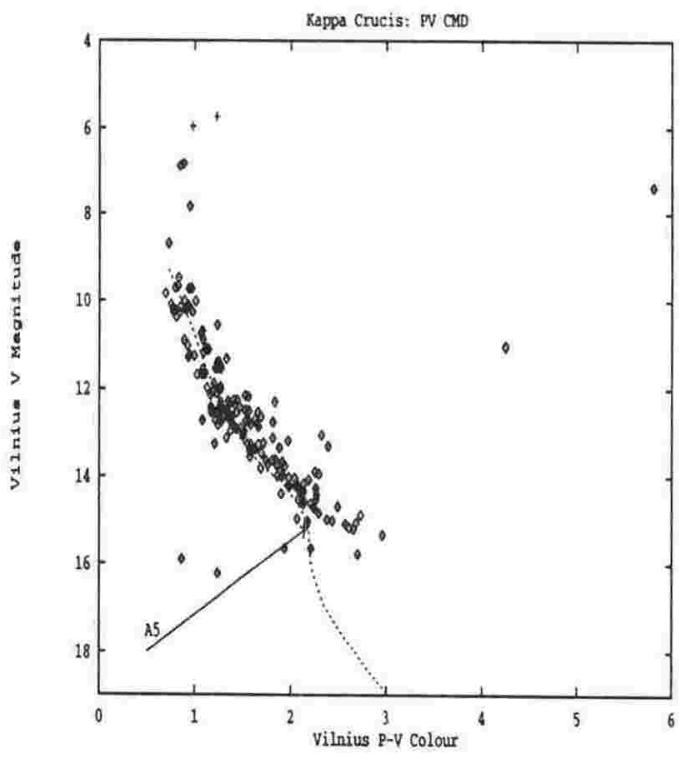

(b)

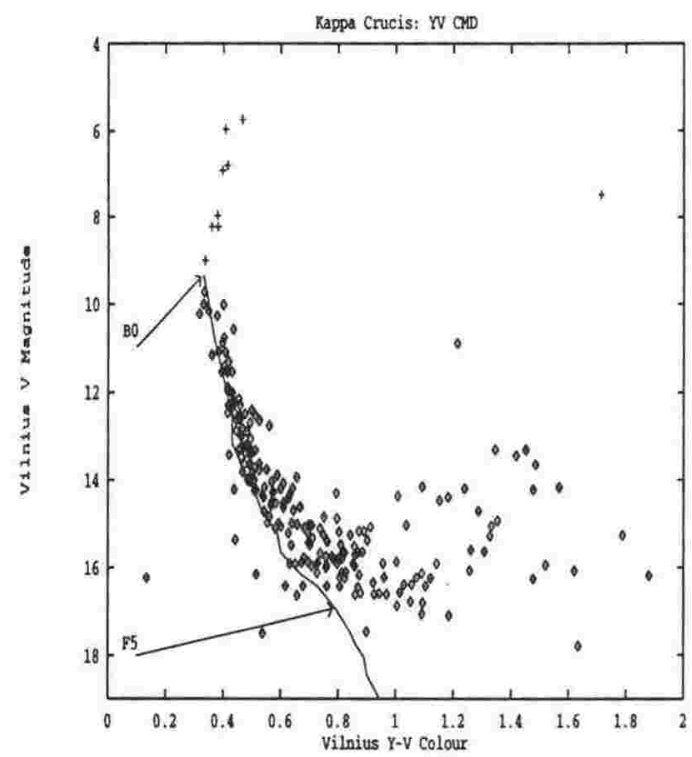

(c)

Figure 6-7: Vilnius Colour-magnitude Diagrams for NGC 4755. Lines representing the main sequence are plotted on each of the colour magnitude diagrams. These have been corrected for reddening, based on the expectation of normal interstellar extinction and the colour excess ratios given in Table 65 of Straižys (1992a). The plus symbols represent data from Forbes (1994). 
Figure 6-7(c) is the YV CMD based on 3.2 minute exposures in the two filters. ${ }^{1}$ It agrees well with the CMDs of Shobbrook (1984), Dachs \& Kaiser (1984), Slettebak (1985), and Frandsen et al. (1989). The density of the brighter stars is lower, due to the small field of view of the CCD, than the cluster-wide studies such as Dachs \& Kaiser (1984). The unusually placed star at V $=10.90$ and $(\mathrm{Y}-\mathrm{V})=1.21$ is star number 104 in Dachs \& Kaiser (DK), with a (V, B - V) of $(11.03,1.57)$, which they excluded as an unlikely member of the cluster. The bright red star $(\mathrm{V}, \mathrm{Y}-\mathrm{V}=7.48,1.71)$ is designated in the DK scheme as D. This star ( $\kappa$ Crucis itself) was used as the centre of the frames taken, since it is effectively in the centre of the cluster.

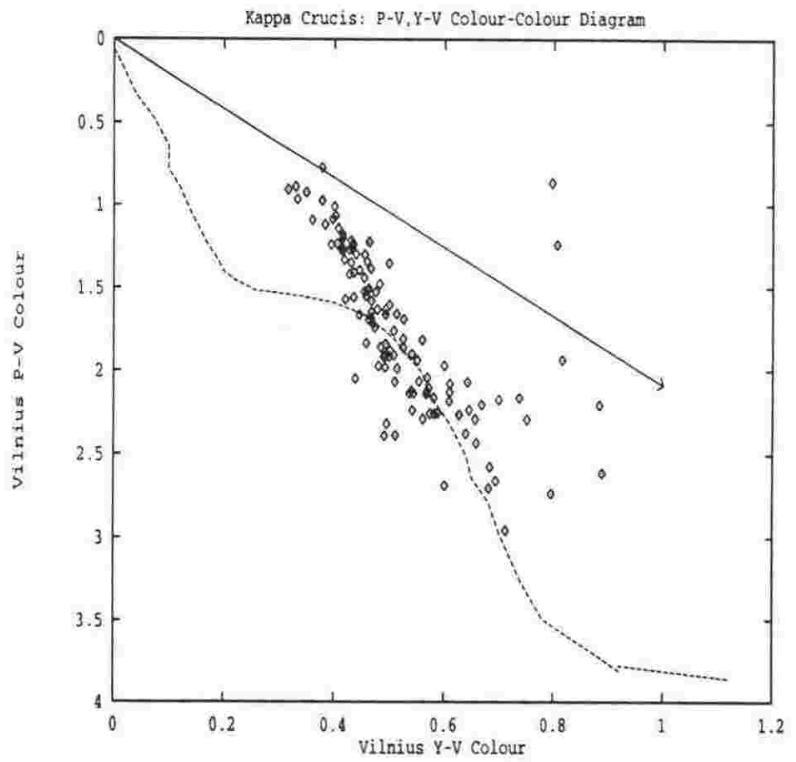

Figure 6-8: P-V, V-Y Colour-Colour Diagram for the $\kappa$ Crucis Cluster. The data has not been dereddened in this figure. The dashed line is the main sequence, and the arrow indicates the direction of reddening.
Reddening was estimated using a colour-colour diagram (see Figure 68) as being $0.69 \pm 0.04$ for $\mathrm{P}-\mathrm{V}$ and $0^{\mathrm{m}} 33 \pm 0^{\mathrm{m}} \cdot 02$ for $\mathrm{Y}-\mathrm{V}$, which correspond to a mean value of $0.42 \pm$ 0 . 03 for $\mathrm{E}(\mathrm{B}-\mathrm{V})$. The reddening is based on the colour excess values tabulated by Straižys (1992a), and is indicated as the arrowed line in Figure 6-8. This degree of reddening was used in conjunction with the main sequence as expected from theory to estimate the distance modulus of the cluster. $\mathrm{V}-\mathrm{M}_{\mathrm{v}}$ is estimated to be $11^{\mathrm{m}} 9 \pm 0 . \mathrm{m} 1$. These values are in reasonable agreement with the literature (see Table 6.4 and Figure 6-9).

Sagar (1987) confirmed the variable extinction across the cluster that Dachs \& Kaiser (1984) had noted to increase with Galactic latitude and angular distance from the Coalsack ${ }^{2}$. The variation in extinction across the cluster could explain the different extinction estimates to some extent. Dachs \& Kaiser (1984) noted that the field studied by Shobbrook (1984) is in a region that they too determined an absorption lower than the mean value of the cluster.

\footnotetext{
${ }^{1}$ The $P, V$ and $Y$ frames were all 3.2 minute exposures, while the $U$ exposure was 0.96 minutes long. The $U$ exposure was the last of the frames to be collected. The cloud front was rapidly approaching the star cluster when the $U$ exposure was being commenced, and hence the observation was kept short.

${ }^{2}$ The Coalsack is a dark nebula, being a large cloud of interstellar gas and dust that is dense enough to obscure the light from objects behind it.
} 


\begin{tabular}{||l|l|l||}
\hline Study & \multicolumn{1}{|c||}{$\mathrm{E}(\mathrm{B}-\mathrm{V})$} & \multicolumn{1}{c||}{$\mathrm{V}-\mathrm{M}_{\mathrm{v}}$} \\
\hline Arp \& van Sant (1958) & 0.31 & 9.6 \\
Feast (1963) & 0.48 & 11.86 \\
Schild (1970) & 0.42 & 11.6 \\
Becker \& Fenkart (1971) & 0.31 & 10.1 \\
Balona \& Crampton (1974) & - & $11.75 \pm 0.08$ \\
Perry et al. (1976) & 0.40 & $11.8 \pm 0.5$ \\
Shobbrook (1984) & $0.380 \pm 0.005$ & $11.4 \pm 0.1$ \\
Dachs \& Kaiser (1984) & $0.41 \pm 0.06$ & $11.82 \pm 0.06$ \\
Balona \& Shobbrook (1984) & - & $11.37 \pm 0.06$ \\
Sagar (1987) & $0.42 \pm 0.05$ & - \\
Kjeldsen \& Frandsen (1991) & $0.40 \pm 0.02$ & $11.85 \pm 0.02$ \\
Balona \& Koen (1994) & $0.376 \pm 0.004$ & $11.51 \pm 0.05$ \\
\hline \multicolumn{2}{|l|}{}
\end{tabular}

Table 6.4: Literature Estimates for Reddening and Distance Modulus of NGC 4755. All units are magnitudes.

\subsubsection{Discussion}

This preliminary work demonstrated that the recently acquired $28 \mathrm{~mm}$ diameter Vilnius filters allow the use of the MJUO CCD Image acquisition system, and the established data reduction pathway at VUW, to produce Vilnius photometry in line with the literature.

The Vilnius filters vary in thickness. The U, P, X, Y, Z, V, and S filters used by this study are $9.79,4.52,3.26,6.25,6.64,6.79$, and $5.91 \mathrm{~mm}$ thick respectively. These different thicknesses lead to a need to refocus the telescope if sharp crowded-field frames are to be collected. This need was considered before the observing run, and not anticipated to be a problem. The focus of the offset guider attached to the MJUO $1 \mathrm{~m}$ telescope is linked to that of the telescope, but the two CCDs do not have the same focal plane. A mirror infront of the filters (i.e., higher in the optic path) directs light to the guider. The mirror was not placed beneath the filter wheel since this arrangement allows the filter wheel to be compact, use of smaller (and therefore cheaper) filters, the use of white light for the guider, and possibly better homogeneity of the glue in glued filters. Refocusing for the $\mathrm{U}$ filter therefore led to the (bright) guide star being taken so far out of focus that it was no longer visible. To focus the guider, it must be rotated around to an access position, the light-tight covering opened, and a micrometer adjusted. The design of the guider has led to the micrometer being difficult to read as it is in a constrained area. Refocusing the guider is therefore time consuming, particularly as often the guider must be rotated around to a star to check that the focus is being adjusted correctly. Unfortunately, the problems in refocusing the guider and the unreliability of the telescope's tracking beyond 10 consecutive minutes (Sullivan 1990), led to the collected 19.1 minute U exposure of NGC 2004 being useless, and so only the short exposure being usable.

The best solution to the focusing problem is to bring the filters all to the same optical depth 


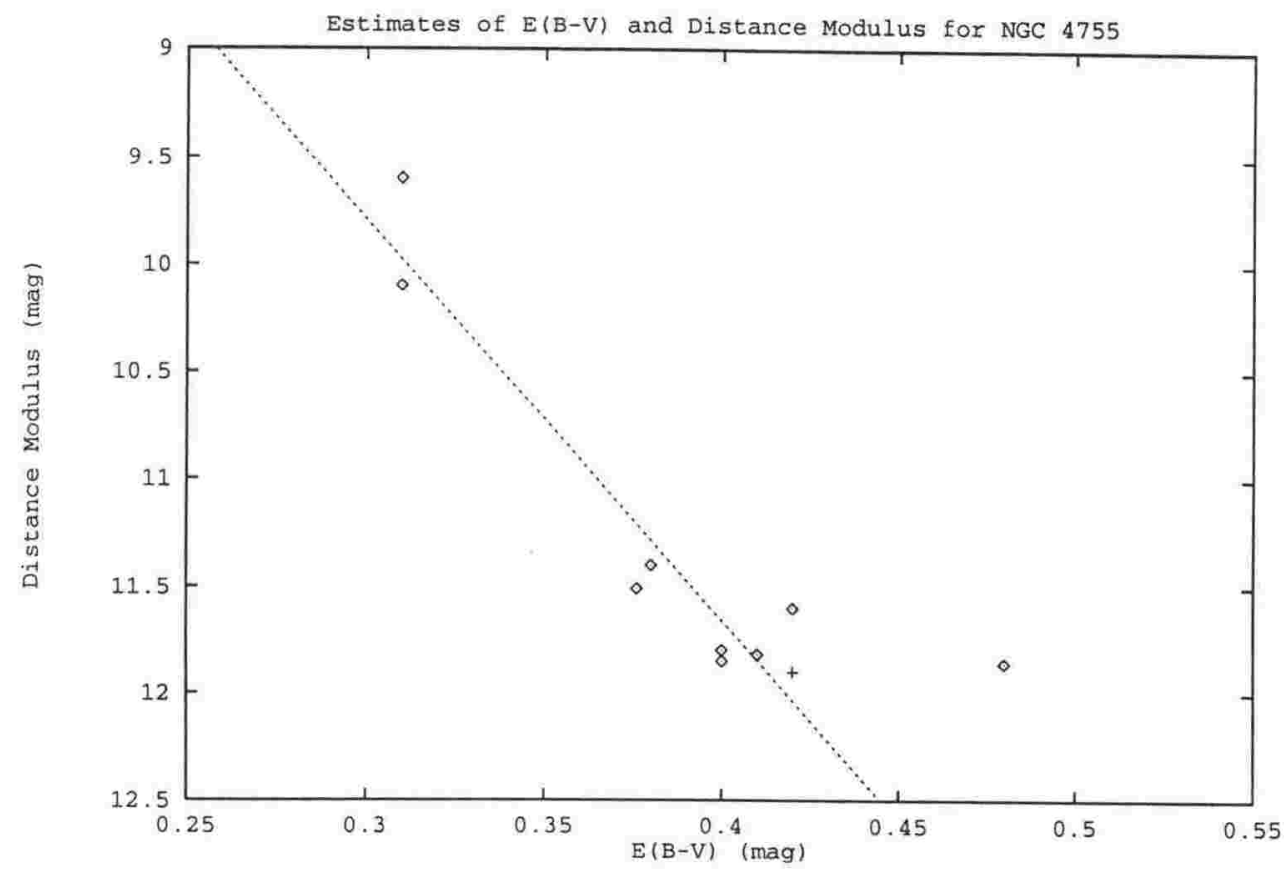

Figure 6-9: Reddening and Distance Modulus Estimates for NGC 4755. These values are taken from Table 6.4 and the text. The plus symbol indicates the values calculated by the current study. The dashed line represents the best linear fit to the data when the value of Feast (1963) is excluded. The distance modulus indicated by this line is given by $4.2+18.7 \mathrm{E}(\mathrm{B}-\mathrm{V})$ mag. The line indicates a correlation between reddening and the distance modulus. When low reddenings for NGC 4755 are derived by a study, the distance modulus is also less.

as the U filter. This has been attempted using Schott FK5 for the P and X filters, and B270 for the remainder, with careful attention paid to not significantly altering the spectral profiles of the filters. Dow Corning Q2-3067 Optical Couplant was used to attach suitably thick, pitchpolished flats to the filters. This Couplant was used in preference to Canadian Balsam since the Couplant is removable. This was considered desirable as further adjustments may need to be made to the blanks, together with the consideration of the difficulty in obtaining the filter set and so not being prepared to risk damage to it. Transmission tests of the filters before and after these modifications showed no significant difference. A glass blank was ground to the same thickness as the other filters, allowing the telescope to be focused in white light. This allowed the telescope to be focused more rapidly than if focus observations were collected through one of the narrow Vilnius bandpasses.

\subsection{The February 1994 Run}

It was intended to collect CCD frames of a LMC star cluster through all of the Vilnius filters, now modified to approximately the same optical depth. The advantages of this filter set for 
the study of star clusters have not yet been definitively demonstrated in the literature. Such a demonstration is essential before observing time on larger instruments can be successfully applied for. The narrow bandpasses of the set combined with the small telescopes available at MJUO, limited the current study to only the brightest of the LMC clusters. NGC 1968/74 was selected, being the cluster whose main sequence extended to the brightest magnitudes of all the clusters covered by the survey. The Galactic cluster Melotte 105 was chosen to be a 'control' cluster, given that the cluster had already been studied (see below). The previous research would then act as a check on the CMDs and parameters derived from the Vilnius observations.

The transformation coefficients for the one clear night of this observing run are given in Table 6.5. The standards were all in the $\kappa$ Crucis cluster, which was rising as the night progressed.

\subsubsection{Melotte 105}

Melotte 105 is an open cluster that has not attracted much attention. It is a compact cluster located in the Galactic plane in the direction of Carina. In the literature, only three studies have produced colour-magnitude diagrams for the cluster. The first was based on the UBV photoelectric and photographic photometry of Sher (1965), who estimated the cluster age via isochrone fits as $\sim 10^{8}$ years. As part of a search for small photometric fluctuations in giant and supergiant stars, Frandsen et al. (1989) produced a Johnson BV CMD based on CCD observations, which extended to $\mathrm{V} \sim 19$. Frandsen et al. (1989) adopted the distance modulus $\left(\mathrm{V}-\mathrm{M}_{\mathrm{v}}\right)=12^{\mathrm{m}} \cdot 7$ and reddening $\mathrm{E}(\mathrm{B}-\mathrm{V})=0^{\mathrm{m}} \cdot 38$ of Sher $(1965)$. Kjeldsen \& Frandsen (1991) re-observed the cluster and derived independent values for these parameters. $\mathrm{E}(\mathrm{B}-\mathrm{V})$ was estimated to be $0^{\mathrm{m}} 52 \pm 0^{\mathrm{m}} 03$, and $\left(\mathrm{V}-\mathrm{M}_{\mathrm{v}}\right)$ as $13^{\mathrm{m}} \cdot 35 \pm 0^{\mathrm{m}} \cdot 20$. The authors considered that the difference was due to the improved detector used, as well as use of DAOphot in this rather crowded field. However, Kjeldsen \& Frandsen (1991) commented that the response of the CCD combined with the U and B filters used by their study differs substantially from the standard responses, and that there were severe problems transforming their data across to the standard system.

Melotte 105 (coordinates $\alpha=11^{\mathrm{h}} 19^{\mathrm{m}} 40^{\mathrm{s}}, \delta=-63^{\circ} 29.5^{\prime}$ for Epoch 2000.0) was observed on February $15^{\text {th }}$ 1994. Seeing was reasonable for MJUO, with a mean of $3.0^{\prime \prime}$ for frames of the cluster. All exposures were 14.35 minutes long. Unfortunately, the flat attached to the Y filter had been accidentally made too thick, and was returned to the optical workshop for regrinding before the observing run. This required the removal of the flat from the $\mathrm{Y}$ filter, and subsequent re-attachment. During the observing run air bubbles increasingly invaded the optical couplant, making the $Y$ filter unsuitable for imaging. Later attempts to separate, clean, and rejoin the flat to the filter proved unsuccessful, again with air penetrating the couplant. The flat has 


\begin{tabular}{||c|c|c|c|c|c|c||}
\hline Filter & $\mathrm{q}_{1}$ & $\mathrm{q}_{2}$ & $\mathrm{q}_{3}$ & RMS & Std RMS & \# Obs \\
\hline $\mathrm{U}$ & $17.531 \pm 0.030$ & $-0.008 \pm 0.007$ & $0.654 \pm 0.019$ & 0.031 & 0.027 & 40 \\
$\mathrm{P}$ & $17.260 \pm 0.031$ & $+0.091 \pm 0.006$ & $0.555 \pm 0.019$ & 0.032 & 0.023 & 47 \\
$\mathrm{X}$ & $17.488 \pm 0.028$ & $+0.015 \pm 0.010$ & $0.365 \pm 0.017$ & 0.030 & 0.018 & 50 \\
Z & $17.622 \pm 0.025$ & $+0.019 \pm 0.004$ & $0.136 \pm 0.017$ & 0.025 & 0.023 & 43 \\
V & $17.484 \pm 0.029$ & $+0.029 \pm 0.003$ & $0.149 \pm 0.015$ & 0.019 & 0.018 & 36 \\
S & $16.611 \pm 0.030$ & $+0.028 \pm 0.004$ & $0.078 \pm 0.025$ & 0.027 & 0.018 & 32 \\
\hline
\end{tabular}

Table 6.5: February 1994 Transformation Coefficients where $\mathrm{q}_{1}, \mathrm{q}_{2}$, and $\mathrm{q}_{3}$ are as given in Equation 6.2, except that the colour is X - V. 'Std RMS' lists the expected RMS given the formal uncertainties listed by Forbes (1994) for the standard stars. The column '\# Obs' indicates the number of standard star observations made per filter. 15 individual standards were observed, with a (Y-V) colour range of 0.31 to 1.71 . The airmass range was 1.0 to 1.8 .

\begin{tabular}{||c|c|c|c||c|c||}
\hline \multicolumn{4}{|c||}{ Sher } & \multicolumn{2}{c||}{ Current Study } \\
\hline Star & V & B - V & X - V & V & X - V \\
\hline 9 & 12.09 & 1.32 & 2.69 & 11.96 & 2.71 \\
10 & 13.00 & 0.37 & 0.99 & 12.95 & 0.92 \\
23 & 14.08 & 1.11 & 1.16 & 14.01 & 1.09 \\
\hline
\end{tabular}

Table 6.6: Comparison of Melotte 105 Photometry. Three stars photoelectrically measured by Sher (1965) were in common with the current study. The (B-V) indices of these stars were converted to the expected Vilnius X-V colours, using Equation 6.3. All units are magnitudes

now been attached to the filter with Canadian Balsam, given that the optical couplant appears to be usable for our purposes once only. The lack of $\mathrm{Y}$ observations meant that the standard reduction technique for Vilnius data could not be followed for Melotte 105, as it centres around the Y filter. This method is described in Straižys \& Sviderskiene (1972), and Straižys (1992a).

Aperture corrections add further uncertainties to the transformations, beyond those given in Table 6.5. For Melotte 105, the uncertainties in the aperture corrections for the U, P, X, Z, $\mathrm{V}$, and S filters are $0.029,0.034,0.013,0.014,0.008$, and 0.024 magnitudes respectively. The transformations for this cluster are in good agreement with Sher (1965). Forbes (1994) has derived the following conversion from the Vilnius (X-V) colour to Johnson (B - V):

$$
(B-V)=a+b(X-V)
$$

where for $(\mathrm{B}-\mathrm{V})>0.8, \mathrm{a}=0.070$ and $\mathrm{b}=0.465$, and for $(\mathrm{B}-\mathrm{V})<0.8, \mathrm{a}=-0.329$ and $\mathrm{b}=0.707$. Only three stars of the current study are in common with the stars Sher (1965) measured via photoelectric photometry. Results of the comparison are given in Table 6.6, and are in good agreement, particularly given the uncertainties in the transformation relations of the current work and that Sher (1965) considered his photoelectric photometry beyond $\sim 13$ th magnitude to be suspect. The photometry of the current study does appear to be somewhat 
brighter.

The cluster has galactic coordinates $(l, b)=(292 \div 90,-2 \div 42)$, and is therefore subject to substantial interstellar absorption. Reddening was estimated using Figure $6-10 . \mathrm{E}(\mathrm{Z}-\mathrm{S})$ is $0.32 \pm 0 . \mathrm{m} 04$, which corresponds to $\mathrm{E}(\mathrm{B}-\mathrm{V})$ being $0.34 \pm 0.04$. This value is within error of that of Sher (1965), but substantially different from the value given by Kjeldsen \& Frandsen (1991). Santos \& Bica (1993) estimated the reddening for Melotte 105 using integrated spectra of cluster stars in the visible and nearinfrared as being $\mathrm{E}(\mathrm{B}-\mathrm{V})=0^{\mathrm{m}} \cdot 30$ $\pm 0^{\mathrm{m}} \cdot 02$, which is within the formal uncertainty of the reddening

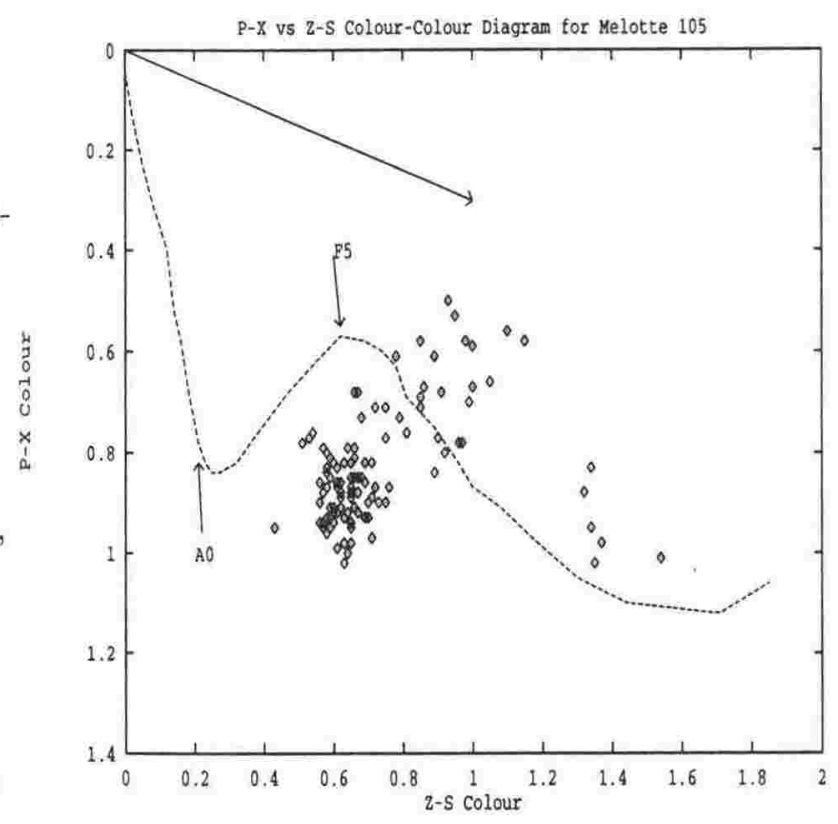

Figure 6-10: P-X, Z-S Colour-Colour Diagram for Melotte 105. The direction of reddening is indicated by the arrow pointing from the origin of the figure. There is a large scatter about the main sequence due to the photometric uncertainties. The clump to the lower right of the figure are stars evolved off the main sequence.

estimated by the present study. The reddening was used in conjunction with the expected luminosity class $\mathrm{V}$ sequence to estimate the distance modulus of the cluster (see Figures 6-11 and 6-12). The true distance modulus ${ }^{1} \mathrm{~V}-\mathrm{M}_{\mathrm{V}}$ was found to be $12^{\mathrm{m}} 9 \pm 0.3$, which is again closer to the value given by Sher (1965).

Given the lack of $Y$ observations, the standard classification technique outlined by Straižys (1992a) could not be followed. However, Straižys (1974) proposed a technique based on the reddening-free energy distributions, i.e., using the $Q$ factors as given in Equation 6.1. This method could be used despite no $\mathrm{Y}$ data being available. The $\mathrm{Q}$ parameters were calculated with respect to the V-S colour index. The parameters Quvs, QPvs, Qxvs, and Qzvs were then plotted against the central wavelength of the first filter in each $Q$ factor. Normally QYvS is included. These $Q(\lambda)$ functions have very different shapes for different spectral types and luminosity classes (see Figure 6-13 on page 162).

While this "poor man's spectroscopy" did not require the Y filter, the effectiveness of the technique was reduced without it. In addition, only the brightest stars in the cluster could be classified, given that the exposures in each filter were of the same length. Hence the U

\footnotetext{
${ }^{1}$ As opposed to the apparent distance modulus which includes the effect of interstellar absorption, i.e., the apparent distance modulus (in $\mathrm{V}$ ) is the true modulus plus $\mathrm{A}_{\mathrm{V}}$.
} 


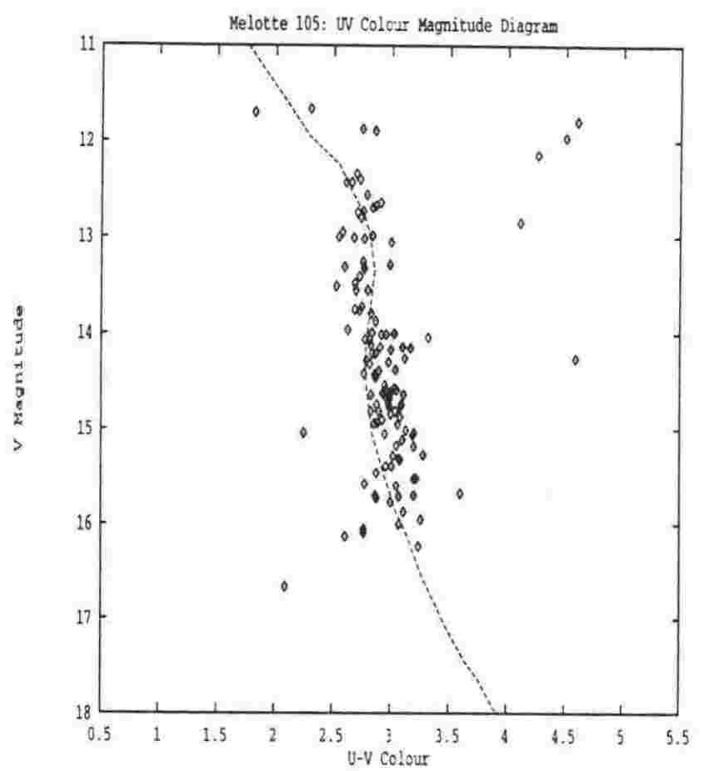

(a)

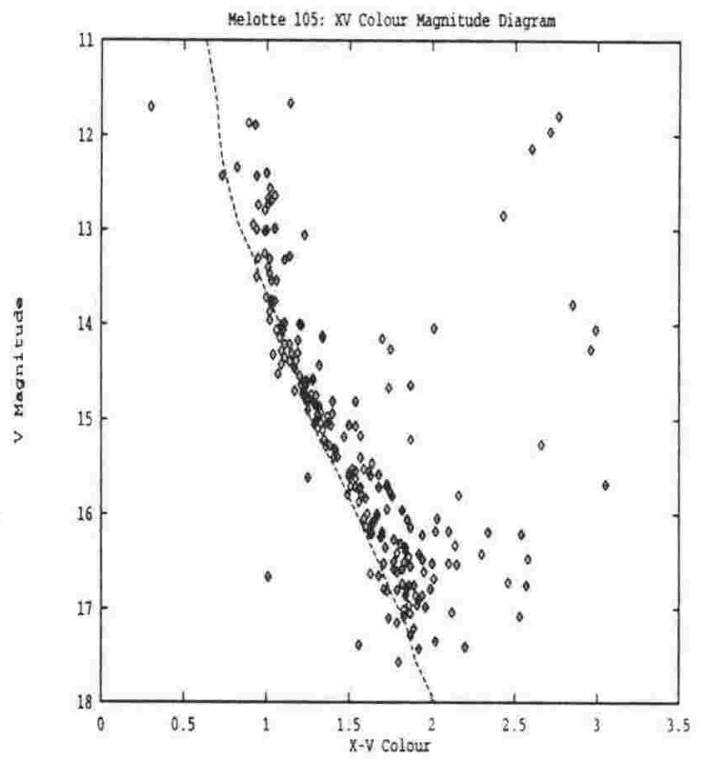

(c)

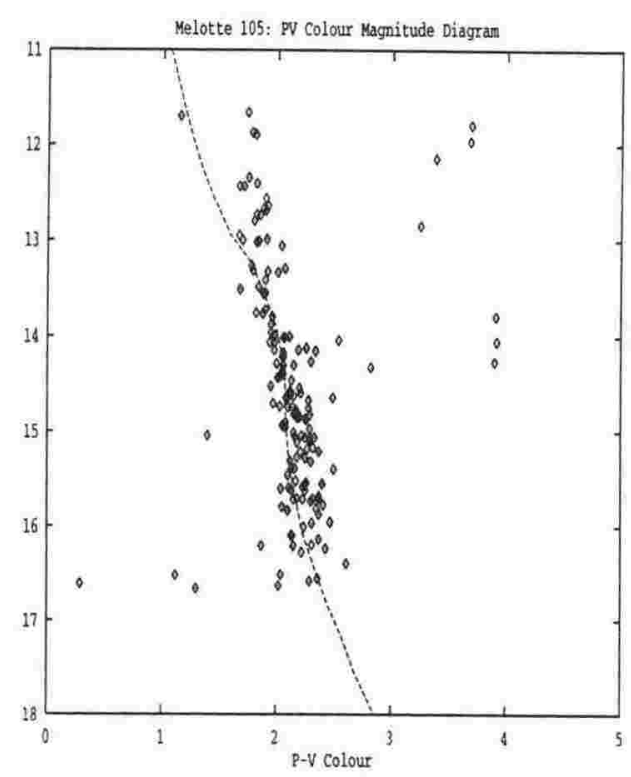

(b)

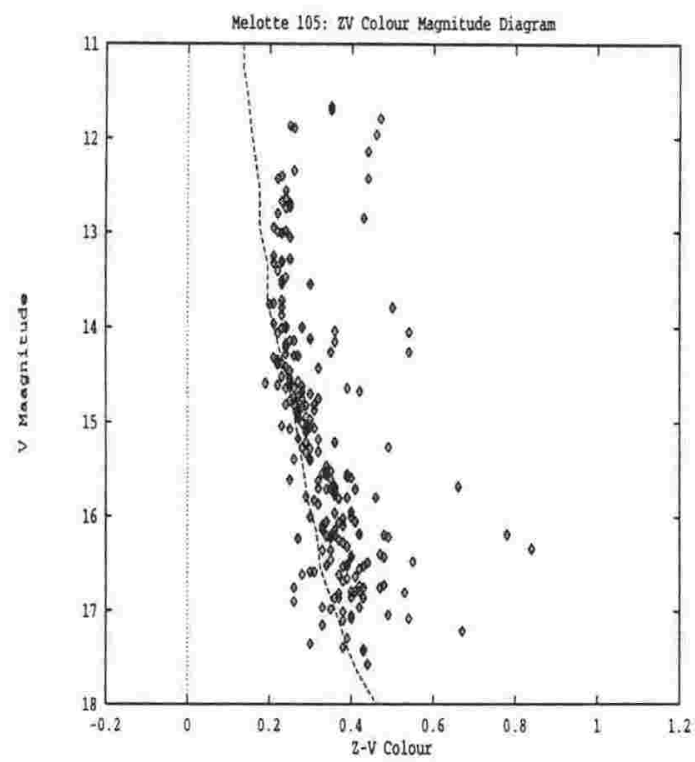

(d)

Figure 6-11: Vilnius Colour-magnitude Diagrams for Melotte 105. Subfigure (a) is a U-V CMD, (b) is P-V, (c) X-V, and (d) Z-V. The V-S CMD for this cluster is given as Figure 612. Lines representing the expected main sequence are plotted on each of the colour-magnitude diagrams. These have been corrected for reddening, as calculated from Figure 6-10. A mean distance modulus $\mathrm{V}-\mathrm{M}_{\mathrm{V}}$ of $12^{\mathrm{m}} \cdot 9 \pm 0 . \mathrm{m}^{\mathrm{m}}$ was derived from fitting the luminosity class $\mathrm{V}$ lines with the data. This value is within error of the $12^{\mathrm{m}} \cdot 7$ value of Sher (1965) and also the $13^{\mathrm{m}} \cdot 35$ \pm 0 m 20 value of Kjeldsen \& Frandsen (1991), although it is closer to the first value. 


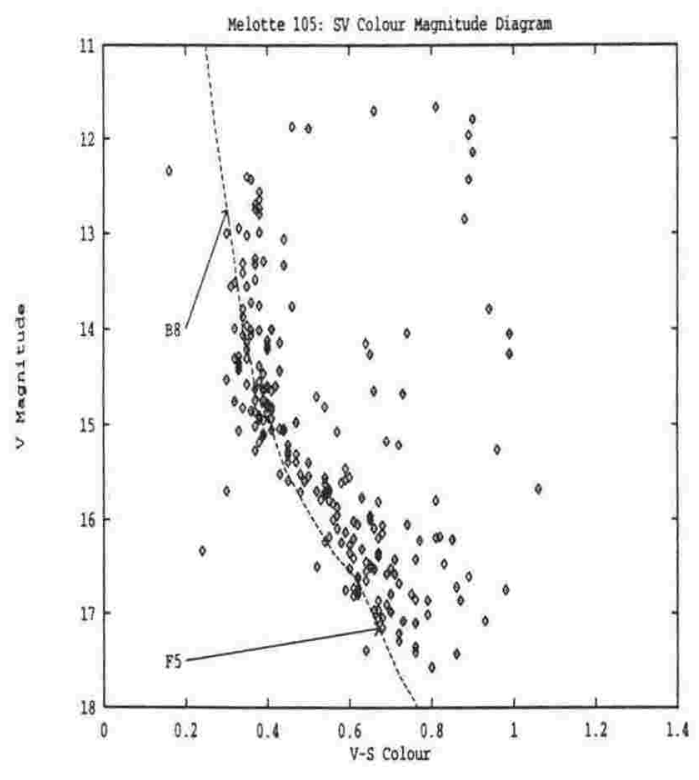

Figure 6-12: Vilnius VS CMD for Melotte 105. A line representing the expected (reddened) main sequence is plotted with the data.

and P filters had brighter limiting magnitudes, given their through-puts. The supergiants were classified as late G stars, while the main sequence extended from $\sim$ B9 through to late A. The magnitudes, positions, and identification numbers of the classified stars are given in Table 6.6 (starting on page 168). Classifications that are uncertain are indicated with a $\dagger$, while a dash indicates stars that could not be classified at all. These stars tended either to be faint, or to be products of images merging together. For instance, the latter was the case of the stars 116 and 117, which are the bright stars to the left of the main sequence tip in Figure 6-11c (see also the star-chart given as Figure 6-14). The table is sorted in order of increasing V magnitude. The errors given are those calculated by DAOphot. The stars with unusual classifications appear to be field stars, located on the CMDs well off the main sequence of the cluster. The major problem with the technique was that the $Q(\lambda)$ functions for most of the spectral range (early to mid A stars) covered by the main sequence do not change greatly (see Figure 6-13), especially given the missing QYvs factor. The XV colour-magnitude diagram was therefore used to select the appropriate spectral type, and so the initial colour excess ratios, in the first iteration of the classification. At least two iterations were used to produce the results listed in Table 6.6. More were required for the unusual stars. Excesses for later iterations were based on the spectral type estimated by the immediately previous step.

The classifications are in good agreement with Frandsen et al. (1989) who commented that the main sequence for Melotte 105 commenced at B8. Kjeldsen \& Frandsen (1991) state that Sher (1965) reached down to the spectral type A5 on the main sequence. The faintest magnitude tabulated by Sher (1965) is 14.99 , which corresponds to A5 V stars in the current analysis. 


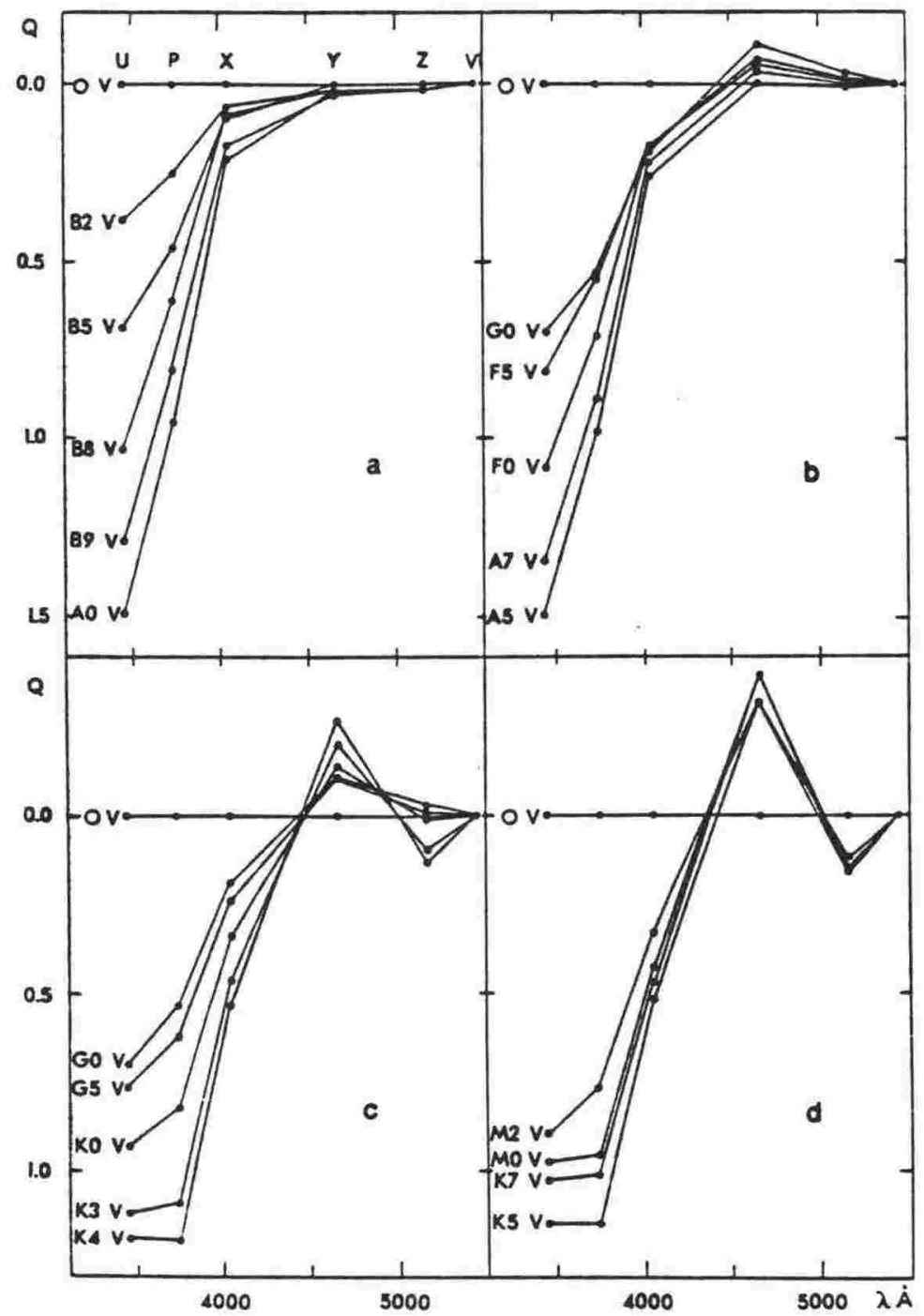

Figure 6-13: Reddening Free $Q(\lambda)$ Functions for Luminosity Class V Stars are shown using all seven Vilnius filters. This classification method uses the information contained in five independent $Q$ parameters, instead of only two as in the standard classification technique. This figure was taken from Straižys (1992a). 
Melotte 105 Star Positions

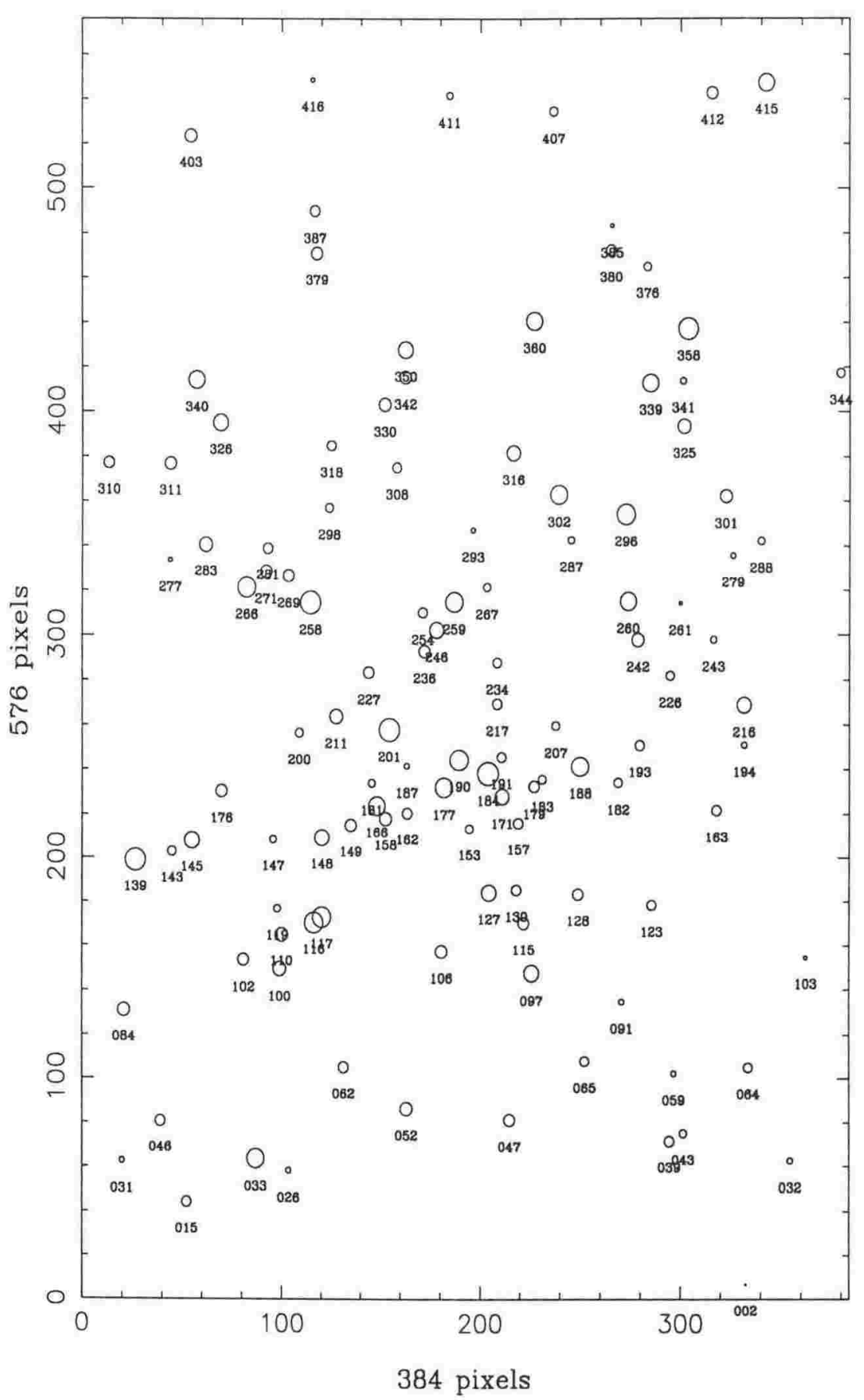

Figure 6-14: Positions and Identifications of Stars in Melotte 105. The radius of a circle indicating the position of a star is proportional to the $\mathrm{V}$ magnitude of that star. The identification number of a star is slightly offset to below the position of the star. The UPXVZS magnitudes, $\mathrm{x}$ and $\mathrm{y}$ positions, and identification numbers of the stars plotted in this figure are given as Table 6.6 (on page 168). North is up, and east is left. 


\subsubsection{NGC 1968/74}

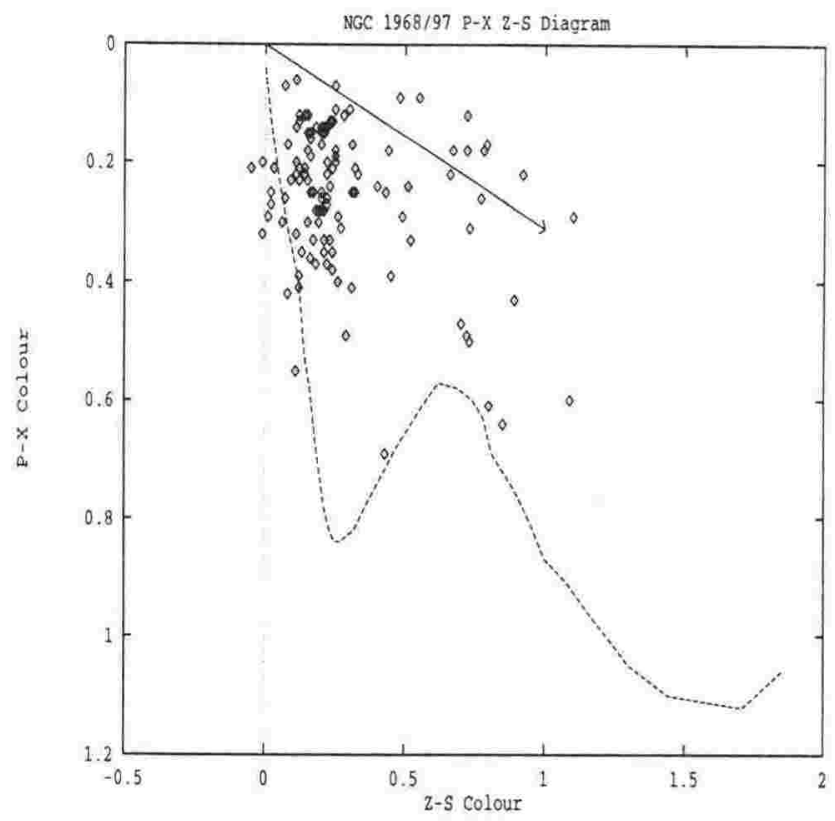

Figure 6-15: P-X, Z-S Colour-Colour Diagram for NGC 1968/74. The direction of reddening is indicated.
These open clusters were imaged together earlier in the night than Melotte 105, being the primary targets and then at low airmasses. Exposures in all filters were 14.35 minutes long. Aperture corrections were estimated using the PSF stars with any neighbouring stars subtracted using the final PSF. Between 5 to 7 bright stars were used to construct the model PSF in the frames. The aperture corrections were estimated to within a standard deviation of 0.02 magnitudes. The mean seeing during the NGC $1968 / 74$ exposures was

$3.2^{\prime \prime}$, at a mean airmass of 1.26 .

The scatter in Figure 6-15 is large. This is partly due to the difficulties of crowded field photometry in frames of poor seeing, and also to the variable extinction expected across the clusters. Variable amounts of gas and dust are particularly obvious in the S observations, but are far less obvious in the other frames. Presumably this is at least partially accountable for the increased scatter in the $\mathrm{S}$ observations, which is particularly evident in Figure 6-15 and also in Figure 6-17. The colour-colour diagram was used to estimate $\mathrm{E}(\mathrm{B}-\mathrm{V})$ as $0^{\mathrm{m}} 13 \pm 0^{\mathrm{m}} 04$, which is larger than expected from the Johnson observations given in the previous chapter, but within error of the previous estimate (which was 0 m09).

Attempts were made to use other colour-colour diagrams, such as $\mathrm{P}-\mathrm{X}$ against $\mathrm{X}-\mathrm{V}$, to estimate the reddening without using the rather noisy S observations. However, either the angle that the reddening lines made with the expected sequences or the colour difference between the observed and expected sequences were too small for an improvement to be made to the estimate. Spectral classification was attempted using the $\mathrm{Q}(\lambda)$ technique. Results are given in Table 6.7 on page 170. Half the total number of stars could be satisfactorily classified. This again appears to be due to the scatter in the $\mathrm{S}$ observations, which affects all the $\mathrm{Q}$ factors used in this technique, and makes classification difficult.

\subsection{Conclusions}

These observations have shown that it is possible to collect usable Vilnius observations using 


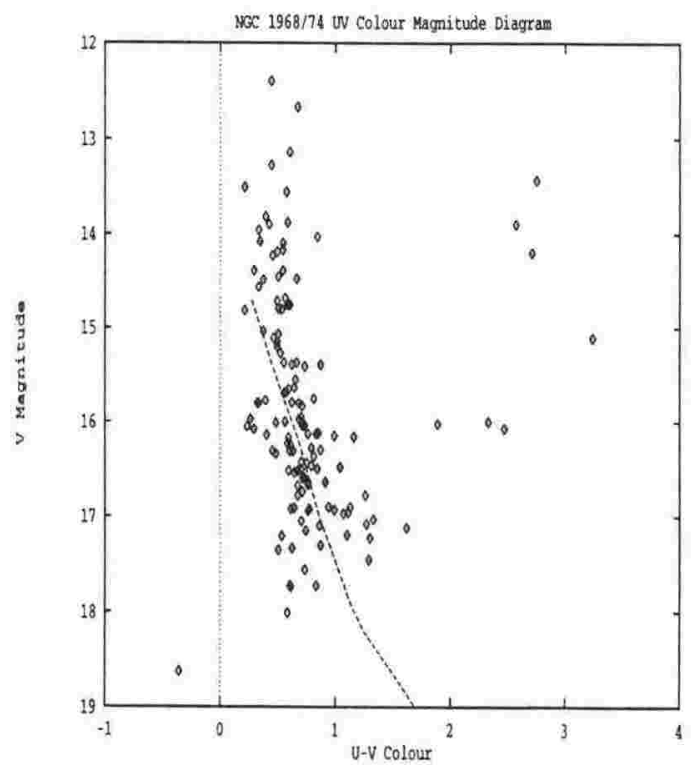

(a)

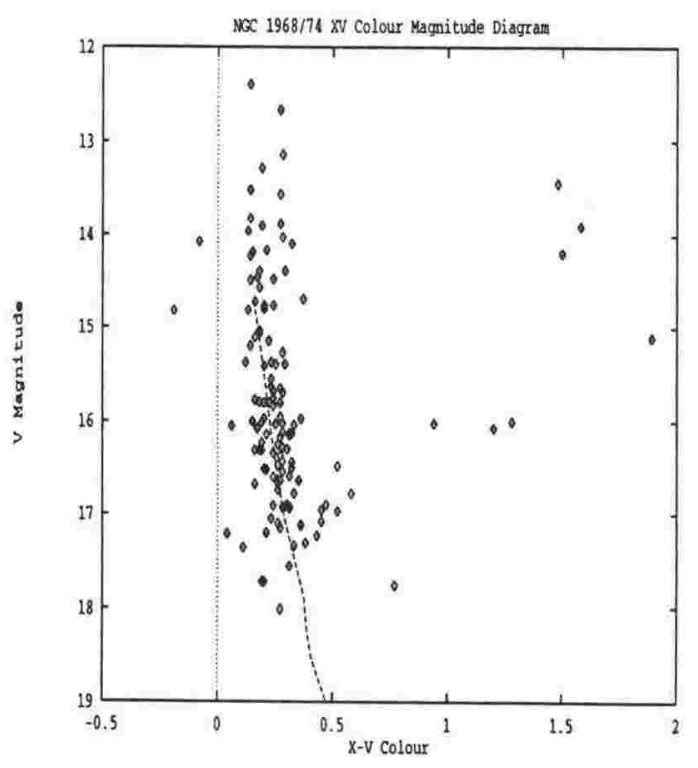

(c)

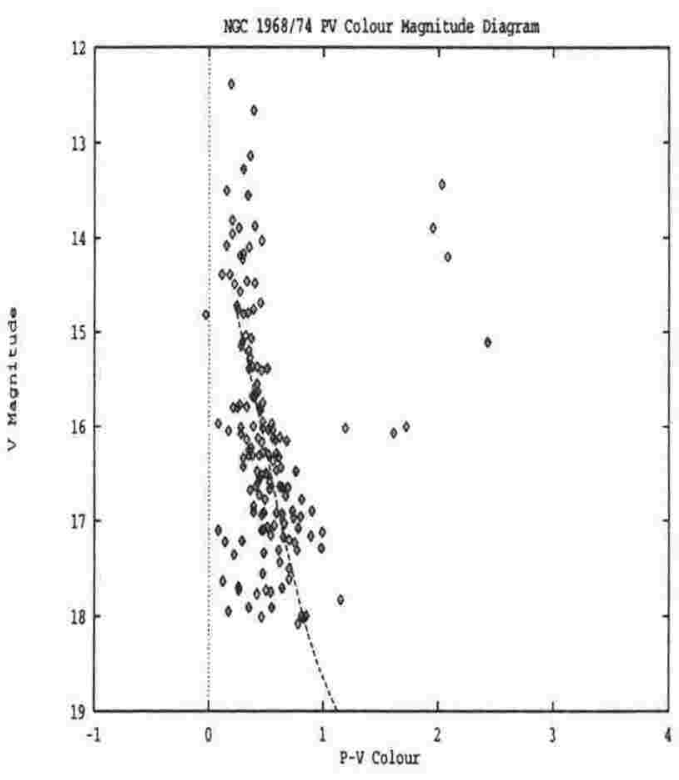

(b)

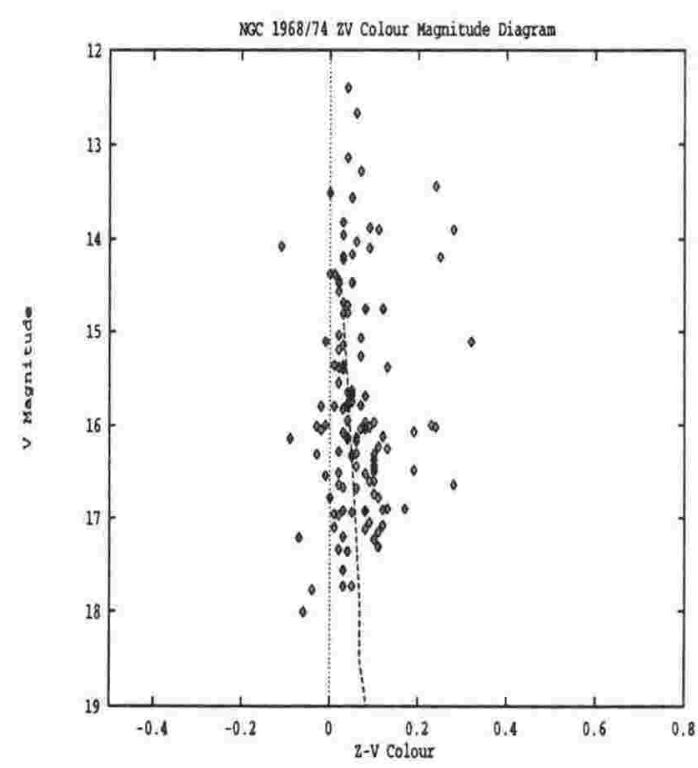

(d)

Figure 6-16: Vilnius Colour-magnitude Diagrams for NGC 1968/74. Subfigure (a) is a U-V CMD, (b) is P-V, (c) X-V, and (d) Z-V. The V-S CMD for the cluster is given as Figure 6-17. Lines representing the expected main sequence (MS) are plotted on each of the colour-magnitude diagrams. These have been corrected for reddening, as given by Figure 615. The MS sequences extend to early B stars, which is the earliest class of star with colours tabulated in Straižys (1992a). A V distance modulus of $18^{\mathrm{m}} \cdot 4$ was assumed. Photometric scatter results in some negative colour indices. It would be worthwhile for a later study to prepare isochrones in the Vilnius system. This should not be too difficult a task for some of the filters which have well-defined transformations from the Johnson B and V bandpasses. This would allow the age of the cluster to be estimated. 


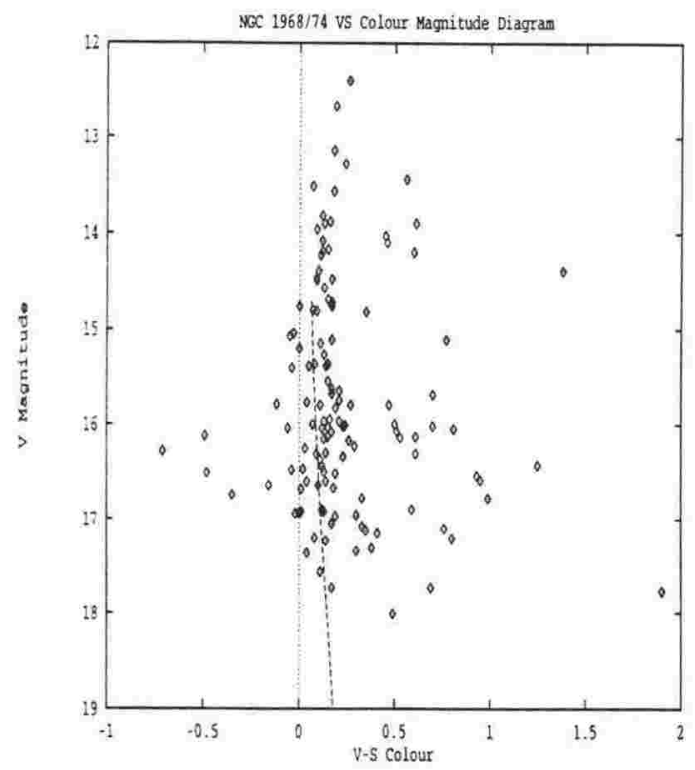

Figure 6-17: Vilnius VS Colour-magnitude Diagrams for NGC 1968/74. A line representing the expected reddened main sequence is plotted with the data.

the MJUO CCD system, and to derive information such as the distance moduli, reddenings, and spectral and luminosity classes of component stars. However, there is a caveat. These tests were performed under moderate seeing conditions for MJUO. For confident estimation of this information, and in particular the spectral and luminosity types, better seeing is required if faint and relatively crowded fields are to be imaged. Future observers should convolve a spectra of the type of star (e.g. from the catalog of Gunn \& Stryker 1983) they expect to observe against the combined response of the Vilnius filters and the CCD. The ratios of the exposure lengths across the different filters could then be calculated, allowing the population of stars to be observed to the same accuracy in all filters.

For the LMC clusters and their population of early stars, the U and P exposure lengths will be substantially longer than that of the $\mathrm{V}$ filter. Given the light grasp of the McLellan telescope, the need for good intensity images, and the short length of the summer nights when these objects are well-placed for observing, it would be worth considering spreading observations of a cluster over several nights. This could make for a long programme, however, considering the experience of this study with the weather conditions at MJUO.

Given the narrow bandpasses of the Vilnius filter system, the relatively small apertures of the available MJUO telescopes and the typical seeing experienced at that site, Vilnius photometry of LMC clusters is limited to only the youngest star clusters. As noted above, the advantages of the Vilnius photometric system for observations of a star cluster have not yet been definitively demonstrated in the literature. Therefore, given the above limitations, it would be worthwhile for a follow-up study to concentrate on a nearby compact Galactic cluster. NGC 4755 would be 
ideal, being small enough that a mosaic of only a few CCD frames would cover the entire object. It is a bright cluster, with the advantage that its brightest members are centrally concentrated. Good photometry of the bright stars could be collected with short integrations, while longer integrations could be performed to the side of these bright stars reaching fainter magnitudes with similar accuracies. The various reduction techniques and analysis methods described by Straižys (1992a) could be applied to the data. Such an illustration of the Vilnius system and its merits could then be used as support in applications for observing time on larger instruments, with which fainter objects such as the LMC clusters could be studied. The increase in aperture would allow good photometry of the faint cluster stars to be collected, and in turn result in increased confidence in parameters derived for the clusters and their component stars. This would be a preferable approach to that suggested in the previous paragraph, being a better application of the available equipment. 
Table 6.6: Melotte 105 Photometry. The magnitude of each filter is listed, together with the formal error estimated by DAOphot. The columns $\mathrm{x}_{\mathrm{c}}$ and $\mathrm{y}_{\mathrm{c}}$ give the centroid position of each stellar image in the $\mathrm{V}$ frame. The column 'ID' gives the $\mathrm{V}$ band identification number assigned to the star by DAOphot, while 'Class' lists the spectral type and luminosity class calculated for each star using the Q-spectra method (see text). The † symbols indicate those classifications that are unreliable.

\begin{tabular}{|c|c|c|c|c|c|c|c|c|c|}
\hline $\mathrm{U} \pm \delta \mathrm{U}$ & $\mathrm{P} \pm \delta \mathrm{P}$ & $\mathrm{X} \pm \delta \mathrm{X}$ & $\mathrm{Z} \pm \delta \mathrm{Z}$ & $\mathrm{V} \pm \delta \mathrm{V}$ & $\mathrm{S} \pm \delta \mathrm{S}$ & $x_{c}$ & $y_{c}$ & ID & Class \\
\hline $16.41 \pm 0.02$ & $15.48 \pm 0.01$ & $14.50 \pm 0.01$ & $12.25 \pm 0.03$ & $11.79 \pm 0.03$ & $10.88 \pm 0.04$ & 203 & 238 & 184 & G8I \\
\hline $14.66 \pm 0.01$ & $13.65 \pm 0.01$ & $12.75 \pm 0.01$ & $12.10 \pm 0.01$ & $11.89 \pm 0.01$ & $11.40 \pm 0.01$ & 154 & 258 & 201 & $\mathrm{~B} 8 \mathrm{III} \uparrow$ \\
\hline $14.79 \pm 0.01$ & $13.71 \pm 0.01$ & $12.81 \pm 0.01$ & $12.13 \pm 0.03$ & $11.91 \pm 0.02$ & $11.38 \pm 0.01$ & 114 & 314 & 258 & B8III $\dagger$ \\
\hline $16.48 \pm 0.01$ & $15.64 \pm 0.01$ & $14.62 \pm 0.01$ & $12.41 \pm 0.01$ & $11.96 \pm 0.02$ & $11.06 \pm 0.02$ & 26 & 198 & 139 & G8I \\
\hline $16.42 \pm 0.02$ & $15.52 \pm 0.01$ & $14.69 \pm 0.01$ & $12.57 \pm 0.01$ & $12.15 \pm 0.01$ & $11.23 \pm 0.01$ & 303 & 437 & 358 & G5I \\
\hline $15.08 \pm 0.01$ & $14.10 \pm 0.01$ & $13.15 \pm 0.01$ & $12.59 \pm 0.01$ & $12.35 \pm 0.01$ & $12.16 \pm 0.05$ & 116 & 170 & 116 & \\
\hline $15.17 \pm 0.01$ & $14.22 \pm 0.01$ & $13.39 \pm 0.01$ & $12.62 \pm 0.01$ & $12.41 \pm 0.01$ & $12.04 \pm 0.01$ & 189 & 244 & 190 & B9V \\
\hline $15.08 \pm 0.01$ & $14.11 \pm 0.01$ & $13.16 \pm 0.01$ & $12.86 \pm 0.01$ & $12.44 \pm 0.01$ & $11.52 \pm 0.03$ & 120 & 172 & 117 & - \\
\hline $15.13 \pm 0.01$ & $14.15 \pm 0.01$ & $13.36 \pm 0.01$ & $12.63 \pm 0.01$ & $12.44 \pm 0.01$ & $12.06 \pm 0.01$ & 272 & 354 & 296 & $\mathrm{~B} 8 \mathrm{~V}$ \\
\hline $15.39 \pm 0.01$ & $14.46 \pm 0.01$ & $13.57 \pm 0.01$ & $12.79 \pm 0.01$ & $12.57 \pm 0.01$ & $12.17 \pm 0.01$ & 82 & 321 & 266 & V \\
\hline $15.59 \pm 0.01$ & $14.57 \pm 0.01$ & $13.68 \pm 0.01$ & $12.87 \pm 0.01$ & $12.65 \pm 0.01$ & $12.25 \pm 0.01$ & 186 & 314 & 259 & V \\
\hline $15.57 \pm 0.01$ & $14.60 \pm 0.01$ & $13.71 \pm 0.01$ & $12.93 \pm 0.01$ & $12.70 \pm 0.01$ & $12.31 \pm 0.01$ & 181 & 232 & 177 & B \\
\hline $15.53 \pm 0.02$ & $14.55 \pm 0.01$ & $13.73 \pm 0.01$ & $12.97 \pm 0.01$ & $12.74 \pm 0.01$ & $12.34 \pm 0.01$ & 249 & 241 & 188 & B \\
\hline $15.49 \pm 0.01$ & $14.60 \pm 0.01$ & $13.68 \pm 0.01$ & $12.96 \pm 0.01$ & $12.75 \pm 0.01$ & $12.36 \pm 0.01$ & 86 & 63 & 33 & V \\
\hline $15.58 \pm 0.01$ & $14.60 \pm 0.01$ & $13.78 \pm 0.01$ & $13.01 \pm 0.01$ & $12.81 \pm 0.01$ & $12.41 \pm 0.01$ & 239 & 363 & 302 & V \\
\hline $16.98 \pm 0.02$ & $16.11 \pm 0.01$ & $15.23 \pm 0.01$ & $13.28 \pm 0.01$ & $12.85 \pm 0.01$ & $11.96 \pm 0.01$ & 147 & 223 & 166 & III \\
\hline $15.57 \pm 0.01$ & $14.63 \pm 0.01$ & $13.86 \pm 0.01$ & $13.14 \pm 0.01$ & $12.97 \pm 0.01$ & $12.61 \pm 0.01$ & 227 & 440 & 360 & V \\
\hline $15.87 \pm 0.01$ & $14.91 \pm 0.01$ & $14.03 \pm 0.01$ & $13.22 \pm 0.01$ & $13.00 \pm 0.01$ & $12.60 \pm 0.01$ & 57 & 414 & 340 & V \\
\hline $15.59 \pm 0.01$ & $14.71 \pm 0.01$ & $13.93 \pm 0.01$ & $13.20 \pm 0.01$ & $13.01 \pm 0.01$ & $12.69 \pm 0.01$ & 284 & 412 & 339 & V \\
\hline $15.83 \pm 0.01$ & $14.84 \pm 0.01$ & $14.00 \pm 0.01$ & $13.24 \pm 0.01$ & $13.03 \pm 0.01$ & $12.66 \pm 0.01$ & 273 & 315 & 260 & V \\
\hline $16.09 \pm 0.01$ & $15.10 \pm 0.01$ & $14.28 \pm 0.01$ & $13.30 \pm 0.01$ & $13.07 \pm 0.01$ & $12.61 \pm 0.01$ & 342 & 547 & 415 & \\
\hline $16.06 \pm 0.01$ & $15.04 \pm 0.01$ & $14.24 \pm 0.01$ & $13.46 \pm 0.01$ & $13.27 \pm 0.01$ & $12.88 \pm 0.01$ & 162 & 427 & 350 & $V$ \\
\hline $16.32 \pm 0.02$ & $15.37 \pm 0.01$ & $14.42 \pm 0.01$ & $13.53 \pm 0.01$ & $13.30 \pm 0.01$ & $12.88 \pm 0.01$ & 69 & 395 & 326 & V \\
\hline $15.95 \pm 0.01$ & $15.11 \pm 0.01$ & $14.25 \pm 0.01$ & $13.52 \pm 0.01$ & $13.32 \pm 0.01$ & $12.96 \pm 0.01$ & 225 & 147 & 97 & V \\
\hline $16.13 \pm 0.01$ & $15.24 \pm 0.01$ & $14.33 \pm 0.01$ & $13.54 \pm 0.01$ & $13.33 \pm 0.01$ & $12.94 \pm 0.01$ & 54 & 207 & 145 & $10 \mathrm{~V}$ \\
\hline $16.14 \pm 0.02$ & $15.34 \pm 0.02$ & $14.43 \pm 0.01$ & $13.53 \pm 0.01$ & $13.34 \pm 0,01$ & $12.87 \pm 0.02$ & 204 & 184 & 127 & B9V \\
\hline $16.18 \pm 0.01$ & $15.31 \pm 0.01$ & $14.41 \pm 0.01$ & $13.62 \pm 0.01$ & $13.42 \pm 0.01$ & $13.06 \pm 0.01$ & 120 & 209 & 148 & AOV \\
\hline $16.21 \pm 0.01$ & $15.32 \pm 0.01$ & $14.49 \pm 0.01$ & $13.71 \pm 0.01$ & $13.49 \pm 0.01$ & $13.10 \pm 0.01$ & 178 & 302 & 246 & B9V \\
\hline $16.08 \pm 0.01$ & $15.20 \pm 0.01$ & $14.44 \pm 0.01$ & $13.72 \pm 0.01$ & $13.52 \pm 0.01$ & $13.18 \pm 0.01$ & 331 & 268 & 216 & B9V \\
\hline $16.29 \pm 0.02$ & $15.46 \pm 0.02$ & $14.60 \pm 0.01$ & $13.84 \pm 0.01$ & $13.56 \pm 0.01$ & $13.23 \pm 0.01$ & 210 & 228 & 171 & $\mathrm{AOV}$ \\
\hline $16.39 \pm 0.02$ & $15.44 \pm 0.01$ & $14.57 \pm 0.01$ & $13.77 \pm 0.01$ & $13.56 \pm 0.01$ & $13.19 \pm 0.01$ & 216 & 381 & 316 & $\mathrm{AOV}$ \\
\hline $16.51 \pm 0.02$ & $15.62 \pm 0.01$ & $14.71 \pm 0.01$ & $13.94 \pm 0.01$ & $13.73 \pm 0.01$ & $13.35 \pm 0.01$ & 61 & 340 & 283 & B9V \\
\hline $16.48 \pm 0.01$ & $15.58 \pm 0.01$ & $14.77 \pm 0.01$ & $13.95 \pm 0.01$ & $13.76 \pm 0.01$ & $13.36 \pm 0.01$ & 301 & 393 & 325 & B9V \\
\hline $16.53 \pm 0.02$ & $15.65 \pm 0.01$ & $14.80 \pm 0.01$ & $13.95 \pm 0.01$ & $13.77 \pm 0.01$ & $13.29 \pm 0.01$ & 127 & 263 & 211 & $\mathrm{~B} 9 \mathrm{~V}$ \\
\hline $16.66 \pm 0.02$ & $15.76 \pm 0.01$ & $14.81 \pm 0.01$ & $14.01 \pm 0.01$ & $13.80 \pm 0.01$ & $13.44 \pm 0.01$ & 98 & 149 & 100 & $\mathrm{AOV}$ \\
\hline $16.78 \pm 0.02$ & $15.82 \pm 0.01$ & $14.88 \pm 0.01$ & $14.09 \pm 0.01$ & $13.88 \pm 0.01$ & $13.52 \pm 0.01$ & 99 & 165 & 110 & A2V \\
\hline $16.63 \pm 0.02$ & $15.91 \pm 0.01$ & $14.97 \pm 0.01$ & $14.16 \pm 0.01$ & $13.97 \pm 0.01$ & $13.60 \pm 0.01$ & 162 & 86 & 52 & $\mathrm{AOV}$ \\
\hline $16.87 \pm 0.02$ & $15.97 \pm 0.01$ & $15.09 \pm 0.01$ & $14.22 \pm 0.01$ & $14.00 \pm 0.01$ & $13.65 \pm 0.01$ & 278 & 298 & 242 & $\mathrm{AOV}$ \\
\hline $17.06 \pm 0.02$ & $16.12 \pm 0.02$ & $15.19 \pm 0.01$ & $14.27 \pm 0.02$ & $14.01 \pm 0.02$ & $13.58 \pm 0.02$ & 152 & 218 & 158 & $\mathrm{AOV}$ \\
\hline $16.97 \pm 0.02$ & $16.08 \pm 0.01$ & $15.09 \pm 0.01$ & $14.24 \pm 0.01$ & $14.02 \pm 0.01$ & $13.63 \pm 0.01$ & 20 & 131 & 84 & $\mathrm{AOV}$ \\
\hline $17.00 \pm 0.02$ & $16.08 \pm 0.01$ & $15.21 \pm 0.01$ & $14.23 \pm 0.01$ & $14.02 \pm 0.01$ & $13.62 \pm 0.01$ & 54 & 523 & 403 & $\mathrm{AOV}$ \\
\hline $17.39 \pm 0.04$ & $16.58 \pm 0.02$ & $16.02 \pm 0.01$ & $14.39 \pm 0.01$ & $14.04 \pm 0.01$ & $13.29 \pm 0.01$ & 151 & 403 & 330 & G8IV \\
\hline $16.88 \pm 0.01$ & $16.05 \pm 0.01$ & $15.11 \pm 0.01$ & $14.27 \pm 0.01$ & $14.07 \pm 0.01$ & $13.71 \pm 0.01$ & 180 & 157 & 106 & A $2 \mathrm{~V}$ \\
\hline $16.92 \pm 0.02$ & $16.00 \pm 0.01$ & $15.15 \pm 0.01$ & $14.27 \pm 0.01$ & $14.07 \pm 0.01$ & $13.68 \pm 0.01$ & 322 & 362 & 301 & $\mathrm{AOV}$ \\
\hline $16.98 \pm 0.04$ & $16.38 \pm 0.02$ & $15.45 \pm 0.01$ & $14.41 \pm 0.01$ & $14.13 \pm 0.01$ & $13.71 \pm 0.01$ & 134 & 215 & 149 & - \\
\hline $17.09 \pm 0.03$ & $16.13 \pm 0.02$ & $15.21 \pm 0.01$ & $14.38 \pm 0.01$ & $14.15 \pm 0.01$ & $13.77 \pm 0.01$ & 162 & 415 & 342 & $\mathrm{AOV} \uparrow$ \\
\hline $17.27 \pm 0.03$ & $16.33 \pm 0.02$ & $15.47 \pm 0.01$ & $14.39 \pm 0.01$ & $14.15 \pm 0.01$ & $13.70 \pm 0.01$ & 92 & 328 & 271 & AOV \\
\hline $17.35 \pm 0.03$ & $16.49 \pm 0.02$ & $15.82 \pm 0.01$ & $14.50 \pm 0.01$ & $14.16 \pm 0.01$ & $13.50 \pm 0.01$ & 44 & 376 & 311 & KOVI \\
\hline $17.20 \pm 0.02$ & $16.24 \pm 0.02$ & $15.35 \pm 0.01$ & $14.40 \pm 0.01$ & $14.18 \pm 0.01$ & $13.75 \pm 0.01$ & 117 & 471 & 379 & $\mathrm{~A} 2 \mathrm{~V}$ \\
\hline $17.09 \pm 0.03$ & $16.26 \pm 0.02$ & $15.31 \pm 0.01$ & $14.44 \pm 0.01$ & $14.22 \pm 0.01$ & $13.79 \pm 0.01$ & 69 & 230 & 176 & A5V \\
\hline $17.12 \pm 0.03$ & $16.28 \pm 0.02$ & $15.34 \pm 0.01$ & $14.44 \pm 0.01$ & $14.22 \pm 0.01$ & $13.84 \pm 0.01$ & 171 & 292 & 236 & AOV \\
\hline $17.41 \pm 0.02$ & $16.57 \pm 0.02$ & $15.98 \pm 0.01$ & $14.60 \pm 0.01$ & $14.27 \pm 0.01$ & $13.60 \pm 0.01$ & 315 & 542 & 412 & $\mathrm{~K} 2 \mathrm{~V}$ \\
\hline $18.86 \pm 0.11$ & $18.16 \pm 0.07$ & $17.15 \pm 0.03$ & $14.80 \pm 0.01$ & $14.27 \pm 0.01$ & $13.26 \pm 0.01$ & 214 & 81 & 47 & KOIII \\
\hline $17.11 \pm 0.03$ & $16.29 \pm 0.02$ & $15.36 \pm 0.01$ & $14.51 \pm 0.01$ & $14.29 \pm 0.01$ & $13.93 \pm 0.01$ & 221 & 170 & 115 & $\mathrm{~A} 2 \mathrm{~V}$ \\
\hline $17.31 \pm 0.03$ & $16.37 \pm 0.03$ & $15.44 \pm 0.01$ & $14.55 \pm 0.01$ & $14.31 \pm 0.01$ & $13.96 \pm 0.01$ & 80 & 153 & 102 & - \\
\hline $17.31 \pm 0.03$ & $16.46 \pm 0.02$ & $15.48 \pm 0.01$ & $14.56 \pm 0.01$ & $14.31 \pm 0.01$ & $13.93 \pm 0.01$ & 103 & 326 & 269 & A5V \\
\hline $17.45 \pm 0.03$ & $16.44 \pm 0.02$ & $15.55 \pm 0.01$ & $14.60 \pm 0.01$ & $14.39 \pm 0.01$ & $13.98 \pm 0.01$ & 143 & 283 & 227 & $\mathrm{~A} 5 \mathrm{~V}$ \\
\hline $17.33 \pm 0.03$ & $16.46 \pm 0.02$ & $15.52 \pm 0.01$ & $14.60 \pm 0.01$ & $14.40 \pm 0.01$ & $14.04 \pm 0.01$ & 13 & 377 & 310 & A5V \\
\hline $17.23 \pm 0.03$ & $16.46 \pm 0.02$ & $15.50 \pm 0.01$ & $14.65 \pm 0.01$ & $14.43 \pm 0.01$ & $14.07 \pm 0.01$ & 248 & 183 & 128 & A2V \\
\hline 17.32 & $16.45 \pm 0.04$ & $15.74 \pm 0.01$ & $14.74 \pm 0.01$ & $14.44 \pm 0.01$ & $13.99 \pm 0.01$ & 227 & 232 & 179 & A5V \\
\hline 17.36 & $16.60 \pm 0.02$ & $15.62 \pm 0.01$ & $14.70 \pm 0.01$ & $14.47 \pm 0.01$ & $14.05 \pm 0.01$ & 131 & 105 & 62 & A5V \\
\hline
\end{tabular}




\begin{tabular}{|c|c|c|c|c|c|c|c|c|c|}
\hline $\mathrm{U} \pm \delta \mathrm{U}$ & $\mathrm{P} \pm \delta \mathrm{P}$ & $\mathrm{X} \pm \delta \mathrm{X}$ & $\mathrm{Z} \pm \delta \mathrm{Z}$ & $\mathrm{V} \pm \delta \mathrm{V}$ & $\mathrm{S} \pm \delta \mathrm{S}$ & $\mathrm{x}_{\mathrm{C}}$ & $y_{c}$ & ID & Class \\
\hline $17.52 \pm 0.03$ & $16.75 \pm 0.02$ & $15.73 \pm 0.01$ & $14.78 \pm 0.01$ & $14.55 \pm 0.01$ & $14.15 \pm 0.01$ & 39 & 80 & 46 & $\mathrm{~A} 2 \mathrm{~V} t$ \\
\hline $17.63 \pm 0.03$ & $16.70 \pm 0.02$ & $15.84 \pm 0.01$ & $14.83 \pm 0.01$ & $14.58 \pm 0.01$ & $14.21 \pm 0.01$ & 116 & 489 & 387 & \\
\hline $17.58 \pm 0.03$ & $16.70 \pm 0.03$ & $15.82 \pm 0.01$ & $14.83 \pm 0.01$ & $14.60 \pm 0.01$ & $14.16 \pm 0.01$ & 265 & 472 & 380 & AOV \\
\hline $17.67 \pm 0.04$ & $16.81 \pm 0.02$ & $15.86 \pm 0.01$ & $14.77 \pm 0.01$ & $14.60 \pm 0.01$ & $14.18 \pm 0.01$ & 163 & 220 & 162 & A $5 \mathrm{~V}$ \\
\hline $17.64 \pm 0.04$ & $16.73 \pm 0.03$ & $15.82 \pm 0.01$ & $14.82 \pm 0.01$ & $14.62 \pm 0.01$ & $14.20 \pm 0.01$ & 92 & 338 & 281 & A $5 \mathrm{~V}$ \\
\hline $17.58 \pm 0.04$ & $16.71 \pm 0.03$ & $15.84 \pm 0.01$ & $14.89 \pm 0.01$ & $14.63 \pm 0.01$ & $14.24 \pm 0.01$ & 218 & 185 & 130 & $\mathrm{~A} 2 \mathrm{~V}$ \\
\hline $17.59 \pm 0.03$ & $16.77 \pm 0.03$ & $15.85 \pm 0.01$ & $14.86 \pm 0.01$ & $3 \pm 0.01$ & $14.22 \pm 0.01$ & 219 & 216 & 157 & A5V \\
\hline $17.50 \pm 0.04$ & $16.72 \pm 0.02$ & $15.84 \pm 0.01$ & $14.89 \pm 0.01$ & $5 \pm 0.01$ & $14.24 \pm 0.01$ & 294 & 71 & 39 & A5V \\
\hline $17.66 \pm 0.03$ & $16.74 \pm 0.02$ & $15.87 \pm 0.01$ & $14.87 \pm 0.01$ & $14.65 \pm 0.01$ & $14.22 \pm 0.01$ & 208 & 269 & 217 & A $5 \mathrm{~V}$ \\
\hline $17.78 \pm 0.05$ & $17.14 \pm 0.03$ & $16.48 \pm 0.02$ & $15.02 \pm 0.01$ & $14.65 \pm 0.01$ & $13.97 \pm 0.01$ & 52 & 44 & 15 & $\mathrm{~K} 4 \mathrm{~V}$ \\
\hline $17.66 \pm 0.04$ & $16.96 \pm 0.05$ & $16.38 \pm 0.05$ & $15.08 \pm 0.06$ & $14.68 \pm 0.05$ & $13.93 \pm 0.06$ & 317 & 221 & 163 & G2V \\
\hline $17.74 \pm 0.04$ & $16.76 \pm 0.03$ & $15.95 \pm 0.01$ & $14.99 \pm 0.01$ & $14.74 \pm 0.01$ & $14.33 \pm 0.01$ & 279 & 250 & 193 & A5V \\
\hline $17.86 \pm 0.04$ & $16.88 \pm 0.02$ & $15.95 \pm 0.01$ & $14.99 \pm 0.01$ & $14.75 \pm 0.01$ & $14.36 \pm 0.01$ & 124 & 384 & 318 & $\mathrm{~A} 5 \mathrm{~V}+$ \\
\hline $17.65 \pm 0.03$ & $16.85 \pm 0.02$ & $16.00 \pm 0.01$ & $15.01 \pm 0.01$ & $14.76 \pm 0.01$ & $14.34 \pm 0.01$ & 285 & 178 & 123 & A $5 V$ \\
\hline $17.87 \pm 0.05$ & $17.04 \pm 0.04$ & $16.04 \pm 0.02$ & $15.06 \pm 0.01$ & $14.76 \pm 0.03$ & $14.42 \pm 0.02$ & 171 & 310 & 254 & - \\
\hline $17.79 \pm 0.04$ & $16.95 \pm 0.03$ & $16.01 \pm 0.01$ & $15.01 \pm 0.01$ & $14.78 \pm 0.01$ & $14.36 \pm 0.01$ & 157 & 375 & 308 & A $5 \mathrm{~V}$ \\
\hline $17.67 \pm 0.03$ & $16.97 \pm 0.02$ & $16.05 \pm 0.01$ & $15.06 \pm 0.01$ & $14.82 \pm 0.01$ & $14.39 \pm 0.01$ & 252 & 107 & 65 & $\mathrm{~A}$ \\
\hline $17.74 \pm 0.03$ & $17.01 \pm 0.03$ & $16.32 \pm 0.01$ & $15.11 \pm 0.01$ & $14.82 \pm 0.01$ & $14.26 \pm 0.01$ & 333 & 105 & 64 & FOV \\
\hline $17.88 \pm 0.04$ & $16.99 \pm 0.03$ & $16.20 \pm 0.01$ & $15.04 \pm 0.02$ & $14.82 \pm 0.02$ & $14.40 \pm 0.02$ & 210 & 245 & 191 & A \\
\hline $17.87 \pm 0.04$ & $17.05 \pm 0.03$ & $16.12 \pm 0.01$ & $15.11 \pm 0.01$ & $14.85 \pm 0.01$ & $14.42 \pm 0.01$ & 208 & 287 & 234 & \\
\hline $17.98 \pm 0.05$ & $17.14 \pm 0.03$ & $16.17 \pm 0.01$ & $15.17 \pm 0.01$ & $14.88 \pm 0.01$ & $14.46 \pm 0.01$ & 44 & 202 & 143 & \\
\hline $17.86 \pm 0.04$ & $16.99 \pm 0.02$ & $16.14 \pm 0.01$ & $15.16 \pm 0.01$ & $14.91 \pm 0.01$ & $14.51 \pm 0.01$ & 268 & 234 & 182 & A5V \\
\hline $17.85 \pm 0.04$ & $16.98 \pm 0.02$ & $16.25 \pm 0.01$ & $15.19 \pm 0.01$ & $14.94 \pm 0.01$ & $14.51 \pm 0.01$ & 379 & 417 & 344 & A5V \\
\hline $17.83 \pm 0.04$ & $17.01 \pm 0.04$ & $16.33 \pm 0.03$ & $15.22 \pm 0.03$ & $14.95 \pm 0.02$ & $14.55 \pm 0.01$ & 294 & 282 & 226 & $\mathrm{~A} 5 \mathrm{~V} \dagger$ \\
\hline $18.04 \pm 0.04$ & $17.04 \pm 0.03$ & $16.25 \pm 0.01$ & $15.21 \pm 0.01$ & $14.96 \pm 0.01$ & $14.55 \pm 0.01$ & 236 & 534 & 407 & KIIV \\
\hline $18.17 \pm 0.06$ & $17.17 \pm 0.03$ & $16.30 \pm 0.01$ & $15.28 \pm 0.01$ & $15.02 \pm 0.01$ & $14.63 \pm 0.01$ & 123 & 357 & 298 & $\mathrm{~A} 5 \mathrm{~V} \dagger$ \\
\hline $17.32 \pm 0.06$ & $16.44 \pm 0.04$ & $16.36 \pm 0.01$ & $15.26 \pm 0.01$ & $15.05 \pm 0.01$ & $14.59 \pm 0.01$ & 231 & 235 & 183 & 一 \\
\hline $18.27 \pm 0.07$ & $17.27 \pm 0.03$ & $16.40 \pm 0.02$ & $15.32 \pm 0.01$ & $15.05 \pm 0.01$ & $14.60 \pm 0.01$ & 108 & 256 & 200 & $\mathrm{~A} 5 \mathrm{~V} \dagger$ \\
\hline $18.03 \pm 0.05$ & $17.22 \pm 0.04$ & $16.33 \pm 0.01$ & $15.34 \pm 0.01$ & $15.06 \pm 0.01$ & $14.63 \pm 0.01$ & 237 & 259 & 207 & A5 \\
\hline $18.28 \pm 0.07$ & $17.40 \pm 0.03$ & $16.53 \pm 0.01$ & $15.36 \pm 0.01$ & $15.07 \pm 0.01$ & $14.60 \pm 0.01$ & 194 & 213 & 153 & A5 \\
\hline $18.24 \pm 0.05$ & $17.29 \pm 0.03$ & $16.44 \pm 0.01$ & $15.39 \pm 0.01$ & $15.12 \pm 0.01$ & $14.71 \pm 0.01$ & 283 & 465 & 376 & $\mathrm{~A} 5 \mathrm{~V}+$ \\
\hline $18.25 \pm 0.05$ & $17.49 \pm 0.05$ & $16.71 \pm 0.03$ & $15.43 \pm 0.03$ & $15.18 \pm 0.03$ & $14.47 \pm 0.04$ & 301 & 75 & 43 & F5V † \\
\hline $18.41 \pm 0.06$ & $17.45 \pm 0.03$ & $16.63 \pm 0.02$ & $15.49 \pm 0.01$ & $15.19 \pm 0.01$ & $14.78 \pm 0.01$ & 203 & 321 & 267 & - \\
\hline $18.58 \pm 0.08$ & $17.46 \pm 0.03$ & $16.64 \pm 0.02$ & $15.54 \pm 0.01$ & $15.28 \pm 0.01$ & $14.89 \pm 0.02$ & 145 & 233 & 181 & - \\
\hline $18.33 \pm 0.05$ & $17.53 \pm 0.04$ & $16.63 \pm 0.02$ & $15.57 \pm 0.01$ & $15.29 \pm 0.01$ & $14.82 \pm 0.01$ & 97 & 176 & 119 & A5V \\
\hline $18.41 \pm 0.07$ & $17.44 \pm 0.04$ & $16.71 \pm 0.02$ & $15.62 \pm 0.01$ & $15.32 \pm 0.01$ & $14.83 \pm 0.01$ & 339 & 342 & 288 & $\mathrm{~A} 7 \mathrm{~V} \dagger$ \\
\hline $18.43 \pm 0.06$ & $17.63 \pm 0.05$ & $16.73 \pm 0.02$ & $15.59 \pm 0.01$ & $15.33 \pm 0.01$ & $14.86 \pm 0.01$ & 95 & 208 & 147 & \\
\hline $18.38 \pm 0.07$ & $17.52 \pm 0.04$ & $16.81 \pm 0.02$ & $15.63 \pm 0.01$ & $15.40 \pm 0.01$ & $14.91 \pm 0.01$ & 184 & 541 & 411 & A \\
\hline $18.43 \pm 0.07$ & $17.56 \pm 0.04$ & $16.79 \pm 0.01$ & $15.68 \pm 0.01$ & $15.40 \pm 0.01$ & $14.93 \pm 0.01$ & 245 & 342 & 287 & $\mathrm{~A} 7 \mathrm{~V} \dagger$ \\
\hline $18.37 \pm 0.06$ & $17.57 \pm 0.04$ & $17.07 \pm 0.02$ & $15.79 \pm 0.01$ & $15.46 \pm 0.01$ & $14.86 \pm 0.01$ & 316 & 298 & 243 & FoV $\dagger$ \\
\hline $18.75 \pm 0.08$ & $17.69 \pm 0.04$ & $17.08 \pm 0.02$ & $15.86 \pm 0.02$ & $15.53 \pm 0.02$ & $15.08 \pm 0.02$ & 301 & 413 & 341 & - \\
\hline $18.39 \pm 0.05$ & $17.81 \pm 0.05$ & $17.23 \pm 0.03$ & $15.96 \pm 0.02$ & $15.59 \pm 0.01$ & $14.98 \pm 0.01$ & 354 & 62 & 32 & $\mathrm{~F} 2 \mathrm{~V}$ \\
\hline $18.68 \pm 0.08$ & $17.65 \pm 0.04$ & $17.07 \pm 0.02$ & $15.94 \pm 0.01$ & $15.61 \pm 0.01$ & $15.09 \pm 0.01$ & 331 & 251 & 194 & Fov $t$ \\
\hline $19.32 \pm 0.21$ & $18.06 \pm 0.07$ & $17.38 \pm 0.03$ & $16.03 \pm 0.01$ & $15.69 \pm 0.02$ & $15.12 \pm 0.01$ & 162 & 241 & 187 & - \\
\hline $18.60 \pm 0.08$ & $17.89 \pm 0.05$ & $17.18 \pm 0.02$ & $16.01 \pm 0.01$ & $15.71 \pm 0.01$ & $15.16 \pm 0.01$ & 325 & 335 & 279 & FOV \\
\hline $18.94 \pm 0.11$ & $18.08 \pm 0.07$ & $17.40 \pm 0.03$ & $16.05 \pm 0.03$ & $15.71 \pm 0.04$ & $15.39 \pm 0.01$ & 270 & 134 & 91 & - \\
\hline $18.81 \pm 0.09$ & $17.86 \pm 0.06$ & $17.25 \pm 0.03$ & $16.04 \pm 0.01$ & $15.72 \pm 0.01$ & $15.15 \pm 0.01$ & 296 & 102 & 59 & $\mathrm{~A} 7 \mathrm{~V} \dagger$ \\
\hline $18.64 \pm 0.08$ & $18.03 \pm 0.05$ & $17.26 \pm 0.03$ & $16.08 \pm 0.02$ & $15.74 \pm 0.02$ & $15.18 \pm 0.01$ & 103 & 58 & 26 & A7V \\
\hline $18.80 \pm 0.11$ & $18.19 \pm 0.06$ & $17.49 \pm 0.03$ & $16.12 \pm 0.02$ & $15.78 \pm 0.01$ & $15.13 \pm 0.01$ & 19 & 62 & 31 & FOV \\
\hline $19.01 \pm 0.10$ & $18.24 \pm 0.07$ & $17.40 \pm 0.03$ & $16.18 \pm 0.01$ & $15.88 \pm 0.01$ & $15.29 \pm 0.01$ & 196 & 347 & 293 & A7V \\
\hline $19.25 \pm 0.16$ & $18.43 \pm 0.08$ & $17.65 \pm 0.03$ & $16.34 \pm 0.02$ & $15.96 \pm 0.02$ & $15.37 \pm 0.01$ & 43 & 333 & 277 & - \\
\hline $19.10 \pm 0.17$ & $18.25 \pm 0.06$ & $17.58 \pm 0.03$ & $16.29 \pm 0.02$ & $16.01 \pm 0.01$ & $15.43 \pm 0.02$ & 115 & 548 & 416 & A7V \\
\hline $18.89 \pm 0.09$ & $18.24 \pm 0.07$ & $17.71 \pm 0.03$ & $16.46 \pm 0.01$ & $16.09 \pm 0.01$ & $15.51 \pm 0.01$ & 362 & 154 & 103 & F2V \\
\hline $18.77 \pm 0.10$ & $18.50 \pm 0.09$ & $17.70 \pm 0.03$ & $16.45 \pm 0.01$ & $16.14 \pm 0.01$ & $15.53 \pm 0.01$ & 265 & 483 & 385 & FoV $\dagger$ \\
\hline $19.50 \pm 0.17$ & $18.66 \pm 0.10$ & $17.90 \pm 0.03$ & $16.49 \pm 0.03$ & $16.24 \pm 0.03$ & $15.68 \pm 0.02$ & 299 & 314 & 261 & - \\
\hline $18.79 \pm 0.08$ & $17.96 \pm 0.07$ & $17.66 \pm 0.05$ & $16.21 \pm 0.04$ & $16.67 \pm 0.41$ & $15.04 \pm 0.05$ & 332 & 6 & 2 & - \\
\hline
\end{tabular}




\begin{tabular}{|c|c|c|c|c|c|c|c|}
\hline $\mathrm{U} \pm \delta \mathrm{U}$ & $\mathrm{P} \pm \delta \mathrm{P}$ & $\mathrm{X} \pm \delta \mathrm{X}$ & $\mathrm{Z} \pm \delta \mathrm{Z}$ & $\mathrm{V} \pm \delta \mathrm{V}$ & $\mathrm{S} \pm \delta \mathrm{S}$ & ID & Class \\
\hline $13.34 \pm 0.01$ & $13.06 \pm 0.01$ & $12.94 \pm 0.01$ & $12.73 \pm 0.01$ & $12.67 \pm 0.01$ & $12.48 \pm 0.01$ & 86 & $\mathrm{O}$ \\
\hline $13.74 \pm 0.01$ & $13.50 \pm 0.01$ & $13.42 \pm 0.01$ & $13.18 \pm 0.01$ & $13.14 \pm 0.01$ & $12.96 \pm 0.01$ & 346 & 0 \\
\hline $13.72 \pm 0.02$ & $13.66 \pm 0.01$ & $13.65 \pm 0.01$ & $13.51 \pm 0.01$ & $13.51 \pm 0.01$ & $13.44 \pm 0.01$ & 190 & 0 \\
\hline $14.13 \pm 0.02$ & $13.90 \pm 0.01$ & $13.83 \pm 0.01$ & $13.61 \pm 0.01$ & $13.56 \pm 0.01$ & $13.38 \pm 0.01$ & 229 & 0 \\
\hline $14.21 \pm 0.01$ & $14.02 \pm 0.01$ & $13.96 \pm 0.01$ & $13.85 \pm 0.01$ & $13.82 \pm 0.01$ & $13.70 \pm 0.01$ & 100 & 0 \\
\hline $14.29 \pm 0.01$ & $14.16 \pm 0.01$ & $14.09 \pm 0.01$ & $13.99 \pm 0.01$ & $13.96 \pm 0.01$ & $13.87 \pm 0.01$ & 203 & 0 \\
\hline $14.71 \pm 0.01$ & $14.47 \pm 0.01$ & $14.38 \pm 0.01$ & $14.22 \pm 0.01$ & $14.17 \pm 0.01$ & $14.02 \pm 0.02$ & 343 & 0 \\
\hline $14.68 \pm 0.01$ & $14.57 \pm 0.01$ & $14.57 \pm 0.01$ & $14.40 \pm 0.01$ & $14.39 \pm 0.01$ & $14.29 \pm 0.01$ & 236 & 0 \\
\hline $14.96 \pm 0.01$ & $14.79 \pm 0.01$ & $14.63 \pm 0.01$ & $14.48 \pm 0.01$ & $14.46 \pm 0.01$ & $14.37 \pm 0.01$ & 134 & $\mathrm{BOV}$ \\
\hline $15.14 \pm 0.02$ & $14.88 \pm 0.01$ & $14.72 \pm 0.01$ & $14.53 \pm 0.01$ & $14.48 \pm 0.01$ & $14.31 \pm 0.01$ & 69 & BOV \\
\hline $14.86 \pm 0.02$ & $14.71 \pm 0.01$ & $14.63 \pm 0.01$ & $14.51 \pm 0.01$ & $14.49 \pm 0.01$ & $14.40 \pm 0.01$ & 189 & $\mathrm{BOV}$ \\
\hline $14.90 \pm 0.02$ & $14.84 \pm 0.01$ & $14.75 \pm 0.01$ & $14.59 \pm 0.01$ & $14.57 \pm 0.01$ & $14.44 \pm 0.01$ & 126 & $\mathrm{BOV}$ \\
\hline $15.30 \pm 0.02$ & $15.14 \pm 0.01$ & $15.00 \pm 0.01$ & $14.84 \pm 0.01$ & $14.80 \pm 0.01$ & $14.73 \pm 0.01$ & 265 & $\mathrm{BOV}$ \\
\hline $15.34 \pm 0.01$ & $15.11 \pm 0.01$ & $14.94 \pm 0.01$ & $14.84 \pm 0.01$ & $14.81 \pm 0.02$ & $14.72 \pm 0.02$ & 131 & $\mathrm{~B} 1 \mathrm{~V}+$ \\
\hline $15.57 \pm 0.02$ & $15.40 \pm 0.01$ & $15.27 \pm 0.01$ & $15.10 \pm 0.01$ & $15.11 \pm 0.01$ & $14.94 \pm 0.02$ & 141 & $\mathrm{~B} 1 \mathrm{Vt}$ \\
\hline $15.64 \pm 0.02$ & $15.43 \pm 0.01$ & $15.37 \pm 0.01$ & $15.18 \pm 0.01$ & $15.15 \pm 0.01$ & $15.04 \pm 0.01$ & 285 & BOV \\
\hline $15.64 \pm 0.02$ & $15.43 \pm 0.01$ & $15.37 \pm 0.01$ & $15.18 \pm 0.01$ & $15.15 \pm 0.01$ & $15.04 \pm 0.01$ & 285 & $\mathrm{BOV}$ \\
\hline $15.69 \pm 0.02$ & $15.55 \pm 0.01$ & $15.34 \pm 0.01$ & $15.22 \pm 0.01$ & $15.20 \pm 0.01$ & $15.20 \pm 0.03$ & 314 & B3V \\
\hline $15.79 \pm 0.02$ & $15.63 \pm 0.01$ & $15.55 \pm 0.01$ & $15.34 \pm 0.01$ & $15.27 \pm 0.01$ & $15.14 \pm 0.02$ & 250 & $\mathrm{~B} 1 \mathrm{~V}$ \\
\hline $15.92 \pm 0.02$ & $15.75 \pm 0.02$ & $15.49 \pm 0.01$ & $15.40 \pm 0.01$ & $15.37 \pm 0.01$ & $15.29 \pm 0.01$ & 237 & $\mathrm{~B} 2 \mathrm{~V}$ \\
\hline $16.01 \pm 0.02$ & $15.74 \pm 0.01$ & $15.64 \pm 0.01$ & $15.41 \pm 0.01$ & $15.39 \pm$ & $15.25 \pm 0.01$ & 206 & $\mathrm{~V} \dagger$ \\
\hline $16.20 \pm 0.02$ & $15.97 \pm 0.02$ & $15.78 \pm 0.01$ & $15.57 \pm 0.01$ & $15.55 \pm 0.01$ & $15.40 \pm 0.02$ & 137 & $\mathrm{~B} 1 \mathrm{V \dagger}$ \\
\hline $16.25 \pm 0.02$ & $16.06 \pm 0.02$ & $15.92 \pm 0.01$ & $15.73 \pm 0.01$ & $15.68 \pm 0.01$ & $15.51 \pm 0.02$ & 78 & BOV \\
\hline $16.16 \pm 0.02$ & $16.04 \pm 0.02$ & $15.93 \pm 0.02$ & $15.81 \pm 0.02$ & $15.77 \pm 0.02$ & $15.73 \pm 0.02$ & 113 & B1V \\
\hline $16.48 \pm 0.02$ & $16.24 \pm 0.02$ & $16.07 \pm 0.02$ & $15.84 \pm 0.01$ & $15.80 \pm 0.01$ & $15.69 \pm 0.02$ & 287 & $\mathrm{~B} 2 \mathrm{~V}$ \\
\hline $16.65 \pm 0.03$ & $16.42 \pm 0.02$ & $16.22 \pm 0.02$ & $15.99 \pm 0.01$ & $15.95 \pm 0.01$ & $15.79 \pm 0.02$ & 227 & $\mathrm{~B} 1 \mathrm{~V} \dagger$ \\
\hline $16.65 \pm 0.02$ & $16.52 \pm 0.02$ & $16.33 \pm 0.02$ & $16.07 \pm 0.02$ & $15.97 \pm 0.02$ & $15.76 \pm 0.03$ & 14 & $\mathrm{BOV}$ \\
\hline $16.56 \pm 0.02$ & $16.39 \pm 0.02$ & $16.15 \pm 0.02$ & $15.99 \pm 0.02$ & $16.00 \pm 0.03$ & $15.93 \pm 0.03$ & 202 & $\mathrm{~B} 2 \mathrm{~V} \dagger$ \\
\hline $18.33 \pm 0.08$ & $17.72 \pm 0.05$ & $17.28 \pm 0.03$ & $16.23 \pm 0.01$ & $16.00 \pm 0.01$ & $15.50 \pm 0.01$ & 67 & B5V \\
\hline $16.74 \pm 0.03$ & $16.55 \pm 0.02$ & $16.28 \pm 0.02$ & $16.11 \pm 0.01$ & $16.03 \pm 0.02$ & $15.88 \pm 0.03$ & 55 & $\mathrm{~B} 1 \mathrm{~V} \dagger$ \\
\hline $16.75 \pm 0.02$ & $16.56 \pm 0.02$ & $16.37 \pm 0.02$ & $16.12 \pm 0.02$ & $16.04 \pm 0.02$ & $15.92 \pm 0.02$ & 35 & $\mathrm{~B} 2 \mathrm{~V}$ \\
\hline $17.14 \pm 0.03$ & $16.73 \pm 0.02$ & $16.46 \pm 0.02$ & $16.19 \pm 0.01$ & $16.15 \pm 0.01$ & $16.02 \pm 0.02$ & 359 & B3V† \\
\hline $17.17 \pm 0.03$ & $16.82 \pm 0.03$ & $16.60 \pm 0.02$ & $16.36 \pm 0.01$ & $16.30 \pm 0.01$ & $16.16 \pm 0.02$ & 221 & $\mathrm{~B} 2 \mathrm{~V} \dagger$ \\
\hline $16.91 \pm 0.02$ & $16.69 \pm 0.03$ & $16.47 \pm 0.01$ & $16.41 \pm 0.01$ & \pm 0.01 & $16.22 \pm 0.02$ & 57 & $\mathrm{~B} 2 \mathrm{~V}$ \\
\hline $17.18 \pm 0.03$ & $16.93 \pm 0.02$ & $16.64 \pm 0.02$ & $16.47 \pm 0.02$ & $16.37 \pm 0.01$ & $16.26 \pm 0.02$ & 262 & $\mathrm{~B} 2 \mathrm{~V} \dagger$ \\
\hline $17.19 \pm 0.03$ & $17.07 \pm 0.03$ & $16.76 \pm 0.02$ & $16.50 \pm 0.01$ & $16.44 \pm 0.01$ & $16.32 \pm 0.02$ & 123 & $\mathrm{~B} 2 \mathrm{~V}$ \\
\hline $17.26 \pm 0.04$ & $17.06 \pm 0.04$ & $16.73 \pm 0.02$ & $16.57 \pm 0.01$ & $16.47 \pm 0.01$ & $16.45 \pm 0.02$ & 83 & $\mathrm{~B} 5 \mathrm{~V} \dagger$ \\
\hline $17.34 \pm 0.04$ & $17.00 \pm 0.03$ & $16.82 \pm 0.03$ & $16.60 \pm 0.02$ & $16.50 \pm 0.03$ & $16.37 \pm 0.03$ & 99 & $\mathrm{~B} 2 \mathrm{~V}$ \\
\hline $17.31 \pm 0.03$ & $17.13 \pm 0.03$ & $16.84 \pm 0.02$ & $16.69 \pm 0.01$ & $16.60 \pm 0.02$ & $16.56 \pm 0.02$ & 174 & B3V† \\
\hline $17.33 \pm 0.04$ & $17.13 \pm 0.03$ & $16.84 \pm 0.01$ & $16.70 \pm 0.01$ & $16.60 \pm 0.02$ & $16.46 \pm 0.02$ & 64 & $\mathrm{~B} 2 \mathrm{~V} \dagger$ \\
\hline $17.55 \pm 0.04$ & $17.26 \pm 0.06$ & $16.91 \pm 0.02$ & $16.66 \pm 0.02$ & $16.64 \pm 0.02$ & $16.54 \pm 0.03$ & 186 & B3V \\
\hline $17.35 \pm 0.02$ & $17.04 \pm 0.03$ & $16.84 \pm 0.02$ & $16.74 \pm 0.03$ & $16.68 \pm 0.02$ & $16.67 \pm 0.03$ & 108 & B3V \\
\hline $17.70 \pm 0.05$ & $17.40 \pm 0.04$ & $17.25 \pm 0.02$ & $16.99 \pm 0.02$ & $16.94 \pm 0.02$ & $16.96 \pm 0.10$ & 135 & B5V† \\
\hline $18.04 \pm 0.05$ & $17.71 \pm 0.04$ & $17.49 \pm 0.09$ & $16.99 \pm 0.02$ & $16.97 \pm 0.02$ & $16.78 \pm 0.03$ & 386 & $\mathrm{~B} 2 \mathrm{~V}$ \\
\hline $18.53 \pm 0.08$ & $17.98 \pm 0.07$ & $17.66 \pm 0.03$ & $17.33 \pm 0.03$ & $17.23 \pm 0.02$ & $17.09 \pm 0.05$ & 338 & $\mathrm{~B} 5 \mathrm{~V} \dagger$ \\
\hline $17.86 \pm 0.05$ & $17.58 \pm 0.05$ & $17.47 \pm 0.03$ & $17.40 \pm 0.03$ & $17.36 \pm 0.02$ & $17.32 \pm 0.06$ & 205 & $\mathrm{~B} 2 \mathrm{~V}$ \\
\hline $18.29 \pm 0.07$ & $18.03 \pm 0.06$ & $17.87 \pm 0.03$ & $17.59 \pm 0.02$ & $17.56 \pm 0.02$ & $17.45 \pm 0.04$ & 25 & $\mathrm{~B} 2 \mathrm{Vt}$ \\
\hline
\end{tabular}

Table 6.7: Vilnius Photometry of NGC 1968/74. The $\dagger$ symbol indicates that the classification of the flagged star is uncertain. The photometric errors are those calculated by DAOphot. 


\section{Chapter 7}

\section{AAT Observations of NGC 2214}

$\mathrm{T}$

his chapter begins with a literature review of NGC 2214 and determinations of the initial mass functions of star clusters in the Magellanic Clouds. Colour-magnitude diagrams for NGC 2214 and a nearby field are presented. It has been suggested that the high ellipticity of NGC 2214 could be due to it being two clusters in the final stages of merging. The CMD and profile of the cluster are examined for evidence to support this hypothesis. Isochrones are fitted to features in the CMD, providing age estimates. Next models are fitted to profiles of the cluster. After this, completeness correction techniques are reviewed, and evaluated with the use of artificial data. The most successful techniques are applied to NGC 2214, and used to estimate the initial mass function of the cluster.

\subsection{Introduction}

$\mathrm{N}$

GC $2214\left(\alpha_{2000}=6^{\mathrm{h}} 12^{\mathrm{m}} 57^{\mathrm{s}}, \delta_{2000}=68^{\circ} 15^{\prime} 33^{\prime \prime}\right.$ South $)$ is a young $\left(32 \times 10^{6}\right.$ years; Elson 1991) populous star cluster situated in a relatively uncrowded field to the far north-east of the bar in the Large Magellanic Cloud. Meylan \& Djorgovski (1987) analysed an intensity profile of the cluster, and found that the core was abnormal. They conjectured that perhaps it had collapsed, although Elson et al. (1987) have shown that the two body relaxation time of the cluster is $\sim 2-6 \times 10^{8}$ years, and so greater than its age. Bhatia \& MacGillivray (1988) found the cluster to have a very elliptical $(e=0.5)$ core with an almost spherical halo, and suggested that this unusual shape could be due to NGC 2214 being a binary star cluster in an advanced stage of merging. Comparison with N-body simulations lent support to this idea. Sagar et al. (1991a, b) used the $1.54 \mathrm{~m}$ ESO Danish telescope in $\sim 2^{\prime \prime}$ seeing, and presented a BV colour-magnitude diagram with two well-defined supergiant branches, separated by $\sim 2$ magnitudes in $\mathrm{V}$. The older population was more centrally condensed than the younger one, and Sagar et al. (1991a) suggested that the first published CMD (Robertson 1974) had failed to detect the older branch due to the problems of photometry in such a crowded region. The shallow BV CMD of Elson (1991) showed no sign of the second turnoff, although the $\sim 5.5^{\prime \prime}$ 
seeing may have obscured it, as could the $3^{\prime \prime}$ seeing in the BV CMD of Banks (1993). However, examination of colour-magnitude diagrams for other LMC clusters presented in Sagar et al. (1991b) showed traces of second (weak) supergiant branches in other clusters. This, combined with the high number of stars blueward of the cluster main Sequence, which are an indicator of crowded field reduction problems (Lee 1992), suggested that there may be problems with the reduction process. Service observations were therefore requested on the Anglo-Australian Telescope (see Figures 7-1 and 7-2) to investigate the reality of this second branch. Short exposure observations taken with the MJUO $1 \mathrm{~m}$ telescope gave some indication of a clump in the main sequence at the $\mathrm{V}$ magnitude corresponding to the older turnoff (Banks 1993, Banks et al. 1994d). After submission of the observing proposal Lee (1992) published a paper describing CCD observations of NGC 2214 in $1.1^{\prime \prime}$ to $1.6^{\prime \prime}$ seeing with the Las Campanas du Pont $2.5 \mathrm{~m}$ telescope. The resulting BV CMD showed only one supergiant branch. The main sequence was matched well by a Maeder \& Meynet (1991) isochrone for 50 Myr, while the supergiant branch was approximately matched by an older $70 \mathrm{Myr}$ isochrone.

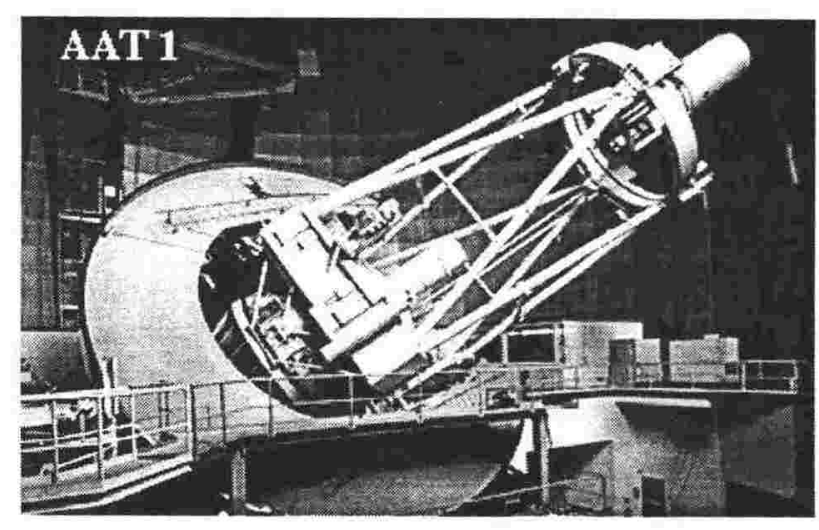

Figure 7-1: The 3.9 metre Anglo-Australian Telescope is located at Siding Spring Mountain outside the town of Coonabarabran in central New South Wales, some $450 \mathrm{~km}$ northwest of Sydney. The telescope was commissioned in 1974. Figure 7-2 is an external view of the site. Photograph by David Malin, 1977 (Reproduced with the permission of the AAO).
A major objective of this study was to derive an estimate of the initial mass function (IMF) of the cluster. The IMF is defined as the frequency distribution of stellar masses on the main sequence at the formation time of a group of stars (Scalo 1986). Mass is one of the primary factors influencing stellar evolution, and a detailed knowledge of the IMF would be important in a wide range of studies ranging from galactic evolution to the spectral properties of binary stars (see Tinsley 1980). A fundamental question about the IMF

is whether it is universal in time and location, or whether the distribution of stars formed is a function of parameters such as metallicity.

Derivation of the IMF is not straightforward. An initial approach might be to use the nearby solar neighbourhood to do this, but this technique is complicated by the fact that these stars have a range of distances, ages, and metallicities. For instance, the random velocities of the stars, combined with their lifetimes, meansy that while massive stars will still be near the site of their formation, low mass stars will have traveled significant distances. Variations 
in composition may result just from such spatial considerations, if not from galactic evolution as well. Scalo (1986) comments that the many assumptions, such as any variation in the star formation rate with time, complicate estimates of the field IMF to the point of impracticality. In addition, a universal nature is assumed for the IMF in such studies.

A better approach is to use clusters, where the component stars will be effectively coeval and of the same composition. Such work is complicated by effects such as dynamical evolution leading to mass segregation in the cluster, tidal stripping (which in the presence of mass segregation will lead to the proportional decrease of low mass stars; see Spitzer 1987), and stellar evolution as stars evolve off the main sequence, which leads to no easily derivable mass function information for stars of a main sequence lifetime less than the age of the cluster. The mass function of the cluster may alter substantially with time, and it is best

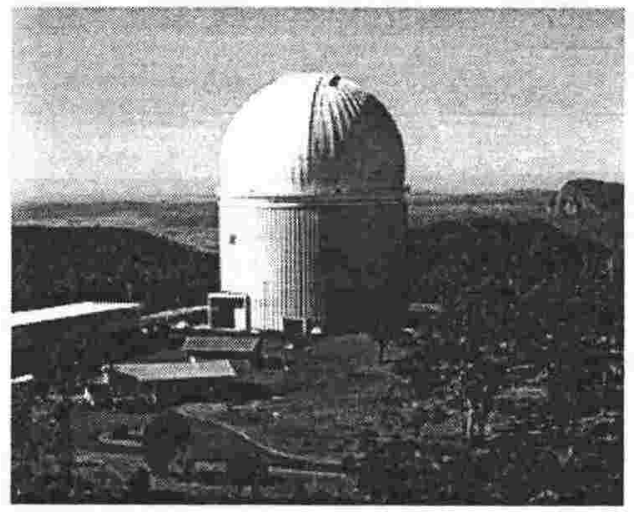

Figure 7-2: The Anglo-Australian Telescope Building is a six-storey structure topped by the dome housing the AAT itself. Photograph by David Malin, 1977 (Reproduced with the permission of the AAO). to select young clusters where these effects have not had time to be significant. Many studies have centred on young Galactic open clusters with their large observable mass range (e.g. Phelps \& Janes 1993, Reid 1992, Stauffer et al. 1991). However, such work is complicated by field star contamination, counting incompleteness, and low number statistics (see Scalo 1986, for more details), as well as the problem that most open clusters suffer substantial and variable reddenings due to their positions in the Galactic disk (Mateo 1988b). There is no strong evidence for variations in the shapes of their mass functions (Sagar \& Richtler 1991). Globular clusters offer better statistics due to the increased number of stars they contain, but the observable mass range is limited due to their distances and age. Evolutionary effects, such as mentioned above, are additional complications. The resulting mass functions appear to vary considerably between clusters, and may be correlated with metallicity (Sagar \& Richtler 1991), although this is clouded by the above problems.

The LMC clusters are effectively a mixture of the best features of these two types of star clusters. They are populous, with resultingly good statistics, and span a wide range of ages and metallicities (Da Costa 1991a). The clusters are distant enough to subtend only a small angle on the sky, and yet not too distant to suffer from resolution problems. Questions, such as the universal nature of the IMF, might be able to be addressed using these clusters, although the very populous nature of both the clusters and their fields leads to counting incompleteness problems. A major portion of this study involved the derivation of counting estimates, in order 
to correct observed luminosity functions to the 'real' distribution.

IMFs have been derived for some LMC clusters by Mateo (1988b), Sagar \& Richtler (1991), Cayrel et al. (1988), and Elson et al. (1987). The results have not been in good agreement. The first three studies were based on CCD frames, and attempted to estimate the counting incompleteness using artificial star trials (see below). A power-law $\frac{d N}{d M}=M^{-(1+x)}$ was assumed for the IMF, where $\mathrm{dN}$ is the number of stars in a given mass interval $\mathrm{dM}$ at mass $\mathrm{M}$. Mateo (1988b) found that the IMF of six Magellanic clusters (the Small Magellanic Cloud cluster NGC 330 was included) could all be fitted with the single power law with $x=2.52 \pm 0.16$ over the mass interval 0.9 to $10.5 \mathrm{M}_{\odot}$. Sagar \& Richtler (1991) used a different method of estimating the incompleteness (see below), and arrived at an $x$ value of $\sim 1.1$, not too different from the 1.35 Salpeter (1955) value and in reasonable agreement with the value of 1.2 for NGC 330 and NGC 1818 derived by Cayrel et al. (1988). They commented that if they used the same incompleteness technique as Mateo (1988b) on NGC 1711, which was the only cluster studied by both, then the mass function estimate of Mateo (1988b) was confirmed. All these values contrast sharply with the photographic star count analysis of Elson et al. (1987), which gave $x$ values between -0.2 to 0.8 (over $1.5-6.0$ solar masses). In light of these differences and the comment of Sagar \& Richtler (1991) about NGC 1711, a review of the incompleteness techniques is obviously of major importance given the effect a chosen method has on the derivation of the mass function slope, and any subsequent conclusions about the universality of the IMF.

\subsection{Observations}

Johnson BV observations of NGC 2214 were collected on the night of March 1/2 1993 using a 1024 by 1050 pixel TEK CCD at the prime focus of the Anglo-Australian Telescope. The pixel scale was $0.39^{\prime \prime}$ per pixel, resulting in a field of view approximately $6.7^{\prime}$ square. The FWHM seeing was $\sim 2.2^{\prime \prime}$. Observations were also made of a field $5^{\prime}$ north of the cluster (see Figures 7-3 and 7-4). Exposure times for both these regions were $30 \& 300$ seconds in V, and 60 \& 600 seconds in B.

The initial reductions of the CCD frames were carried out at the Anglo-Australian Observatory (AAO), and included trimming the frames of the overscan rows, subtraction of the mean of the overscan (the CCD has negligible bias structure), and flat-field division using sky flats. Given already derived extinction coefficients (Da Costa 1993), selected E2 and E3 standards from Graham (1982) were observed. These were used by the IRAF photcal package to derive the zero point shift in the following transformation equations:

$$
\mathrm{b}=\mathrm{B}+(25.02 \pm 0.01)+0.12(\mathrm{~B}-\mathrm{V})-(0.4-0.02(\mathrm{~B}-\mathrm{V})) \mathrm{X}
$$




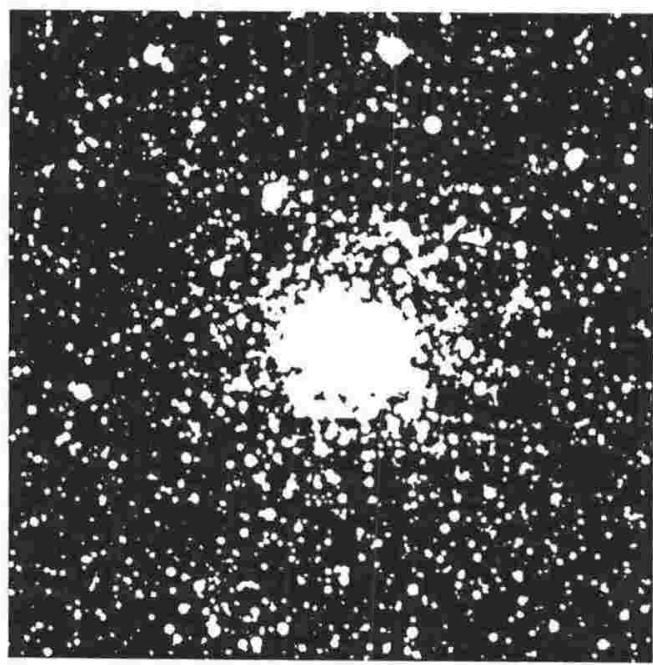

Figure 7-3: Cluster Frame. Processed 60 second B CCD frame of the cluster NGC 2214. North is up, and East to the left.

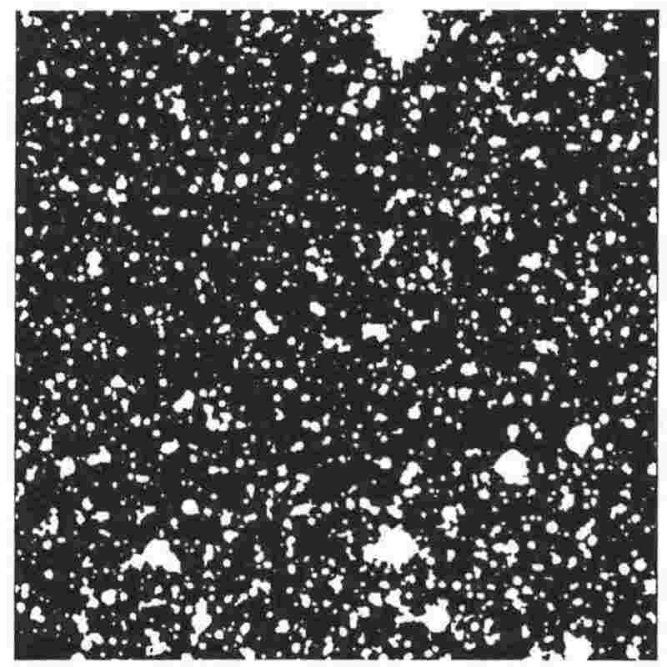

Figure 7-4: Field Frame. Processed 60 second $\mathrm{B}$ exposure of the accompanying field $5^{\prime}$ to the north of the cluster. Note the overlap of some $1.7^{\prime}$ which allowed matching of magnitudes between the two regions.

and

$$
\mathrm{v}=(24.63 \pm 0.01)-0.27 \mathrm{X}+\mathrm{V}
$$

where $\mathrm{X}$ is the airmass of the observation, and the lower-case letters refer to the observed instrumental magnitudes. The root mean squares of the fits were 0.018 and 0.016 magnitudes for $\mathrm{B}$ and $\mathrm{V}$ respectively. These transformation equations were used throughout this chapter.

At the end of the project an extra 19 standard star frames obtained on March 1/2 were made available by the AAO, resulting in $45 \mathrm{~V}$ and $49 \mathrm{~B}$ standard star observations. These were reduced using standard synthetic aperture photometry techniques. The airmass range was from 1.04 to 1.80 , while the colour range was $-0^{\mathrm{m}} \cdot 271 \leq(\mathrm{B}-\mathrm{V}) \leq 1 \mathrm{~m} \cdot 261$. The standards were chosen from the E2, E3, and E5 regions of Graham (1982), and the PG0918+029 and PG1323-086 regions of Landolt (1992). 23 separate standard stars were observed. The following transformation equations were derived from the frames:

$$
\begin{gathered}
\mathrm{b}=\mathrm{B}+(24.915 \pm 0.016)-((0.271 \pm 0.012) \mathrm{X})+((0.158 \pm 0.010)(\mathrm{B}-\mathrm{V})) \text { and } \\
\mathrm{v}=\mathrm{V}+(24.615 \pm 0.015)-((0.205 \pm 0.011) \mathrm{X})-((0.007 \pm 0.008))
\end{gathered}
$$

The RMS values were $0^{\mathrm{m}} 017$ and $0^{\mathrm{m}} 016$ for $\mathrm{B}$ and $\mathrm{V}$ respectively. The transformation values previously supplied by the AAO were obtained late in 1992, when the extinction was greater being closer to the time of the Mt. Pinatubo eruption. However, the NGC 2214 data is not affected much by the choice between the two sets of transformation equations. The second set 


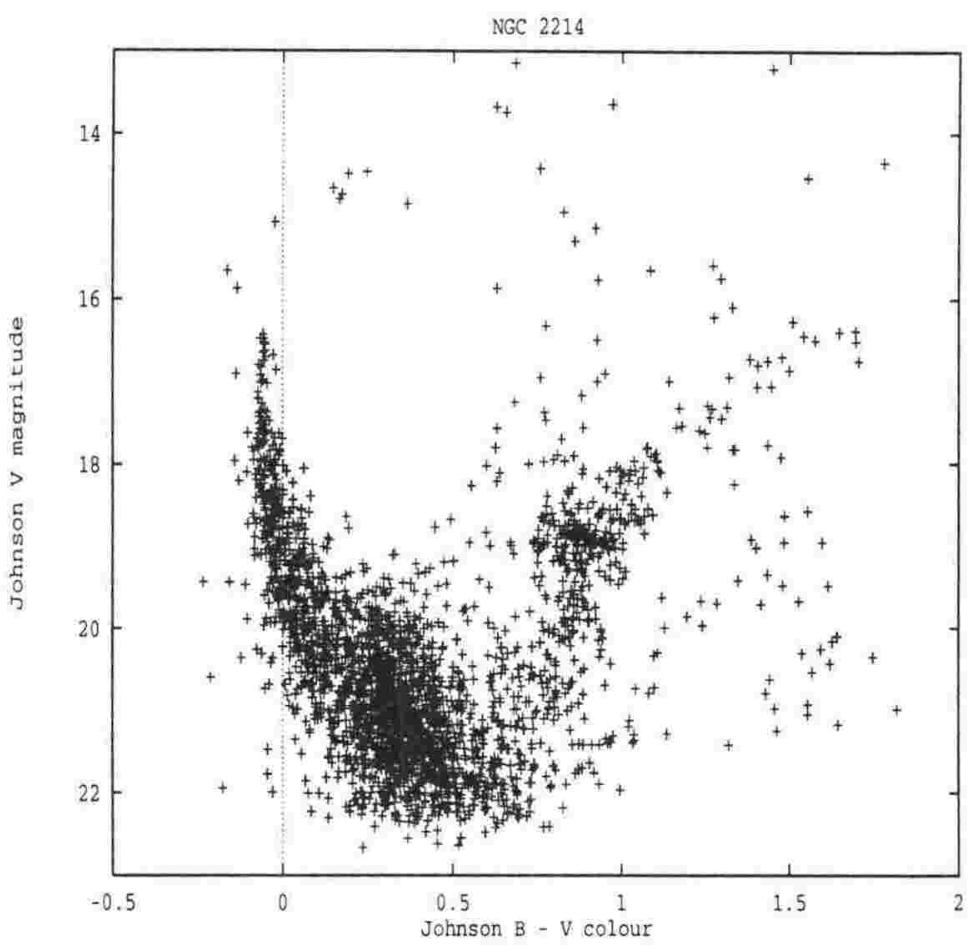

Figure 7-5: Colour-magnitude Diagram for NGC 2214.

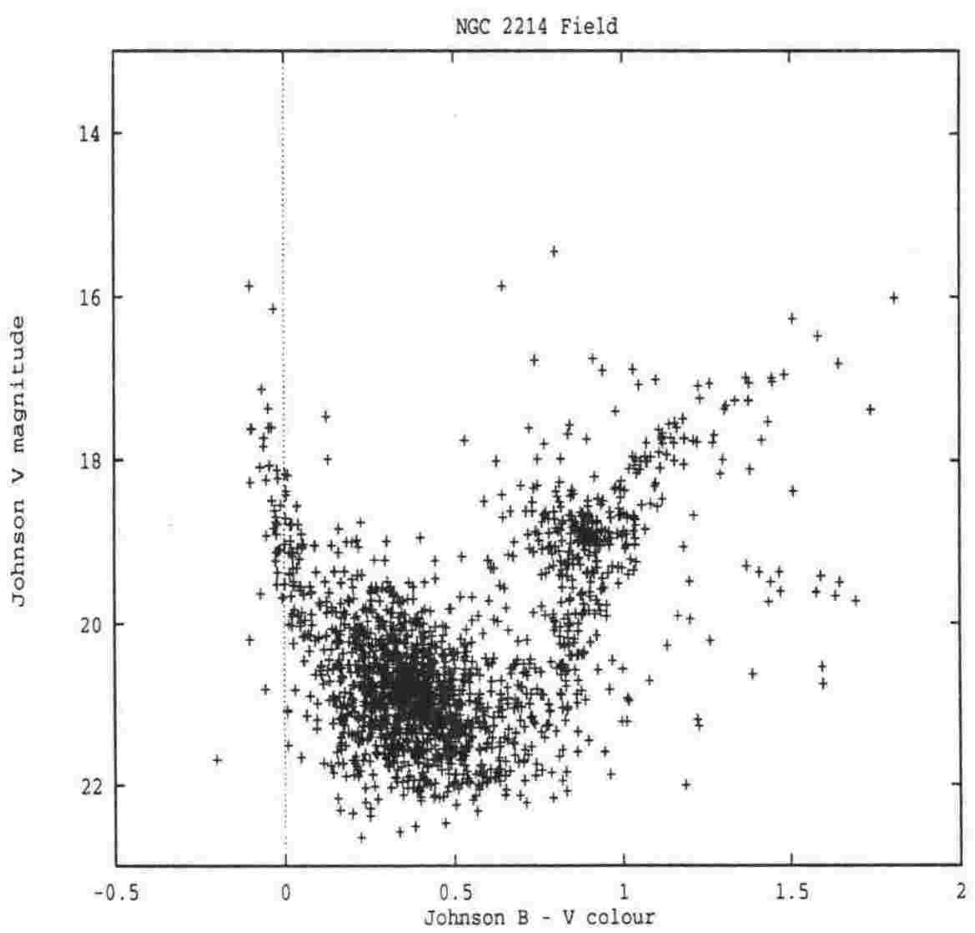

Figure 7-6: Colour-magnitude Diagram for the Field. 
of equations transfer the upper main sequence to within 0.04 and 0.015 magnitudes (in $\mathrm{V}$ and $(\mathrm{B}-\mathrm{V})$ ) of the positions reached using the original transformations. The $\mathrm{V}$ is slightly dimmed, while the colours are more red.

\section{$7.3 \quad$ Results}

\subsubsection{Colour-magnitude Diagrams}

The digiphot package of IRAF, which includes DAOphot (Stetson 1987), was used to reduce the crowded frames, perform aperture corrections, and transform the data across to the standard system. Figures 7-5 and 7-6 are the final colour-magnitude diagrams. 2919 and 1832 stars are plotted in the cluster and field diagrams respectively. The matching point between the long and short exposures was chosen to be the region where the data sets had similar errors, and was magnitude 16 and 17 for $\mathrm{V}$ and $\mathrm{B}$ respectively.

$\chi$ is the ratio of the actual scatter

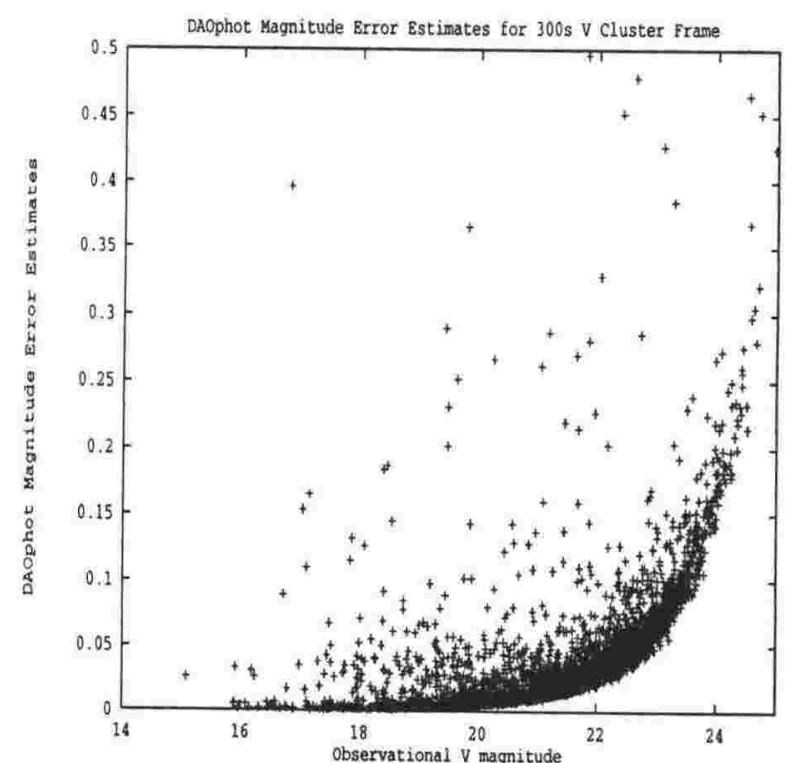

Figure 7-7: Photometric Errors for the 300 second V Exposure of NGC 2214.

about a point spread function fit divided by the expected scatter given the star and background sky brightness combined with the CCD readout noise. A value of $\chi$ near unity indicates a good fit. Only stars with a $\chi$ value of 3.0 or less were accepted (as in Mateo \& Hodge 1986). Examination of $\chi$ against observational magnitude showed this to be an acceptable limit, with the vast majority of detected 'stars' being within it. A further constraint was the use of the 'sharpness' measure of the difference between the square of the width of the object and the square of the width of PSF. Values should be close to zero for single stars, large positive values for blended doubles and partially resolved galaxies, and large negative values for cosmic rays and blemishes. Examination of this parameter for all detected 'stars' showed that the majority had values inside $|0.2|$. The final selection criterion was the use of photometric error estimates from $D A O$ phot. Photometric errors, as determined from least-square fitting by DAOphot, naturally increase with magnitude (see Figure 7-7). If a low threshold was set for the acceptable errors across all the data, the fainter magnitudes would be excluded. In order to retain as many as possible of these fainter stars in the CMD, the acceptable photometric uncertainty was relaxed 


\begin{tabular}{||c|c|c|r||c|c|r|r||}
\hline \multicolumn{4}{||c||}{ Johnson V } & \multicolumn{4}{c||}{ Johnson B } \\
\hline Magnitude & Limit & Cluster & Field & Magnitude & Limit & Cluster & Field \\
\hline $10-16$ & 0.010 & 16 & 1 & $10-17$ & 0.001 & 22 & 2 \\
$16-18$ & 0.011 & 56 & 13 & $17-18$ & 0.005 & 33 & 5 \\
$18-19$ & 0.016 & 111 & 54 & $18-19$ & 0.013 & 109 & 22 \\
$19-20$ & 0.025 & 349 & 139 & $19-20$ & 0.018 & 236 & 103 \\
$20-21$ & 0.040 & 504 & 291 & $20-21$ & 0.028 & 550 & 317 \\
$21-22$ & 0.060 & 925 & 644 & $21-22$ & 0.040 & 886 & 578 \\
$22-23$ & 0.090 & 1045 & 811 & $22-23$ & 0.063 & 1055 & 873 \\
$23-24$ & 0.200 & 287 & 207 & $23-24$ & 0.120 & 568 & 349 \\
\hline Total: & - & 3293 & 2160 & Total: & - & 3459 & 2249 \\
\hline
\end{tabular}

Table 7.1: Adopted Error limits. The majority of the photometric errors derived by $D A O p h o t$ fall onto an exponential function. The error adopted for each observational magnitude range (as given in the table) corresponds to the value of this function at the fainter magnitude limit of each interval. The number of stars recovered in each bin is also indicated under the headings 'cluster' and 'field' for each filter.

as magnitude increased. These values are given in Table 7.1.

The main features of the cluster CMD are:

1. A conspicuous main sequence of $(\mathrm{B}-\mathrm{V}) \sim 0$ extending to $\mathrm{V} \sim 16.5$;

2. A single and well-defined supergiant branch;

3. A subgiant branch of $(B-V) \sim 0.85$, extending from $V \sim 19$ to $\sim 21$;

4. A giant star clump at $(\mathrm{V}, \mathrm{B}-\mathrm{V}) \sim(19,0.9)$ which is slightly extended in $(\mathrm{B}-\mathrm{V})$ by some 0.25 magnitudes. This extension is well above the accepted photometric error at this V magnitude (see Table 7.1);

5. A uniformly populated giant branch extending from the giant clump up to (V, B - V) $\sim(16.5,1.7)$;

6. Several stars blueward of the main sequence, which are artifacts of the crowded field reduction (Lee 1992), being stars in dense regions whose parameters could not be estimated accurately by DAOphot. Magnitudes tend to be under-estimated. This is the basis of the bin migration phenomena noted by Mateo (1988b), amongst others;

7. A clump peaking at $(\mathrm{V}, \mathrm{B}-\mathrm{V}) \sim(19.7,0.3)$ of older stars;

8. and many faint red stars in the lower right of the CMD, which have been tentatively identified as giant stars by Chiosi (1989), and considered to be field stars.

Only items 1 and 2 are features associated with the cluster, the others being due to field stars. Being at a reasonable Galactic latitude $\left(\sim-30^{\circ}\right)$ the majority of field stars will be in the LMC. The Field CMD appears to be contaminated to an extent by cluster stars, as evidenced by the 


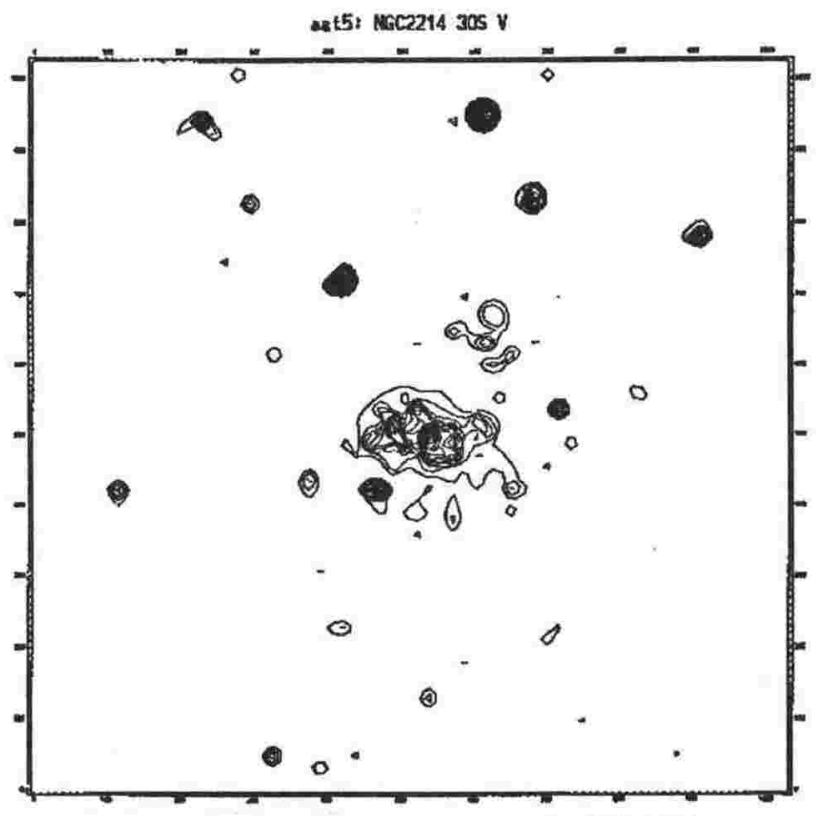

Figure 7-8: Contours for the Inner Regions of NGC 2214, in which contour lines range from 196 to $3196 \mathrm{ADU}$, with an increment of 100 . Note the apparent double nature to the core, which should be compared with Figure 2b of Bhatia \& MacGillivray (1988)

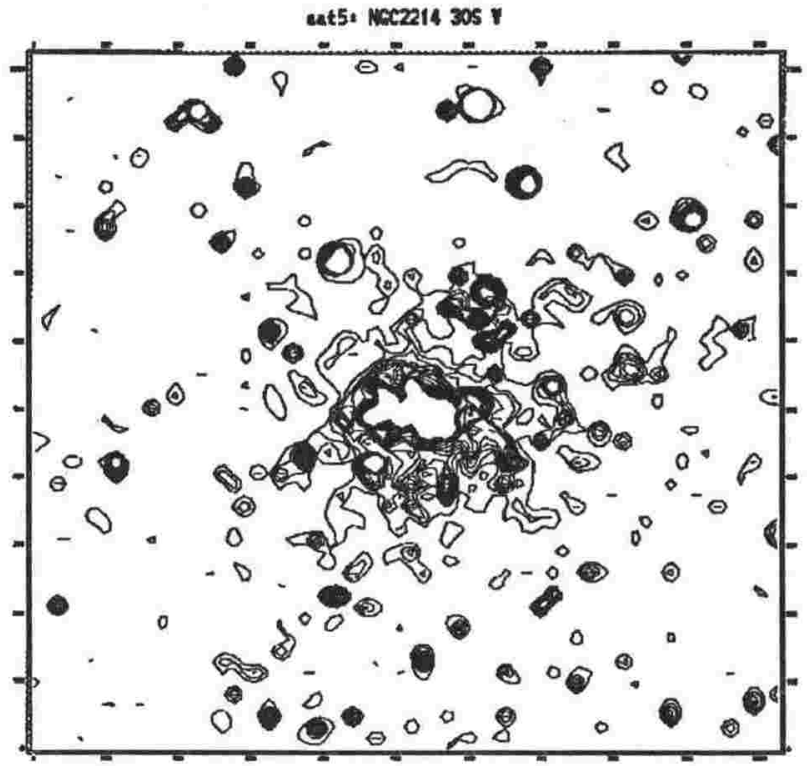

Figure 7-9: Contours for the outer regions of NGC 2214. The contour lines are increments of 20 counts per pixel over the range 96 to $496 \mathrm{ADU}$. 
reasonably strong main sequence. This would be expected from the results of the profile fitting discussed in Chapter 5.

CMDs were also generated for subsections of the frame, in order to search for differences across the cluster which might be expected for two merging clusters as in Bhatia \& MacGillivray (1988). First the frame was split vertically about the cluster centre, which was estimated as the mean value derived from the ellipse fitting described below, as was the rotation of the cluster. Marginal distributions peak close by. Given the position angle was $\sim 100$ degrees this division effectively split NGC 2214 into the suggested merging halves. No difference was found in the shape of the two CMDs, nor of those for four 300 pixel $^{2}$ regions placed at 90 degree increments about the cluster centre, suggesting that if the merger idea is correct the two bodies are of similar age (see also Bhatia \& Piotto (1993a, b), who came to the same conclusion).

\subsubsection{Contours}

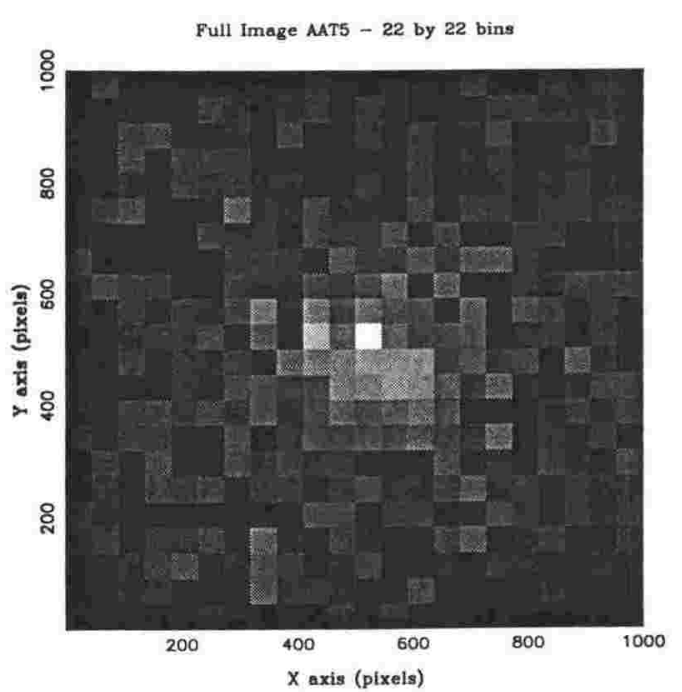

Figure 7-10: $\quad 30$ second $V$ cluster Frame Star-counts in 19.5 arcsecond Bins. White corresponds to the greatest number of stars (220 or more per square arcminute), while black corresponds to 15 or less stars per square arcminute.
Bhatia \& MacGillivray (1988) presented contours of NGC 2214 based on a IIIaF UK Schmidt survey plate, which exhibited two lobes in the core of the cluster. Noting the possible criticism that isophotal maps may be influenced by the placement of bright stars, Bhatia \& MacGillivray (1988) processed the COSMOS (MacGillivray \& Stobie 1984) scans of the plates using a crowded field package in order to obtain star counts. They claimed these would not be strongly influenced by bright stars and so reflect more appropriately the distribution of stars in the cluster. Again a double peak was evident, although it can be seen that the contour delineating the peaks is not statistically different from the next 'lower' contour, which does not show lobes, if a Poisson distribution is assumed. It should also be noted that the IIIaJ plate contours presented by Bhatia \& MacGillivray (1988) do not show a double peak, but rather a slightly offset centre. This suggests that the lobes are due to a small number of stars, with markedly different colour indices.

The results of contouring the 30 second V frame of NGC 2214 are shown in Figures 7-8 and 7-9. As in Bhatia \& MacGillivray (1988), two components appear to be in the cluster centre. Following Bhatia \& MacGillivray (1988), stars were counted into 0.3 arcminute square 


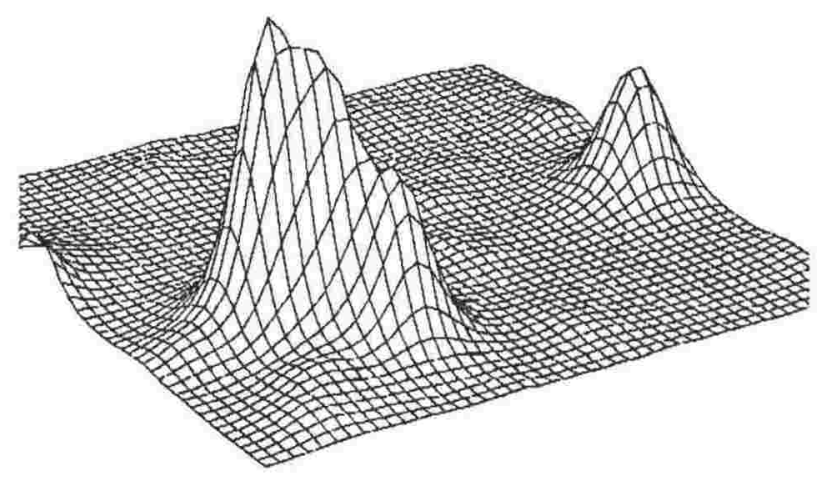

Figure 7-11: Grid Diagram of the Blended Region. A surface diagram, or a three dimensional representation of a portion of the frame with intensity as the $z$ (vertical) axis, shows that the gap between the two 'cores' contains the blended product of several bright stars. Each grid square represents one pixel.

bins, and each scaled to the number of stars per arcminute. A central condensation is evident, with a slight indication of a lobe, however a grey-scale representation of the data shows that the contour diagram tends to over-interpret the data (see Figure 7-10). The central peak bin contained 20 stars, while the alleged secondary peak and gap between the two peaks contained 15 and 11 stars respectively. Assuming a Poissonian distribution, these latter two values are not statistically different.

Different selection effects apply to each of the bins, and so like is not being compared with like. The gap contains three of the four brightest stars in the bins. These stars, and the blended product shown in Figure 7-11, could be obscuring fainter stars. This would explain why the faintest star recovered in this bin is 2.5 magnitudes brighter than that recovered in the less crowded 'second lobe'. Certainly only the two outer stars were detected in the blended peak, with another further possible star being lost in outer regions of the brightest star. The latter 'star' appears as a small rise in the outer regions of the PSF of the bright star when it is compared with the generic PSF derived for the frame.

The second peak is mainly outside the populated central region of the cluster, where blending is less severe. Fainter stars can be resolved, and so the star count increased. Star counts in populous clusters can not naively be considered to reflect the underlying stellar distribution due to problems deconvolving extremely crowded regions. A worst-case scenario for a populous spherical cluster could show only a few star counts in the heavily blended inner regions, surrounded by a ring of greatest counts. From the centre of such a cluster, star counts would increase with radius until the radius of this ring was reached. Beyond this radius, the counts would drop away again.

Increasing the number of bins along each axis of the frame resulted in grey-scale plots of greater resolution, but with the tradeoff of less counts and greater noise. None of these plots 
AAT3 Ellipse Fitting Output

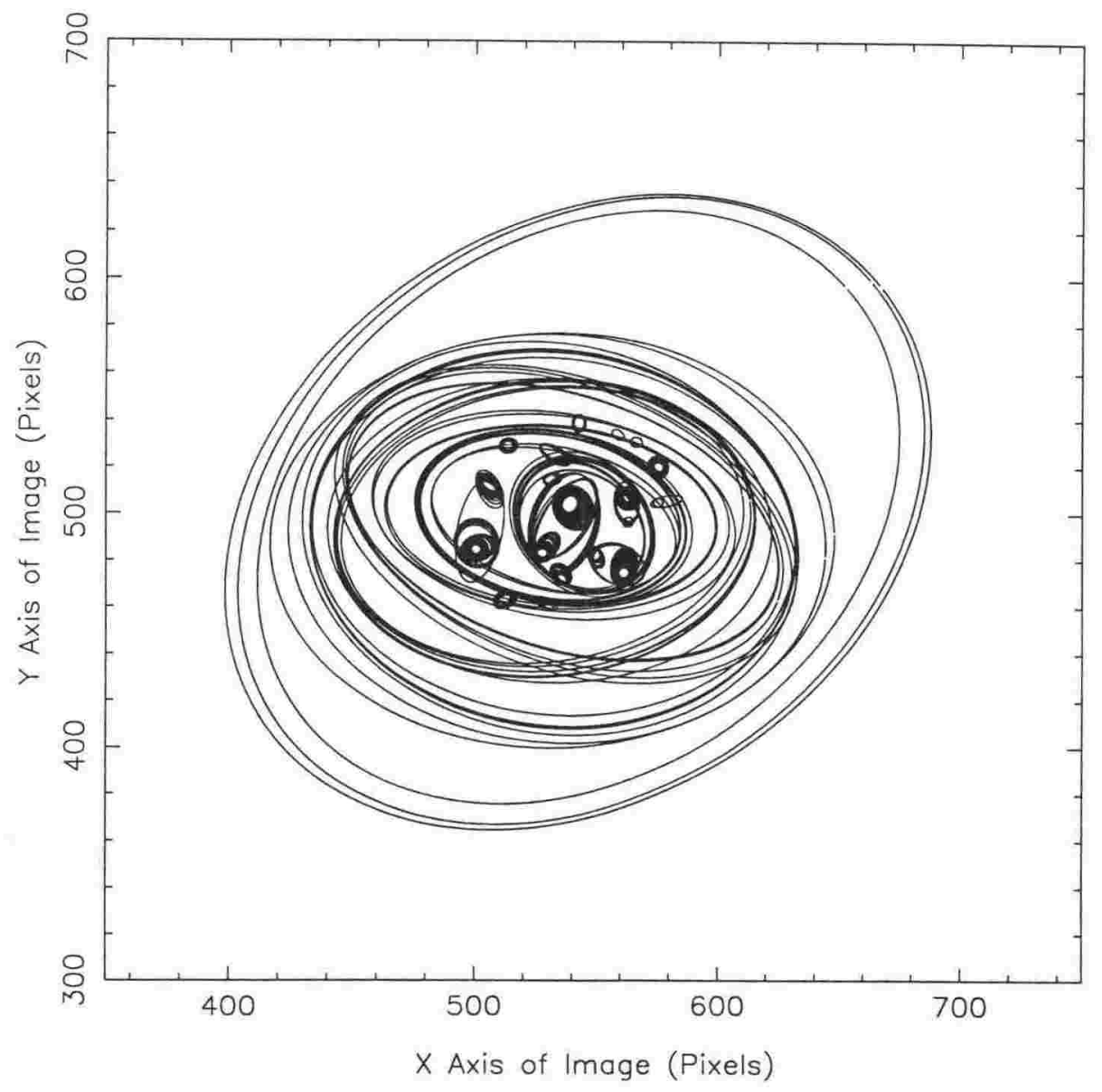

Figure 7-12: Ellipse Fits to the 60 second B Frame. The 'lowest' threshold used to detect pixels for ellipse fitting was 3 times the standard deviation of the background (which was in turn found by using the IRAF imex task in 21 regions across the frame).

showed any substantial evidence of a second component to the core. It is contended that there is no evidence from the star counts discussed here to support the notion that two cores are present in this cluster.

\subsubsection{Ellipse Fitting}

Moment-based ellipse fitting was performed on the 60 second B frame of NGC 2214 using the routines described in Chapter 5 (and Banks et al., 1994b). A generally constant ellipticity of 0.4 was found out to a radius of $\sim 30^{\prime \prime}$ from the cluster centre (see Figure 7-12), before falling away to an ellipticity of 0.2 by $\sim 40^{\prime \prime}$. Beyond this radius the ellipticity is constant (out to the maximum measurement of $62^{\prime \prime}$ ). The position angle was constant at $\sim 100^{\circ}$ out to a radius of $\sim 40^{\prime \prime}$, and then climbed to another plateau at $\sim 150^{\circ}$. This may have been caused by the lower detection thresholds, used for the greater radii, encountering a grouping of a few stars to 


\begin{tabular}{||c|c|c|c||c|c||}
\hline CMD & \multicolumn{2}{|c||}{ Swiss models at Z } & \multicolumn{2}{c||}{ Bertelli et al. at Z= } \\
\cline { 2 - 6 } Feature & 0.001 & 0.008 & 0.020 & 0.004 & 0.020 \\
\hline Main Sequence: & - & 8.0 & 8.0 & 8.3 & 8.2 \\
Supergiant Branch: & - & 7.8 & 7.8 & 8.0 & 7.9 \\
Field Main Sequence Turnoff: & 9.2 & 9.2 & 9.0 & - & 9.6 \\
Red Giant Clump: & 8.7 & 8.7 & 8.6 & - & 9.0 \\
\hline
\end{tabular}

Table 7.2: Isochrone Fit Ages. Log ages are given for the best isochrone fits to these features in the CMDs. A distance modulus of $18^{\mathrm{m}} \cdot 4$ was used. The long and short moduli of $18^{\mathrm{m}} \cdot 2$ and $18^{\mathrm{m}} \cdot 7$ were also fitted, with no difference in ages for $18^{\mathrm{m}} \cdot 2$, while the $18^{\mathrm{m}} \cdot 7$ ages were $\log 0.2$ younger. The intermediate distance of $18^{\mathrm{m}} \cdot 4$ was a compromise. The Swiss models are those of Schaerer et al. 1993, Schaller et al., 1992, and Meynet et al. 1993.

the side of the main body of the cluster, and so skewing the ellipse fit.

Zepka \& Dottori (1987) fitted ellipses to photographic observations of NGC 2214, and found that the cluster ellipticity was constant at around 0.4 out to their maximum radius of $43^{\prime \prime}$, and that the position angle of the clusters was constant. Frenk \& Fall (1982) give an ellipticity of 0.29 for their eye measurements of the burnt-out centre of NGC 2214 in a SRC sky survey plate. This result is in accord with those of the current study, as the measurement would correspond to outer regions of the cluster.

However, these apparent trends should be treated with caution, given the results of Chapter 5 in which synthetic frames were generated of clusters with known ellipticities. Spurious trends were found by both the moment analysis technique used here and the method of Jedrzejewski (1987), as implemented in STSDAS, due to bright stars, some of which were so badly blended in crowded regions that they could be neither detected nor cleanly subtracted.

The ellipse fitting provides no definitive support for a double core to the cluster.

\subsubsection{Isochrone Fits}

Initially both non-overshooting and overshooting isochrones were constructed with $\mathrm{Z}=0.001$, 0.008 , and 0.020 (Schaerer et al. 1993, Schaller et al. 1992, Meynet et al. 1993) ${ }^{1}$. Fits to features in both the cluster and field CMDs were attempted (see Table 7.2). The data points were transformed for a distance modulus of $18^{\mathrm{m}} \cdot 4$ and reddening of $0^{\mathrm{m}} 08$ (see Lee 1992, Castella et al. 1987, Elson 1991). V extinction was taken to be 3.1 times E(B - V) (Rieke \& Lebofsky 1985, Mateo 1988b, Sagar \& Richtler 1991).

The 0.001 isochrones did not fit the features of the CMD at all well, being too blue. The fit to the red giant clump was poor, due to different gradients. Similarly the gradient of the giant branch was too steep. Non-overshooting models, at all three metallicities, did not fit this feature well either and were not considered further. However, the overshooting $Z=0.020$

\footnotetext{
${ }^{1}$ Hereafter referred to as the Swiss isochrones.
} 


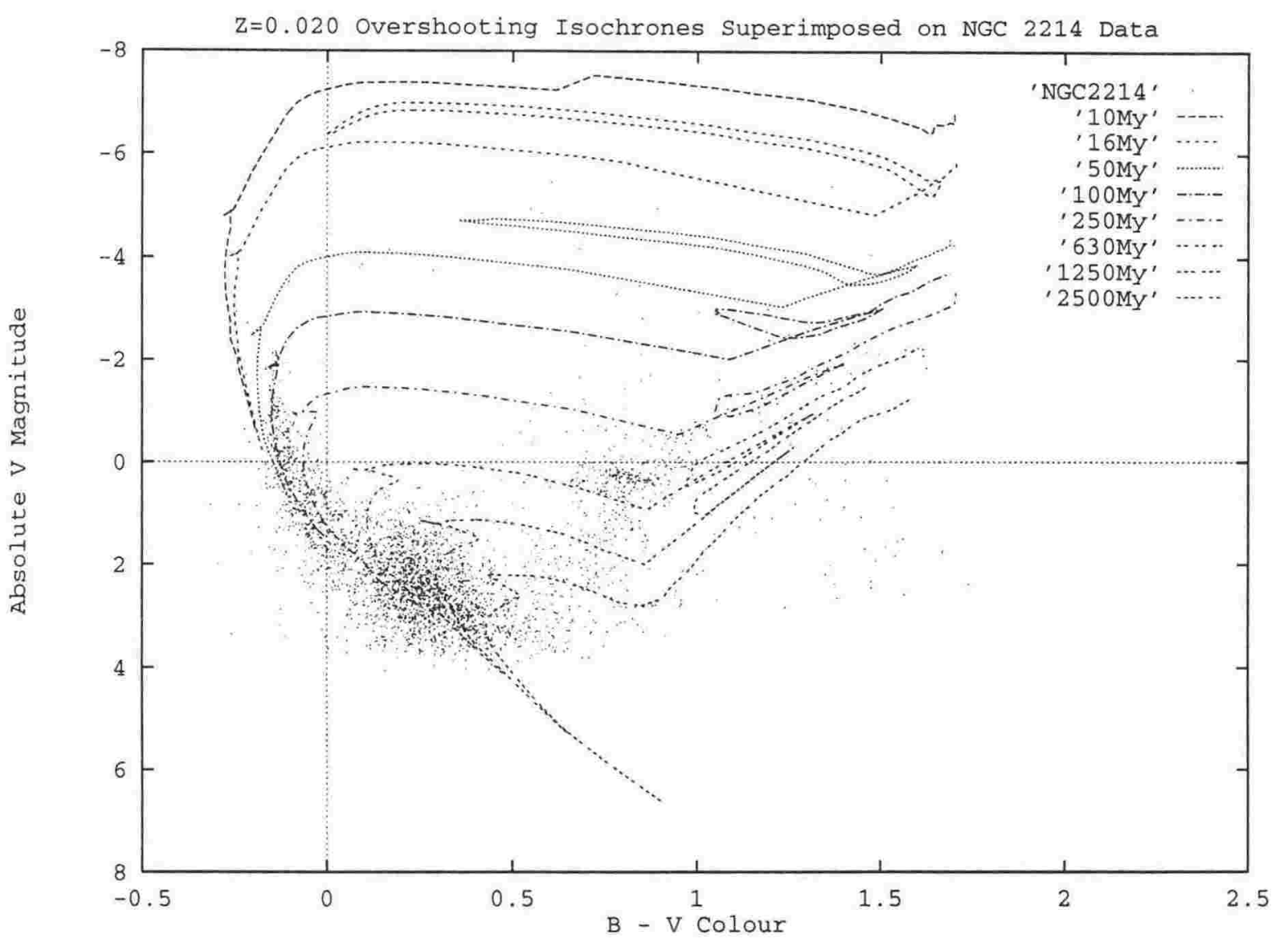

Figure 7-13: $\quad Z=0.02$ Isochrones Superimposed onto the BV CMD of NGC 2214 . Isochrones based on the Schaller et al. (1993) evolution models are plotted over the CMD derived for NGC 2214. The cluster data points have been shifted for a distance modulus of 18.4 and reddening of 0.08 (see Lee 1992, Castella et al. 1987, Elson 1991). V extinction was taken to be 3.1 times $\mathrm{E}(\mathrm{B}-\mathrm{V})$.

isochrones did fit the branch well, but not the field giant clump or the main sequence at the fainter magnitudes.

$\mathrm{Z}=0.020$ isochrones fitted all features of the CMD best (see Figure 7-13), with the $\mathrm{Z}$ $=0.008$ isochrones being slightly too blue. The ages in Table 7.2 tend to depend more on the $\mathrm{V}$ magnitude axis than colour, explaining the similarity of ages derived using the different metallicities. It was not possible with any of the isochrones to have one fit simultaneously the main sequence and the supergiant branch of the cluster. Lee (1992) used the Maeder \& Meynet (1991) isochrones, and encountered the same problem, having to assign different ages of $\log 7.7$ and 7.8 respectively to these features. Sagar et al. (1991) gave a range of ages for their 'bright' supergiant branch, ranging from log 7.5 using the Castellani et al. (1990) isochrones, to log 8.0 using Bertelli et al. (1990). The lower age was also derived by Elson (1991), based on the models of Becker (1981) and Brunish \& Truran (1982). The results of the current study tend towards the upper end of this age range since models incorporating convective overshooting 
were used, being, as are the other age estimates, dependent on the model used to derive the isochrones (see Figure 7-14 for a comparison between same age isochrones based on different models).

Turning to another set of models, the $\mathrm{Z}=0.020$ isochrones of Bertelli et al. (1990) fitted the majority of the CMD features well, confirming the comment of Sagar et al. (1991) that this metallicity is the best fit from the Bertelli et al. (1990) tables of $\mathrm{Z}=0.020,0.004$, and $0.001{ }^{1}$ Again (see Table 7.2) two isochrones were needed to fit the upper main sequence and the supergiant branch well, although a shift of $\sim+0.035$ in $(\mathrm{B}-\mathrm{V})$ would allow the $100 \mathrm{My}$ isochrone to fit both. ${ }^{2}$ Such a shift is just outside the formal errors in the

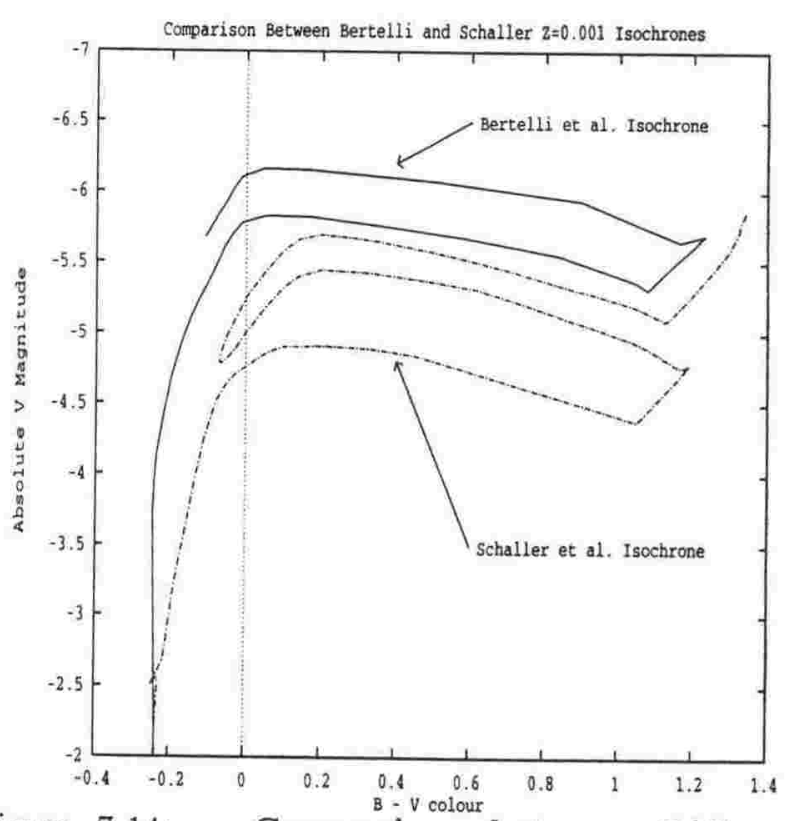

Figure 7-14: Comparison between Different Isochrones. The $\mathrm{Z}=0.00140$ million year isochrones of Bertelli et al. (1990) and Schaller et al. (1992) are combined aperture corrections and pho-plotted together.

tometric transformations. The same

shift would allow the $\mathrm{Z}=0.0206 \times 10^{7} \mathrm{yr}$ isochrone of Schaller et al. (1993) to fit both features well. However, fits to the upper main sequence by lower metallicity isochrones can also be made with appropriate extra reddenings, although the fit to fainter features in the CMD worsens. NGC 2214 lies in a region of $\sim 5 \times 10^{19} \mathrm{H} \mathrm{I}$ atoms per $\mathrm{cm}^{2}$ (a column density), according to the Mathewson \& Ford (1984) map of the Magellanic Stream. Sauvage \& Vigroux (1991) give the relation of $\mathrm{E}(\mathrm{B}-\mathrm{V})$ increasing by one magnitude for every $2.4 \times 10^{22}$ atoms per $\mathrm{cm}^{2}$ in the LMC. Reddening by this gas would therefore not be detected. Mould et al. (1994) give the foreground reddening $\mathrm{E}(\mathrm{B}-\mathrm{V})$ for the LMC as 0.07 , in agreement with the Galactic reddening maps of Burstein \& Heiles (1982), which place NGC 2214 between the 0.06 and 0.09 reddening contours.

\footnotetext{
${ }^{1}$ This is interesting in light of the Richtler \& Nelles (1983) Strömgren estimate of $[\mathrm{Fe} / \mathrm{H}]=-1.2 \pm 0.2$, which would make NGC 2214 the least metal abundant of all the young LMC clusters (see Da Costa 1991a). Richtler \& 330 (the latter also give $[\mathrm{Fe} / \mathrm{H}]$ values of -1.6 and $-1.8 \pm 0.2$ for the Magellanic Cloud clusters NGC 1818 and (Spite et alter being in the SMC). Later values for these clusters are -0.9 (Richtler et al. 1989) and -1.3 (Spite et al. 1986), both using high dispersion spectra, suggesting that the Richtler \& Nelles (1983) estimate $-0.55 \pm 0.04$ for the two clusters.

${ }^{2}$ This is possible using the second set of transformation equations.
} 


\begin{tabular}{||l||c|c|c|c|c|c||}
\hline \multicolumn{1}{|c||}{} & (B-V) & \multicolumn{5}{|c||}{ V Magnitude Range } \\
\cline { 3 - 7 } & Colour & $13-15$ & $15-17$ & $17-19$ & $19-21$ & $21-23$ \\
\hline Obs & $0.8-1.3$ & 2 & 10 & 132 & 120 & 27 \\
Exp & $0.8-1.3$ & 0.7 & 3.5 & 7.3 & 5.9 & 10 \\
\hline Obs: & $1.3+$ & 3 & 14 & 13 & 19 & 4 \\
Exp: & $1.3+$ & 0.1 & 0.8 & 5.0 & 17.7 & 39.5 \\
\hline
\end{tabular}

Table 7.3: Expected Star Counts. The predicted starcounts of Ratnatunga \& Bahcall (1985) for given regions of a CMD for the direction of the LMC are compared against actual star-counts from the CMD of the cluster produced by this study. The region limits are those given in the tables of Ratnatunga \& Bahcall (1985). The 'Obs' rows give the actual star counts, while the 'Exp' rows contain the expected numbers.

The field red giant clump fell on the Swiss 1 Gy isochrone, although a continuous series of older clumps was present. However, good fits to the lower sections of the subgiant branch required the lower metallicity of 0.004 . The $Z=0.001$ isochrones were too blue, and could not fit this feature well, whereas the 0.004 isochrones could also fit the slight clumping of stars to the upper left of the giant clump, as well as the few stars above the giant branch (i.e., as being on the AGB). This would be in keeping with the general nature of the age-metallicity relation of Da Costa (1991a) for the LMC. The relative densities along the giant clumps also suggest that there was a final burst of field star formation $\sim 1$ Gy ago, after what appears to be continuous formation as evidenced by the extended subgiant branch.

No isochrone could be fitted to the faint red stars given the adopted distance modulus, although their distribution in the CMD resembled a giant branch. The same feature can be seen in the field CMD. If this is a giant branch, then it must belong to a population with a distance modulus greater than that of the cluster. A program was used to assign an identification number to each star, and could be used to plot the distribution of these stars on either a CMD or on the CCD frame itself, allowing the physical distributions of CMD features to be examined. The faint red stars were not clumped, so another more distant cluster could not be the cause of the feature. The photometry of these stars was good. None of them exceeded the sharpness and $\chi$ limits of Bhatia \& Piotto (1993a), who explained the older supergiant branch of Sagar et al. (1991) as being due to photometric errors primarily caused by crowding in the central regions of the cluster. For 'good' photometry Bhatia \& Piotto (1993a) required $\chi$ to be under 2 and the absolute value of sharpness to be under 0.5. The faint red stars in the current CMDs had mean sharpness values of $0.95 \pm 0.13$ and $1.00 \pm 0.15$, and mean $\chi$ values of $-0.01 \pm 0.05$ and $0.00 \pm 0.02$, for the long exposure $\mathrm{B}$ and $\mathrm{V}$ frames respectively.

Mateo \& Hodge (1986) noted a similar 'arm' in the remote LMC cluster NGC 1777, and considered them to be a subgiant branch at least 3 Gy old, based on Vandenberg (1985) isochrones. Bertelli et al. (1992) considered that similar stars in their CMDs were likely foreground ob- 


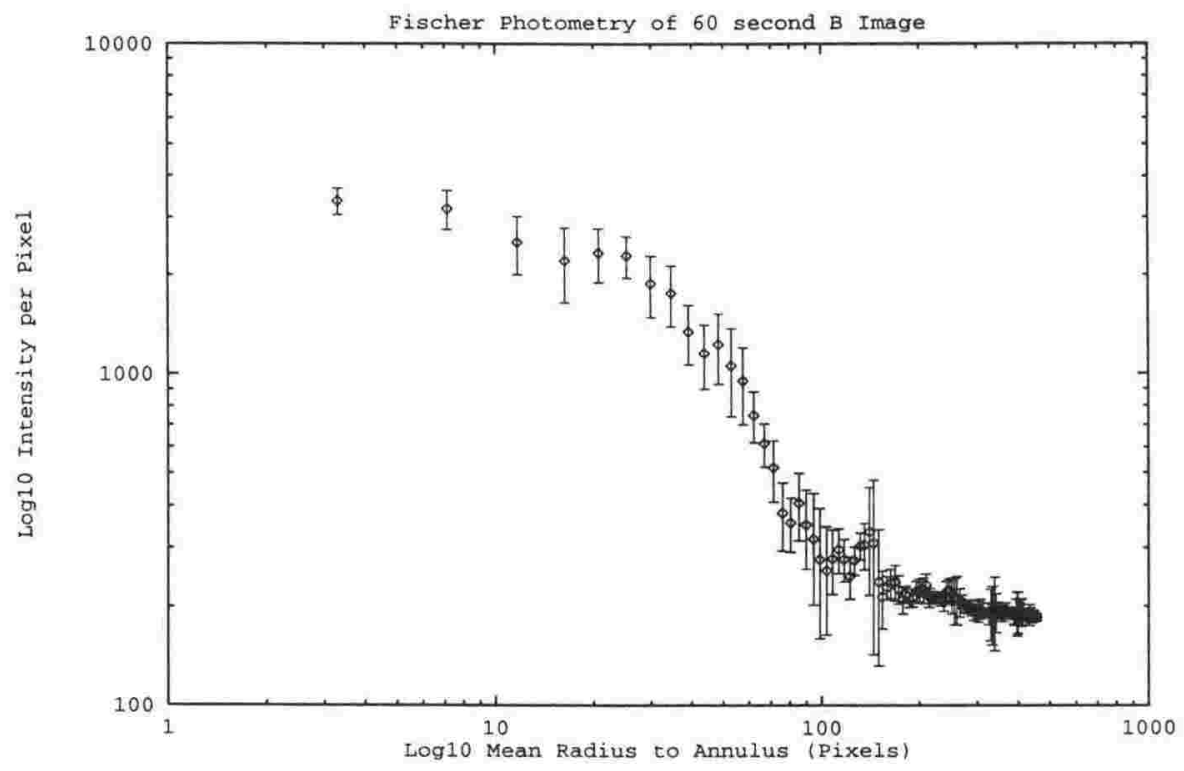

Figure 7-15: Luminosity Profile. A luminosity profile for the 60 second B exposure of NGC 2214. The kink at a radius of $\sim 10$ pixels was not apparent in NGC 1978. This second cluster is also elliptical ( $e \sim 0.3$ ), and so the kink does not seem to be due to the elliptical apertures, but rather the data.

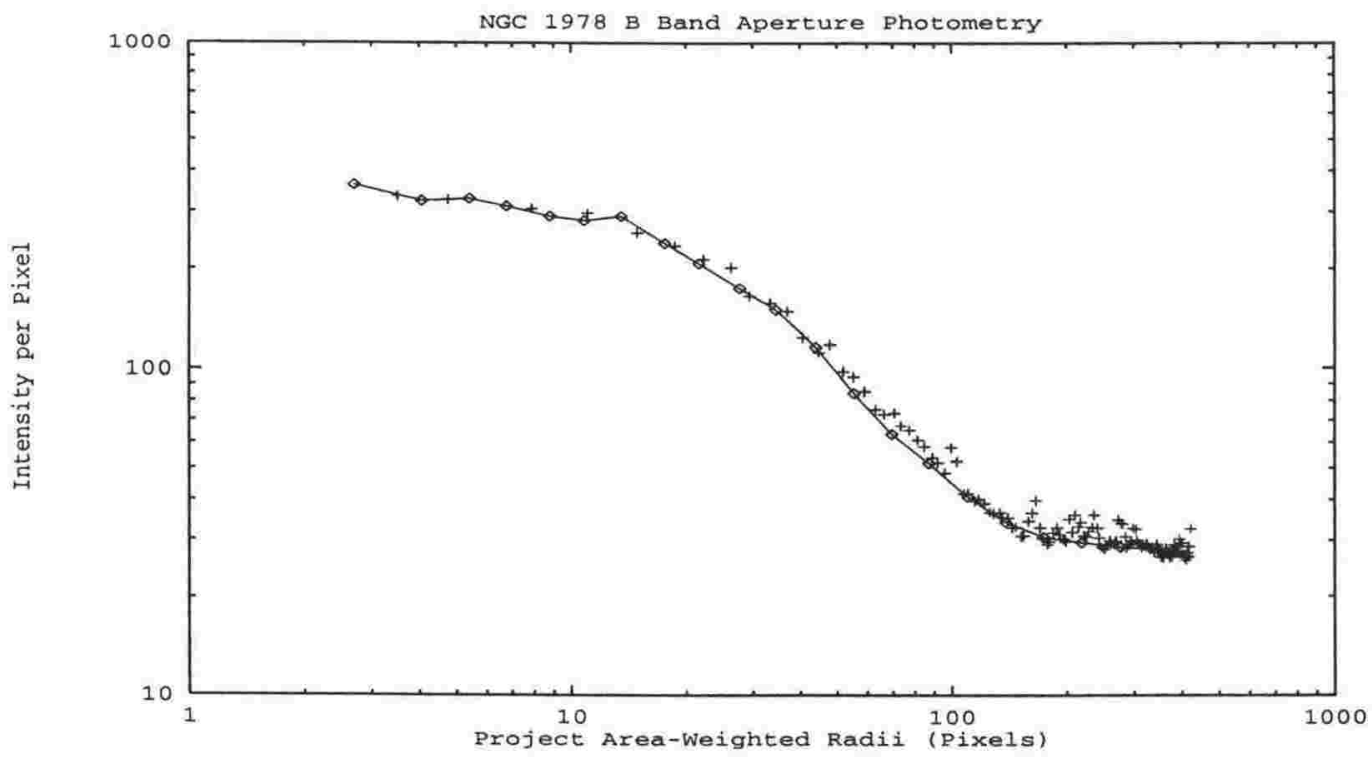

Figure 7-16: NGC 1978 Johnson B Band Aperture Photometry Comparison. The $\mathrm{B}$ band surface photometry of Fischer et al. (1992b) has been rescaled for comparison against the VUW test results for the same data, confirming that despite modifications the program is producing similar results. The cluster centre was estimated by eye. The data of Fischer et al. (1992b) are plotted as diamonds joined together by a line, while the VUW test data are shown as plus symbols. 
jects, according to the counts predicted by Ratnatunga \& Bahcall (1985) for the direction of the LMC. A comparison of the cluster CMD given in this study, with the predicted star counts given by the Bahcall \& Soniera $(1980,1984)$ galaxy model was made. Observational counts exceeded the predicted ones in all but the region $\mathrm{V}=21$ to 23 and $(\mathrm{B}-\mathrm{V})$ greater than 1.3 where the observational limit was met (see Table 7.3). The majority of the enhancements were obviously due to features such as the giant branch. The region $(\mathrm{V}, \mathrm{B}-\mathrm{V})=(21-23,0.8-1.3)$ is to the red of the field main sequence (MS) by more than any of the individual observational errors of MS stars at these magnitudes, and it contains 3 times as many stars as expected from the model, even though the count is not corrected for completeness (see below) which will be significant at such faint magnitudes. The photometric errors of these stars do not overlap with those of similarly faint MS stars. The region $(19-21,1.3+)$ is barely above the expected value, and not statistically significant. However, when counting incompleteness is considered, using the Lesser Completeness Factor Method discussed below, some 26 stars are expected in this region. It appears that foreground contamination or photometric uncertainty (such as these being stars 'scattered' off the main sequence) can not account for all the faint red stars, leaving their origin still unclear.

\subsubsection{Aperture Photometry}

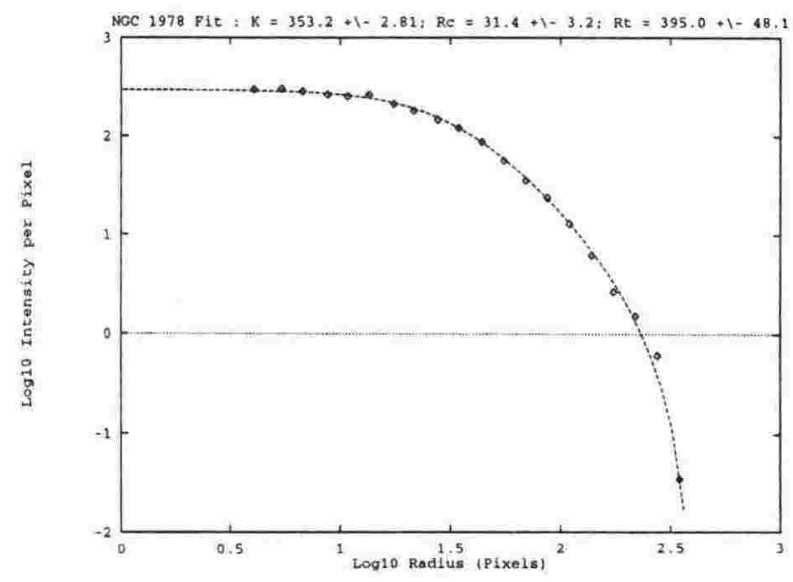

Figure 7-17: King Model fit to NGC 1978. The $\log$ binned V profile for NGC 1978 is shown, with the best fit King model.
The IRAF IMFORT program of Fischer et al. (1992a, b, 1993) was slightly modified to handle the AAT data and used to perform photometry, in a manner similar to Djorgovski (1987), on the 30 and 60 second $V$ and $B$ exposures of both NGC 2214 and the field region. The cluster frames were broken up into a series of concentric elliptical annuli centred on the cluster centre. The same central pixel coordinates were used for the field frames. Each annulus was divided into eight sectors, and the intensity summed within each (see Figure 7-15 for an example profile). The median value was adopted as being representative of the sectors at the weighted average radius, in an attempt to reduce the effect of bright supergiants in the profile (Fischer et al. 1993). The standard error of the sectors is equal to the standard error of the mean multiplied by $\sqrt{\pi / 2}$, leading to the photometric 
uncertainty for the annulus.

The program was used with the NGC 1978 Johnson B band data of Fischer et al. (1992b), as a check to see that it was performing correctly (see Figure 7-16). In this case an ellipticity of 0.3 was adopted. A smooth luminosity profile was derived, very similar to Figure 10 of Fischer et al. (1992b), which was reassuring. Tests using a circular aperture showed no significant systematic differences from results using the elliptical aperture, confirming Fischer et al. (1992b), and lending support to the contention of Elson et al. (1987) that circular apertures could be used on LMC clusters despite the (small on average) cluster ellipticities.

The non-linear least-squares profile fitting program, described in Chapter 5, was used to fit King models to the V band profile of NGC 1978, and the V and B ones of NGC 2214. NGC 1978 was fitted well by the models. A typical result was $r_{c}=12.2 \pm 1.4^{\prime \prime}$ and $r_{t}=154 \pm 15^{\prime \prime}$ (see Figure 7-17). Slightly different starting parameters led to different final values, with ranges of around $10^{\prime \prime}<\mathrm{r}_{\mathrm{c}}<14^{\prime \prime}$ and $140^{\prime \prime}<\mathrm{r}_{\mathrm{t}}<170^{\prime \prime}$, suggesting that the minimum was rather flat. This was confirmed by a grid search, in which $r_{c}$ and $r_{t}$ were fixed, leaving only $k$ to be optimised. A shallow, but fully enclosed, minimum was revealed. The core radius value for NGC 1978 agrees well with the 11.2" estimate of Mateo (1987).

The NGC 2214 data were collected into equal-width $\log$ radius bins, with the mean being taken for each populated bin, to avoid implicit weighting of the model fit by more data points being at the outer regions. Fits to both the binned and unbinned data required large values for the tidal radius. Grid searches were also performed on the B and V NGC 2214 data. They revealed that while the core radius was constrained, there was only a lower boundary for the tidal radius. The best $\mathrm{B}$ fit was for radii $\mathrm{r}_{\mathrm{c}}=9.9 \pm 2.4^{\prime \prime}$ and $\mathrm{r}_{\mathrm{t}}=296 \pm 67^{\prime \prime}$

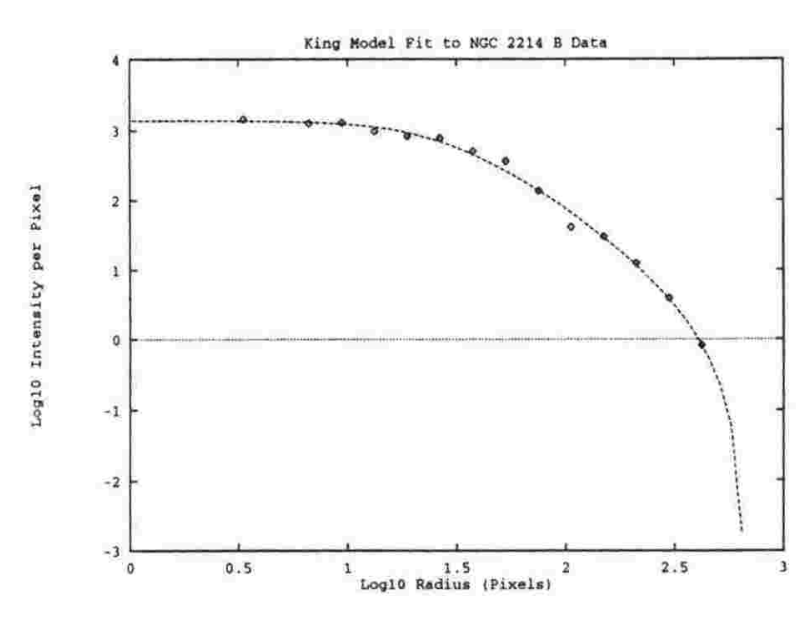

Figure 7-18: King Model fit to NGC 2214. The log binned B profile for NGC 2214 is shown with the best fit King model. The curvature for NGC 2214 is shallow and insufficient to provide good constraints on the tidal radius, unlike NGC 1978.

(see Figure 7-18). It was repeatedly reached from various starting values, as long as $r_{t}$ was initially small. The $\mathrm{V}$ band result was similar in $\mathrm{r}_{\mathrm{c}}$, being $10.6 \pm 2.2^{\prime \prime}$, and the tidal radius was $258 \pm 190^{\prime \prime}$. The tidal radii were very dependent on the background values adopted - a variation of $\sim 1 \%$ could cause the radius to vary by nearly a factor of 2 . The NGC 2214 radii agree well with the value of $10.5 \pm 0.7^{\prime \prime}$ 
given by Elson (1991) for the core radius, and the tidal radius range of $125^{\prime \prime}$ to $630^{\prime \prime}$ reported by Elson et al. (1987).

The model proposed by Elson et al. (1987) gave inconclusive results when it was used as the fitting function to the current NGC 2214 data. The $\chi^{2}$ for the B band was $\sim 75 \%$ better than that of the best King model fit, but the $\mathrm{V}$ band was $\sim 44 \%$ worse making it unclear if the model fitted the data better. The values of the radius ' $a$ ' were $14.0 \pm 0.2^{\prime \prime}$ and $16.6 \pm$ $0.2^{\prime \prime}$ respectively, which is larger than the value of $11^{\prime \prime}$ (no error given) of Elson et al. (1987). However, $\gamma$ values of $2.65 \pm 0.19$ and $3.05 \pm 0.22$ were obtained for the two profiles. The first value of $\gamma$ is in reasonable agreement with the value of $2.40 \pm 0.24$ derived by Elson et al. (1987) for the cluster, while the second falls into the range of $\gamma$ covered by their young cluster sample. Elson (1991) fitted such models to CCD observations of NGC 2214, but did not present the $\gamma$ values, commenting that they too fell into the same range found by Elson et al. (1987).

King models fit the intermediate age cluster NGC 1978 reasonably well, but not the young cluster NGC 2214. Elson et al. (1987) noted similar problems with constraining the tidal radius in young LMC clusters, and commented that an unbound 'halo' of stars about the clusters could be responsible. The profile fits of the AAT data support this idea.

There is a potential problem with the aperture photometry program used in this section, in that it simply checks to see if the centre of a given pixel falls within an annulus. It does not consider how much of a pixel may fall within the annulus, and scale the intensity appropriately. Such a ragged edge to the annuli may become more important with decreasing radius from the centre, in a region possibly critical for fitting King (1966) models, although these values tend to be within the core radius and so of essentially uniform intensity. This may be countered by the observation above that elliptical and circular apertures resulted in similar profiles. Despite these comments, the program can be used with no reservations to search for colour gradients in the cluster, provided the same parameters are used for the B and V frames, and so the same pixels fall into the same apertures. The field showed no colour variation, nor any gradient in star counts. The cluster showed no variation, beyond random scatter, at large radii, and then became increasingly more red with decreasing radius as the ratio of cluster stars to field increased. Finally, the colour leveled out within the main body of the cluster, although the cluster is really too small for these results to be definitive. Small radii colours can be easily affected by individual bright stars.

\subsubsection{Completeness}

The brighter stars in a frame are almost certainly all recovered by the crowded field reduction software. However certainly not all of the faintest magnitude stars are identified and recovered, with the recovery rate decreasing with increasing magnitude. Problems affecting image recovery include: 
1. simply being so faint that their identification is adversely affected by stochastic variations of the background;

2. being too close to a comparably bright star, leading to a blended elliptical object which will be rejected as not being a star;

3. being lost in the profile of a much brighter star.

A luminosity function that is not 'corrected' for these effects will have a more shallow gradient, and with increasing magnitude increasingly underestimate the actual function. Empirical methods of estimating these 'completeness factors' generally centre around the addition of artificial stars, or scaled versions of the PSFs derived from selected images, into the CCD frames. These frames are then reduced in an identical manner to the original frame. The efficiency with which these false stars are recovered is taken to estimate the completeness factors, which are then used to correct the observed main sequence star counts to the values expected if the recovery rate had been $100 \%$. For a given magnitude interval $i$ the completeness factor is given as:

$$
\Lambda_{i}=\frac{n_{i, \text { recovered }}}{n_{i, \text { added }}}
$$

On a single frame the completeness correction can easily be derived since the variation of the completeness factors with magnitude and crowding can be empirically determined. However it is not possible to separate main sequence stars out of the mass of stars. Two colours, such as $\mathrm{B}$ and $\mathrm{V}$, are required for their identification. The resulting problem of having to match the star image in both colours leads to a further incompleteness. Mateo (1988b) and Mateo \& Hodge (1986) did not account for this last point, although mention was made of it, and simply considered that the completeness correction for a point $V_{i},\left(B_{i}-V_{i}\right)$ would be given by:

$$
\Lambda\left(V_{i}\right) \times \Lambda\left(B_{i}\right) .
$$

Sagar \& Richtler (1991) argued that the two frames were not independent and that the multiplicative assumption of Mateo (1988b) could not be justified. Instead, as the spatial distribution of stars in the frames is the same and the magnitude distribution is slightly modified, the completeness at a given point in the CMD would be mainly controlled by the lesser of the two completeness factors. There has been no test of the ability of these techniques to recover a known luminosity function.

An IRAF script was written which placed a small number of artificial stars into a frame. 50 stars was the maximum number added each iteration by the script (typically $\sim 5 \%$ of the detected stars), which meant that the image crowding and luminosity function would not be greatly affected by the introduction of the artificial stars. A user-selected magnitude range 


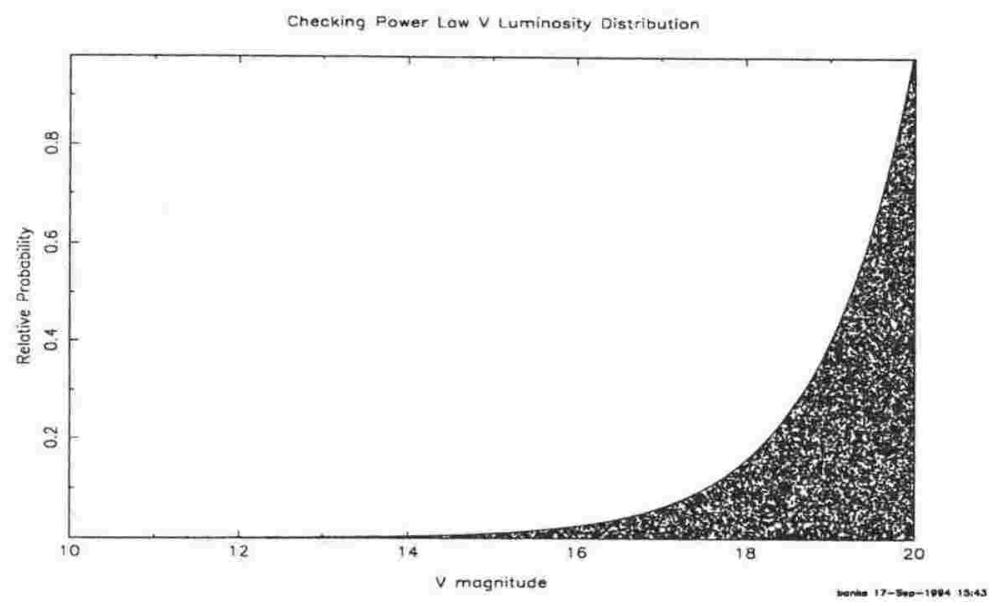

Figure 7-19: Monte-Carlo Luminosity Function. A normalised power-law for a V luminosity function is plotted as a smooth line. The dots are the 15,000 'successful' random numbers generated.

was divided up into bins, typically 0.5 mag wide. 'Random' magnitudes and ( $\mathrm{x}, \mathrm{y})$ positions were generated for stars in each bin, using the "Minimal Standard" pseudo-random number generator of Park \& Miller (1978). Press et al. (p.279, 1992) note that the period of this generator is $2^{31}-1$.

Several sets of false stars would be added to a given frame (in different iterations) and reduced until a user-set limit of recovered false stars was met. This limit was set high since the completeness factors vary with crowding. In a frame of a LMC star cluster, the degree of crowding naturally varies across the frame. Mateo (1988b), for instance, divided frames into three rings centred on the cluster, surrounded by field, and formed a mean luminosity function from the normalised ones of the annuli. Rather than adopt this technique, and its assumptions, many stars were placed into the frames in an attempt to derive the average. Once the recovery limit had been met, the magnitudes of the false stars in the next iteration would fall within the boundaries of the next magnitude bins, until all the subsections had been used.

\subsubsection{Tests}

In an attempt to decide which of the techniques proposed by Mateo (1988b) and Sagar \& Richtler (1991) was correct (or closer to correct), artificial star field frames of known luminosity functions were created. These frames were then subjected to the completeness factor program described above. The basic idea was that the different completeness factors could be applied to the recovered luminosity functions, and then compared with the input (and known) function in order to see which was the best fit. Simple functions, rather than more physically correct ones, were chosen to simplify what was effectively a computational exercise. Magnitudes, and the $\mathrm{x}$ and y positions of the 'fake' star on the frame, were randomly generated using the "Minimal 
Standard" pseudo-random number generator. Several tests of this routine used different initial seed values and involved 2 to 20 thousand random numbers. Flat distributions were produced by these tests, within the expected Poissonian uncertainty and with no evidence for periodicity. A simple power-law was used to define the luminosity function. The program randomly generated a magnitude. Another random number (between 0 and 1) was then generated and used in a Monte-Carlo test with the luminosity function, which acted as a probability function. If the number was less than the value of the normalised luminosity function for the given magnitude, then a star image was considered to exist (see Figure 7-19). The $\mathrm{x}$ and $\mathrm{y}$ values of the image were then randomly generated, and the three values ( $\mathrm{x}, \mathrm{y}$, and magnitude) written to file. Otherwise, another magnitude was generated. This process continued until the required number of stellar images was met.

It was necessary to make the luminosity function of a second frame different for the tests. The completeness factors, which depend on the luminosity function and the associated crowding, could not be the same. The relationship between the two magnitudes was arbitrarily assumed to be linear on a colour-magnitude diagram. Shallow gradients were adopted in the tests to force a substantial colour difference over the majority of the magnitude range (see Figure 7-20).

1000 by 1000 pixel frames were generated by the IRAF $m k o b$ task. 5000 images were present in the frames, covering the $\mathrm{V}$ magnitude range of 10 to 22. A Gaussian PSF and

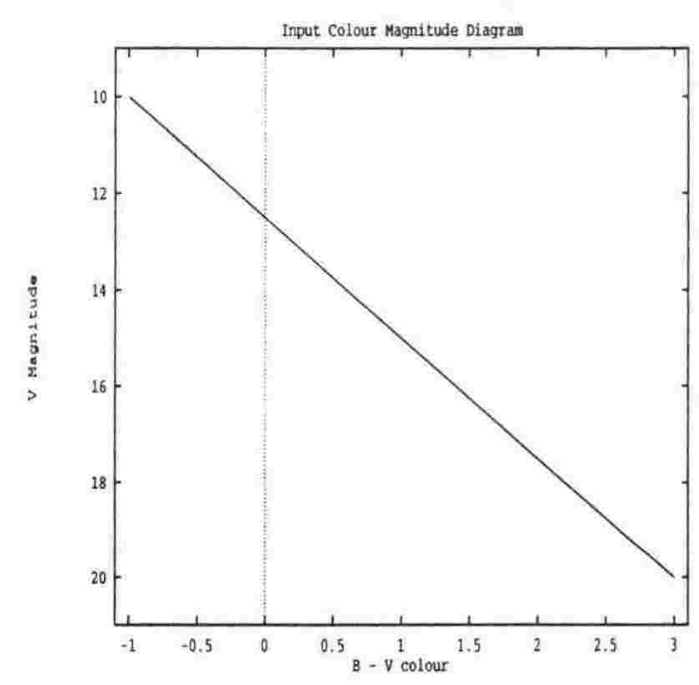

Figure 7-20: The Test Frames Magnitude Relationship. A linear relation on the CMD was adopted for the completeness factor tests, forcing the luminosity functions to differ in each 'colour'. 1.6 arcsec seeing was adopted, along with a CCD read noise and gain of $5.5 \bar{e}$ and $7.35 \overline{\mathrm{e}}$ per ADU, corresponding to the real values of the AAT frames (Da Costa 1993). A zero magnitude point of 21 was used, along with an 'exposure' time of 300 seconds. Poissonian noise was assumed. Additional B and V frames were also generated with the same parameters as the artificial field frames, but containing only 16 bright stars placed in an equally spaced grid. These stars were used to generate the semi-empirical PSF used by the artificial star recovery script, and indicated the aperture correction ( 0.262 magnitudes for a 7 pixel radius aperture). The problem of incomplete subtraction of neighbouring stars, usually requiring the iterative approach described by Stetson (1987), was therefore avoided. 


\begin{tabular}{||c|c|c|c|c||c|c|c||}
\hline \multirow{2}{*}{ Min } & \multirow{2}{*}{ Mag } & \multicolumn{3}{|c||}{ B Test Frame } & \multicolumn{3}{c||}{ V Test Frame } \\
\cline { 3 - 8 } & & Recovered & Input & Percent & Recovered & Input & Percent \\
\hline 9.0 & 9.5 & 927 & 950 & $97.6 \%$ & - & - & - \\
9.5 & 10.0 & 923 & 950 & $97.2 \%$ & - & - & - \\
10.0 & 10.5 & 2040 & 2100 & $97.1 \%$ & 1276 & 1300 & $98.2 \%$ \\
10.5 & 11.0 & 2066 & 2150 & $96.1 \%$ & 1266 & 1300 & $97.4 \%$ \\
11.0 & 11.5 & 2166 & 2250 & $94.0 \%$ & 1257 & 1300 & $96.7 \%$ \\
11.5 & 12.0 & 1626 & 1750 & $92.9 \%$ & 1242 & 1300 & $95.5 \%$ \\
12.0 & 12.5 & 1638 & 1800 & $91.0 \%$ & 1272 & 1400 & $90.9 \%$ \\
12.5 & 13.0 & 1345 & 1500 & $89.7 \%$ & 2156 & 2250 & $91.4 \%$ \\
13.0 & 13.5 & 2582 & 2950 & $87.5 \%$ & 1240 & 1400 & $88.6 \%$ \\
13.5 & 14.0 & 2320 & 2750 & $84.4 \%$ & 1216 & 1450 & $83.9 \%$ \\
14.0 & 14.5 & 1309 & 1600 & $81.8 \%$ & 1213 & 1500 & $80.9 \%$ \\
14.5 & 15.0 & 1302 & 1700 & $76.6 \%$ & 1230 & 1650 & $74.5 \%$ \\
15.0 & 15.5 & 2285 & 3150 & $72.5 \%$ & 1232 & 1800 & $68.4 \%$ \\
15.5 & 16.0 & 2238 & 3200 & $69.9 \%$ & 1212 & 1850 & $65.5 \%$ \\
16.0 & 16.5 & 1320 & 2050 & $64.4 \%$ & 1223 & 2050 & $59.7 \%$ \\
16.5 & 17.0 & 412 & 1200 & $34.3 \%$ & 1217 & 3650 & $33.3 \%$ \\
17.0 & 17.5 & - & - & - & 401 & 8250 & $4.9 \%$ \\
\hline Total & - & 26499 & 35250 & - & 18553 & 24200 & - \\
\hline
\end{tabular}

Table 7.4: Test Frame Artificial Stars Recovery Rates. The numbers of artificial stars input per magnitude bin are given, together with the numbers of these artificial stars actually recovered. 705 and 484 iterations were required for the B and $\mathrm{V}$ frames respectively.

\begin{tabular}{||r|c|c|c|c|c|c|c|c|c|c||}
\hline Min & Mag & Input & Recover & Lesser & Greater & Mateo & $\delta \mathrm{L}$ & $\delta \mathrm{G}$ & $\delta \mathrm{M}$ & Err \\
\hline 9.0 & 9.5 & 18 & 18 & 18.45 & 18.34 & 18.79 & -0.02 & -0.02 & -0.04 & 1.45 \\
9.5 & 10.0 & 41 & 40 & 41.17 & 40.99 & 42.19 & -0.00 & 0.00 & -0.03 & 2.90 \\
10.0 & 10.5 & 50 & 50 & 51.60 & 51.41 & 53.06 & -0.03 & -0.03 & -0.06 & 3.25 \\
10.5 & 11.0 & 55 & 50 & 52.03 & 51.71 & 53.81 & +0.05 & +0.06 & +0.02 & 2.00 \\
11.0 & 11.5 & 45 & 41 & 43.60 & 42.89 & 45.61 & +0.03 & +0.05 & -0.01 & 3.02 \\
11.5 & 12.0 & 48 & 48 & 52.44 & 51.19 & 55.93 & -0.09 & -0.07 & -0.17 & 3.20 \\
12.0 & 12.5 & 56 & 47 & 51.71 & 51.56 & 56.74 & +0.08 & +0.08 & -0.01 & 3.15 \\
12.5 & 13.0 & 63 & 56 & 62.45 & 61.02 & 68.05 & +0.01 & +0.03 & -0.08 & 2.40 \\
13.0 & 13.5 & 69 & 67 & 76.55 & 75.17 & 85.89 & -0.11 & -0.09 & -0.24 & 3.68 \\
13.5 & 14.0 & 84 & 66 & 78.42 & 75.98 & 90.28 & +0.07 & +0.10 & -0.07 & 3.65 \\
14.0 & 14.5 & 89 & 69 & 84.34 & 82.21 & 100.49 & +0.05 & +0.08 & -0.13 & 3.73 \\
14.5 & 15.0 & 103 & 84 & 109.68 & 103.70 & 135.40 & -0.06 & -0.01 & -0.31 & 4.10 \\
15.0 & 15.5 & 83 & 61 & 84.09 & 79.63 & 109.77 & -0.01 & +0.04 & -0.32 & 3.53 \\
15.5 & 16.0 & 92 & 55 & 78.92 & 74.58 & 107.03 & +0.14 & +0.19 & -0.16 & 3.38 \\
16.0 & 16.5 & 117 & 62 & 96.29 & 90.34 & 140.31 & +0.18 & +0.23 & -0.20 & 3.55 \\
16.5 & 17.0 & 145 & 46 & 133.99 & 68.97 & 200.89 & +0.08 & +0.52 & -0.39 & 3.15 \\
\hline
\end{tabular}

Table 7.5: B Test Frame Completeness. Columns 'Min' and 'Mag' give the magnitude limits for each bin. The column labeled 'input' gives the number of stars per magnitude bin that formed the artificial field frame. 'Recover' lists the number of stars per bin actually recovered by DAOphot. The 'Lesser', 'Greater', and 'Mateo' columns give the expected number of stars using three different completeness methods - with the recovery probability being given by the lesser of $\Lambda\left(V_{i}\right)$ and $\Lambda\left(B_{i}\right)$, the greater of the two, or their product. The columns $\delta \mathrm{L}, \delta \mathrm{G}$ and $\delta \mathrm{M}$ give the fractional error $\frac{\text { Input - Calculated }}{\text { Input }}$ where 'Calculated' is the completeness-corrected value for the three techniques. 'Err' gives the error in the adjusted values expected for a $2.5 \%$ variation in the completeness probabilities combined with the variation of one per BV bin. Repeated trials gave probabilities well within $2.5 \%$ of each other. 


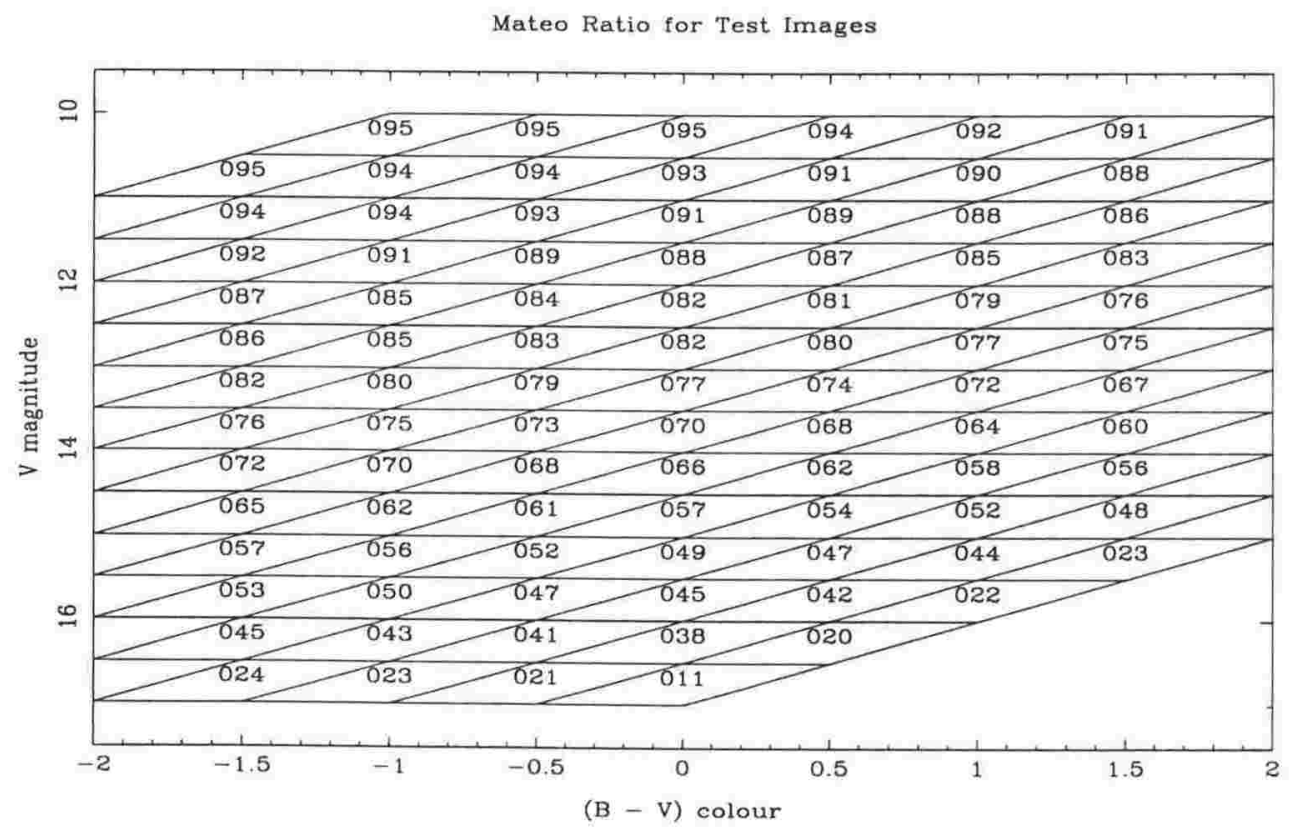

Figure 7-21: Mateo (1988b) Ratio for the Test Frames. The one-dimensional recovery rates of the $\mathrm{B}$ and $\mathrm{V}$ frames are combined to form a two-dimensional distribution, based on the assertion by Mateo (1988b) that the frames are independent and that a star can only be considered to be found if both $\mathrm{B}$ and $\mathrm{V}$ magnitudes are determined successfully. The number in each region is its percentage completeness factor.

\begin{tabular}{||c|c|c|c|r|r|r|c|c|c||}
\hline Min & Mag & Input & Recover & Lesser & Greater & Mateo & \multicolumn{1}{c}{$\delta$} & $\delta \mathrm{G}$ & $\delta \mathrm{M}$ \\
\hline 10.0 & 10.5 & 27 & 27 & 28.74 & 28.53 & 29.28 & -0.06 & -0.06 & -0.08 \\
10.5 & 11.0 & 52 & 52 & 54.55 & 54.42 & 56.02 & -0.05 & -0.05 & -0.08 \\
11.0 & 11.5 & 85 & 79 & 82.08 & 81.57 & 84.76 & +0.03 & +0.04 & +0.00 \\
11.5 & 12.0 & 57 & 55 & 58.69 & 57.57 & 61.43 & -0.03 & -0.01 & -0.08 \\
12.0 & 12.5 & 78 & 70 & 77.04 & 76.20 & 83.87 & +0.01 & +0.02 & -0.08 \\
12.5 & 13.0 & 90 & 77 & 86.05 & 83.90 & 93.76 & +0.04 & +0.07 & -0.04 \\
13.0 & 13.5 & 107 & 96 & 111.48 & 108.39 & 125.86 & -0.04 & -0.01 & -0.18 \\
13.5 & 14.0 & 120 & 98 & 119.34 & 116.67 & 142.09 & +0.01 & +0.03 & -0.18 \\
14.0 & 14.5 & 134 & 101 & 133.40 & 124.90 & 164.97 & +0.00 & +0.07 & -0.23 \\
14.5 & 15.0 & 124 & 88 & 124.02 & 118.05 & 166.37 & -0.00 & +0.05 & -0.34 \\
15.0 & 15.5 & 170 & 88 & 161.67 & 128.29 & 235.80 & +0.05 & +0.25 & -0.39 \\
15.5 & 16.0 & 198 & 16 & 78.64 & 41.21 & 120.04 & +0.60 & +0.79 & +0.39 \\
15.5 & 15.7 & 83 & 16 & 78.64 & 41.21 & 120.04 & +0.05 & +0.50 & -0.45 \\
\hline
\end{tabular}

Table 7.6: V Test Frame Completeness. The columns are the same as in the previous table. The low recovery rate in the magnitude range $(15.5-16.0)$ is due to the fact that the $\mathrm{B}$ magnitude limit falls into this bin, demonstrating that such limits must be noted or unexpected results occur. When the correct number of 83 stars input into the test frame, in the recoverable range of $\mathrm{V}=(15.5-15.72)$, is noted the Lesser Ratio method gives the best result, as could be expected from the other bins. The totals exclude the last bins with $\mathrm{V}$ greater than 15.5 . 


\begin{tabular}{||l|l|c|c|c|c|c|c|c||}
\hline \multicolumn{1}{|c|}{ V Range } & B Range & Input & $\begin{array}{c}\text { Expected } \\
\text { V\% }\end{array}$ & $\begin{array}{c}\text { Expected } \\
\text { B\% }\end{array}$ & Mean & C\% & B\% & V\% \\
\hline $10.0-10.5$ & $09.0-09.7$ & 850 & 100.0 & 100.0 & 100.0 & $97.0 \pm 2.4$ & $97.2 \pm 2.1$ & $99.5 \pm 1.0$ \\
$10.5-11.0$ & $09.7-10.4$ & 850 & 100.0 & 100.0 & 100.0 & $97.9 \pm 2.8$ & $98.2 \pm 2.1$ & $98.9 \pm 2.1$ \\
$11.0-11.5$ & $10.4-11.1$ & 850 & 100.0 & 100.0 & 100.0 & $96.7 \pm 2.3$ & $97.2 \pm 1.9$ & $97.4 \pm 1.9$ \\
$11.5-12.0$ & $11.1-11.8$ & 850 & 100.0 & 100.0 & 100.0 & $95.3 \pm 3.2$ & $95.5 \pm 3.3$ & $96.3 \pm 2.6$ \\
$12.0-12.5$ & $11.8-12.5$ & 950 & 97.0 & 94.3 & 95.65 & $94.3 \pm 2.8$ & $94.7 \pm 2.8$ & $95.5 \pm 2.1$ \\
$12.5-13.0$ & $12.5-13.2$ & 900 & 89.8 & 93.2 & 91.50 & $90.4 \pm 4.7$ & $91.0 \pm 4.7$ & $92.9 \pm 3.5$ \\
$12.75-13.25$ & $12.85-13.55$ & 900 & 83.2 & 83.7 & 83.45 & $84.7 \pm 5.8$ & $85.9 \pm 6.0$ & $90.1 \pm 3.7$ \\
$13.0-13.5$ & $13.2-13.9$ & 1000 & 76.5 & 72.0 & 74.25 & $75.8 \pm 6.7$ & $79.6 \pm 6.1$ & $86.9 \pm 4.5$ \\
$13.25-13.75$ & $13.55-14.25$ & 850 & 63.7 & 66.3 & 65.00 & $66.2 \pm 7.4$ & $71.5 \pm 8.3$ & $80.9 \pm 4.7$ \\
$13.5-14.0$ & $13.9-14.6$ & 900 & 52.3 & 58.2 & 55.25 & $56.5 \pm 6.9$ & $64.2 \pm 8.7$ & $75.7 \pm 6.7$ \\
$14.0-14.5$ & $14.6-15.3$ & 1800 & 36.1 & 38.7 & 37.40 & $22.2 \pm 7.0$ & $28.1 \pm 6.8$ & $61.9 \pm 9.0$ \\
\hline
\end{tabular}

Table 7.7: Dual Frame Completeness Test Results. The artificial stars generated by the completeness script fell within the ranges specified in the first two columns, i.e., into boxes on the CMD. These boxes were chosen to follow the line on the CMD that the test frame stars were generated along. The columns entitled 'expected' give the expected completeness factors calculated from the number of test frame stars retrieved within each box compared with the number used in the creation of the test frames. 'Mean' gives the mean of the two expected values. Columns $\mathrm{C}, \mathrm{B}$, and $\mathrm{V}$ give the completeness factors calculated by the script for the two frames together, the B frame, and the $\mathrm{V}$ frame. The combined correction is always equal to or smaller than the lesser of the individual B and V correction factors. The errors are based on the standard deviations of the trials in each box on the CMD. Each trial placed 50 stars into the test frames, with the total number of artificial stars used per box given in the 'Input' column.

The completeness program was then run on the frames. After the trials were completed, another computer program took the completeness factors for each frame, and appropriately scaled the number of stars in each $(B, V)$ box across the CMD. See Figure 7-21, which shows such boxes on the CMD. A heapsort (see Press et al. 1992) was used to sort these boxes into increasing magnitude order in both $\mathrm{B}$ and $\mathrm{V}$, leading to estimates of the input $\mathrm{B}$ and $\mathrm{V}$ luminosity functions. This program was carefully tested with artificial data to make sure it was scaling the data and performing the sorts correctly. Tables 7.4, 7.5, and 7.6 present the results of artificial star recovery on the test frames. Using the lesser of the $B / V$ recovery factors fitted the input luminosity function better than the technique of Mateo (1988b). The latter technique always gave an over-estimate which naturally became worse with increasing magnitude (and decreasing recovery factors for each frame). However, the lesser factor method did not recover the actual function that well, with estimates off by up to $18 \%$ and $6 \%$ of the input value in $\mathrm{B}$ and $\mathrm{V}$ respectively. The mean error over all the bins for the technique was $2 \%$ and $1 \%$ in $\mathrm{B}$ and V, or effectively zero, as could be expected if the errors are random and not systematic (as with the products method). However, towards the fainter magnitudes even the Lesser Ratio Method tended to underestimate the luminosity function, suggesting that the completeness factors were slightly too large. The technique assumes that all the false stars recovered on the frame producing the lower of the two completeness factors would be recovered on the other frame. As Mateo (1988b) notes, this may not be true. 
In order to test this, another IRAF command language script was written, which divided the Colour-magnitude diagram up into regions. These regions were bounded by lines of constant $\mathrm{V}$ magnitude and either constant $\mathrm{B}$ or $(\mathrm{B}-\mathrm{V})$. False 'stars' were then generated, using the minimal pseudo-random number generator discussed above, within each region in turn. Artificial images were assigned these random $\mathrm{V}$ magnitudes and colours, and input into the $\mathrm{V}$ and $\mathrm{B}$ CCD frames. The Images were then reduced in the standard manner by DAOphot. The false stars were recovered in each frame independently, and then matched in the same manner as the 'real' stars were. An IMFORT program was called by the script to perform this, with a substantial increase in speed over the algorithm being implemented in the interpreted script. This program and the script were carefully tested. Tests included giving the IMForT program known lists to match, and the use of small artificial frames with a few dozen bright stars in them. The script successfully recovered the stars, and the stars input by the script itself, in all trials (except in cases when two stars were badly blended together, and therefore both rejected). Table 7.7 gives results of the script being used on the same test frames as the previous completeness script, but employing an additional stringent matching criterion that stars with an estimated magnitude error greater than 0.10 were rejected. The dual frame method estimated the recovery rates better than the other techniques, except when the factor fell below 50\%, which Stetson (1991) defines as the limiting magnitude of a frame. However the technique is extremely computer intensive, making use of it prohibitive if it was to be used over the entire magnitude range of the frames. Fortunately, the matching problem will only affect completeness estimates near the faint limit of the Images, allowing the simpler Lesser Ratio Method to be used for the bulk of the calculations.

The low number of 'real' stars per magnitude range (as given in Tables 7.5 and 7.6) causes statistical problems. It is not practical to extend a magnitude bin too wide to increase the number of stars in it, as the completeness factor will then vary substantially inside the bin itself. In selecting the bin ranges, a balance needs to be struck between the variation in the factor across the magnitude range of the bin and the number of recovered 'real' stars in the bin.

While many artificial star trials can be performed to calculate the appropriate recovery rates, the low number of stars recovered in a given magnitude range leads to problems. The distribution of faint stars, and the level of obscuration by brighter stars, affects the number of these stars that is recovered. If, in a given magnitude range, the stars were fortuitously distributed their recovery rate would be greater than would be expected. The application of a completeness factor based on many artificial star trials, and representing the mean recovery rate for many distributions, would then result in the parent population being over-estimated. 


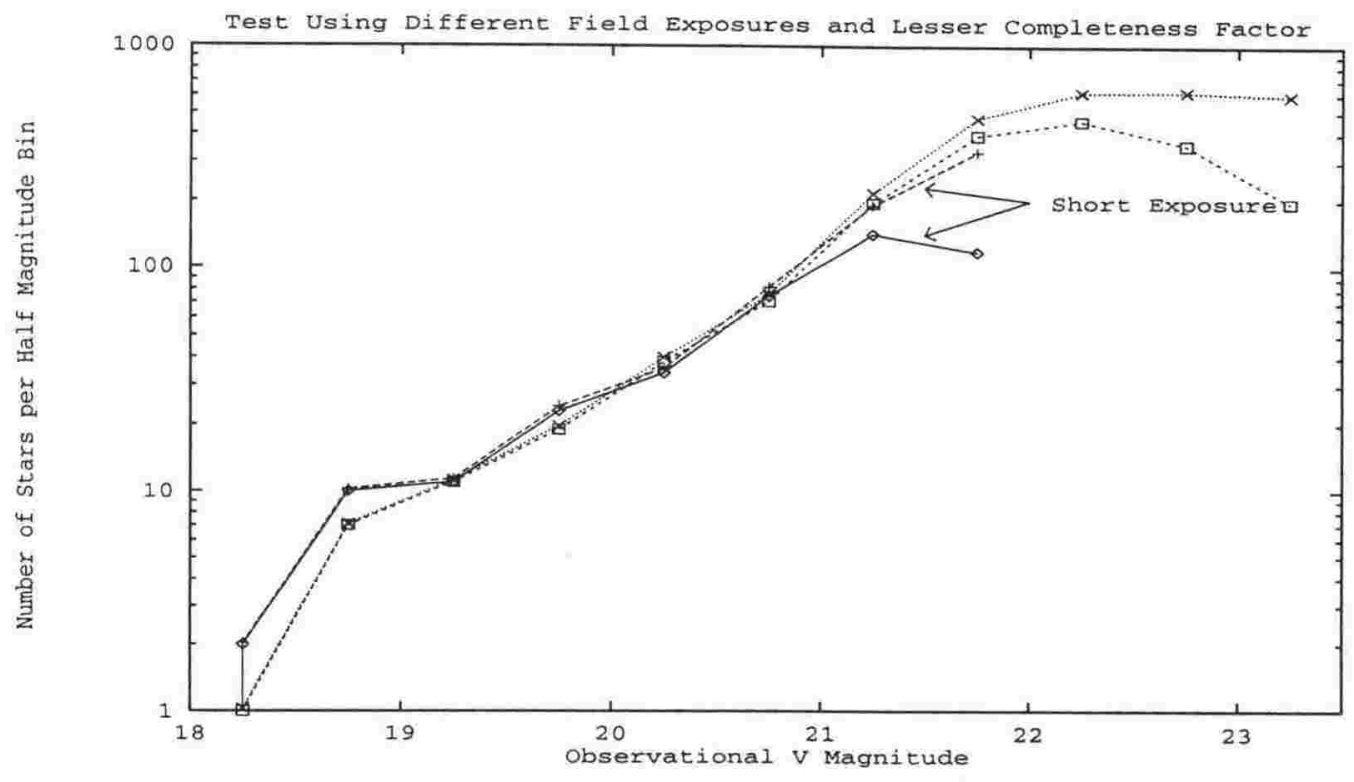

Figure 7-22: Observational V Band Luminosity Functions. The long and short exposure $\mathrm{V}$ band luminosity functions, with no attempt to remove non-main sequence stars, are plotted both with and without the appropriate completeness corrections applied. The uncorrected (raw) data is given by $\diamond$ and $\square$ for the short and long exposures respectively, while the corrected values are indicated by + and $x$ symbols.

The lower calculated recovery rates at brighter magnitudes are likely to be due to too many bright artificial stars being input. Interference between artificial stars becomes more likely with decreasing magnitude. Such blended products would be rejected, and so the recovery rate lowered. Recovery rates for all methods (Lesser, Greater, Mateo, and Combined) should be very similar at brighter magnitudes, and only differ at the fainter end. While the completeness corrected estimates were given as real numbers in the tables, converting them to integers could be preferable. In the brighter magnitude bins with small star numbers and a completeness correction slightly below $100 \%$, such a conversion would suitable given that it is likely that all the stars were recovered. In increasingly populous (and less complete) bins the rounding would proportionally less important.

\subsubsection{Application}

Tables 7.8 and 7.9 present the results of the completeness factor trials on the long exposure AAT frames. More artificial stars were added to the cluster frames in an attempt to account for the variation in crowding across the frames. This work was a large computing effort, involving months of CPU time. All six computers in the IRAF network were used simultaneously to run the completeness scripts.

Table 7.10 contains the completeness factors for the short exposure field frames. These 


\begin{tabular}{||c|c||c|c|c||c|c|c||}
\hline \multicolumn{2}{||c||}{ Range } & \multicolumn{3}{c||}{ B field } & \multicolumn{3}{c||}{ V field } \\
\hline Min & Mag & Out & In & $\%$ & Out & In & $\%$ \\
\hline 14.0 & 14.5 & 887 & 900 & 98.6 & 529 & 550 & 96.2 \\
14.5 & 15.0 & 443 & 450 & 98.4 & 531 & 550 & 96.5 \\
15.0 & 15.5 & 442 & 450 & 98.2 & 526 & 550 & 95.6 \\
15.5 & 16.0 & 444 & 450 & 98.7 & 537 & 550 & 97.6 \\
16.0 & 16.5 & 441 & 450 & 98.0 & 535 & 550 & 97.3 \\
16.5 & 17.0 & 448 & 450 & 99.6 & 539 & 550 & 98.0 \\
17.0 & 17.5 & 443 & 450 & 98.4 & 534 & 550 & 97.1 \\
17.5 & 18.0 & 437 & 450 & 97.1 & 535 & 550 & 97.3 \\
18.0 & 18.5 & 442 & 450 & 98.2 & 543 & 550 & 98.7 \\
18.5 & 19.0 & 443 & 450 & 98.4 & 541 & 550 & 98.4 \\
19.0 & 19.5 & 441 & 450 & 98.0 & 537 & 550 & 97.6 \\
19.5 & 20.0 & 433 & 450 & 96.2 & 533 & 550 & 96.9 \\
20.0 & 20.5 & 429 & 450 & 95.3 & 529 & 550 & 96.2 \\
20.5 & 21.0 & 420 & 450 & 93.3 & 514 & 550 & 93.5 \\
21.0 & 21.5 & 409 & 450 & 90.9 & 543 & 600 & 90.5 \\
21.5 & 22.0 & 423 & 500 & 84.6 & 515 & 600 & 85.8 \\
22.0 & 22.5 & 1060 & 1350 & 78.5 & 538 & 700 & 76.9 \\
22.5 & 23.0 & 422 & 650 & 64.9 & 727 & 1200 & 60.6 \\
23.0 & 23.5 & 400 & 850 & 47.0 & 526 & 1550 & 33.9 \\
23.5 & 24.0 & 291 & 1050 & 27.7 & 236 & 1500 & 15.7 \\
24.0 & 24.5 & 60 & 450 & 13.3 & 61 & 550 & 11.1 \\
\hline \multicolumn{2}{||c||}{ Total: } & 9658 & 12050 & - & 10609 & 14400 & - \\
\hline
\end{tabular}

Table 7.8: Long Exposure Field Completeness Factors are given for the observational magnitudes of the long $\mathrm{B}$ and $\mathrm{V}$ field exposures.

values were used with the lesser value method of Sagar \& Richtler (1991) to 'correct' the short exposure V luminosity function. Similarly, values from Tables 7.8 and 7.9 were used to correct the long exposure $V$ function. This was to test whether the same 'real' function would be recovered from the two exposures. It was found to be true in general, except for the brightest and faintest magnitudes (see Figure 7-22). The latter difference is likely to be due to the matching problem mentioned above, which will become more apparent near the limiting magnitude. Use of the dual frame completeness script seems to be warranted when the individual frame completeness factors fall below $\sim 75 \%$. The flattening of the long exposure $\mathrm{V}$ luminosity function shown in Figure 7-22 lies in such a range. The former difference is due to saturation of stars in the longer exposures, which may have a dependence on the position of the star within the cluster depending on the magnitude of the star. Bright stars, no matter where they are in a frame, will be saturated in a suitably long exposure. Hence a luminosity function for the long exposures would be zero in this range, and less than the function based on the short magnitudes. However, there will be a magnitude range where location does matter. If such a star is in a relatively uncrowded region of the frame, it will not be saturated. However, if it is in a crowded region then its light, combined with the fainter stars it is covering, will 


\begin{tabular}{||c|c||c|c|c|c||c|c|c|c||}
\hline \multicolumn{2}{||c||}{ Range } & \multicolumn{3}{c||}{ B cluster } & \multicolumn{4}{c||}{ V cluster } \\
\hline Min & Mag & Out & In & $\%$ & Err & Out & In & $\%$ & Err \\
\hline 14.0 & 14.5 & 3069 & 3100 & 99.0 & 1.8 & 2435 & 2450 & 99.4 & 2.0 \\
14.5 & 15.0 & 2220 & 2250 & 98.7 & 2.1 & 2225 & 2250 & 98.9 & 2.1 \\
15.0 & 15.5 & 3485 & 3550 & 98.2 & 1.7 & 2457 & 2500 & 98.3 & 2.0 \\
15.5 & 16.0 & 1717 & 1750 & 98.1 & 2.4 & 2168 & 2200 & 98.6 & 2.1 \\
16.0 & 16.5 & 3052 & 3100 & 98.4 & 1.8 & 3434 & 3500 & 98.1 & 1.7 \\
16.5 & 17.0 & 2591 & 2650 & 97.8 & 1.9 & 2354 & 2400 & 98.1 & 2.0 \\
17.0 & 17.5 & 3169 & 3250 & 97.5 & 1.5 & 3573 & 3650 & 97.9 & 1.7 \\
17.5 & 18.0 & 2038 & 2100 & 97.0 & 2.2 & 3066 & 3150 & 97.3 & 1.8 \\
18.0 & 18.5 & 3801 & 3950 & 96.2 & 1.6 & 2832 & 2900 & 97.7 & 1.9 \\
18.5 & 19.0 & 2733 & 2850 & 95.9 & 1.9 & 2649 & 2750 & 96.3 & 1.9 \\
19.0 & 19.5 & 3222 & 3350 & 96.2 & 1.7 & 3151 & 3250 & 96.9 & 1.7 \\
19.5 & 20.0 & 2489 & 2650 & 93.9 & 1.9 & 4633 & 4800 & 96.5 & 1.4 \\
20.0 & 20.5 & 2910 & 3200 & 90.9 & 1.8 & 2268 & 2450 & 92.6 & 2.0 \\
20.5 & 21.0 & 2921 & 3350 & 87.2 & 1.7 & 1442 & 1600 & 90.1 & 2.5 \\
21.0 & 21.5 & 2921 & 3500 & 83.5 & 1.7 & 1674 & 2000 & 83.7 & 2.2 \\
21.5 & 22.0 & 1986 & 2500 & 79.4 & 2.0 & 1633 & 2100 & 77.8 & 2.2 \\
22.0 & 22.5 & 3231 & 4550 & 71.0 & 1.5 & 1781 & 2450 & 72.7 & 2.0 \\
22.5 & 23.0 & 1990 & 3200 & 62.2 & 1.8 & 771 & 1250 & 61.7 & 2.8 \\
23.0 & 23.5 & 1003 & 2150 & 46.7 & 2.2 & 1627 & 4000 & 40.7 & 1.5 \\
23.5 & 24.0 & 536 & 2250 & 23.8 & 2.1 & 566 & 2400 & 23.6 & 2.0 \\
24.0 & 24.5 & - & - & - & - & - & - & - & - \\
\hline \multicolumn{2}{|c||}{ Total: } & 51084 & 59250 & - & - & 46739 & 54050 & - & - \\
\hline
\end{tabular}

Table 7.9: Long Exposure Cluster Completeness Factors are given for the observational magnitudes of the long $\mathrm{B}$ and $\mathrm{V}$ cluster exposures. The completeness software was altered in between the field and cluster studies to record the results of individual iterations. This modification allowed the calculation of the error estimates given for the cluster factors.

cause the star image to saturate. Therefore, the long exposures function would not be zero in this region, although less than the short exposure function.

\subsubsection{Field Star Subtraction}

Field stars need to be statistically subtracted from the luminosity function of the cluster. Cluster membership itself is hard to assess in the LMC. Even if radial velocities were available for all the stars, Freeman et al. (1983) showed young clusters to have disk dynamics, making such information rather uninformative about membership, only excluding Galactic stars. Flower et al. (1980) and Olszewski (1984) subtracted a star from the cluster CMD for every star within the same given region in the field CMD. This technique ignored completeness factors being different between the field and cluster. Mateo \& Hodge (1986) adjusted the number of stars in both the field and cluster by their completeness factors and also the relative areas of the field and cluster regions.

A computer program was written to subtract field stars from the cluster frame listing. Both lists were based on the long exposure frames. The observational CMD could either be divided 


\begin{tabular}{||c|c|r|r|r|r|r|r||}
\hline \multicolumn{2}{||c|}{ Range } & \multicolumn{3}{c|}{ B Field } & \multicolumn{3}{c||}{ V Field } \\
\hline Min & Mag & Out & \multicolumn{1}{c|}{ In } & $\%$ & Out & \multicolumn{1}{l|}{ In } & $\%$ \\
\hline 14.0 & 14.5 & 1155 & 1170 & 98.7 & 711 & 720 & 98.8 \\
14.5 & 15.0 & 1157 & 1170 & 98.9 & 709 & 720 & 98.5 \\
15.0 & 15.5 & 1923 & 1950 & 98.6 & 707 & 720 & 98.2 \\
15.5 & 16.0 & 1932 & 1950 & 99.1 & 705 & 720 & 97.9 \\
16.0 & 16.5 & 1890 & 1900 & 99.5 & 771 & 780 & 98.9 \\
16.5 & 17.0 & 1937 & 1950 & 99.3 & 59 & 60 & 98.3 \\
17.0 & 17.5 & 1223 & 1230 & 99.4 & 769 & 780 & 98.6 \\
17.5 & 18.0 & 509 & 510 & 99.8 & 766 & 780 & 98.2 \\
18.0 & 18.5 & 1217 & 1230 & 98.9 & 767 & 780 & 98.3 \\
18.5 & 19.0 & 768 & 780 & 98.5 & 762 & 780 & 97.7 \\
19.0 & 19.5 & 1146 & 1170 & 97.9 & 811 & 840 & 96.6 \\
19.5 & 20.0 & 1184 & 1230 & 96.3 & 799 & 840 & 95.1 \\
20.0 & 20.5 & 1180 & 1230 & 95.9 & 2294 & 2400 & 95.6 \\
20.5 & 21.0 & 414 & 450 & 92.0 & 1446 & 1560 & 92.7 \\
21.0 & 21.5 & 1148 & 1450 & 79.2 & 732 & 840 & 87.1 \\
21.5 & 22.0 & 1112 & 2790 & 39.9 & 1431 & 1860 & 76.9 \\
22.0 & 22.5 & 107 & 1050 & 10.2 & 1416 & 2700 & 52.4 \\
22.5 & 23.0 & - & - & - & 322 & 1800 & 17.9 \\
\hline \multicolumn{2}{|c|}{ Total: } & 20002 & 23210 & - & 15917 & 19680 & - \\
\hline
\end{tabular}

Table 7.10: Short Exposure Field Frames Completeness Factors are given for the observational magnitudes.

up into a $(\mathrm{B}, \mathrm{V})$ or $(\mathrm{V}, \mathrm{B}-\mathrm{V})$ grid, or into circular regions about each star in the cluster frame. Regardless of which of the three methods was used to bound a region in the CMD, the numbers of stars within this region in the field and cluster CMDs were counted. The completeness factors appropriate for the centre of the region in both the field and cluster CMDs were used to adjust the star counts. The selected regions therefore should not to be too large, otherwise the completeness factors would vary within the region itself. The ratio of the completeness corrected cluster to field ratio was taken as the probability that a given star (within the region) in the cluster star-list was actually a cluster member. If a grid was being used then a random number was generated for each cluster-list member within the region. If the random number was greater than the probability given by the corrected ratio, then the star was considered to be a cluster member, otherwise it was removed from the list. In the case of the circular regions, the probability was only applied to the central star, as all the stars in the cluster frame would be checked in turn. It should be noted that the cluster star-list was not altered during execution of the program. Artificial star and completeness factor lists were used to test the software, which performed as expected. Given the result of the completeness tests, the lesser of the appropriate $\mathrm{B}$ and V completeness factors was used in the analysis of NGC 2214.

\subsubsection{Luminosity Functions}

The field-star-subtracted star-lists need to be corrected for counting incompleteness, before a luminosity function can be estimated. The field-star subtraction discussed above only compared the cluster and field ratios in order to calculate a probability that a given star in the cluster 
CMD was a field member. Mateo (1988b) commented that the calibration of the false-star magnitudes was problematic as transformation equations explicitly involve a colour term, and so assumed that all his false-stars had a $(B-V)$ colour of 0.5 .

To overcome this problem a bin distribution was selected for the $\mathrm{V}$ and $\mathrm{B}$ luminosity functions. The standardized magnitudes of each star were in turn transferred back to the observational system using the transformation equations given above. The relevant $\mathrm{B}$ and $\mathrm{V}$ completeness factors were then referenced, and the appropriate completeness ratio (Mateo, Lesser, or Greater) chosen. The standardised magnitudes were used to determine which luminosity function bins should be altered. The appropriate bin counts were then incremented by the inverse of the completeness ratio. This technique avoided the problem of standardising the false-star magnitudes. The program output results at every step of the process during testing. These values were compared with manual calculations, and found to be correct.

In addition, only main sequence stars should be included in a luminosity function. Following Mateo (1988b), a line was arbitrarily drawn between main sequence and evolved stars, with the evolved stars being discarded. The importance of using two-colour photometry to define main sequence stars belonging to a cluster can be illustrated by the Da Costa (1982) luminosity function for the Galactic globular cluster 47 Tuc. A series of single-colour plates was used, producing a relatively steep luminosity function. Later BV CCD photometry of the cluster by Hesser et al. (1987) revealed that there was severe contamination of the cluster photometry by faint SMC stars. Removal of these stars flattened the luminosity function to the point where 47 Tuc appears to have one of the flattest luminosity functions amongst the globular clusters.

Several 'subtractions' were performed with different seeds for the pseudo-random number generator, producing similar results. The individual search option was used, with a search radius of 0.282 magnitudes. This corresponds to the same area as that of 0.5 square magnitude box, or roughly 1.4 times the area of a given combined completeness bin. The intention was to use a search area large enough to collect a reasonable number of stars to avoid low number statistics, yet not so large that the completeness corrections varied substantially within the region. Given that completeness factors were calculated for 0.5 magnitude bins in each frame, it was an arbitrary decision to use a region slightly larger than a completeness 'diamond' (see Figure 7-21 on page 195) in order to satisfy these two requirements.

\subsubsection{Mass Functions}

The luminosity functions were converted into mass functions using the best fitting Swiss isochrone discussed above ( $\mathrm{Z}=0.020$ with an age of $\log 8.0$ years) to derive a mass-luminosity function for the cluster. The evolution in luminosity of a star still on the main sequence can be quite substantial. The change in luminosity between ZAMS and at the turnoff of a star can corre- 
spond to between 0.5 and 1.6 magnitudes, depending on the mass of a star (Mateo 1988b). The global mass function slope does not sensitively depend on the evolutionary models used (see Mateo (1988b) and Sagar \& Richtler (1991) who compare mass functions derived using classical and overshooting stellar models). As Mateo (1993) pointed out, the mass range being studied changes, but the gradient remains effectively the same with the greatest variation occurring for metallicities below $\mathrm{Z}=0.004$.

The resulting mass functions derived from the long exposure frames are given in Figure 7-23. Functions were calculated from both the B and V luminosity functions, and are displayed. The errors shown are the Poisson error in the number of actually retrieved stars (i.e., before the star numbers were corrected for completeness) combined with uncertainty in the completeness correction. The error in the completeness factors themselves is hard to quantify. Poisson errors were assumed for them (as in Table 7.9). Their inclusion could be quite involved - as the stars falling into a given bin could belong to subgroups each with a different completeness correction. In order to have an upper estimate, the largest of the completeness correction errors was used for the entire group. Linear least-square fitting was performed on the functions. Using the expression for the IMF introduced in the introduction to this chapter, $x$ values of $1.18 \pm 0.18$, $1.47 \pm 0.21$, and $1.32 \pm 0.14$ were derived for the B, V, and combined BV data. These gradients are in reasonable agreement with Sagar \& Richtler (1991), who calculated $x$ values of $1.1 \pm$ $0.3,2.2 \pm 0.3,1.4 \pm 0.5$, and $1.3 \pm 0.3$ for NGC 2214, using the lesser of the BV completeness factors and different stellar evolution models.

Like the results of Sagar \& Richtler (1991), the mass function for NGC 2214 appears to have a steep gradient at the higher masses. They made no comment about this feature, and applied a single straight line. In the current study, the steep decline in the two most massive $\mathrm{V}$ bins, and the most massive $\mathrm{B}$ bin, is an artifact of the reduction process. While an isolated star with a magnitude that fell into one of these bins would not be saturated on the real frame, it would be if the star were in a region with an enhanced background level, e.g. the cluster centre. Unfortunately, no clipping level corresponding to that used in the reduction of actual observations was set for the artificial-star trials. The artificial-stars were therefore not rejected even if they were in the cluster centre, leading to an over-estimate of the completeness factor in these, the brightest of bins. It is possible that the same effect was present in the artificial-star trials of Sagar \& Richtler (1991).

The short exposure frames were examined for the number of stars that fell into these bins. The numbers were substantially larger, and increased the mass spectrum values of the bins (see Figure 7-24). There is still some hint that two lines could be fitted to the high and low mass stars respectively. An $x$ of 2 fits the greater than $\sim 3$ solar mass stars well. Below $\sim 3$ solar masses a shallower gradient appears reasonable, corresponding to an $x$ of 0.8 . The 


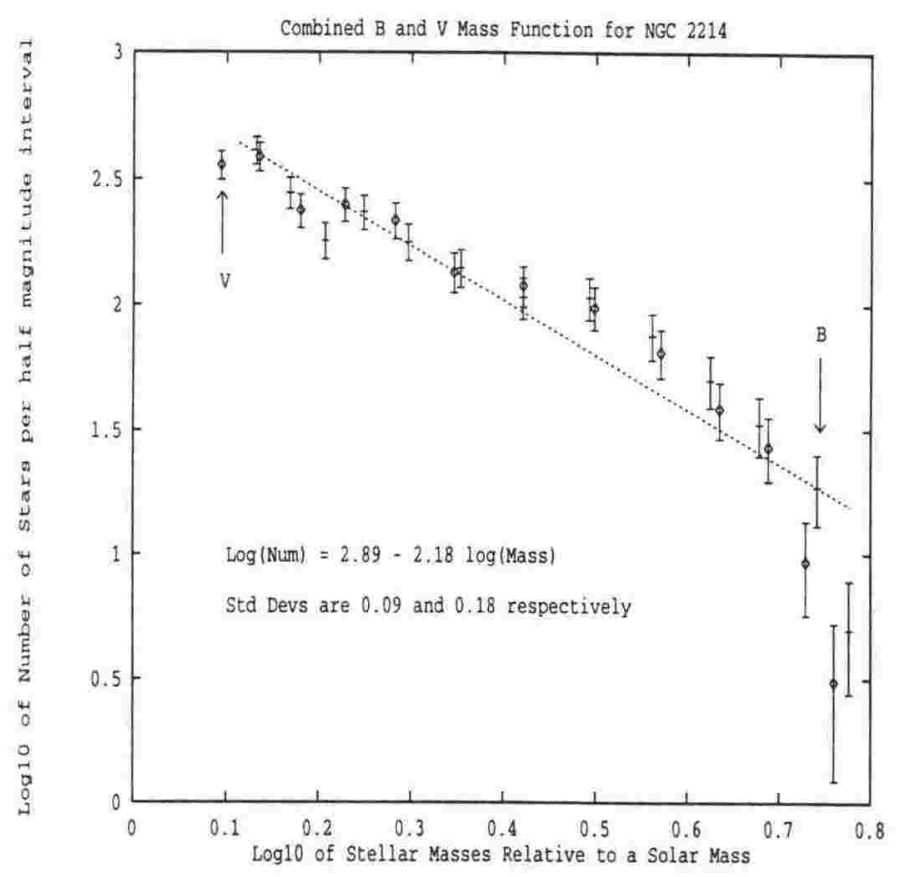

Figure 7-23: Long Exposure Frames Mass Function for NGC 2214. Values calculated from $\mathrm{V}$ magnitudes are given as diamonds, while the B-based ones are marked by a horizontal dash. The dashed line is the linear least-squares fit to the B data, and its parameter values are given.

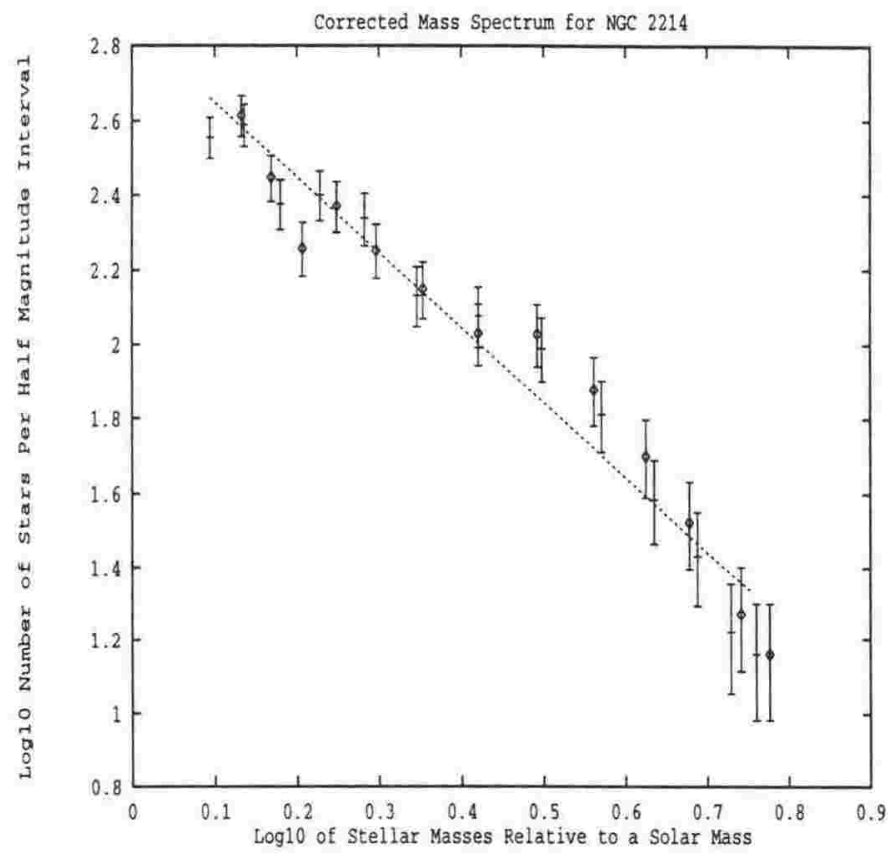

Figure 7-24: Mass Function for NGC 2214. The same symbols are used as in Figure 7-23, but the high mass bins are based on the short-exposure frames. The line of best fit given in the previous figure is shown, to ease comparison between the diagrams, and to demonstrate just how uncertain the gradient is. 
combined cluster mass function of Mateo (1988b) was over effectively the same range as that of this study. He suggested that the low mass end $\left(\log \mathrm{M} / \mathrm{M}_{\odot}<0.45\right)$ was slightly steeper. The incompleteness technique of Mateo (1988b) under-estimates recovery rates, with the discrepancy increasing as the completeness rates decline. Therefore the gradients of the current study are substantially less than those of Mateo (see Sagar \& Richtler 1991, who applied the same completeness technique corrections to NGC 1711, which was the only cluster common with Mateo (1988b), and derived a similar gradient to the present study). The steep gradients of Mateo are not confirmed; nor are the very shallow gradients of Elson et al. (1989).

The possibility that the change in gradient was due to incorrect estimation of the recovery rates at faint magnitudes was considered. The dual frame completeness script was therefore used on the cluster and field frames. The bins were half a magnitude wide over the observational $\mathrm{V}$ magnitude range 20-23, and were centred on the main sequence with a $\mathrm{B}-\mathrm{V}$ width of 0.30 . The ranges of these bins were selected as per the discussion at the end of Section 7.3.8. In the case of the current data, no advantage was gained. The estimated completeness factors were within $1.2 \pm 0.7 \%$ of those estimated by the lesser completeness factor method. This result raises the question of whether more complicated dual frame completeness techniques, employing a variant of the matrix method proposed by Drukier et al. (1988) to measure the effect of the bin migration discussed above, would be worthwhile in practice.

Linear least-square fits to the B, V, and combined B and V mass spectra (of all the data points) resulted in $x$ values of $0.96 \pm 0.14,1.06 \pm 0.13$, and $1.01 \pm 0.09$ respectively. While this is shallower than the Sagar \& Richtler (1991) values for NGC 2214, it should be noted that similar values were recovered before the saturation problem discussed above was corrected, and that the new values are in reasonable agreement with the average value of $x=1.1$ of Sagar \& Richtler (1991) for their five LMC clusters overall for the mass range 1.9-6.3 $\mathrm{M}_{\odot}$.

Sagar \& Richtler (1991) investigated the effect that binary stars have on mass functions, finding that the slope of the actual mass function determined the effect itself. Steep mass functions are weighted towards low mass stars. Therefore the secondary component of a binary is likely to be less massive than the primary, and so causes little change in the luminosity of the system. Alternatively, flat distributions will produce binaries with mass ratios closer to unity, dramatically affecting the system luminosity (Mateo 1993). Sagar \& Richtler (1991) found that mass functions with $x$ values of 2.5 or steeper were essentially unaffected by binaries, while a mass function of 'slope' $(x) 1.5$ could be lowered by 0.4 in the extreme case of every star being binary. While the actual proportion of binary stars in LMC clusters is unknown, the work of Sagar \& Richtler (1991) indicates that the gradients derived by studies similar to this one are underestimates of the actual gradient.

Further support for the reasonably shallow mass function derived by this study may be 
found in several studies. Grondin et al. (1992) derived an $x$ value of $1.3 \pm 0.1$ over the mass range 1.5 to $12 \mathrm{M}_{\odot}$ for three small Associations located in the bridge between the Magellanic Clouds. Like the IMF derived for NGC 2214, that of Grondin et al. (1992) was steeper for the low mass end, with a similar slight flattening over the mass interval 0.3-0.5 $\log \left(\mathrm{M} / \mathrm{M}_{\odot}\right)$. Cayrel et al. (1988) gave a value of 1.2 for NGC 1818 and the SMC Cluster NGC 330. Being in the SMC, this last cluster (which Mateo (1988b) also found agreed well with his LMC cluster sample) has a lower metallicity than many LMC clusters (see Jasniewicz \& Thévenin 1994), pointing towards the IMF having no metal-dependency. Lapierre \& Moffat (1991) derived an $x$ value of $0.8 \pm 0.4$ for 30 Doradus (over $\sim 10-50 \mathrm{M}_{\odot}$ ). Bencivenni et al. (1991) found that the cumulative mass function of NGC 2004 was well described by a Salpeter (1955) IMF (i.e. $x=$ 1.35), while Chiosi et al. (1989) found that the same distribution described well the morphology of NGC 1866 on a CMD. The LMC Association LH 10 has a slope of $1.1 \pm 0.1$ over $0.9-1.7 \mathrm{M}_{\odot}$, although the same paper found that LH 9 was well described by an $x$ of $1.6 \pm 0.1$ over a similar ranges of masses (Parker et al. 1992). Conti (1992) studied NGC 2070, the core of 30 Dor, deriving a slope of $1.5 \pm 0.3$ over the mass interval $20-100 \mathrm{M}_{\odot}$.

Dubath et al. (1990) fitted multi-mass King models to NGC 1835 and concluded that $x$ lay between 1.0 and 1.8. Similarly, Fischer et al. (1992a, b, 1993) modeled NGC 1850, NGC 1866, and NGC 1978 using radial velocities for individual stars. Structural parameters were constrained by surface photometry of the clusters. While the value of $x$ for NGC 1866 was determined to fall between 1.3 and 1.8, NGC 1850 and NGC 1978 were found to have considerably flatter mass functions, with $x$ being in the range 0.0 to 1.0 .

It is interesting to consider the different environments of the LMC clusters and associations mentioned above, and the fact that their determined mass functions are all similar - suggesting the possibility of a global IMF in the LMC independent of factors such as metallicity.

The values derived for the Magellanic Clouds should be compared with IMF studies of Galactic stars, to see if there is any difference between mass functions in the three galaxies. Taff (1974) analysed 62 Galactic open clusters, finding no statistically significant difference between the clusters, and an $x$ of 1.8 for the range 1-10 $\mathrm{M}_{\odot}$. Piskunov (1976, from Scalo 1986) performed a similar study on 61 clusters, and found that a slope of 1.3 fitted the data over 1 to $25 \mathrm{M}_{\odot}$, whereas Tarrab (1982) found $x=1.6$ fitted 75 open clusters well. Similarly, Sagar et al. (1986) investigated the IMF of 11 young clusters, again finding no evidence for inter-cluster differences, and that $x=1.4$ for the mass interval 1.3 to $60 \mathrm{M}_{\odot}$. This is in good agreement with the value of $1.3 \pm 0.2$ found by Kjeldsen \& Frandsen (1991) in their UBV CCD survey of 13 southern hemisphere clusters (over 1-6 $\mathrm{M}_{\odot}$ ). These values are smaller than those determined by many studies of high mass stars, such as Brown et. al. (1994) and Claudius \& Grosbøl (1980), who found $x$ values of $1.7 \pm 0.2$ and $1.9 \pm 0.2$ for Orion OB1 respectively 
(4-120 $\mathrm{M}_{\odot}$ ). However, Lapierre \& Moffat (1991) derived an $x$ value of $1.3 \pm 0.4$ for NGC 3603 over the range $\sim 10-50 \mathrm{M}_{\odot}$.

Other investigators have claimed that mass functions do differ between open clusters. These include Burki (1977) who analysed 27 young clusters finding that the mass function appeared to steepen as the total radius of the clusters decreased, and Stecklum (1985) who found the opposite. Further problems were met with mass segregation (Burki 1977, Pandey et al. 1991) altering the measured IMFs. The average value of $x$ over the sample of Burki (1985) was 1.5 (from a population ranging between 1.7 and 1.2) for 6.3 to $50 \mathrm{M}_{\odot}$. Both Stecklum (1985) and Tarrab (1982) used data from Mermilliod (1976), but there is poor agreement between them, emphasizing the difficult nature of this work (see Scalo 1986, for a full discussion of the problems and assumptions involved). The Scalo (1986) IMF for the solar neighbourhood has a 'slope' of $\sim 1.7 \pm 0.5$ (Larson 1992) for the solar mass range 2-20.

It is unclear whether the IMF of young Magellanic clusters (MC) and associations is different from Galactic values, and no definite conclusions can be made on the universality of the IMF, based on these results, at the present. It has been suggested that the IMF will flatten with decreasing metallicity (see e.g. Terlevich \& Melnick 1985, Piotto 1991), and it could be argued that the MC IMFs are flatter - although it should be noted that isochrones of solar neighbourhood metallicity provided the best fit to the NGC 2214 CMD, and there does not appear to be any difference between Galactic halo and disk field star IMFs over the small mass range of 0.3 to $0.8 \mathrm{M}_{\odot}$ (Scalo 1986). Further study is required, including the estimation of the mass function of LMC clusters with substantially different metallicities.

\subsection{Conclusions}

No evidence was found to support the contention that NGC 2214 is a merging cluster. Models have been fitted to radial profiles of the cluster, and support the contention of Elson et. al. (1987) that an unbound halo of stars causes poor fits by King models. Techniques for estimating completeness factors have been evaluated, with the method of Mateo (1988b) being shown to underestimate completeness factors. The best techniques were employed on the AAT data, leading to an estimate of the mass function of the cluster, with an $x$ value of $\sim 1$, which is in good agreement with other studies of young Magellanic Cloud clusters. IMF estimates for Galactic regions are unreliable, making it difficult to draw any conclusions about the universality of the IMF. However, there are no substantial differences between IMFs derived for the Magellanic Clouds and our Galaxy, and it is likely that star formation in these three galaxies can be described by a 'universal' IMF, at least over the mass interval $\sim 1$ to $\sim 10 \mathrm{M}_{\odot}$. 
Chapter 7 


\section{Appendix A}

\section{Flexible Image Transport System}

\section{A.1 Preface}

$\mathrm{T}$ he Flexible Image Transport System (FITS) tape transfer format was developed to allow the movement of digital Image data between Institutions in a very flexible but unambiguous format. The large-scale use of such data had led to problems in continuing analysis away from the acquisition site, with different researchers developing an individual system constrained by their locally available facilities. The cost in resources of the development of a large quantity of software, based on these individual formats, made the global adoption of a single fixed format for data storage untenable. A feasible approach was to adopt a common transfer format, where each site (or package) had the smaller task of preparing a translation program into and out of this format. The IAU now recommends that all astronomical computer facilities support FITS, and the format is the default of NASA. The original standard for FITS is in Wells et al. (1981).

\section{A.2 Introduction}

A FITS file is made up of three basic sections:-

- The Primary Header and Data Unit (HDU),

- Extensions, and

- Special records.

The logical record size is 23,040 bits, which is a length evenly divisible by the bytes and word lengths of all commercially marketed computers. Each physical record usually corresponds to a logical one. After the original development of the FITS standard, provision was made to increase the ratio of logical to physical records adopted in accordance with ANSI outlines.

Both the extensions and special records sections are optional. They are similar in structure to the HDU, consisting of a header followed by the data. The contents of special records are 


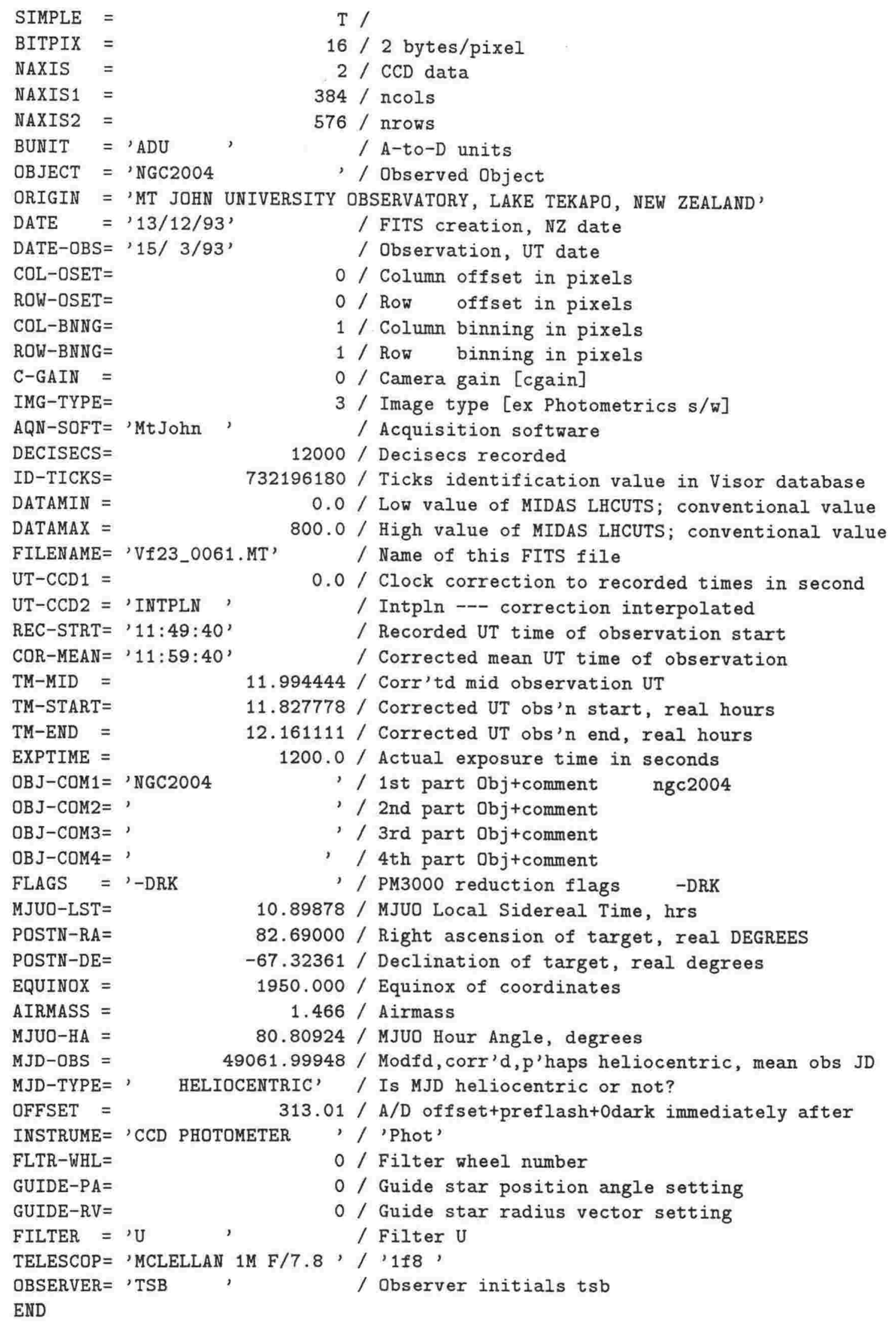

Figure A-1: MJUO FITS Header as written by the program which converts the Image files from Photometrics format on tape across to FITS format on disk. 
not specified in the FITS standard, although the first 8 bytes must not contain the strings XTENSION or SIMPLE, and the records must be of the standard FITS record length.

\section{A.3 The Primary Header}

The Primary Header begins a FITS file. It contains information on the data structure, coordinate system, and auxiliary parameters. Comment cards allow the transmission of an unlimited amount of text. They are based on a 80 character-wide card format. One logical record in the header contains 36 cards (or 36 lines). There is no upper limit to the number of header records possible. The basic structure for these strictly formatted lines is:

$$
\text { keyword = value } / \text { comment. }
$$

The keyword is a left-justified ASCII string up to 8 characters in length, with no embedded blanks or lowercase characters. All digits, the hyphen, underscore, and uppercase Roman letters are valid. No lowercase letters should be used. Identical restrictions apply to the value field. The following keywords are absolutely necessary, and must be given in the following fixed order:

SIMPLE: a logical variable defining if the file is a standard FITS file.

BITPIX: an integer specifying the number of bits representing each pixel. Options are:

$$
\begin{aligned}
& 8: \text { unsigned binary integer, } \\
& 16: 16 \text { bit Two's Complement binary integer, } \\
& 32: 32 \text { bit Two's Complement binary integer, } \\
& -32: \text { single precision floating point, } \\
& -64: \text { double precision floating point. }
\end{aligned}
$$

NAXIS: requires an integer specifying the number of coordinate axes in the Image, e.g. two for the MJUO CCD.

$\square$ NAXISn: needs an integer corresponding to the number of pixels along the nth axis. Up to 999 dimensions are permitted.

A variety of other keywords can follow in any order after the final NAXIS, but before the END statement which closes the header. These keywords can be information about the Image such as the date and time of observation, details on the observed object or the instruments being used, and so on (see Wells et al. (1981) for a list of recommended keywords). Use of these optional keywords is important, as the standard (required) keywords do not even specify the orientation of the Image. Keywords such as BSCALE and BZERO are used to convert the recorded values into the real ones via: 


$$
\text { Physical scale }=\text { BZERO }+(\text { BSCALE } \mathrm{x} \text { array value }) .
$$

The equals sign must be in column 9 of a keyword line if a corresponding value to the keyword is present, with column 10 blank. Otherwise column 9 may have any character present (except $=$ ) with any ASCII text in the columns 10 to 80 . The slash $(/$ ) is optional (as is the comment following it) and may appear in any column after the parameter value. At least one blank each side of the slash is recommended, improving the format and enhancing the legibility of the header. It is sometimes also recommended that the slash always should be placed in column 32 for the same reasons. The following format should be adhered to for variables:

$\square$ logical: a $\mathrm{T}$ or $\mathrm{F}$ written in Column 30 indicating true or false

$\square$ integer: the real part is right justified in columns 11-30, and any imaginary section is right justified in columns 31-50.

$\square$ real: If exponential notation is used then the real and imaginary parts must be right justified in columns $11-30$ and 31-50 respectively. A decimal point is required.

$\square$ character: Normally eight letters in length, although longer strings are allowed if a single quote is placed in column 11, followed by a closing quote that can not occur before column 20.

The 'END' keyword indicates the end of the header. The remaining cards in a header record must be filled with ASCII blanks, to complete the 36 (or a multiple thereof) card images. Comment cards can also be used to transmit an amount of text before END. An example FITS header is given as Figure A-1 on Page 210.

\section{A.4 The Data Unit}

The data records follow the primary header records, in one of two forms:

1. The first form is the basic multidimensional regular array, while

2. The second is the "random group" format consisting of a series of arrays, each accompanied by a set of associated parameters. This structure was intended for applications such as radio interferometry, where data does not occur in a uniform grid on all axes. Use of 
the axis order indicated in the header. The pixels are packed into the data records with no attention given to the row or column length of the Image. That is to say, that the data values are stored as a byte stream with no embedded fills or spaces. Unused pixel values after the last data array values in the final data record are filled with zeroes. The actual length (in bytes) of the data can be calculated by a FITS reader program using NAXISn values and BITPIX. Within a pixel value, the bytes are in order of decreasing significance for binary representation, i.e., the byte containing the 1 bit of a 16 or 32 bit integer will be last. The bit positions for a floating point representation are (from left to right):

$\square$ Bit 1: Sign (0 = negative),

$\square$ Bits 2-9: Exponent, and

$\square$ Bits 10-32: Mantissa.

for 32-bit representation (see also Figure 3-3 on Page 71). The bit structure for 64-bit floating point is similar, but with bits 2-12 and 13-64 representing the exponent and the mantissa respectively.

The data representation in 32-bit is given by:

$$
\text { value }=-1^{\text {sign }} \times 2^{\text {(exponent -127) }} \times \text { Mantissa }
$$

and for 64 is:

$$
\text { value }=-1^{\text {sign }} \times 2^{(\text {exponent }-1023)} \times \text { Mantissa. }
$$

The 127 and 1023 values in the exponents are to allow for negative exponents, as there are 256 and 2048 possible representations respectively, i.e., in 32 floating point the "exponent" of 127 is really zero, while 128 means $2^{1}$.

\section{A.5 Extensions}

If the data record is the end of the file, then a double tape mark must be written if the files are being written to tape. Otherwise the file is simply closed. However extensions, special records, or both may be written between the end mark and the primary data. The primary header must have the EXTEND keyword set to $\mathrm{T}$ (for true) for these structures to be in the Image file. The keyword should be immediately after the last NAXIS, although its presence does not guarantee, but rather only permits, the existence of any extensions. Again there is an inflexible keyword order which is:

- XTENSION, gives the extension type (TABLE or BINTABLE)

\section{- BITPIX}


- NAXIS, as in the primary header.

- NAXISn

- PCOUNT, which must be set to 0 if the extension is a table extension.

- GCOUNT, which must be set to 1 if the extension is a table extension.

PCOUNT and GCOUNT are keywords originally from the Random Groups Format, which now specify the total number of bits in the extension data array, excluding any fill at the end, i.e.,

$$
\text { \#bits }=\mid \text { BITPIX } \mid \times \text { GCOUNT } \mathrm{x}(\text { PCOUNT }+ \text { NAXIS1 } \times \text { NAXIS2 } \mathrm{x} \ldots \mathrm{x} \text { NAXISn }) .
$$

The keyword END indicates the end of the header record.

So far only the ASCII table extension (type Table) is formally supported, with the binary table extension (type BINTABLE) still under review. See Grosbøl et al. (1988) for a discussion of generalised extensions in FITS. Extensions enable the transmission of new data structures within the basic FITS rules, along with the movement of collections of related data structures in an organized manner, e.g. observing logs, analysis results, or position lists associated with the data matrix which would be better given in a tabular form than in comment lines. An extension hierarchy, similar to a directory tree, can be identified by use of the optional keywords EXTNAME, EXTVER, and EXTLEVEL giving the name of an extension, its version, and level in the hierarchy.

\section{A.5.1 ASCII Table Extension}

The basic table stores characters in ASCII, rather than a binary format, due to problems with the internal binary format between different computers. At first this may appear confusing, as why is the Data Unit in binary, and not ASCII then? The distinction lies in the data unit being numbers, while the Table can contain characters whose internal codes may vary between machines. Hence a standard encoding must be adopted. Each row of the table consists of a sequence of fields, which must be the same for every row. The required keyword TFIELDS contains an integer representing the number of fields in a row. There is a maximum of 99 fields. The TFIELDS keyword must come before keywords such as TBCOLnnn. Formats between fields in a row can be different, with each field being specified by a series of keywords in the extension header, e.g. for field nnn the required keywords are:

- TBCOLnnn : starting column of field. 
- TFORMnnn : Fortran format of the field ( A: char, I: int, F: real, E: single precision real, exponential notation, D: double precision real, exponential notation).

- TTYPEnnn : Heading of field.

- TUNITnnn : Physical Unit of the field.

- TSCALnnn : scale factor applicable to the value, with a non-declaration default of 1.0.

- TZEROnnn : the zero offset applied to the value, with a default of zero.

Again, the final keyword in this header must be END, with the last records suitably padded.

Characters must be left justified within the table, while integers and real numbers must be right justified to avoid the problem of how trailing blanks are treated by different computers. It is recommended that a blank be placed between each field within a row. Extra blank characters may need to be appended to rows so as to ensure that each row contains the same number of characters, since the data records are stored as a large character array NAXIS1 by NAXIS2 characters across in a binary bit string (only 2 dimensions are allowed). All information is stored as printable 8 bit ASCII with the eighth (parity) bit set to zero. Characters outside hexadecimal 20 to $7 \mathrm{E}$ should be avoided as their meaning is often computer dependent. Again, records are 2880 bytes long, with no attempt made to prevent partial rows, and the final data record needs to be padded with ASCII blanks. Further details on the format of this extension type may be found in Harten et al. (1988).

\section{A.5.2 Binary Table Extension}

The Binary table extension can store vectors as fields, giving "depth" to the table. This type of extension has a header identical to that of the standard table. BITPIX must be set to 8 . There is no need for TBCOL, TZERO and TSCALE. TFORMn has types:

- rL : logical,

- rX : bit array,

- rA : ASCII character,

- rI : 16 bit integer,

- rJ : 32 bit integer,

- $\mathrm{rE}: 32$ bit floating point, and

- rD : 64 bit floating point. 
The $r$ preceding the type indicator is the repeat count, or how many times the data type appears within field $n$. A zero $r$ means that the header specified entry does not exist in the table. Standard FITS rules apply to the data types, and their storage. See Cotton \& Tody (1991) for further details on the format of this type of extension. 


\section{Appendix B}

\section{IRAF Documentation}

$\mathrm{T}$

he printed documentation for IRAF is extremely thorough and covers every aspect of the system, from the level of novice users up to the information required for maintenance and development of an IRAF installation. Given this high level of support and a nearly complete set of manuals at VUW, this appendix is intended to provide 'pointers' to the appropriate documents rather than supplying explicit command sequences. In particular, the appendix is aimed at those at VUW who will be using the system well after the author is no longer the IRAF Site Manager. An extremely useful document for the beginner, which provides references to more detailed sources, is the IRAF Frequently Asked Questions (FAQ) list. This document is available in the World Wide Web at the universal resource locator 'http://iraf.noao.edu/FAQ.html', and is updated frequently.

While the manual collection at VUW should be adequate for the next few years, IRAF is an evolving system and the manuals will date. Fortunately, the IRAF support team updates manuals as well as providing completely new ones. These manuals can be obtained using the anonymous File Transfer Protocol (FTP) across the computer networks from the IRAF network archive (iraf.noao.edu), which also contains the IRAF distributions themselves together with add-on packages and user-contributed software. IRAF, the add-on packages, and documentation are available free of charge. The manuals tend to be compressed PostScript files, which must be retrieved as binary (not ASCII) files in a FTP transfer. These can be unpacked with the 'uncompress' command, and then submitted to a PostScript printer. In addition to the manuals, the IRAF group provides a 'hotline' ( $\mathrm{Ph}$ : 00-1-602-323-4160 or iraf@noao.edu) to assist users. An answering machine is connected to the telephone line outside of business hours. The group aims to answer queries within 24 hours of receipt. A final source of help is several network Newsgroups discussing IRAF under a hierarchy called 'adass'. These groups are not yet carried by VUW, but can be received directly from NOAO by the command 'trn -nntpserver iraf.noao.edu'. The groups can also be accessed using an e-mail list-server. Further information about the server can be obtained by sending e-mail to listproc@iraf.noao.edu with the word help as the entire body of the message. The subject-line can be omitted. 
IRAF is currently supported on a variety of platforms, including Ultrix, VMS, AIX, IRIX, HPUX, Solaris, and Apple Macintosh A/UX. Port attempts are in progress for Alpha-based operating systems and an IBM PC Unix. Installation of IRAF is straight-forward with source code, object libraries and HSI executables being all included in a distribution. The appropriate files for a given host system should be retrieved in a binary FTP transfer. These will be compressed tar (ㅁape archive) files. Installation Guides and Instructions for Site Managers are available in the same directory as each distribution (e.g. iraf/v210/aux/). IRAF can be installed by simply following the instructions, although root access is required for the final steps. Very little knowledge of the host computer's operating system is required. It is possible to attempt to port IRAF across to an unsupported platform, but this is a difficult task given that IRAF is a large and complex virtual operating system. The IRAF group does not have the resources to assist in such attempts. Test procedures for a new installation of IRAF, to confirm that it is functioning correctly, may be found in the manual 'Preliminary Test Procedure for IRAF' (testproc2.ps.Z in the IRAF archive).

The IRAF group recommends that a single-user installation of IRAF would require at least $16 \mathrm{Mb}$ of memory, 32-48 $\mathrm{Mb}$ of swap space, and around $100 \mathrm{Mb}$ of disk space (although this will vary between sites depending on how many additional packages are installed, and whether the system is stripped of source code or not).

Before a user can run IRAF, they should execute the command 'mkiraf' in an appropriate directory (which they own), and answer the questions that follow. This directory is now the IRAF home directory for the user. Mkiraf creates a script and the subdirectories required to store user-defined parameter files. The user can now start IRAF by entering the command 'cl'. This command may be issued in any directory, although the script installed by Mkiraf will only be run if the command is issued in the IRAF home directory. It is best to start IRAF from the home directory given that the script starts IRAF with several packages loaded, provides links to commands in the host operating system, and determines the terminal type that is being used. To exit IRAF issue the command 'logout'.

The best way to learn how to use IRAF is to actually use it! The manuals 'A Beginner's Guide to using IRAF' and 'A User's Introduction to the IRAF Command Language Version 2.3 ', the IRAF external package called 'demo' which provides demonstrations using playback scripts, and exercises (file 'exer2102.tar.Z' in directory iraf.old/archive at iraf.noao.edu) can help in this. The exercises provide real data and a step-by-step guide to the procedures for the reduction and calibration of photometric and spectroscopic data. An additional source of help, in an operational IRAF, is the IRAF help-pages. Information on an IRAF task (say digiphot) can be accessed by typing 'help digiphot' inside IRAF. As well as a description of the command or package, the help-pages point towards other related help-pages and provide worked examples. 
Many manuals are available for IRAF and IRAF-related software. A selection of useful manuals is listed in Table B.1. More manuals, especially for given packages, can be retrieved from the sites mentioned in the caption to Table B.1.

There are many add-on packages for IRAF, which are 'layered' onto the IRAF core system. The network sites that contain the latest versions of the more popular packages and their documentation are given in Table B.2. Only once the base system is installed can these packages themselves be implemented, as they are dependent on the VOS. These packages extend the capabilities of IRAF, often to different types of data.

At VUW, the sources for the Sun and IRIX versions of IRAF are stored on a gigabyte disk attached to the Silicon Graphics Parallel Processor 'Kauri'. This disk is regularly backed up by the Information and Technology Services group, as part of their standard maintenance of this computer. Following the recommended procedures of the IRAF group, a maintenance usercode (IRAF) has been created on each machine that IRAF is installed on. The IRAF system belongs to this account (all the usercodes share the same identification codes making them one account). Any alterations to the IRAF system, such as the addition of an external package, must be performed by this account. A log detailing the maintenance performed on IRAF can be found in the home directory of the Sun IRAF maintenance usercodes. The passwords to these usercodes are stored in sealed envelopes in the office of the Computing Support Technician in the Physics Department. 


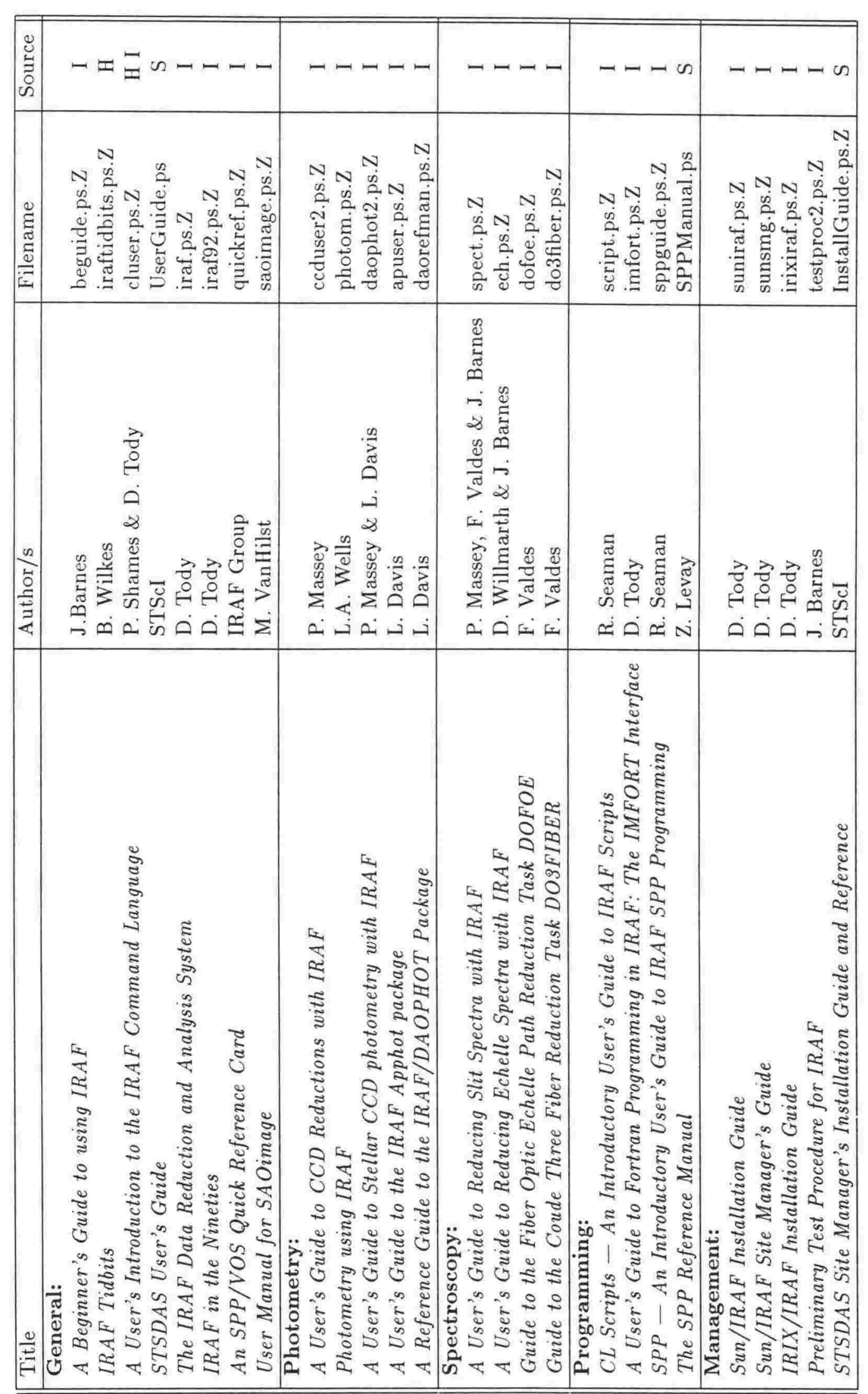

苛总

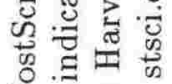

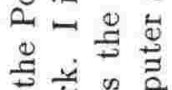
ธे है

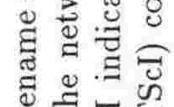

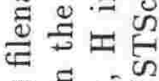
무응 봄 응 홍 政 跑 ヨ 咩

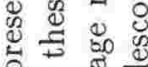
吨 要

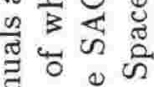

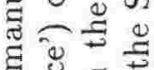
द्व 解 ช 芩

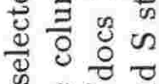
记

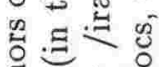

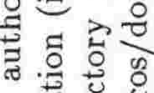
듀.ّ. 造寻 저ㅇㅖㅕ

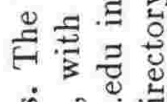
帘

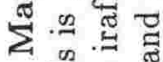
达苛

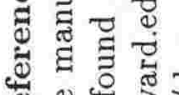
월 环 등

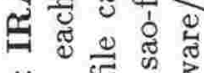

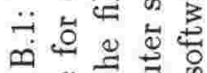

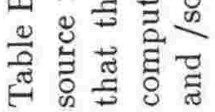




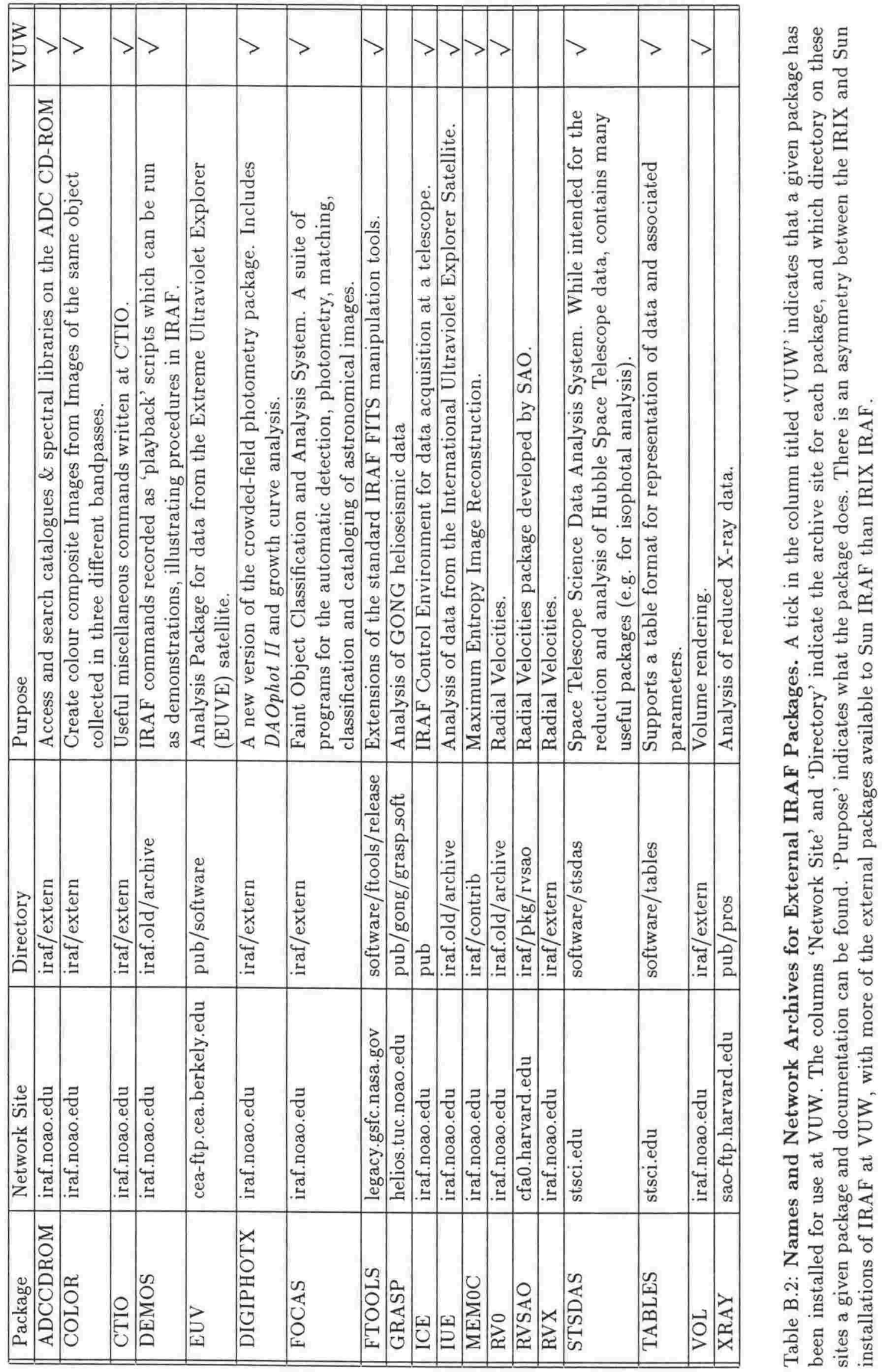




\section{Glossary of Acronyms}

A\&L: Alcaino \& Liller (1987).

AAT: Anglo-Australian Telescope.

AAO: Anglo-Australian Observatory.

ADC: Analogue to Digital Converter.

ADU: Analogue to Digital Unit.

AGB: Asymptotic Giant Branch.

ANSI: American National Standards Institute.

ASCII: American Standard Code for Information Interchange.

AUX: Apple Unix.

BPI: Bits Per Inch.

BS: Bright Star.

CCD: Charge-Coupled Device.

CE: Camera Electronics.

CH: Camera Head.

CL: Command Language (IRAF).

CMD: Colour-Magnitude Diagram.

CO: Carbon monoxide.

COSMOS: Coordinates, Sizes, Magnitudes, Orientations and Shapes.

CPU: Central Processing Unit.

CTE: Charge Transfer Efficiency.

CTIO: Cerro Tololo Interamerican Observatory.

DAO: Dominion Astrophysical Observatory.

DAT: Digital Audio Tapes.

DK: Dachs \& Kaiser (1984).

EFF: Elson, Fall \& Freeman (1987).

ESO: European Southern Observatory.
EUVE: Extreme UltraViolet Explorer.

FAQ: Frequently Asked Questions.

FITS: Flexible Image Transport System.

FOCAS: Faint Object Classification and Analysis System.

FTP: File Transfer Protocol.

FWHM: Full-Width Half-Maximum.

GEC: General Electric Corporation.

GMST: Greenwich Mean Sidereal Time.

GSC: Hubble Space Telescope Guide Star Catalog.

H.A.: Hour Angle.

HB: Horizontal Branch.

HC: Heliocentric Correction.

HD: Henry Draper catalogue.

HDU: Header and Data Unit of a FITS file.

HPUX: Hewlett Packard Unix.

H-R Diagram: Hertzsprung-Russell Diagram.

HSI: Host System Interface (IRAF).

HVC: High Velocity Clouds.

IAU: International Astronomical Union.

ICE: IRAF Control Environment.

IEEE: Institute of Electrical and Electronic Engineers.

IMF: Initial Mass Function.

I/O: Input/Output.

IRAF: Image Reduction and Analysis Facility.

IUE: International Ultraviolet Explorer.

JD: Julian Date. 
K: Kontizas et al. (1987).

KPNO: Kitt Peak National Observatory

LED: Light Emitting Diode.

LH: Lucke \& Hodge (1970).

LMC: Large Magellanic Cloud.

LN: Liquid Nitrogen.

LST: Local Sidereal Time.

M: Messier.

MC: Magellanic Clusters.

MIDAS: Munich Image Data Analysis System.

MJD: Modified Julian Date.

MJUO: Mount John University Observatory.

MOS: Metal-oxide Semiconductor.

MS: Main Sequence.

MT: Magnetic Tape.

NASA: National Aeronautics and Space Administration.

NFS: Network File System.

NGC: New General Catalog.

NOAO: National Optical Astronomy Observatories.

OIF: Old IRAF Format.

P.A.: Position Angle.

PC: Personal Computer.

PSF: Point-Spread Function.

PTM: Photometrics Ltd

Q.E.: Quantum Efficiency.

QIC: Quarter-Inch Cartridge.

R.A.: Right Ascension.

RAM: Random Access Memory.

RCA: Radio Corporation of America.

RGB Red Giant Branch.

RMS: Root-Mean-Square.
RNGC: Revised New General Catalog (Sulentic \& Tifft 1973).

ROE: Royal Observatory Edinburgh.

SAAO: South Africa Astronomical Observatory.

SAO: Smithsonian Astrophysical Observatory.

SBm: Spiral, Barred - Magellanic subclass.

SGB: Supergiant Branch.

SRC: Science Research Council.

SIMBAD: Set of Identifications, Measurements, and Bibliography for Astronomical Data.

SL: Shapley \& Lindsay (1963) catalogue.

SLIP: Serial Line Internet Protocol.

SMC: Small Magellanic Cloud.

SPP: The Subset PreProcessor programming language (IRAF).

STScI: Space Telescope Science Institute.

STSDAS: Space Telescope Science Data Analysis System.

UK: United Kingdom.

UKST: United Kingdom Schmidt Telescope.

UoC: University of Canterbury.

US: United States (of America).

UT: Universal Time.

UUCP: Unix to Unix Copy.

UV: UltraViolet.

VAX: Virtual Address eXtension.

VMS: Virtual Memory System.

VOS: Virtual Operating System (IRAF).

VUW: Victoria University of Wellington.

ZAMS: Zero Age Main Sequence.

ZD: Zepka \& Dottori (1987). 


\section{References}

Aaronson, M., \& Mould, J., 1982, Astrophys. J. Suppl. Ser., 48, 161.

Abbot, C.G., 1925, The Earth and the Stars, Third Printing, Van Nostrand, New York.

Abott, D.C., 1982, Astrophys. J., 263, 723.

Adobe Systems Inc., 1985a, PostScript Language Reference Manual, Addison-Wesley, Reading (Mass.).

Adobe Systems Inc., 1985b, PostScript Language Tutorial and Cookbook, Addison-Wesley, Reading (Mass.).

Adobe Systems Inc., 1985c, PostScript Language Program Description, Addison-Wesley, Reading (Mass.).

Alcaino, G., \& Liller, W., 1984, Astron. J., 98, 1712.

Alcaino, G., \& Liller, W., 1985, Astron. Astrophys., 146, 389.

Alcaino, G., \& Liller, W., 1986, Astron. J., 91, 87.

Alcaino, G., \& Liller, W., 1987, Astron. J., 89, 1712.

Allen, C.W., 1973, Astrophysical Quanities, Athlone Press, London.

Andersen, J., Blecha, A., \& Walker, M.F., 1985, Astron. Astrophys., 150, L12.

Anton, H., 1980, Calculus, John Wiley, Canada.

Arnett, W.D., Bahcall, J.N., Kirshner, R.P., \& Woosley, S.E., 1989, Ann. Rev. Astron. Astrophys., 27, 629 .

Arp, H.C., 1961, Science, 134, 810.

Arp, H.C., \& van Sant, C.T., 1958, Astron. J., 63, 341.

The Astronomical Almanac for 1990, 1990, Joint publication of the Nautical Almanac Office (USNO) and Her Majesty's Nautical Almanac Office (RGO), U.S. Government Priniting Office, Washington.

Bahcall, J.N., \& Soniera, R.M., 1980, Astrophys. J. Suppl. Ser., 44, 73.

Bahcall, J.N., \& Soniera, R.M., 1984, Astrophys. J. Suppl. Ser., 55, 67.

Balona, L.A., \& Crampton, D., 1974, Mon. Not. R. Astron. Soc., 166, 203.

Balona, L.A., \& Jerzykiewicz, M., 1993, Mon. Not. R. Astron. Soc., 260, 782.

Balona, L.A., \& Koen, C., 1994, Mon. Not. R. Astron. Soc., 267, 1071.

Balona, L.A., \& Shobbrook, R.R., 1984, Mon. Not. R. Astron. Soc., 211, 375. 
Banks, T., 1993, Southern Stars, 35, 33.

Banks, T., \& Jansen, F., 1992, Astrophys. Space. Sci., 190, 155.

Banks, T., Sullivan, D.J., Forbes, M.C., \& Dodd, R.J., 1994a, Southern Stars, 36, 69.

Banks, T., Dodd, R.J., \& Sullivan, D.J., 1994b, accepted by Mon. Not. R. Astron. Soc..

Banks, T., Dodd, R.J., \& Sullivan, D.J., 1994c, accepted by Mon. Not. R. Astron. Soc..

Banks, T., Sullivan, D.J., \& Dodd, R.J., 1994d, submitted to Southern Stars.

Banse, K., Crane, D., Grosbøl, P., Middelburg, F., Ounnas, C., Ponz, D., \& Waldthausen, H., 1982, Messenger, 31, 26.

Bateson, F.M., 1962, Sky Telesc., 24, 4.

Bateson, F.M., 1964, Pub. Univ. Penn. Ast. Series, 10, 1.

Batinelli, P., \& Capuzzo-Dolcetta, R., 1989, Astrophys. J., 347, 794.

Becker, S.A., 1981, Astrophys. J. Suppl. Ser., 45, 475.

Becker, W., \& Fenkart, R., 1971, in The Spiral Structure of our Galaxy, IAU Symposium \#38, Eds: W. Becker \& G. Contopoulos, Reidel, Dordrecht, 42.

Bemporad, A., 1904, Zur Theorie der Extinktion des Lichtes in der Erdatmosphäre, Grossh, Sternwarte Heidelberg, 4, 1.

Bencivenni, D., Brocato, E., Buoanno, R., \& Castellani, V., 1991, Astron. J., 102, 137.

Bertelli, G., Bressan, A., Chiosi, C. \& Angerer, K., 1986, Astron. Astrophys. Suppl. Ser., 66, 191.

Bertelli, G., Betto, R., Bressnan, A., Chiosi, C., Nasi, E., \& Vallenari, A., 1990, Astron. Astrophys. Suppl. Ser., 34, 229.

Bertelli, G., Mateo, M., Chiosi, C., \& Bressan, A., 1992, Astrophys. J., 388, 400.

Bessel, M.S., 1990, Publ. Astron. Soc. Pac., 102, 1181.

Bessel, M.S., 1993, in Precision Photometry, Eds: D. Kilkenny, E. Lastovica, \& J. W. Menzies, SAAO, 46 .

Best, E., 1987, The Astronomical Knowledge of the Maori, Reprint of Dominion Museum Monograph \#3, Government Print, Wellington.

Bettwieser, E., \& Sugimoto, D., 1984, Mon. Not. R. Astron. Soc., 208, 493.

Bevington, P.R., 1969, Data Reduction \& Error Analysis in the Physical Sciences, McGraw-Hill, New York.

Bhatia, R.K., \& MacGillivray, H.T., 1987, ESO Conference Workshop Proceedings \#27, 485.

Bhatia, R.K., \& MacGillivray, H.T., 1988, Astron. Astrophys., 203, L5.

Bhatia, R.K., \& MacGillivray, H.T., 1989, Astron. Astrophys., 211, 9.

Bhatia, R.K., \& Piotto, G., 1993a, The Globular Cluster-Galaxy Connection, ASP Conf. Ser. \#48, Eds: G.H. Smith \& J.P. Brodie, Astronomical Society of the Pacific, 400.

Bhatia, R.K., \& Piotto, G., 1993b, Astron. Astrophys., 283, 424.

Binney, J., 1981, Mon. Not. R. Astron. Soc., 196, 455. 
Binney, J., 1992, Annual Reviews of Astronomy \& Astrophysics, 30, 51.

Bok, B.J., 1966, Ann. Rev. Ast. Astrophys., 4, 95.

Bok, B.J., 1969a, Annual Reviews of Astronomy \& Astrophysics, 4, 95.

Bok, B.J., 1969b, J. R. Astron. Soc. Can., 63, 105.

Boyle, R.P., Smriglio, F., Nandy, K., \& Straižys, V., 1990, Astron. Astrophys. Suppl. Ser., 84, 1.

Boyle, R.P., Dasgupta, A.K., Smriglio, F., Straižys, V., \& Nandy, K., 1992, Astron. Astrophys. Suppl. Ser., 95, 51.

Brown, A.F.A., de Gues, E.J., \& de Zeeuw, P.T., 1994, accepted by Astron. Astrophys..

Brunish, W.M., \& Truran, J.W., 1982, Astraphys. J. Suppl. Ser., 49, 447.

Buonanno, R., Coluzzi, R., Corsi, C.E., De Biase, G.A., \& Ferraro, I., 1979, Mem. Soc. Astron. Ital., 50, 451 .

Buonanno, R., Buscema, G., Corsi, C.E., Ferraro, I., \& Iannicola, G., 1983, Astron. Astrophys., 126, 278.

Burki, G., 1977, Astron. Astrophys., 57, 135.

Burki, G., 1985, Astron. Astrophys., 62, 159.

Burstein, D., \& Heiles, C., 1982, Astron. J., 87, 1165.

Butcher, H., 1977, Astrophys. J., 216, 372.

Carignan, C., 1983, Unpublished PhD Thesis, Australian National University, Canberra (cited by Freeman, 1984).

Castella, A., Barbero, J., \& Geyer, E.H., 1987, Astrophys. J. Suppl. Ser., 64, 83.

Castellani, V., Chieffi, A., \& Straniero, O., 1990, Astrophys. J. Suppl. Ser., 64, 83.

Cayrel, R., Tarrab, I, \& Richtler, T., 1988, Messenger, 54, 29.

Černies, K., Meištas, E., Straižys, V., \& Jasevičius, V., 1989, Bull. Vilnius Obs., 84, 9.

C̆ernies, K., \& Jasecvičus, V., 1992, Baltic Astronomy, 1, 83.

Chernoff, D., \& Weinberg, M., 1990, Astrophys. J., 351, 121.

Chiosi, C., 1989, Rev. Mexicana Astron. Astrof., 18, 125.

Chiosi, C., \& Pigatto, L., 1986, Astrophys. J., 308, 1.

Chiosi, C., Bertelli, G., Meylan, G., \& Ortolani, S., 1989, Astron. Astrophys., 219, 167.

Christian, C.A., \& Schommer, R.A., 1982, Astrophys. J., 351, 121.

Chrysovergis, M., Kontizas, M., \& Kontizas, E., 1989, Astron. Astrophys. Suppl. Ser., 77, 357.

Chun, M.S., 1978, Astron. J., 83, 1062.

Clark, M., 1994, Pers. Comm.

Claudius, M., \& Grøsbol, P.J, 1980, Astron. Astrophys., 87, 339.

Cohen, J., 1982, Astrophys. J., 258, 143. 
Cohen, J., Frogel, J.A., Persson, S.E., \& Elias, J.H., 1981, Astrophys. J., 249, 481.

Conti, P.S., 1992, in The Stellar Populations of Galaxies, Eds: B. Barbuy \& A. Renzini, Kluwer, Dordrecht, 93.

Cotton, W.D., \& Tody, D.B., 1991, preprint.

Coveney, P., \& Highfield, R., 1990, The Arrow of Time, Ballantine, New York.

Cowley, A. P., 1992, Ann. Rev. Astron. Astrophys., 30, 287.

Cowley, A.P., \& Hartwick, F.D.A., 1982, Astrophys, J., 259, 89.

Crawford, D.L., 1958, Astrophys. J., 128, 185.

Da Costa, G., 1982, Astron. J., 87, 990.

Da Costa, G., 1991a, in The Magellanic Clouds, IAU Symposium \#148, Eds: R. Haynes \& D. Milne, Kluwer, Dordrecht, 183.

Da Costa, G., 1991b, AAO Preprint \#265.

Da Costa, G., 1992, in The Astronomy and Astrophysics Encylopedia, Ed: S.P. Maran, Cambridge University Press, Cambridge, 681.

Da Costa, G., 1993, Pers. Comm.

Da Costa, G.S., Mould, J.R., \& Crawford, M.D., 1985, Astrophys. J., 297, 582.

Dachs, J., \& Kaiser, D., 1984, Astron. Astrophys. Suppl. Ser., 58, 411.

Danziger, I.J, 1973, Astrophys. J., 181, 641.

de Vaucouleurs, G., 1954, Observatory, 74, 158.

de Vaucouleurs, G., 1957, Astron. J., 62, 69.

de Vaucouleurs, G., 1963, Astrophys. J., 137, 720.

de Vaucouleurs, G., \& de Vaucouleurs, A., 1963, Astrophys. J., 137, 363.

de Vaucouleurs, G., \& Freeman, K.C., 1972, Vistas Astr., 14, 163.

Dickens, R., \& Woolley, R. v. d. R., 1967, Royal Observatory Bulletin, \#128

Djorgovski, S., 1987, in Globular Cluster Systems in Galaxies, IAU Symposium \#126, Eds: J. Grindlay \& A.G.D. Philips, Reidel, Dordrecht, 333.

Dodd, R.J., \& MacGillivray, H.T., 1986, Astron. J., 92, 706.

Dodd, R.J., Forbes, M.C., \& Sullivan, D.J., 1993, in Stellar Photometry - Current Techniques and Future Developments, IAU Colloquium \#136, Eds: C.J. Butler \& I. Elliot, Cambridge University Press, Cambridge, 51.

Druiker, G.A., Fahlman, G.G., Richer, H.B., \& VandenBerg, D.A., 1988, Astron. J., 95, 1415.

Dubath, P., Meylan, G., Mayor, M., \& Magain, P., 1980, Astron. Astrophys., 239, 142.

Duffett-Smith, P., 1984, Astronomy with your Personal Computer, Cambridge University Press, Cambridge.

Dzérvitis, U., \& Paupers, O., 1993, Astron. Astrophys., 199, 77.

Einasto, J., Haud, U., Joeveer, M., Kaasik, A., 1976, Mon. Not. R. Astron. Soc., 177, 357. 
Elsässer, H, 1959, Zeit. f. Astrophys., 47, 1.

Elsässer, H., \& Haug, V., 1960, Zeit. f. Astrophys., 50, 121.

Elson, R.A.W., 1991., Astrophys. J. Suppl. Ser., 185, 76.

Elson, R.A.W., \& Freeman, K.C., 1985, Astrophys. J., 288, 521.

Elson, R.A.W., Fall, S.M., \& Freeman, K.C., 1987, Astrophys. J., 323, 54.

Elson, R.A.W., Fall, S.M., \& Freeman, K.C., 1989, Astrophys. J., 336, 734.

Fall, S.M., \& Frenk, C.S., 1984, in Structure and Evolution of the Magellanic Clouds, IAU Symposium \#108, Eds: S. van den Bergh \& K.S. de Boer, Reidel, Dordrecht, 27.

Feast, M.W., 1963, Mon. Not. R. Astron. Soc., 126, 213.

Feast, M.W., 1964, Mon. Not. R. Astron. Soc., 127, 195.

Feast, M.W., 1989, in Recent Developments of Magellanic Cloud Research, Eds: K.S de Boer, F. Spite, \& G. Staninska, Obervatoire de Paris, Paris, 75.

Feast, M.W., 1991, in The Magellanic Clouds, IAU Symposium \#148, Eds: R. Haynes, \& D. Milne, Kluwer, Dordrecht, 1.

Feast, M.W., Thackeray, A.D., \& Wesselink, A.J., 1955, Observatory, 75, 216.

Feast, M.W., Thackeray, A.D., \& Wesselink, A.J., 1960, Mon. Not. R. Astron. Soc., 121, 337.

Feast, M.W., Thackeray, A.D., \& Wesselink, A.J., 1961, Mon. Not. R. Astron. Soc., 122, 433.

Fich, M., \& Tremaine, S., 1991, Annual Reviews of Astronomy and Astrophysics, 29, 409.

Fischer, P, Welch, D.L., Côté, Mateo, M., \& Madore, B.F., 1992a, Astron. J., 103, 857.

Fischer, P., Welch, D.L., \& Mateo, M., 1992b, Astron. J., 104, 1086.

Fischer, P., Welch, D.L., \& Mateo, M., 1993, Astron. J., 105, 938.

Flower, P.J., 1976, Unpublished PhD Thesis, University of Washington, Seattle(cited by Flower 1982).

Flower, P.J., 1982, Publ. Astron. Soc. Pac., 94, 894.

Flower, P.J., 1984, Astrophys. J., 278, 582.

Forbes, M.C., 1992, Southern Stars, 35, 1.

Forbes, M.C., 1993, Southern Stars, 35, 69.

Forbes, M.C., 1994, Pers. Comm.

Forbes, M.C., Dodd, R.J., \& Sullivan, D.J., 1993, Baltic Astronomy, 2, 246.

Forbes, M.C., Dodd, R.J., \& Sullivan, D.J., 1994a, accepted by Baltic Astronomy.

Forbes, M.C., Banks, T., Sullivan, D.J., Dodd, R.J., Gilmore, A.C., \& Kilmartin, P., 1994b, accepted by Observatory.

Frandsen, S., Dreyer, P., \& Kjeldsen, H., 1989, Astron. Astrophys., 215, 287.

Freeman, K.C., 1974, in ESO/SRC/CERN Conf. Res. Programs for the New Large Telescopes, Ed: A. Reiz, Geneva, ESO, 177. 
Freeman, K.C., 1977, in The Evolution of Galaxies and Stellar Populations, Eds: B.M. Tinsley \& R.B. Larson, Yale University Observatory, New Haven, 133 (cited by Elson et al. 1987).

Freeman, K.C., 1980, in Globular Clusters, Ed: D. Hanes \& B. Madore, Cambridge University Press, Cambridge, 103.

Freeman, K.C., 1981, Ann. Rev. Astron. Astrophys., 19, 319.

Freeman, K.C., 1984, in Structure and Evolution of the Magellanic Clouds, IAU Symposium \#108, Eds: S. van den Bergh \& K.S. de Boer, Reidel, Dordrecht, 107.

Freeman, K.C., Illingworth, G.D., \& Oemler, A., 1983, Astrophys. J., 272, 488.

Frenk, C.S., \& Fall, S.M., 1982, Mon. Not. R. Astron. Soc., 199, 565.

Frogel, J.A., 1984, Publ. Astron. Soc. Pac., 96, 856.

Frogel, J.A., \& Blanco, V.M., 1983, Astrophys. J., 274, L57.

Fusi Pecci, F., 1988, in Globular Cluster Systems in Galaxies, IAU Symposium \#126, Eds: J.E. Grindlay \& A.G.D. Philip, Kluwer, Dordrecht, 173.

Gascoigne, S.C.B., 1966, Mon. Not. R. Astron. Soc., 134, 59.

Gascoigne, S.C.B., 1980, in Star Clusters, IAU Symposium \#85, Ed: J.E. Hesser, Reidel, Dordrecht, 305 .

Gascoigne, S.C.B., Norris, J., Bessel, M.S., Hyland, A.R., \& Visvanathan, N., 1976, Astrophys. J., 206, L25.

Geisler, D., \& Hodge, P.W., 1980, Astrophys. J., 242, 66.

Geyer, E.H., \& Cassatella, 1984, in Structure and Evolution of the Magellanic Clouds, IAU Symposium \#108, Eds: S. van den Bergh \& K.S. de Boer, Reidel, Dordrecht, 55.

Geyer, E.H., \& Hänel, A., 1984, in Structure and Evolution of the Magellanic Clouds, IAU Symposium \#108, Eds: S. van den Bergh \& K.S. de Boer, Reidel, Dordrecht, 29.

Geyer, E.H. \& Richtler, T., 1984, in Astrophysical Parameters of Globular Clusters, Eds: A.G.D. Philips \& D.S. Hayes, Davis, Schenectady, 239.

Geyer, E.H., Hopp, U., \& Nelles, B., 1983, Astron. Astrophys., 125, 359.

Geyer, E.H., Hopp, U., Kiehl, M., \& Witzigmann, S., 1979, Astron. Astrophys., 77, 61.

Gilmore, A.C., \& Tobin, W., 1991, Mt. John Photometrics CCD System: Use \& Performance Note \#5.

Gilmore, A.C., \& Kilmartin, P.M., 1994, submitted to Southern Stars.

Gilmore, G., 1989, in The Milky Way as a Galaxy, Eds: R. Buser, \& I. King, Geneva Observatory, 370 .

Graham, J., 1982, Publ. Astron. Soc. Pac., 94, 244.

Graham, J.A., 1984, in Structure and Evolution of the Magellanic Clouds, IAU Symposium \#108, Eds: S. van den Bergh \& K.S. de Boer, Reidel, Dordrecht, 207.

Greisen, E.W., \& Harten, R.H., 1981, Astron. Astrophys. Suppl. Ser., 44, 371.

Grondin, L., Demers, S., \& Kunkel, W.E., 1992, Astrophys. J., 103, 1234. 
Grosbøl, P., Harten, R.H., Greisen, E.W. \& Wells, D.C., 1988, Astron. Astrophys. Suppl. Ser. 73,359 .

Grothues, H.G., \& Gochermann, J., 1992, Messenger, 68, 43.

Gum, C.S., \& de Vaucouleurs, G., 1953, Observatory, 73, 152.

Gunn, J.E., 1980, in Globular Clusters, Eds: D. Hanes \& B. Madore, Cambridge University Press, Cambridge, 271.

Gunn, J.E., \& Stryker, L.L., 1983, Astrophys. J. Suppl. Ser., 52, 121.

Hardie, R.H., 1962, in Astronomical Techniques, Ed: W.A.Hiltner, Volume II of Stars \& Stellar Systems, University of Chicago Press, Chicago, Illinois.

Hardy, E., Buonanno, R., Corsi, C.E., Janes, K.A., \& Schommer, R.A., 1984, Astrophys. J., 278, 592.

Harris, W.E., 1990, Publ. Astron. Soc. Pac., 102, 966.

Harris, W.E., \& Deupree, G., 1976, Bull. Am. Astron. Soc., 8, 289.

Harris, W.E., \& Hesser, J.E., 1985, in Dynamics of Star Clusters, IAU Symposium \#113, Eds: J. Goodman \& P. Hut, Reidel, Dordrecht, 81.

Harris, W.E., \& Racine, R., 1979, Annual Rev. Astron. Astrophys., 17, 241.

Harris, W.E., FitzGerald, M.P., \& Reed, B.C., 1981, Publ. Astron. Soc. Pac., 93, 507.

Harten, R.H., Grosbøl, P., Greisen, E.W, \& Wells, D.C, 1988, Astron. Astrophys. Suppl. Ser., 73, 365 .

Hartwick, M., 1973, Astrophysical Concepts, John Wiley \& Sons, New York.

Hatzidimitriou, D., \& Bhatia, R.K., 1988, Astron. Astrophys., 230, 11.

Hearnshaw, J., 1992, Southern Astronomy, 5, 12.

Heggie, D.C., 1975, Mon. Not. R. Astron. Soc., 173, 729.

Hendon, A.A., \& Kaitchuk, R.H., 1989, Astronomical Software for the IBM PC, Willmann-Bell, Richmond VA.

Herschel, J., 1847, Results of Astronomical Observations Made During the Years 1834,5,7,8 at the Cape of Good Hope, Smith \& $\mathrm{C}^{\circ}$, London.

Hertzsprung, E., 1920, Mon. Not. R. Astron. Soc., 80, 782.

Hertzsprung, E., 1923, Mon. Not. R. Astron. Soc., 83, 348.

Hesser, J.E., Harris, W.E., VandenBerg, D.A., Allwright, J.W.B., Schott, P., \& Stetson, P.B., 1987, Publ. Astron. Soc. Pac., 99, 739.

Hindman, J.V., 1967, Astrophys. J., 20, 147.

Hindman, J.V., Kerr, F.J., \& McGee, R.X., 1963, Aust. J. Phys., 16, 570.

Hodge, P.W., 1961, Astrophys. J., 133, 413.

Hodge, P.W., 1980, Astron. J., 85, 423.

Hodge, P.W., 1983, Astrophys. J., 264, 470.

Hodge, P.W., 1984, Publ. Astron. Soc. Pac., 96, 947. 
Hodge, P.W., \& Flower, P.J., 1973, Astrophys. J., 185, 829.

Hodge, P.W., \& Lee, S.O., 1984, Astrophys. J., 276, 509.

Hodge, P.W., \& Wright, F., 1966, The Large Magellanic Cloud, Smithsonian Press, Washington D.C.

Ibata, R.A., Gilmore, G., \& Irwin, I.J., 1994, Nature, 370, 194.

Illingworth, V., 1979, (Editor) The Macmillan Dictionary of Astronomy, Macmillan, London.

Janesick, J., \& Blouke, M., 1987, Sky Telesc., 74, 238.

Jaschek, C., \& Jaschek, M., 1987, The Classification of Stars, Cambridge University Press, Cambridge.

Jasniewicz, G., \& Thévenin. F., 1994, Astron. Astrophys., 282, 717.

Jedrzejewski, R.I., 1987, Mon. Not. R. Astron. Soc., 226, 747.

Johnson, H.L., 1962, Stars and Stellar Systems, 2, 157.

Johnson, H.L., 1963, Stars and Stellar Systems, 3, 120.

Johnson, H.L., 1965, Com. L. Planet Lab., 3, 73.

Johnson, H.L., \& Morgan, W.W., 1953, Astrophys. J., 114, 522.

Jones, B.G., Klemola, A.R., \& Lin, D.N.C., 1991, Bull. Am. Astron. Soc., 21, 1107.

Jones, K.G., 1968, Messier's Nebulae and Star Clusters, Faber \& Faber, London.

Kaburaki, M., 1931, Proc. Phys. Math. Soc. of Japan, 13, 151.

Kerr, F.J, 1965, in Symp. on Magellanic Clouds, Eds: J.V. Hindman \& B.E. Westerlund, Mt. Stromlo, ANU, Canberra, 8.

Kerr, F.J., Hindman, J.V., \& Robinson, B.J., 1954, Aust. J. Phys., 7, 297.

Kilmartin, P.M., 1992, Pers. Comm.

King, I.R., 1962, Astron. J., 67, 471.

King, I.R., 1966a, Astron. J., 71, 64.

King, I.R., 1966b, Astron. J., 71, 276.

King, I.R., 1971, Publ. Astron. Soc. Pac., 83, 199.

King, I.R., 1980, in Star Clusters, IAU Symposium \#85, Ed: J.E. Hesser, Reidel, Dordrecht, 139.

King, I.R., 1989, in The Milky Way as a Galaxy, Eds: R. Buser \& I. King, Geneva Observatory, 1.

Kinman, T.D., \& Rosino, L, 1962, Publ. Astron. Soc. Pac., 74, 499.

Kjeldsen, H., \& Frandsen, S., 1991, Astron. Astrophys. Suppl. Ser., 87, 119.

Kontizas, M., Chrsyovergis, M., \& Kontizas, E., 1987, Astron. Astrophys. Suppl. Ser., 68, 147.

Kontizas, E., Kontizas, M., Sedmak, G., \& Smareglia, R., 1989, Astron. J., 98, 590.

Kontizas, E., Chrsyovergis, M., \& Kontizas, E., 1991, in The Magellanic Clouds, IAU Symposium \#148, Eds: R. Haynes \& D. Milne, Kluwer, Dordrecht, 234. 
Kristian, J., \& Blouke, M., 1982, Sci. Am., 247, 48.

Lamla, E., 1982, in Landolt-Börnstein Neue Serie, Gp VI, Vol. 2b, Eds: K. Schaifers \& H.H Voigt, Springer-Verlag, Berlin, 71.

Landolt, A.U., 1992, Astron. Astrophys., 104, 340.

Landolt, A.U., \& Blondeau, K.L., 1972, Publ. Astron. Soc. Pac., 84, 784.

Lapierre, N., \& Moffat, A.F.J., 1991, The Formation and Evolution of Star Clusters, ASP Conf. Ser. \#13, Ed: K. Janes, Astronomical Society of the Pacific, 155.

Larson, H.J., 1982, Introduction to Probability Theory and Statistical Inference, 3rd Edition, Wiley, New York.

Larson, R.B., 1992, in The Astronomy and Astrophysics Encylopedia, Ed: S.P. Maran, Cambridge University Press, Cambridge, 672.

Lasker, B.M, Sturch, C.R., MacLean, B.J., Russel, J.L., Jenkner, H., \& Shara, M.M., 1990, Astron. J., 99, 2019.

Lauer, T.R., 1983, Unpublished PhD Thesis, University of California, Santa Cruz.

Leach, R.W., \& Gursky, H., 1979, Publ. Astron. Soc. Pac., 91, 855.

Lee, M.G., 1991, in The Magellanic Clouds, IAU Symposium \#148, Eds: R. Haynes \& D. Milne, Kluwer, Dordrecht, 207.

Lee, M.G., 1992, Astrophys. J., 399, L133.

Lin, D.N.C., \& Lynden-Bell, D., 1982, Mon. Not. R. Astron. Soc., 198, 705.

Lindoff, U., 1967, Arkiv för Astronomi, 5, 1.

Liu, Y., 1991, Astron. Astrophys., 257, 505.

Lucke, P.B., \& Hodge, P.W., 1970, Astron. J., 75, 171.

Lupton, R.H., \& Gunn, J.E., 1986, Astrophys. J., 91, 317.

Lynden-Bell, D. \& Wood, R., 1968, Mon. Not. R. Astron. Soc., 138, 495.

Lyngà, G., 1981, Astron. Data Cen. Bul., 2, 1.

MacGillivray, H.T., \& Stobie, R.S., 1984, Vistas Astron., 27, 433.

MacKay, C.D., 1986, Ann. Rev., 24, 255.

McCall, M.L., English, J., \& Shelton, I., 1989, J. R. Astron. Soc. Can., 83, 179.

McCuskey, S.W., 1966, Vistas Astron. 7, 141.

McGee, R.X., \& Milton, J.A., 1966, Aust. J. Phys., 19, 343.

Mclean, I.S., 1989, Electronic and Computer-aided Astronomy, Wiley, New York.

Maeder, A., \& Mermilliod, J.C., 1981, Astron. Astrophys., 93, 136.

Maeder, A., \& Meynet, G., 1991, Astron. Astrophys. Suppl. Ser., 89, 451.

Massey, P., \& Davis, L.E., 1992, A User's Guide to Stellar CCD Photometry with IRAF, NOAO, Tucson. 
Mateo, M., 1988a, in Globular Cluster Systems in Galaxies, IAU Symposium \#126, Eds: J.E. Grindlay \& A.G.D. Philip, Kluwer, Dordrecht, 557.

Mateo, M., 1988b, Astrophys. J., 331, 261.

Mateo, M., 1993, The Globular Cluster-Galaxy Connection, ASP Conf. Ser. \#48, Eds: G.H. Smith \& J.P. Brodie, Astronomical Society of the Pacific, 387.

Mateo, M., \& Hodge, P., 1986, Astrophys. J. Suppl. Ser., 60, 893.

Mateo, M., \& Hodge, P., 1987, Astrophys. J., 320, 626.

Mateo, M., \& Schecter, P., 1989, in Proceedings of the First ESO ST-ECF Data Analysis Workshop, Eds: P.J. Grosbøl, F. Murtagh, \& R.M., Warmels, ESO, Garching, 69.

Mateo, M., Udalski, A., Szymanik, M., Kaluzny, J., Kubiak, M., \& Krzemiński, W., 1994, submitted to Astrophys. $J$.

Mathewson, D.S., 1985, Proc. Astron. Soc. Aust., 6, 104.

Mathewson, D., \& Ford, V., 1984, in Structure and Evolution of the Magellanic Clouds, IAU Symposium \#108, Eds: S. van den Bergh \& K. de Boer, Reidel, Dordrecht, 127.y

Mathewson, D.S., Ford, V.L., Schwarz, M.P., \& Murray, J.D., 1979 in The Large-scale Characteristics of the Galaxy, IAU Symposium \#84, Ed: W.P. Burton, 547.

Mathewson, D.S., Wayte, S.R., Ford, V.L., \& Ruan, K., 1987, Proc. Astron. Soc. Aust., 7, 19.

Meeus, J., 1982, Astronomical Formulae for Calculators, Willman-Bell, Richmond, Virginia.

Menzies, J.W., Banfield, R.M., Cousins, A.W.J., \& Laing, J.D., 1989, S. Afr. Astron. Obs. Circ., 13, 1 .

Menzies, J.W., Marang, F., Laing, J.D., Coulson, I.M., \& Engelbrecht, C.A., 1991, Mon. Not. R. Astron. Soc., 248, 642 .

Mermilliod, J.C., 1976, Astron. Astrophys. Suppl. Ser., 24, 159.

Meurer, G.R., Cacciari, C., \& Freeman, K.C., 1990, Astron. J., 99, 1124.

Meylan, G., 1988, Astrophys. J., 331, 718.

Meylan, G., 1989, Astron. Astrophys., 214, 106.

Meylan, G., \& Djorgovski, S., 1987, Astrophys. J., 322, L91.

Meylan, G., Dubath, P., Mayor, M., \& Magion, P., 1989, Messenger, 55, 55.

Meylan, G., Dubath, P., \& Mayor, M., 1991, in The Magellanic Clouds, IAU Symposium \#148, Eds: R. Haynes \& D. Milne, Kluwer, Dordrecht, 211.

Meynet, G., Mermilliod, J-C., \& Maeder, A., 1993, Astron. Astrophys., 98, 447.

Moore, B., \& Davis, M., 1994, Preprint.

Moore, C.H., 1974, Astron. Astrophys, Suppl. Ser. ,15, 497.

Morgan, D.H., Hatzidimitriou, D., Kontizas, M., \& Kontizas, E., 1989, in Recent Developments of Magellanic Cloud Research, Eds: K.S. de Boer, F. Spite, \& G. Stasinska, Observatoire de Paris, 95.

Morgan, W.W., \& Keenan, P.C., 1973, Ann. Rev. Astron. Astrophys., 11, 29. 
Mould, J.R., \& Aaronson, M., 1983, Astrophys. J., 232, 421.

Mould, J.R., Da Costa, G.S., \& Crawford, M.D., 1984, Astrophys. J., 280, 595.

Mould, J.R., Xystus, D.A., \& Da Costa, G.S., 1993, Astrophys. J., 408, 108.

Murai, T., \& Fujimoto, M,, 1980, Publ. Astron. Soc. Jpn., 32, 581.

Murai, T., \& Fujimoto, M., 1986, Astrophys. Space. Sci., 119, 169.

Nankivell, G.R., \& Rumsey, N.J., 1986, in Instrumentation and Research Programmes for Small Telescopes, IAU Symposium \#118, Eds: J.B. Hearnshaw \& P.L. Cottrell, Reidel, Dordrecht, 101.

Nelles, B., \& Richtler, T. 1984, in Structure and Evolution of the Magellanic Clouds, IAU Symposium \#108, Eds: S. van den Bergh \& K.S. de Boer, Reidel, Dordrecht, 33.

Nelson, M., \& Hodge, P., 1983, Publ. Astron. Soc. Pac., 95, 5.

Norris, J., 1983, Preprint as reported by Van den Bergh (1984).

North, P., 1980, Astron. Astrophys. Suppl. Ser., 41, 497.

Olszewski, E., 1984, Astrophys. J., 284, 108.

Olszewski, E., 1988, in Globular Cluster Systems in Galaxies, IAU Symposium \#126, Eds: J.E. Grindlay \& A.G.C. Philips, Reidel, Dordrecht, 159.

Olszewski, E.W., Mateo, M., \& Madore, B.F., 1991, in The Formation and Evolution of Star Clusters, ASP Conf. Ser. \#13, Ed: K. Janes, Astronomical Society of the Pacific, 588.

Pandey, A.K, Mahra, K.W.,\& Sagar, R., 1991, The Formation and Evolution of Star Clusters, ASP Conf. Ser. \#13, Ed: K. Janes, Astronomical Society of the Pacific, 170.

Park, S.K., \& Miller, K.W., 1988, Communications of the ACM, 31, 1192.

Parker, J.W., Garmany, C.D., Massey, P., \& Walbourn, N.R., 1992, Astron. J., 103, 1205.

Penny, A.J., \& Dickens, R.J., 1989, Mon. Not. R. Astron. Soc., 220, 845.

Perry, C.L., Franklin, C.B. Jnr., Landolt, A.U., \& Crawford, D.L., 1976, Astron. J., 81, 632.

Petersen, C.J., \& King, I.R., 1975, Astron. J., 80, 427.

Phelps, R.L., \& Janes, K.A., 1993, Astron. J., 106, 1870.

Photometrics Ltd., 1989, CCDs for Quantitative Electronics Imaging, Promotional Booklet.

Piotto, G., 1991, The Formation and Evolution of Star Clusters, ASP Conf. Ser. \#13, Ed: K. Janes, Astronomical Society of the Pacific, 200.

Piskunov, A.E., 1976, Nauch. Inf., 22, 47.

Pollard, K., 1989a, Mt. John Photometrics CCD System: Use E Performance Note \#2.

Pollard, K., 1989b, Mt. John Photometrics CCD System: Use \& Performance Note \#3.

Press, F., \& Siever, R., 1982, Earth, Third Edition, W.H. Freeman, San Francisco.

Press, W.H., Teukolsky, S.A., Vetterling, W.T., \& Flannery, B.P., 1992, Numerical Recipes in C: The Art of Scientific Programming, Second Edition, Cambridge University Press.

Pritchard, J.D., 1993, Mt. John Photometrics CCD System: Use E Performance Note \#12. 
Rabin, D., 1982, Astrophys. J., 261, 85.

Ratnatunga, K.U., \& Bahcall, J.N., 1985, Astrophys. J. Suppl. Ser., 59, 63.

Reid, N., 1992, Mon. Not. R. Astron. Soc., 257, 257.

Richtler, T., \& Nelles, B., 1983, Astron. Astrophys., 119, 57.

Richtler, T., \& Seggeweiss, W., 1988, in Globular Cluster Systems in Galaxies, IAU Symposium \#126, Eds: J.E. Grindlay \& A.G. Davis Philip, Kluwer, Dordrecht, 553.

Richtler, T., Spite, M., \& Spite, F., 1989, Astron. Astrophys., 225, 351.

Rieke, G.H., \& Lebofsky, M.J., 1985, Astrophys. J., 288, 615.

Robertson, J.W., 1974, Astron. Astrophys. Suppl. Ser., 15, 261.

Rogers, D.F., 1988, Procedural Elements for Computer Graphics, Fourth Printing, McGraw-Hill, Singapore.

Sagar, R., 1987, Mon. Not. R. Astron. Soc., 228, 483.

Sagar, R., \& Richtler, T., 1991, Astron. Astrophys., 250, 324.

Sagar, R., Piskunov, A.E., Myakutin, V.I., \& Joshi, U.C., 1986, Mon. Not. R. Astron. Soc., 220,383 .

Sagar, R., Richtler, T., \& de Boer, K.S., 1991a, Astron. Astrophys., 249, L5.

Sagar, R., Richtler, T., \& de Boer, K.S., 1991b, Astron. Astrophys. Suppl. Ser., 90, 387.

Salpeter, E.E., 1955, Astrophys. J., 121, 161.

Sandage, A.R., \& Tammann, G., 1974, Astrophys. J., 190, 525.

Santos Jr., J.F.C., \& Bica, E., 1993, Mon. Not. R. Astron. Soc., 260, 915.

Sauvage, M., \& Vigroux, L., 1991, in The Magellanic Clouds, IAU Symposium \#148, Eds: R. Haynes \& D. Milne, Kluwer, Dordrecht, 407.

Scalo, J.M., 1986, Fundam. Cosmic Phys., 11, 1

Schaerer, D., Meynet, M., Maeder, A., \& Schaller, G., 1993, Astron. Astrophys., 98, 523.

Schaller, G., Shaerer, G., Meynet, G., \& Maeder, A., 1992, Astron. Astrophys., 96, 269.

Schecter, P., Mateo, M., \& Saha, A., 1993, Publ. Astron. Soc. Pac., 105, 1342.

Schild, R.E., 1970, Astrophys. J., 161, 855.

Schommer, R.A., 1991, in The Magellanic Clouds, IAU Symposium \#148, Eds: R. Haynes \& D. Milne, Kluwer, Dordrecht, 171.

Schommer, R.A., Olszewski, E.W., \& Aaronson, M., 1984, Astrophys. J., 285, L53.

Scott, J.L., 1976, Unpublished M.Sc Thesis, University of Canterbury, Christchurch.

Searle, L., 1984, in Structure and Evolution of the Magellanic Clouds, IAU Symposium \#108, Eds: S. van den Bergh \& K.S. de Boer, Reidel, Dordrecht, 13.

Searle, L., Wilkinson, A., \& Bagnuolo, W.G., 1980, Astrophys. J., 239, 803.

Seggewiss, W., \& Richtler, T., 1989, in Recent Developments of Magellanic Cloud Research, Eds: K.S. de Boer, F. Spite, \& G. Stasinska, Observatoire de Paris, 45. 
Seidel, E., Da Costa, G.S., \& Demarque, P., 1987, Astrophys. J., 313, 192.

Shapley, H., 1918, Astrophys. J., 48, 154.

Shapley, H., 1924, Harv. Coll. Obs. Bull., 225, 1.

Shapley, H., 1930a, Harv. Coll. Obs. Bull., 884, 1.

Shapley, H., 1930b, Star Clusters, McGraw-Hill, New York.

Shapley, H., 1953, P.N.A.S., 39, 1161.

Shapley, H., \& Ames, M., 1932, Annals of the Harvard College Observatory, 88, 41.

Shapley, H., \& Lindsay, E.M., 1963, Ir. Astron. J., 6, 74.

Shapley, H., \& Nail, V.M., 1953a, P.N.A.S., 39, 1.

Shapley, H., \& Nail, V.M., 1953b, P.N.A.S., 39, 358.

Sher, D., 1965, Mon. Not. R. Astron. Soc., 129, 17.

Shobbrook, R.R., 1984, Mon. Not. R. Astron. Soc., 206, 273.

Slettebak, A., 1985, Astrophys. J. Suppl. Ser, 59, 769.

Smith, H.A., Searle, L., \& Manduca, A., 1988, in Globular Cluster Systems in Galaxies, IAU Symposium\# 126, Eds: A.G.D. Philip \& J.E. Grindlay, Reidel, Dordrecht, 563.

Spite, M., 1989, in Recent Developments of Magellanic Cloud Research, Eds: K.S de Boer, F. Spite, \& G. Staninska, Obervatoire de Paris, Paris, 37.

Spite, M., Cayrel, R., Francois, P., Richtler, T., \& Spite, F., 1986, Astron. Astrophys., 168, 197.

Spitzer Jr., L., 1987, Dynamical Evolution of Globular Clusters, Princeton Series in Astrophysics, Princeton University Press, New Jersey.

Spitzer Jr., L., \& Hart, M.H., 1971, Astrophys. J., 163, 399.

Stallings, W., 1990, Computer Organisation and Architecture, Second Edition, Macmillan, New York.

Stauffer, J., Klemola, A., Prosser, C., \& Probst, R., 1991, Astron. J., 101, 980.

Stecklum, B., 1985, Astron. Nachr., 306, 45.

Sterken, C., \& Manfroid, J., 1992, Astronomical Photometry, Kluwer, Dordrecht.

Stetson, P.B., 1987, Publ. Astron. Soc. Pac., 99, 191.

Stetson, P.B., 1989, Highlights of Astronomy, 8, 635.

Stetson, P.B., 1990, Publ. Astron. Soc. Pac., 102, 932.

Stetson, P.B., 1991, in The Formation and Evolution of Star Clusters, ASP Conf. Ser. \#13, Ed: K. Janes, Astronomical Society of the Pacific, 88.

Stobie, R.S., 1980a, J. Br. Interplanet. Soc., 33, 323.

Stobie, R.S., 1980b, Proc. SPIE, 264, 208.

Stowe, L.L., Carey, R.M., \& Pellegrino, P.P., 1992, Geophysical Research Letters, 19, 159. 
Straižys, V., 1973, Bulletin of the Vilnius Observatory, 36, 3.

Straižys, V., 1974, Astron. Astrophys., 36, 435.

Straižys, V., 1992a, Multicolor Stellar Photometry, Vol 15, Astronomy \& Astrophysics Series, Pachart, Tucson.

Straižys, V., 1992b, Baltic Astronomy, 1, 107.

Straižys, V., \& Sviderskienè, Z., 1972, Bull. Vilnius. Astron. Obs., 35, 1.

Strömgren, B., 1963, Basic Astronomical Data, University of Chicago Press, Chicago.

Strömgren, B., 1966, Ann. Rev., 4, 433.

Stryker, L., 1981, Unpublished PhD Thesis, Yale University (cited by Stryker 1983).

Stryker, L., 1983, Astrophys. J., 266, 82.

Stryker, L., 1984, in Structure and Evolution of the Magellanic Clouds, IAU Symposium \#108, Eds: S. van den Bergh \& K.S. de Boer, Reidel, Dordrecht, 79.

Subramaniam, A., Sagar, R., \& Bhatt, H.C., 1993, Astron. Astrophys, 273, 100.

Sulentic, J.W., \& Tifft, W.G., 1973, The Revised New General Catalogue of Nonstellar Astronomical Objects, University of Arizona Press, Tucson.

Sullivan, D.J., 1990, Pers. Comm.

Sullivan, D.J., 1993, in Precision Photometry, Eds: D. Kilkenny, E. Lastovica, \& J. W. Menzies, SAAO, 159 .

Sweigart, A.V., \& Gross, P.G., 1976, Astrophys. J. Suppl. Ser., 32, 367.

Taff, L.G., 1974, Astron. J., 94, 1280.

Tarrab, I., 1983, Astron. Astrophys., 109, 285.

Terlevich, R., \& Melnick, J., 1985, Mon. Not. R. Astron. Soc., 213, 841.

Thackeray, A.D., 1963, Adv. Astron. Astrophys., 2, 263.

Thackeray, A.D., \& Wesselink, A.J., 1953, Nature, 171, 693.

Thackeray, A.D., \& Wesselink, A.J., 1955, Observatory, 75, 33.

Tinsley, B.M., 1980, Fundam. Cosmic Phys., 5, 287.

Tobin, W., 1990, Mt. John Photometrics CCD System: Use \& Performance Note \#1.

Tobin, W., 1992a, Southern Stars, 34, 421.

Tobin, W., 1992b, Mt. John Photometrics CCD System: Use \& Performance Note \#9

Tobin, W., 1993, Mt. John Photometrics CCD System: Use E̛ Performance Note \#11

Tobin, W., Kershaw, G.M., Ritchie, R.A., Ma, L., Graham, G.J., \& Hemmingsen, S.B., 1993, in Stellar Photometry - Current Techniques and Future Developments - Poster Papers, IAU Colloquium No. 136, Eds: I. Elliott \& C. J. Butler, Cambridge University Press, 153.

Tody, D.C., 1986, in "Instrumentation in Astronomy IV", Proc. SPIE, Ed: D.L. Crawford, 627, 733 . 
Tucholke, H.J., \& Hiesgen, M. 1991, in The Magellanic Clouds, IAU Symposium \#148, Eds: R. Haynes \& D. Milne, Kluwer, Dordrecht, 491.

Vallenari, A., Chiosi, C., Bertelli, G., Meylan, G., \& Ortolani, S., 1991, Astron. Astrophys. Suppl. Ser., 87, 517.

Vallenari, A., Apracio, A., Fagotto, F., Chiosi, C., Ortolani, S., \& Meylan, G., 1994, Astron. Astrophys., 284, 447

Vandenberg, D.A., 1981, Astron. Astrophys. Suppl. Ser., 46, 79.

Vandenberg, D.A., 1985, Astrophys. J. Suppl. Ser., 558, 711.

van den Bergh, S., 1981, Astron. Astrophys. Suppl. Ser., 46, 79.

van den Bergh, S., 1983, Publ. Astron. Soc. Pac., 95, 839.

van den Bergh, S., 1984, in Structure and Evolution of the Magellanic Clouds, Eds: S. van den Bergh \& K.S. de Boer, 1.

van den Bergh, S., 1991, Astrophys. J., 369, 1.

van den Bergh, S., \& Morbey, C.L., 1984, Astrophys. J., 283, 598.

Wadsworth, A., \& Cole, C.S., 1994, submitted to Southern Stars.

Walker, M.F., 1974, Mon. Not. R. Astron. Soc., 169, 199.

Walker, M.F., 1987, Publ. Astron. Soc. Pac., 84, 365.

Wannier, P. \& Wrixon, G.T., 1972, Astrophys. J., 179, 461.

Webster, B.L., 1965, in Symp. on Magellanic Clouds, Eds: J.V. Hindman \& B.E. Westerlund, Mt. Stromlo, ANU, Canberra, 29.

Wells, D.C, Greisen, E.W. \& Harten, R.H., 1981, Astron. Astrophys. Suppl. Ser., 44, 363.

Wenger, M., Bonnarel, F., Lesteven, S., \& Watson, J.M., 1990, SIMBAD User's Guide, Centre de Données astronomiques de Strasbourg, Strasbourg.

West, S.R.D., 1991, Unpublished M.Sc Thesis, University of Canterbury, Christchurch.

West, S.R.D., Tobin, W., \& Gilmore, A.C., 1992, Mon. Not. R. Astron. Soc., 254, 419.

Westerlund, B.E., 1964, in The Galaxy and the Magellanic Clouds, IAU Symposium \#20, Eds: F.J. Kerr \& A.W. Rodgers, Australian Academy of Sciences, Canberra, 239.

Westerlund, B.E., 1989, in Recent Developments of Magellanic Cloud Research, Eds: K.S. de Boer, F. Spite, \& G. Stasinska, Observatoire de Paris, Paris, 159.

Westerlund, B.E., 1990, Astron. Astrophys. Rev., 2, 29.

Wielin, R., \& Fuchs, B., 1984, in The Milky Way Galaxy, IAU Symposium \#106, Eds: H. Van Woerden, R.J. Allen \& W.B. Burton, Reidel, Dordrecht, 481.

Wilson, R.E., 1918, Lick Obs. Bull., 13, 187.

Wilson, R.E., 1944, Publ. Astron. Soc. Pac., 56, 102.

Wolf, B., \& Reiermann, A., 1989, in Recent Developments of Magellanic Cloud Research, Eds: K.S. de Boer, R. Spite, \& G. Stasinska, Observatoire de Paris, Paris, 23.

Young, A.T., 1974, in Astrophysics Part A: Optical and Infrared, Volume 12 of 'Methods of Experimental Physics', Ed: N. Carleton, Academic Press, New York, 123. 
Young, A.T., 1993, in Precision Photometry, Eds: D. Kilkenny, E. Lastovica, \& J. W. Menzies, SAAO, 25 .

Zdanavičus, K., Sūdžius, J., Sviderskienè, Z., Straižys, V., Burnašov, V., Drazdys, R., Bartkevičius, A., Kakaras, G., Kavalianskaite, G., \& Jasevičius, V., 1969, Bull. Vilnius Obs., 26, 3 .

Zepka, A.F., \& Dottori, H.A., 1987, Rev. Mexicana Astron. Astrof., 14, 172. 